\title{
WHOI-86-44
}

\section{Description of W.H.O.I. Rock Dredge Samples Volume II}

\author{
Edited by
}

J. E. Broda and P. J. Andrew

Woods Hole Oceanographic Institution

Woods Hole, Massachusetts 02543

September 1986

\section{Technical Report}

\section{Prepared for the National Science Foundation} under grant No. OCE 85-19889.

Reproduction in whole or in part is permitted for any purpose of the United States Government. This report should be cited as: Woods Hole Oceanog. Inst. Tech. Rept. WHOI-86-44.

Approved for publication; distribution unlimited.

\section{Approved for Distribution:}

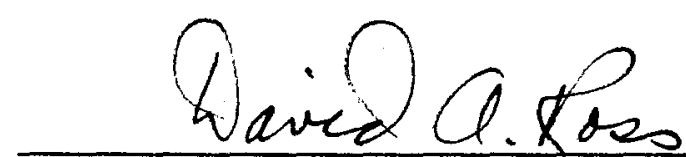

David A. Ross, Chairman

Department of Geology \& Geophysics 


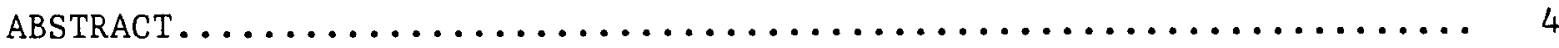

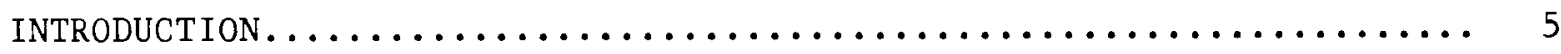

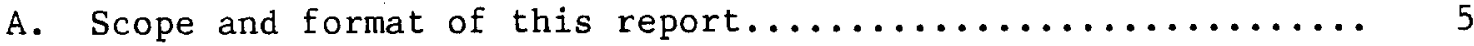

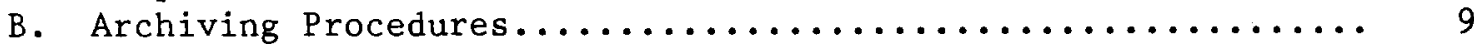

C. Digitization of Dredge Sample Data.................. 10

D. Sample Distribution Policy......................... 12

E. Responsibilities of Persons Receiving Samples.............. 13

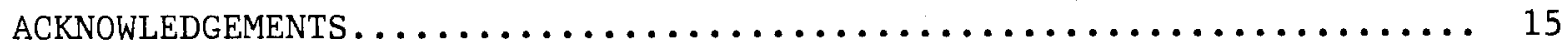

DESCRIPTIONS OF W.H.O.I. ROCK DREDGE SAMPLES, VOLUME II.......... 39

\section{ATLANTIS II}

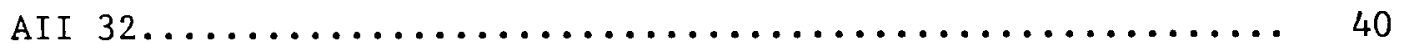

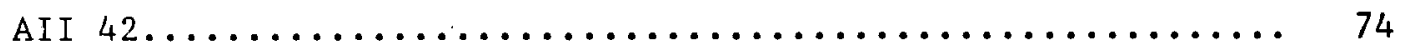

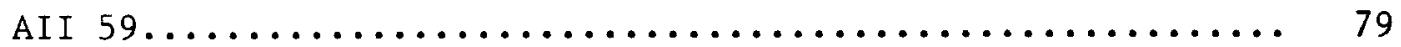

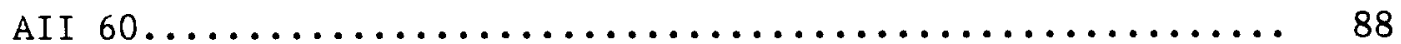

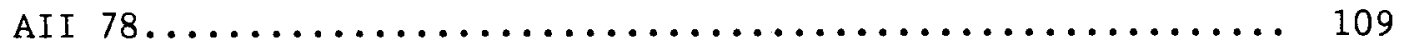

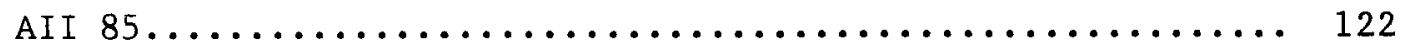

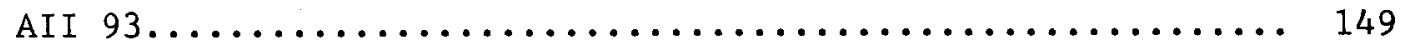

CHAIN

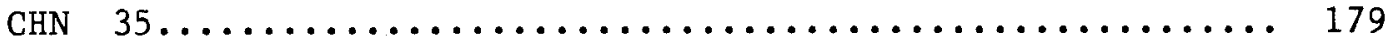

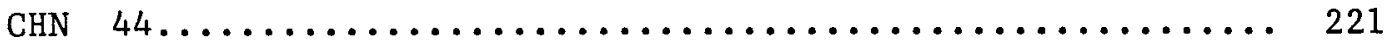

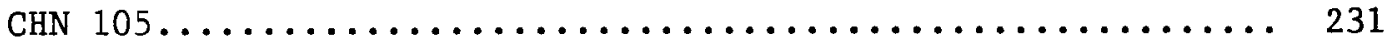

\section{$\underline{\text { GILLIS }}$}

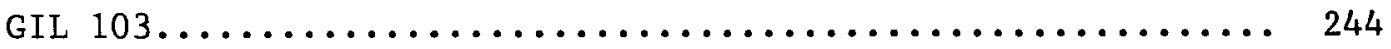

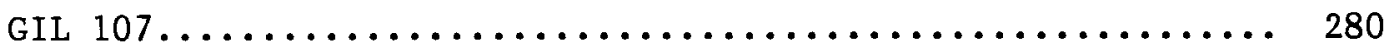

\section{ISLAS ORCADAS}

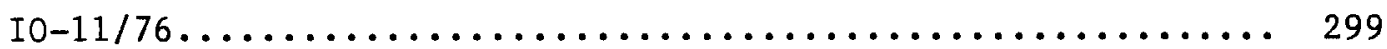

$\underline{\text { KNORR }}$

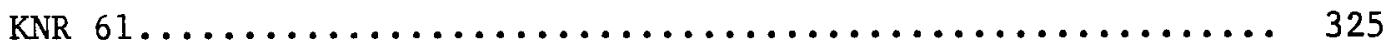

\section{OCEANUS}

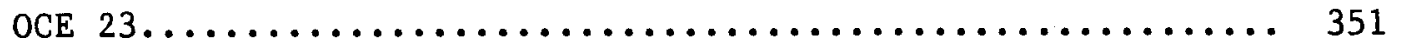

\section{RESEARCHER}

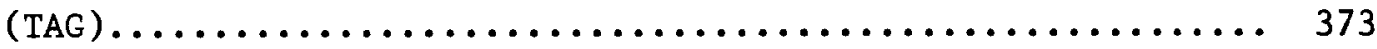


LIST OF TABLES

Page

TABLE 1. CRUISE INDEX OF DESCRIPTIONS OF W.H.O.I. ROCK DREDGE SAMPLES, VOLUMES I-III.................... 6

TABLE 2. NOTES ON DETAILED ROCK DESCRIPTIONS............. 16

TABLE 3. COMPUTER LISTING OF W.H.O.I. DREDGES, ARRANGED

BY MARSDEN SQUARE (Includes Map).............. 17 


\section{ABSTRACT}

This report is Volume II in the series of reports entitled "DESCRIPTIONS OF W.H.O.I. ROCK DREDGE SAMPLES". This volume represents the final step in the major effort to catalog and prepare initial descriptions for all rock dredge samples in the W.H.O.I. Sea Floor Samples Collection, and to distribute this information throughout the scientific community. The distribution of this report completes the initial description of the backlog of W.H.O.I. Dredge Samples. The data contained in this volume is an accumulation and transcription of initial descriptions made at sea, along with post-cruise descriptions performed at the $1 \mathrm{ab}$ by the curatorial staff.

Volume II contains individual stations executed during the period 1963 through 1986. It also presents a digitized listing of all dredge station data for the entire W.H.O.I. Dredge Collection through 1986. The data are sorted by Marsden Squares and can serve as a regional index for all rock descriptions included in Volumes I-III. 


\section{INTRODUCTION}

\section{A. Scope \& Format of this Report}

"Descriptions of W.H.O.I. Rock Dredge Samples" is an ongoing series of reports that present station data and detailed descriptions of the dredge samples in the W.H.O.I. Sea Floor Samples Collection.

The first three volumes in this series represent the completion of a major effort to describe the entire back-1og of 790 dredge stations in the W.H.O.I. dredge collection as of 1986. Volume 1 includes a variety of cruises with a wide geographic distribution executed during the period 1960 to 1977 (Table 1). Many of the cruises in Volume I preceded the establishment of a central archiving facility in Woods Hole. The material in Volume I has been prepared by the W.H.O.I. curatorial staff who have painstakingly verified station locations and slabbed and described representative suites from each of these dredge hauls.

Volume III includes material collected from September 1978 - December 1980 and represents a new procedure where most of the descriptive work is executed onboard ship by participating scientists. As such, it represents a model for future dredging cruises and descriptive reports and, therefore, was prepared first (in May 1981). Volume II fills the gap and include the years 1966 through 1986.

This volume is organized alphabetically by research vessel and then chronologically within each of the vessels' many cruises. Each cruise "chapter" includes a generalized map of the ship's track, a digitized summary of the station location and samples recovered, and finally the detailed descriptions. Notes on the descriptive format and abbreviations used are provided in Table 2 (Notes on Detailed Rock Descriptions). 
TABLE 1

CRUISE INDEX OF DESCRIPTIONS OF W.H.O.I. ROCK DREDGE

SAMPLES, VOLUMES I-III

Cruise No. General Location Date

VOLUME I

ATLANTIS

260 Hydrographer Canyon

Oct.

June

266 Blake Plateau

280 New England Seamounts

281 New England Seamounts

June

June

Aug .

60

296 New England Seamounts

61

162

' 62

163

ATLANTIS II

1 Continental Slope off New York

Feb. $\quad 163$

11 Puerto Rico Trench

13 Mid-Atlantic Ridge

15 Bitter Lakes Regions-Suez Cana1/Continental

July

$' 64$

Sept.

' 64

Slope of Ethiopia

42 Mid-Atlantic Ridge

$73 \mathrm{Mid}-A t$ lantic Ridge

86 Continental Rise off Atlantis Canyon

92 Median Valley, Mid-Atlantic Ridge

Feb.-Mar. ' '65

July $\quad 168$

Nov. $\quad 172$

Mar. $\quad 175$

Sept. $\quad ' 75$

Leg 693 Indian Ocean Triple Junction/Banda Sea

Feb., Oct. $\quad 176$

96 Kane Fracture Zone

Nov.

$\cdot 77$

CHAIN

7 New England Seamounts

9 Plantagenet Bank (S. of Bermuda)

11 Caribbean Sea

13 Rockal1 Bank/Continental Slope of England

19 Puerto Rico Trench

21 New England Seamounts/Mid-Atlantic Ridge

34 Puerto Rico Trench

36 Barracuda Fault

39 Abyssa1 Hil1s S.E. of Bermuda

43 Seychelles (Indian Ocean)/Mid-Atlantic Ridge

46 Mona Canyon/Blake Plateau

52 Blake Plateau

57 Puerto Rico Trench

58 Bermuda Rise

61 Mediterranean/Red Seas

75 Caribbean-Aves Ridge, Mid-Atlantic Ridge

May

oct. $\quad 159$

Feb. $\quad 60$

Sept. $\quad 160$

June $\quad ' 61$

Aug. $\quad ' 61$

Dec. $\quad 62$

June '63

Sept. $\quad 163$

May, Aug. '64

Feb. $\quad 65$

Sept. $\quad 65$

Apri1 $\quad 166$

May $\quad 166$

Aug. $\quad 166$

oct. ' 67 
TABLE 1 (Cont'd)

Cruise No.

General Location

Date

VOLUME I (Cont'd)

CHAIN (Cont'd)

82 Mid-Atlantic Ridge

Aug.

168

100 Samoan Passage, Ninety East Ridge, Slope of $f$ Australia

115 Bouvet Triple Junction

Apr., Sept. ' 71

Feb. $\quad ' 74$

119 Eastern Mediterranean Sea

Apr.

175

GOSNOLD

73 Blake Plateau

Ju1y

.65

97 Off Jamaica

Mar.

' 67

$\underline{\text { KNORR }}$

42 Mid-Atlantic Ridge

54 Cayman Trough

Aug. $\quad 174$

Dec. $\quad ' 76$

\section{VOLUME II}

\section{ATLANTIS II}

32 Mid-Atlantic Ridge $42^{\circ}-43^{\circ} \mathrm{N}$

Ju1y

167

42 Romanche Fracture Zone

June

.68

59 Mid-Atlantic Ridge

Dec.

$\cdot 70$

60 Romanche Fracture Zone

June

$\cdot 71$

78 Mid-Atlantic Ridge, Kane Fracture Zone

oct.

$\cdot 73$

85 New England Seamounts

Leg 293 Walvis Ridge

Sept. $\quad 174$

Nov.

$\cdot 75$

CHAIN

35 St. Paul's Rocks

44 Mid-Atlantic Ridge, $22^{\circ} \mathrm{N}$

105 King's Trough

Mar.

Oct.

GILLIS

103 Mid-Atlantic Ridge

Aug.

178

107 Tamayo Fracture Zone

oct.

$\cdot 79$

ISLAS ORCADAS

11/76 Far South Atlantic

Nov.

$\cdot 76$ 
TABLE 1 (Cont'd)

Cruise No.

General Location

Date

VOLUME II (Cont'd)

$\underline{\text { KNORR }}$

61 New England Seamounts

Nov.

176

$\underline{\text { OCEANUS }}$

23 Cayman Trough

Apr .

$\cdot 77$

RESEARCHER

(Tag) Mid-Atlantic Ridge

Ju1y

' 82

\section{VOLUME III}

\section{ATLANTIS II}

107-6 Deep South Atlantic

107-7 Deep South Atlantic

Mar., Apr. ' 80

May

$' 80$

GILLIS

104 Kane Fracture Zone

Sept .

$\cdot 78$

$\underline{\text { KNORR }}$

79 Kane Fracture Zone

June

$' 80$

VULCAN

5 Far South Atlantic

Dec.

' 80 


\section{B. Archiving Procedures}

The standard format for archiving W.H.O.I. rock dredge samples is as follows: Each rock (and slabs derived from it) is labelled with the ship symbol, cruise number, and station number of the dredge haul, followed by a unique number for every rock within the dredge. For example, CHN 115 - 26 16 refers to rock 16 from station 26 of cruise Chain 115 . Some samples are also labelled with their respective dredge numbers as well as with the information previously described. Thus AII 32-1-2-5 refers to rock number 5 from station number 1 , dredge number 2 of cruise Atlantis II 32. The assignment of identification numbers for individual rocks from a given dredge station was done on a random basis. Thus rocks numbered in order do not necessarily possess similar lithologies.

In describing a dredge haul all specimens of reasonable size were sorted, and many were slabbed to obtain a fresh surface for description. If the dredge haul was very large and homogeneous, a representative suite of rocks was selected and described. Al1 rock samples from this collection should be referenced in the literature by their Woods Hole identification number.

As a rule the station positions listed in this report approximate the beginning of the station or the best estimate between several fixes of the dredge's location during the station. The quality of these fixes vary with the accuracy and precision in ship positioning capabilities. In recent years the end depth or $f$ ix has been taken when the dredge leaves the sea floor, not where it actually arrives on deck. All depths included in this volume are given in corrected meters. More detailed navigation information such as additional fixes or length of wire-out during stations is available from the curator's office. Detailed sampling records (including names of investigators, 
proposed analyses, and copies of published papers) are also kept at the curator's office.

\section{Digitization of Dredge Sample Data}

A11 logistical information about geological samples in the W.H.O.I. Sea Floor Samples Collection is stored on disc and is accessible through a rapid retrieval computer program. This data can also be obtained through the NGDC by writing to the following address or by calling them directly:

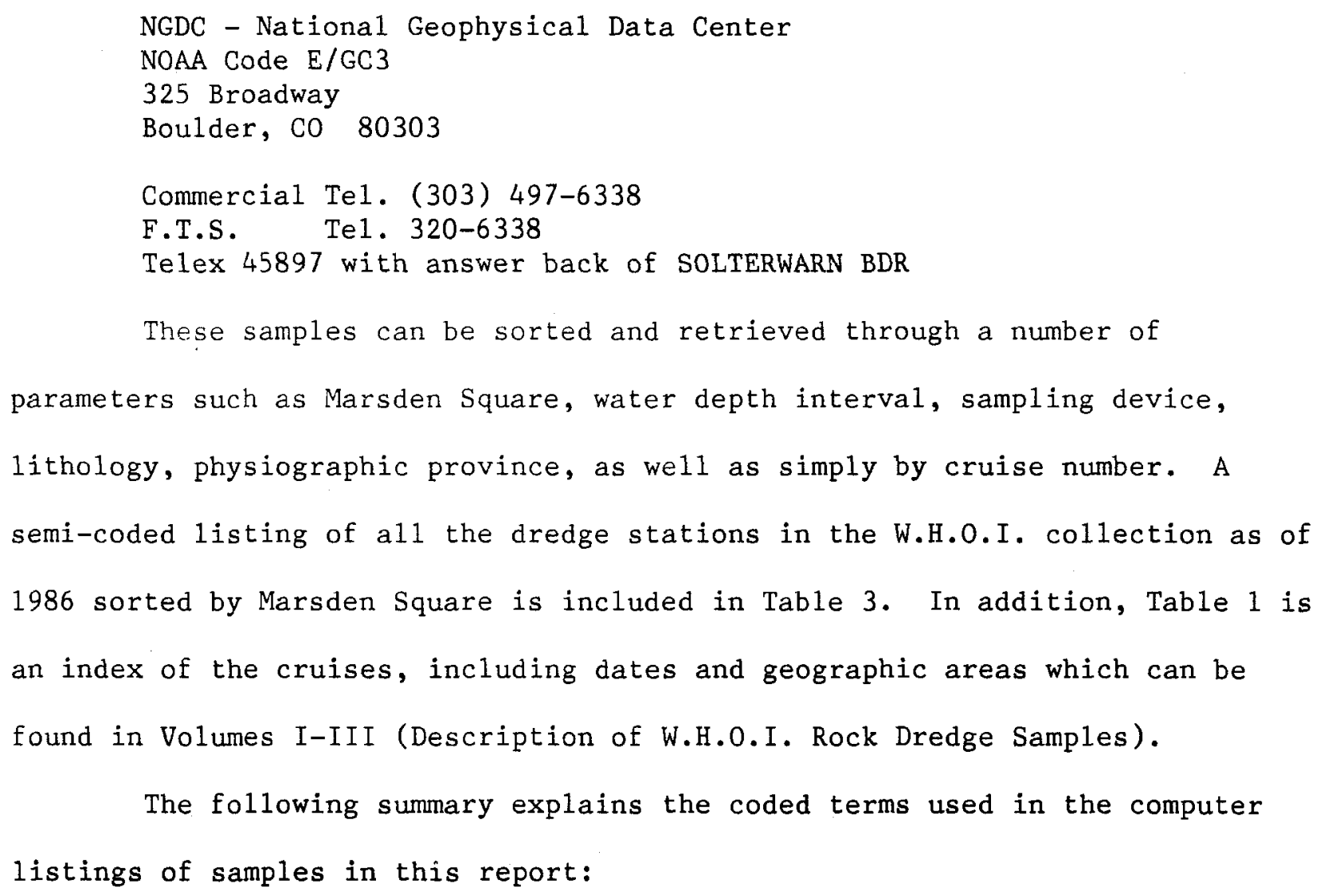


C. Digitization of Dredge Sample Data. (Cont'd)

Sample Recovery Devices:

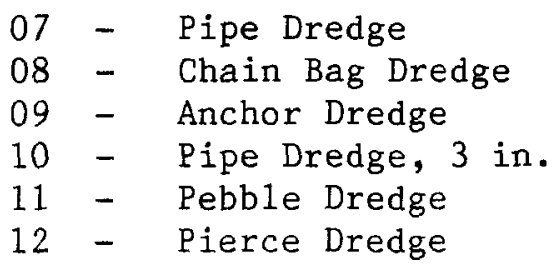

Fix Types:

Types of navigational equipment used to determine the sample location are as follows:

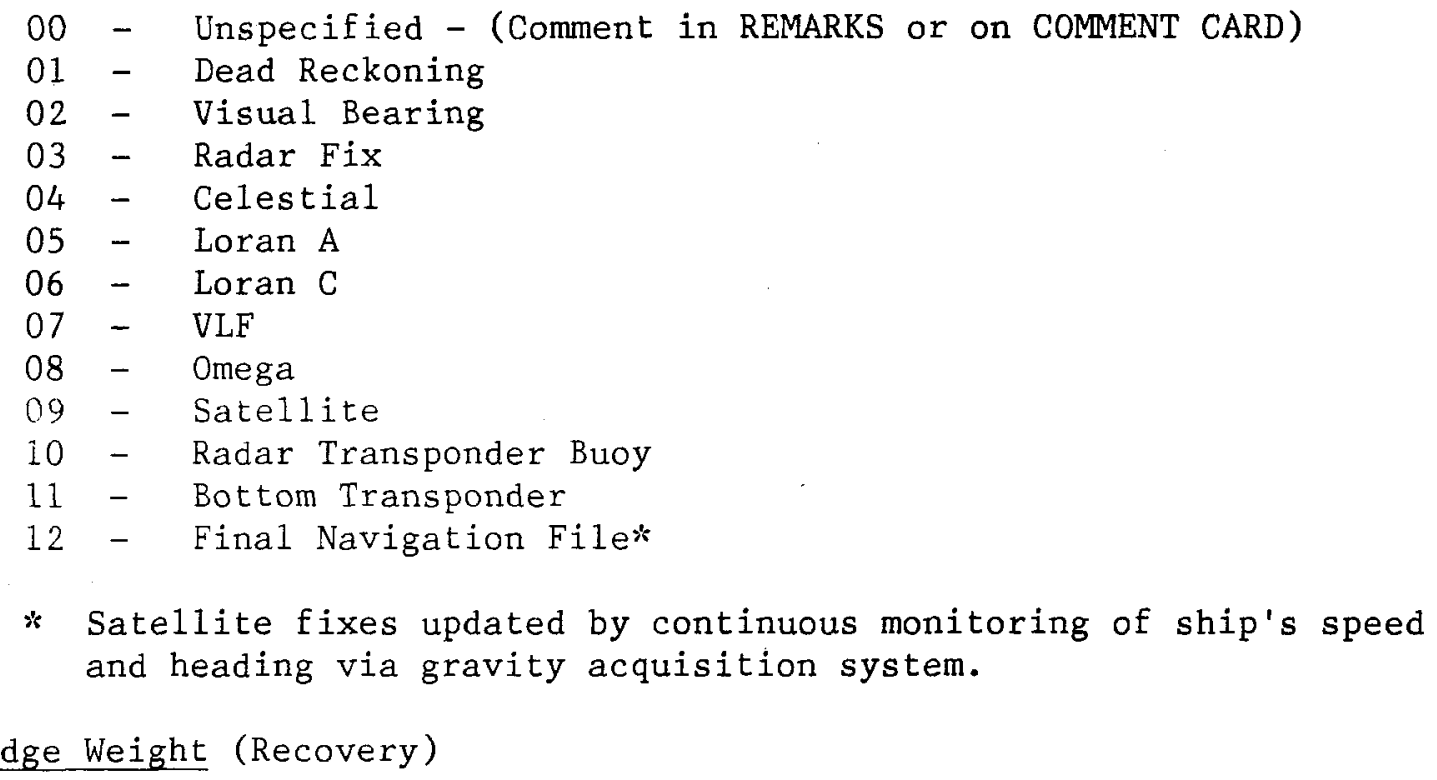

These are always quoted in kilograms unless the code specifically includes a $G$ for grams (i.e. $010 G=10$ grams).

\section{Physiographic Province}

A general physiographic location has been assigned to each of the samples 1isted, and can be decoded as follows:

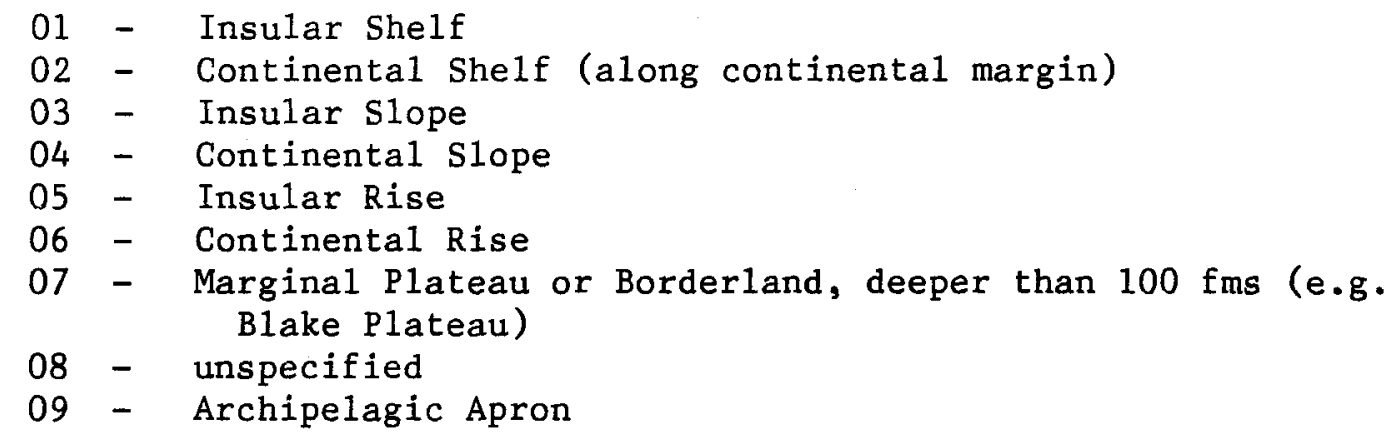


C. Digitization of Dredge Sample Data. (Cont'd)

Physiographic Province (Cont'd)

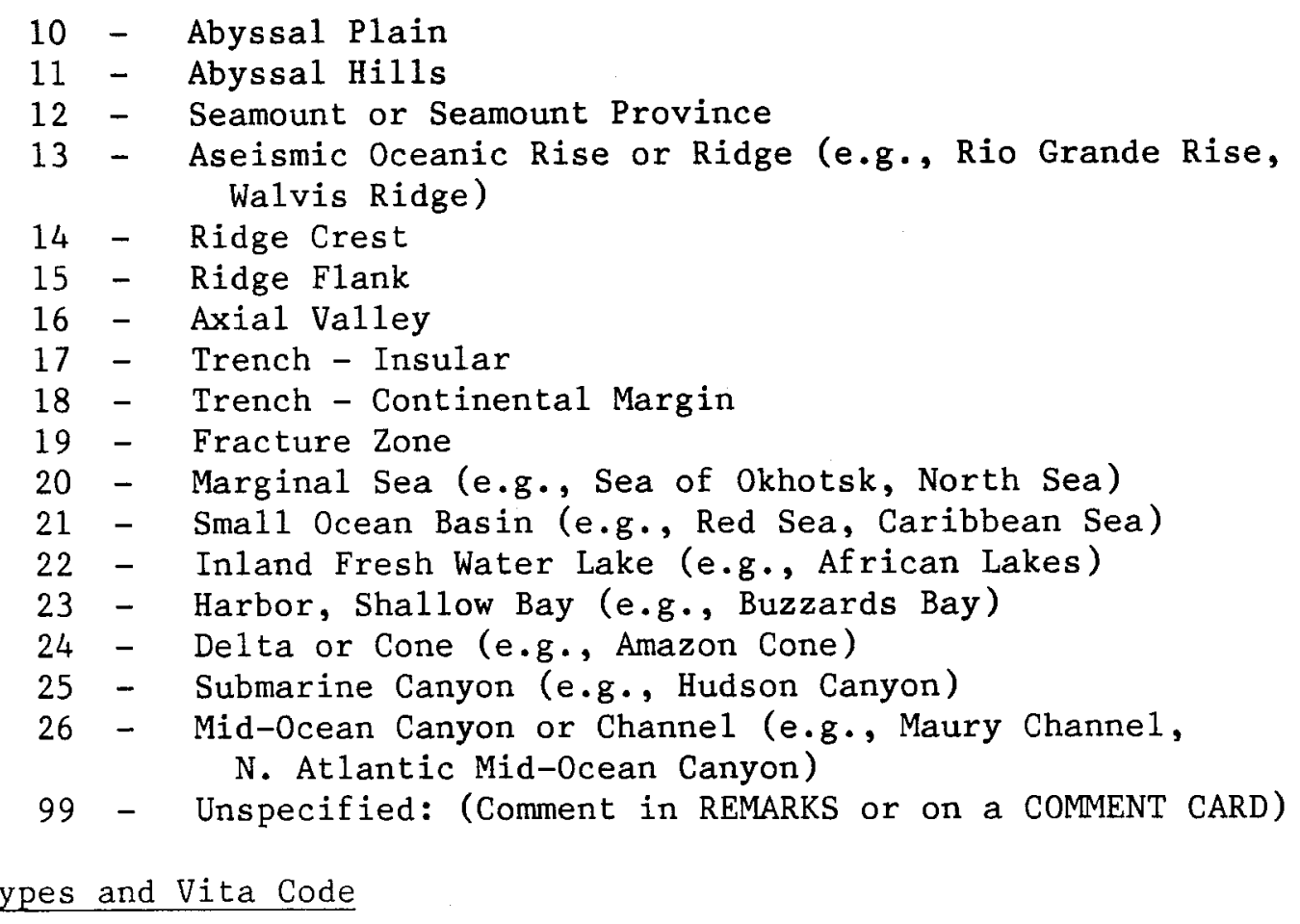

These columns have not been coded as they are undergoing some revision in order to be more descriptive of rock samples.

\section{Sample Distribution Policy}

The W.H.O.I. Sea Floor Samples Laboratory is prepared to furnish samples and data to intertested researchers and students within the scientific community who express a legitimate interest and need.

Requests for samples may be sent to the Geological Sample Curator's Office, McLean Laboratory, W.H.O.I., Woods Hole, MA 02543. These should include a summary of the intended research and the laboratory facilities available. Requests will be reviewed by the Principal Investigator responsible for collecting the samples, and may be approved if the proposed studies are not in conflict with concurrent laboratory studies. The Principal 


\section{Sample Distribution Policy. (Cont'd)}

Investigator will retain authority to approve sample requests until expiration of the relevant research grant or until two years from the date of termination of the cruise. Following the period of proprietary access, sample requests will be approved by the Curator's office in consultation with the appropriate staff scientists.

Persons obtaining samples will also be given a statement explaining the "Responsibilities of Persons Receiving Samples" (Part E). Further documentation regarding this distribution policy may be found in W.H.O.I. Institutional Memorandum 非-75 ("Distribution Policy for Geological Samples").

\section{E. Responsibilities of Persons Receiving Samples.}

1. The original alpha-numeric samples label should be used in published papers, or any departure from this scheme should be clearly equated with the original labeling system in published papers or data summaries. This labeling system will be explained in the information supplied with the samples.

2. Published papers should acknowledge the source of samples and the appropriate grant or funding agency which supported the cruise recovering the samples. This information will be supplied at the time the samples are sent. These papers should also acknowledge the financial support responsible for maintaining the Woods Hole geological samples (NSF Grant OCE85-19889).

3. Copies of all published papers, reports or data summaries utilizing Woods Hole samples should be sent to the appropriate W.H.O.I. staff scientist and the W.H.O.I. curator. 
E. Responsibilities of Persons Receiving Samples. (Cont'd)

4. The researcher should return all unused samples or portions of samples to the curator at the completion of his work.

5. Recipients of samples should not co-opt the services of other investigators or undertake research projects which differ substantially from work originally proposed, without obtaining the approval of the curator and the appropriate staff scientist. 


\section{ACKNOWLEDGEMENTS}

The editors of this report would like to recognize all those persons contributing to the rock descriptions provided herein (especially D. Bergersen and L. Peirson). We also wish to thank other members of the W.H.O.I. Curatorial Staff for their persistence in carrying out routine archival procedures which maintain the high quality of this collection. (David A. Johnson provided much of the guidance and drive for this effort through the years.) Alice Tricca did a great job typing the complex forms. Support for the preparaton of this report was provided by a grant to the Woods Hole Oceanographic Institution, Sea Floor Samples Laboratory (National Science Foundation Grant No. (OCE85-19889). The petrologists on the scientific staff at Woods Hole (W. Bryan, G. Thompson, H. Dick, P. Meyer, S. Humphris, and M. Mott1) have been of continuing assistance to us in providing logistical and descriptive information from their respective shipboard programs, and in implementing an effective sample distribution policy. 
TABLE 2

\section{NOTES ON DETAILED ROCK DESCRIPTIONS}

Lithology: Rock name; i.e. Basa1t, Gabbro, Greenstone, etc.

Wt.: $\quad$ Weight in kilograms

G.S.: $\quad$ Grain size: $G=$ glassy

$A=$ aphanitic; individual grains not visible to the naked eye

$\mathrm{F}=\mathrm{f}$ ine; $<\mathrm{lmm}$

$\mathrm{M}=\operatorname{medium} ; 1$ to $5 \mathrm{~mm}$

$\mathrm{C}=$ coarse $;>5 \mathrm{~mm}$

Mineralogy: Phases present in groundmass if apparent in hand specimen

Phenocrysts: Type and estimated amount in \%; use abbreviations:

$$
\begin{aligned}
& \text { Pg - Plagioclase, Px - Pyroxene, Amph - Amphibole, } \\
& \text { Mt - Magnetite, Py - Pyrite, 01 - Olivine, } \\
& \text { I1 - Ilmenite, Ep - Epidote, Pr - Prehnite, Mi - Mica, } \\
& \text { Hb or Hnb1 - Hornblende, Fs - Feldspar, etc. }
\end{aligned}
$$

Ve: Vesicles - give an estimate of the percent in the rock

Am:

Amygdules - filled vugs or vesicles; estimate abundance:

$$
\begin{array}{ll}
T-\text { trace } & S \text { - scattered } \\
C \text { - common } & \text { A - abundant }
\end{array}
$$

Mn: $\quad$ Manganese coating - give thickness (in $\mathrm{mm}$ )

We: $\quad$ Weathering F - Fresh, no discoloration

L - Light, discolored at edges

M - Moderately discolored

$\mathrm{H}$ - Heavy, clayey

VH - Very Heavy, disaggregating

Alteration: Metamorphism - facies and degrees

$$
\text { i.e. } \quad \begin{aligned}
& \text { Ze } \text { - Zeolite (prehnite - pumpellyite) } \\
& \text { Gr } \text { - Greenschist } \\
& \text { Amph } \text { - Amphibolite } \\
& \text { Gran. - Granulite }
\end{aligned}
$$

$\underline{\text { Remarks: }}$

Note if glass is present, indicate visible structures (internal or external), and describe distinct morphologies. For example: "pillow rind fragments". 
TABLE 3

COMPUTER LISTING OF W.H.O.I. DREDGES, ARRANGED BY MARSDEN SQUARES 


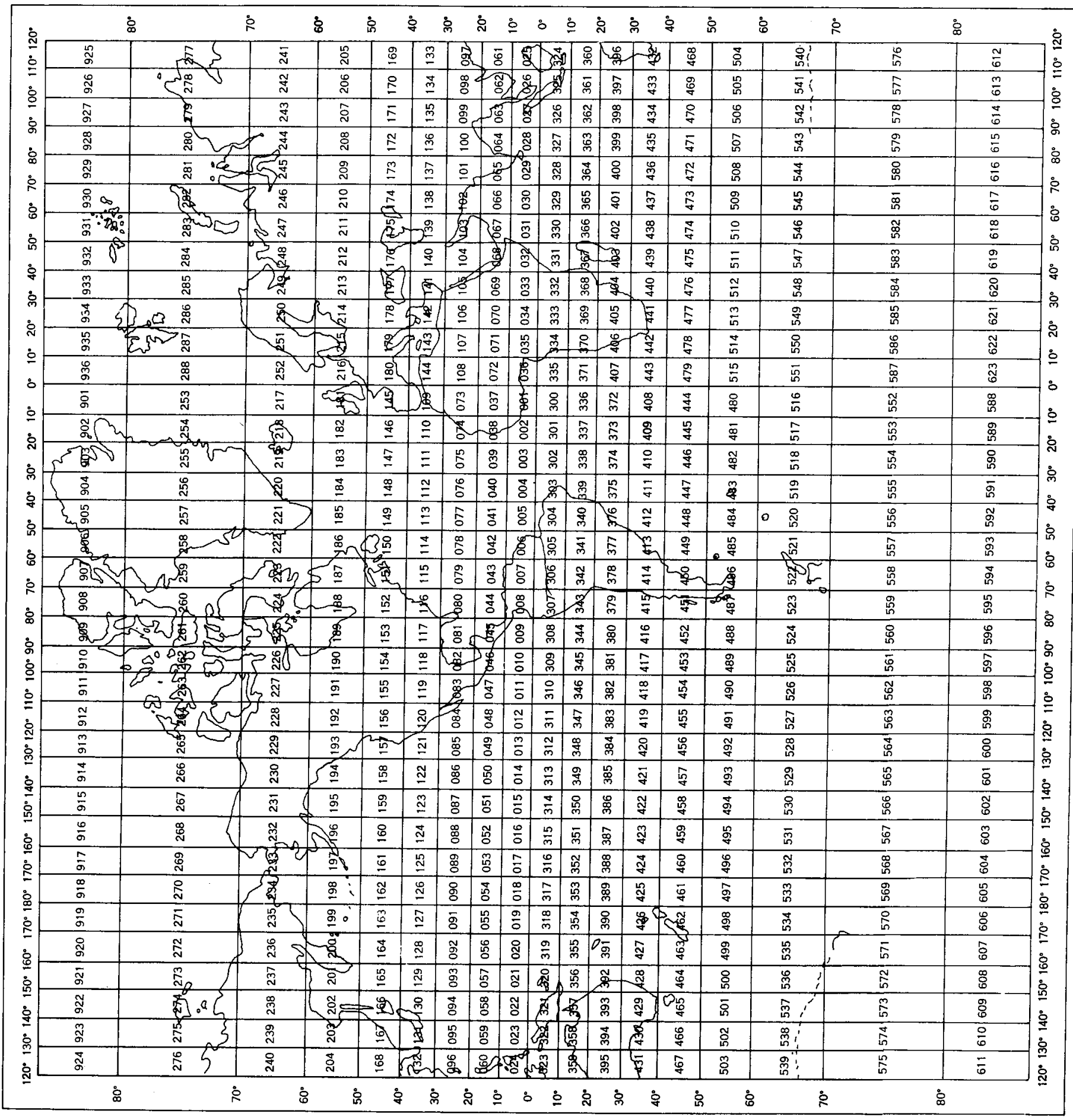


เค่

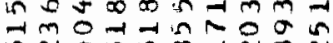
ம் $\dot{0} \dot{0} \dot{0} \dot{0} \dot{\sim} \dot{0}$

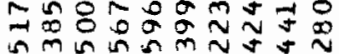
占z莘

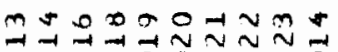
경ㅎㅇㅇㅇㅇㅇㅇㅇㅇㅇㅇㅇㅇㅇ

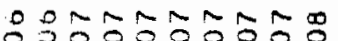
$\dot{\nu} \dot{\sim} \dot{\sim} \dot{\sim} \dot{v} \dot{v}$

$+4-0000000$ 323353333 005050500 - $\dot{0} \therefore \dot{0} 0 \dot{0}=$

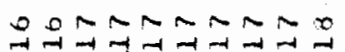
$z z z z z z z z z z$ 0000000000

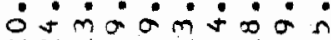

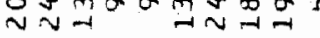

0000000000

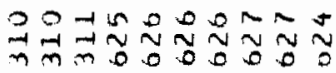

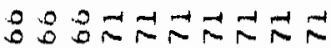

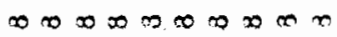

윽응ㅇㄱㅇㅇㅇ영용여

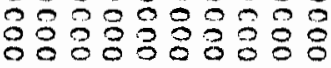

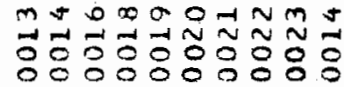
NNND000000

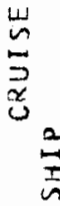

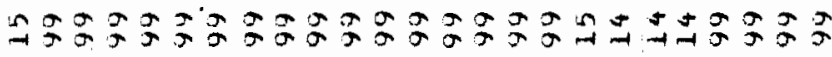

$\ddot{n}$

$\underset{0}{x} \underset{0}{x} \underset{n}{x}$

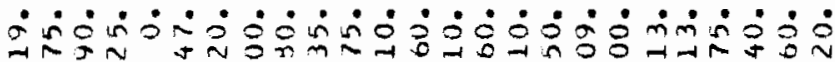

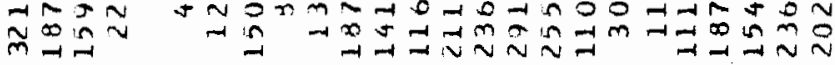

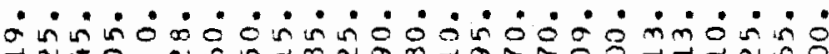

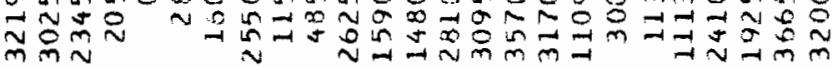

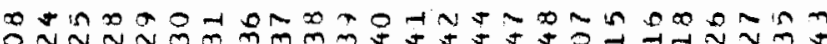

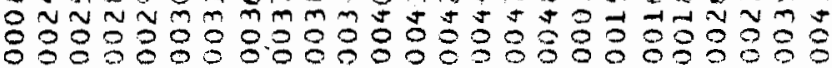

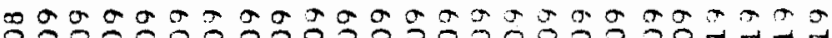

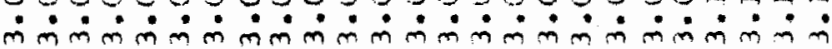

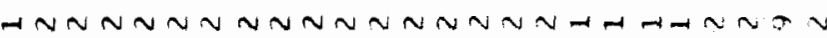

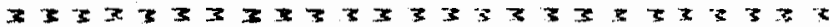

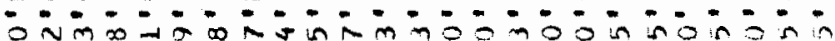

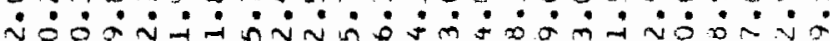

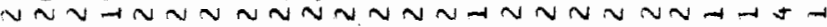

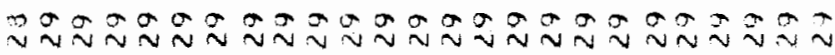
$z z \geq z z z \geq z z z z z z z z z z z z z z z \geq z z$

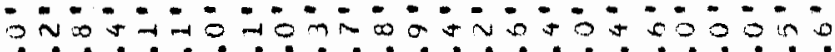

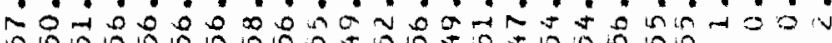
$000000000000000000000-4-1$

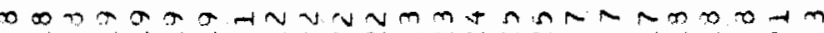

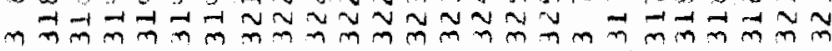

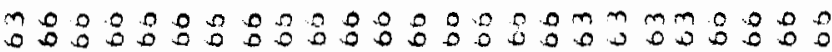
on

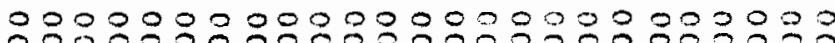

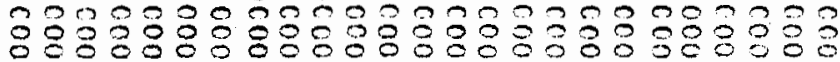

TIn NNำm

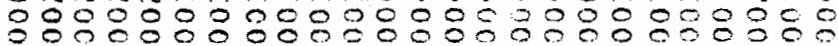

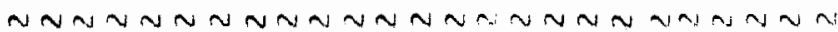

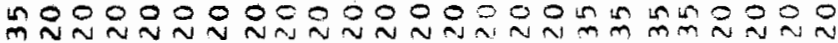

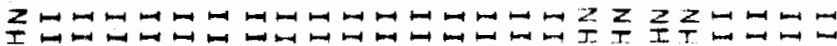

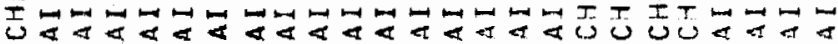




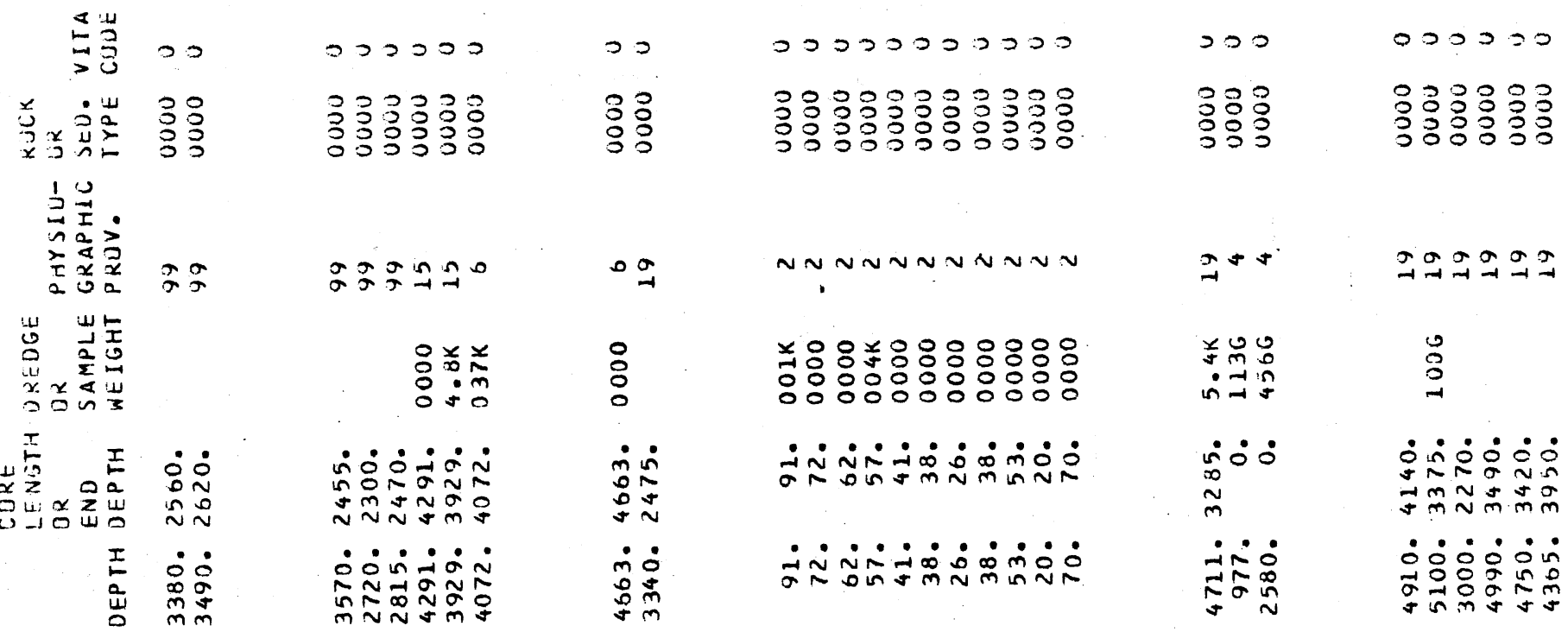

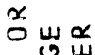

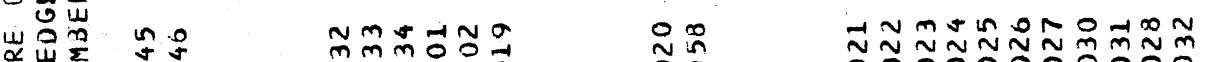

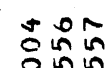

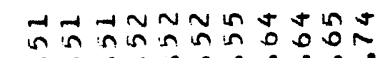

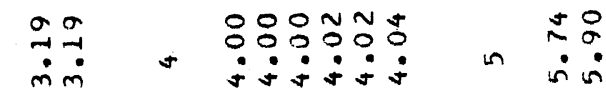

$a \sigma$
$\dot{m} \dot{m}$

8ूo

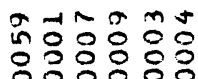

in $\vec{\sigma} \vec{\sigma}$

Nôñ ${ }_{0}^{m}=m$

$x_{i}$

*

$\underset{4}{4}$

(u

NN $\frac{w}{\alpha} \sigma \sigma a r-1$

$3=$ a 33333

0 in 0000

rivisis

을으ำก

$z \geq z \geq z z$

岁 $2 \geq$

in 00000

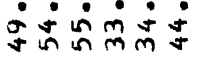

o 00000

$+a$

$3=$

in

in $m$

20

$\dot{0} \dot{0} \dot{0} \dot{0} \dot{0} \dot{0} \dot{0} \dot{0} \dot{0}$

iो $\frac{\dot{m}}{m}$

$2=$

$\dot{m}:$

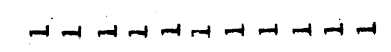

3. $353 \times 33333$

000000 in in 000

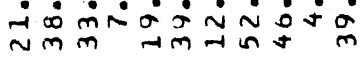

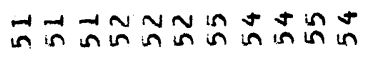

$z z z z z z z z z z z$

00 N000000\%

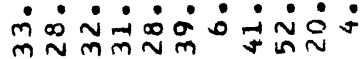

in inininin o o o on

$\sim o$

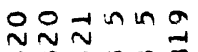

$\frac{\pi}{20}$

00000000000

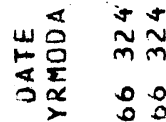

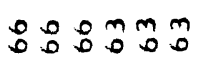

กิ:

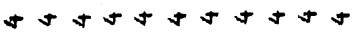

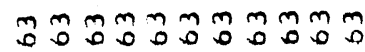

$\simeq \infty$

$\simeq \simeq \simeq \simeq \simeq \simeq \simeq \simeq \simeq \simeq$

0
8
8
0

응응ㅇㅇㅇㅇㅇㅇㅇ응

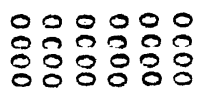

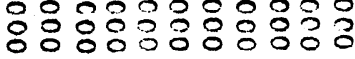

$$
\text { +o }
$$

然

\subsection{0}

$\dot{m i n}$

in $\vec{n} \vec{\Omega}$

$\geq \geq \geq$

in 0 :

injo

$n \rightarrow 0$

$\tan x$

$\sin$

o

$\infty 0$

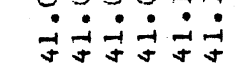

岁总 $\infty$

$\infty \infty \infty \infty$

요용

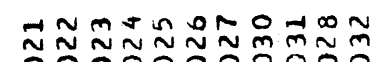

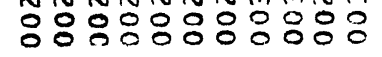

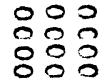

응응으으을 응ㅇㅇㅇㅇ

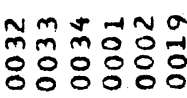
$m m$

$\mathrm{mmmmmmmmmm}$

요요

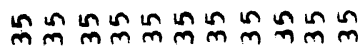

$\min _{\rightarrow \rightarrow \infty}$

윤유유

壬怘

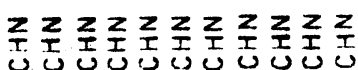

录ロロ

ジロショコ 
$=\begin{array}{ll}\square \\ =3\end{array}=0000000000$

苛出希

b.

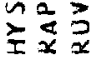

$\frac{7}{2} \frac{x}{a}$

岁崖等

x 0 in

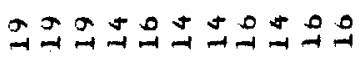

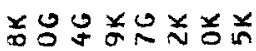

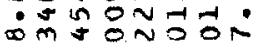

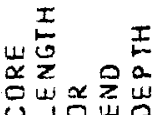

மீं $\dot{0} \dot{0} \dot{0} \dot{0} \dot{\sim} \dot{\sim} \dot{\sim} \dot{0}$

$m a-1 m 5 t \rightarrow 00$

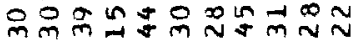

吉

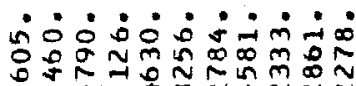

号

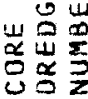

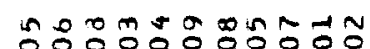

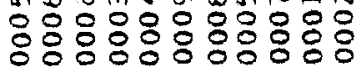

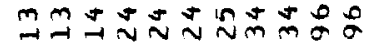

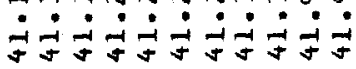

×㟔

$3 \times 33 \times 3 \times 3 \times 3$

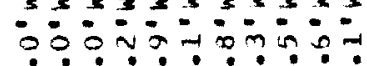

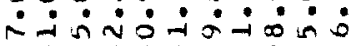

A $a$ a that

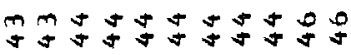

岁

$z z z z z z z z z z z$

ல0ं

$\dot{\sigma} \dot{0} \dot{0} \dot{\sim} \dot{\infty} \dot{\infty} \dot{\infty} \dot{\sim}$

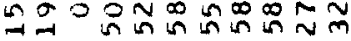

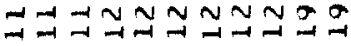

$\underset{2}{4}$

Dod-ATNNANmm

Nลี

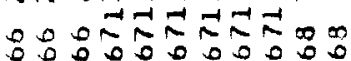

岁㟔

$\infty \omega \infty \infty 00000$

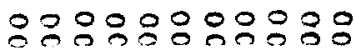

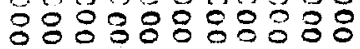

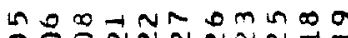

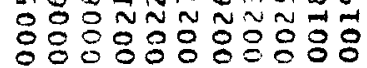

u

HATNNNNNNA-

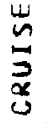

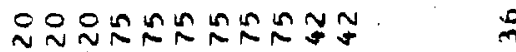

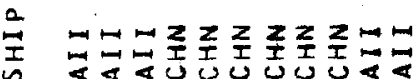

$\dot{0}$
3
00900000000000000

200002000

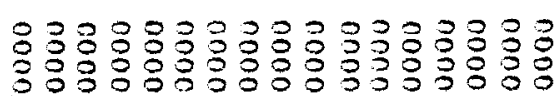

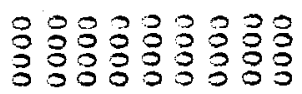

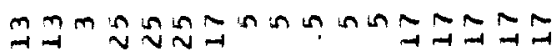

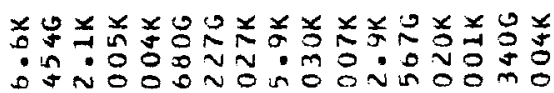

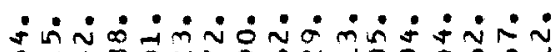

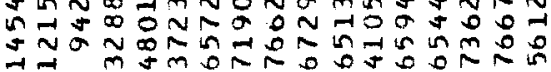

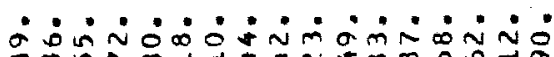

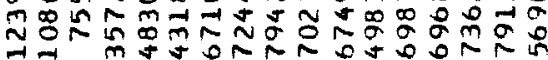

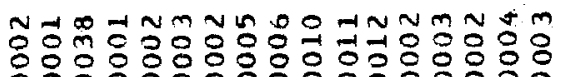

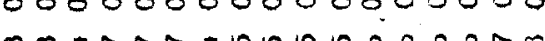

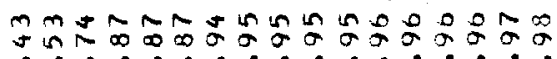

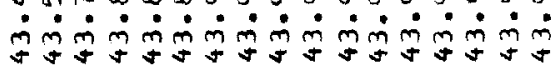

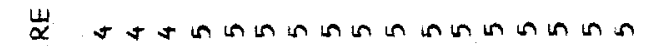

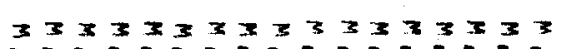

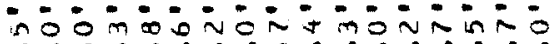

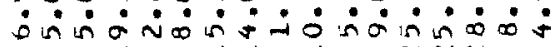
की $z \geq z z z z z z z z z z z z z z z$

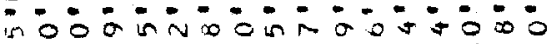
- $\dot{0} \dot{0} \dot{0} \dot{0} \dot{0} \dot{0} \dot{0} \dot{0}$

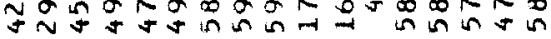

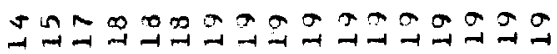

OONNNmSORG

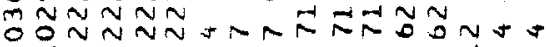

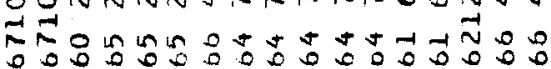

ONT

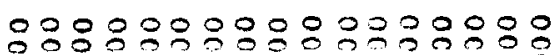

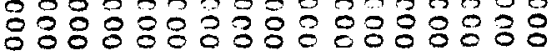

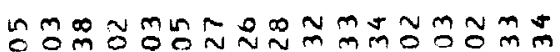

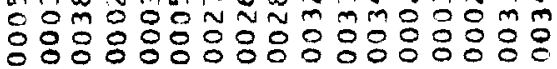

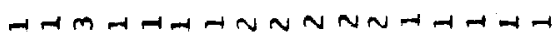

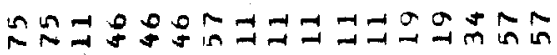

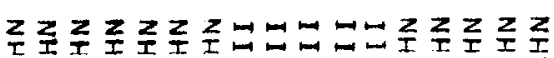

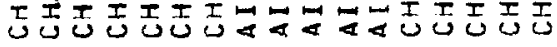

$m-n+1+1+n$

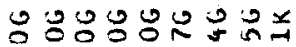

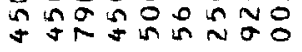

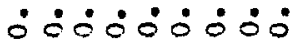

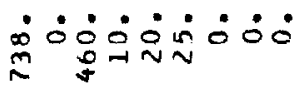

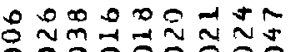

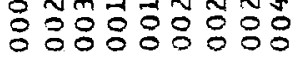
윳N소 - $\dot{a} \dot{2} \dot{4}+\dot{4} \dot{2}$

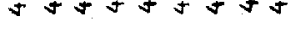

तカ-

$33 x 333_{3}$

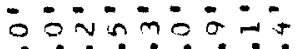

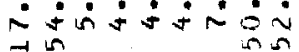
ㅇNニำ 特 $z \geq z \geq z \geq z z z$ i00000 im in

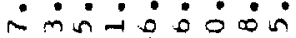
o vongmang ニニュニニニュニュ

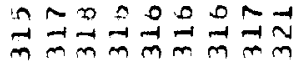

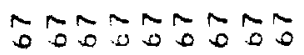

윽응영영영영

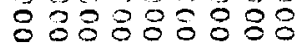

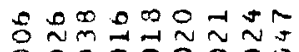

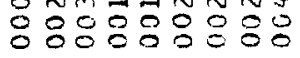
000000000

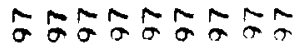

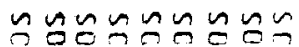

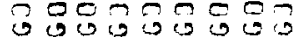

NANANRN 


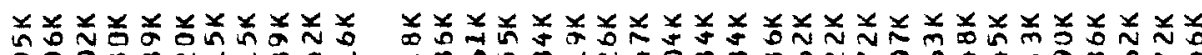

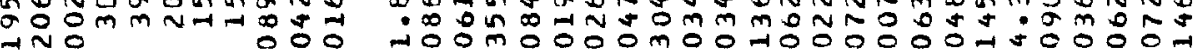

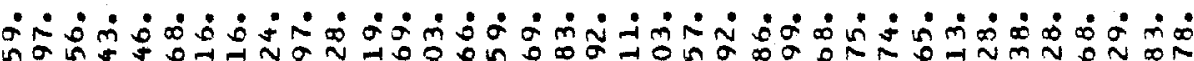

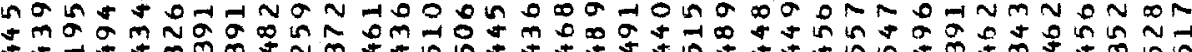

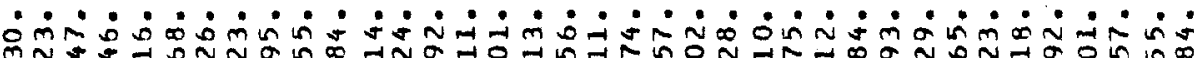

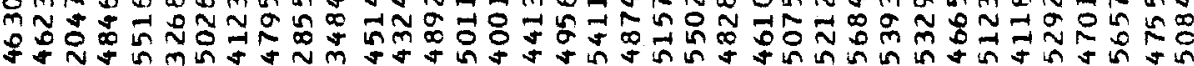

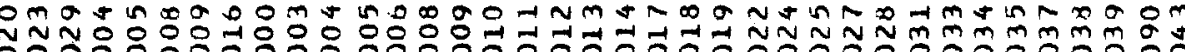

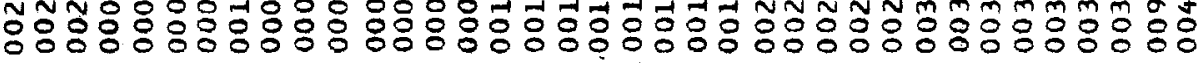

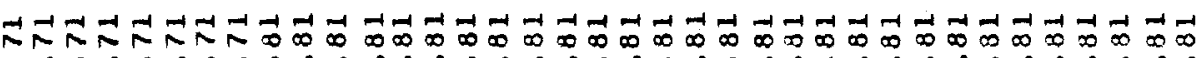

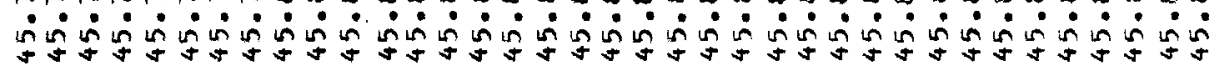

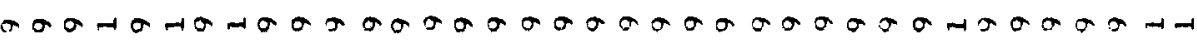

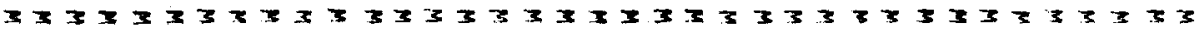

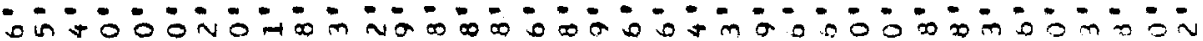

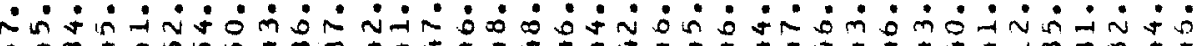

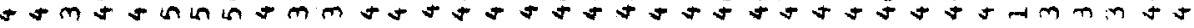

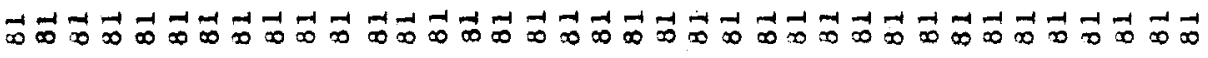

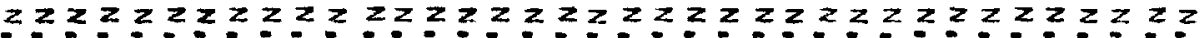

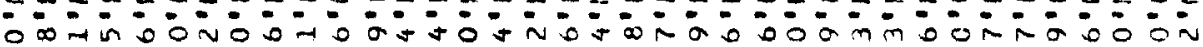

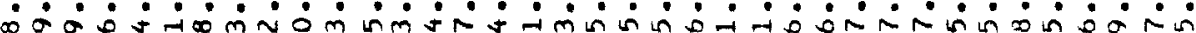

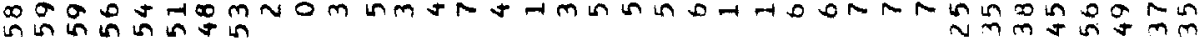

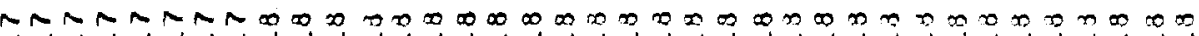

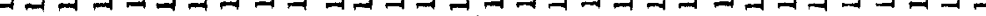

긍 MNNGง

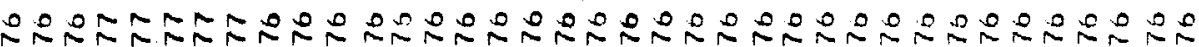

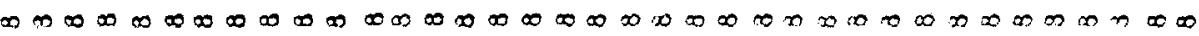

$0000000000000000000000000000 \% 20000000$

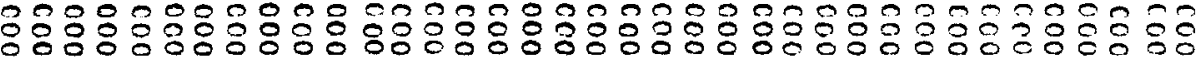

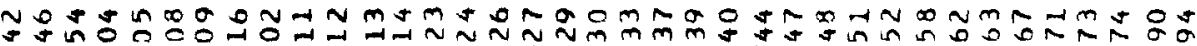

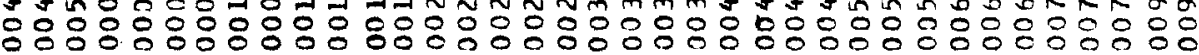

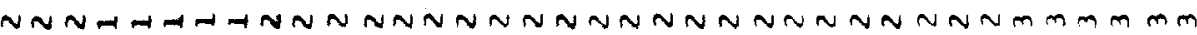

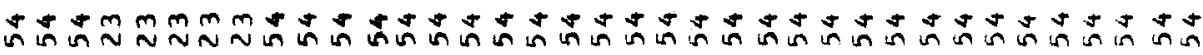

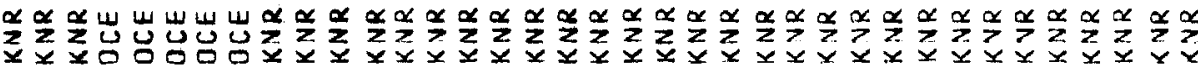




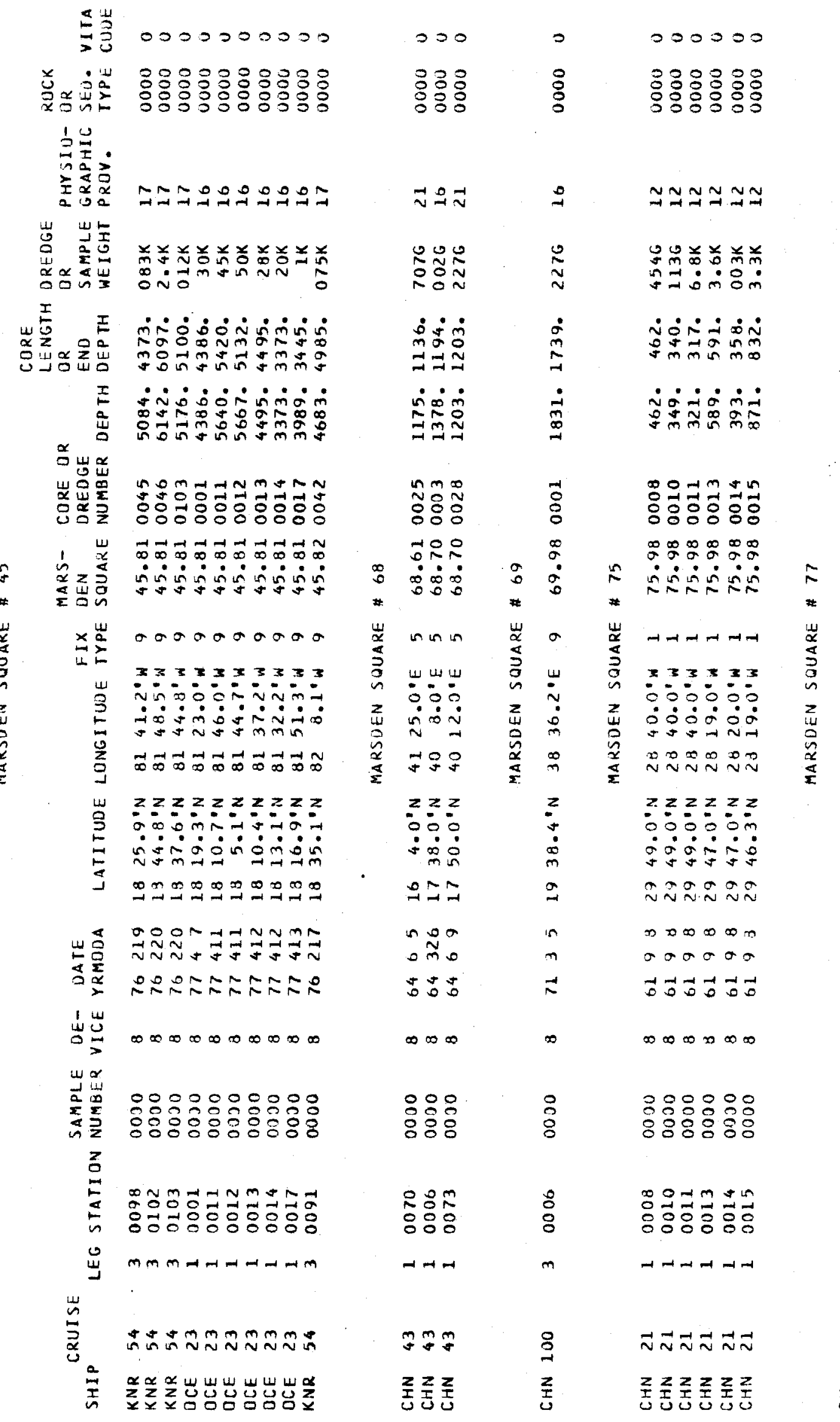

00000000000000

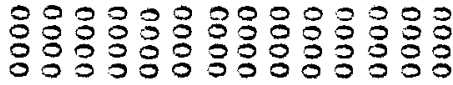

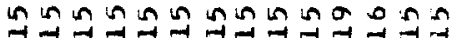

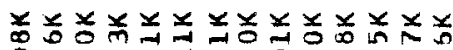

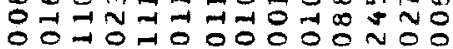

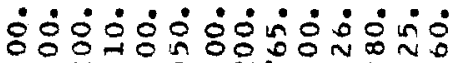

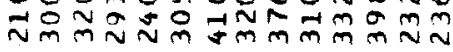

ச் $\dot{0} \dot{0} \dot{0} \dot{0} \dot{0} \dot{0} \dot{0} \dot{0}$

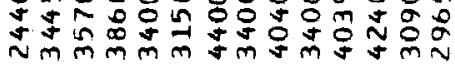

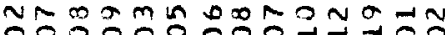

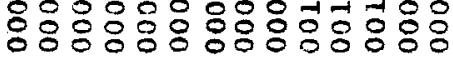

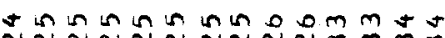

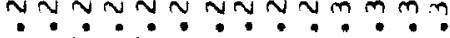

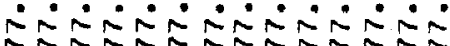

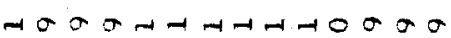
$3333 \times 3 \times 3 \times 333 \times 3$

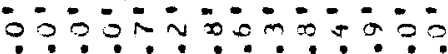

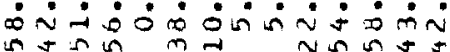

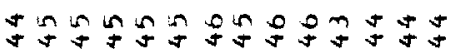
$z \geq z z \geq z \geq z \geq z z z z z$ 00000ल

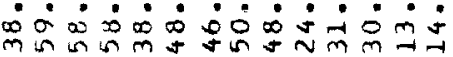

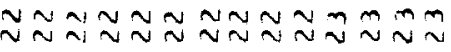

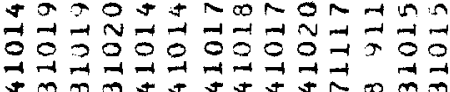
ง

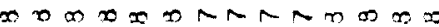

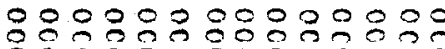

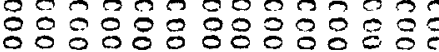

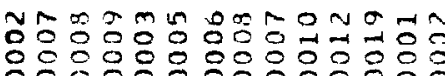
$\rightarrow N N N \rightarrow-1+\rightarrow \rightarrow m-N a$

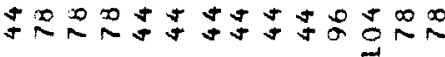

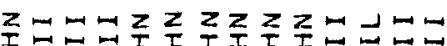

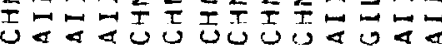




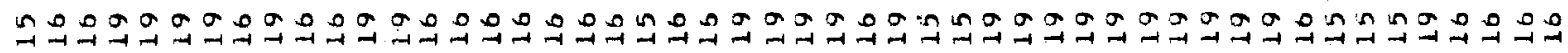
岁岁壱

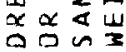

崖吉至

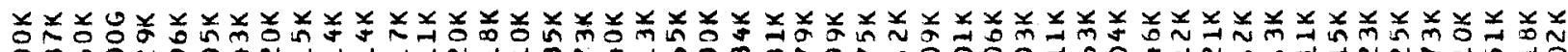

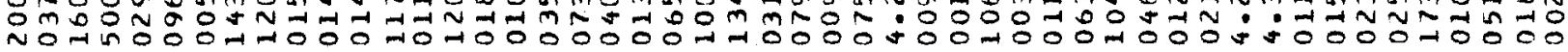

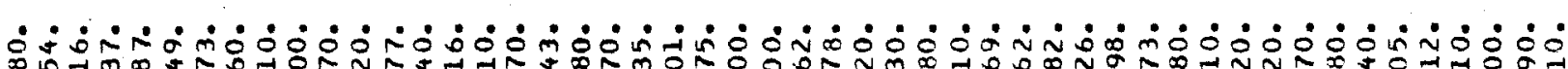

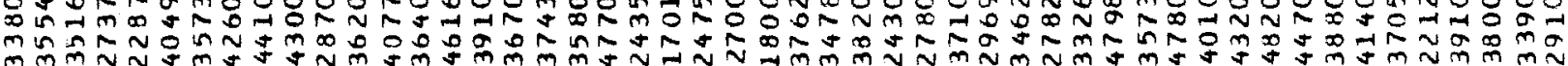

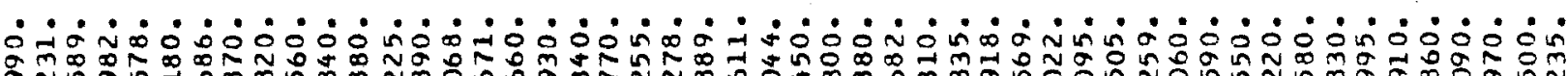

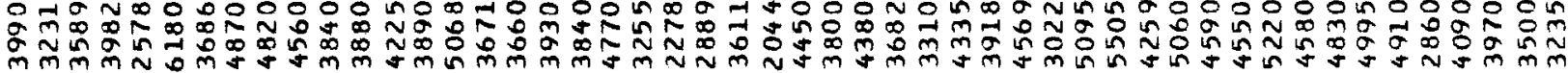

$\stackrel{\circ}{\circ}$

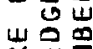

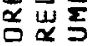

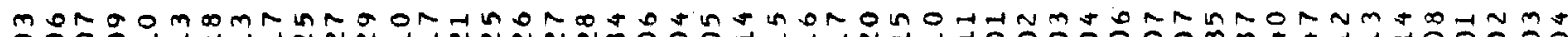

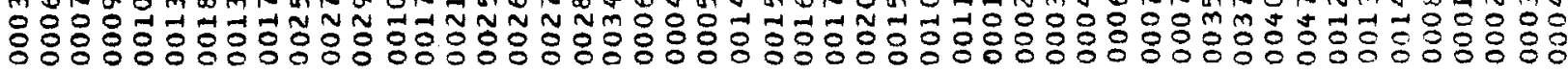

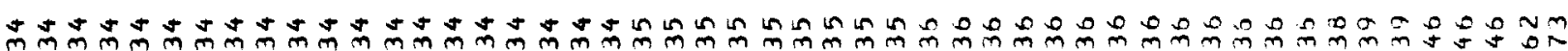

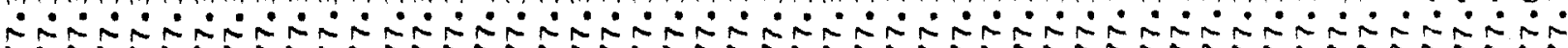

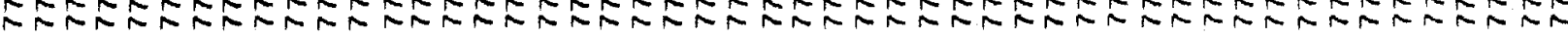

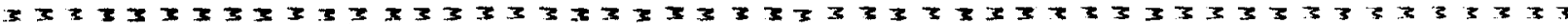
oj

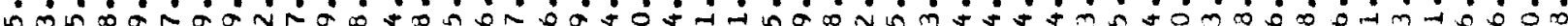

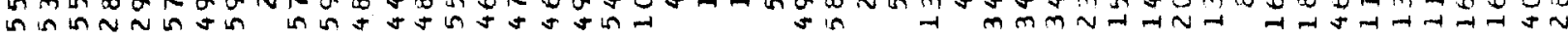

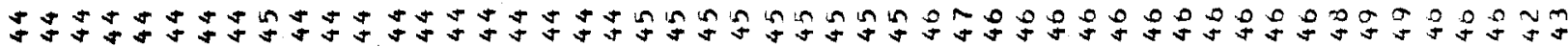

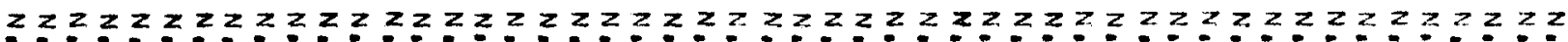

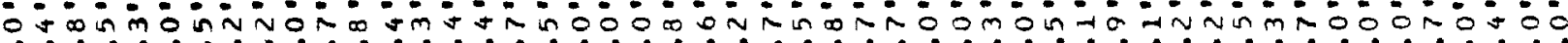

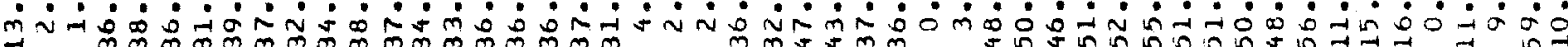
m

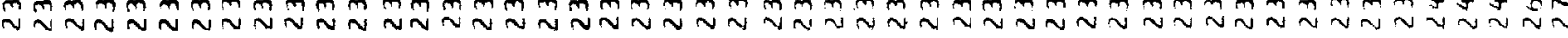

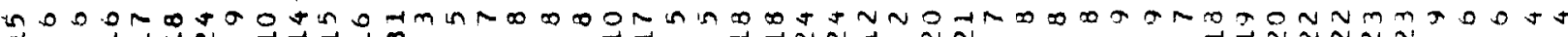

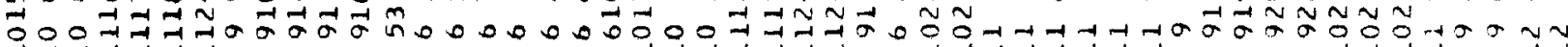
ヘ

岂

O

$\underline{\alpha}$

$\frac{1}{2} \stackrel{\infty}{i}$

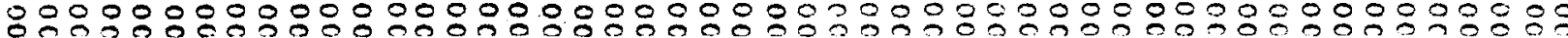

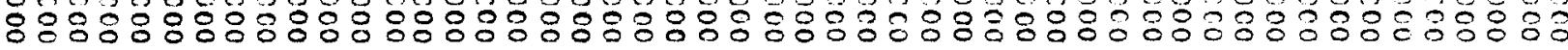

$z$

F

$\stackrel{\leftrightarrow}{u}$

$\stackrel{\omega}{2}$

$\frac{a}{n}$

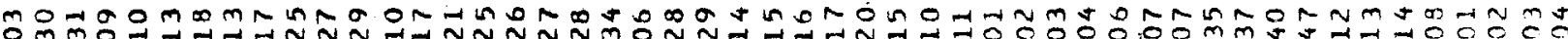

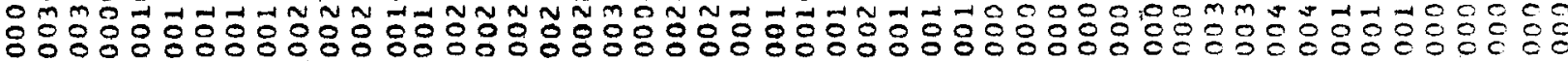

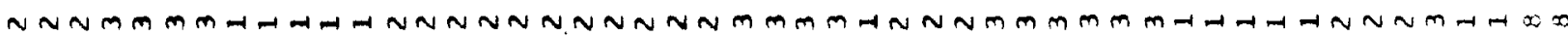

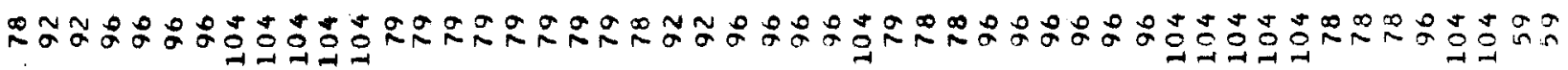

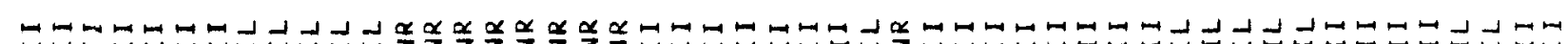

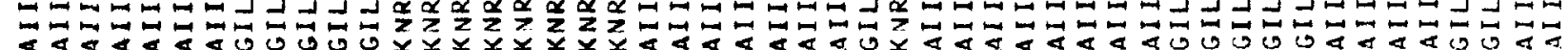


$\stackrel{200000000000000000}{ }$

苛芯岕

$\stackrel{1}{\Theta}$

논

I

岁出至

㟧 $x<$

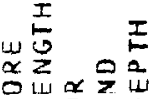

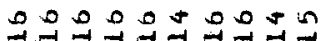

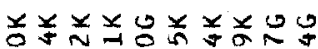
in

$\stackrel{\alpha}{b} u \alpha$

山宫㟧

岁崖

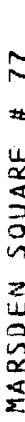

$\dot{0} \dot{0} \dot{0} \dot{0} \dot{0} \dot{0} \dot{\sim} \dot{0}$ N N N N N

I $\dot{0} \dot{0} \dot{0} \dot{0} \dot{\sim} \dot{5} \dot{0}$ 岀

0

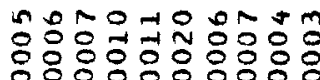

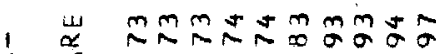

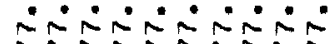
nanNARNARA

$\times$

$\rightarrow \rightarrow-\rightarrow-1$ in $\rightarrow-\rightarrow-1$

$3333 \times 3 \times 33$ 0.000 $\dot{S} \dot{0} \dot{0} \dot{\sim} \dot{\sim} \dot{\sim} \dot{m} \dot{\sim}$

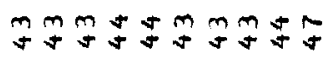
$z \geq z z z \geq z z \geq z$ 00000 in $00 \mathrm{~m} 0$ ن் $\dot{0} \dot{0} \dot{0} \dot{0} \dot{0}$ NNNNNoga

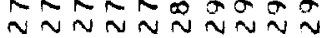

Sก $n M N \sigma$ nM $\sim \sigma$

nNNNNNara

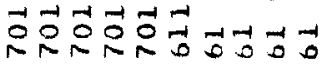

岁岂

$\infty \infty \infty \infty \infty \infty \infty \infty$

응ㅇㅇㅇㅇㅇㅇㅇㅇ

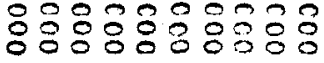

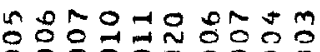

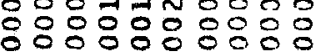

$\infty \infty \infty \infty \infty \sqcap \leadsto-\neg$

岕

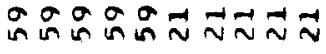

은

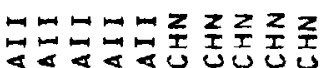

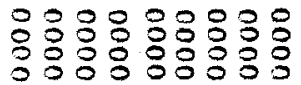

ヘヘヘココココささ

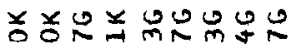
○워.

$\dot{0} \dot{0} \dot{0} \tilde{N} \tilde{N}$ Dañ

$\dot{0} \dot{0} \dot{0} \dot{0} \dot{0} \dot{0}$

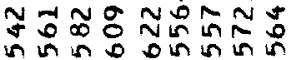

in 0 in + NMN

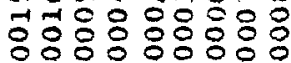
ON n $\cos \operatorname{ta} \sigma a$ m. m $\div$ in $\dot{\infty} \dot{\infty} \dot{\infty} \dot{\infty} \dot{\infty} \dot{\infty}$

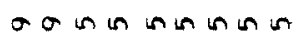

$x 3 \times 3 \times 3333$ -5050000 in் $\dot{0} \dot{0} \dot{0} \dot{0}$ 은 $z z z z z z z z$ 000500005 $\dot{j} \dot{\sim} \dot{\infty} \dot{\mathrm{s}} \dot{\mathrm{j}} \dot{\mathrm{N}}$ $\sim \sim \sim n \sim ⿻-N=$

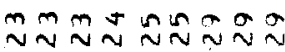

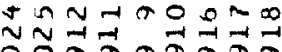

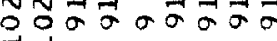

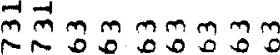

$\infty \infty \infty \infty \infty \infty \infty$

응응응영웅융ㅇㅇㅇ

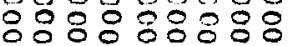

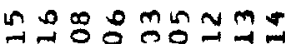

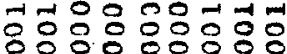

NNNNNNNNN

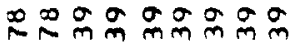

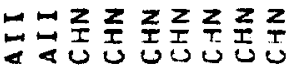

00000000000000

00000

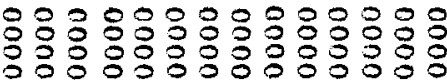

83989
88989

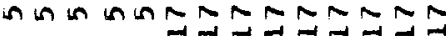

$\pm 0000$

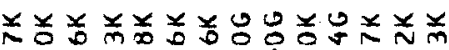

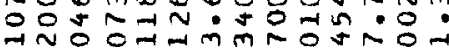

西前前希 ज़ि0

$\dot{\sim} \dot{\sim} \dot{\sim} \sim \dot{\sim} \dot{\sim} \dot{\sim} \dot{\sim} \dot{\sim} \dot{\sim}$ テर̃

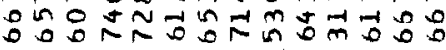

$\dot{0} \dot{0} \dot{0} \dot{\sim} \dot{\sim} \dot{\sim} \dot{0} \dot{0}$

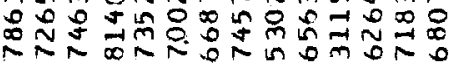

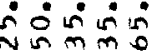
लㅇํำ

ம் $\dot{0} \dot{0} \dot{0}$ N

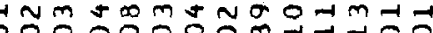

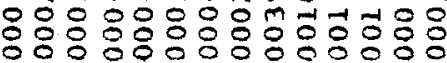

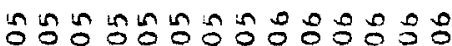

$\stackrel{9}{9}$

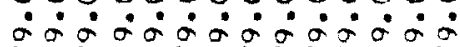

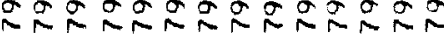

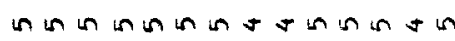

$33333 \times 333 \times 3333$

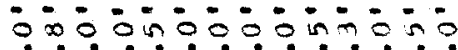

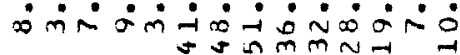
คู $z \geq z \geq z \geq z z z z z z z z$

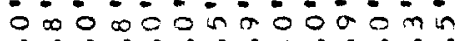
$\dot{0} \dot{0} \dot{0} \dot{0} \dot{\sim} \dot{\sim} \dot{0} \dot{0} \dot{\dot{v}} \dot{\sim}$

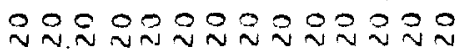
in ON $\sim \sim \sim \sim N$ N T.

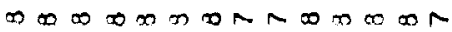

응응ㅇㅇㅇㅇㅇㅇㅇㅇㅇㅇㅇㅇㅇ응

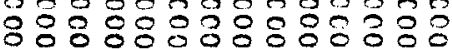

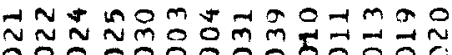

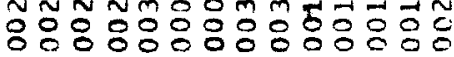
NNN NNMA-

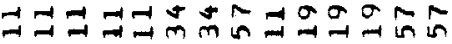

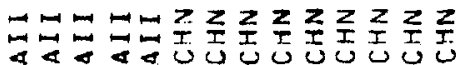

tNm v 88808 $\infty \infty \infty \infty \infty$ $N N N N N$ $\dot{m} \dot{m} \dot{m} \dot{m} \dot{m}$ $\infty \infty \infty \infty$

o. 000

$33 \times 1=$ $\therefore 0$ in $\dot{0} \dot{0} \dot{j} \dot{j}$

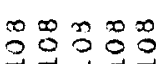
$z z z z z$ $\because \simeq m$

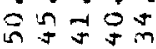
$\approx \sim N \approx N$

ป v v

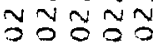
केन्त

and

응응융응 88080

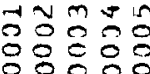
$\rightarrow-1-1$

$\widehat{O} \hat{0} \tilde{O}$ $\rightarrow-2 \rightarrow=$

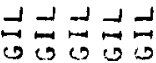




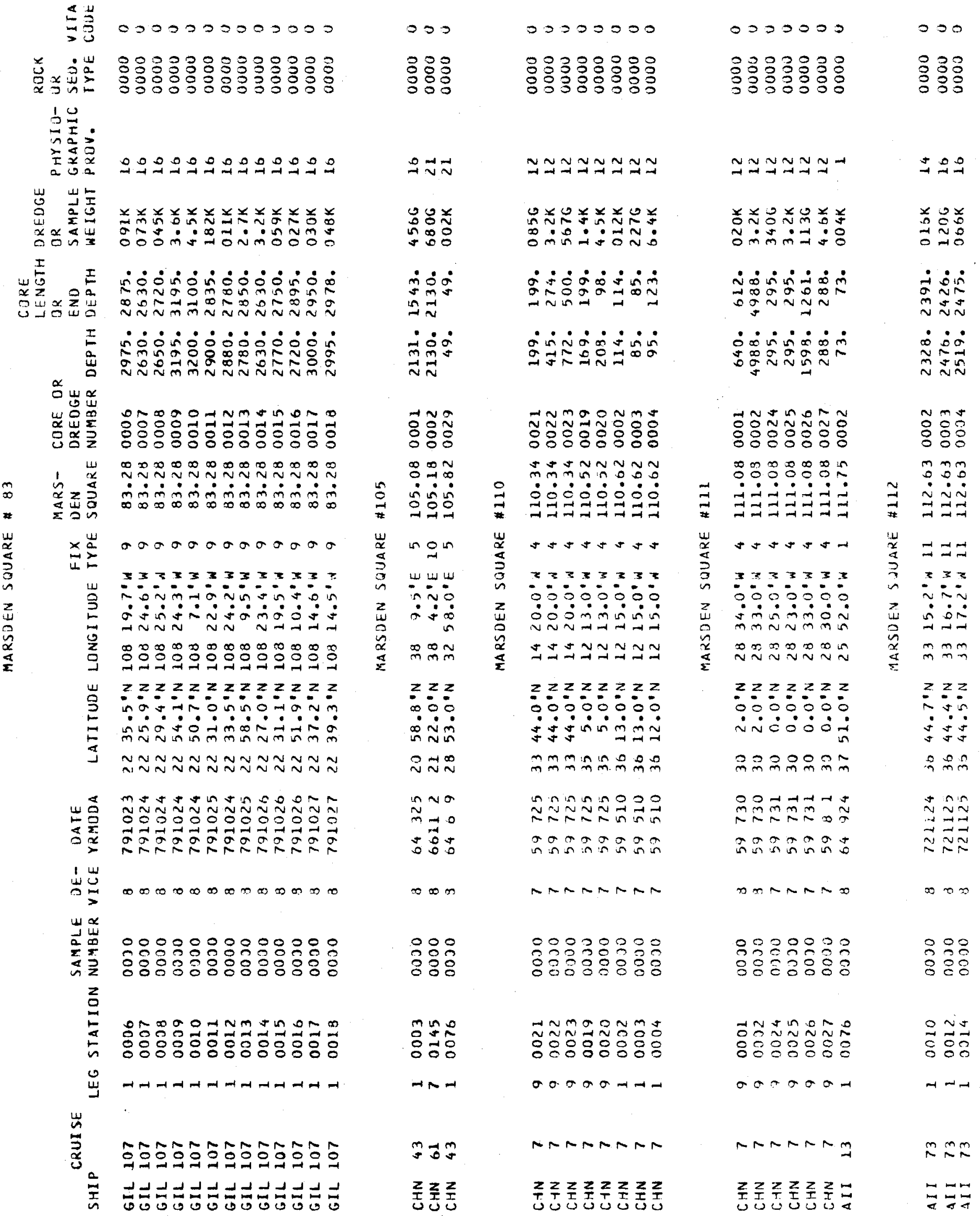




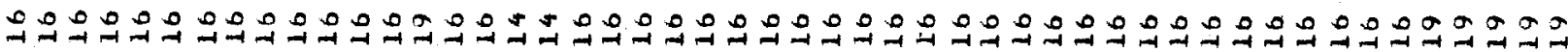

岁岁売 $\stackrel{x}{0}$ in

崖吉吉

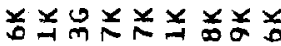

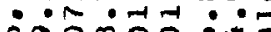

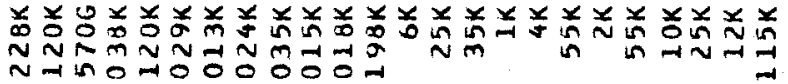

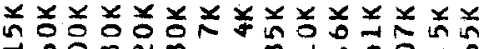

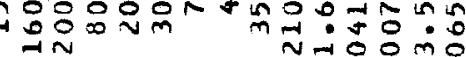

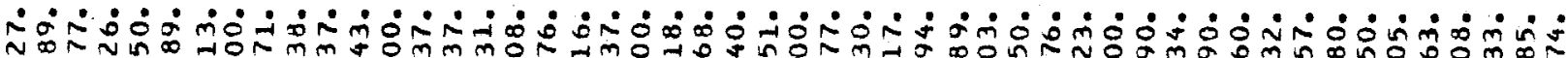

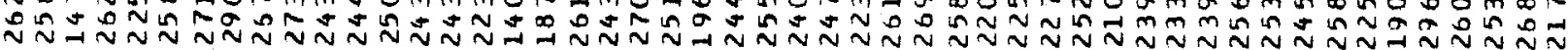

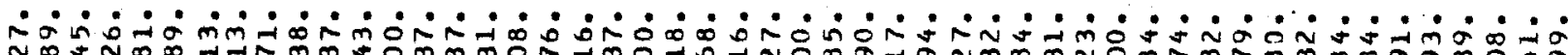
$\stackrel{x}{\Delta} \amalg \propto$

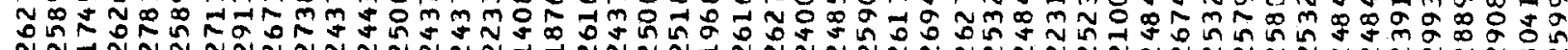

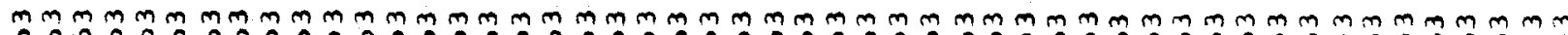

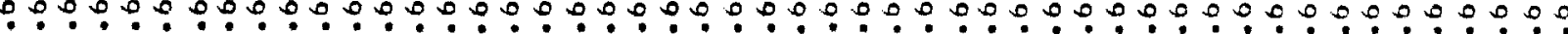

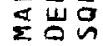

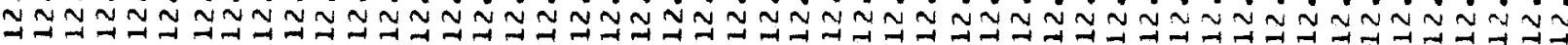

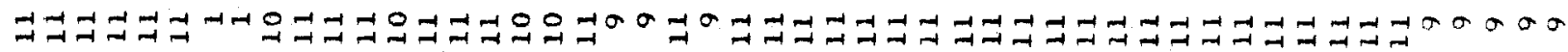

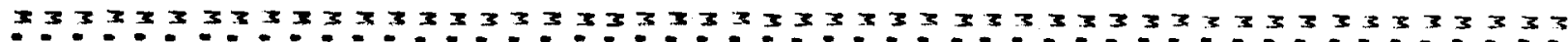

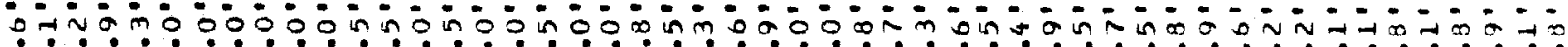

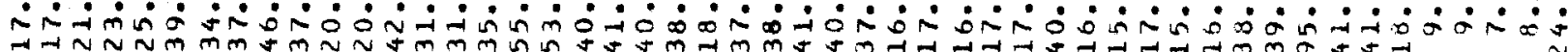
ANNNM mm

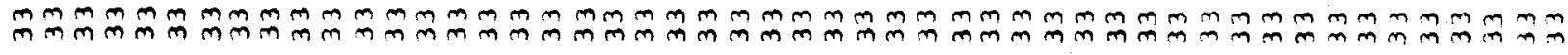

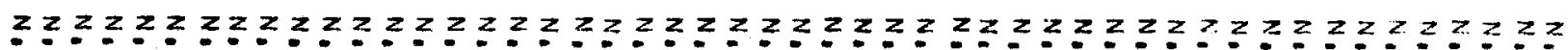
on in ?. ษ T

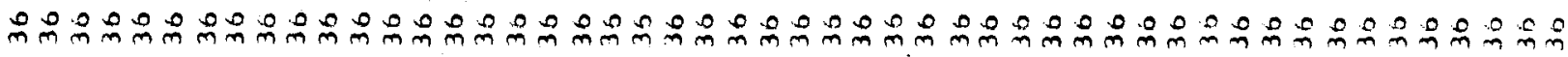

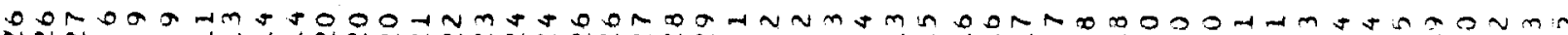

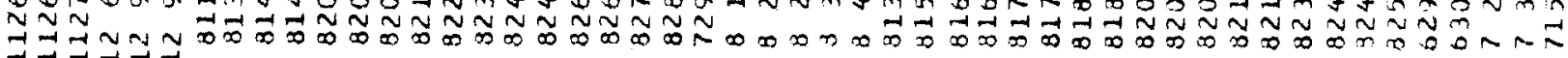

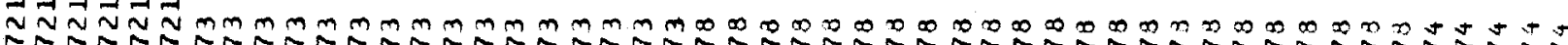

岂

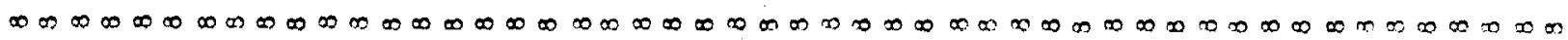

$\omega \approx$

党岕

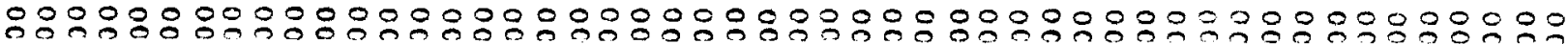

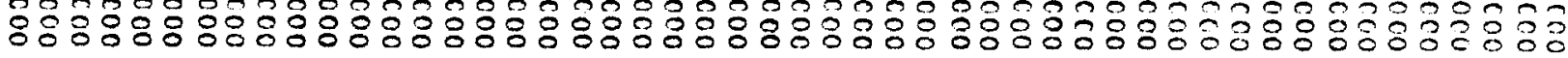

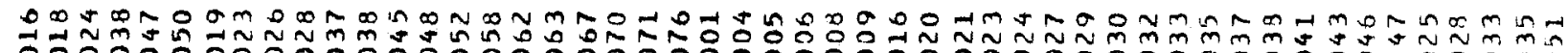

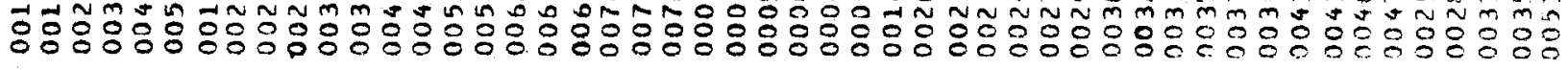

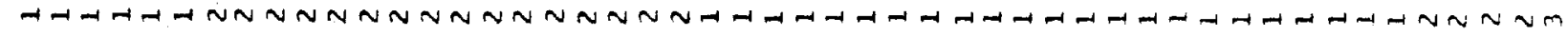
$\stackrel{u}{a}$

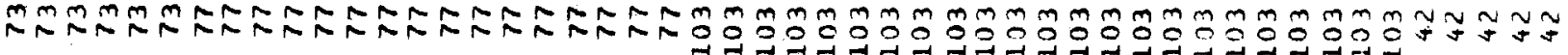
$\stackrel{2}{\sim}$

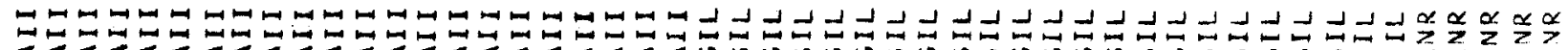

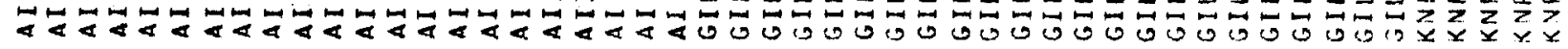


$-28-$

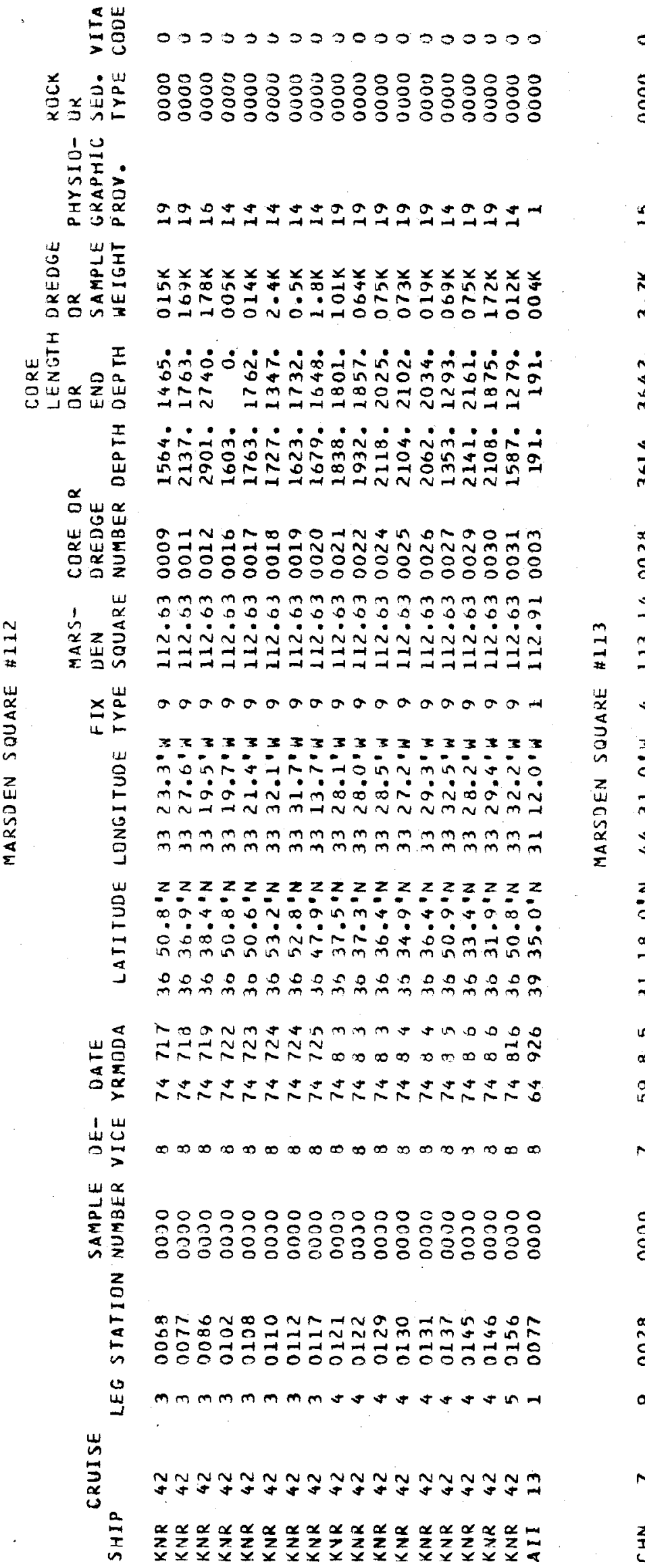

0
00000000000000000000000

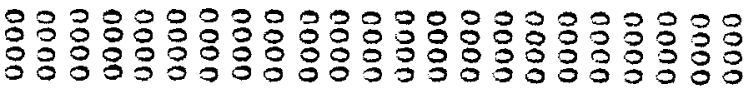

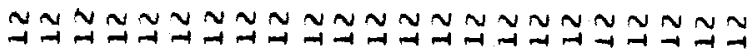

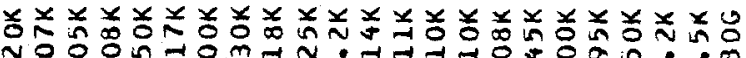

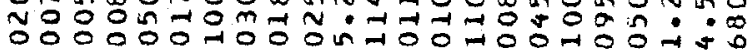

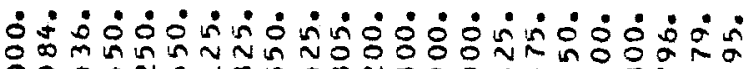

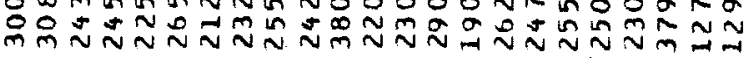

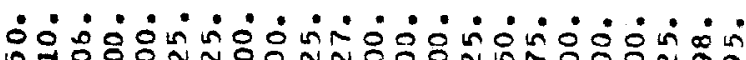

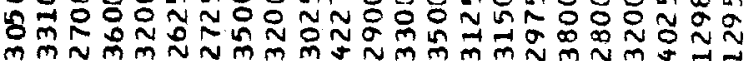

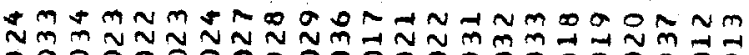

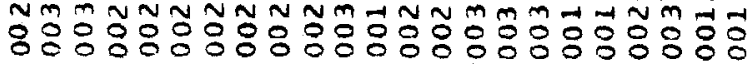

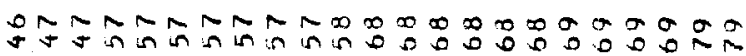

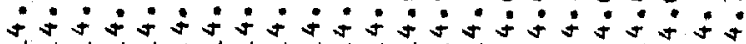

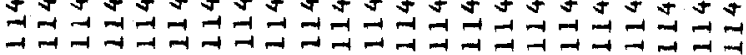

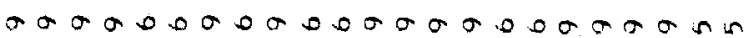

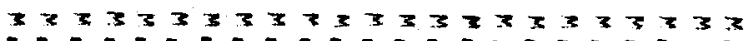

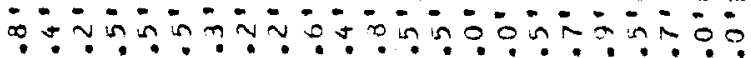

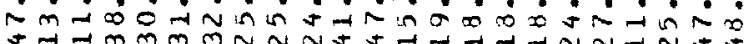

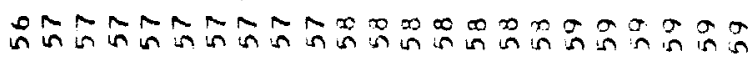

$z z \geq z z z z z z z z z z z z z z z \geq z z \geq z$

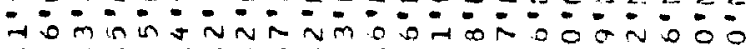

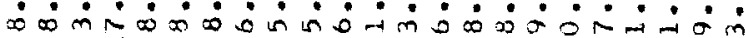

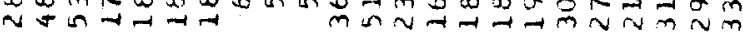

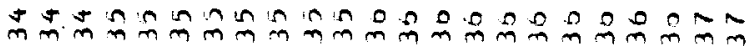

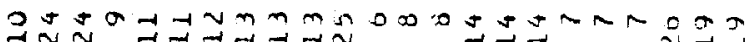
व NaNa유.

क

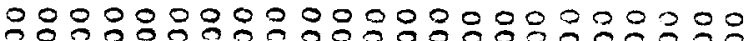

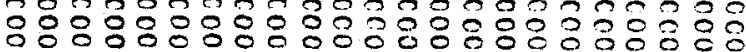

स

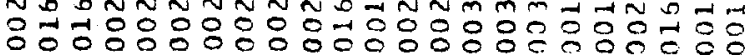

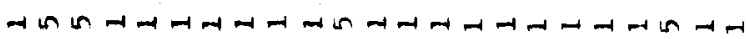

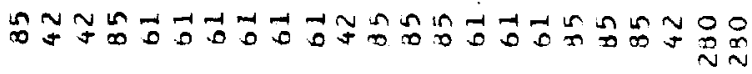

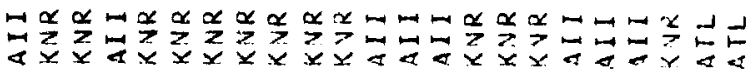


용 000

弟岀崖 8988

by

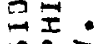

녕

I $x \alpha$

岁步志

至分的出

崖点

号出出出

mõ

I $\dot{m} \dot{0} \dot{0}$

嵌 กั

$\stackrel{f}{\mu} \alpha$

山岁

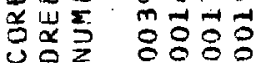

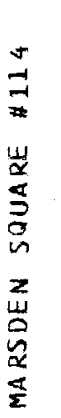

1 य 중

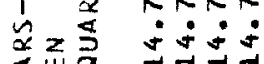

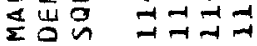

$\times$

0000

$3=3$

$\sin =$

int

$\ln 2 \pi$

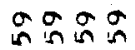

$z \geq z z$

oñ

$\dot{0} \dot{0} \dot{\sim} \dot{n}$

ำกำ

กิM

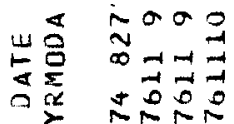

岂岕 $\infty \infty \infty$

a

a

응ㅇㅇ

8:88:

Hor 등명ㅎㅇㅇㅇㅇㅇ

n $-1-1$

岕 点

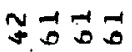
$\sum_{x}^{\infty} \sum_{x}^{\infty} \frac{\alpha}{2} \sum_{x}^{\frac{\alpha}{2}}$
000000000000000000000000000000000000000000

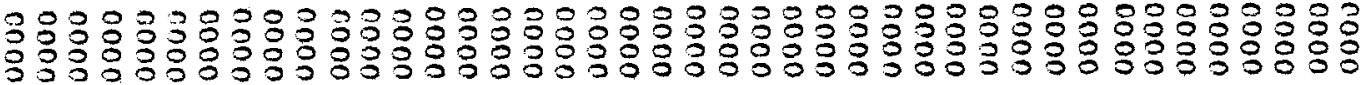

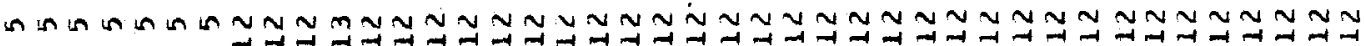

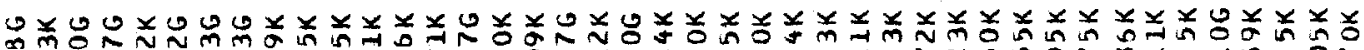
NOONNOSOA-

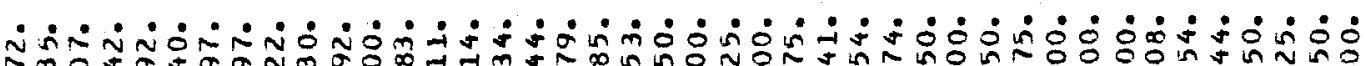
môn

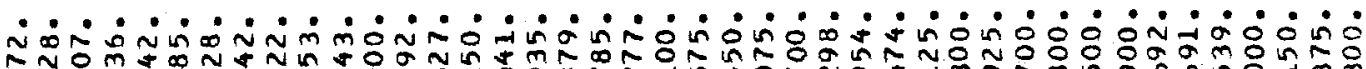

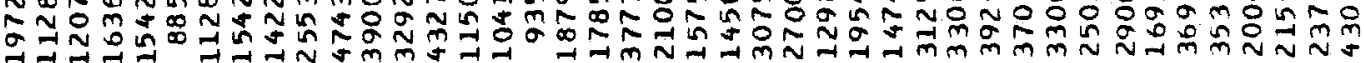

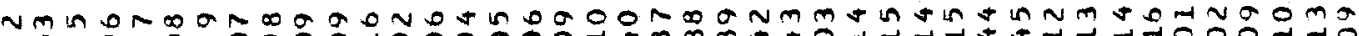

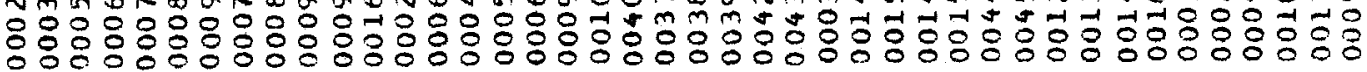

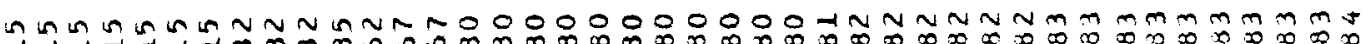

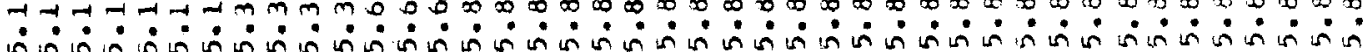

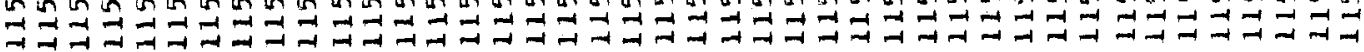

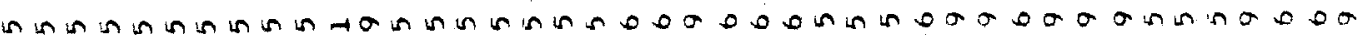

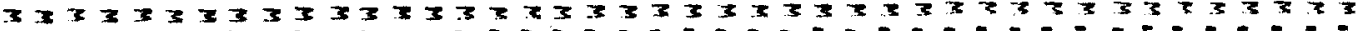
mä in

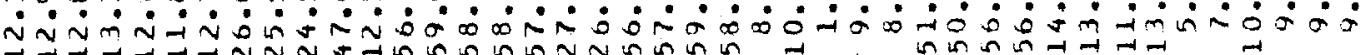
ถู่ $z z z z z z z z z z z z z z z z z z z z z z \geq z z z z z z z z z z z z z z z z z z z$

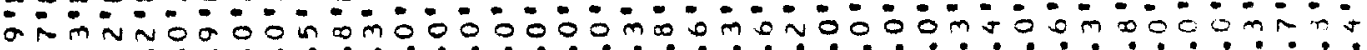

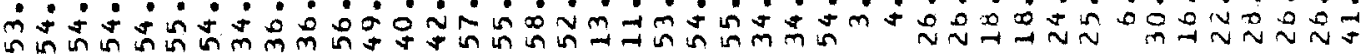

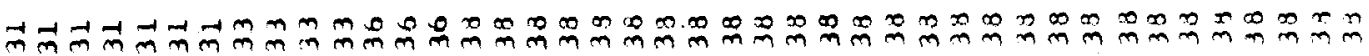
क

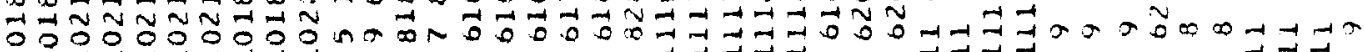

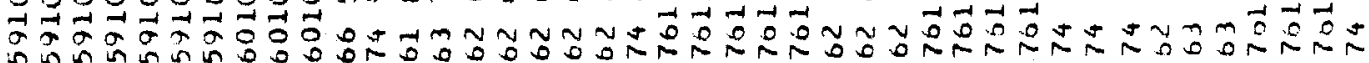
rNR N

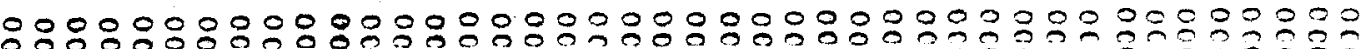

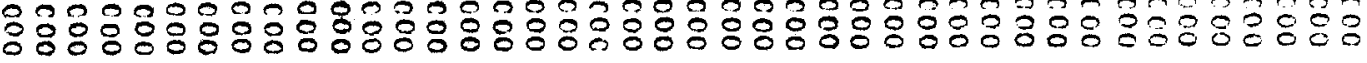

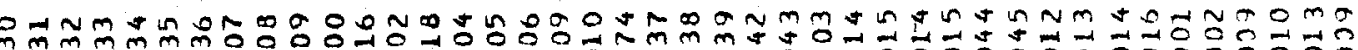

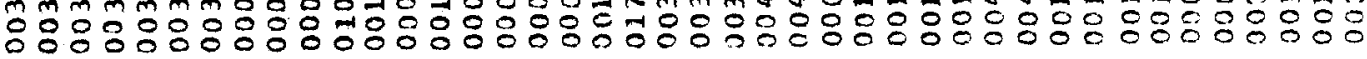

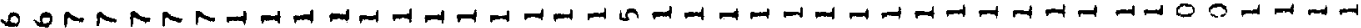

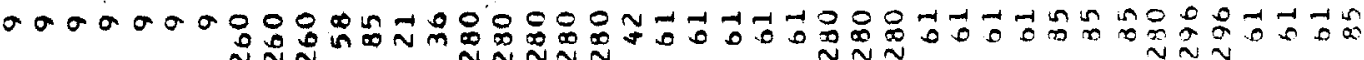

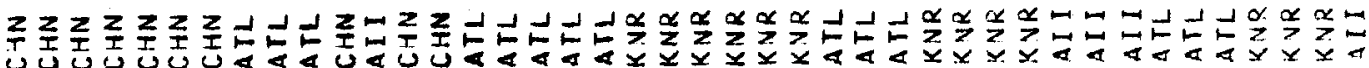


$-30-$

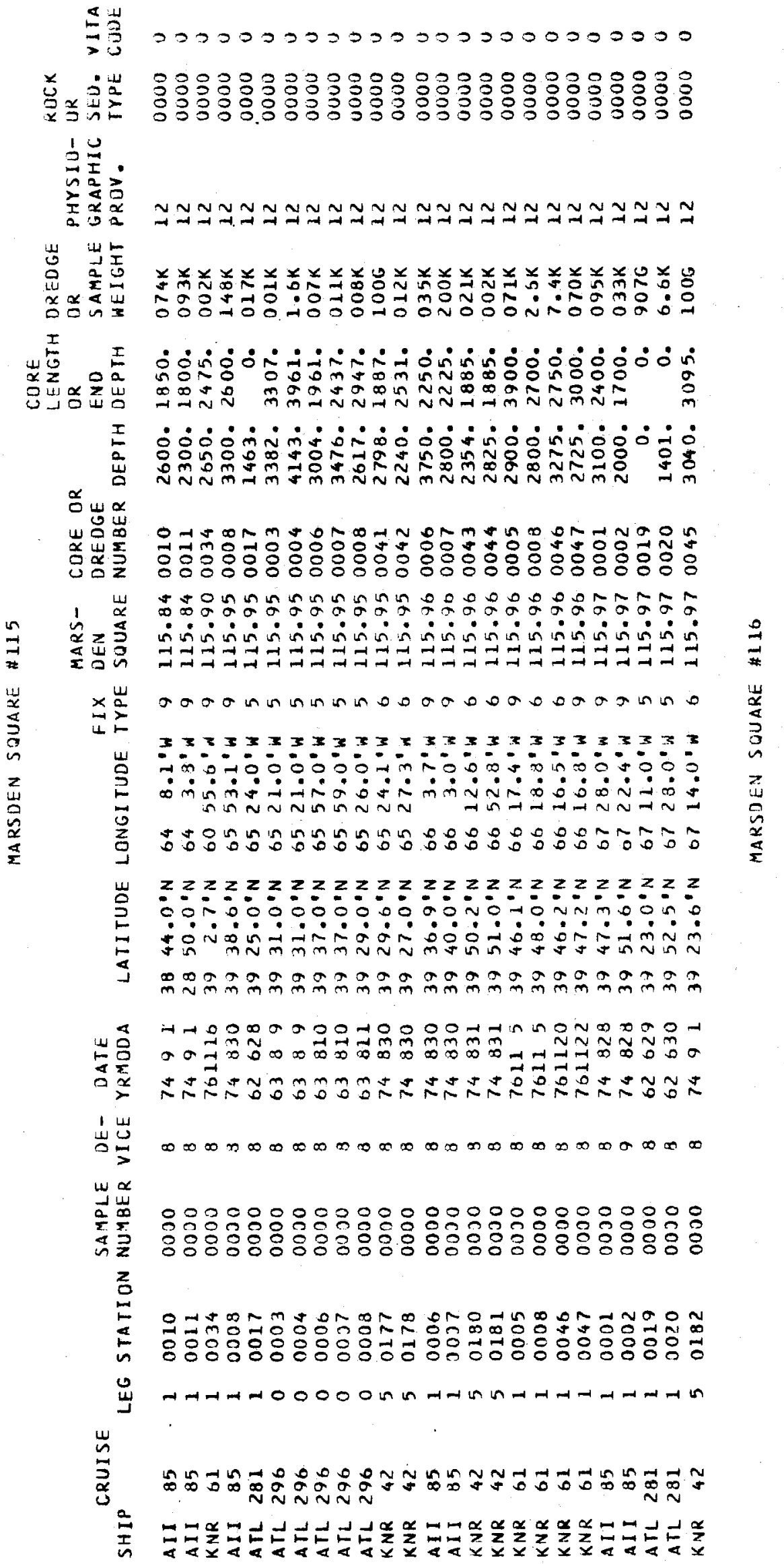

000000000000000000000

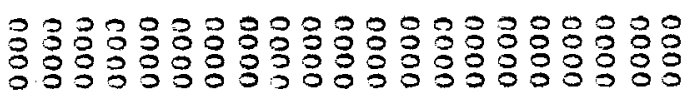

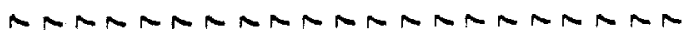

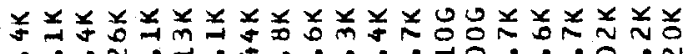

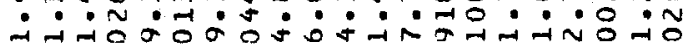

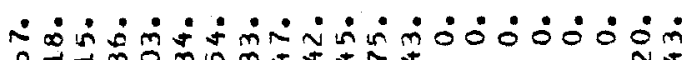

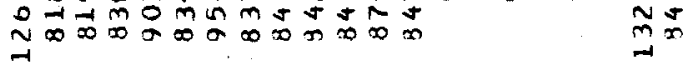

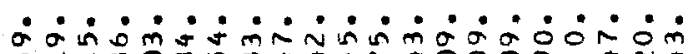

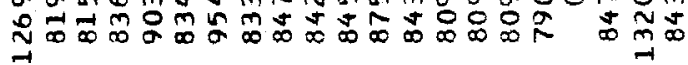

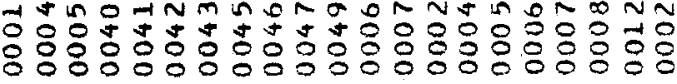
○ :ㅂ.

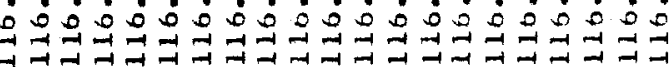

in in in in in in in in in in in in in in in in in in in in in

$3 \times 3 \times 3=3 \times 3 \times 333 \times 33 \times 33 \div 3$

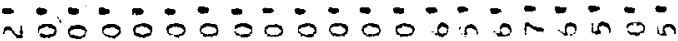

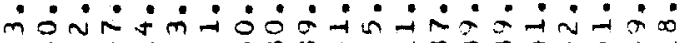

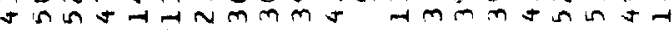

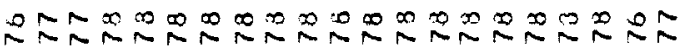

$z z z z z z z z z z z z z z \geq z 2 z \geq \geq z$

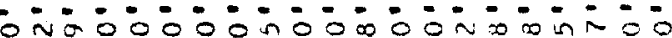
- $\dot{0} \dot{0} \dot{0} \dot{0} \dot{0} \dot{0} \dot{0} \dot{0} \dot{0} \dot{0} \dot{0}$

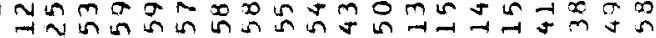

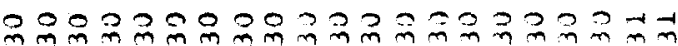

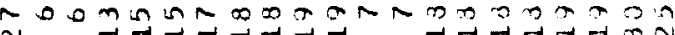

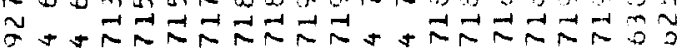
น

NNR

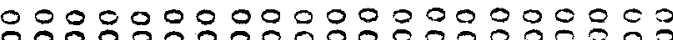

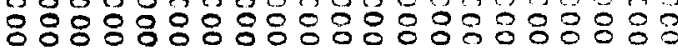

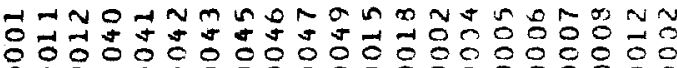

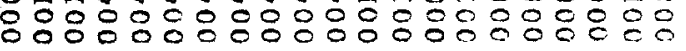

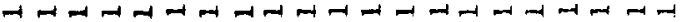

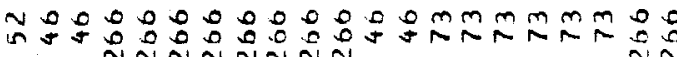

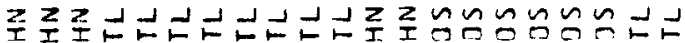

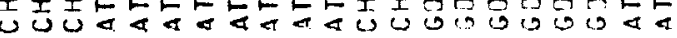


$-31-$

吉苟 000000000000000000000000000000

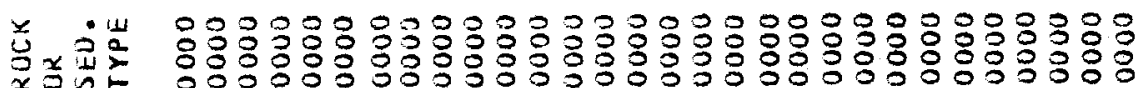

10

$2 \stackrel{1}{2}$

는

co

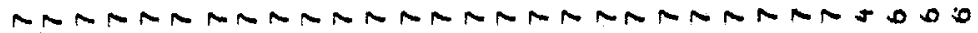

岁出管 品我出

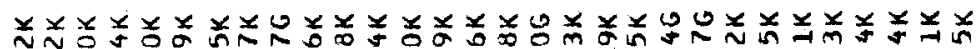

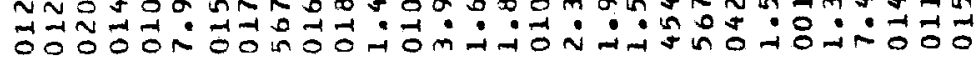
岕志志党

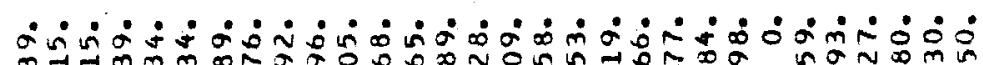

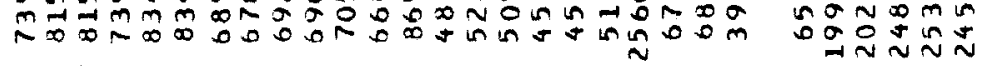

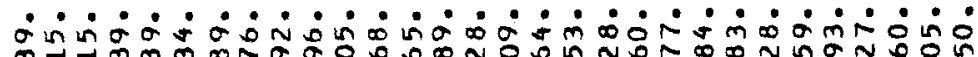
$\stackrel{\alpha}{\sigma} \propto$

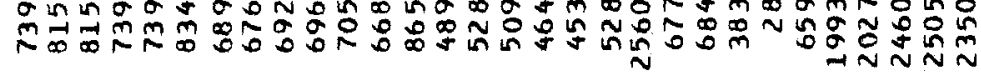

岃虽

若岩要

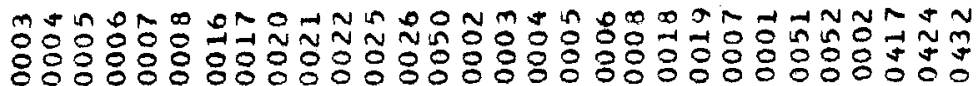

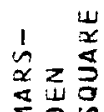

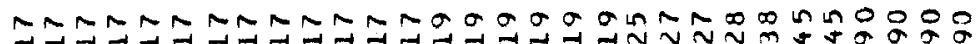

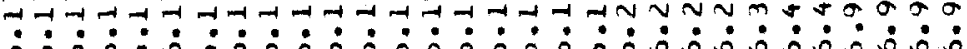

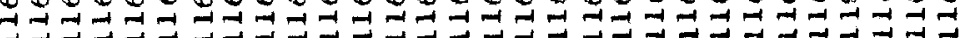

$\times \underset{a}{4}$

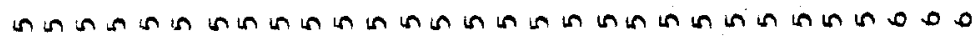

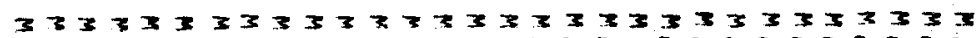

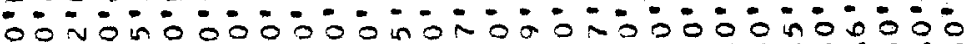

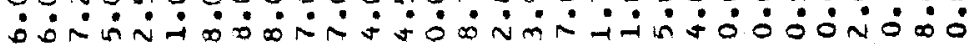
N NNNNNDNmmmmañ

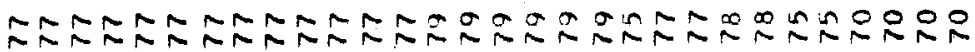

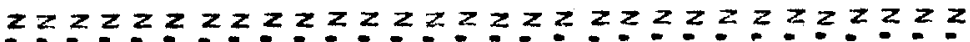
in 0000000 in in 0 in in $v$ in $=0$ in $m$ in 0 movio:0

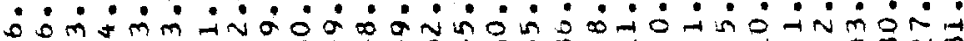
Un जी

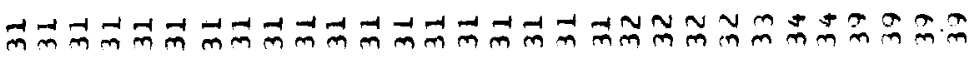

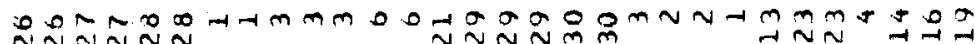

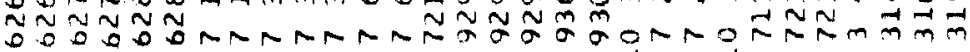

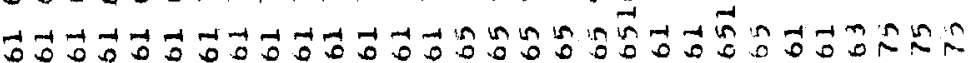

1 U

崩岕

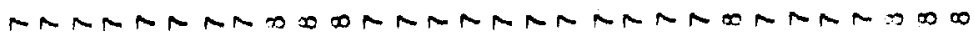

W $\alpha$

$\sum \underset{\infty}{1}$

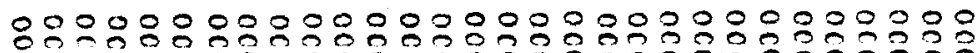

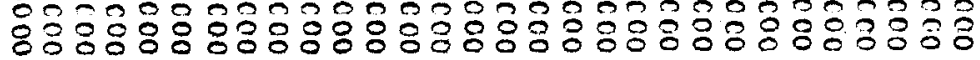

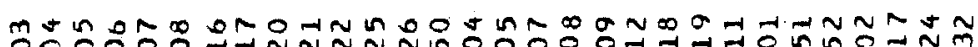

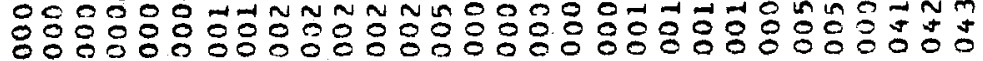

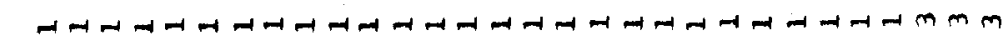

虫

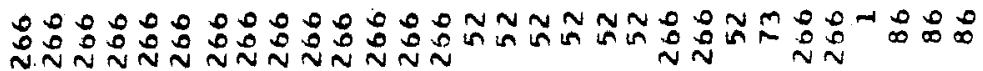
宣

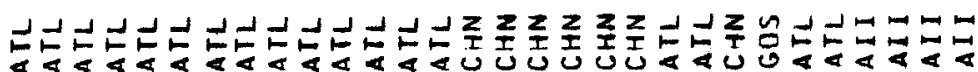

000000000000000

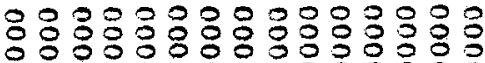

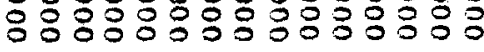

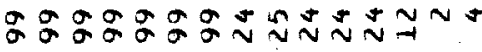

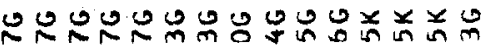

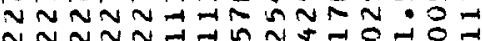

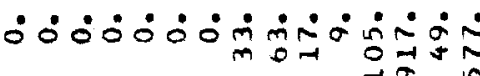

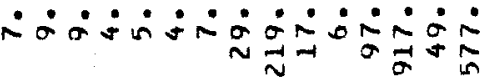

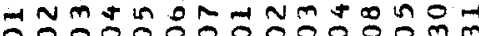

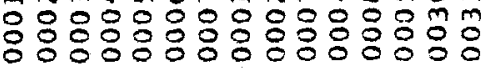

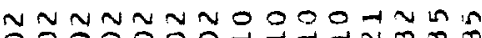

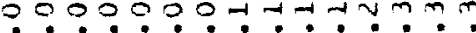

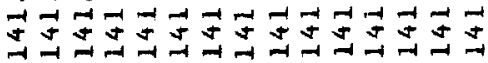

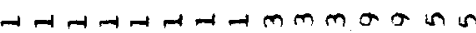

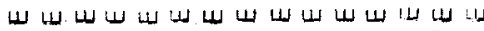

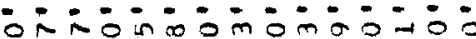
$\dot{\sigma} \dot{\sigma} \dot{\sim} \dot{\sim} \dot{0} \dot{0} \dot{\sim} \dot{\sim} \dot{\sim} \dot{\sim} \dot{\sim}$ $\pi \rightarrow \sim N N \rightarrow-m$ a InNJA

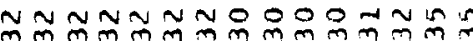

$z z z z z \geq z z \geq z z \geq z z z$

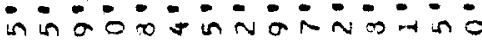
$\dot{\sim} \dot{\sigma} \dot{\sim} \dot{\sim} \dot{\sim} \dot{\sim} \dot{\sim} \dot{0} 0 \dot{0} \dot{0} \dot{\sim}$

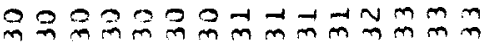

mmatatamanmodn

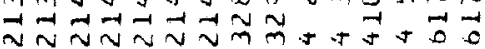
ng

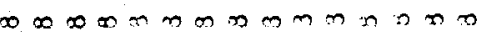

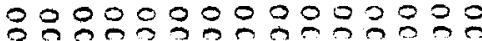

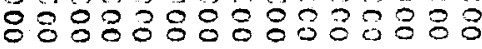

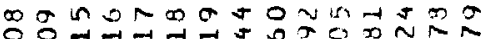

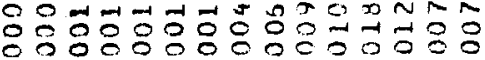

MmMmMMMNNNNNNH-

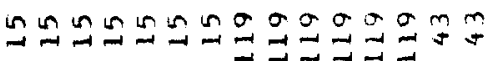

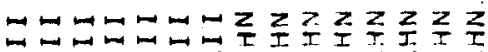

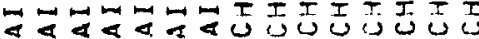


$-32-$

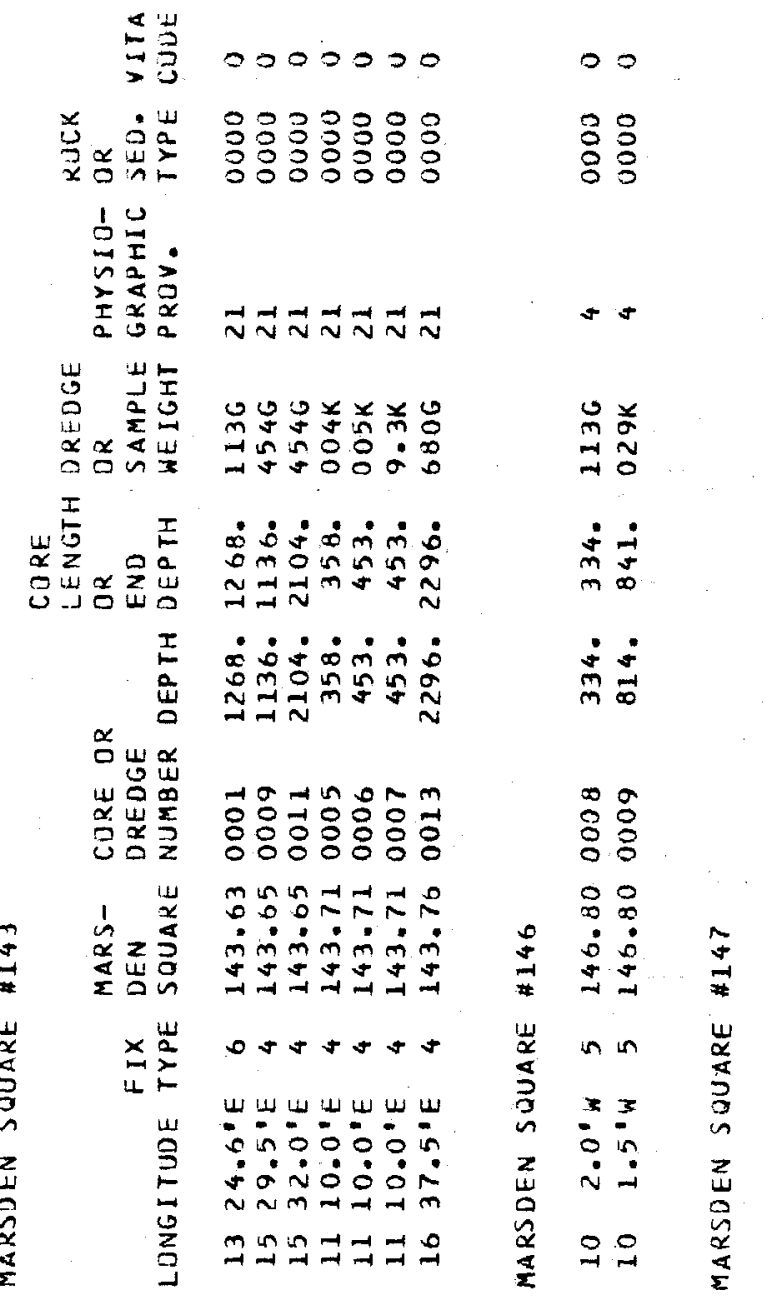

000000000000000000000000000000000

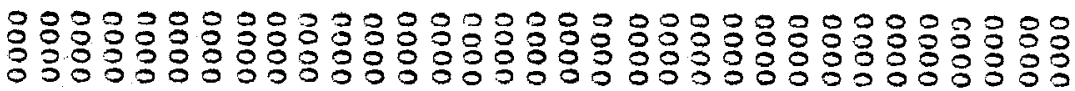

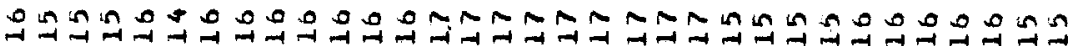

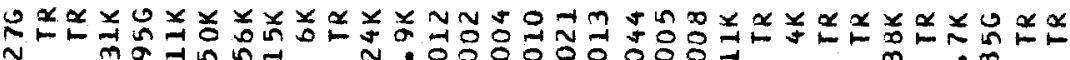
N

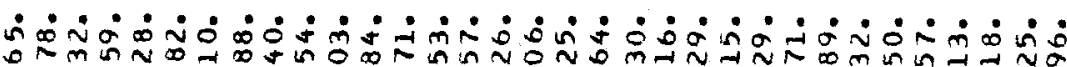
ON $n \backsim N$ N

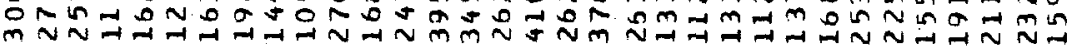

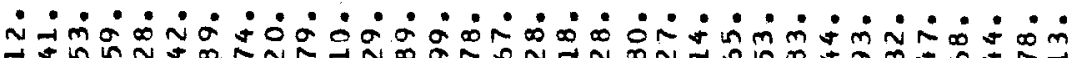

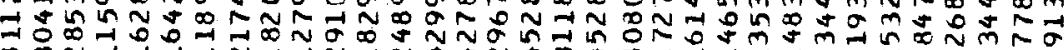

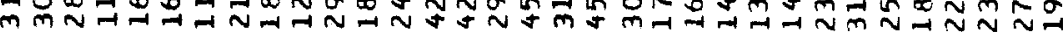

$4 \quad z \geq z \geq z z$

$\geq 2$

00

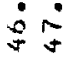

is

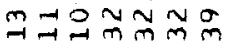

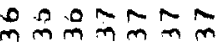

$\infty$

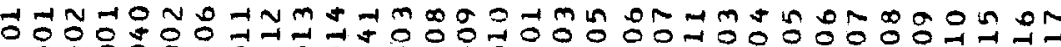

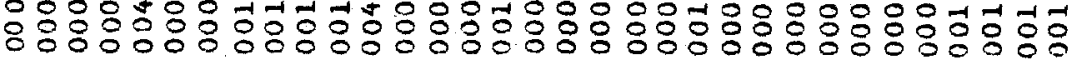

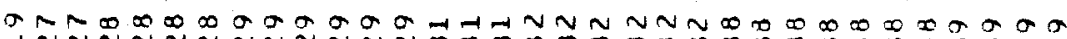

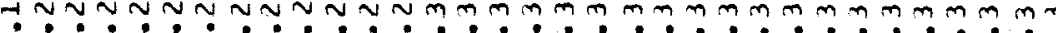

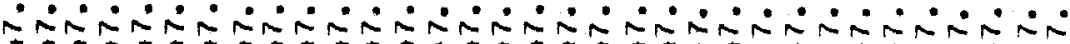

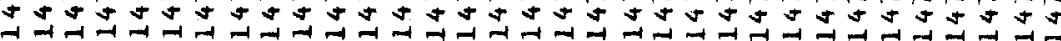

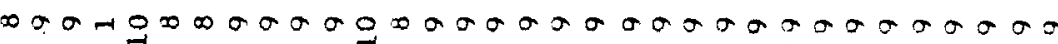

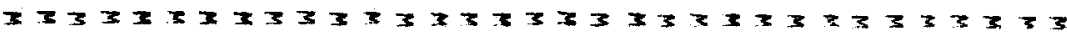

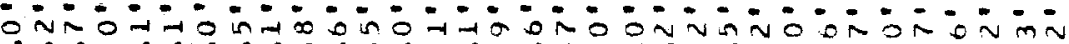

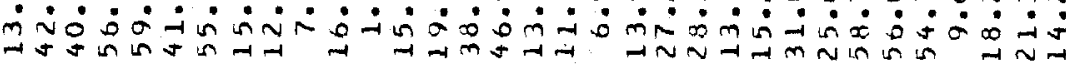

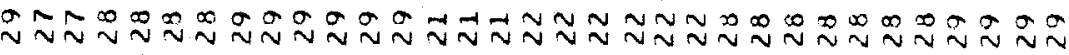

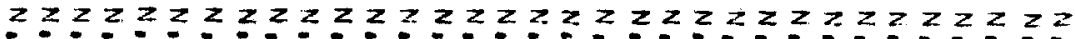

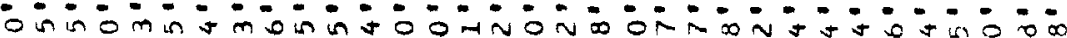

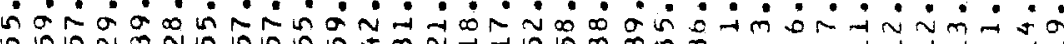

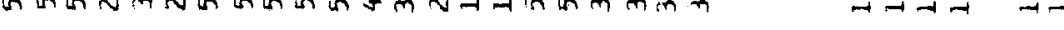

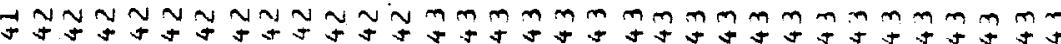

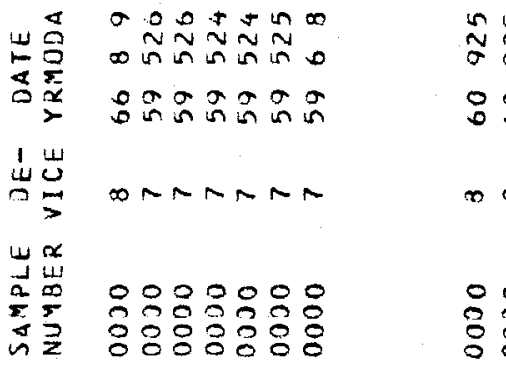

$\stackrel{n}{N}$

$8 ?$

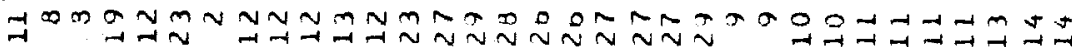

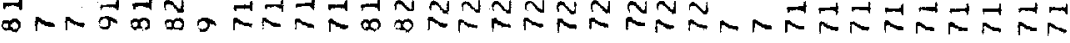

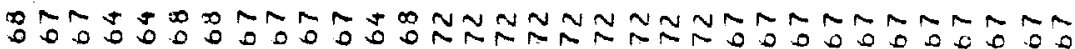

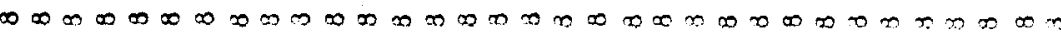

$=-\sigma=n \pi N m$

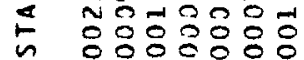

$\begin{array}{ll}\infty & 0 \\ 0 & 0 \\ 0 & 0 \\ 0 & 0\end{array}$

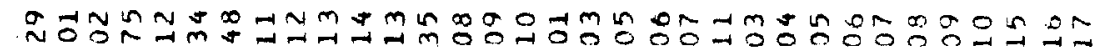

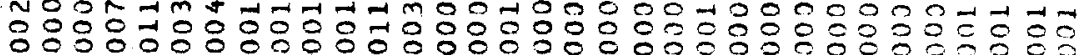

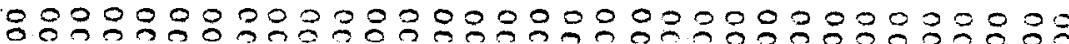

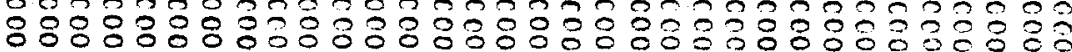

W Nmmment

$\sim \sim$ OMmA-T চ

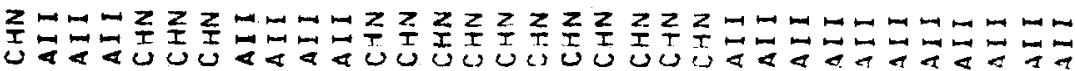




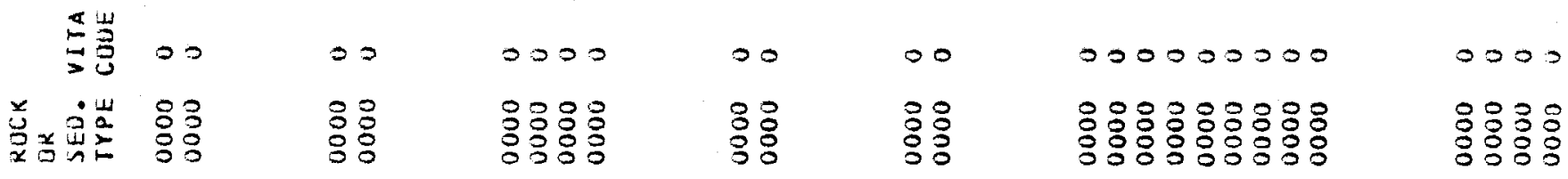

b品

in 2

I

$\stackrel{0}{0}=$

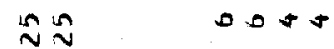

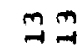

$\stackrel{+1}{\rightarrow+1}$

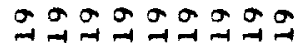

$x_{-1}^{\infty} a_{-1}^{n}$

岁岃

읨

$\stackrel{x}{m}$

?ำ

Q

$\stackrel{x}{\stackrel{x}{=}}$

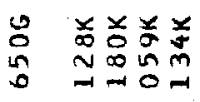

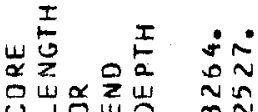

$\dot{\sim}$

ㄷํ요

爻亭

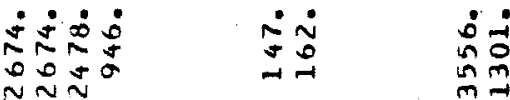

ஓं் 寸

$\dot{0} \dot{0} \dot{0} \dot{0} \dot{\sim} \dot{\infty}-\dot{-}$

遖

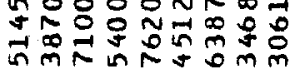

吕w $\propto$

in

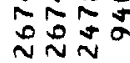

$\exists \stackrel{1}{ \pm}$

㟧㟔票

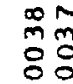

m:

ผn

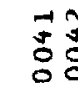

$m$.

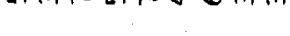

00 $\quad$ ñ̃m

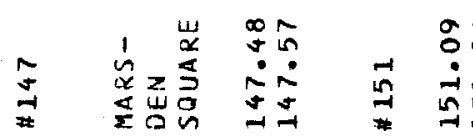

$\sim \stackrel{\sim}{\sim} \dot{\sim} \quad m \quad \dot{m}$

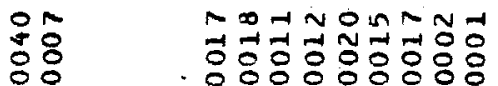

กิธิ์:

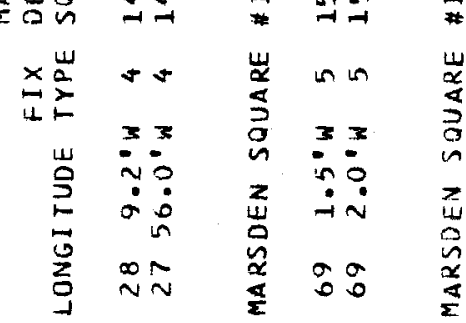

$\infty_{-1}^{\infty} \bigodot_{-1}^{\infty} \infty_{-1}^{\infty}$

$\rightarrow \infty$

in in in in

山ய山

$\stackrel{4}{\frac{4}{2}}$

in?

mm n

$\sim \sim \infty$

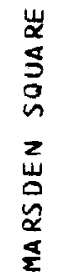

$n-1$
$x=$
$0 \dot{0}$
$\dot{m} m$
$m=$

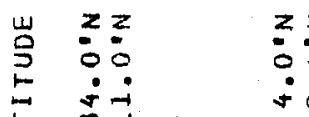

$z \geq z z$

in 0

$\dot{0} \sim \dot{\sim}$

$\mathfrak{q} \mathfrak{q}$

99

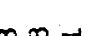

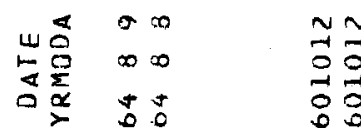

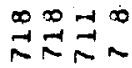

त.

in

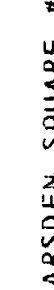

㟧

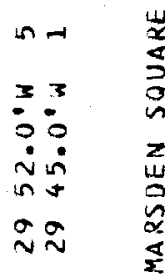

$\geq$

in

23

$\pm \infty$

in $\mathrm{in}$

in in

$\stackrel{n}{\sim} N$

$\vec{N} \tilde{N}$

80

00

岂岕

$n$

$\sim \sim$

$\sim \infty$

$8 \%$

80

8000
880
880

88

88

윽웅영영영영

x $33 x 33 x$

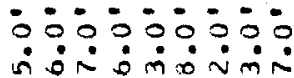

MTNNMAN

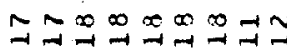

nnn nnn nn

- in 0000000

$\dot{m} \dot{n}=\dot{0} \dot{0} \dot{0}$

$0000000 \mathrm{mt}$

$\Rightarrow \exists \infty \sigma a \pm \exists=$

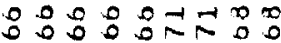

minm

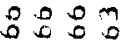

$\infty \infty \infty \infty \infty \infty \infty$

$\infty$ an

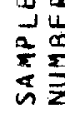

89

mas

$\frac{1}{2}$

90
$0 \%$

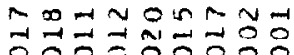

명영응응ㅇㅇㅇㅇㅇㅇ

$N N$

NNNNNDO Inin

$m a$

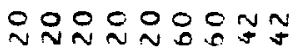

요요ำ

론로른

$\stackrel{m}{-1}$

$\geq \underset{1}{2}$

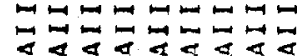

シேッ

2835

$\frac{2}{1}$

a 1 


\section{$\stackrel{\square}{5}$

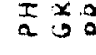 \\ 岁出壱}

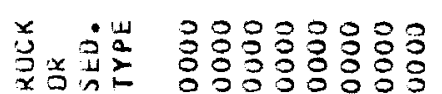

古至.

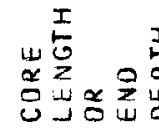

壱

各山。

崖岕䀁

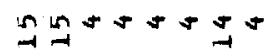

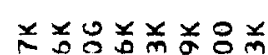

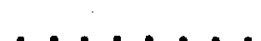

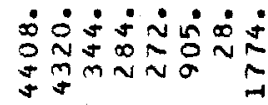

$\dot{0} \dot{\circ} \dot{0} \dot{0} \dot{0} \dot{\infty} \dot{N}$

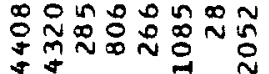

政

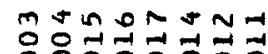

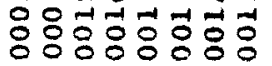

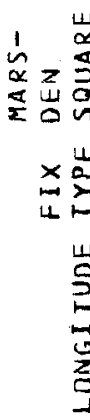

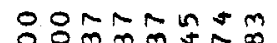

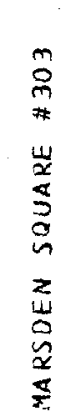

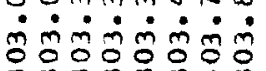

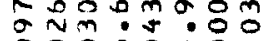

$>0$

咅

8

:

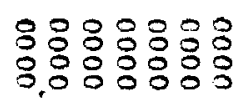

$000=$

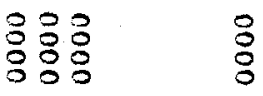

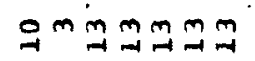

$\vec{n} \mathfrak{N}$

$\vec{\sim}$

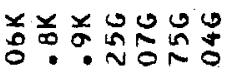

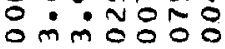

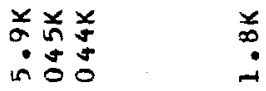

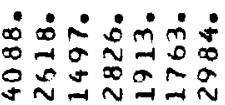

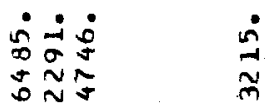

$\dot{0} \dot{0} \dot{0} \dot{m} \dot{a} \dot{a}$

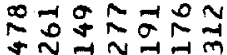

这突家交

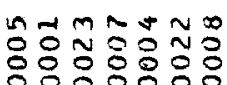

minn

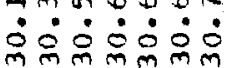

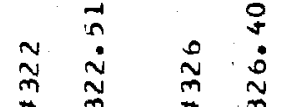

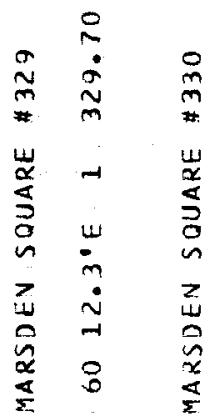

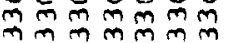

tat-A-th

山

-0 in 0500

- $\dot{\sim} \dot{\alpha} \dot{\sim} \dot{\sim}$

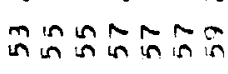

nnunnun

0000000

ímjojón

tmingor

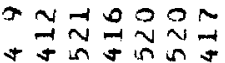

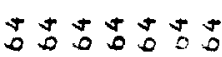

ำㅇํㅇํㅇ

in

in $\dot{n} \dot{0}$

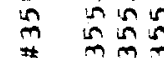

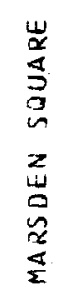

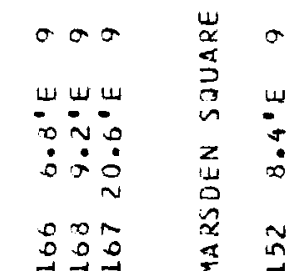

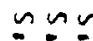

in

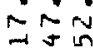

man

$\rightarrow-7$

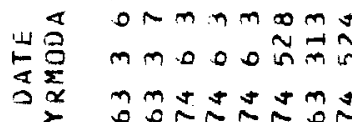

$\begin{array}{ll}a & \overrightarrow{0} \\ 0 & \vec{n}\end{array}$

in

s

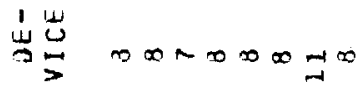

$\infty$

$\infty$

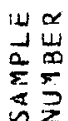

응ㅇㅇㅇㅇㅇㅇㅇㅇ

영영요영

$\stackrel{8}{8}$

$\stackrel{8}{8}$

웅융영융융응

$=\prod_{0}^{n} \infty$

$\vec{A} \vec{i}$

$n \sim \infty \infty ⿻ \infty$

웅

00000

고ำำำㅇำ

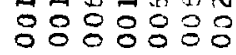

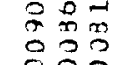

울웅

3

ㅇํㅇำ

$\stackrel{5}{8}$

กี

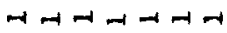

$a+a$

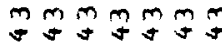

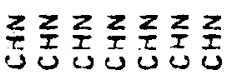

808

\begin{tabular}{l}
$\stackrel{8}{3}$ \\
\hdashline \\
$\vdots$
\end{tabular} 


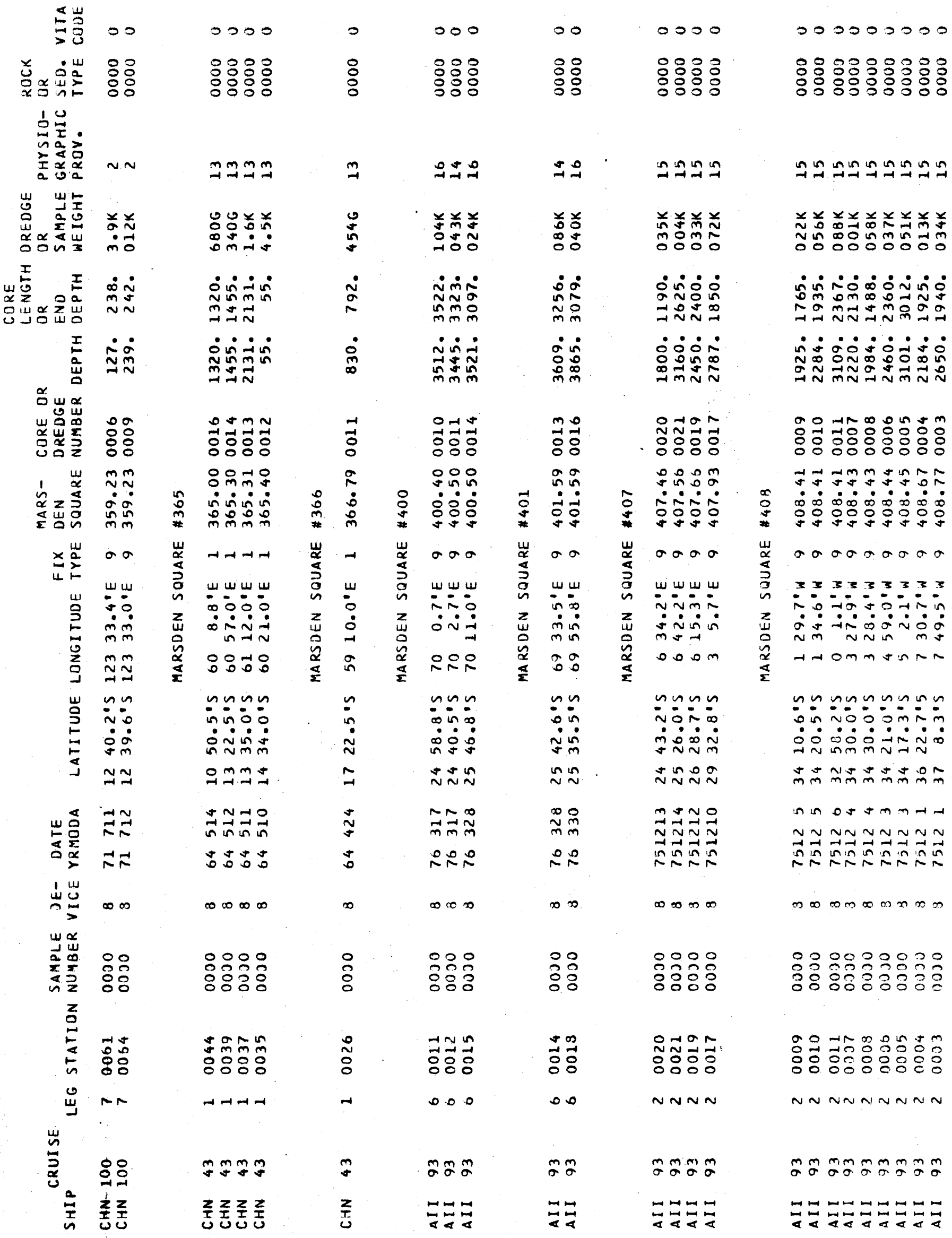




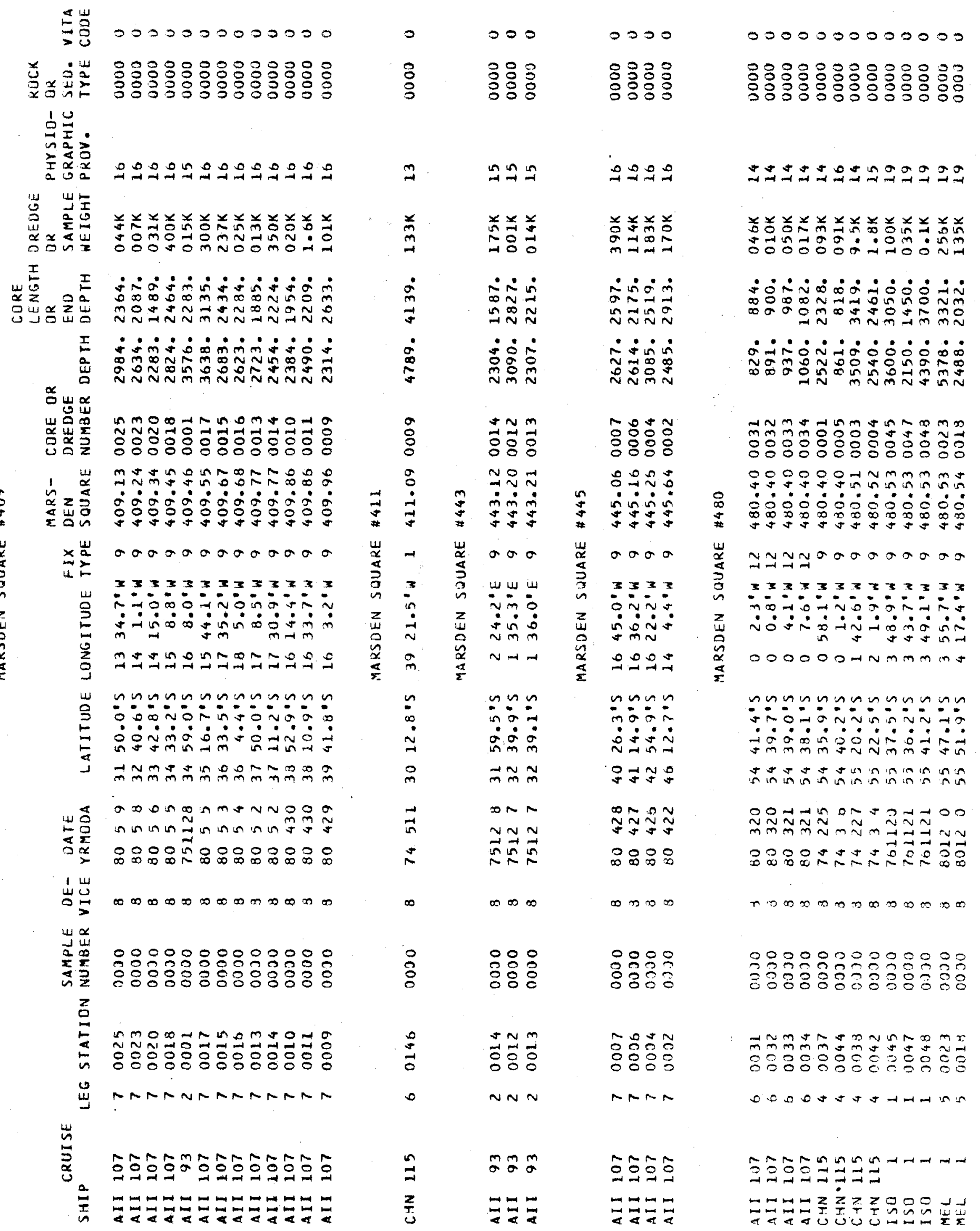


$\$ \frac{U}{3} 00000000000000000$

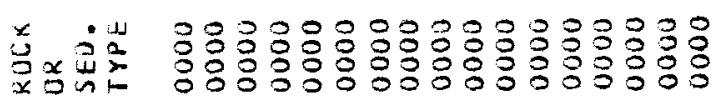<smiles>C[Si]=[Ti]</smiles>

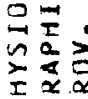
ta

岁出志 峞乐崖

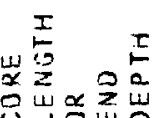

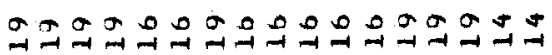

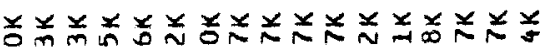

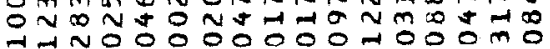

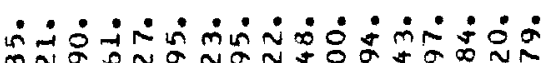

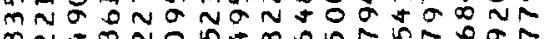

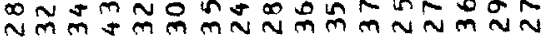

㟒

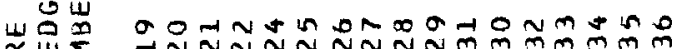

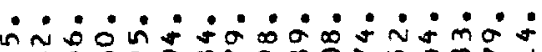

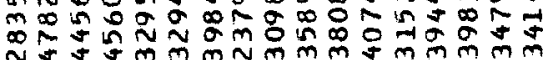

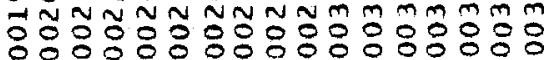

1) $\dot{0} \dot{0} \dot{0} \dot{0} \dot{0} \dot{0} \dot{0} \dot{0} \dot{0} \dot{0} \dot{0} \dot{0} \dot{0} \dot{0}$

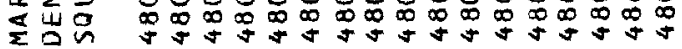

$\times$ 㟧 000000000000000 4

$333333 \leq x 3333 \times 3 \times 3$

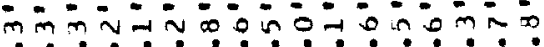

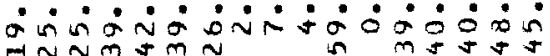

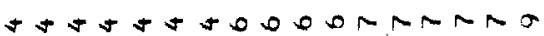

nunnunnennnnunnnu

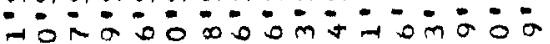

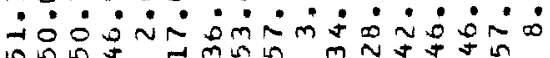

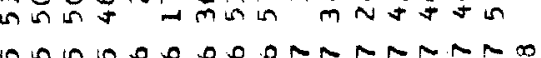

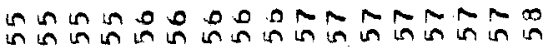

t) 00000000000000000 NNNNNNNNNNNNNNNNN

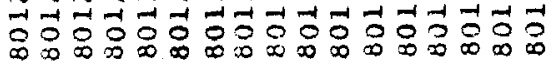

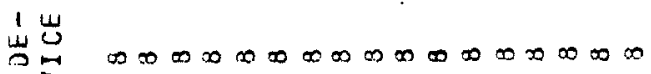

岃湈 00000000000000000

出

z

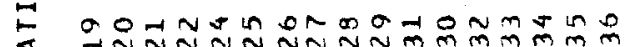

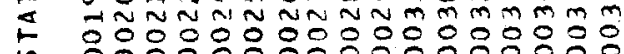
w

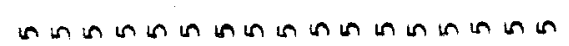

$\stackrel{w}{\stackrel{w}{a}}$

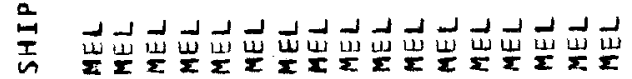

0000000

00

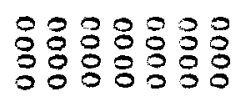

88

응

8898989
8898989

$\log _{\rightarrow+\infty}^{0} 000$

$\underset{-1}{\sim}$

$a$

ำำำ

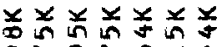

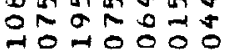

$x$
0
0
$0-1$

$\dot{\sigma} \dot{\sim} \dot{\sim} \dot{\sim} \dot{\sim} \dot{N}$

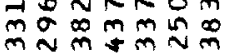

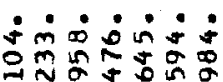

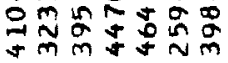

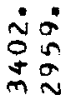

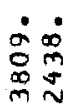

กิำ

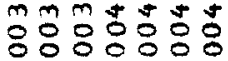

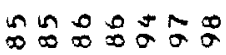

$\dot{-1} \dot{-1} \dot{-1}-\dot{1}$

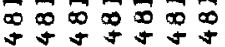

o. 0000

$3 x_{3} 33$

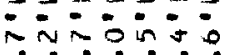

ல்

$n=000 \cong 0$

nnnแnnn

-ivizio

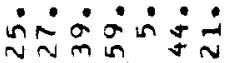

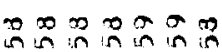

0000000

$\sim \sim \sim \sim \sim n n$

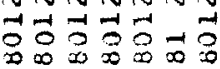

$\infty \infty \infty \infty \infty$

허융

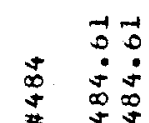

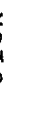

$\stackrel{0}{\circ}$

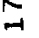

$\dot{0}$

岁

$2=1$
$=3$
$3=$
$0=5$
$5=5$
$5=5$

$\min$

$\because \frac{\pi}{0} \div$

$\therefore 0$

$\rightarrow d$

$m m$

옹

$\rightarrow n$

영웅응용응

8888588

응용

80

$\frac{9}{8}$

30

mm $m=n$ v

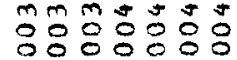

80

00

un min in in in

50

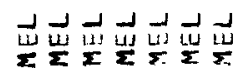

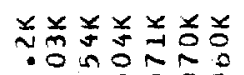
ن융ํㅇㅇㅇㅇ

îㅇㅇㅇㅇㅛ NजSN

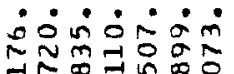

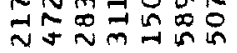

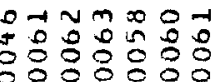
moD 0 a

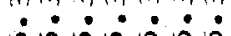

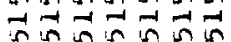
$\simeq 000 \cong \simeq$ 山山以 山以!

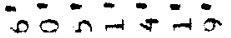
$\dot{m} \sim \dot{\sim} \dot{\sim} \dot{\sigma} \dot{\sigma}$ molona

innunnnu - ㄴ.? in a

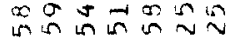
जी मी मी मी?

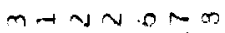
ที่ง ข

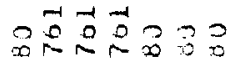
$n x \rightarrow \infty x m$ 긍응응응 $389398 \%$

O-1 Nm $20-1$

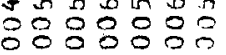
$n \rightarrow \rightarrow-\infty 0$ $\hat{0}^{+1+\hat{0}} \hat{\mathrm{O}} \tilde{\mathrm{O}}$ ロ路品ロニ゙ 
$-38-$

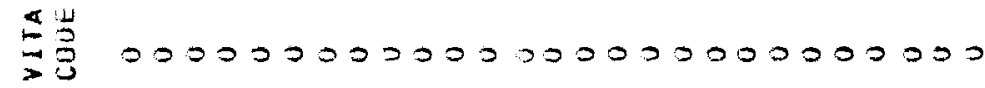

苍

尚至:

i 2

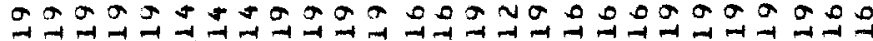

岁步占

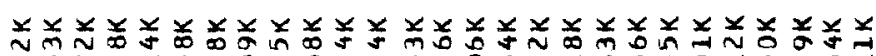

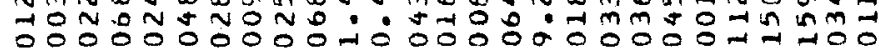

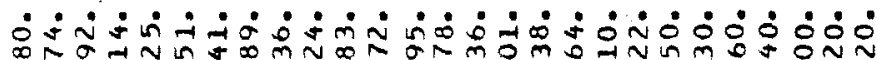

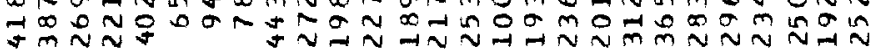

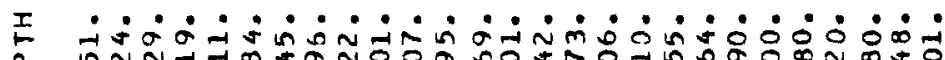
岃

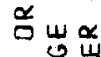

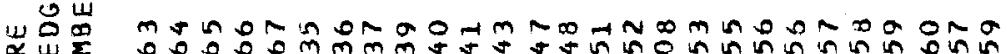

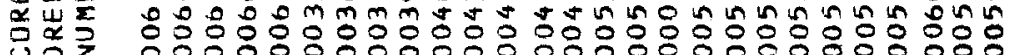
1 य

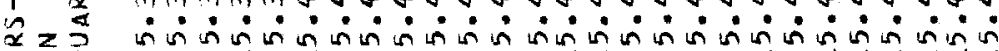

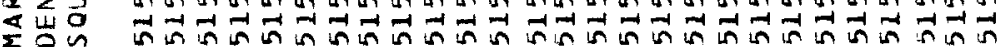

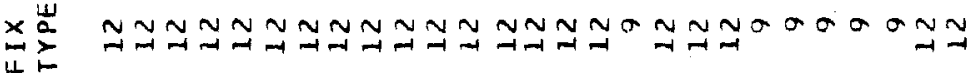

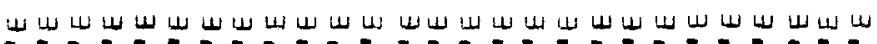

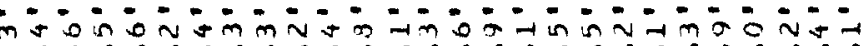

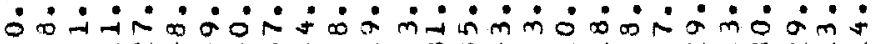
NHANG

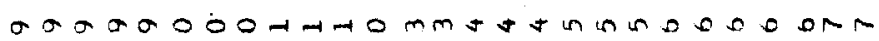

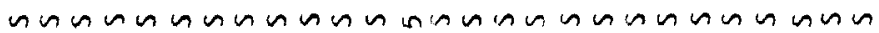
- ind - o

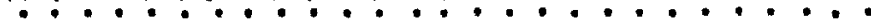

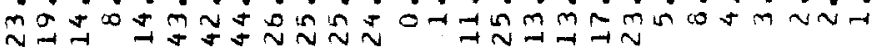

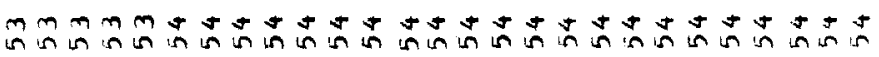

感

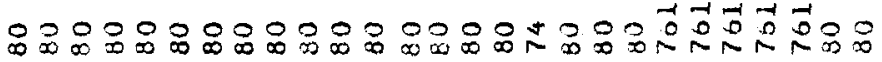

岁

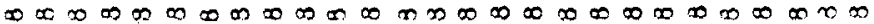

岸崫

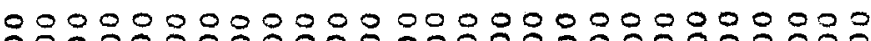

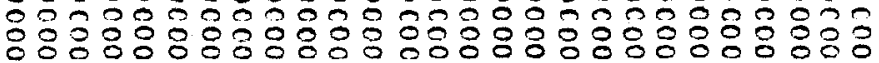

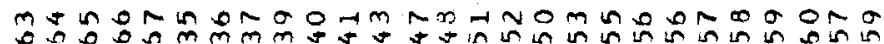

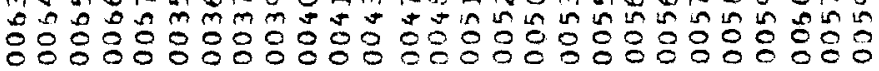

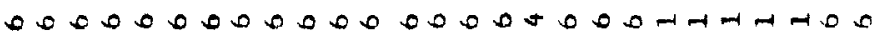

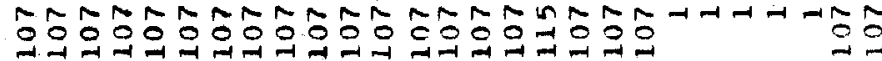

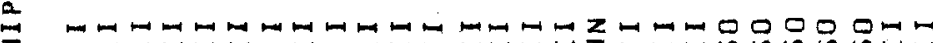

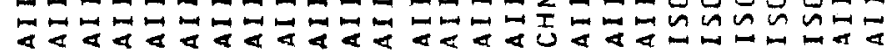


DESCRIPTIONS OF W.H.O.I. ROCK DREDGE SAMPLES, VOLUME II. 


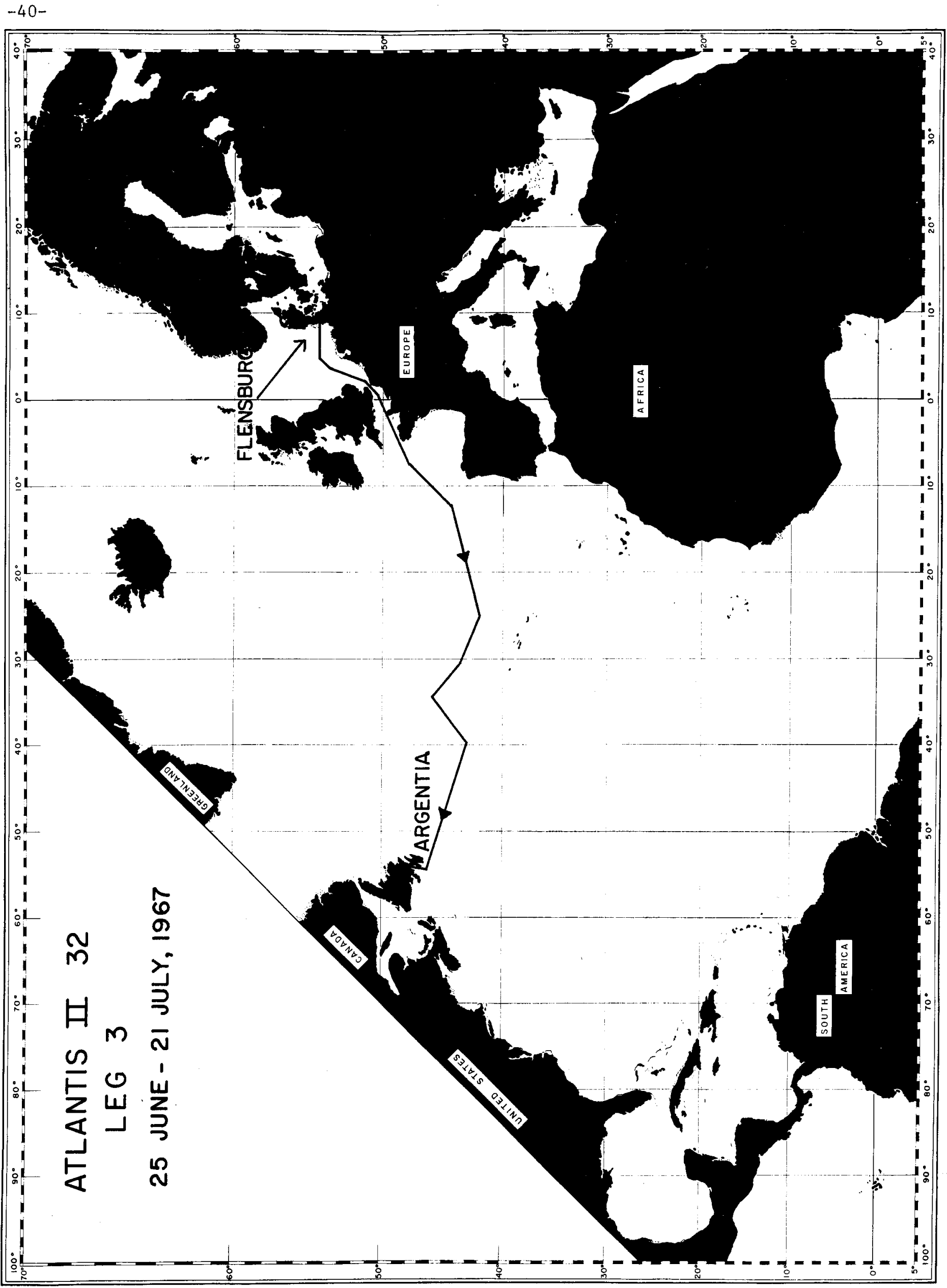




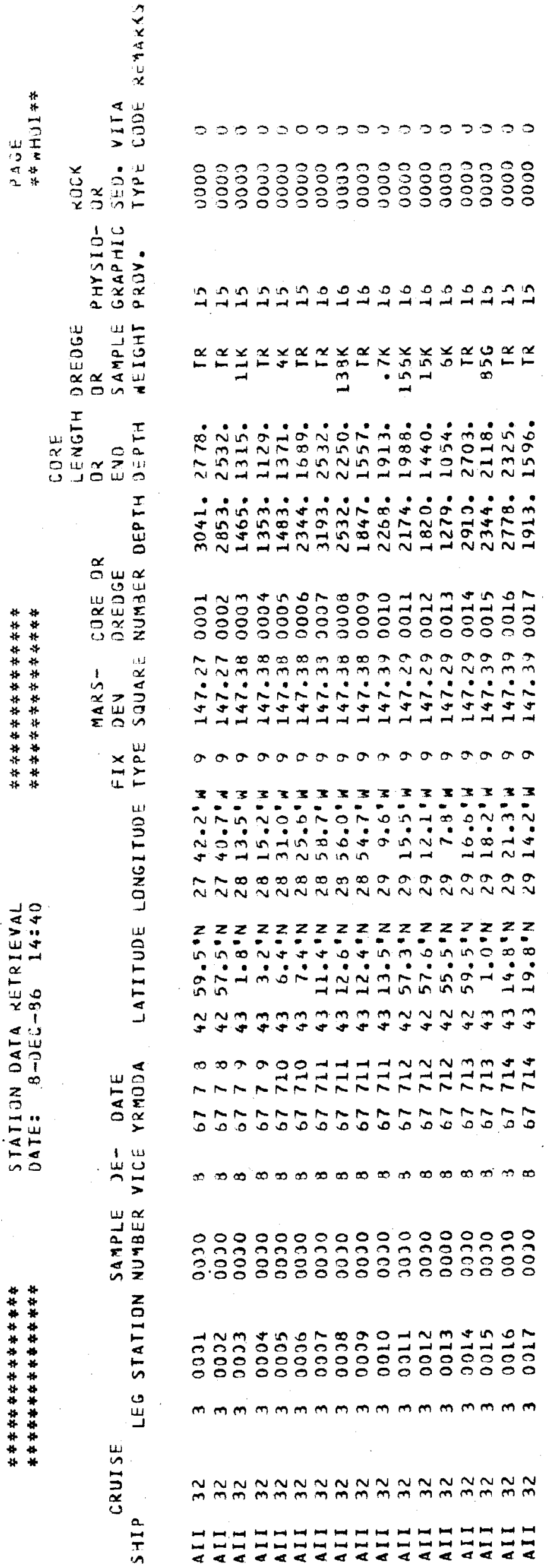




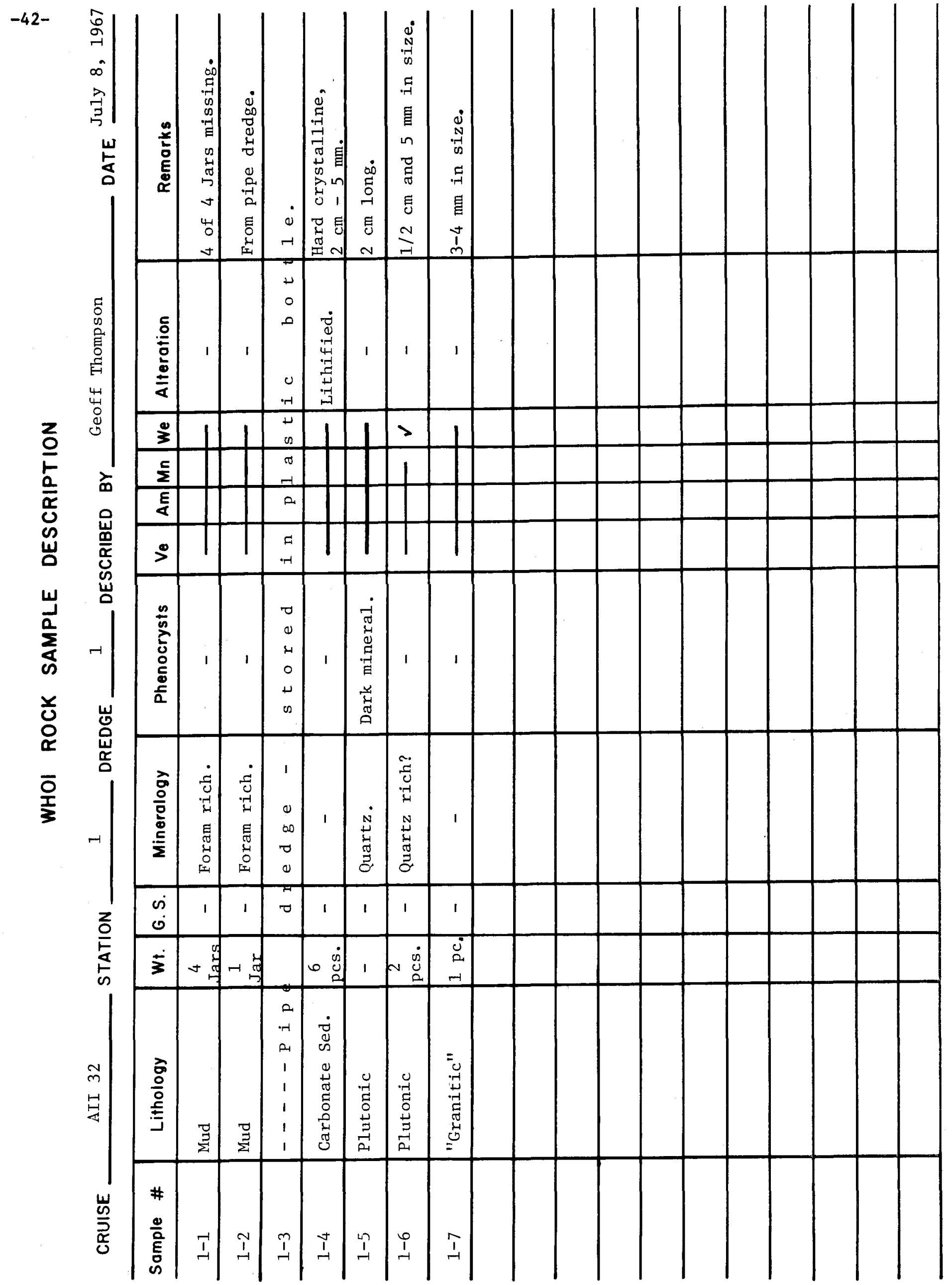




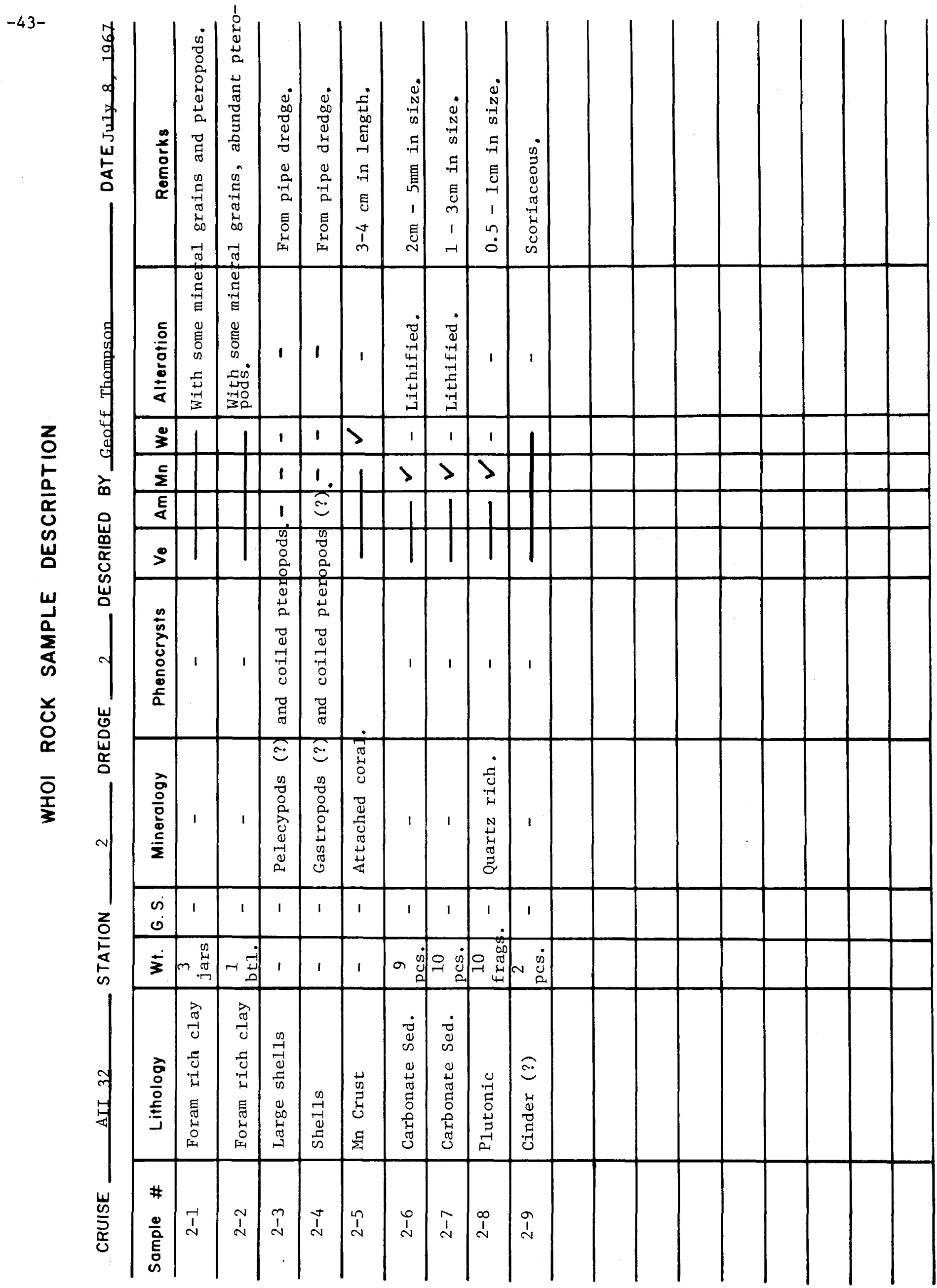




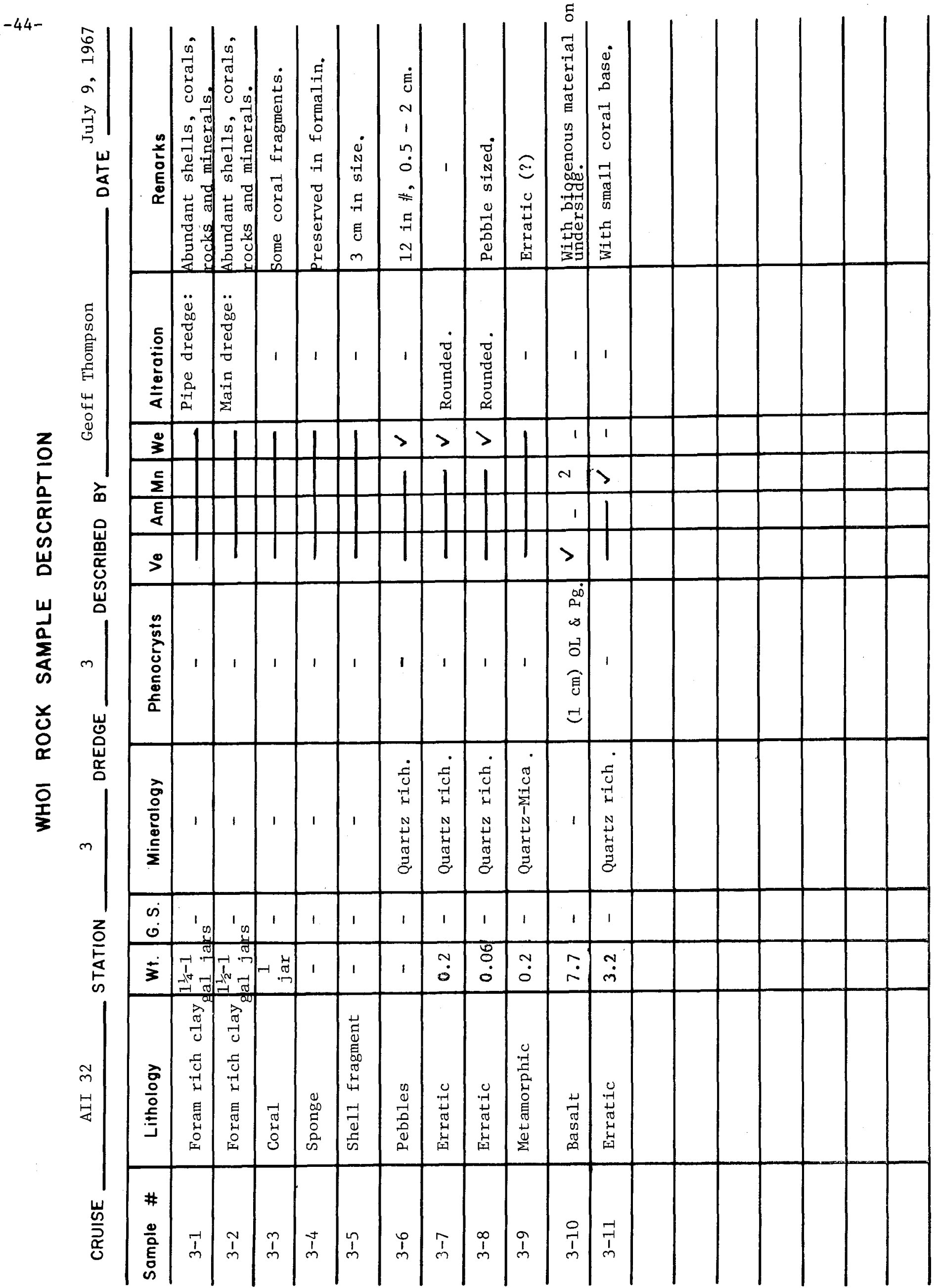




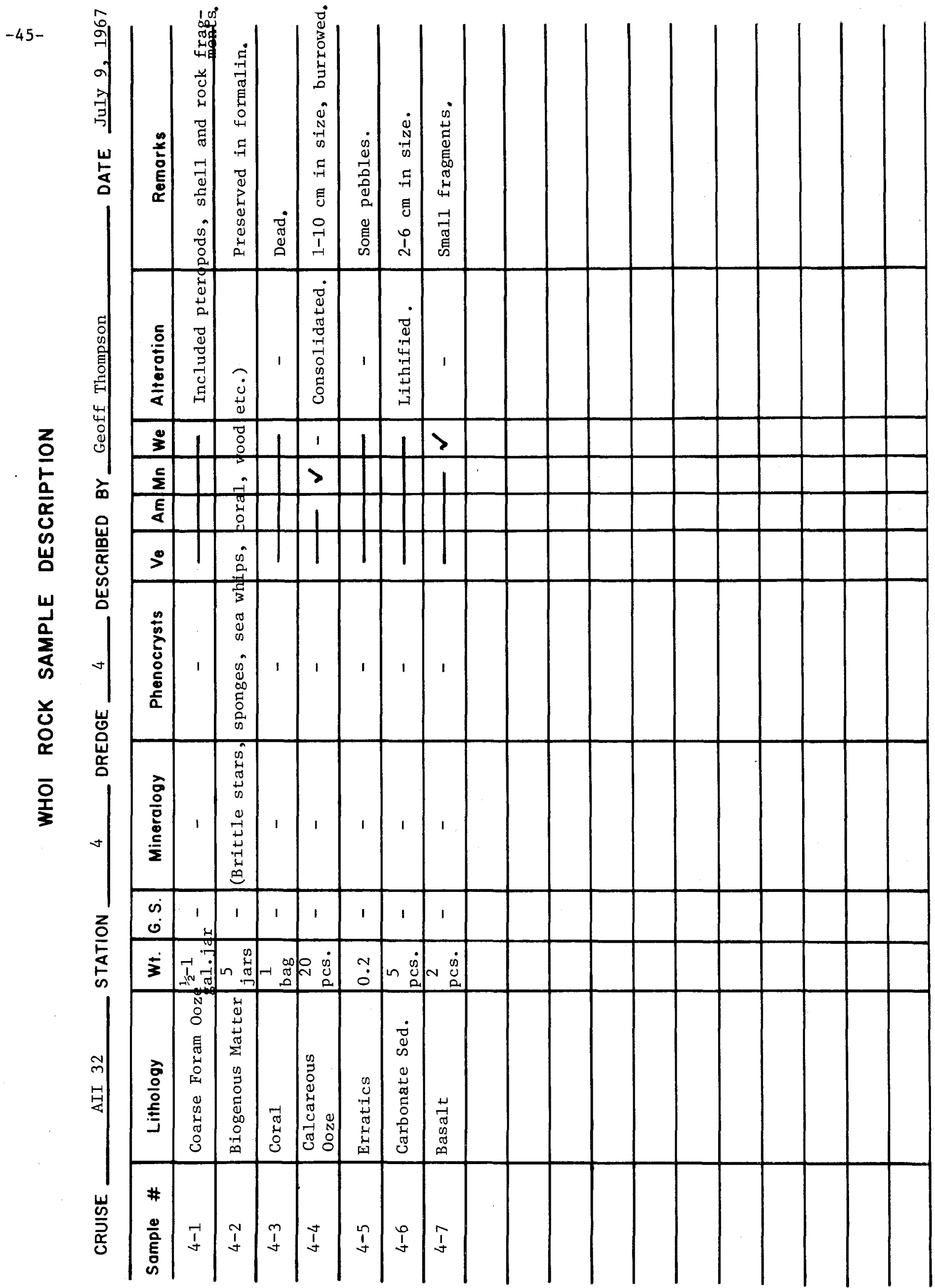




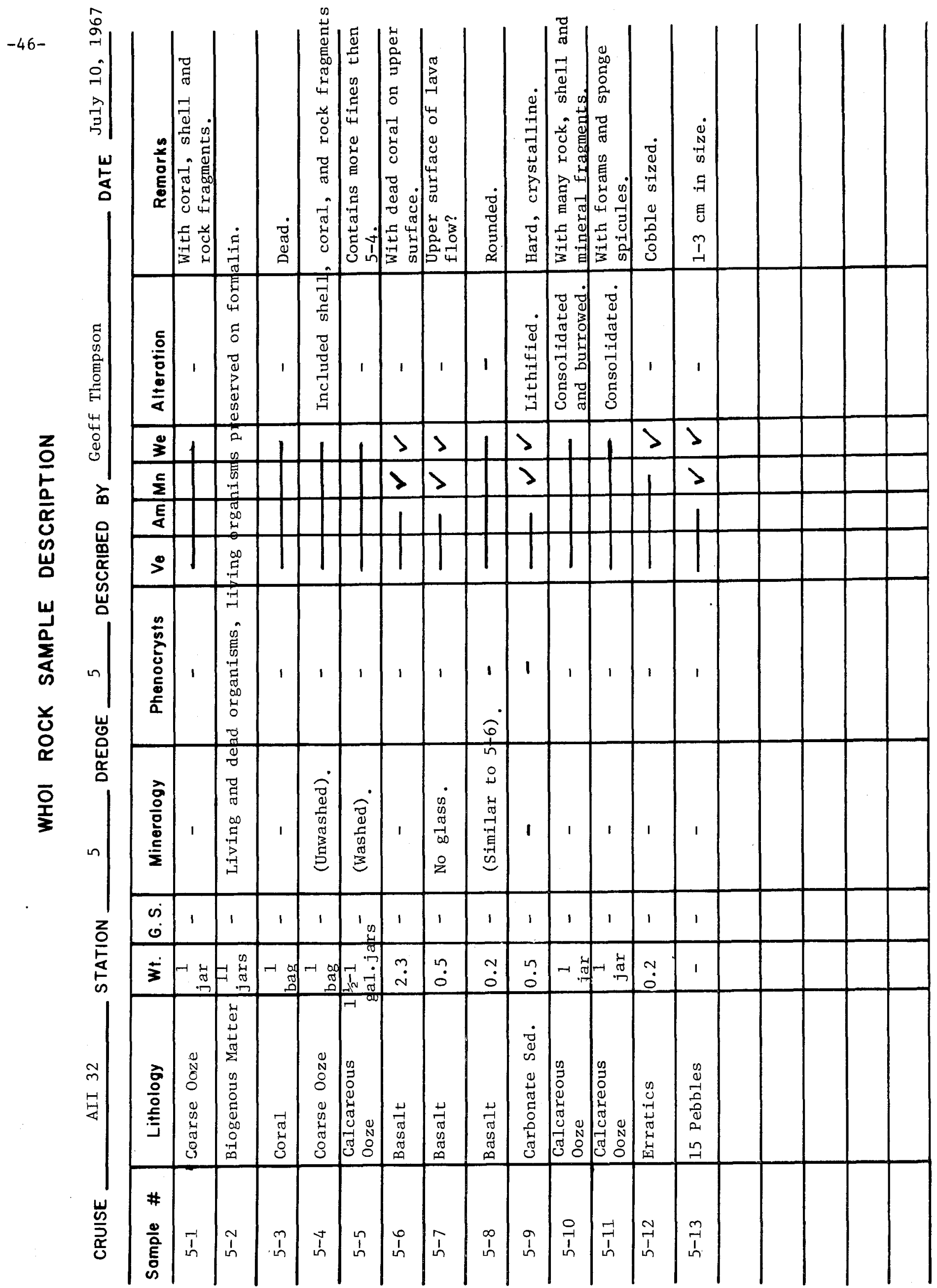



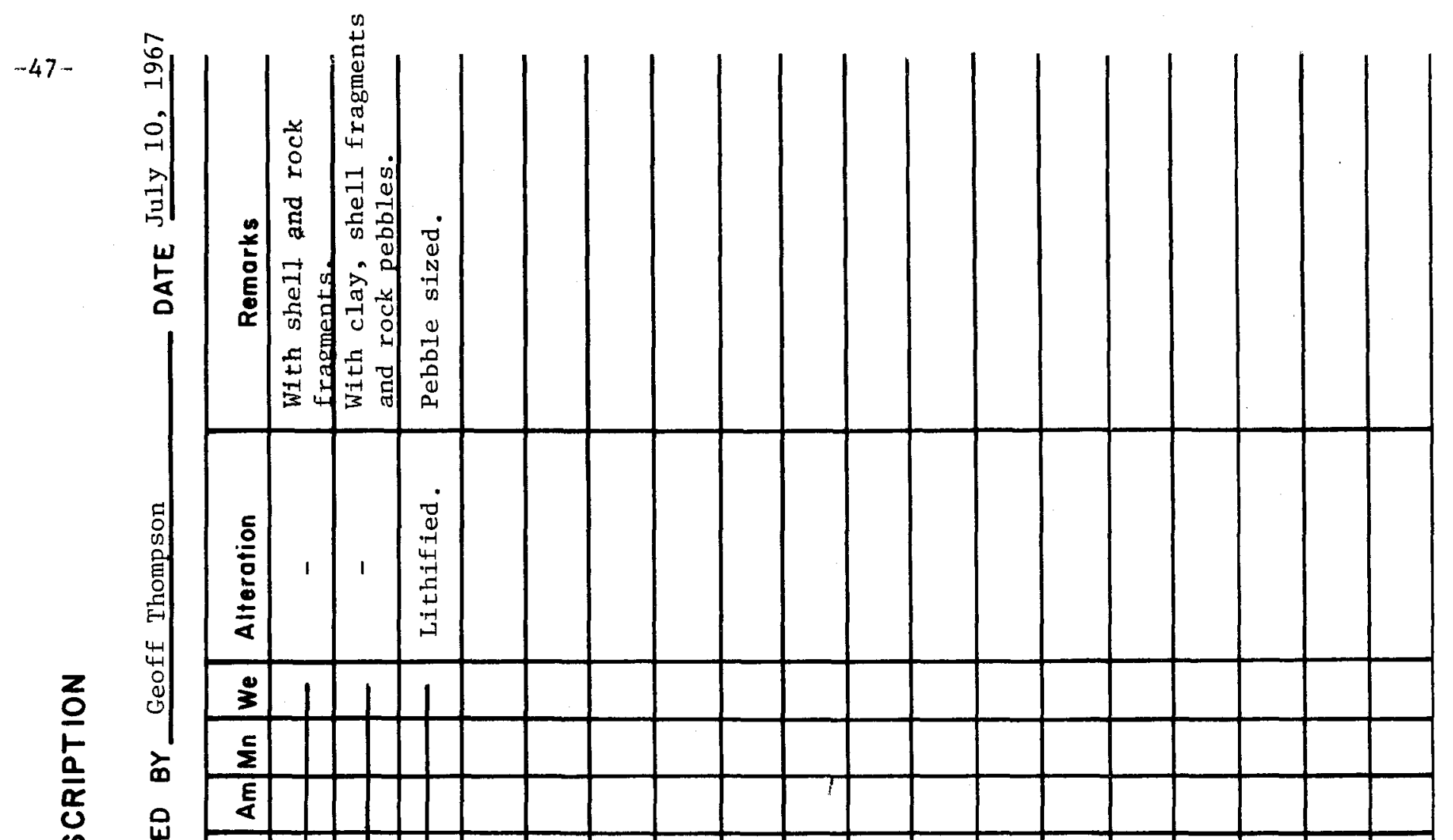

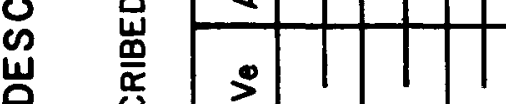

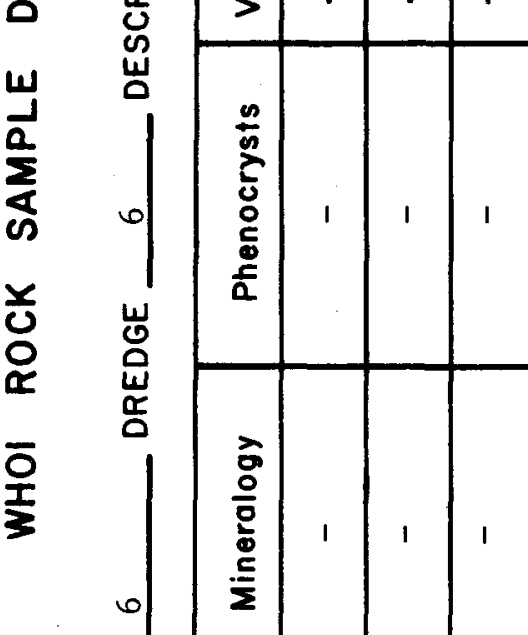

(

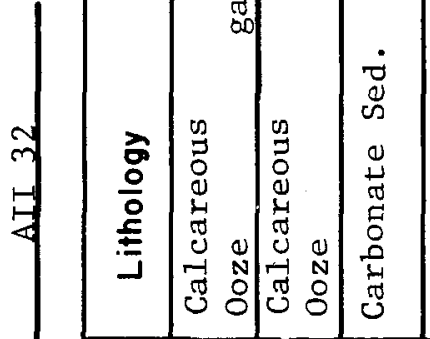

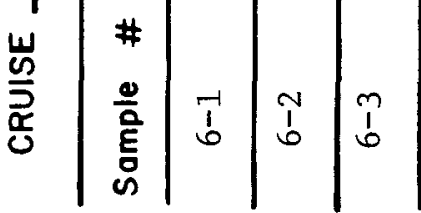




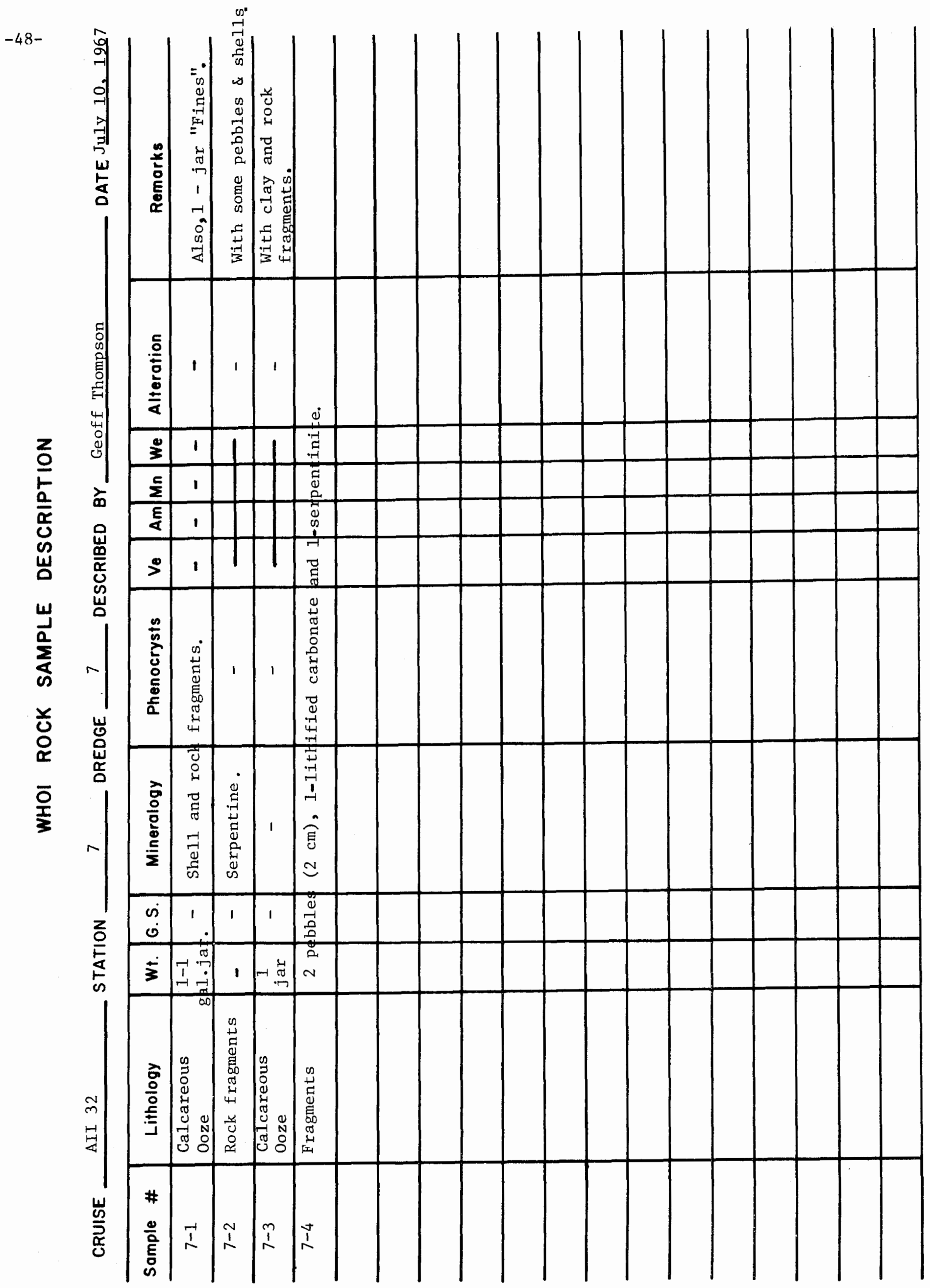




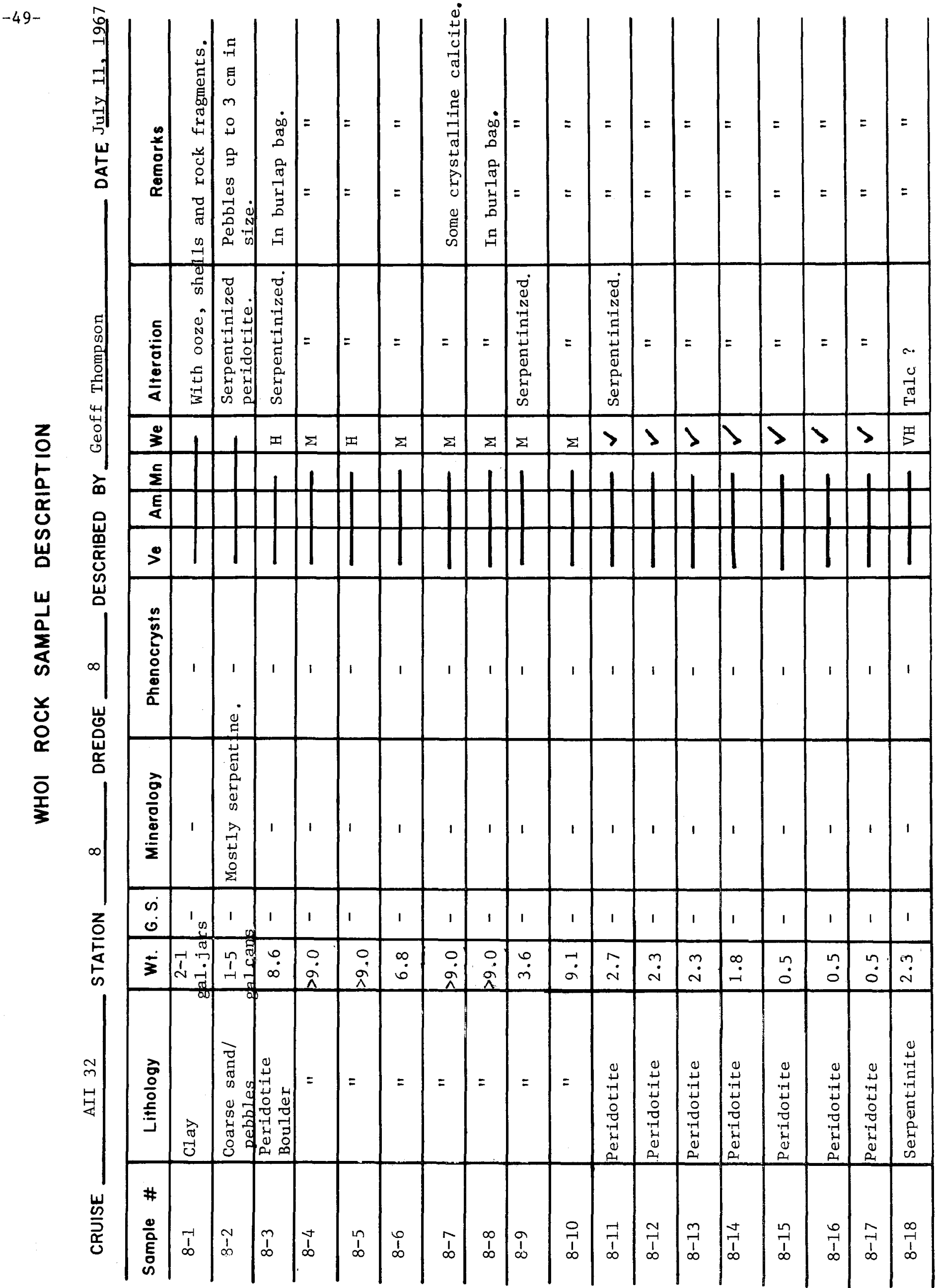




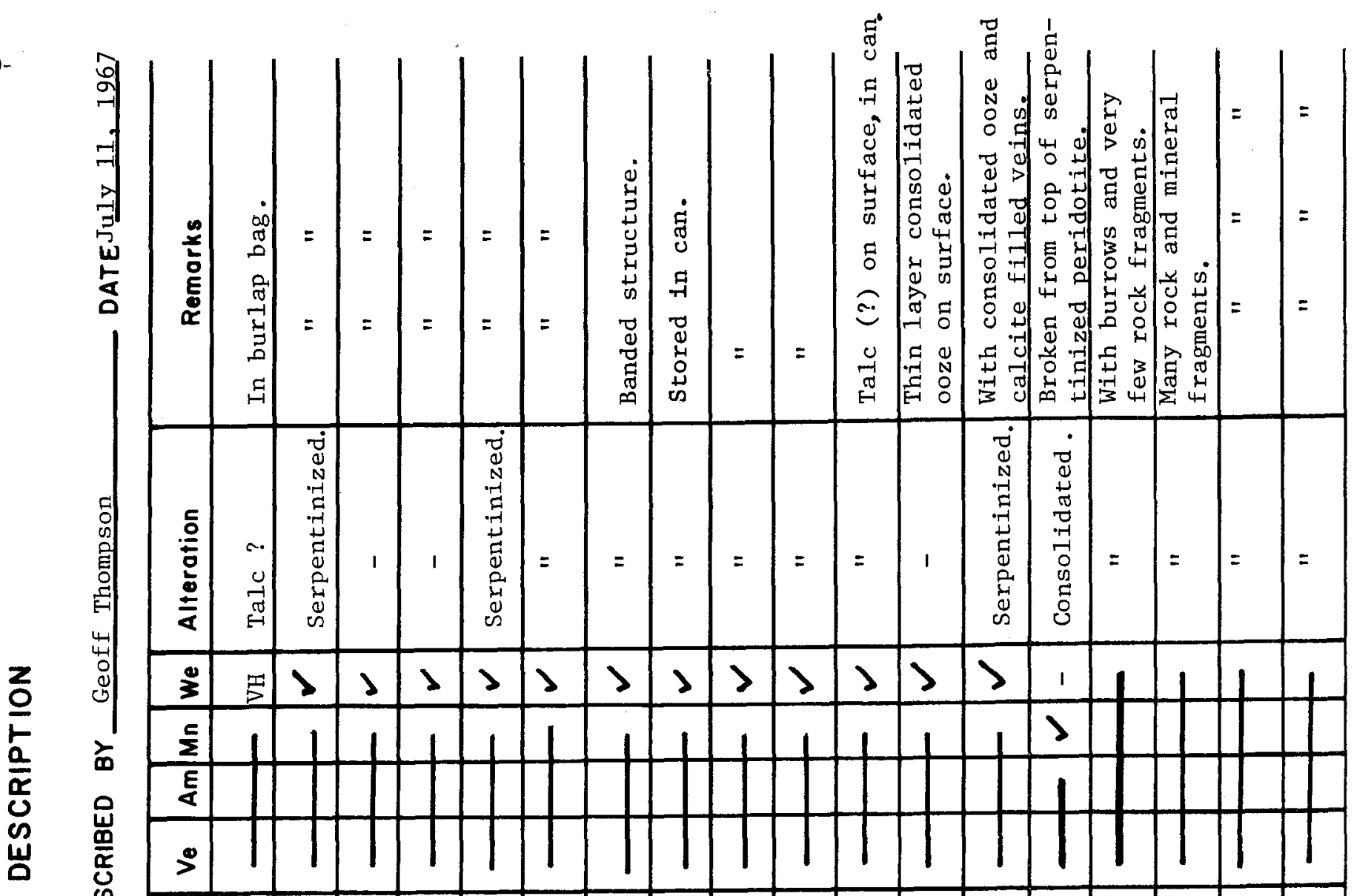

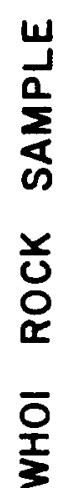

竞

㟔

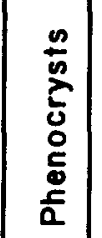

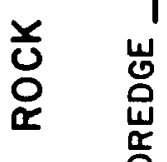

$\infty$

$\frac{7}{\frac{0}{2}}$

古

is

\begin{tabular}{|c|c|c|c|c|c|c|c|c|c|c|c|c|c|c|c|c|c|c|}
\hline$\dot{\xi}$ & $\stackrel{m}{\sim}$ & $\vec{\sigma}$ & $\tilde{0}$ & ? & $\ddot{?}$ & $=$ & $\stackrel{9}{0}$ & $=$ & $\stackrel{0}{0}$ & $\dot{m}$ & $=$ & $1=$ & $\stackrel{0}{0}$ & $\dot{0}$ & $\ddot{0}$ & $\stackrel{\sim}{0}$ & $\stackrel{v}{\vdots}$ & $\because$ \\
\hline
\end{tabular}

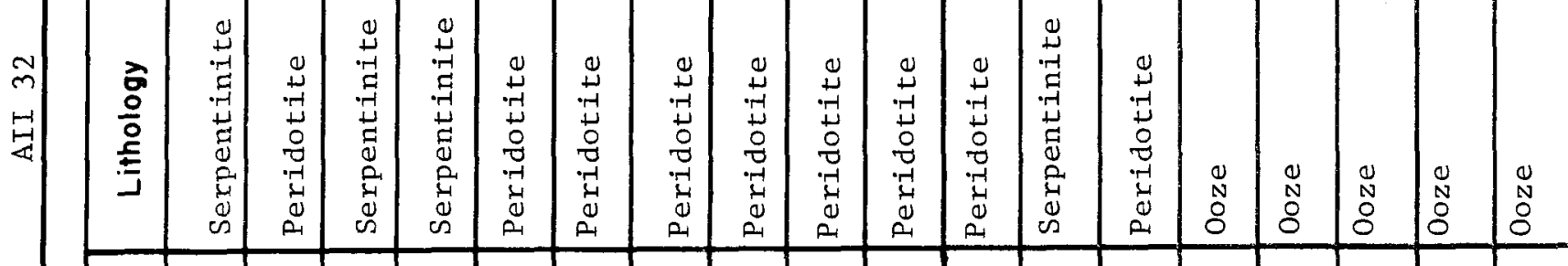

岕

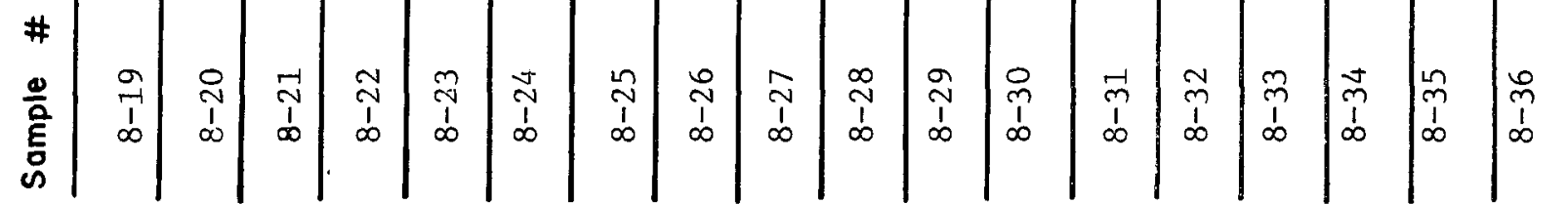




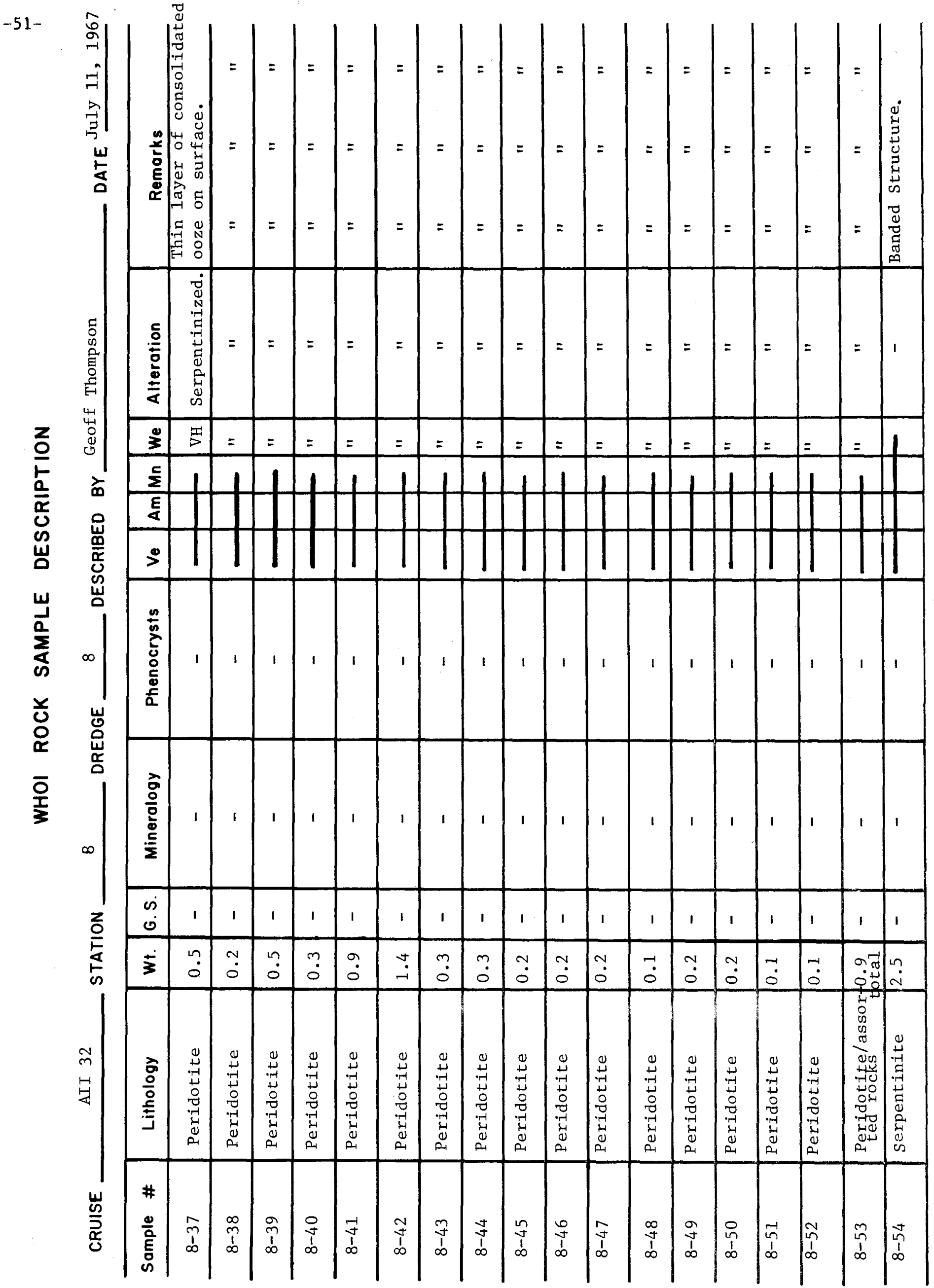




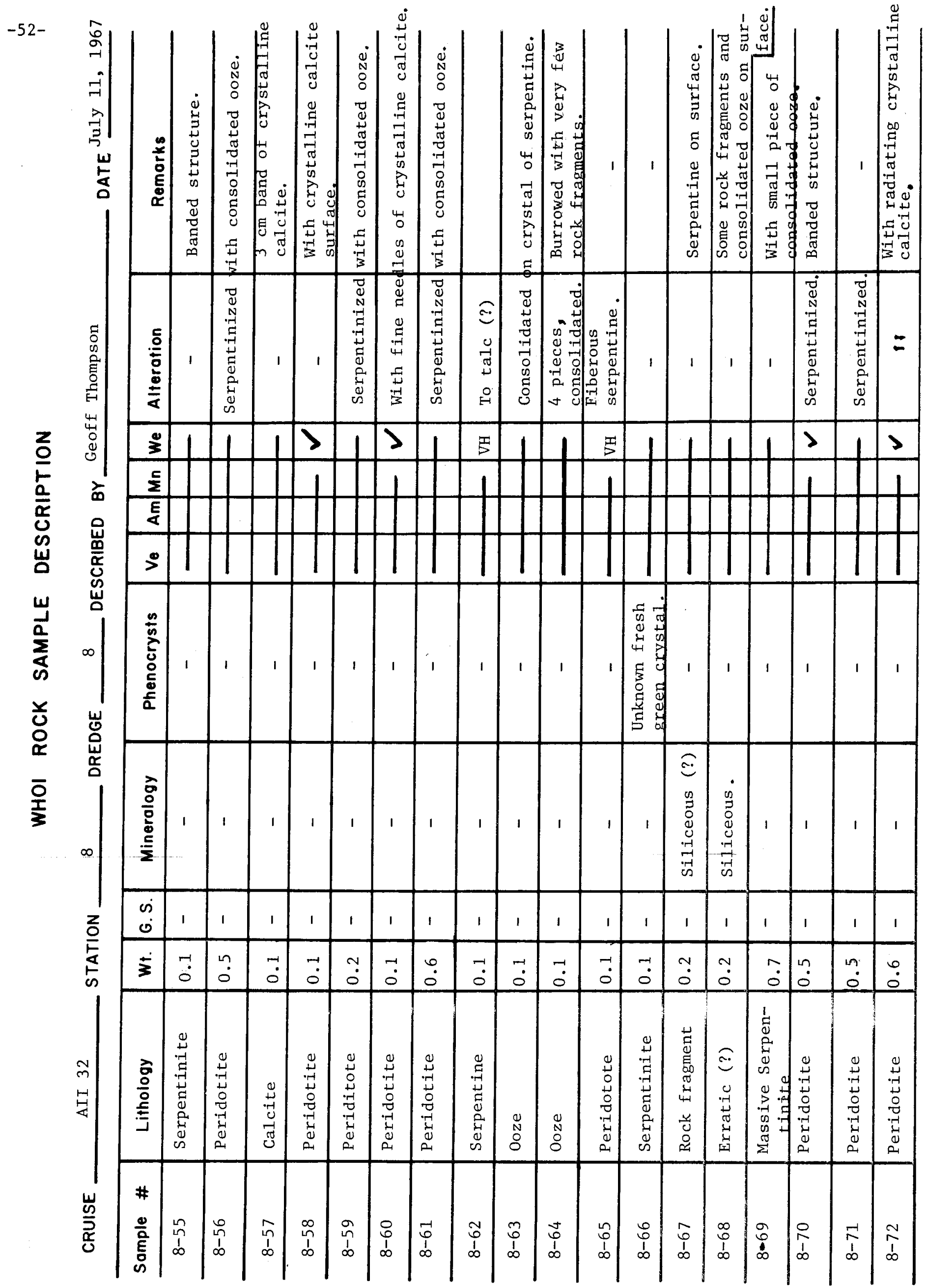




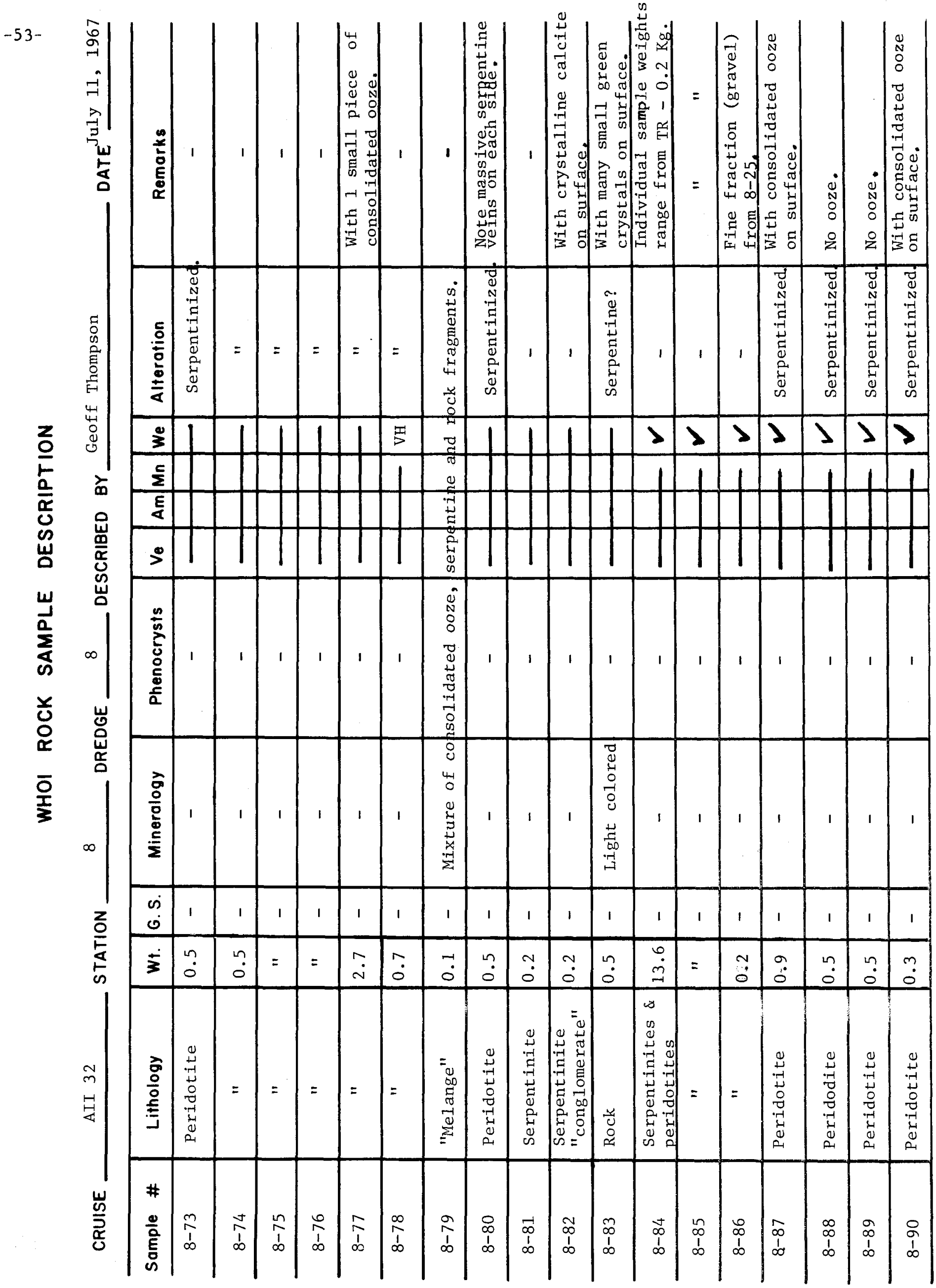




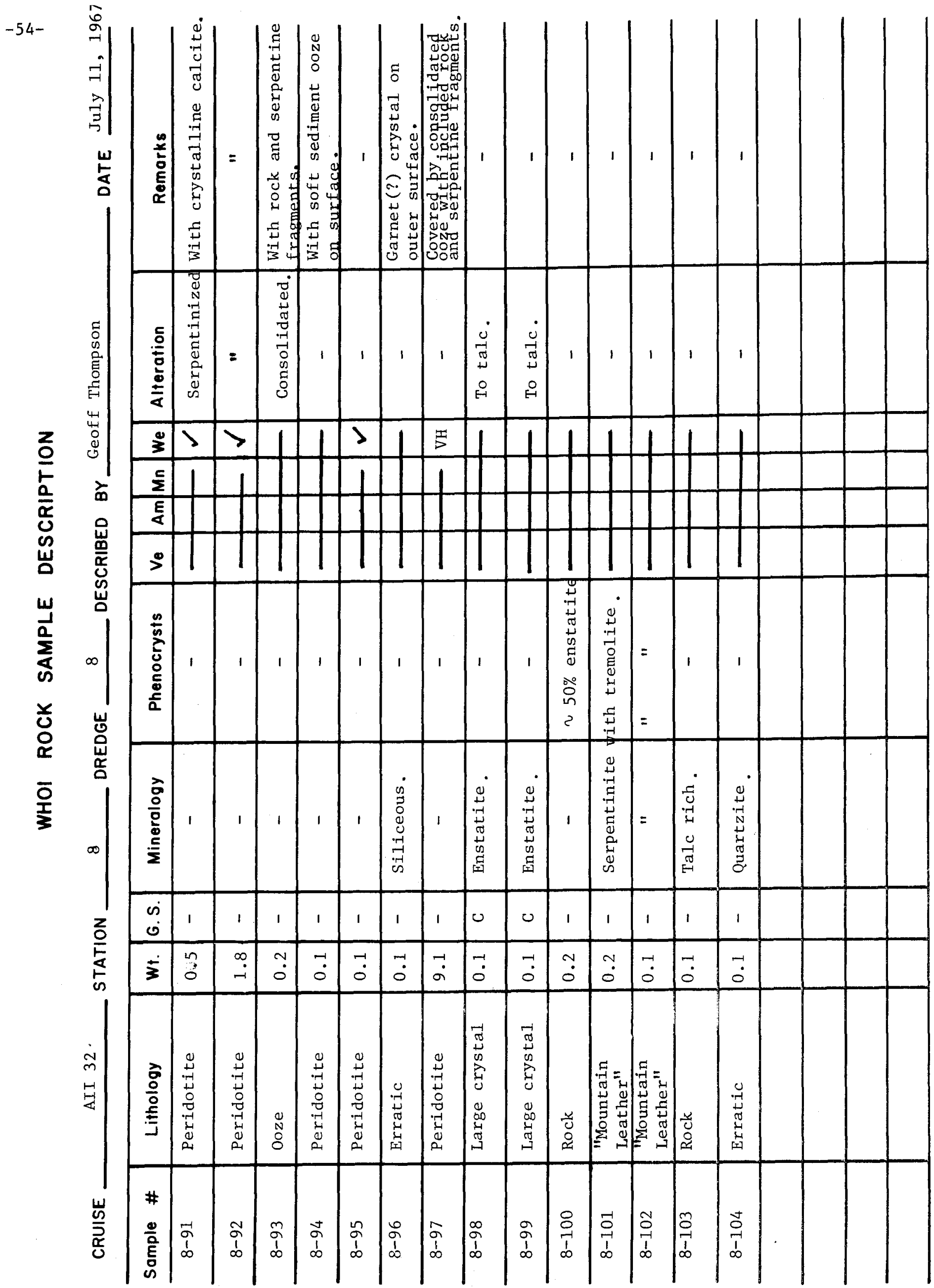




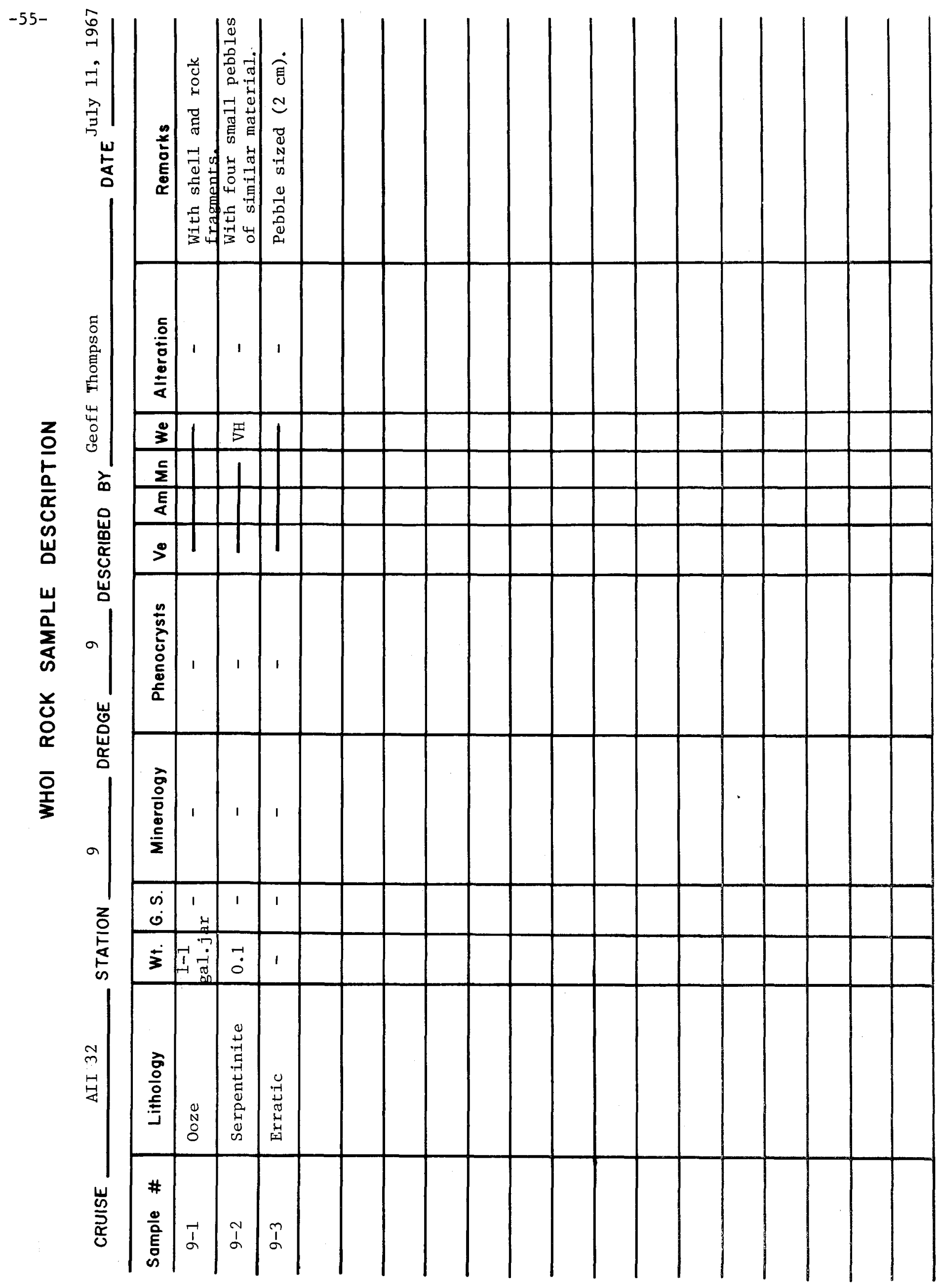




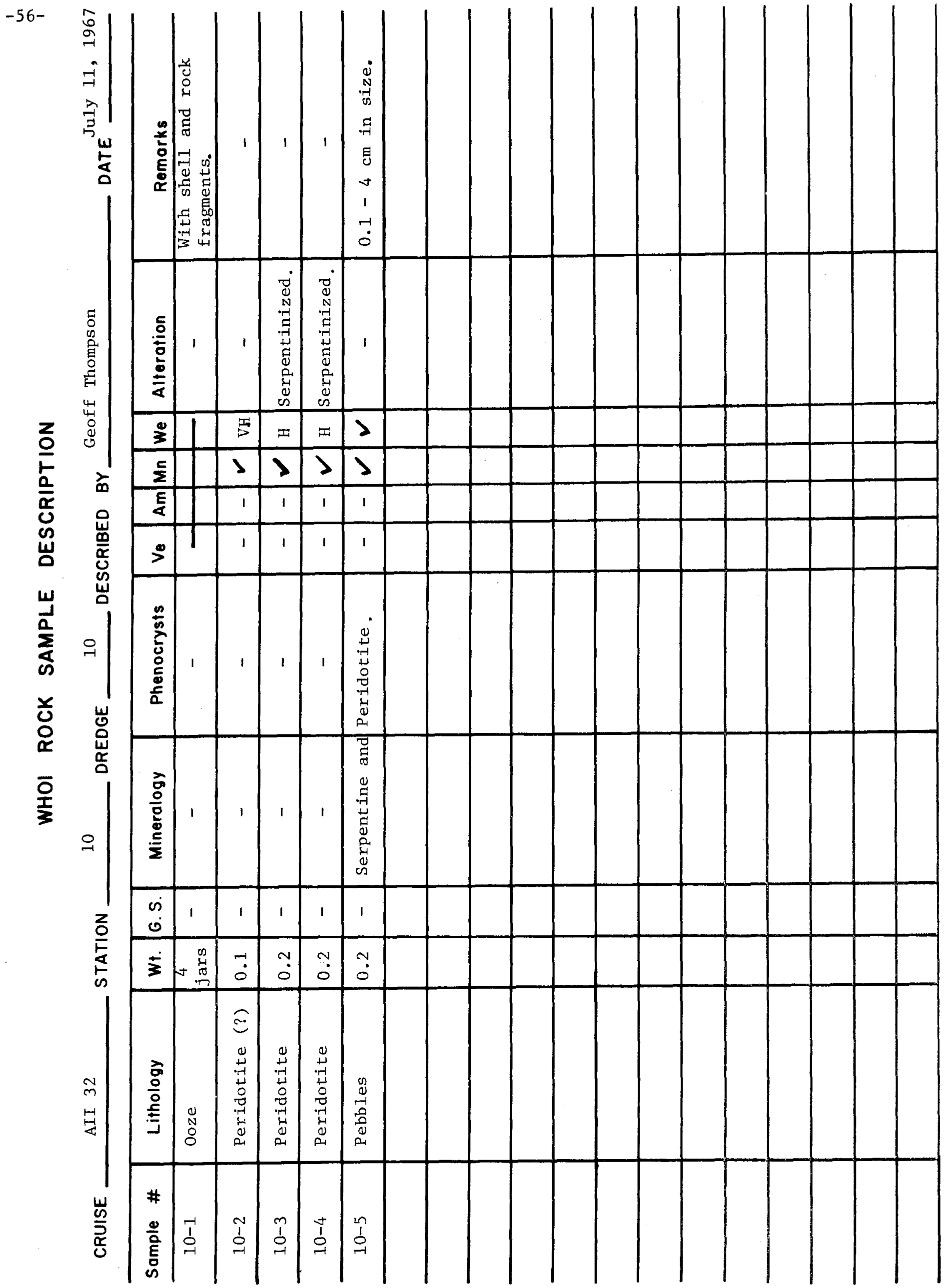




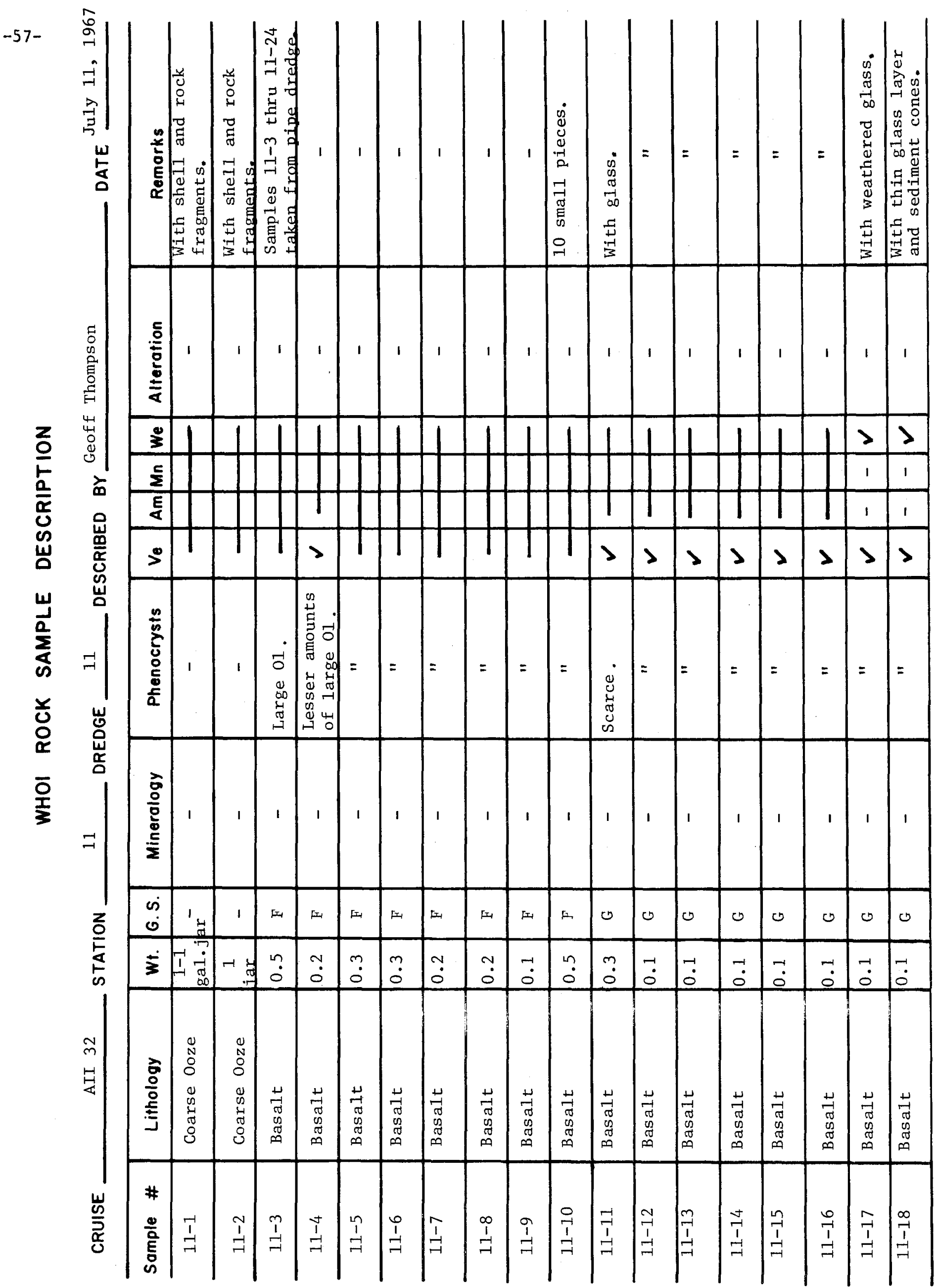




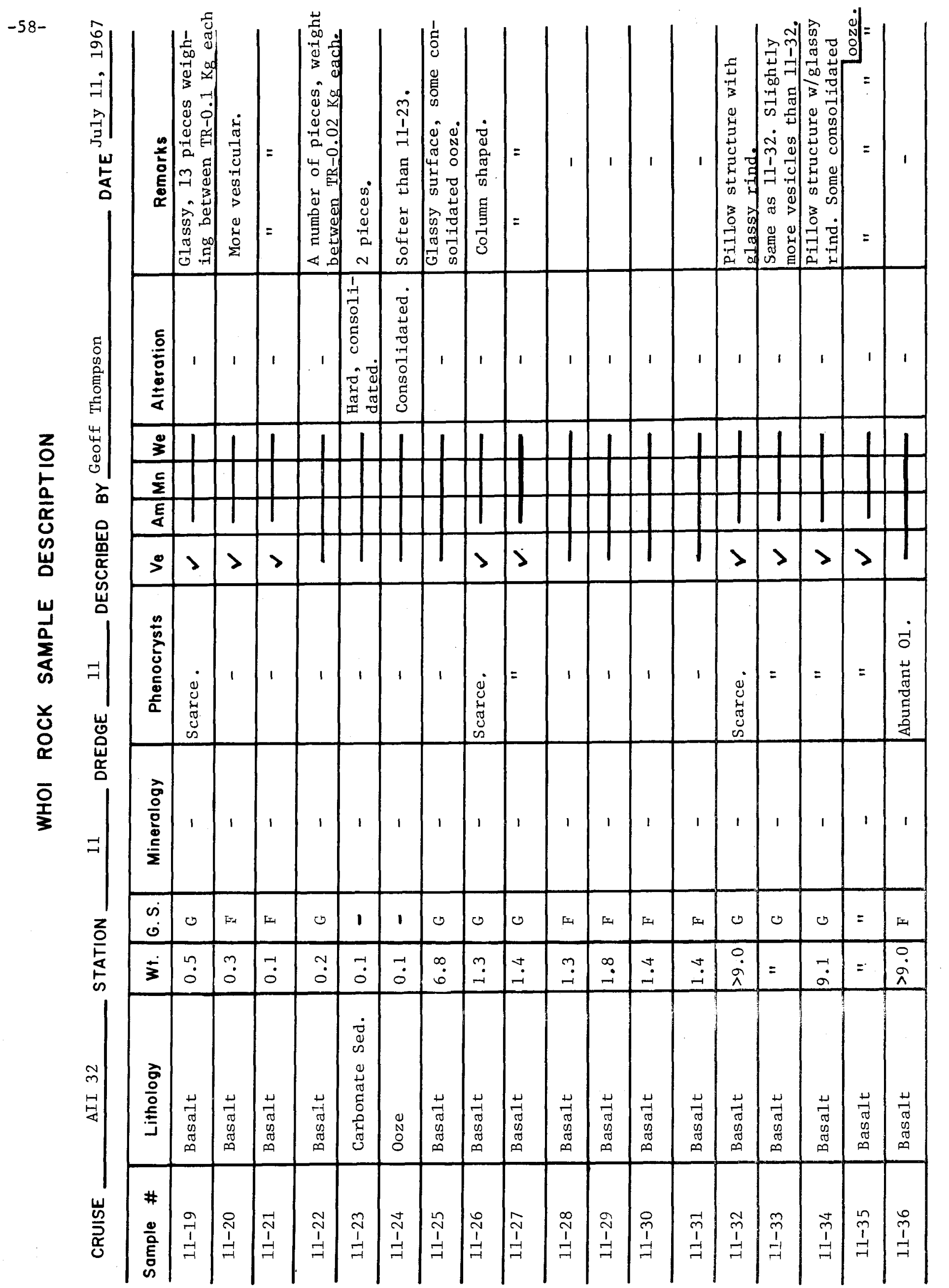




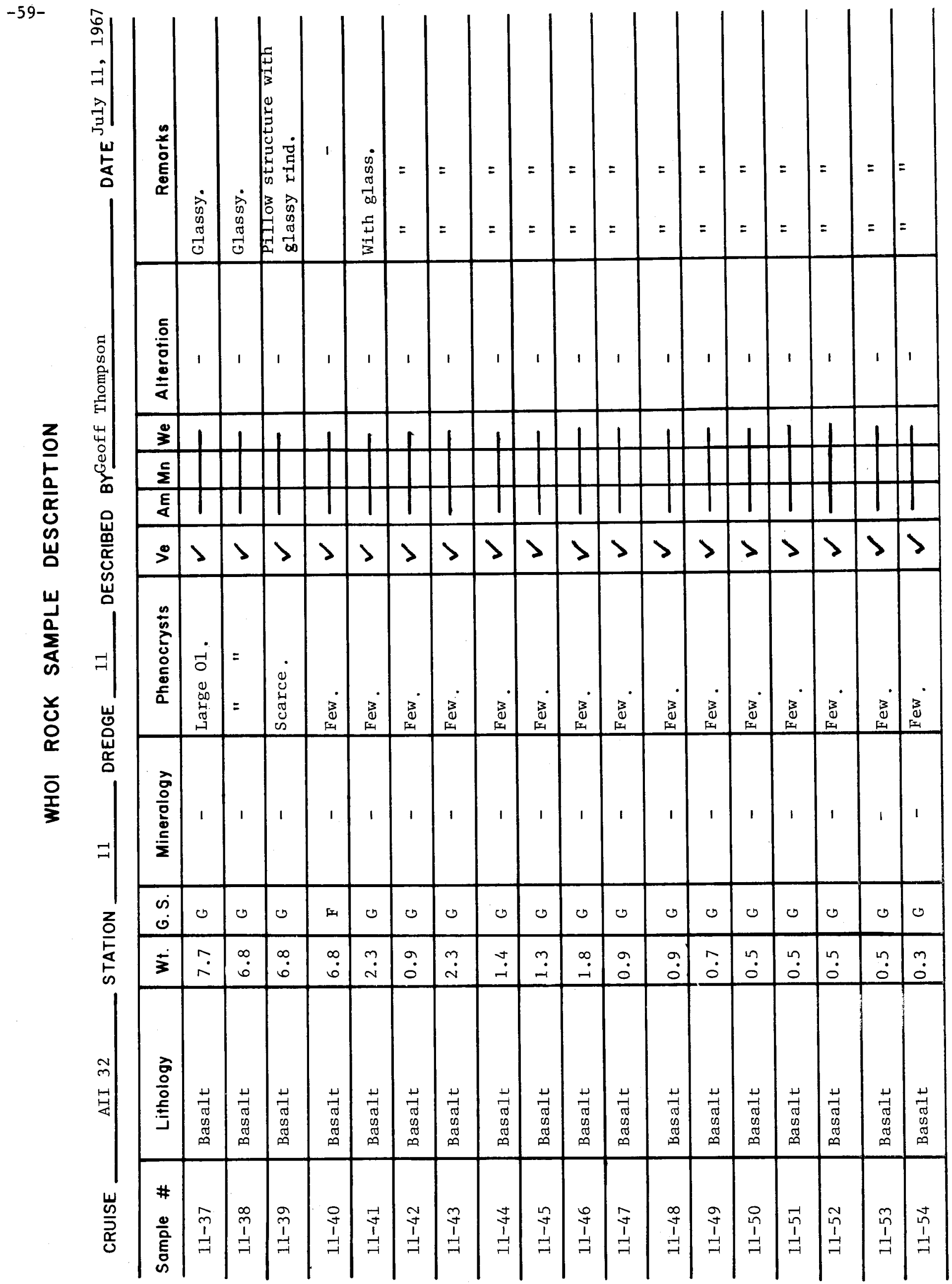




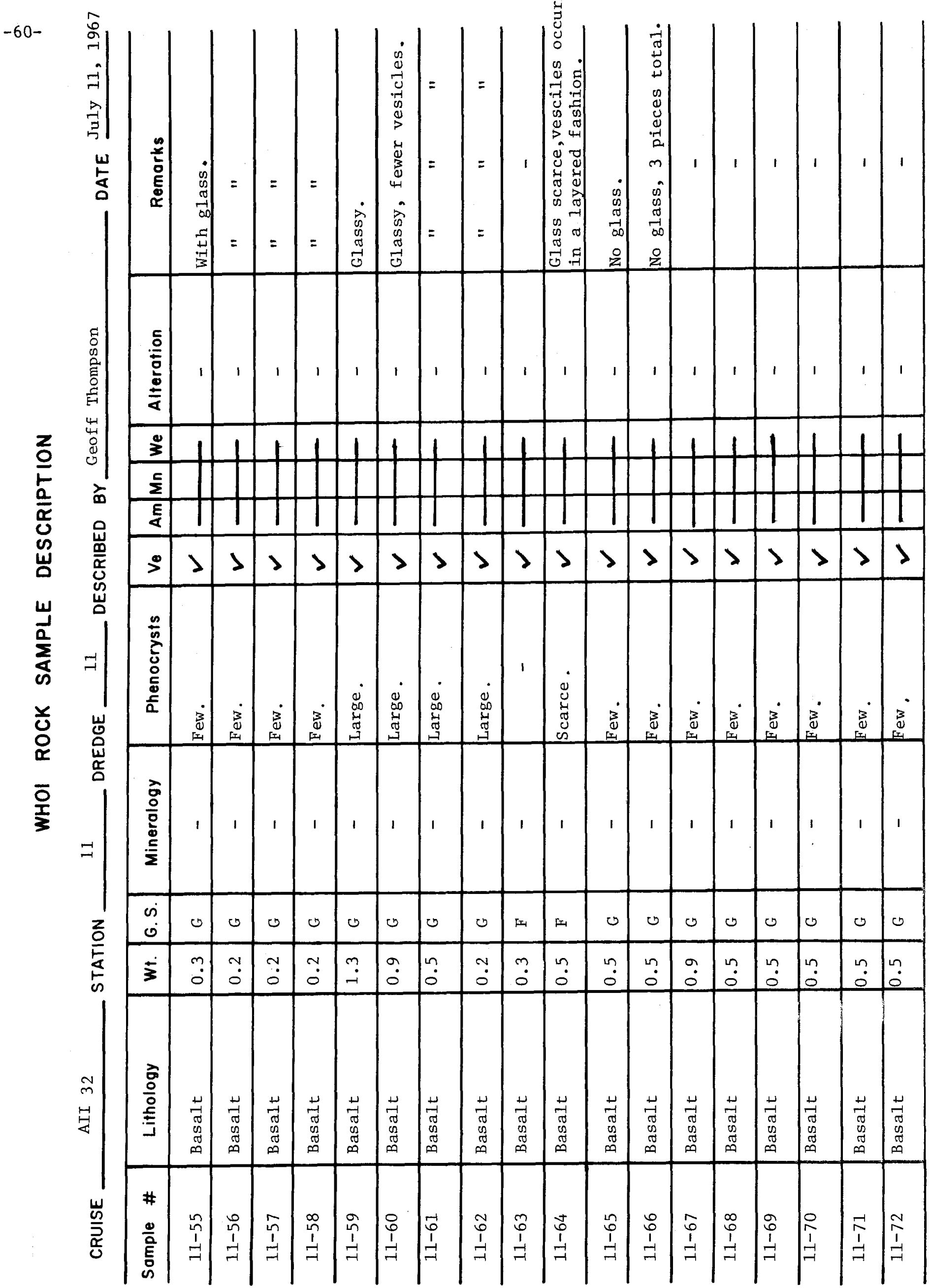




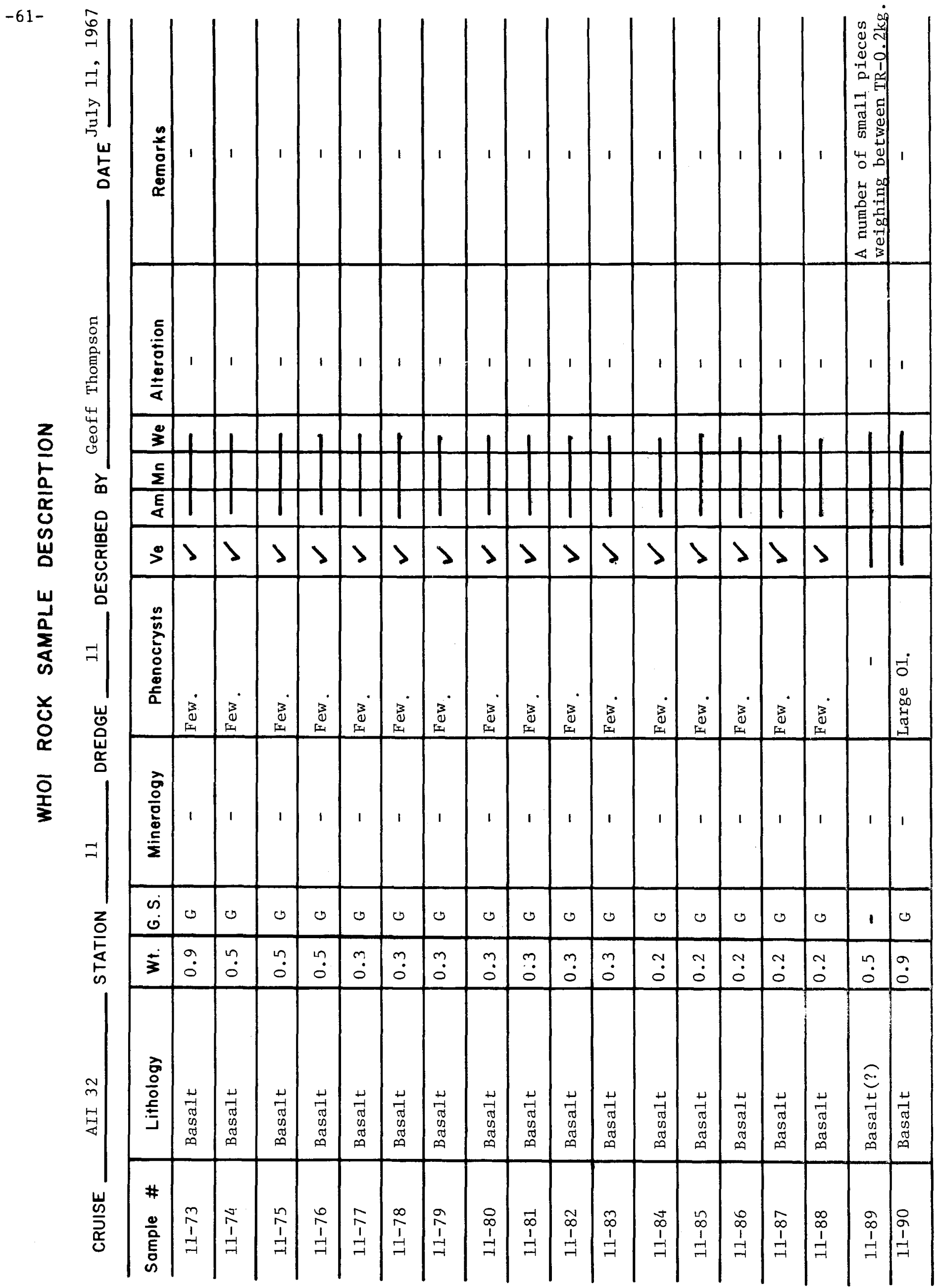




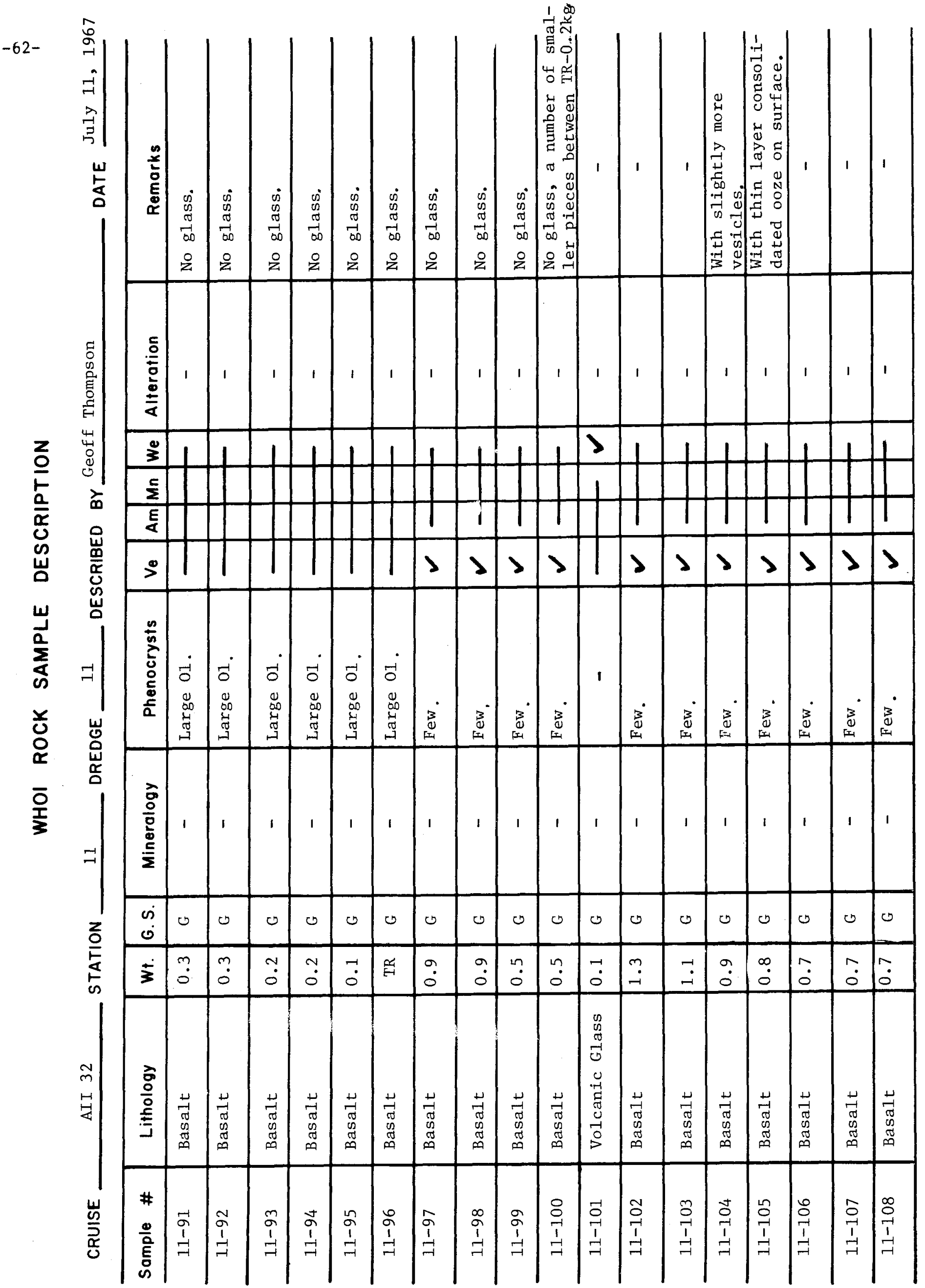




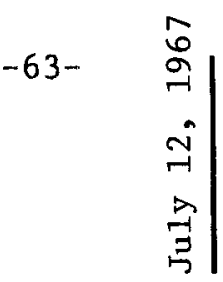

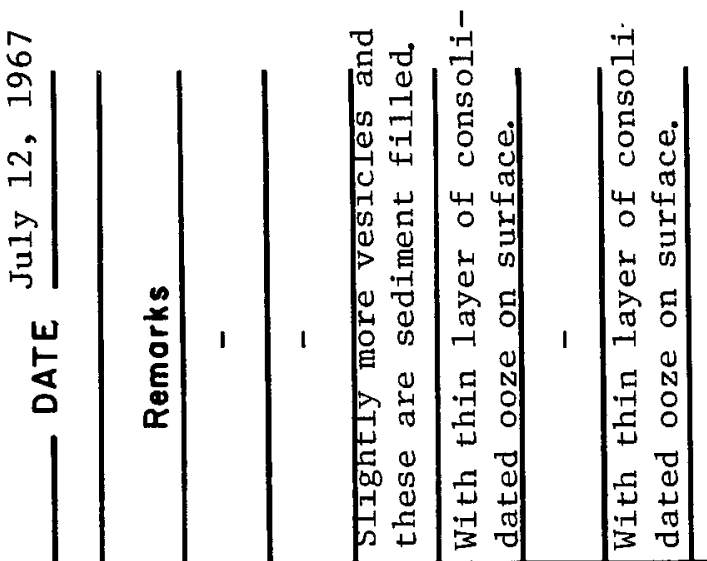

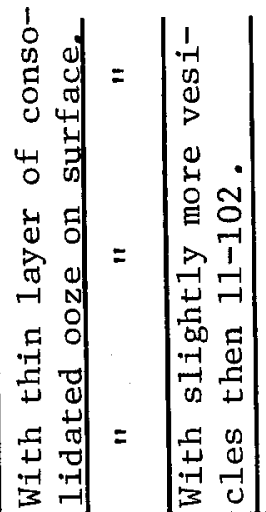

ह

$2 \begin{array}{r}4 \\ 0 \\ 0\end{array}$

占

동

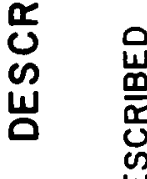

w

$\sum_{\infty}=1$

ํㅡㅇ
옴
움

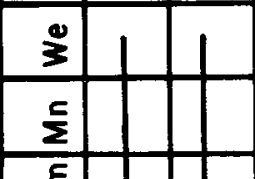

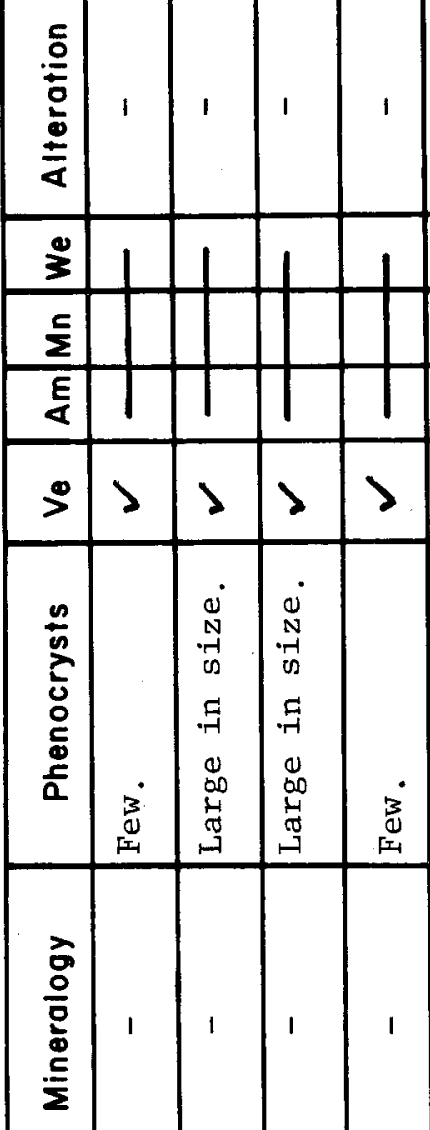

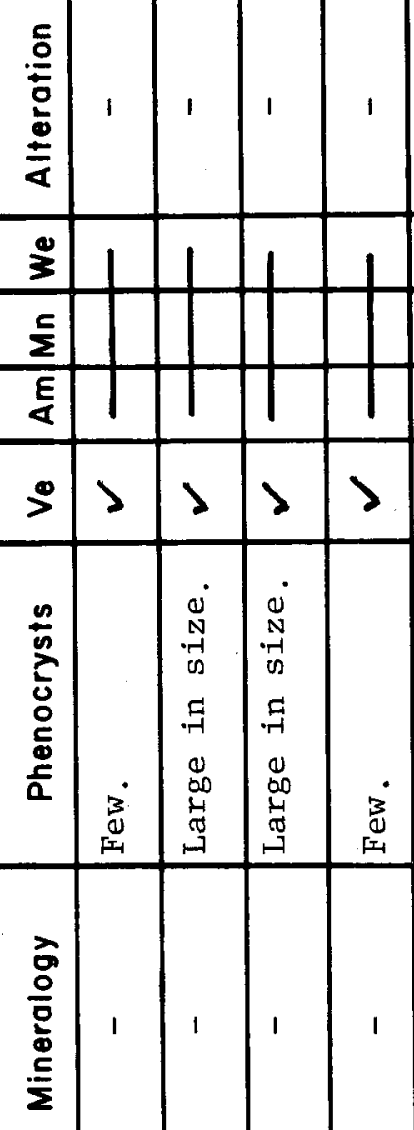

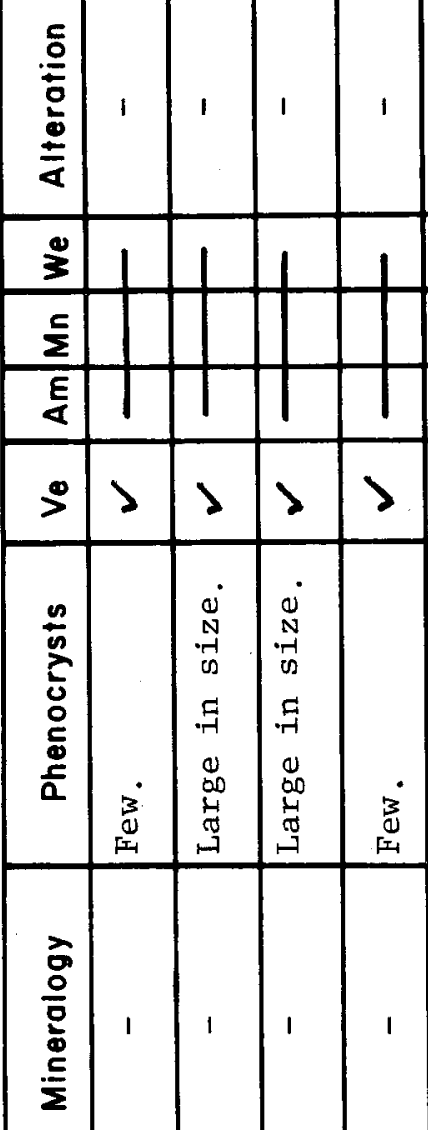

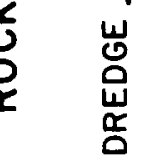

옹

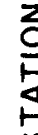

is

은

กี

占

$\$$

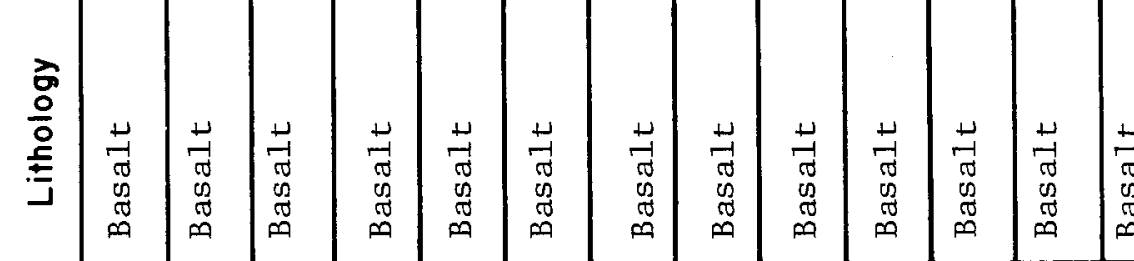

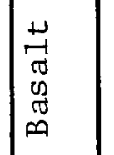

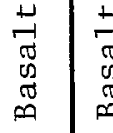

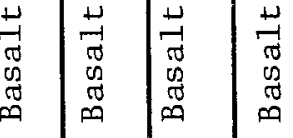

帹

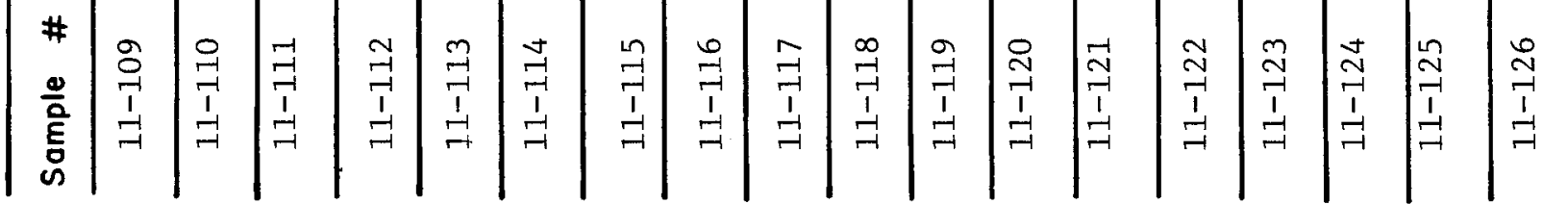




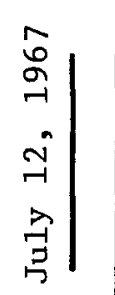

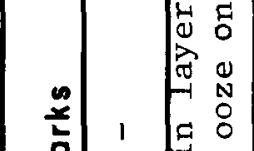

炭

产

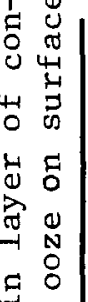

过

为

番

$0-1$
3

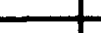

\begin{tabular}{ll}
$z$ & 4 \\
0 & 0 \\
\hline & 0
\end{tabular}

a

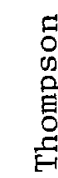

客

岳

山

$\sum_{\infty}^{\frac{1}{a}} ت-1$

爻 岁

옥

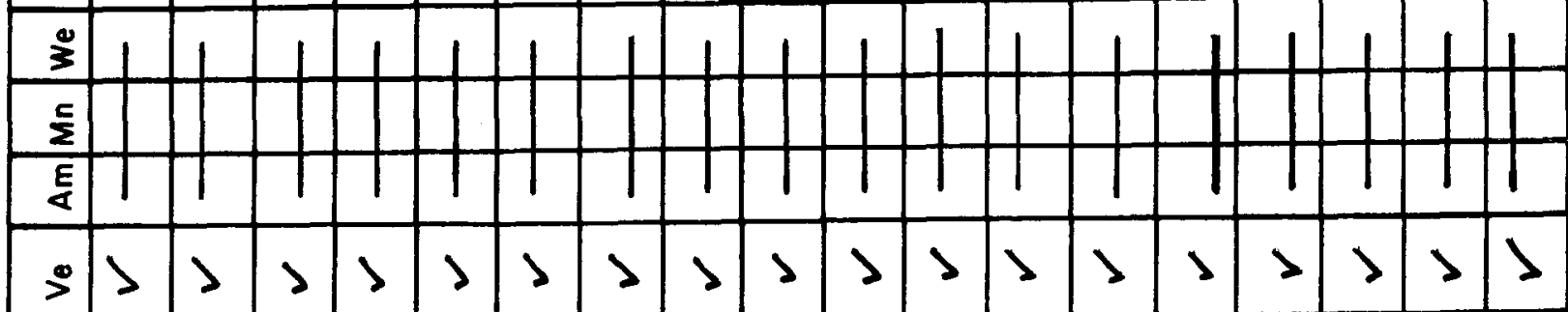

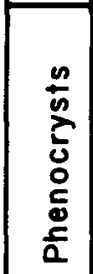

焉

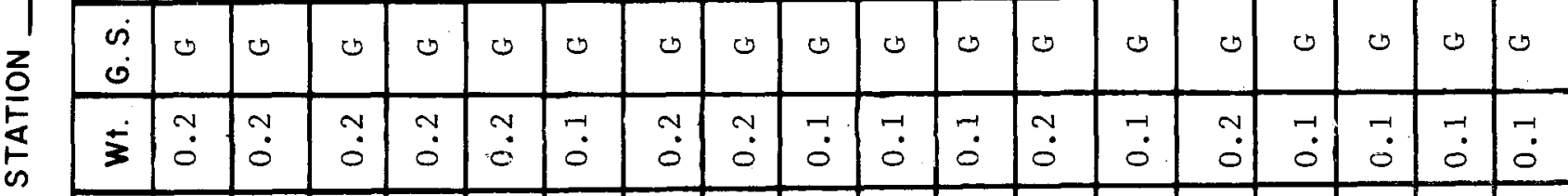

$\Rightarrow \quad \stackrel{\frac{\Phi}{\Sigma}}{\Sigma}$

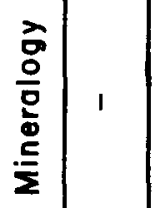

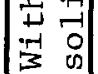

$\sum_{i=1}^{-7} \cdot$

斗祭 

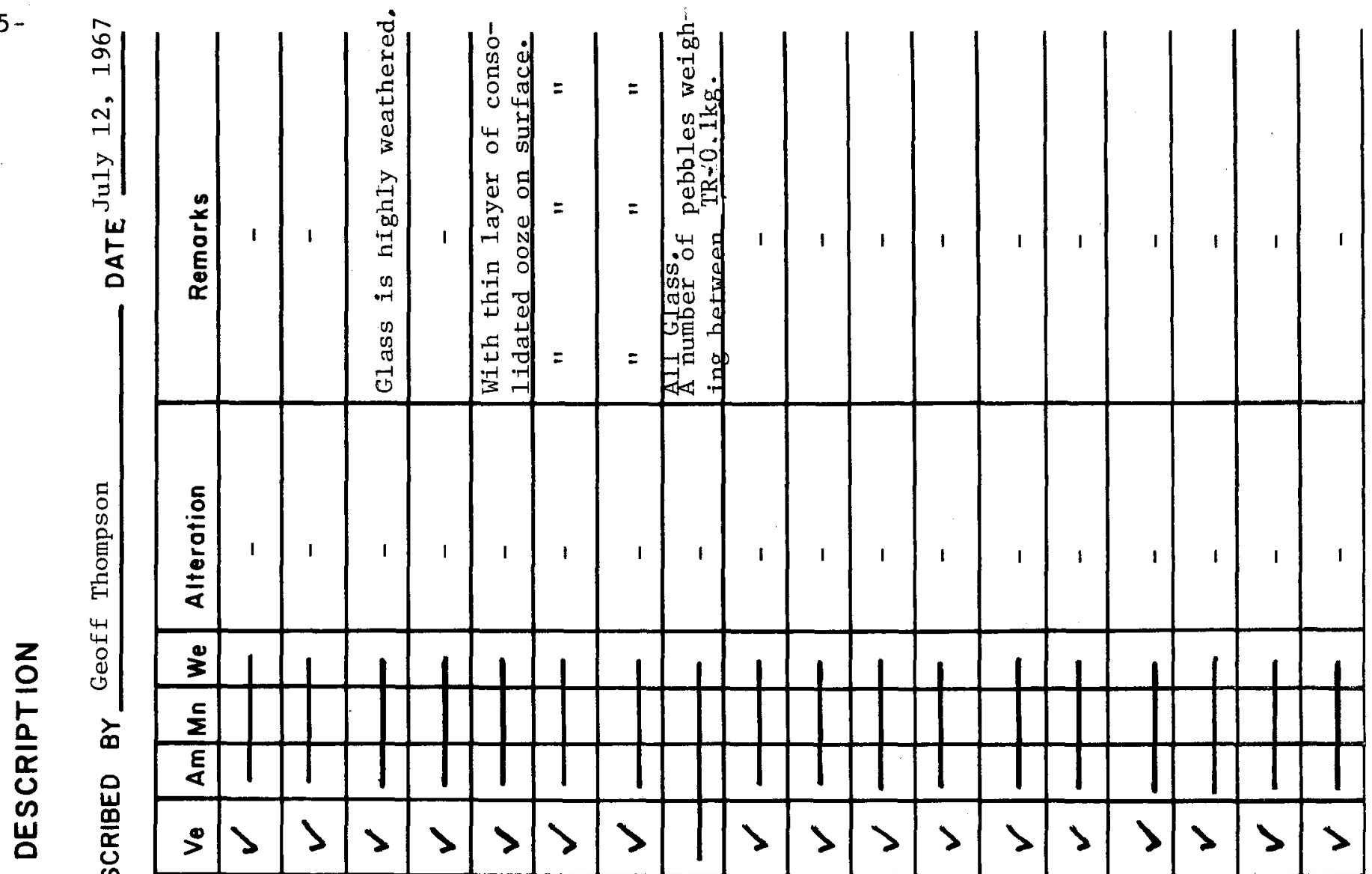

(4)

$\sum_{\infty}^{\frac{1}{a}}{ }_{-1}^{-1}$

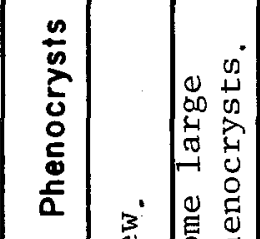

บ

놈

울

$\frac{\widehat{0}}{\frac{0}{0}}$

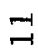

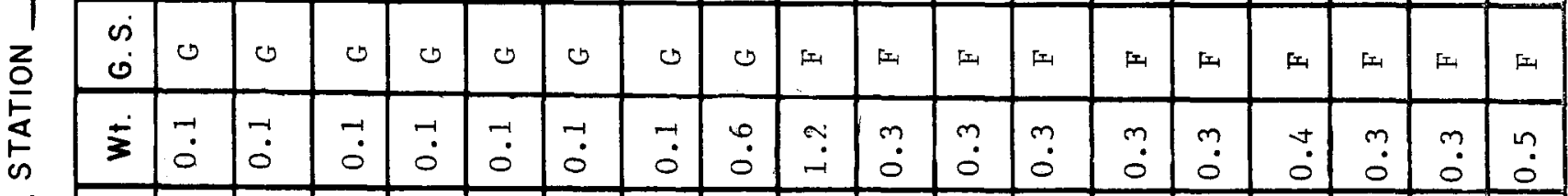

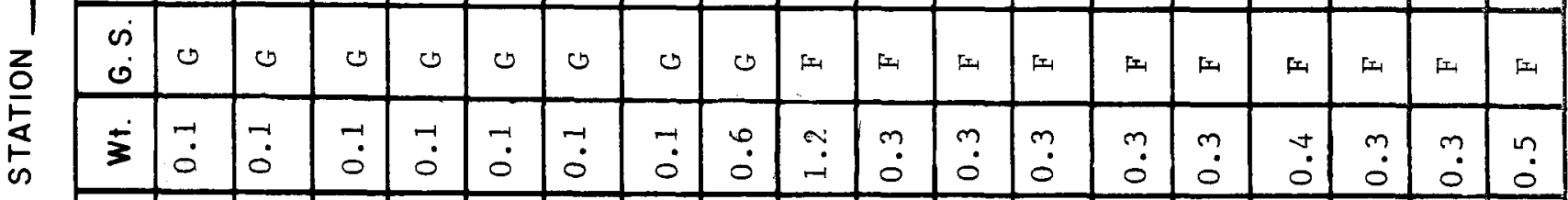

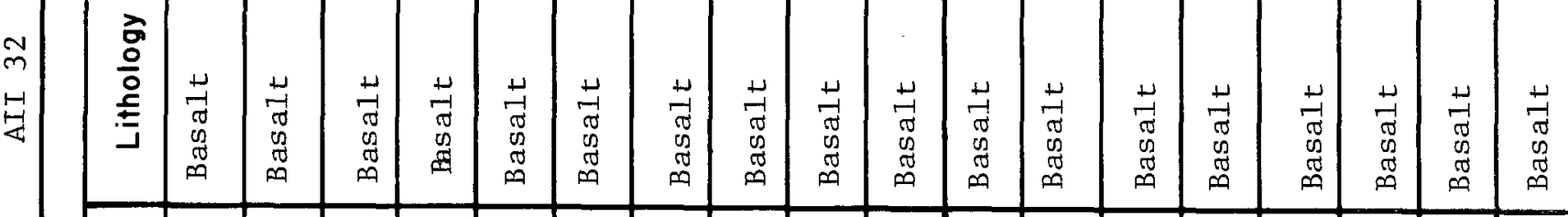

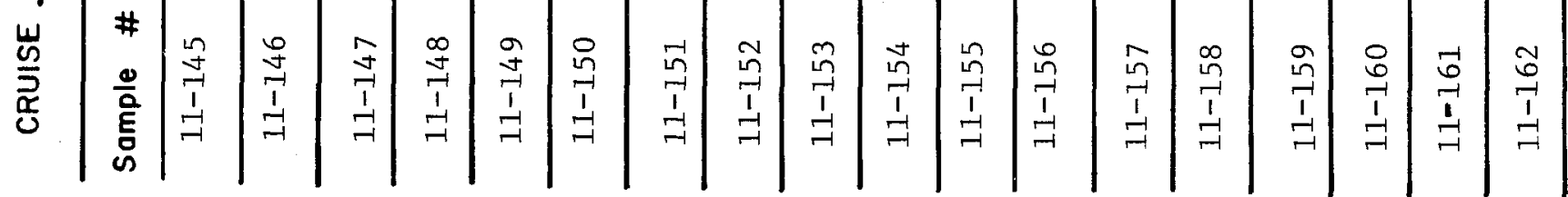



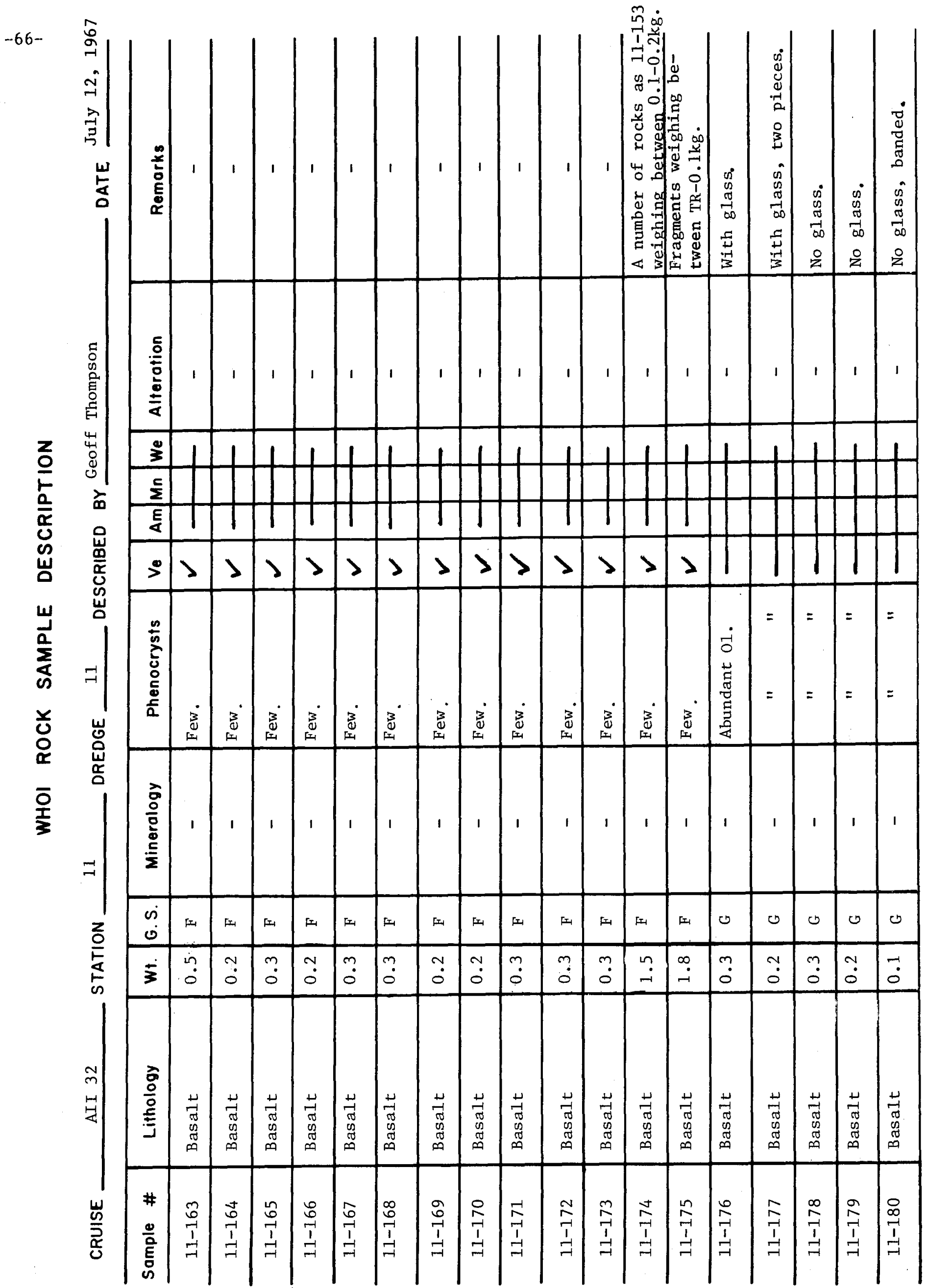


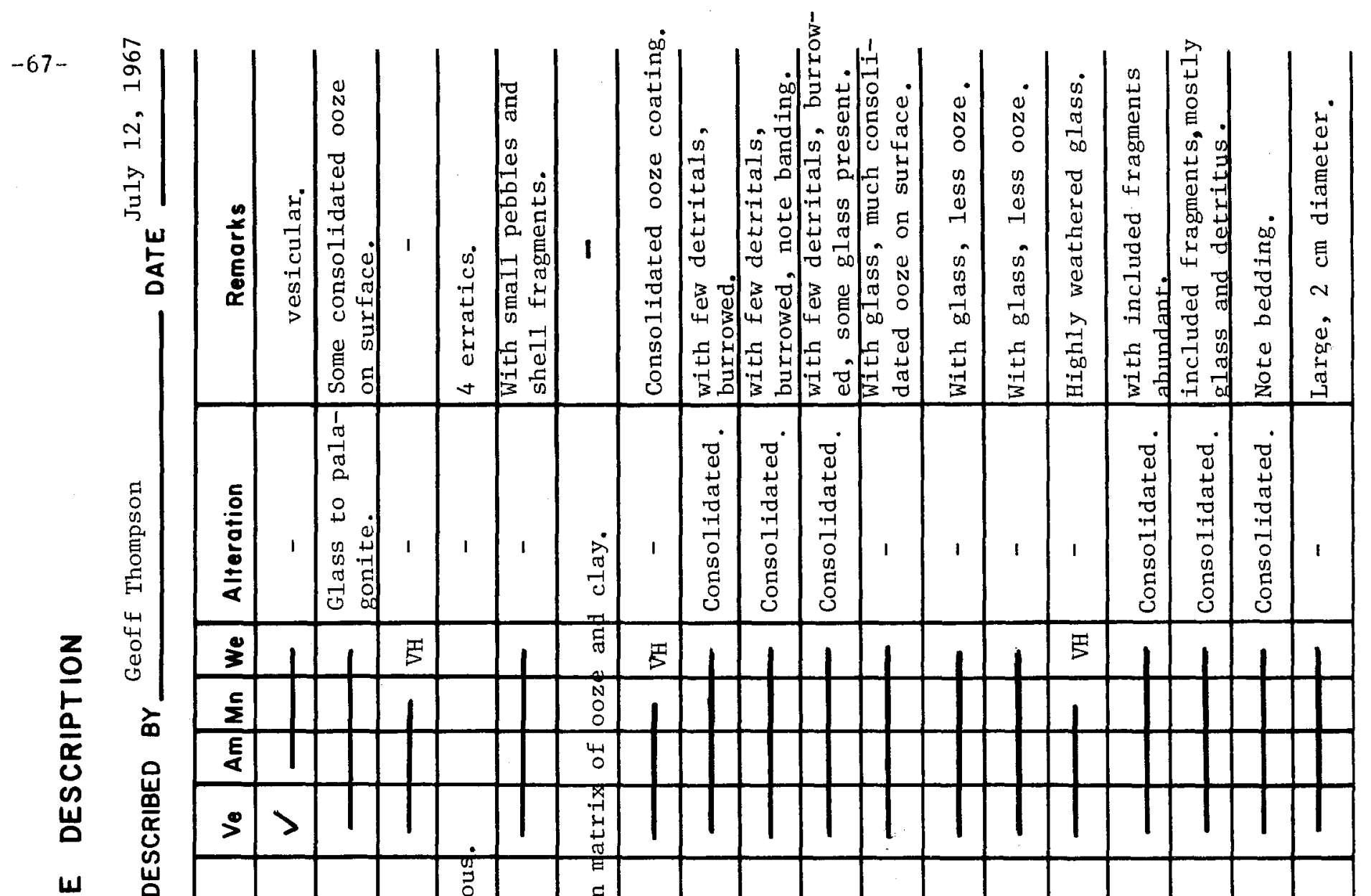

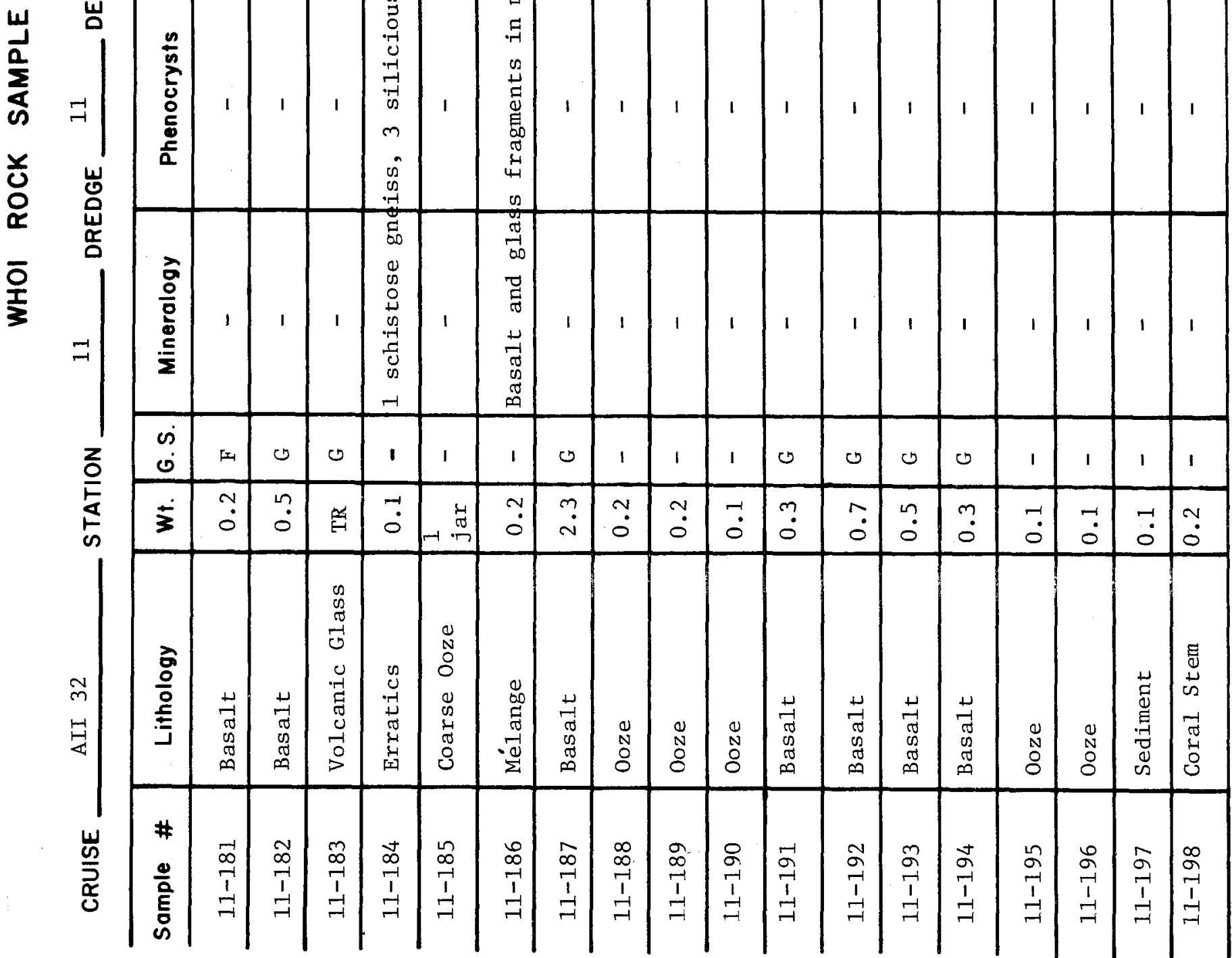




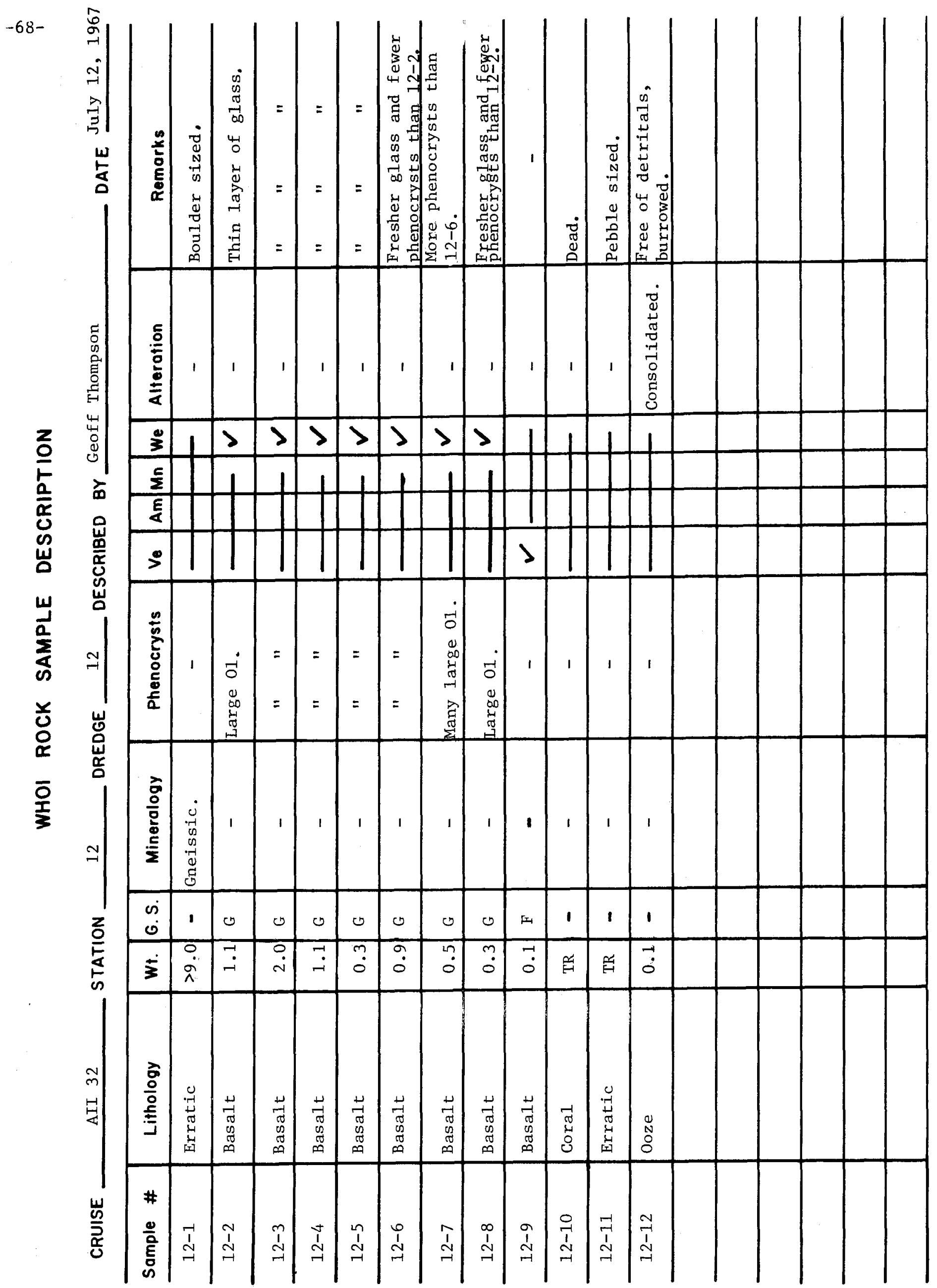




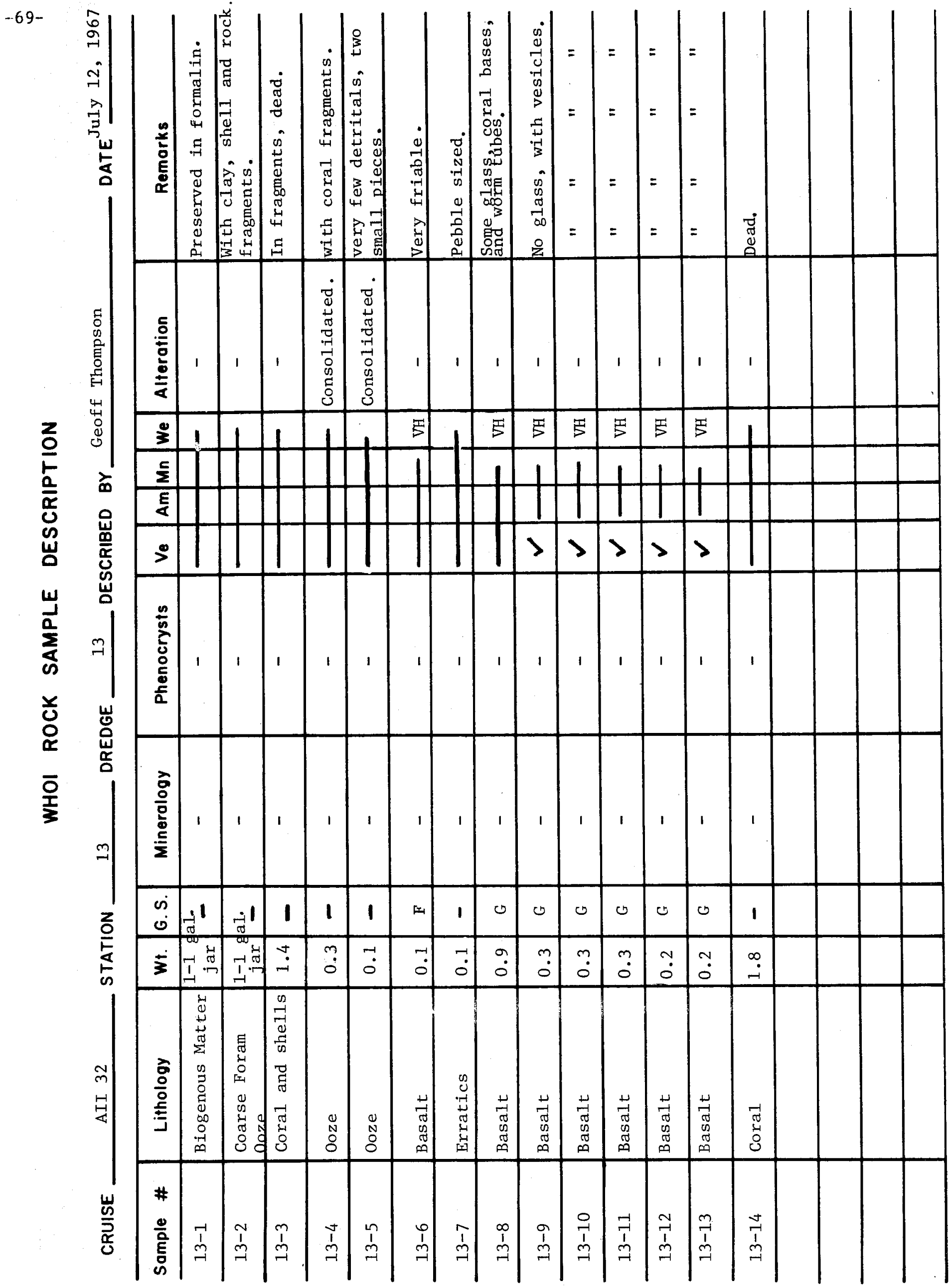




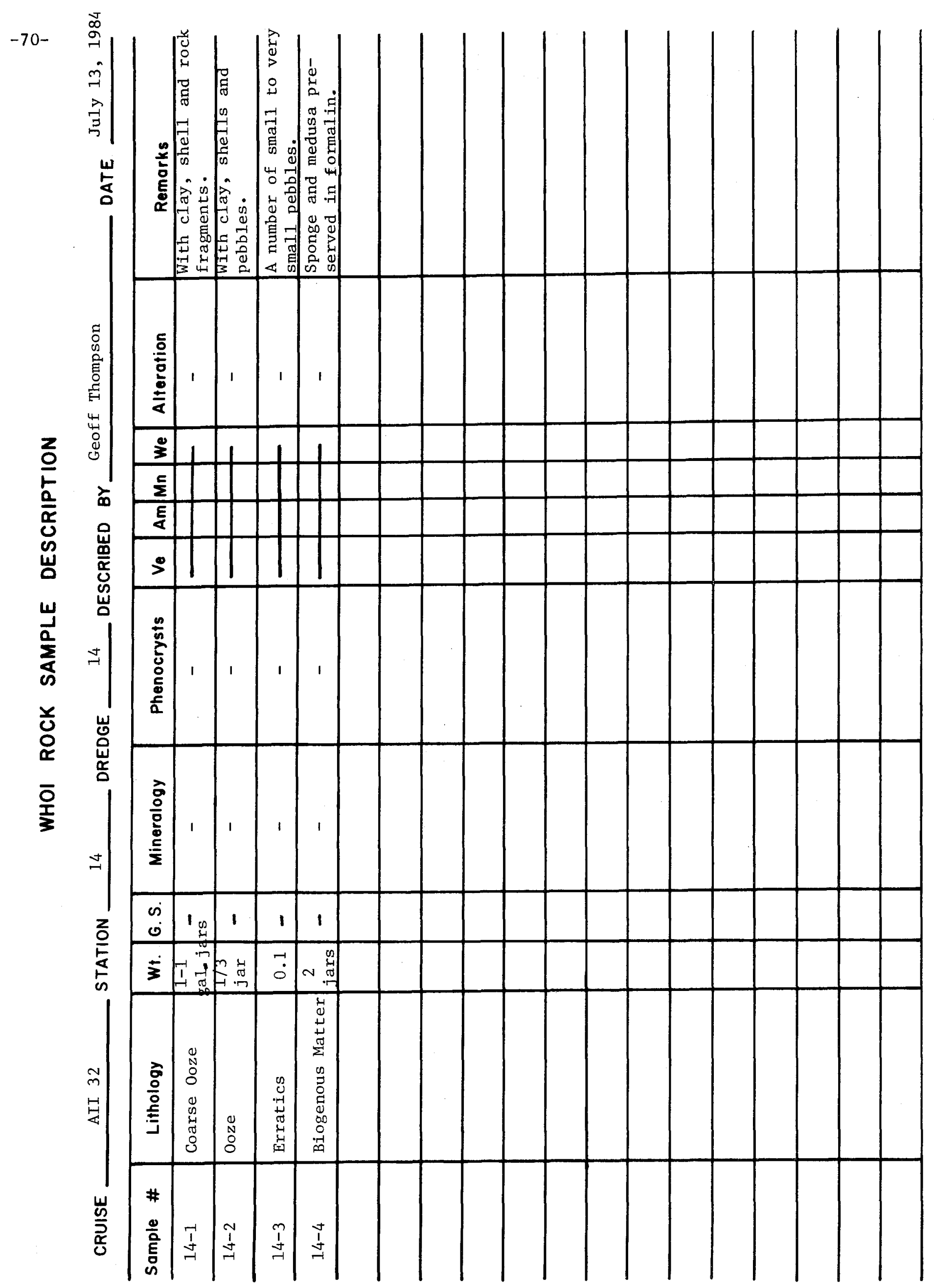




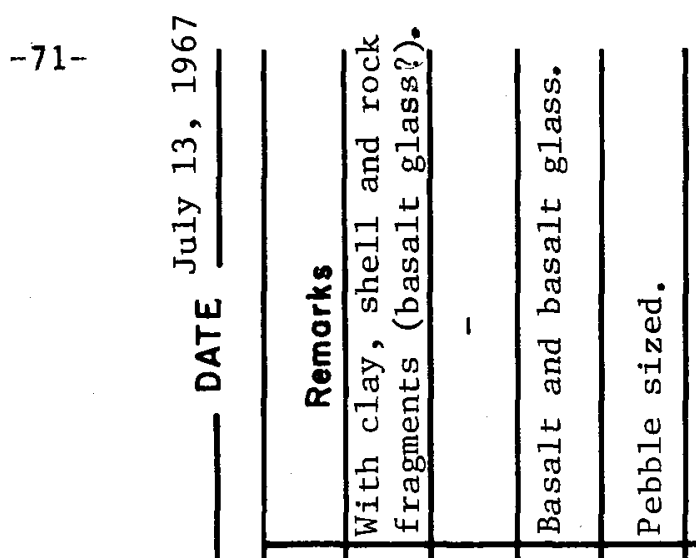
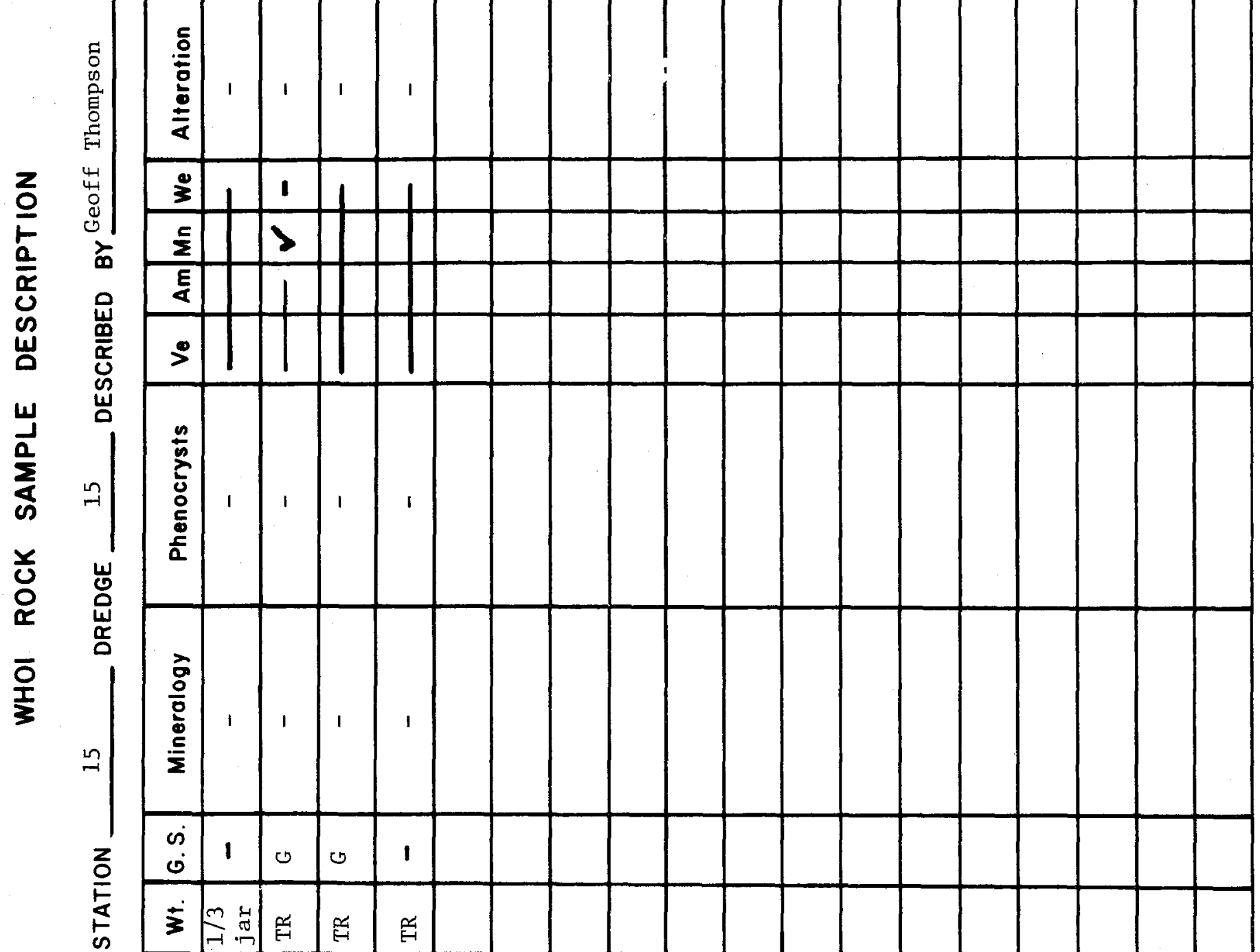

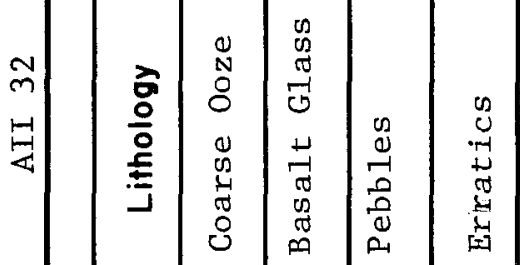

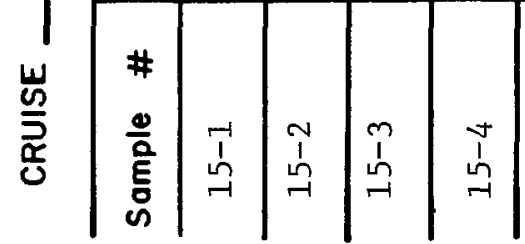




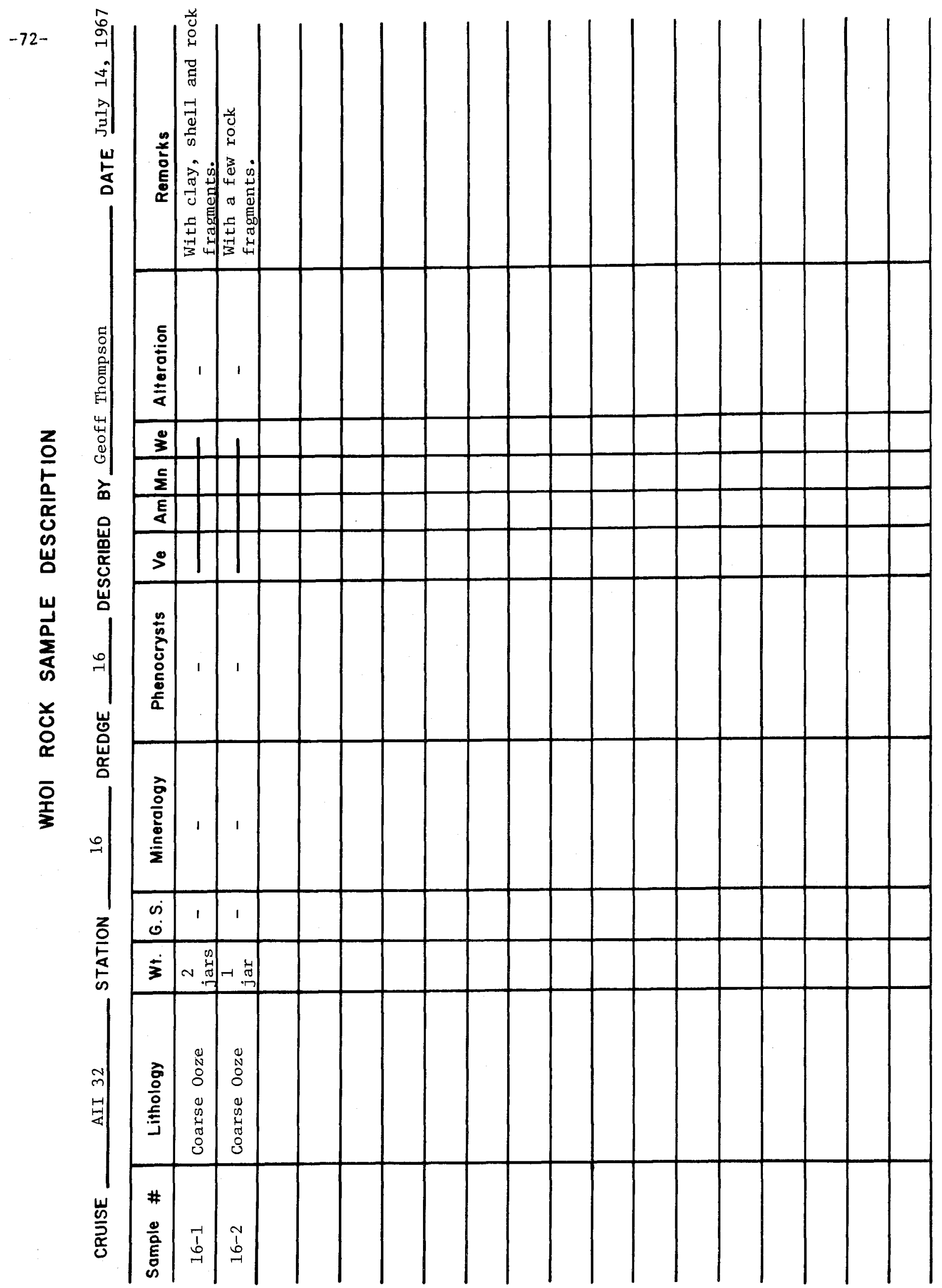




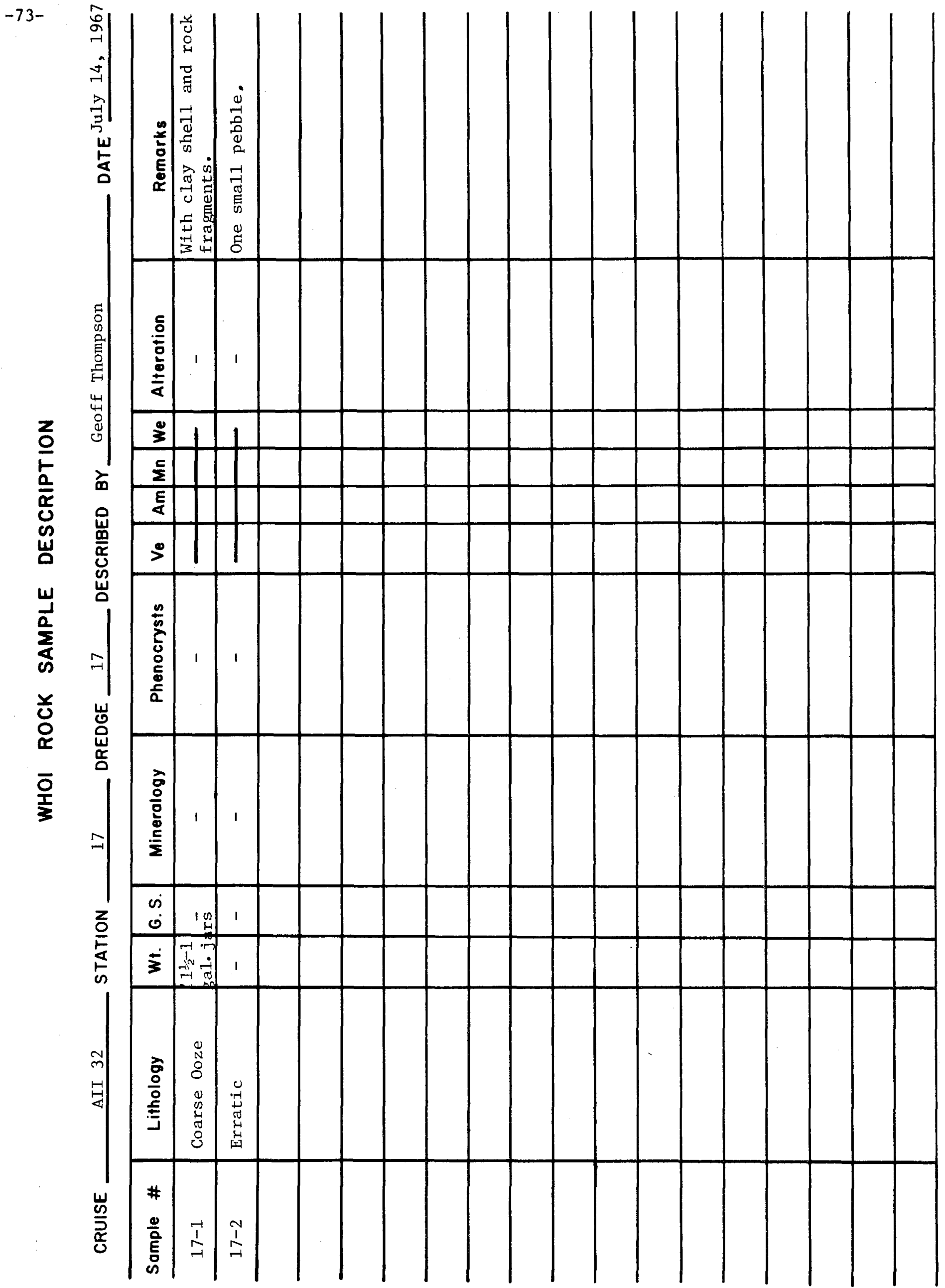




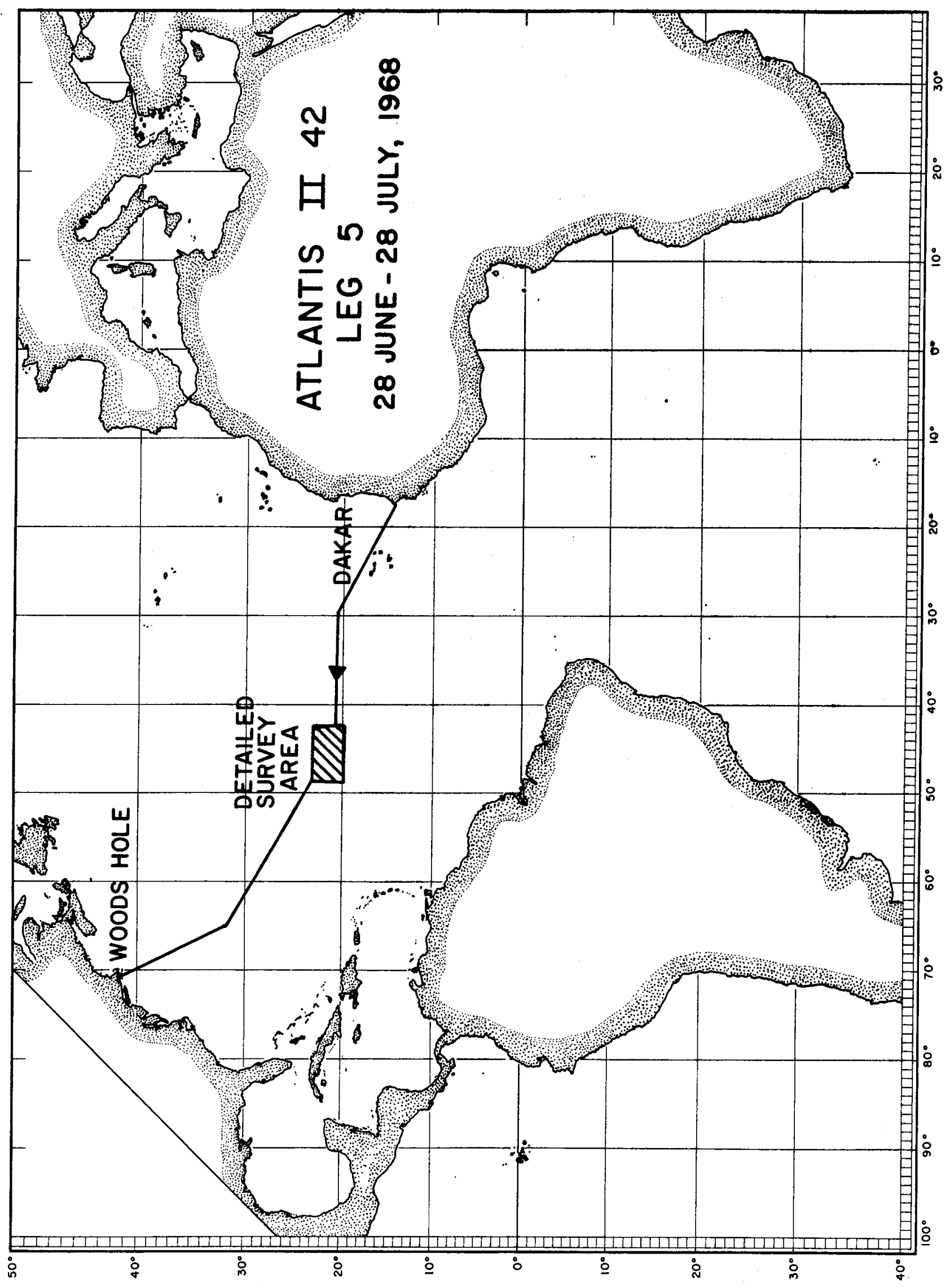




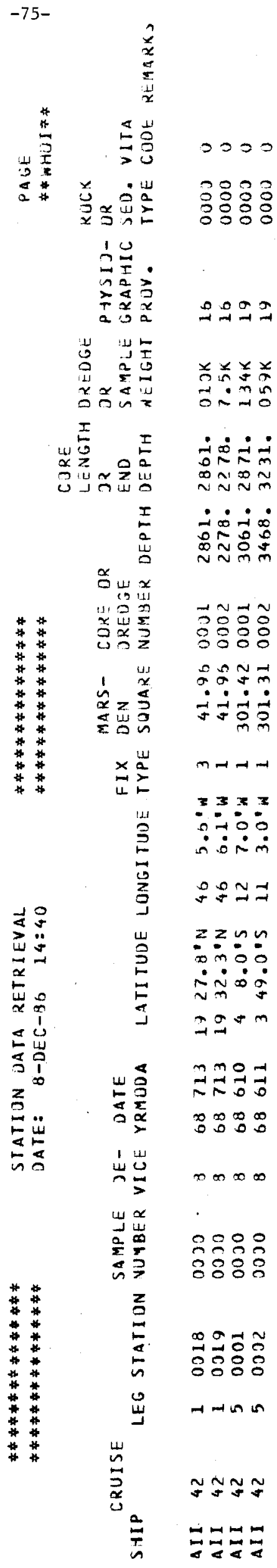




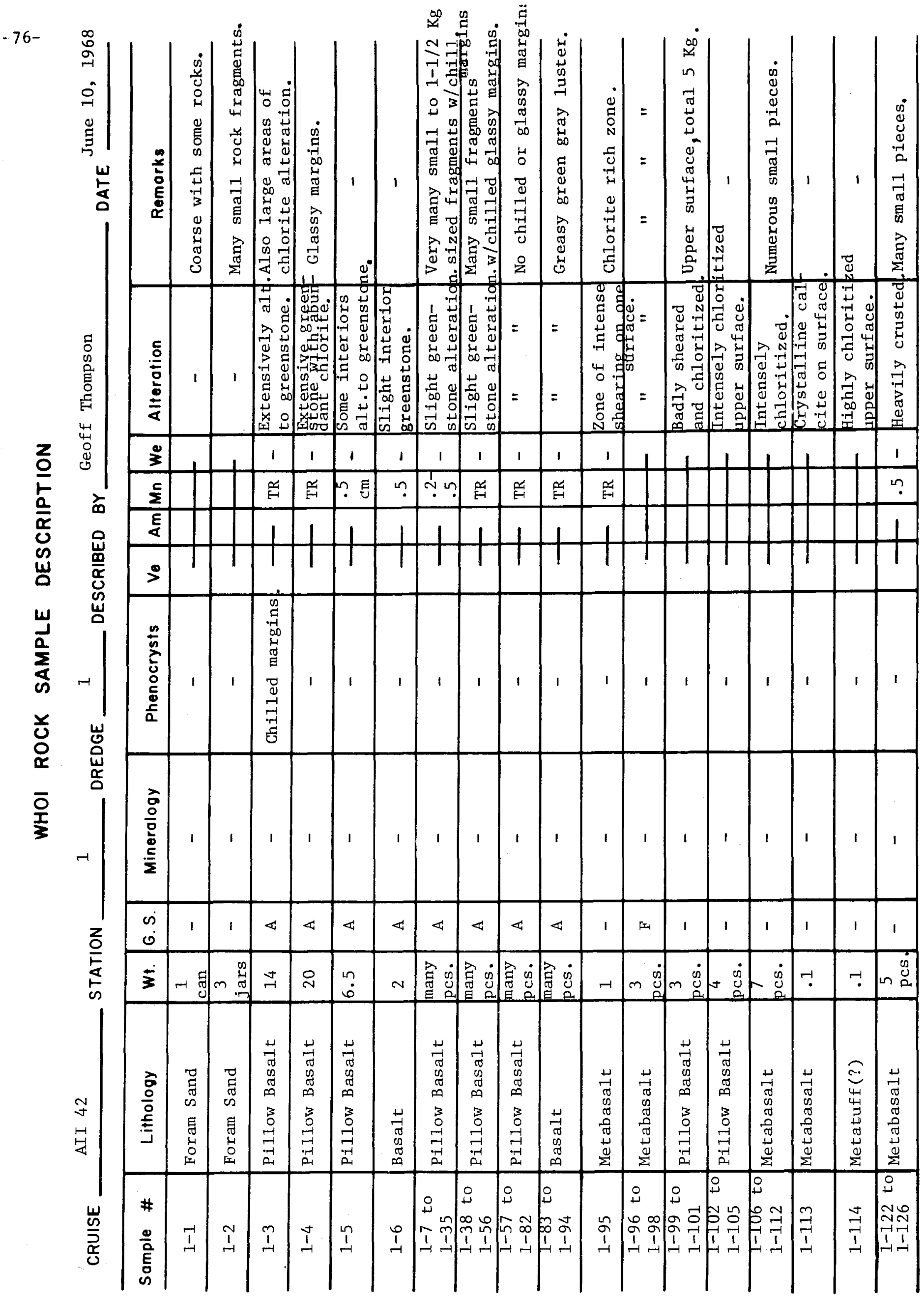




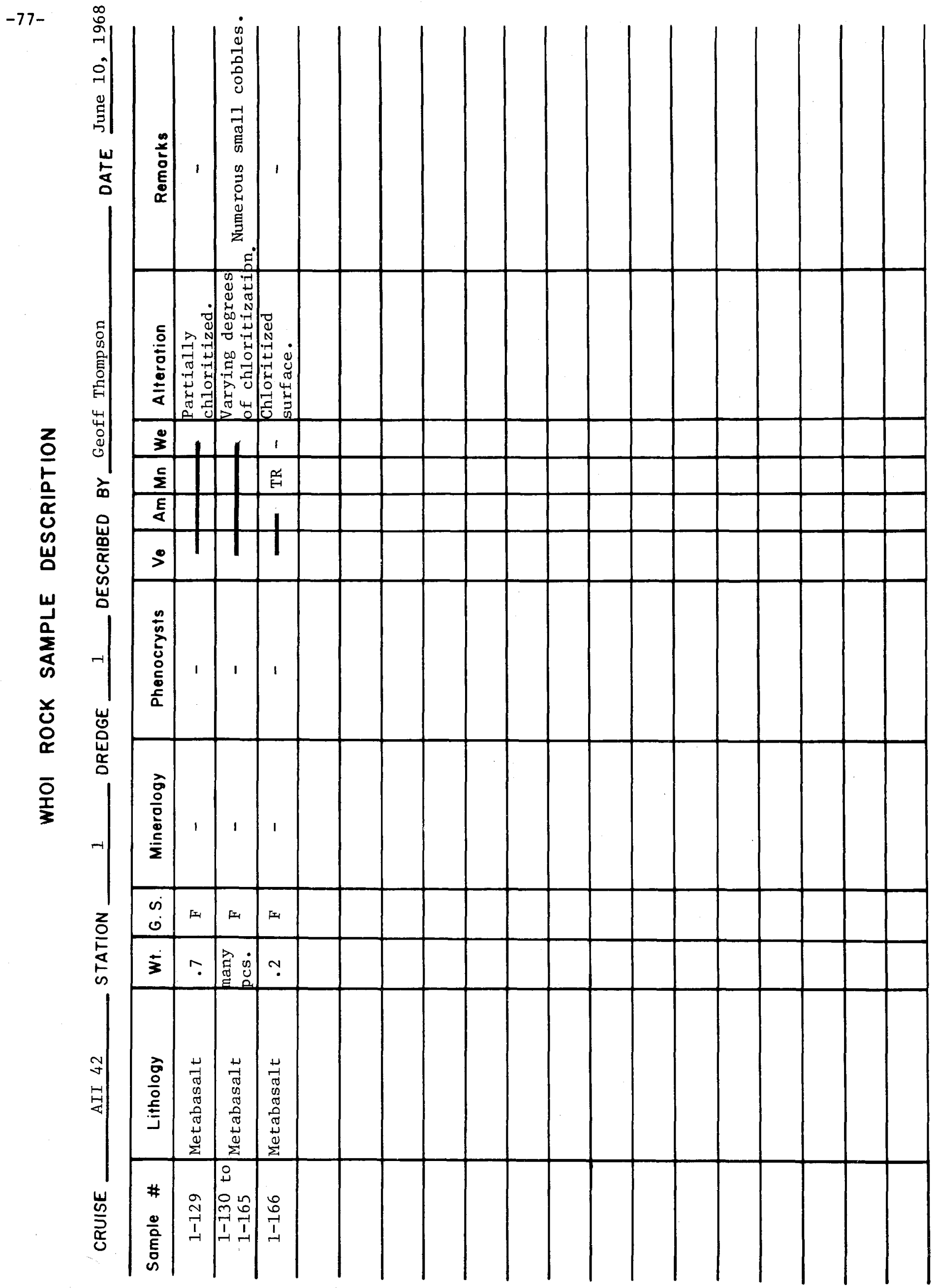




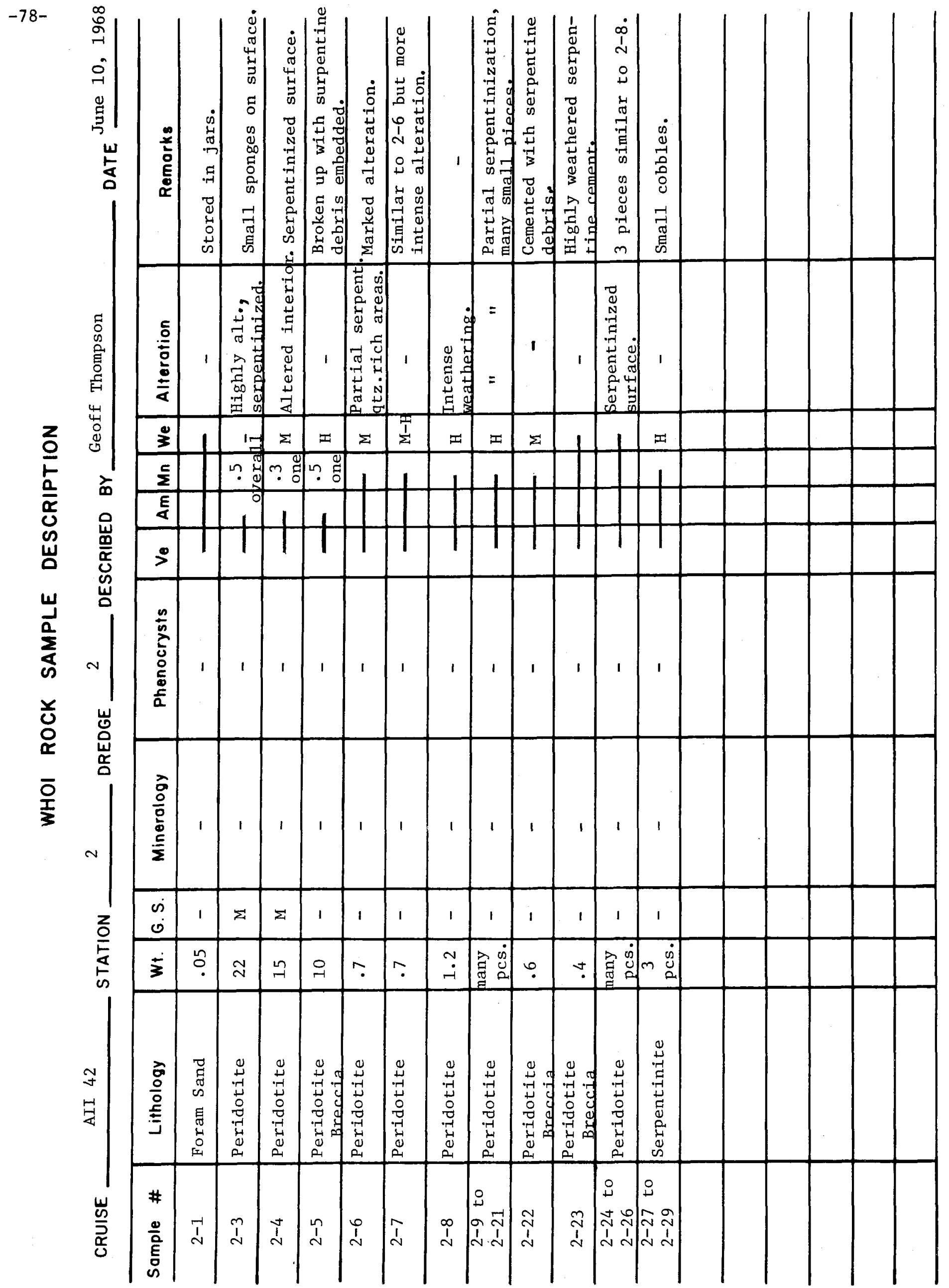




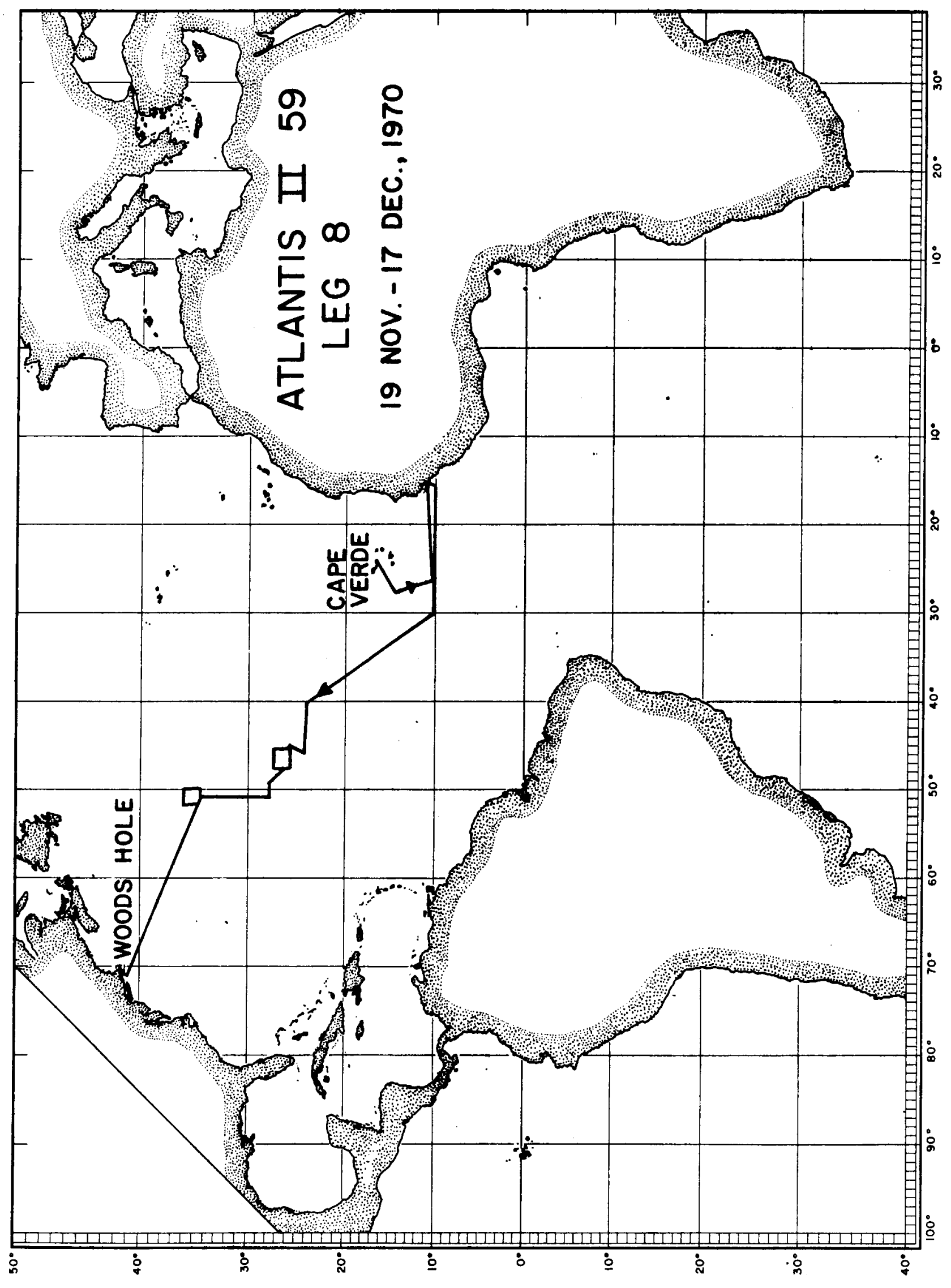




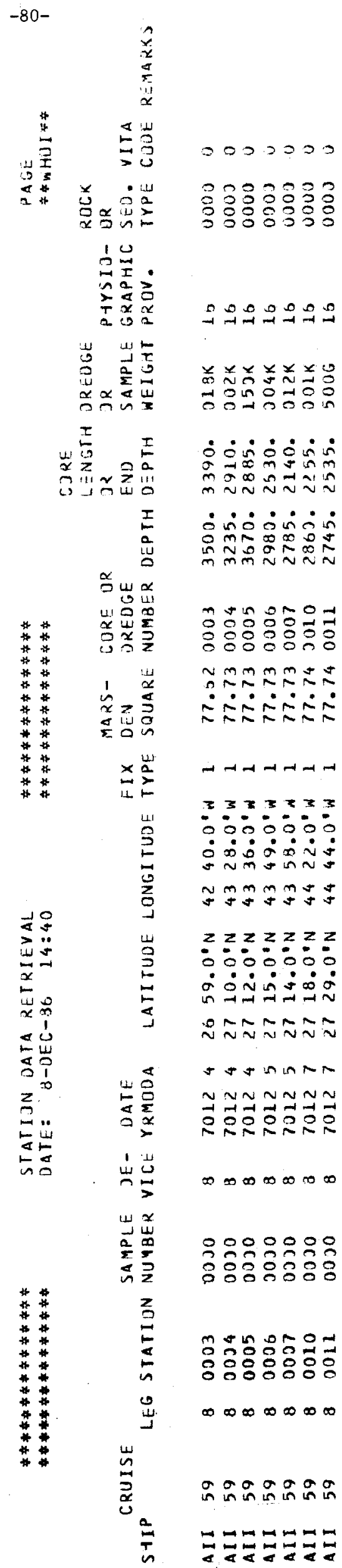




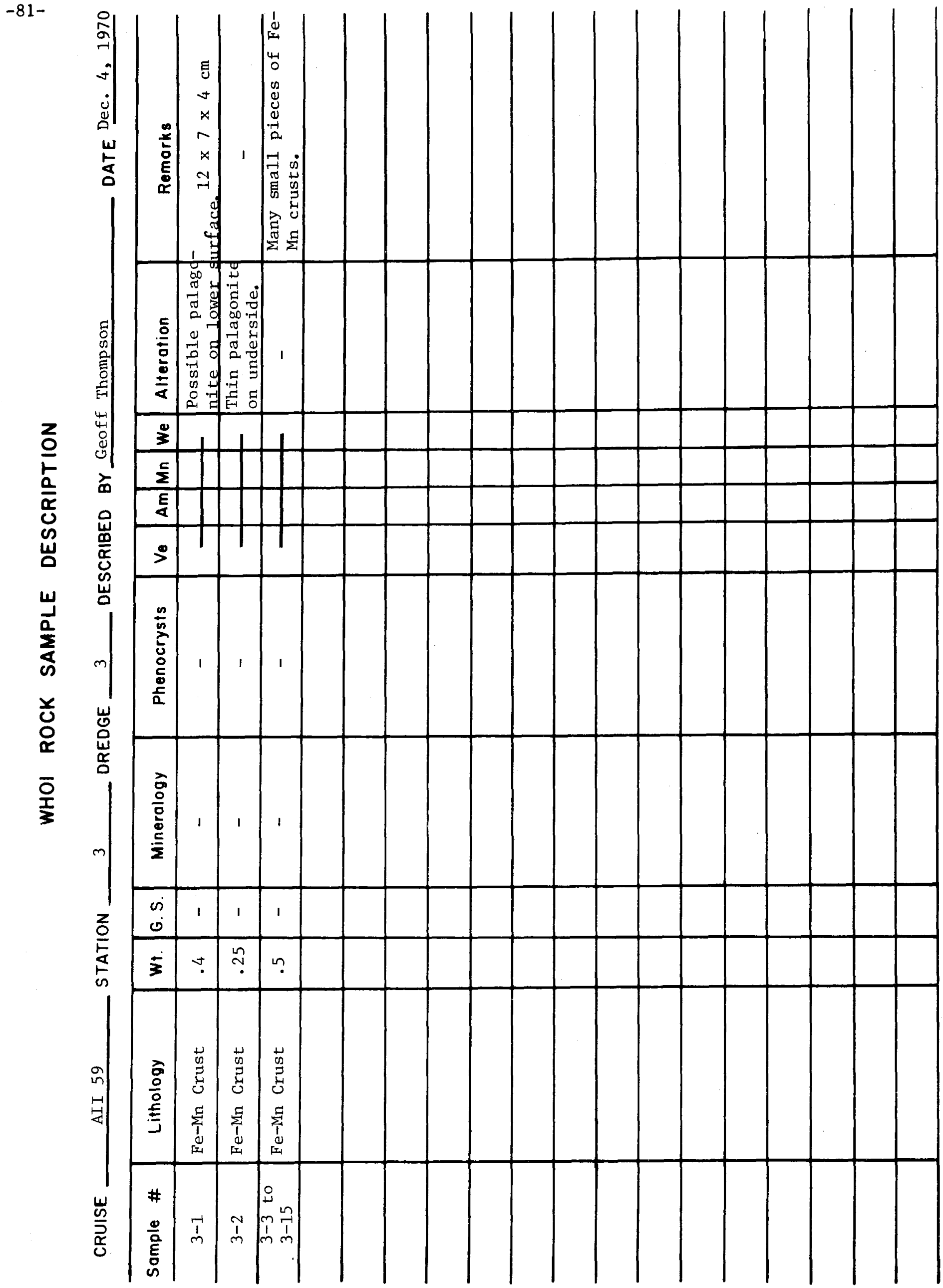




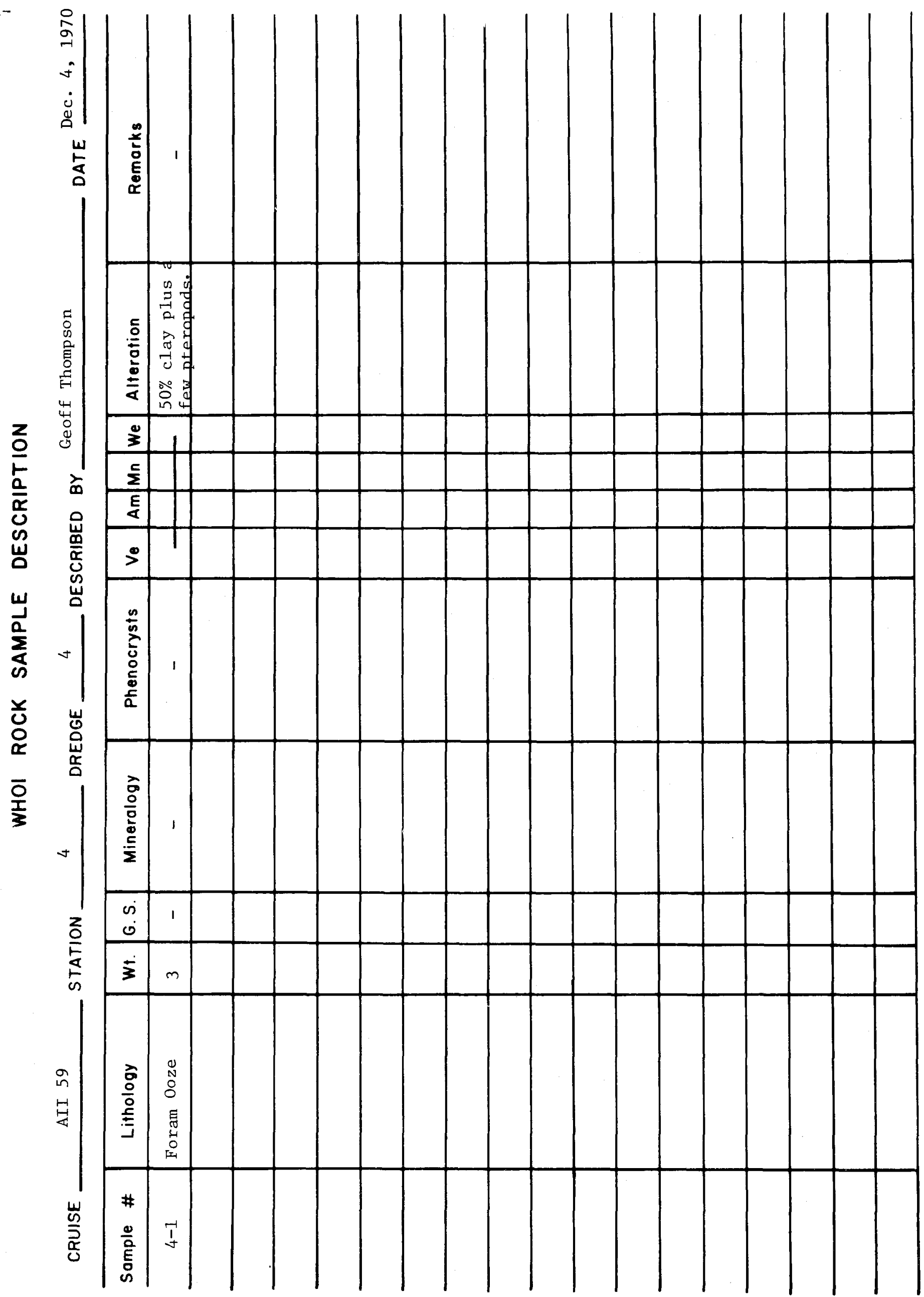




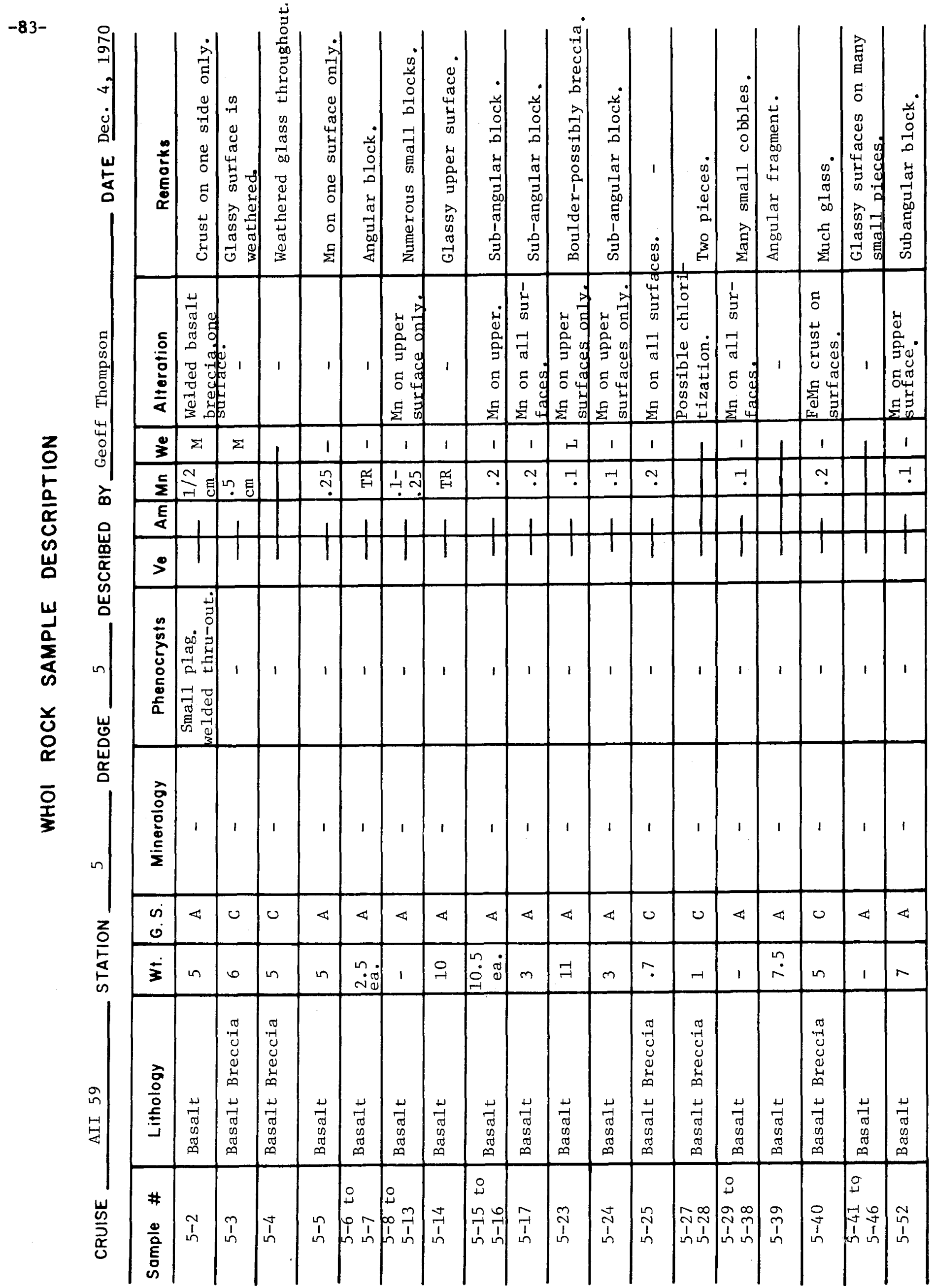




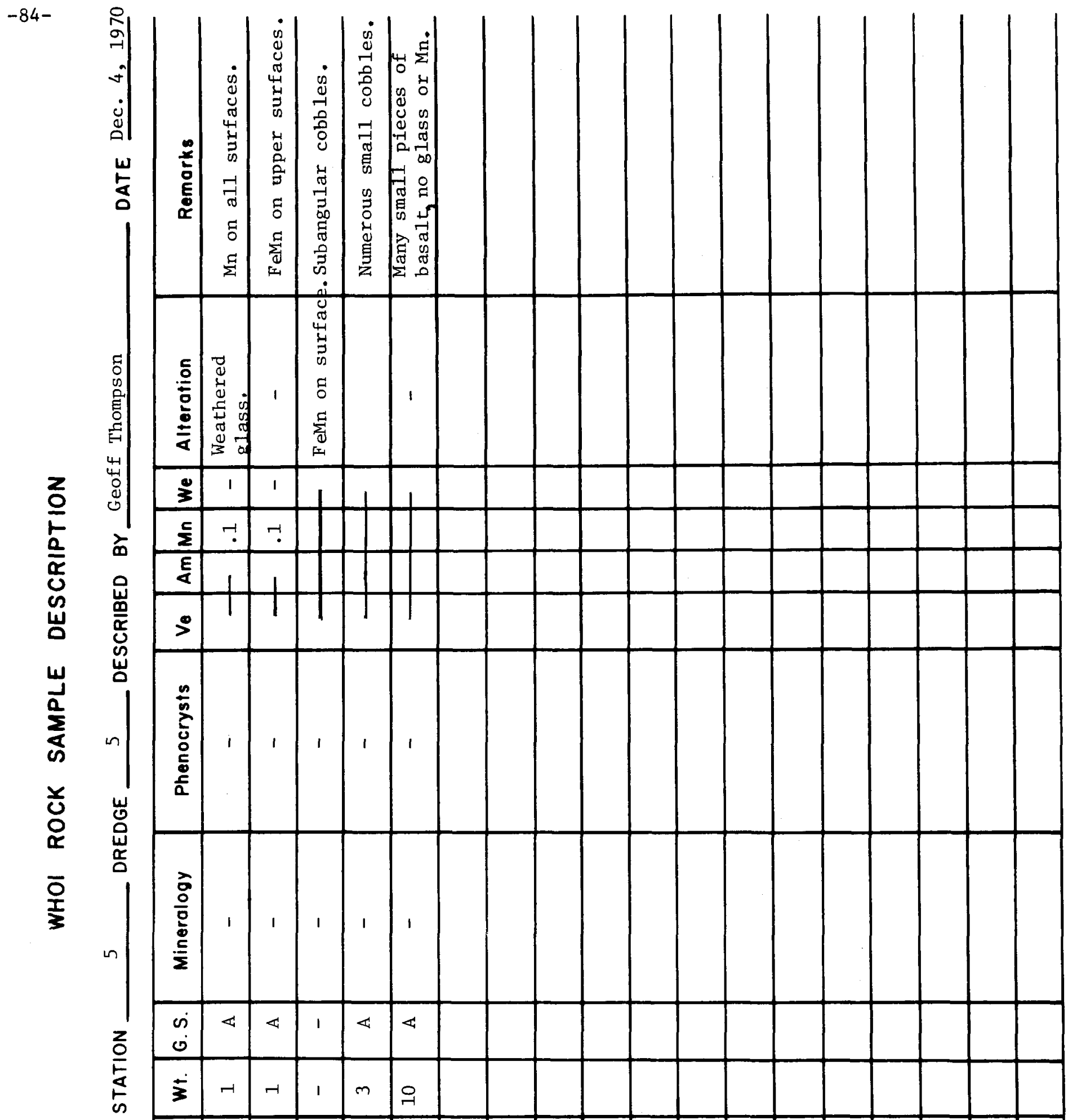

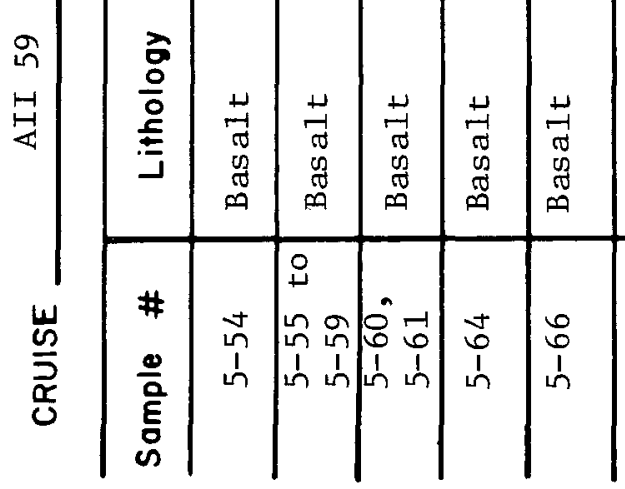




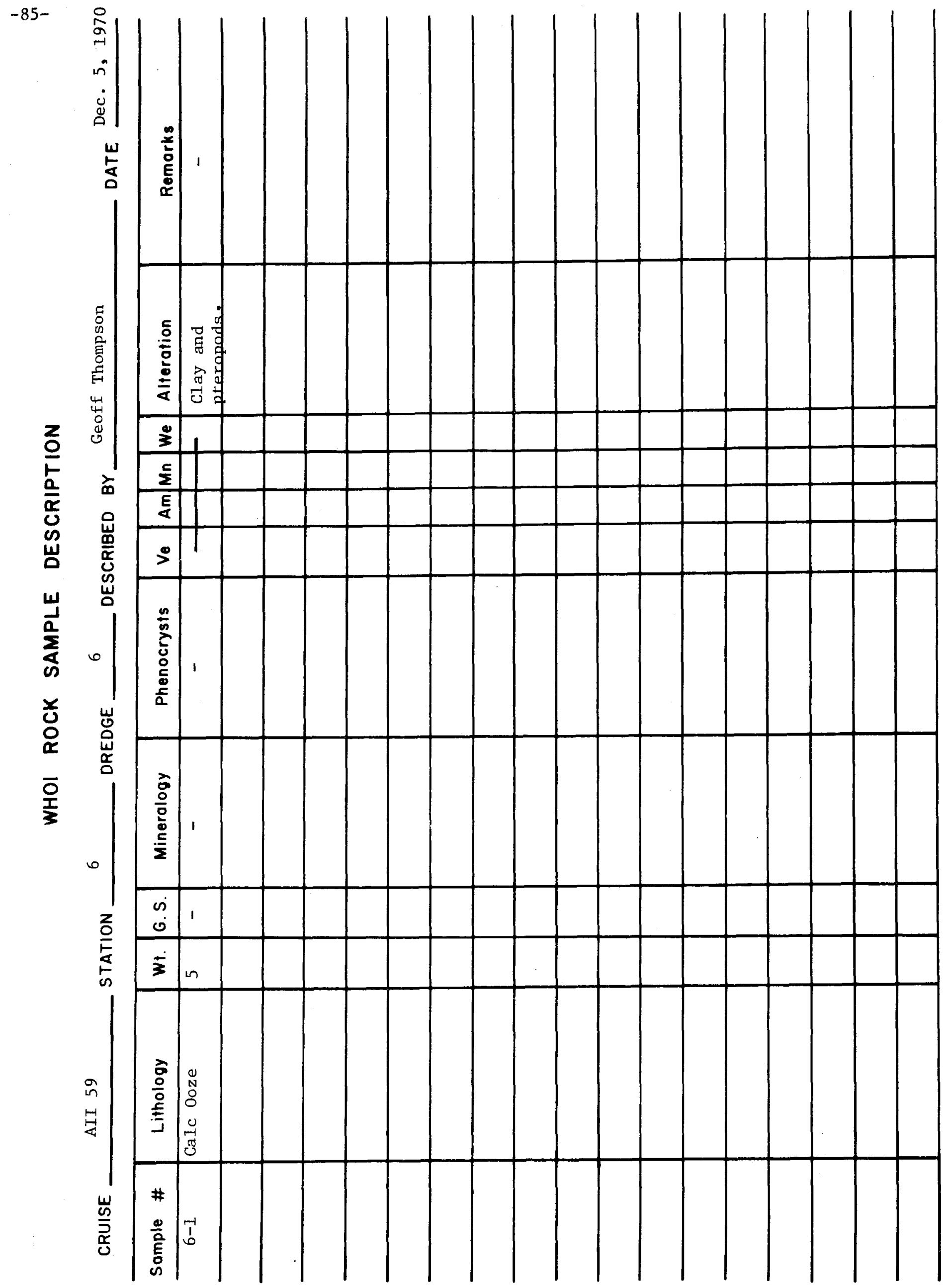




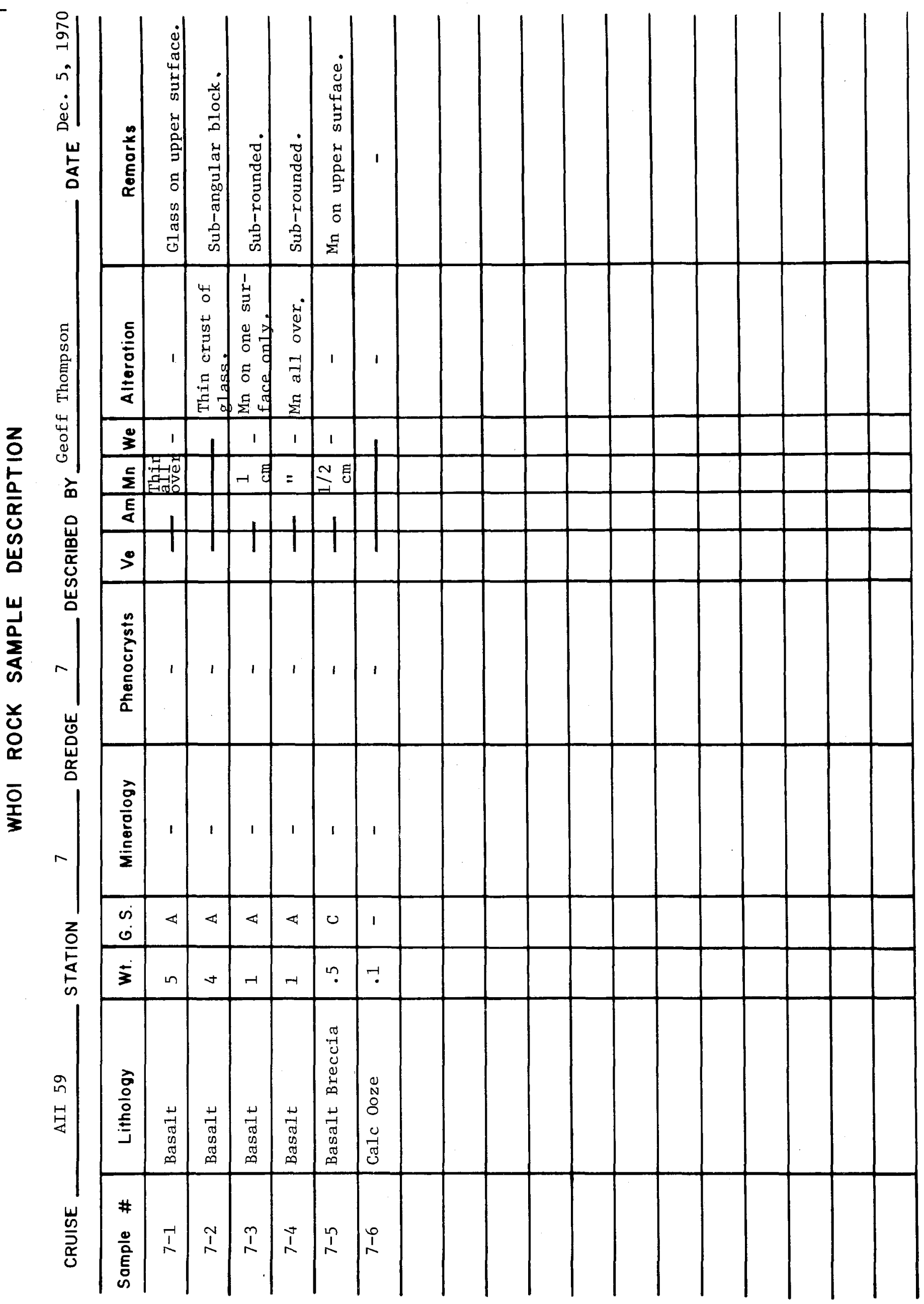




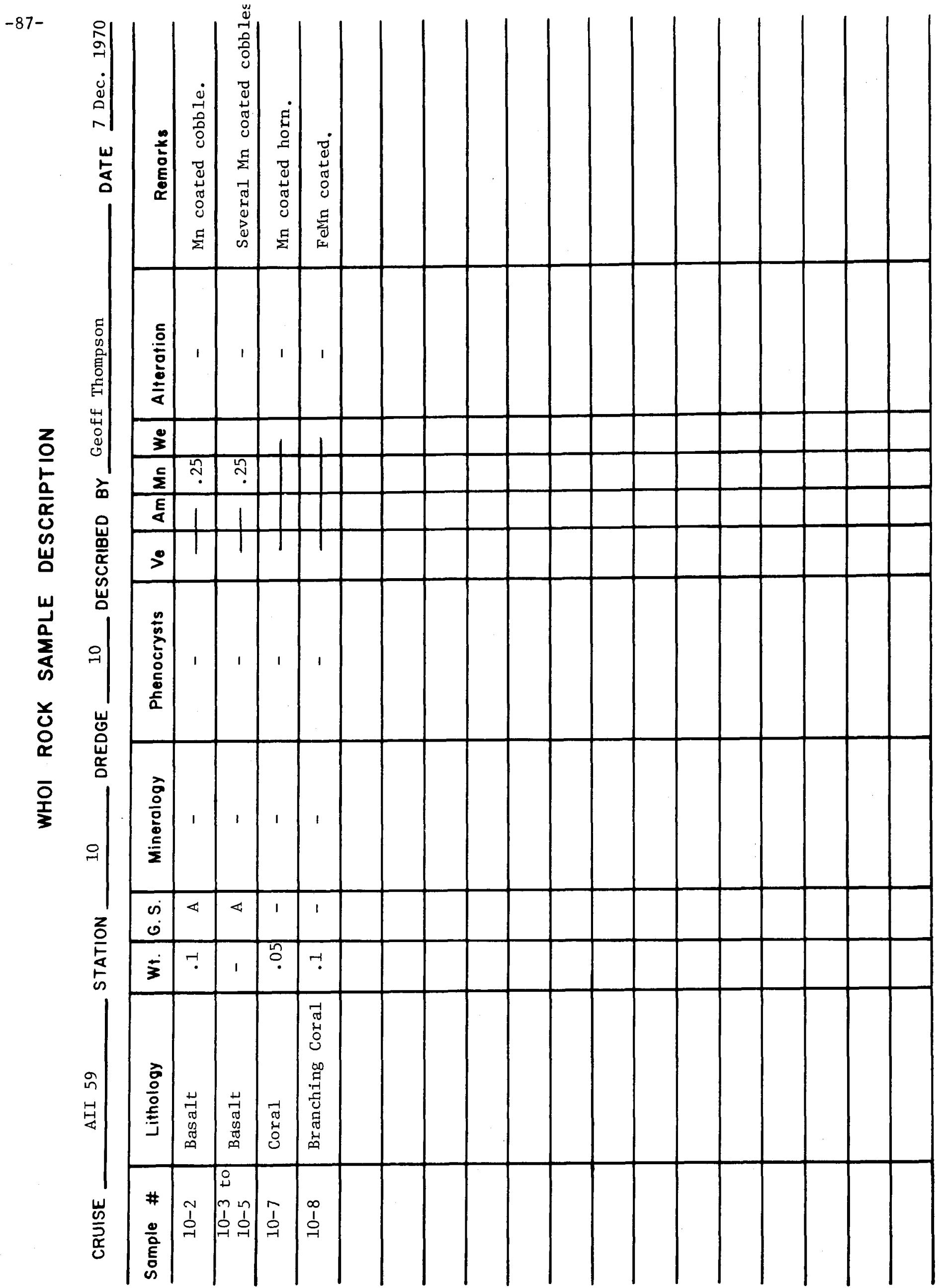




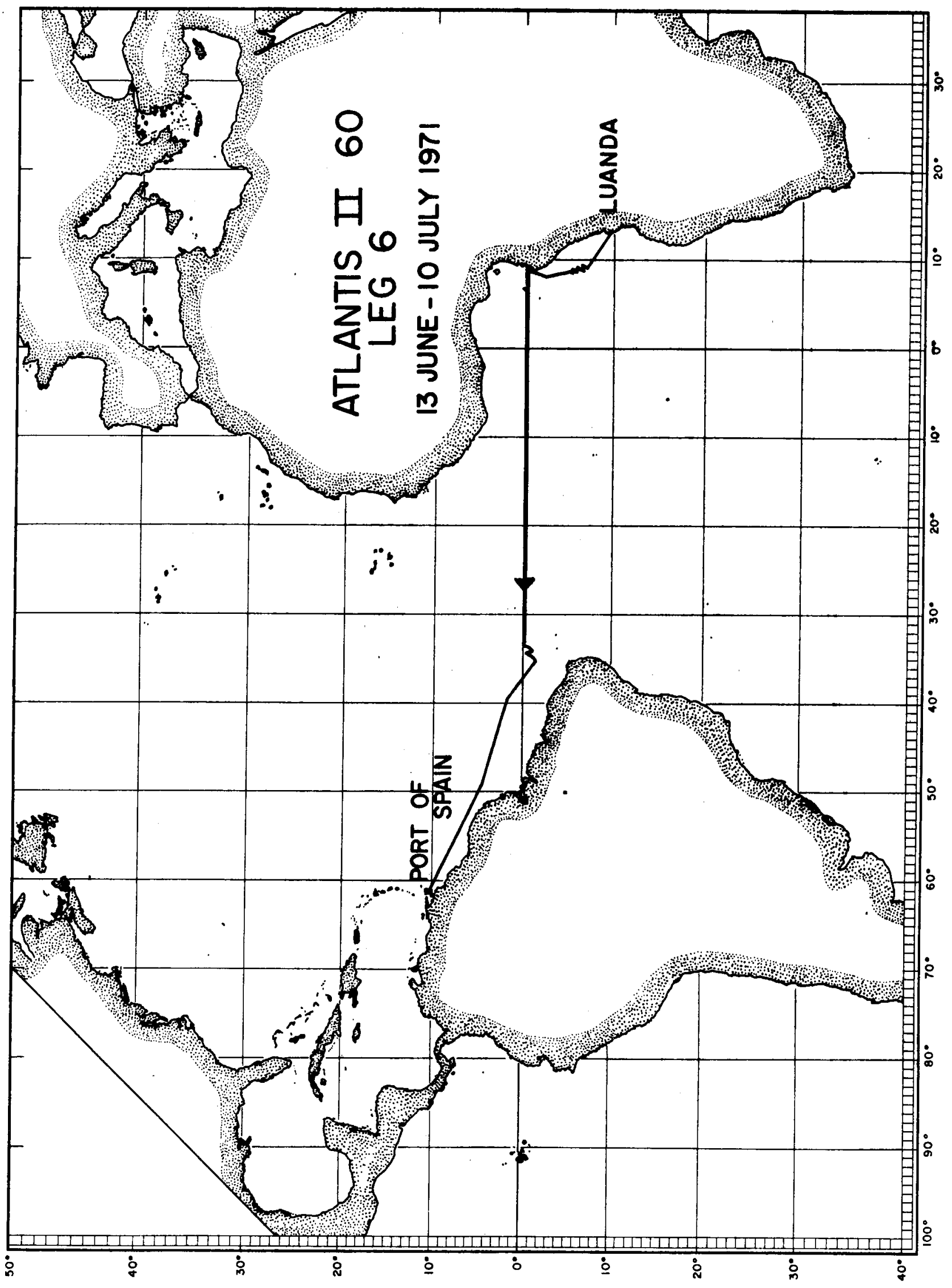


$-89-$

希出 $\alpha$

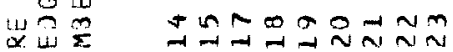

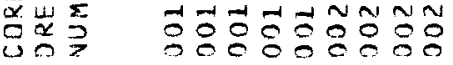
1 湈 300500000

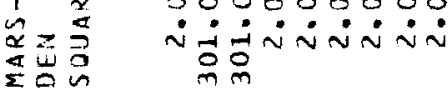
$\times \frac{2}{2}$

000000000

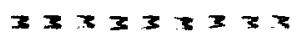
300505050 $\sim \dot{\sim} \dot{\sim} \dot{0} \dot{0} \dot{0}$ $\pi n-a n$

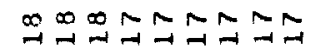
$z \sim n z \geq z \geq z z$ 000000050 in 0.0

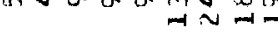
000000000

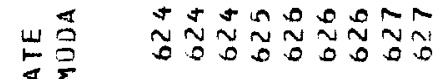

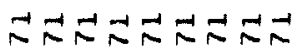
ח

แ1 $\alpha$

$\frac{1}{2}+\infty$

윽음응윽역용응 응응ㅇㅇㅇㅇ

$z$

20080080

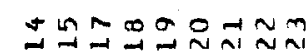
응영ㅇㅇㅇㅇㅇㅇㅇㅇㅇㅇㅇ

㟧 000000000

$u$
$\mathfrak{5}$
$\mathfrak{x}$
0

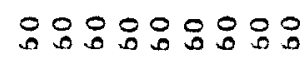

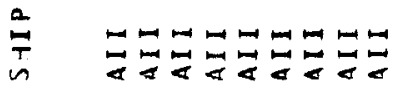


$-90$

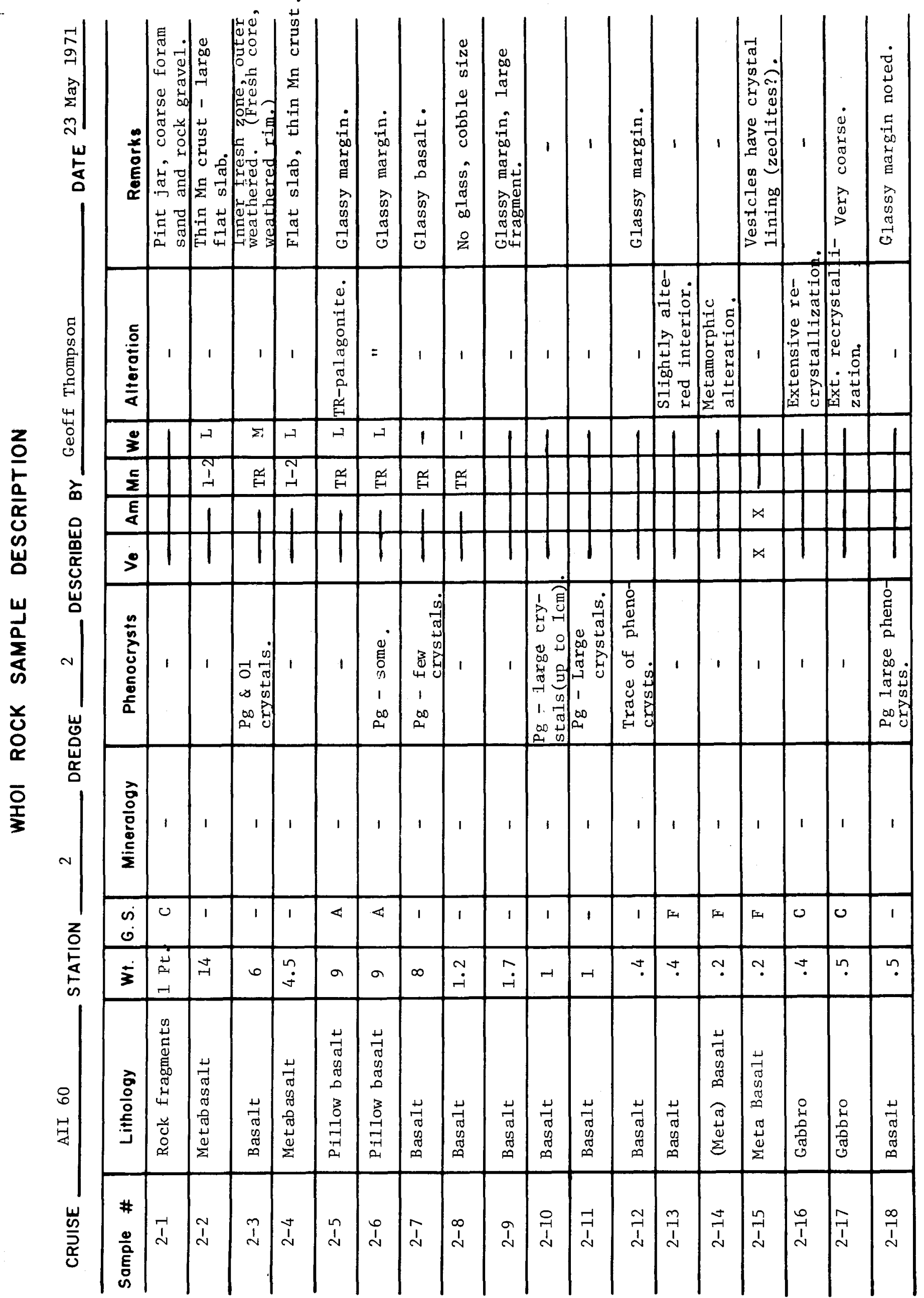




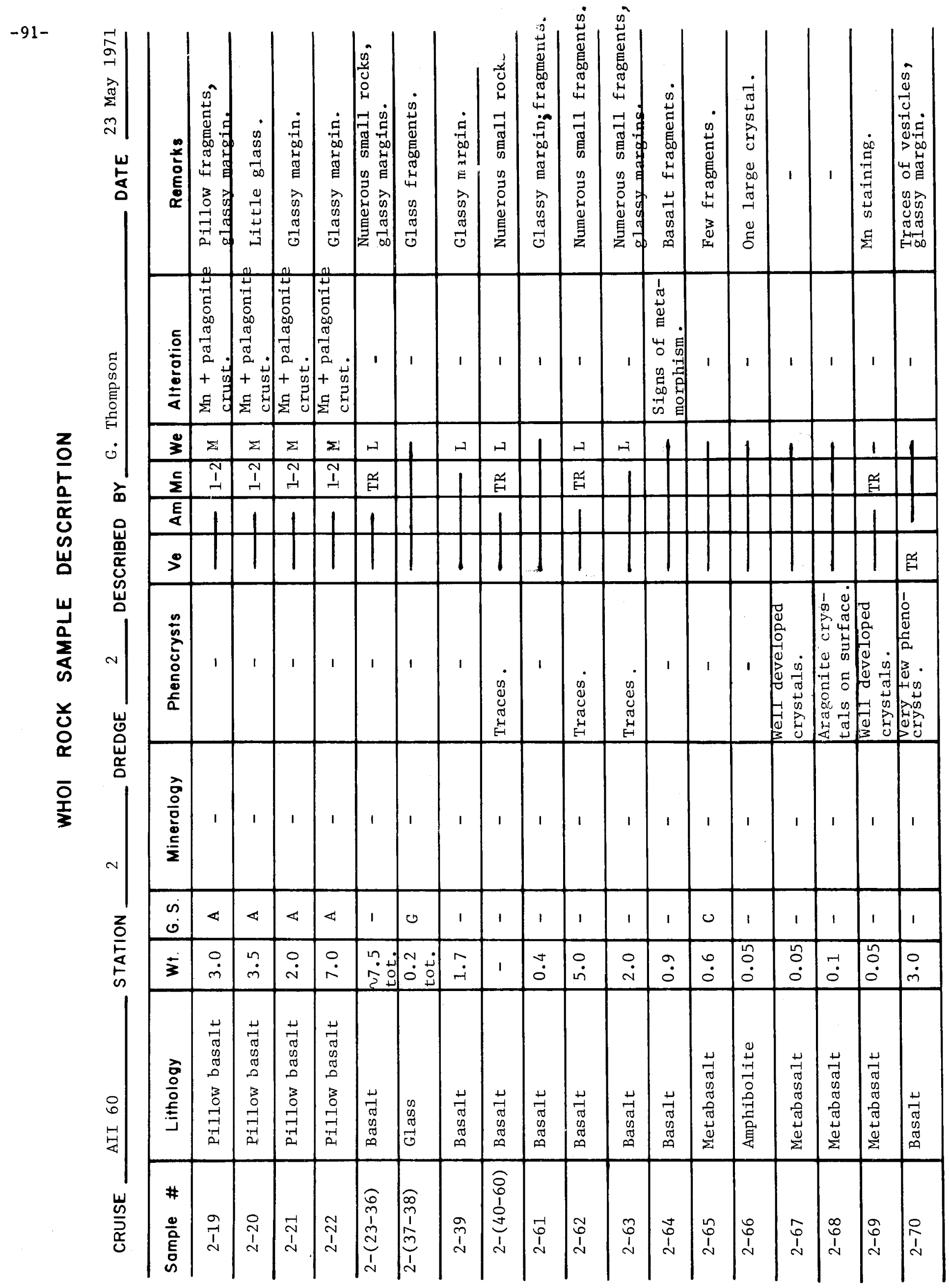




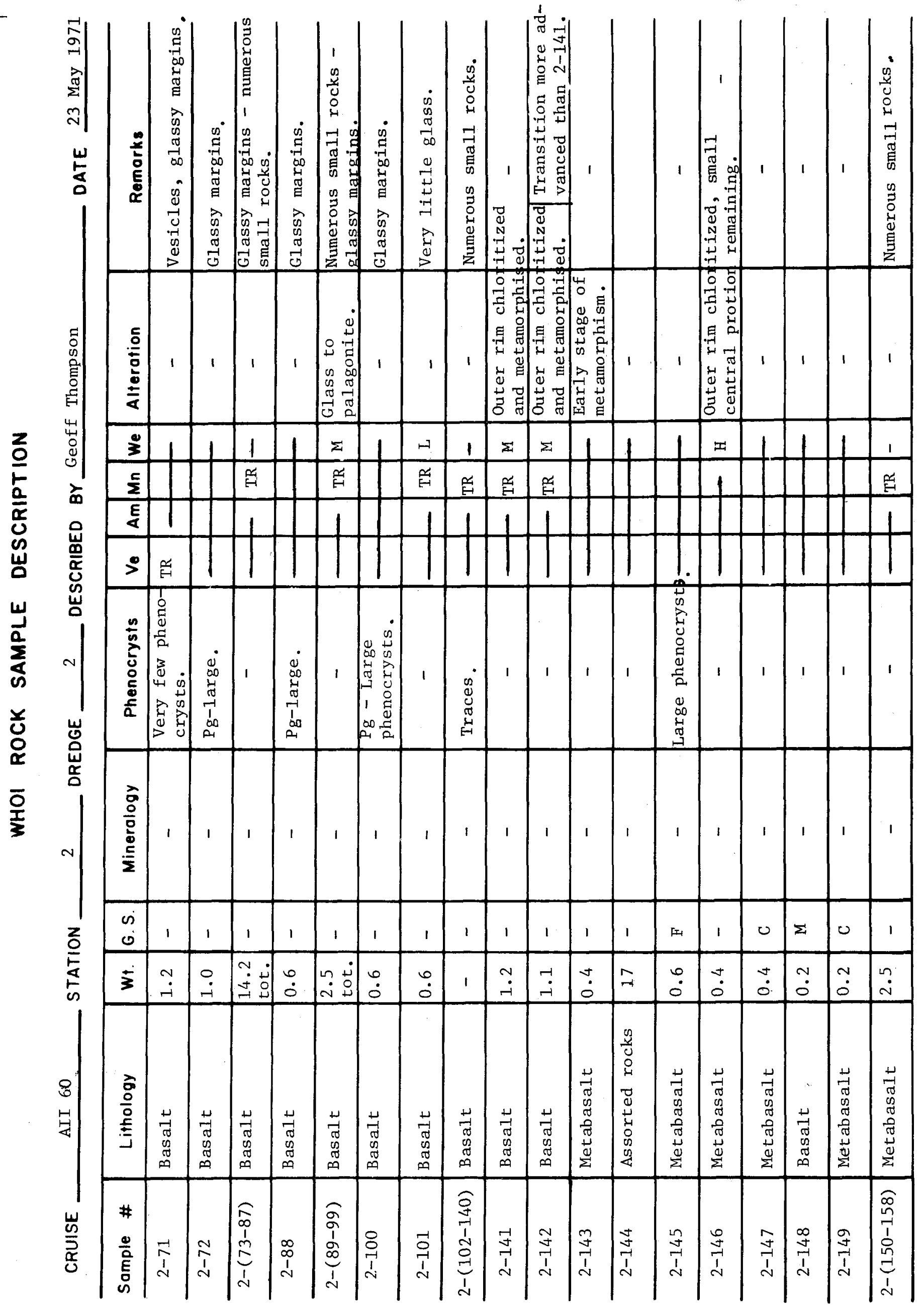



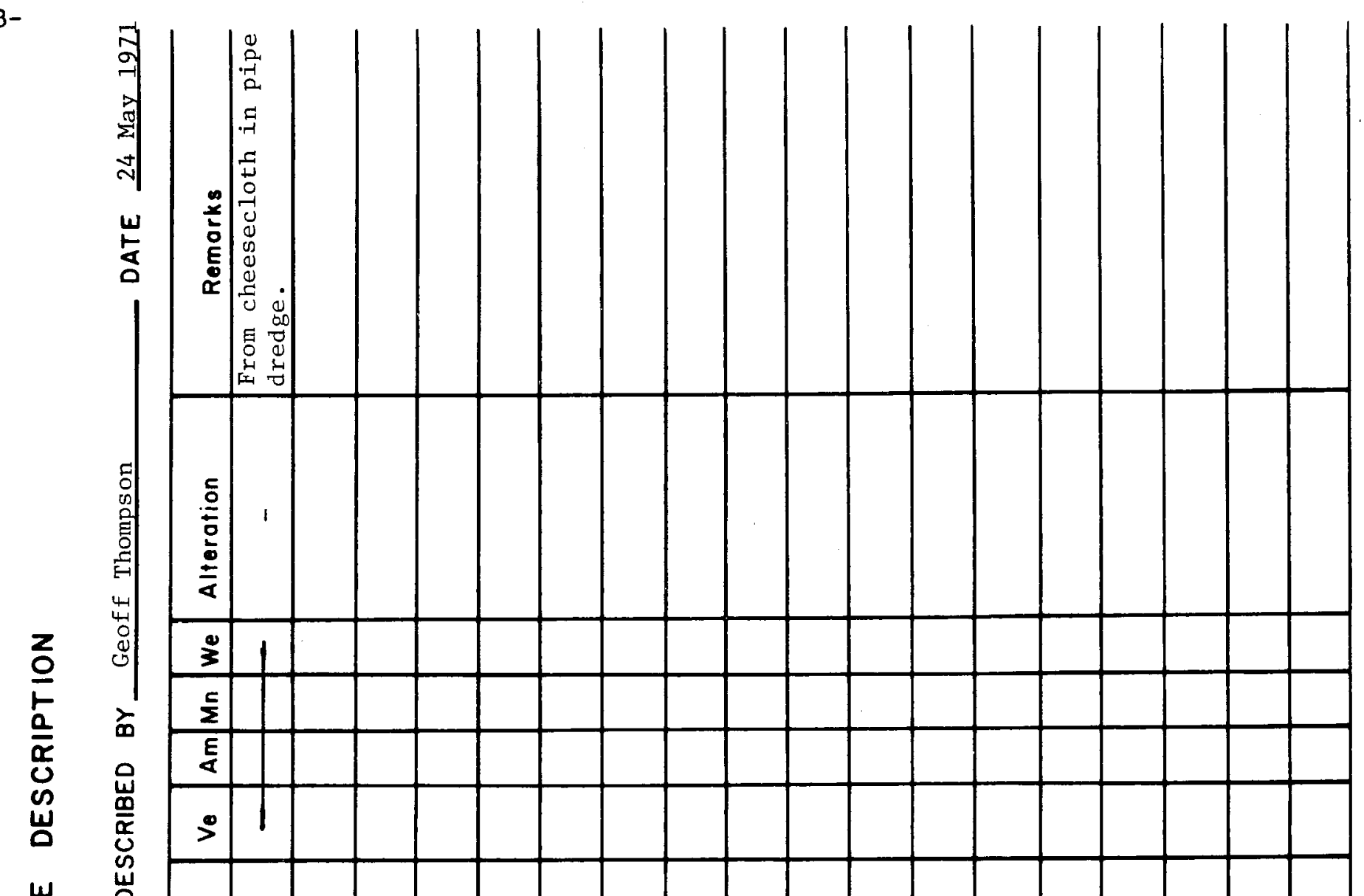

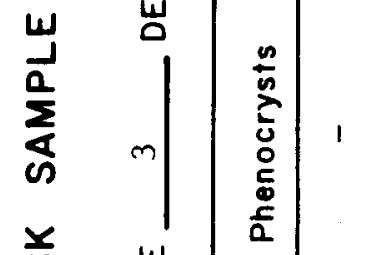

崖

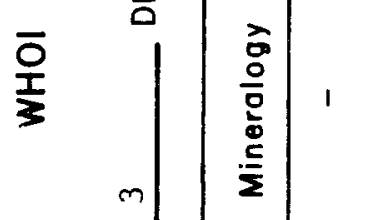

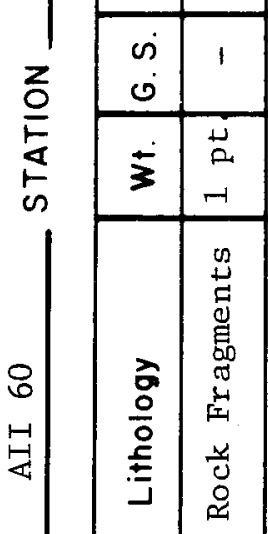

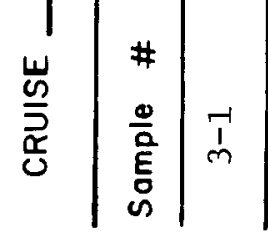


$-94-$

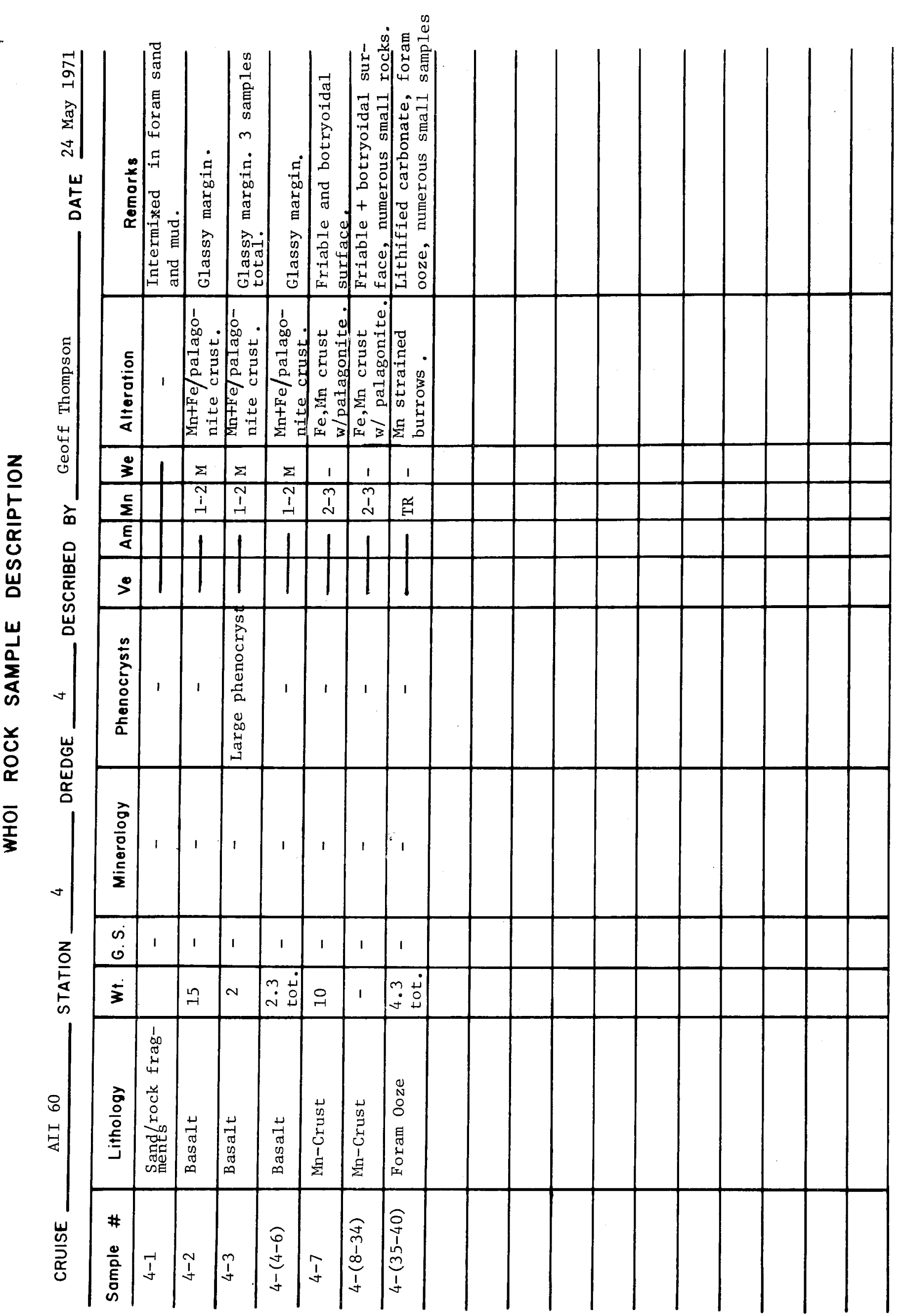




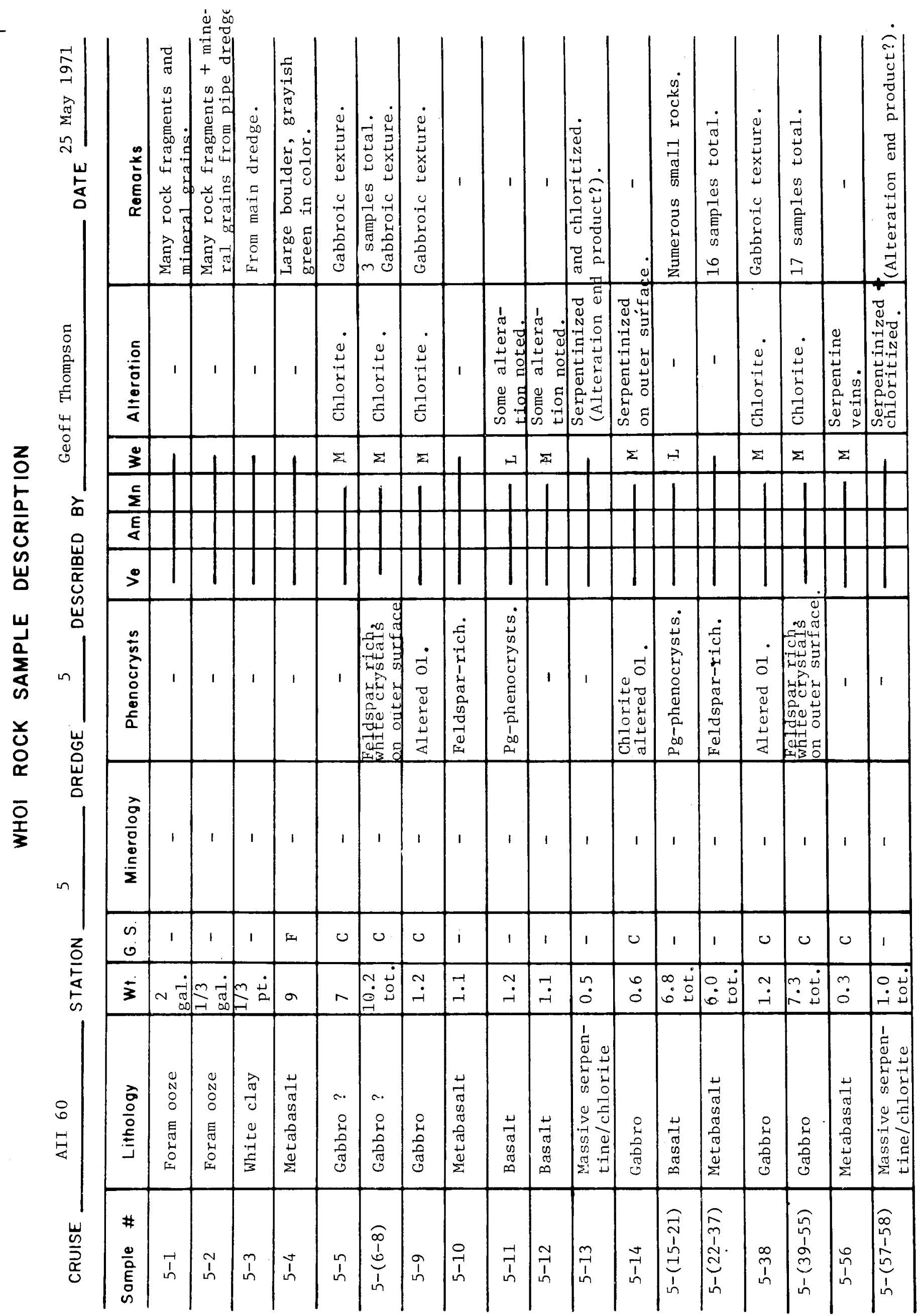




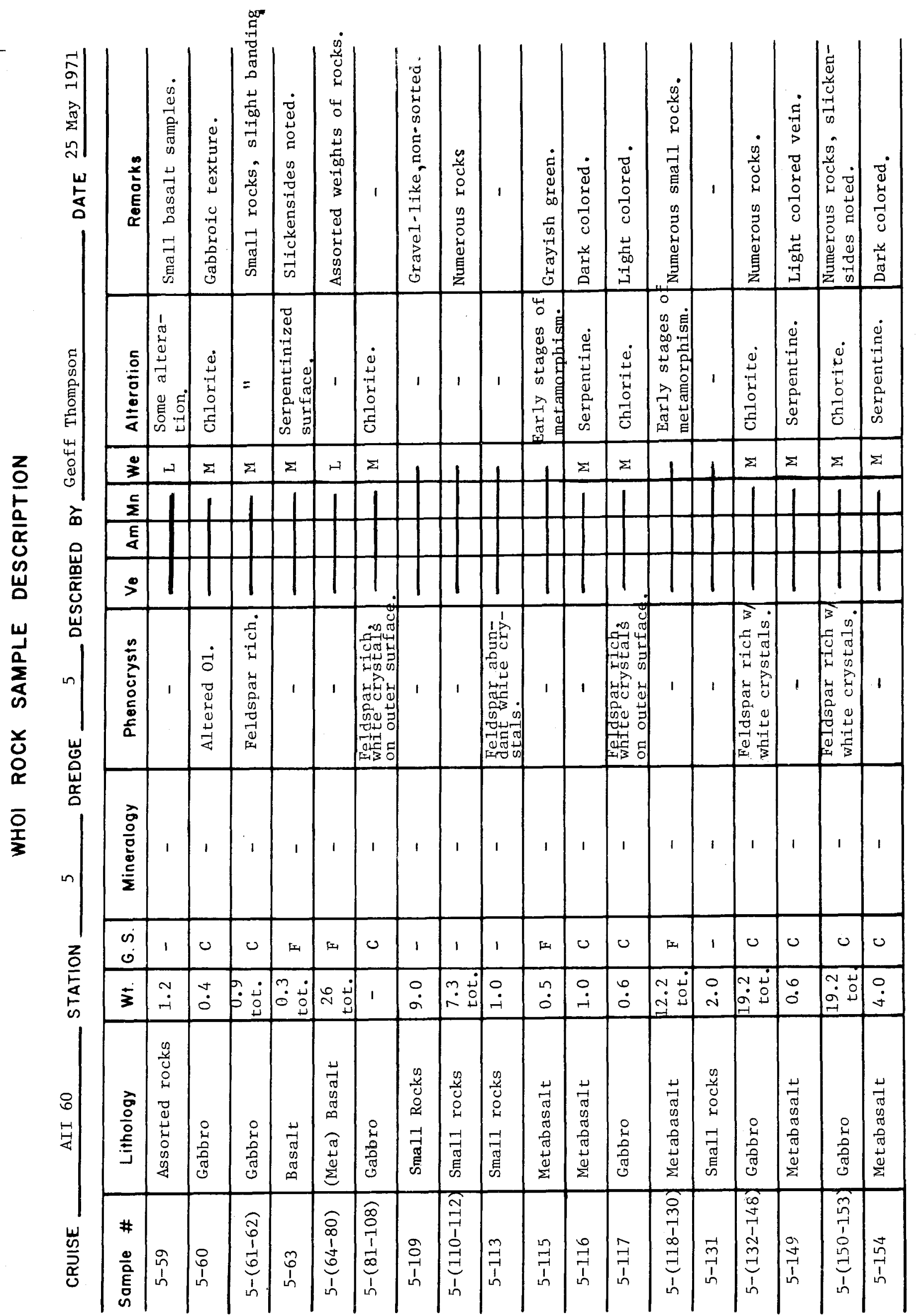


$-97-$

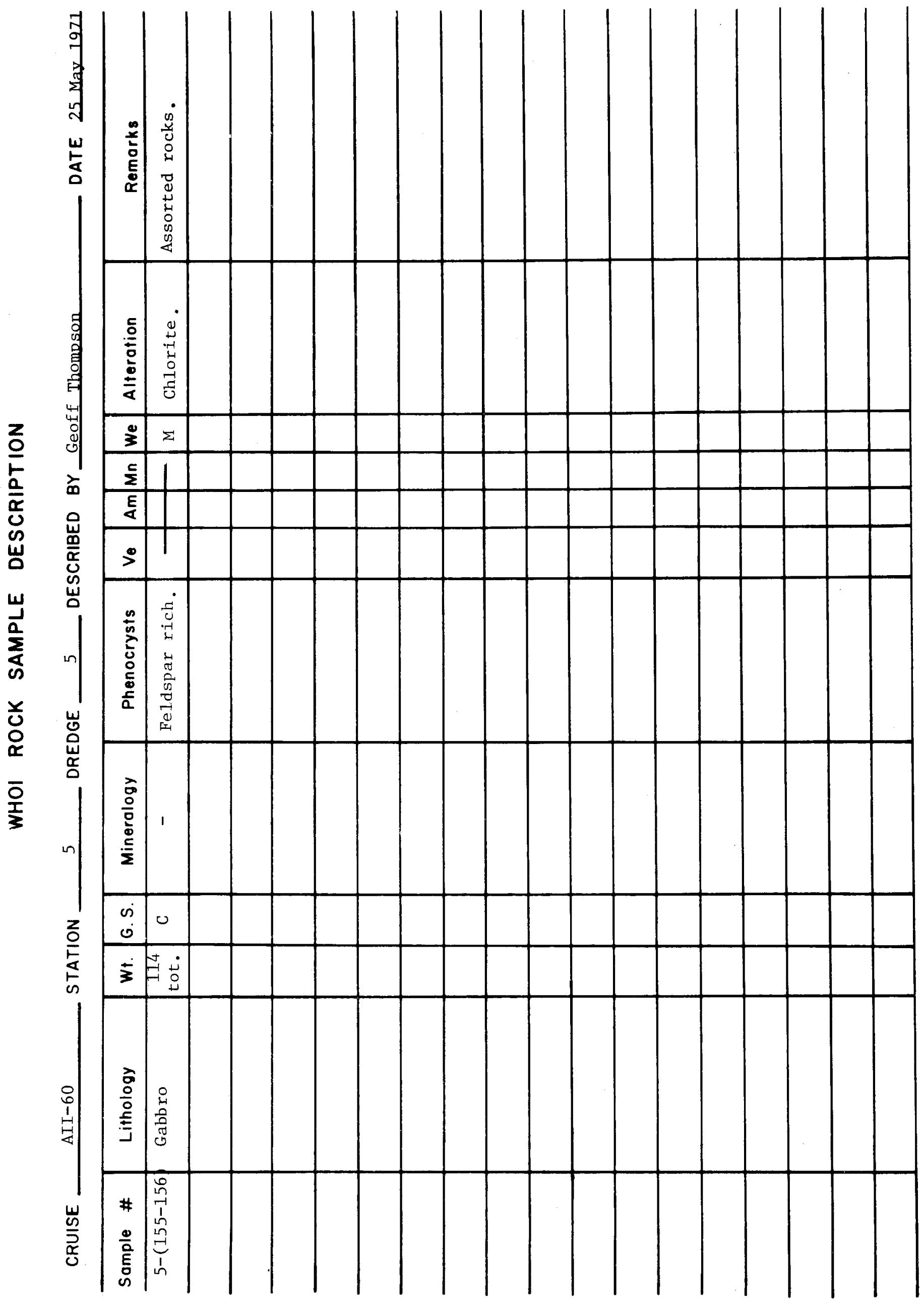




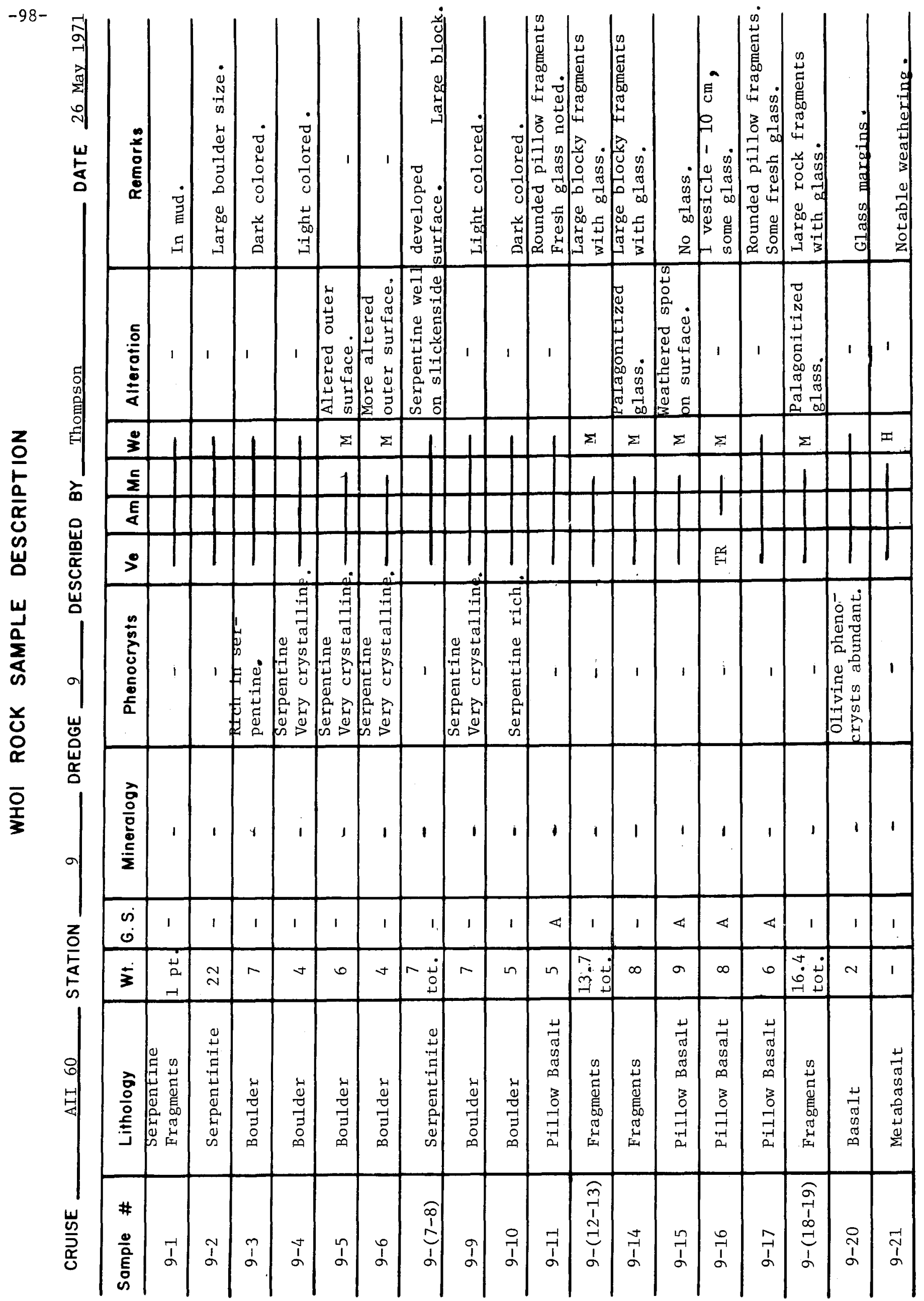




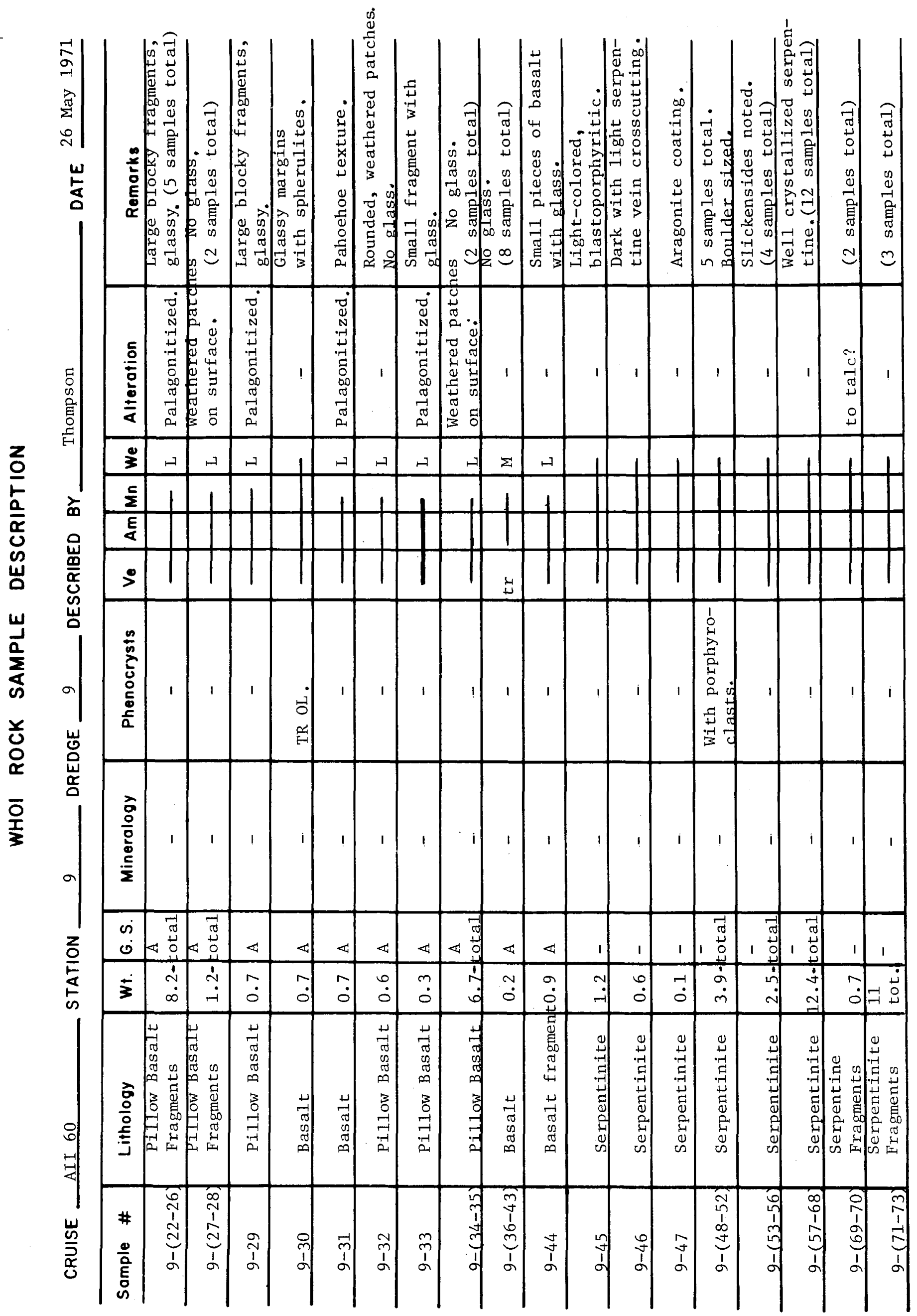


$-100$

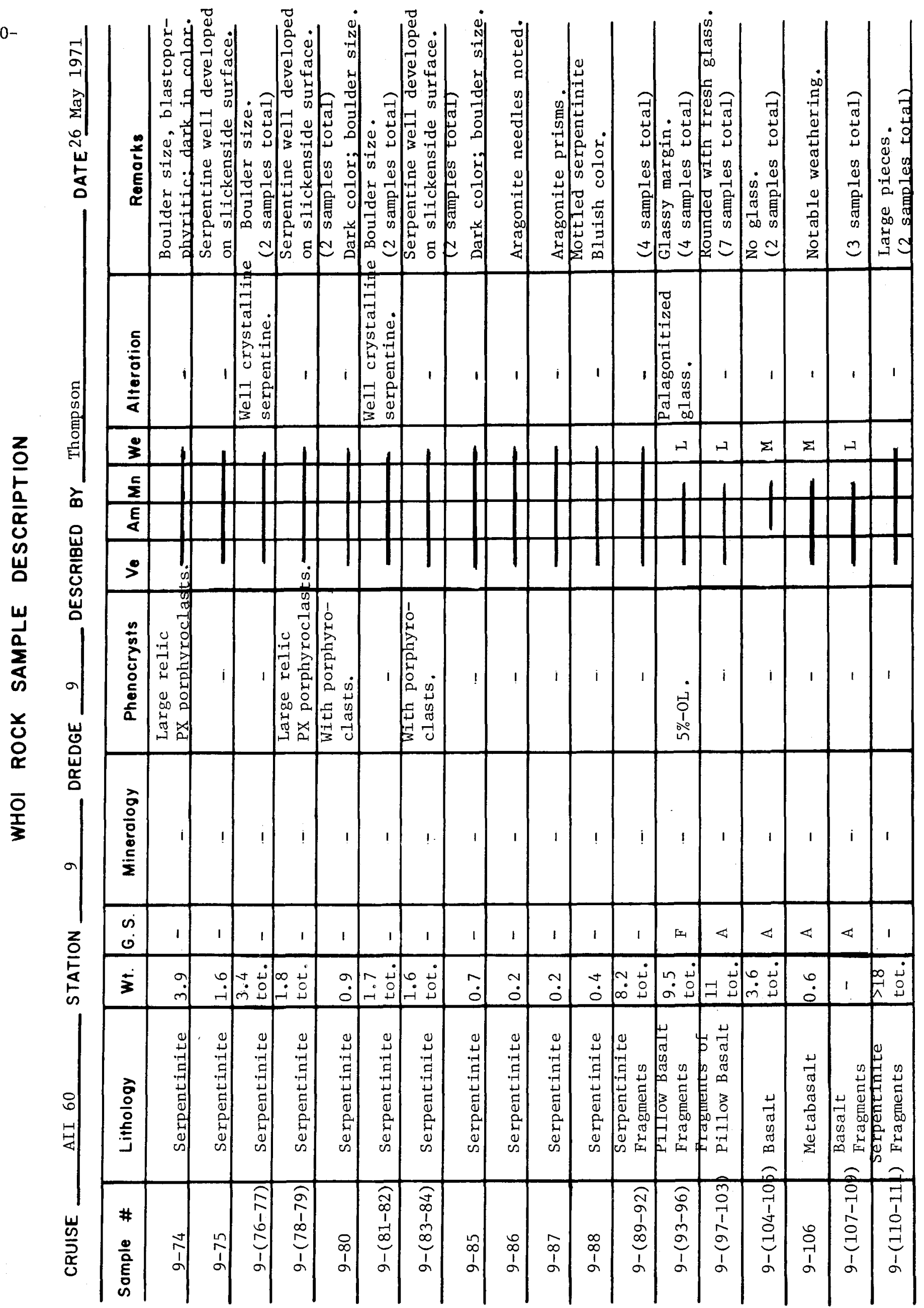




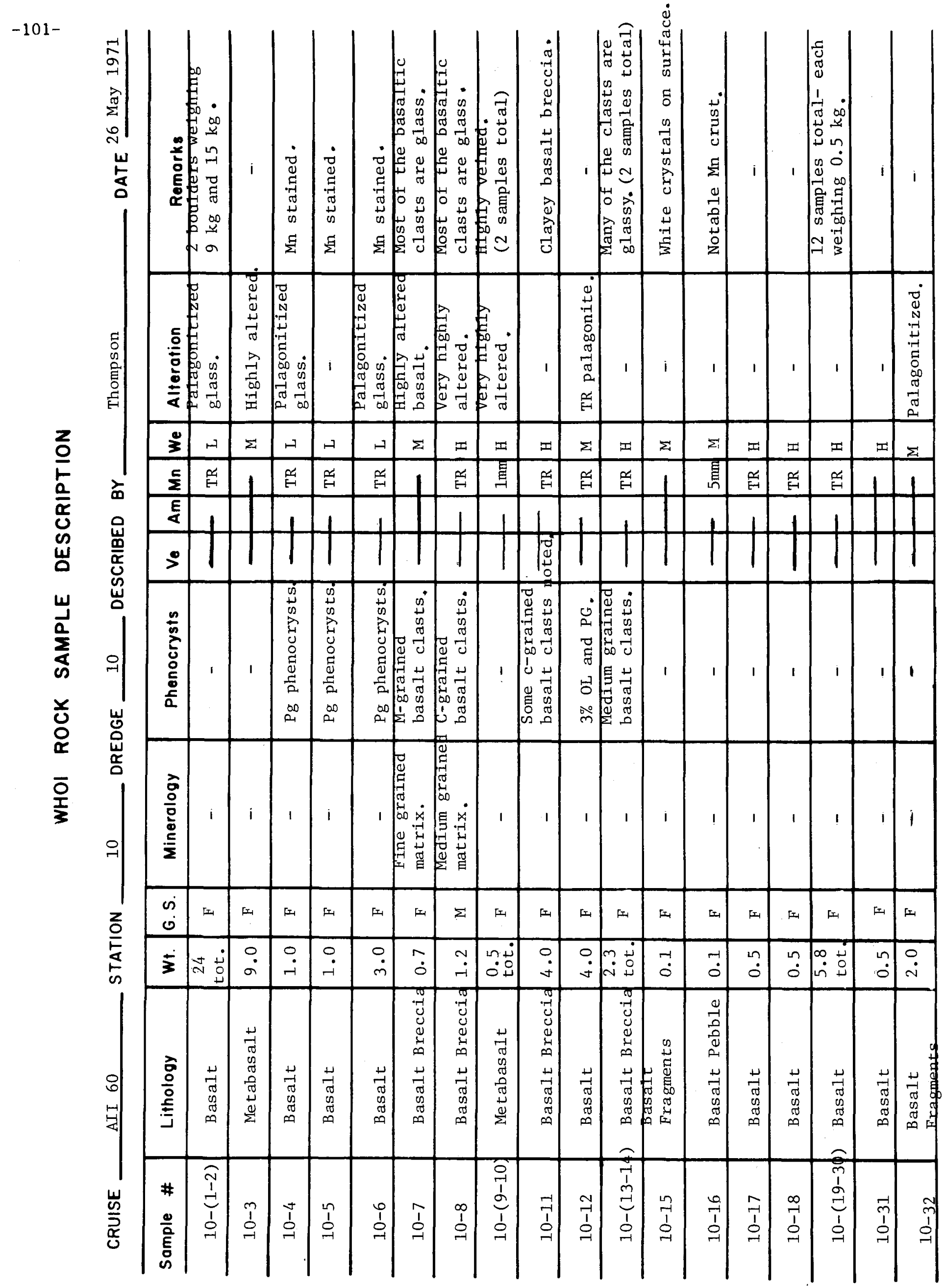




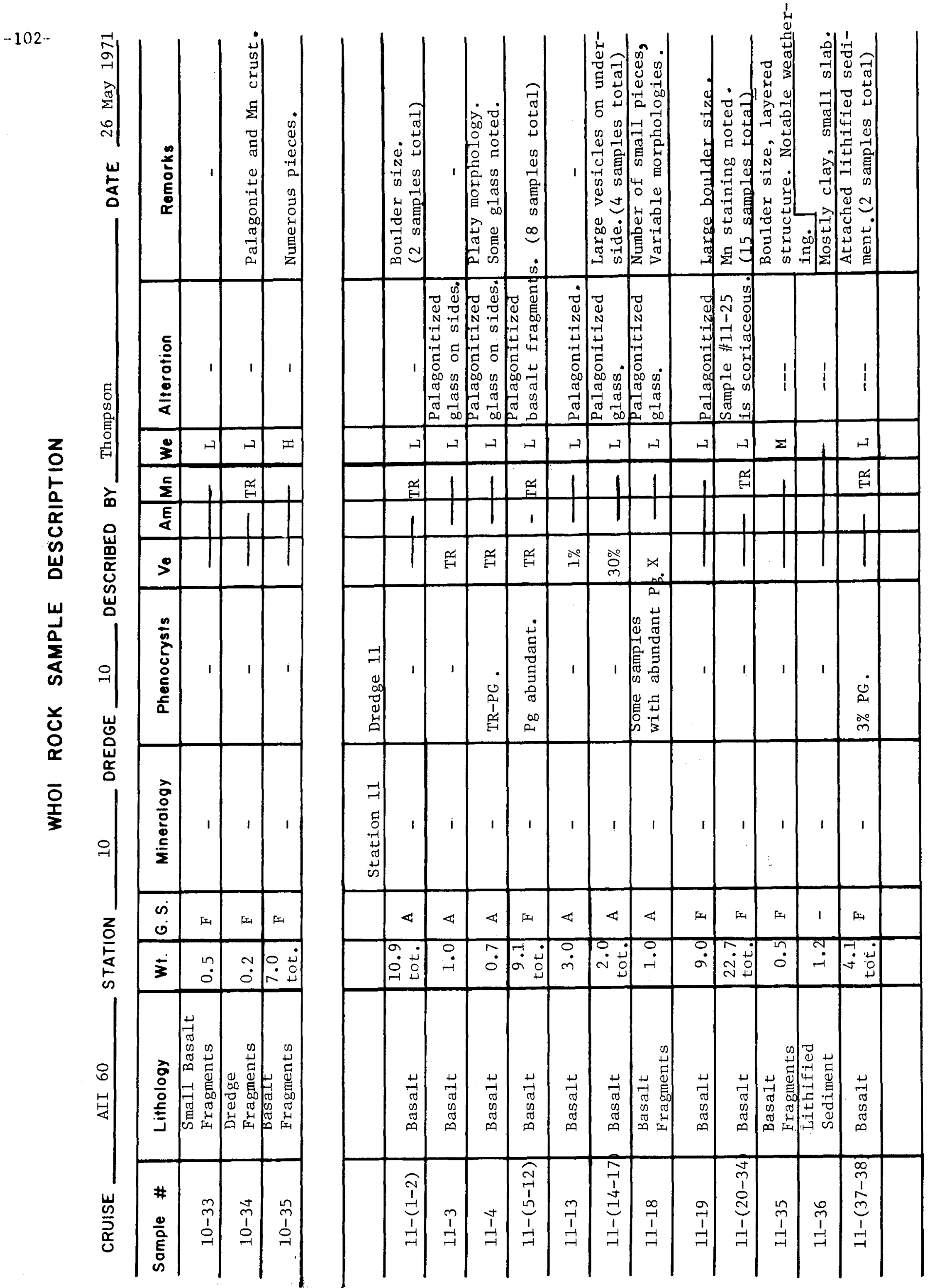


部

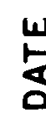

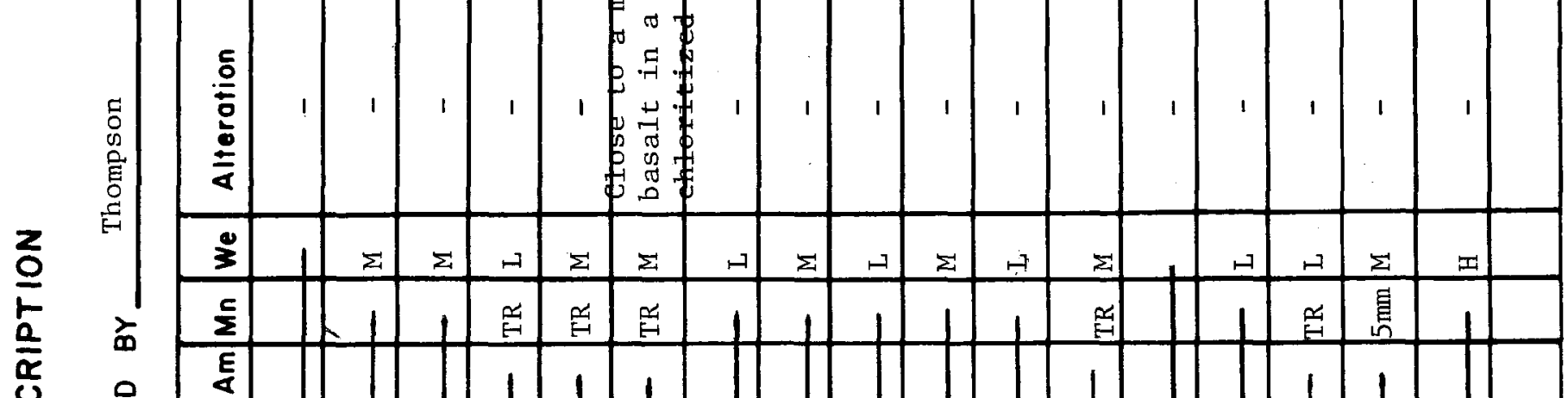

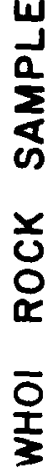

量

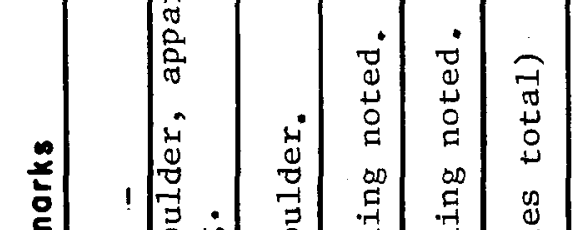

.

赵

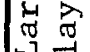

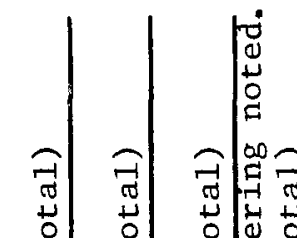

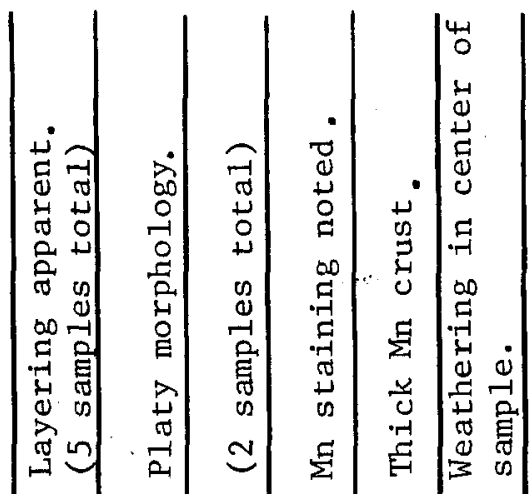

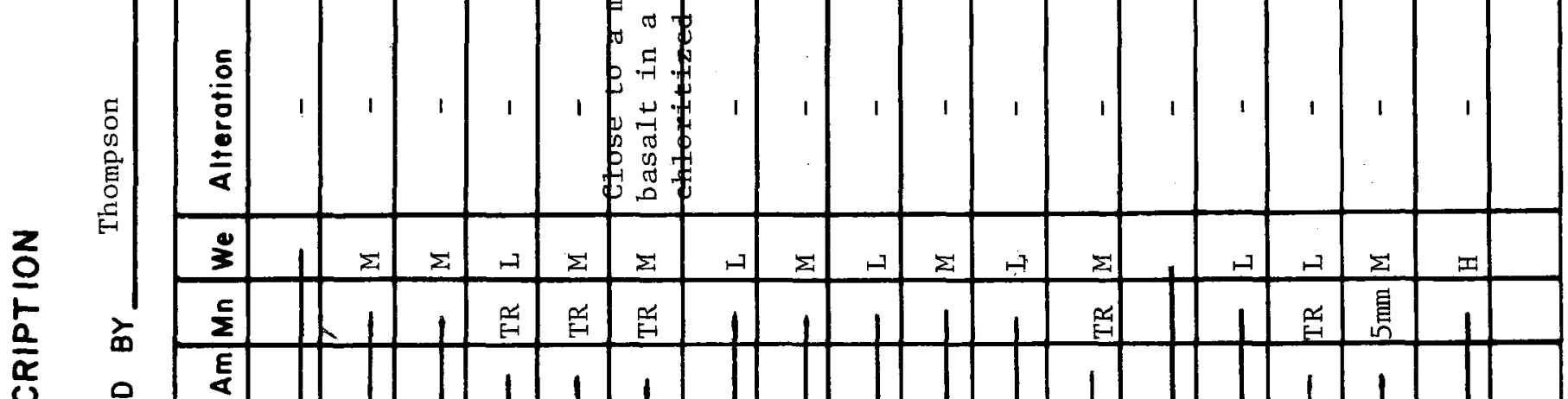

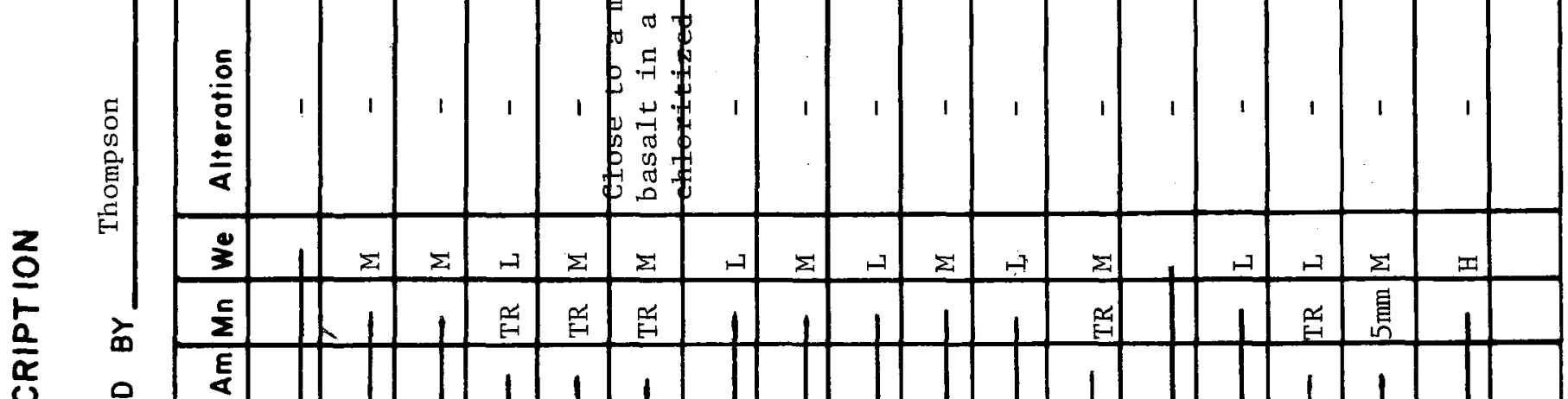

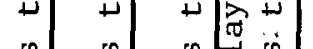

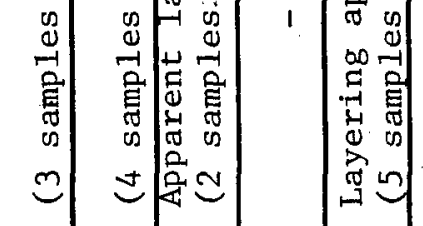

.

N 㭡

热

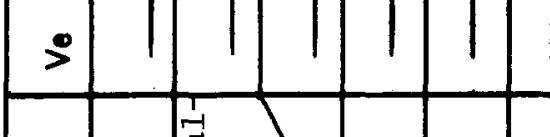

实

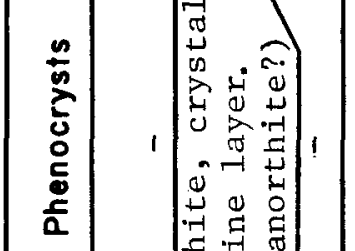

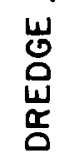

点

$\frac{2}{0}$

N

은

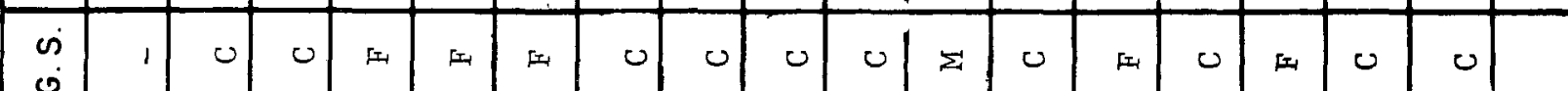

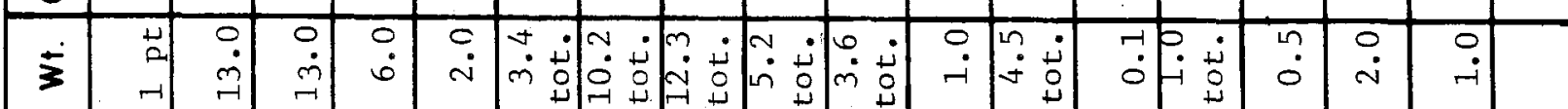

8

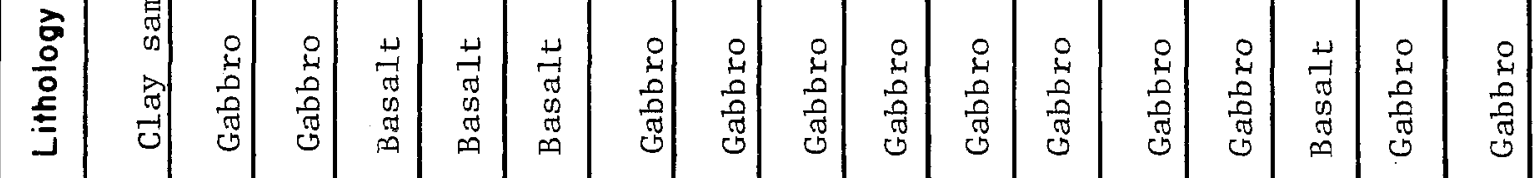

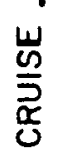

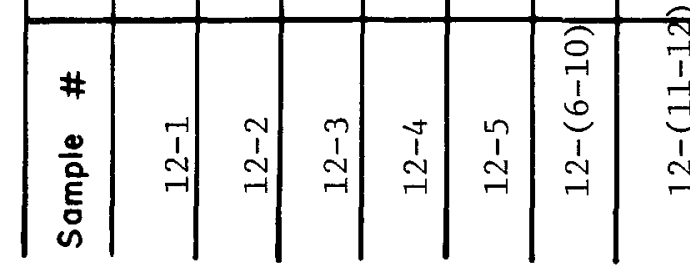

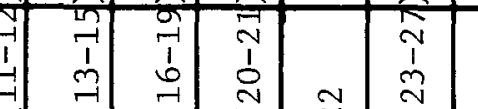




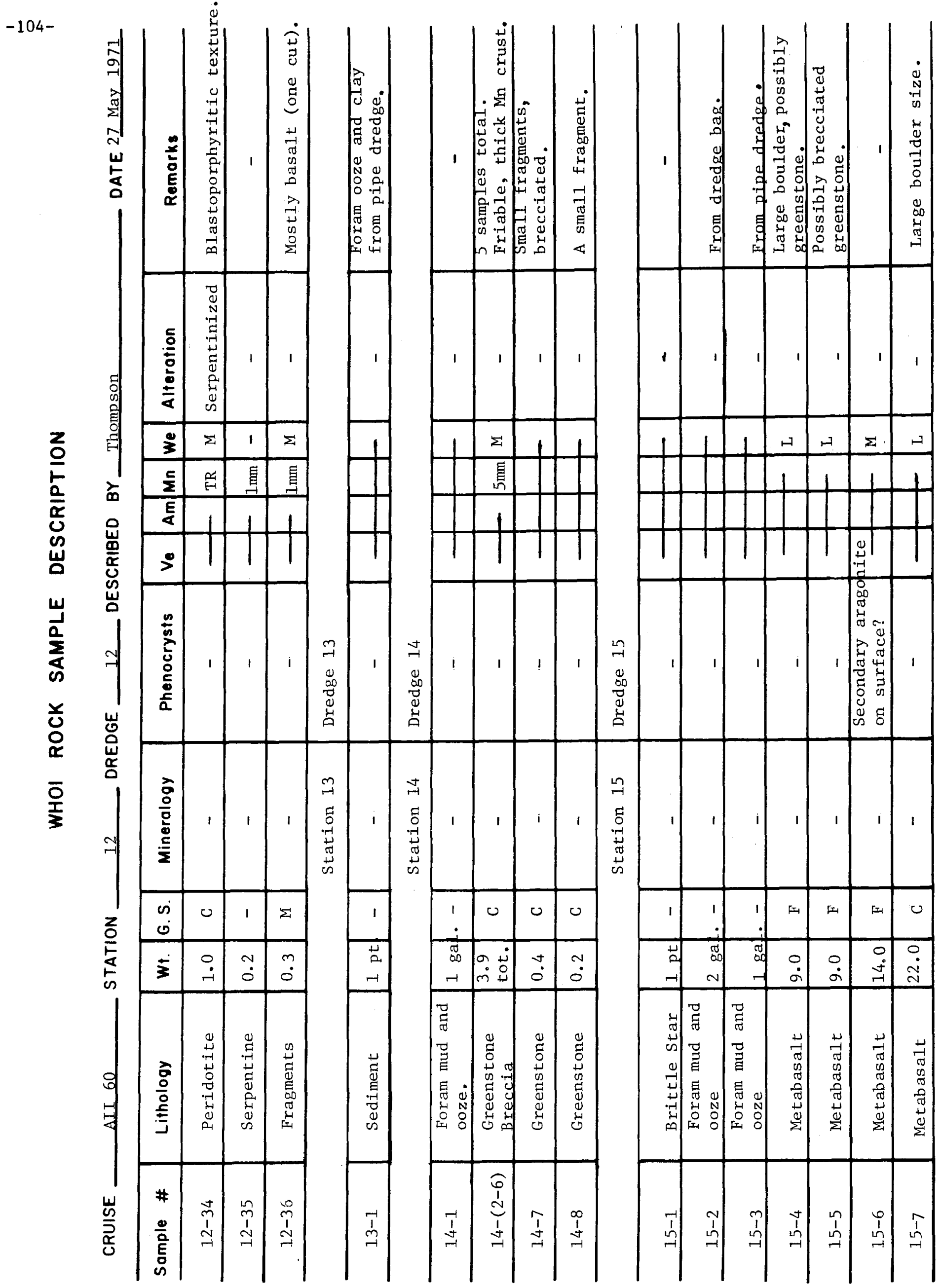




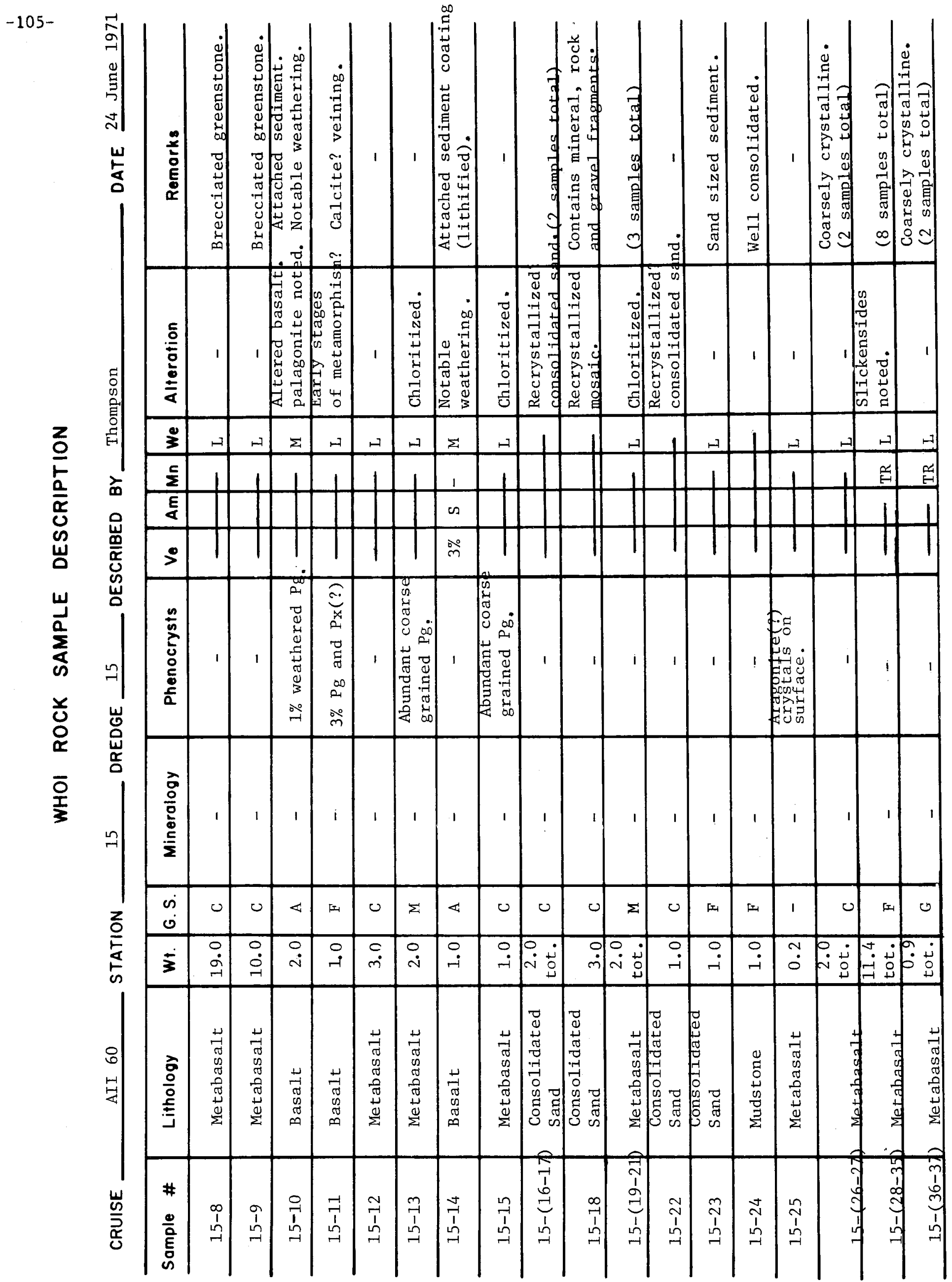




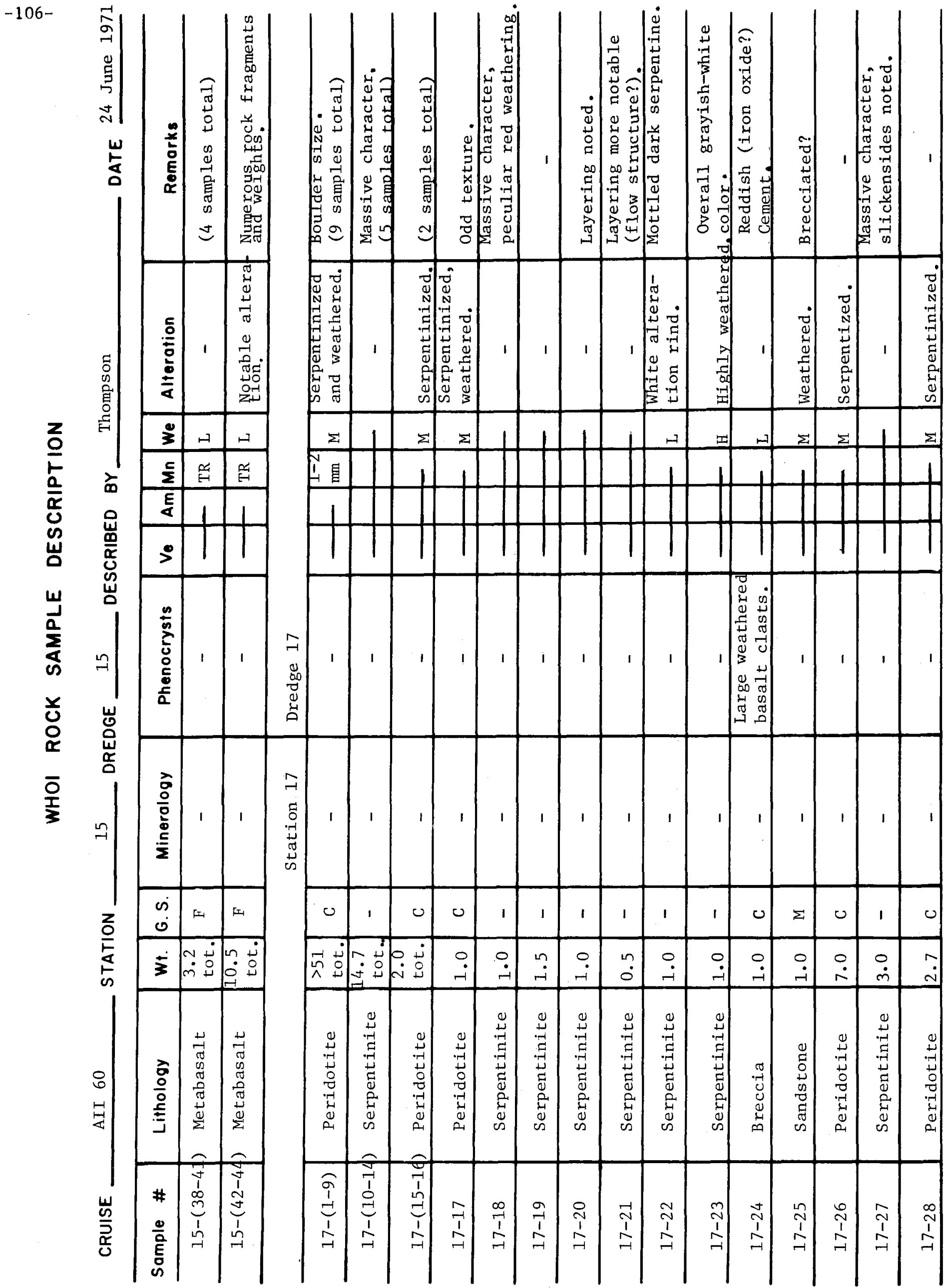




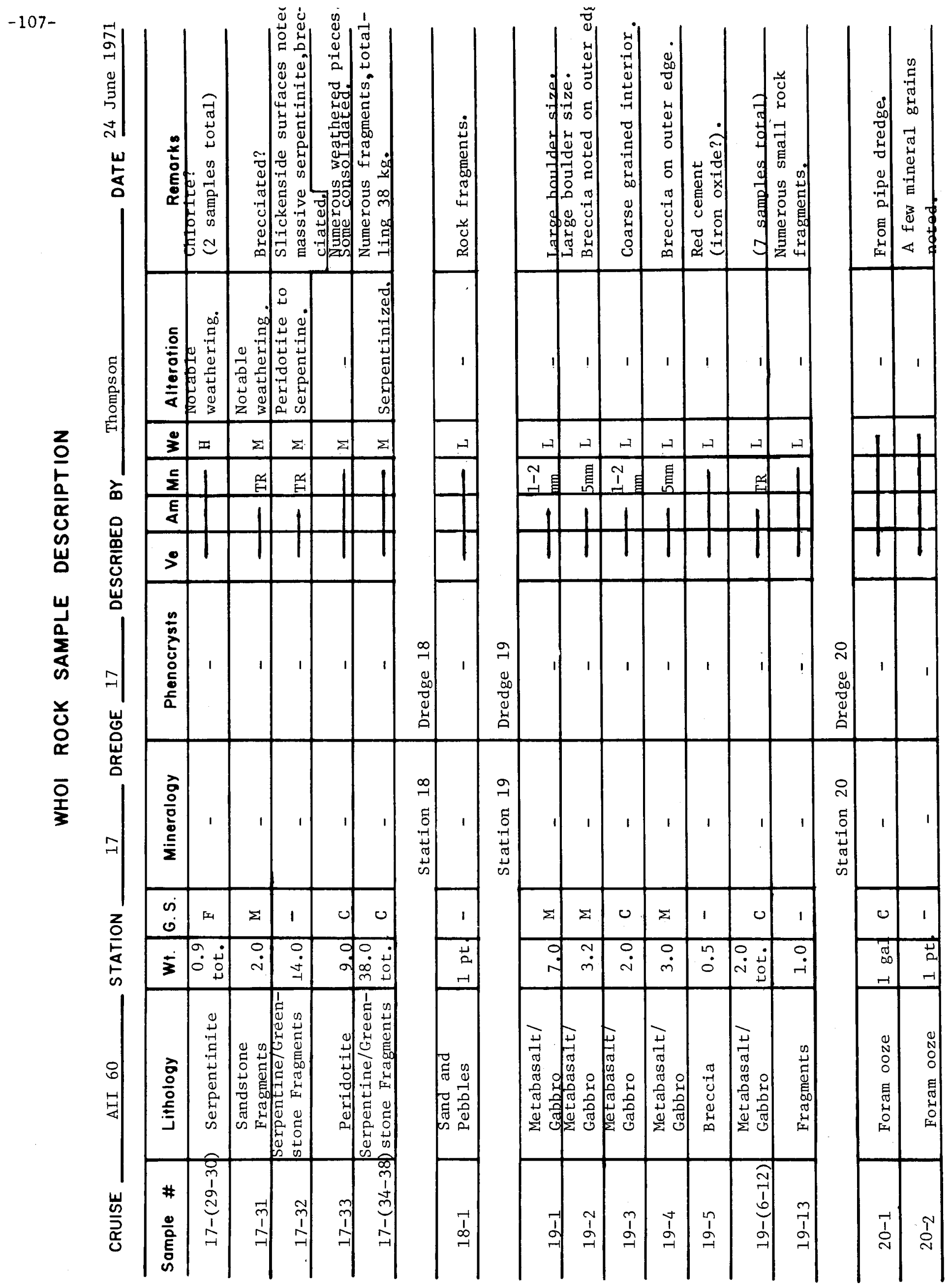




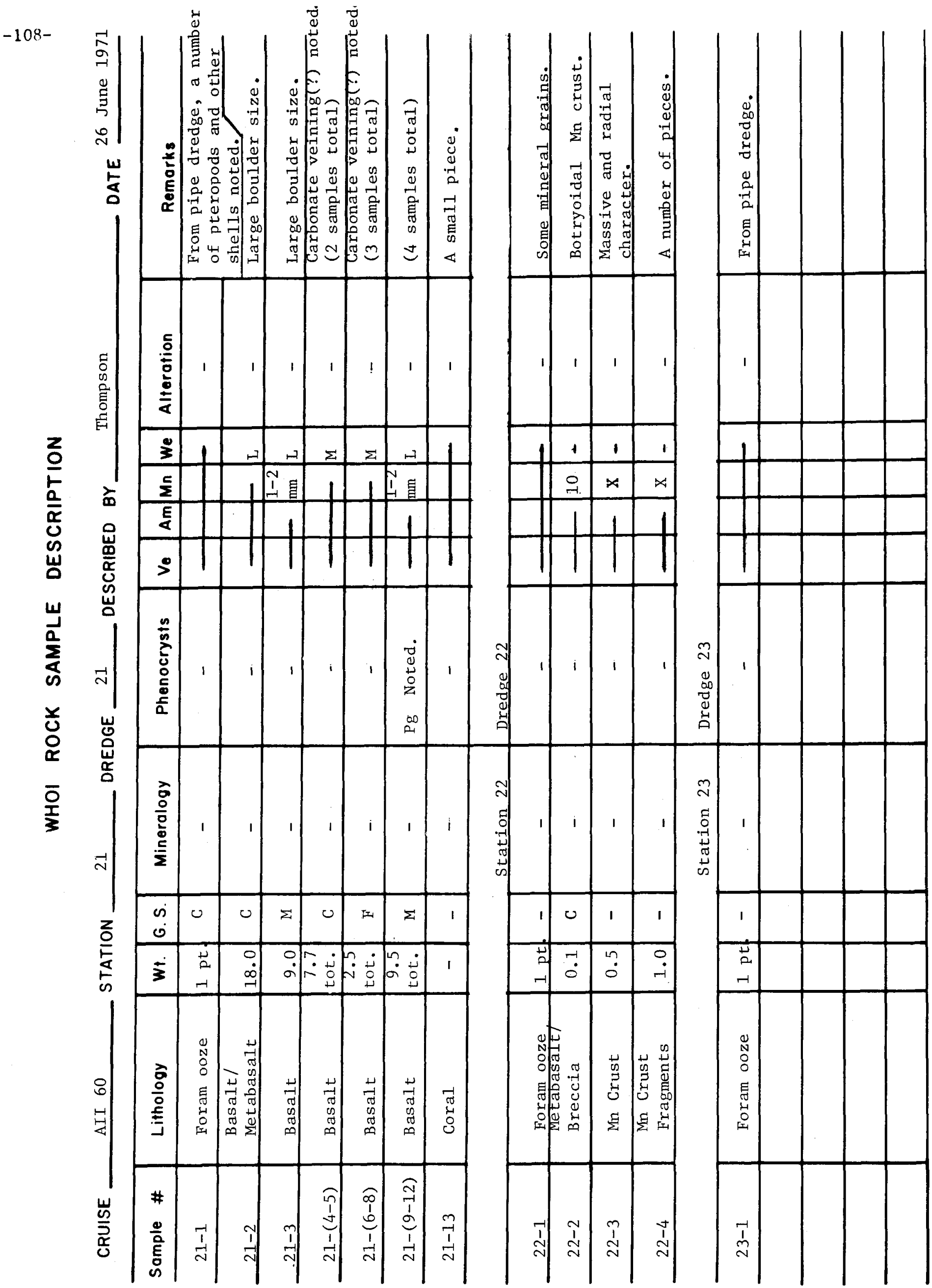




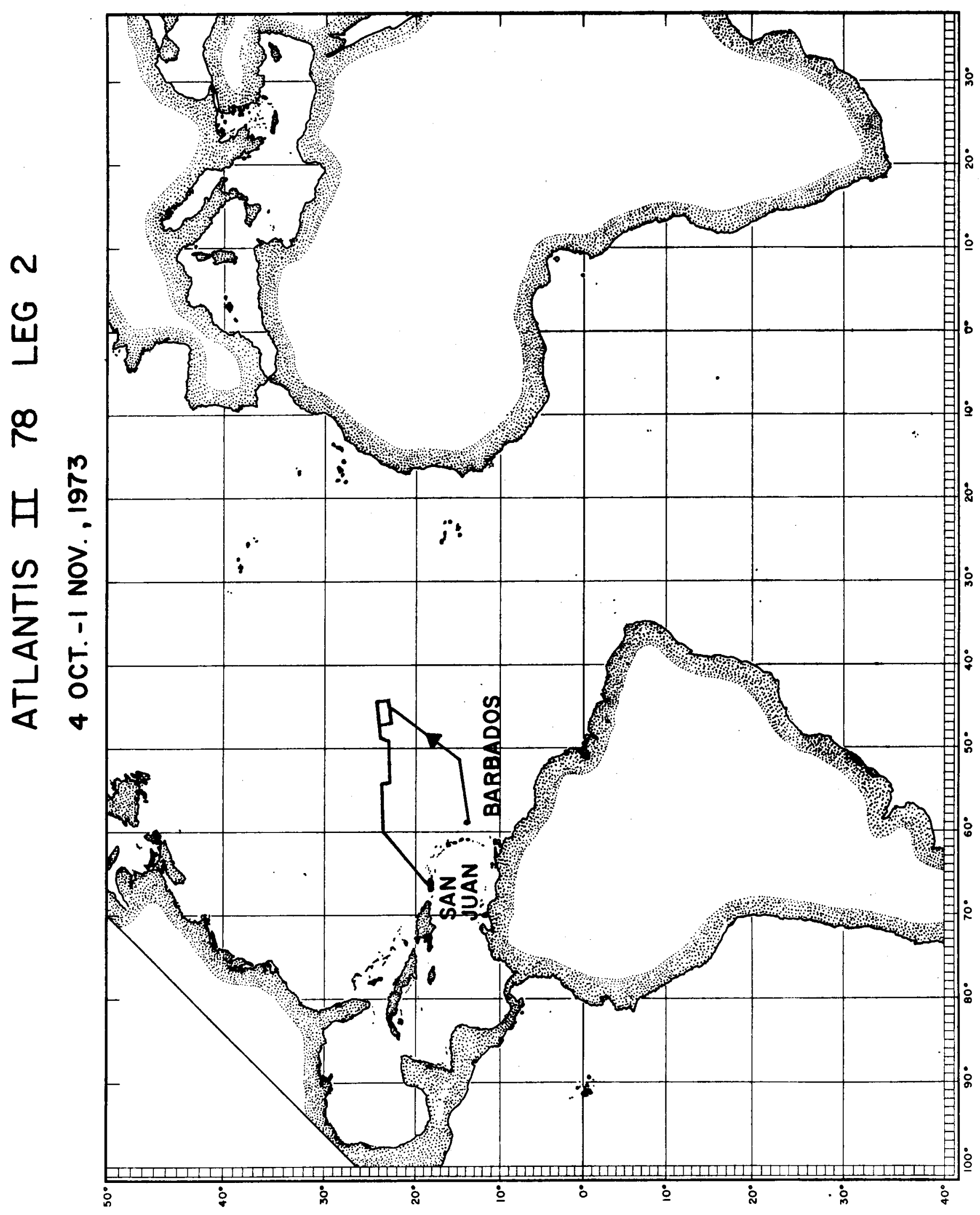




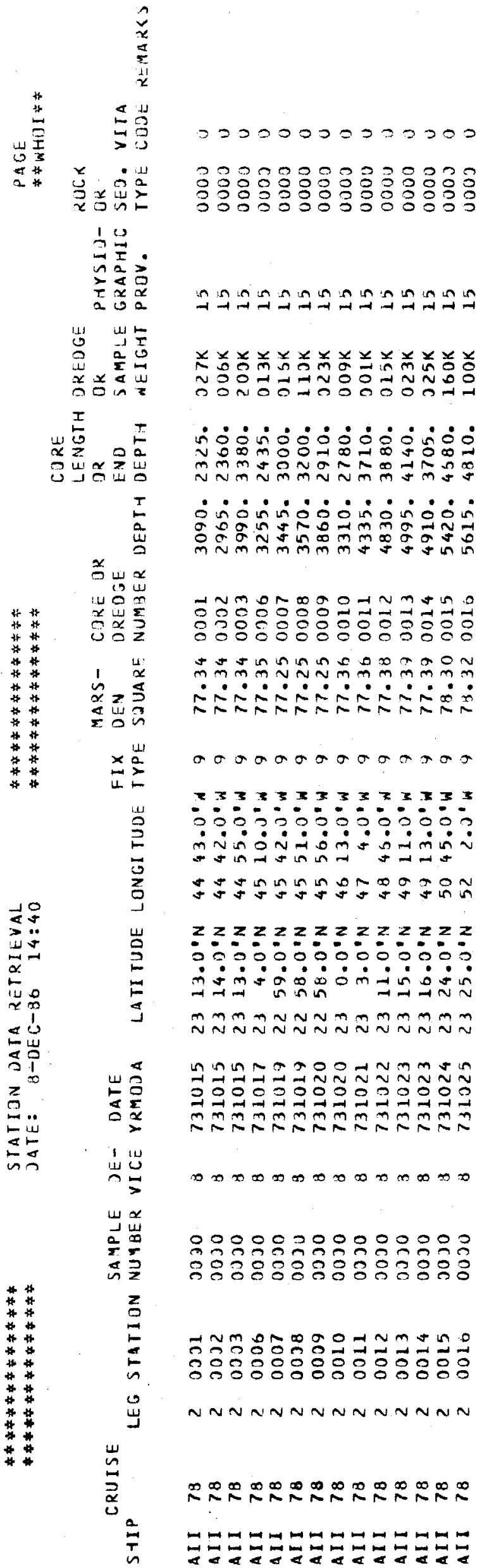




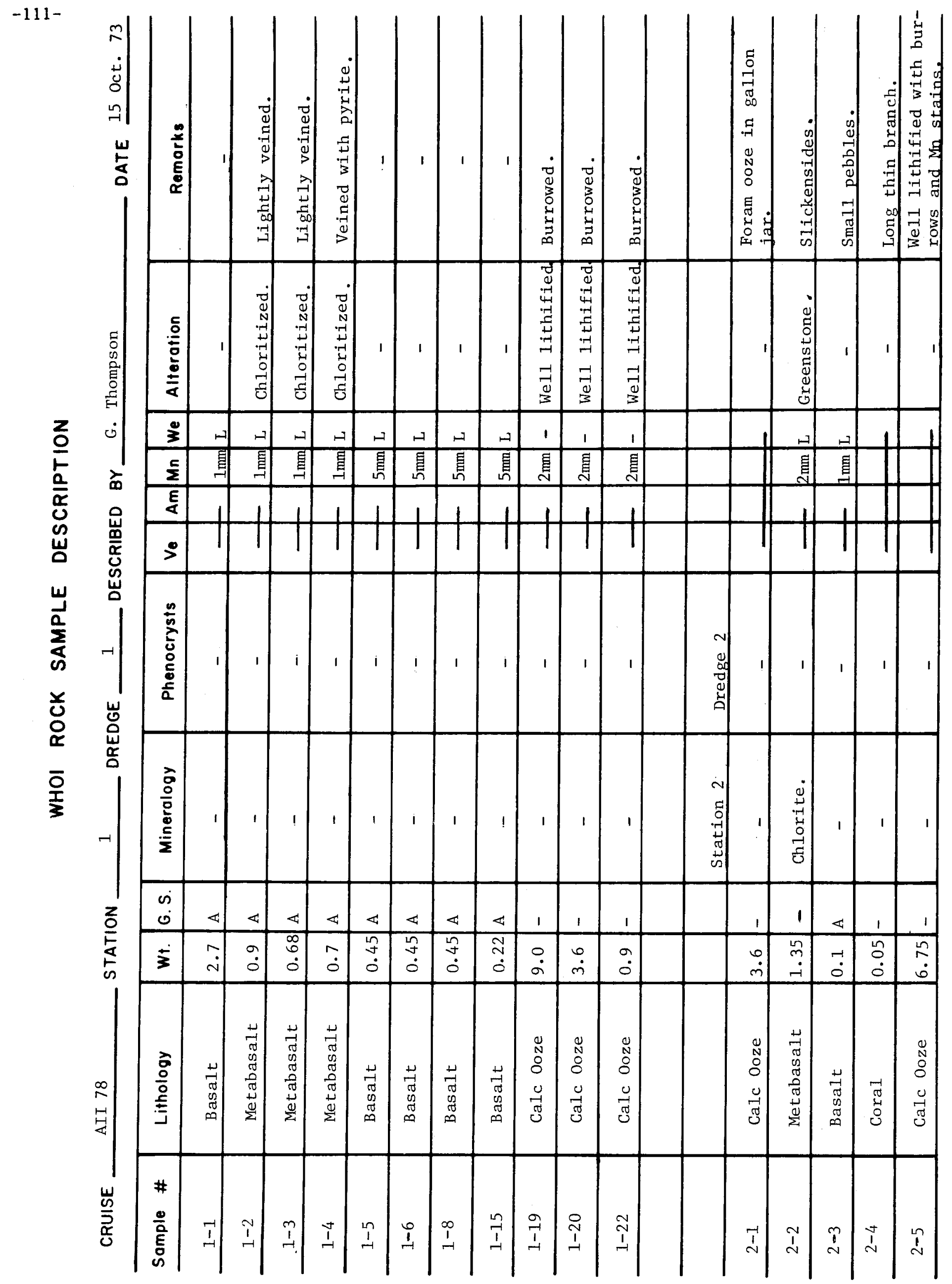




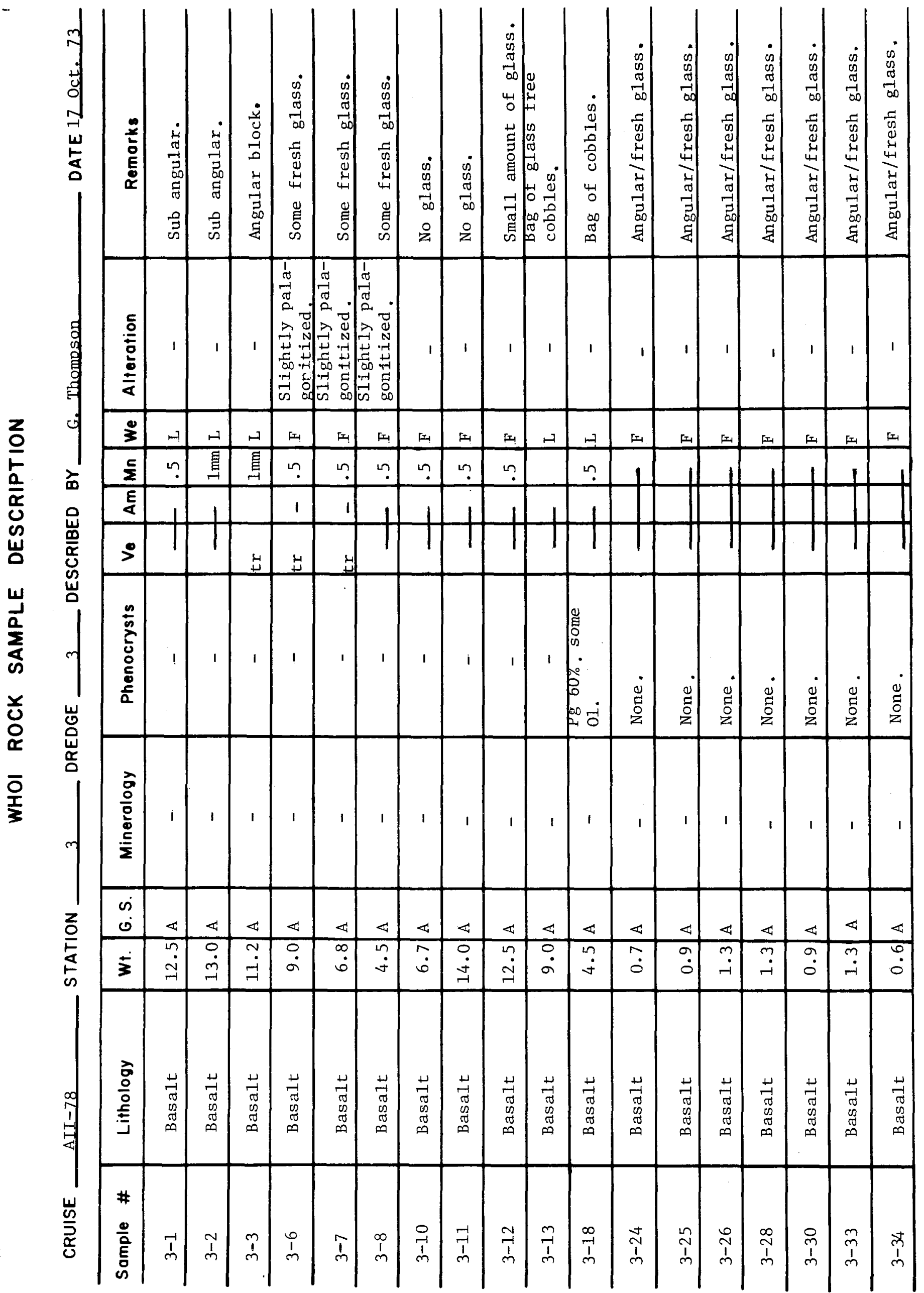




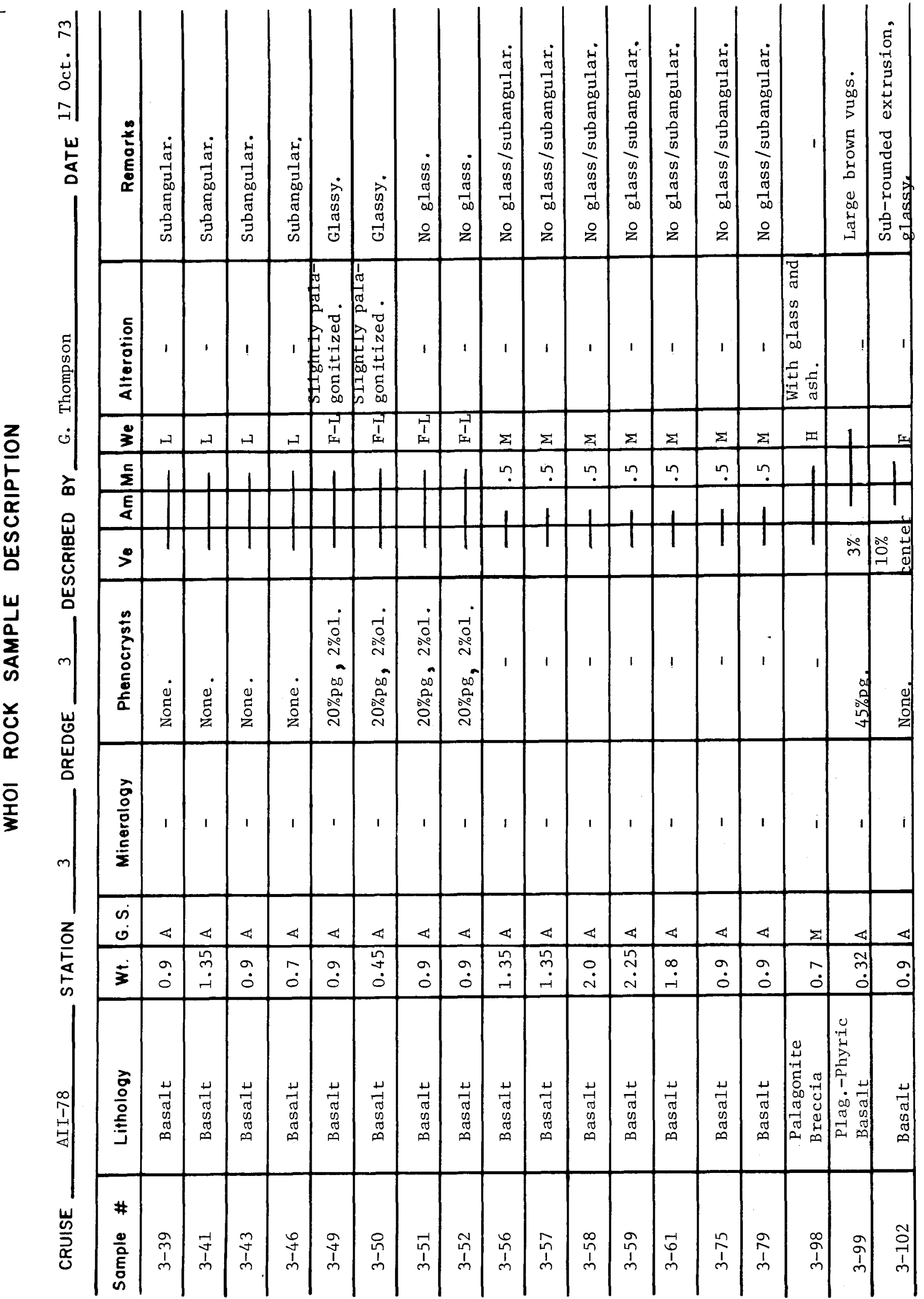




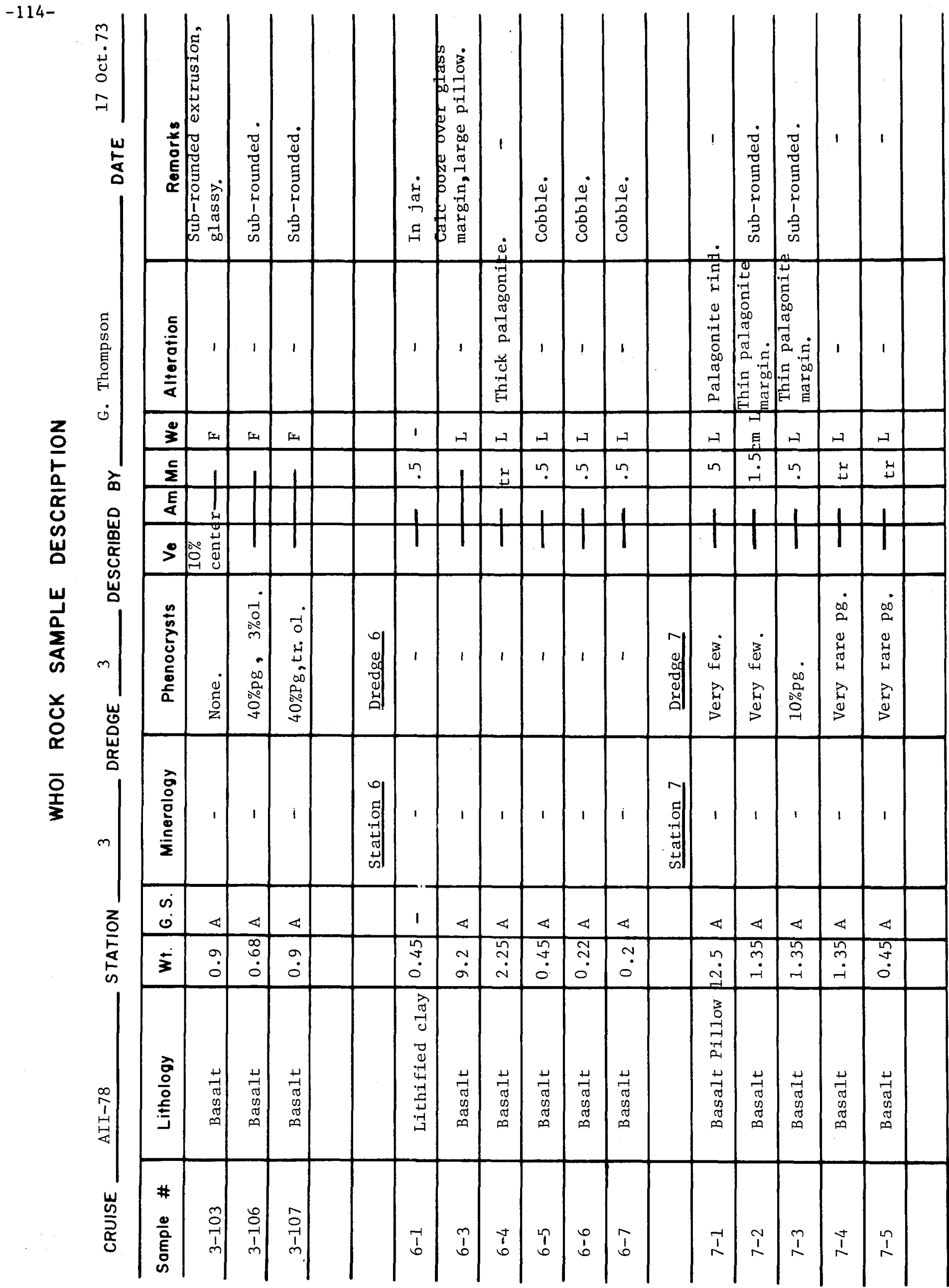




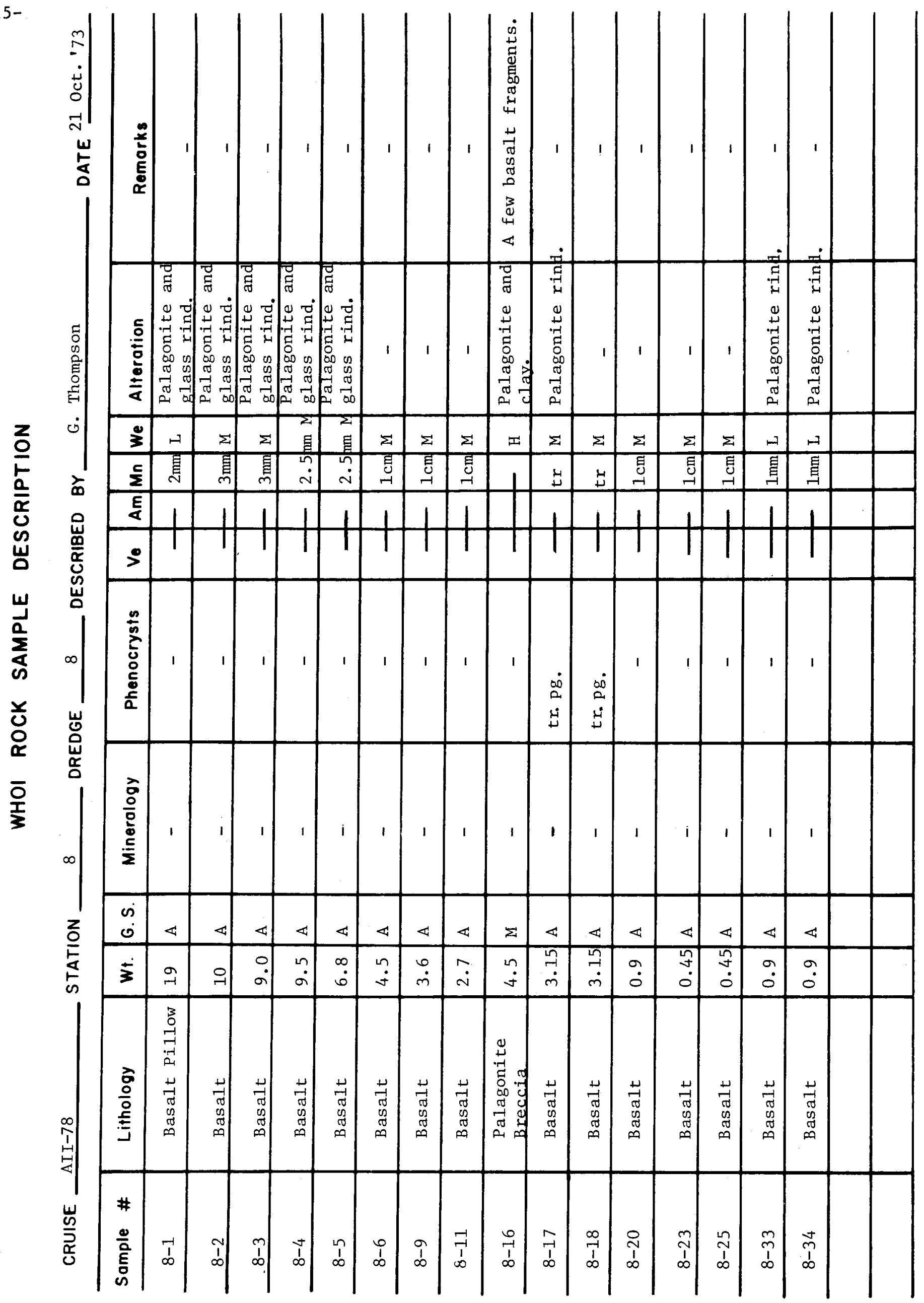




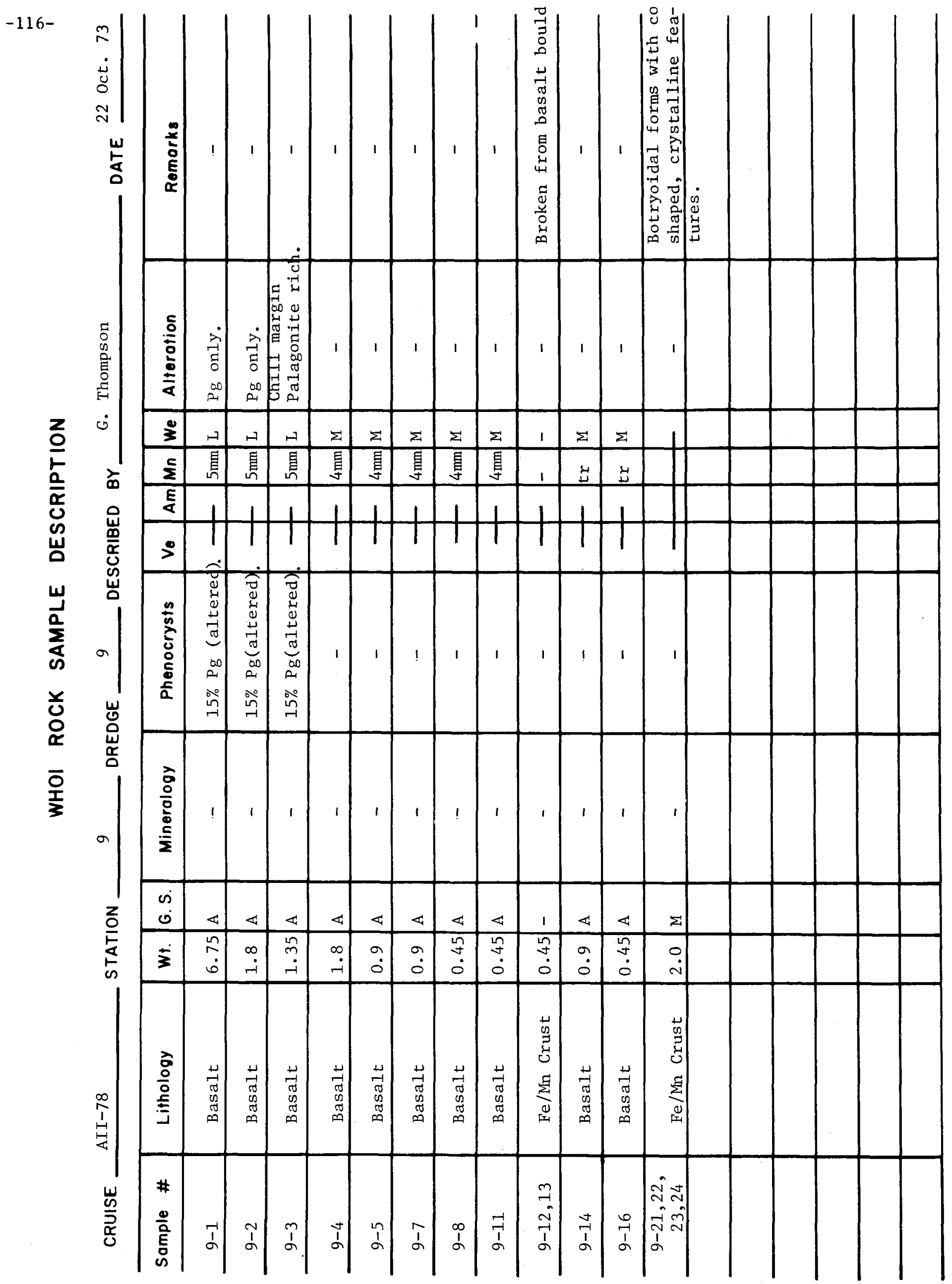




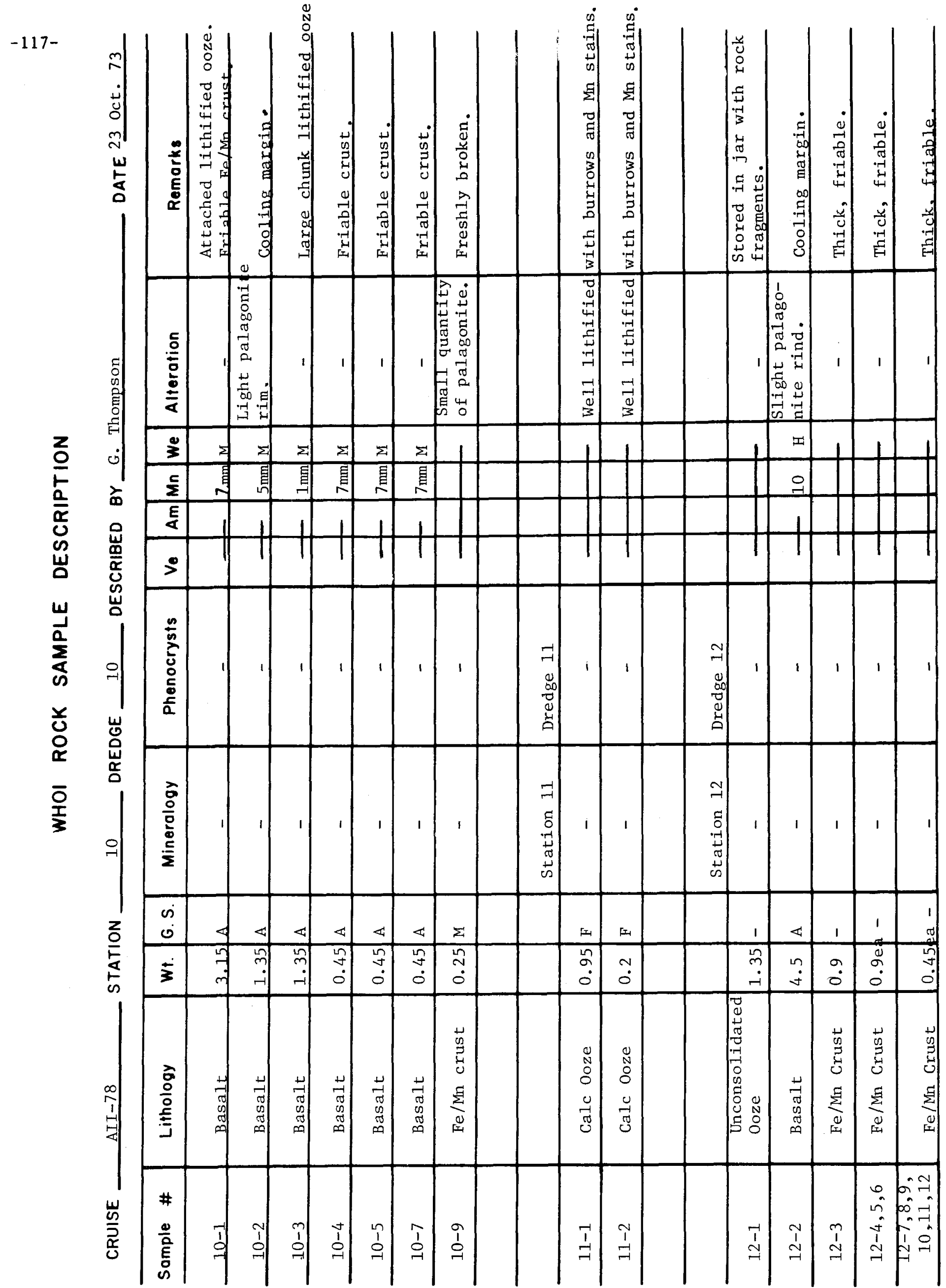




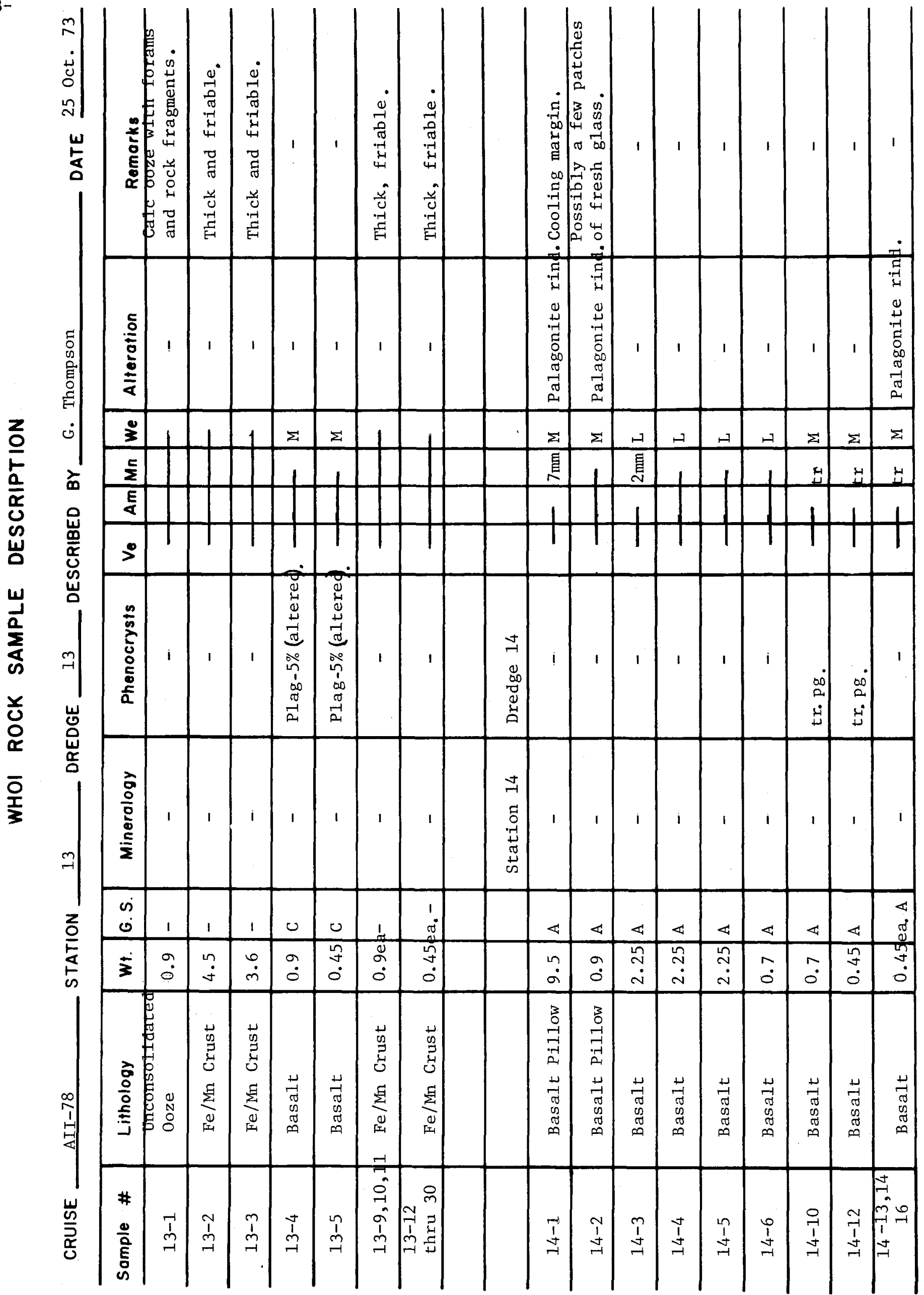




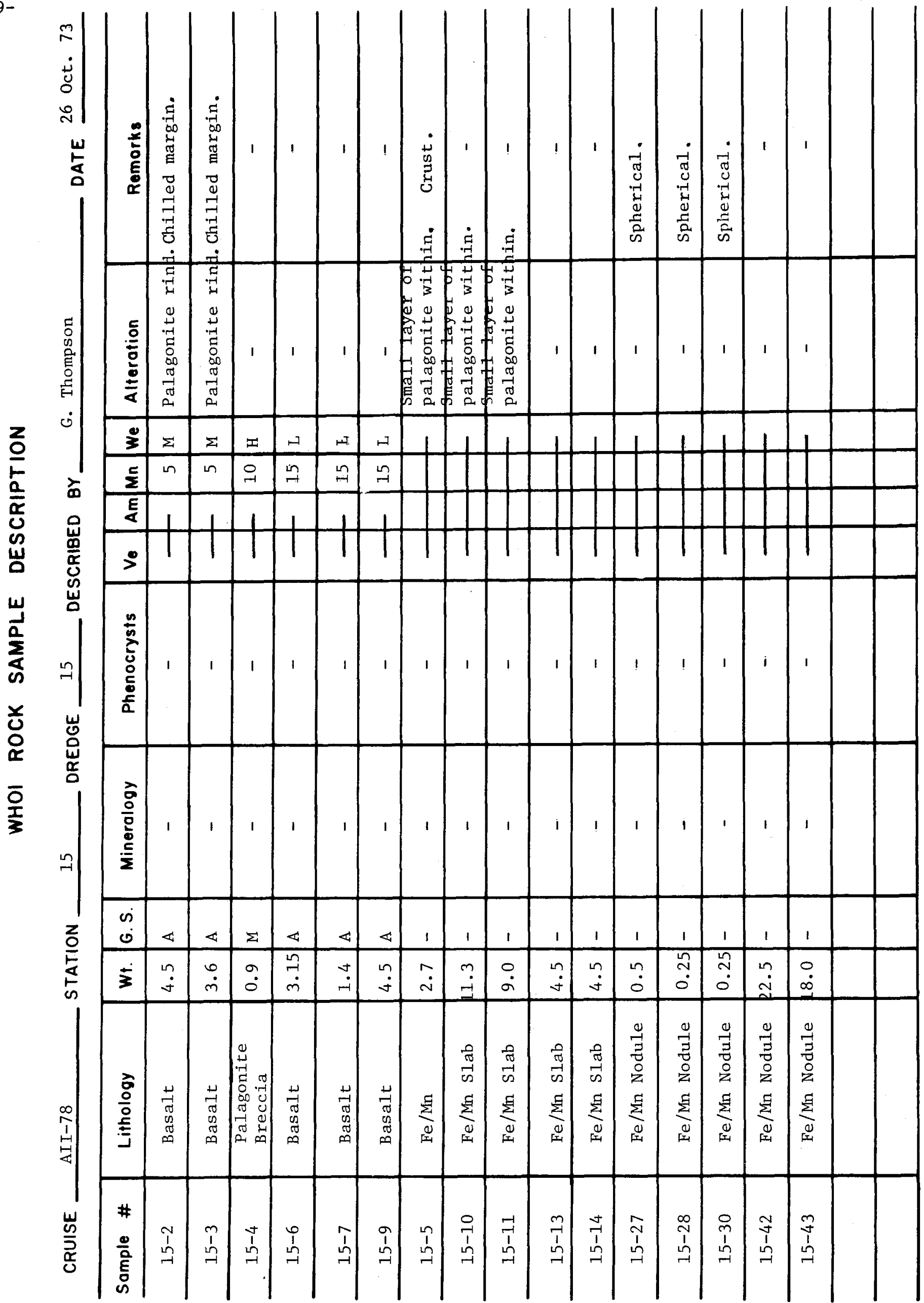




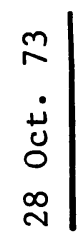

$\mid$

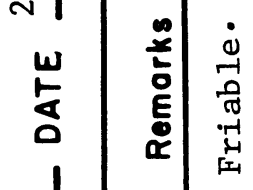

.

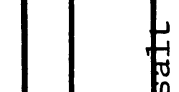

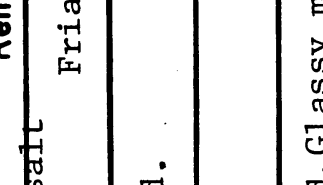

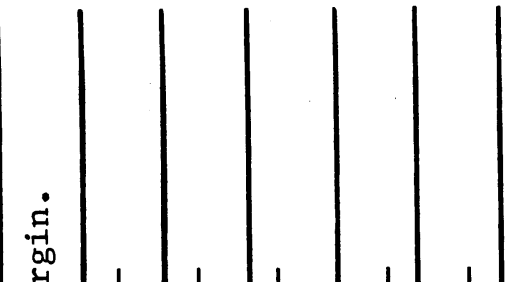

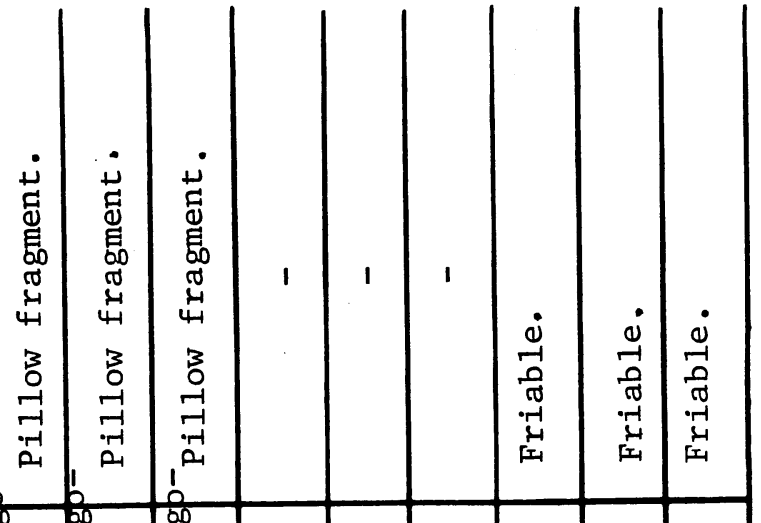

으

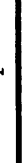

एँ

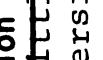

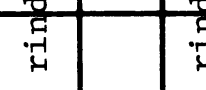

की

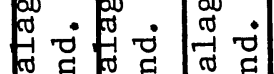

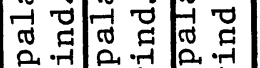

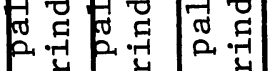

$\stackrel{0}{\sim}$

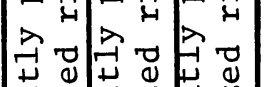

过娄

E

4

每

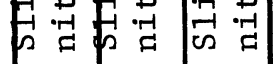

竞

w

$\sum_{\infty}^{\frac{1}{a}}$

일

亩

1

ర Uू

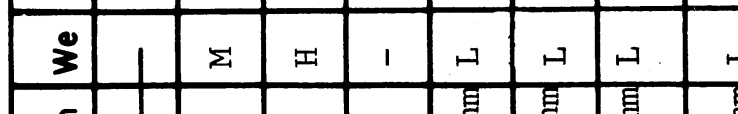

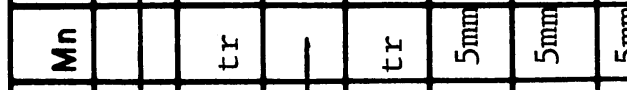

\begin{tabular}{l|l|l}
\hline$\varepsilon$ & & \\
\hline
\end{tabular}

$>$

\begin{tabular}{l|l|l|l|}
\hline & \\
\hline & &
\end{tabular}

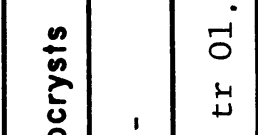

응

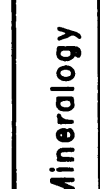

$\rightarrow$

is

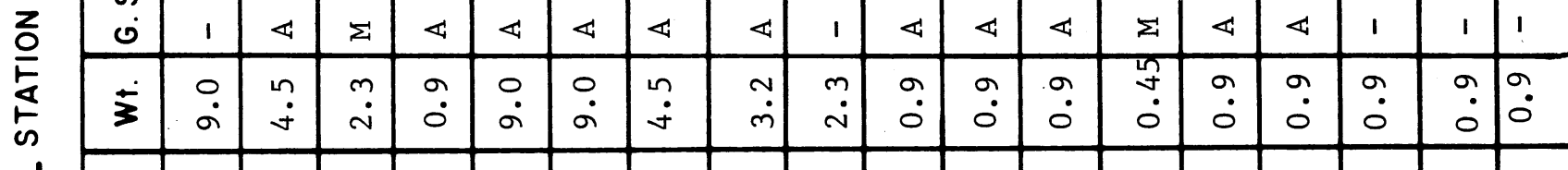

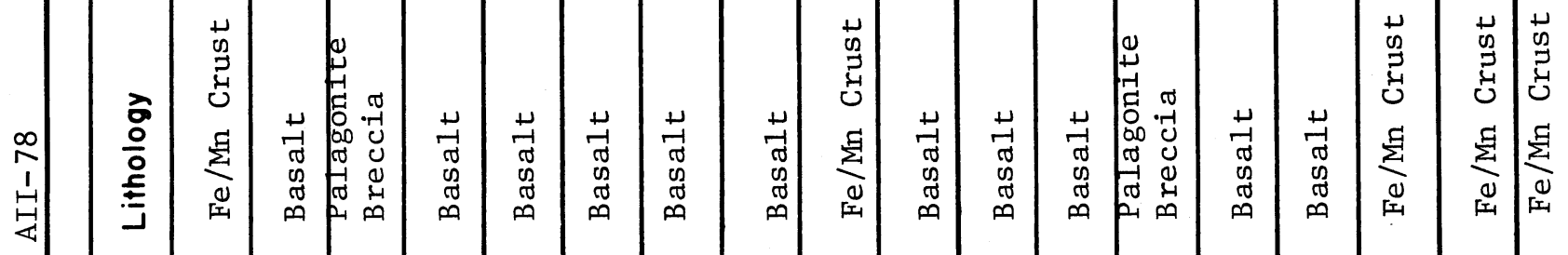

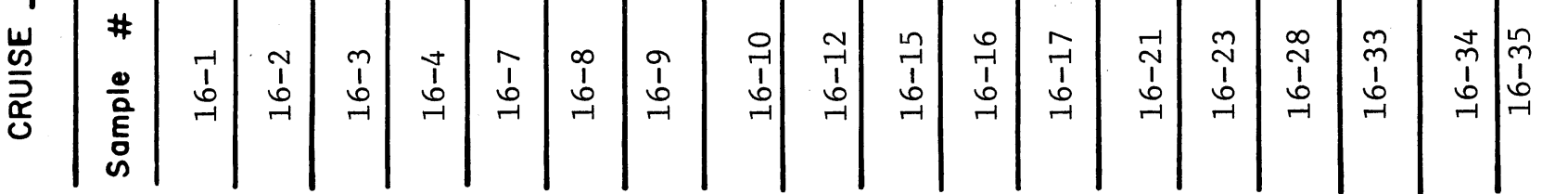




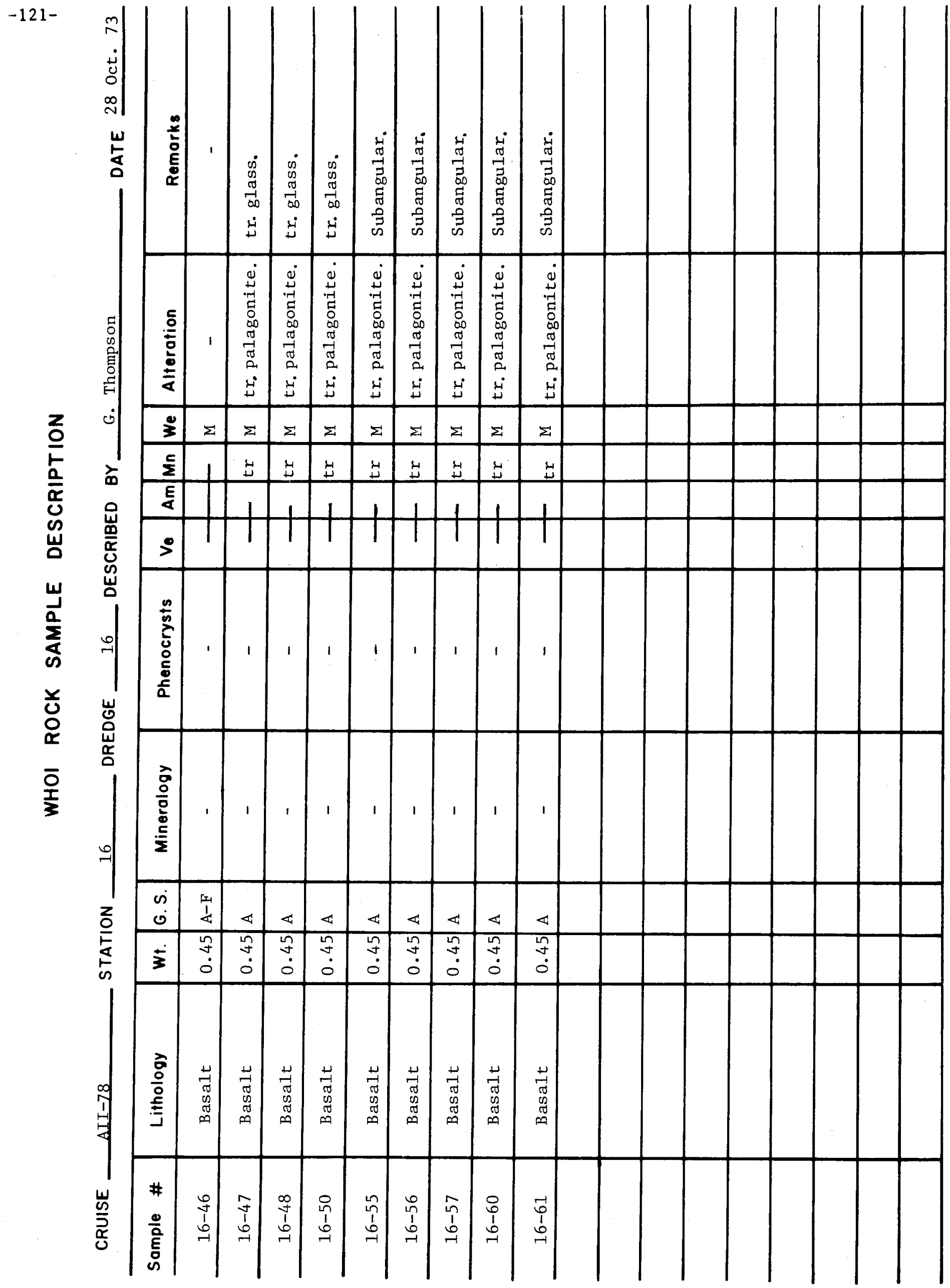




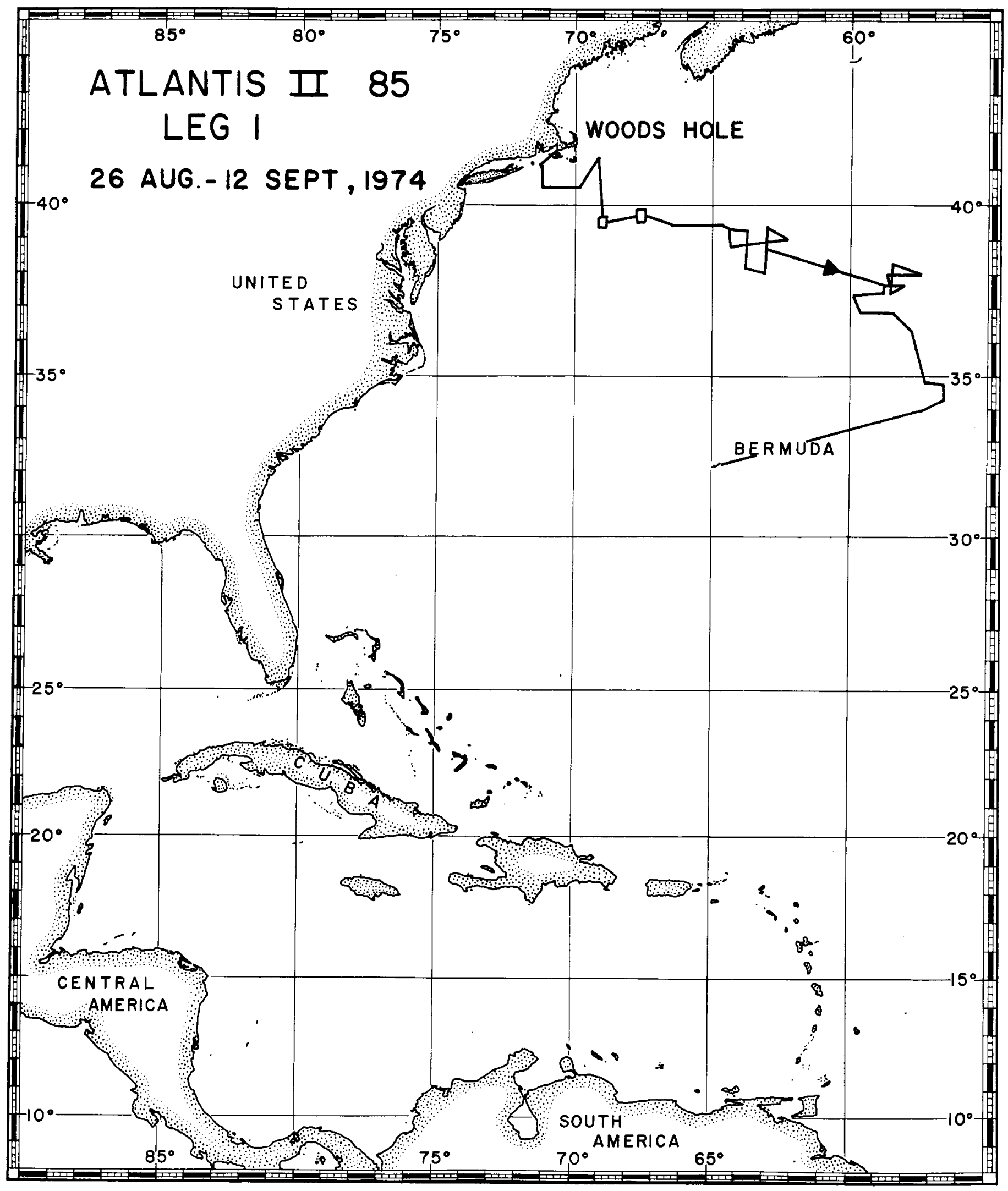


00000000000000000000

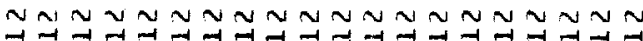

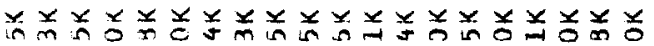
की

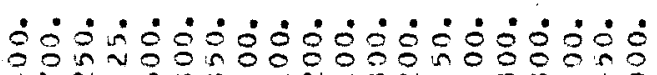

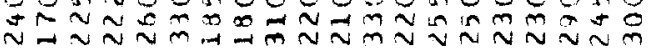
$\dot{0} \dot{0} \dot{0} \dot{0} \dot{0} \dot{0} \dot{0} \dot{0} \dot{0} \dot{0} \dot{0} \dot{0} \dot{0} \dot{0}$

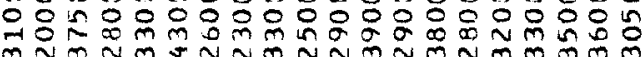
号山

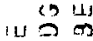

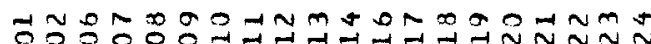

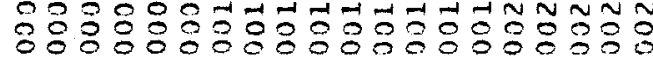

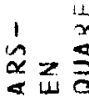

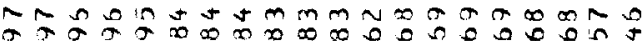

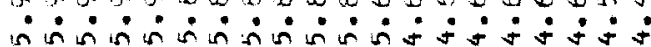

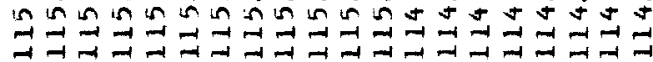

on

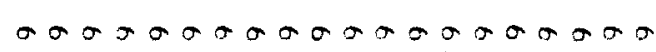

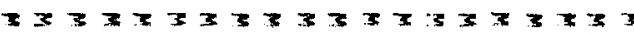

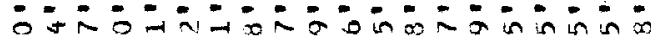

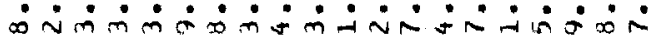
$\sim N$ in กิด

$z z z z z z z z z z z z z z z z z z z z$

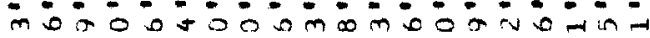

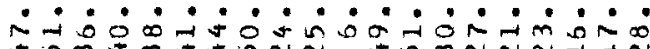
$\operatorname{tin} m \sim N N-1+\infty$

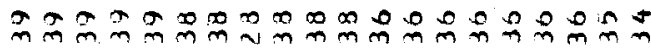

48

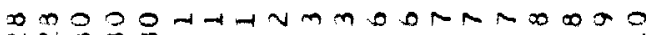

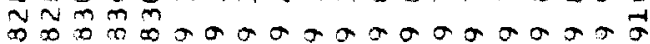

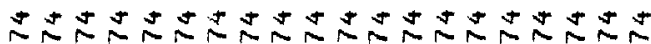

岁岕

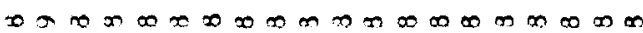

$11 \alpha$

虫

00000000000000000000

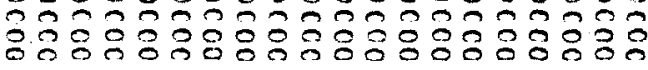

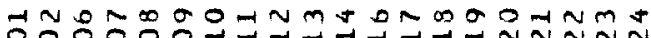

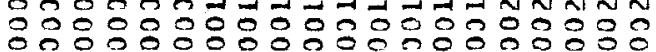

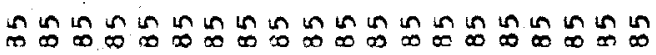
章

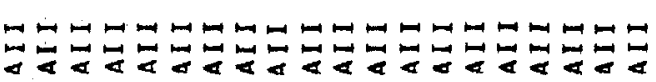


$-124-$

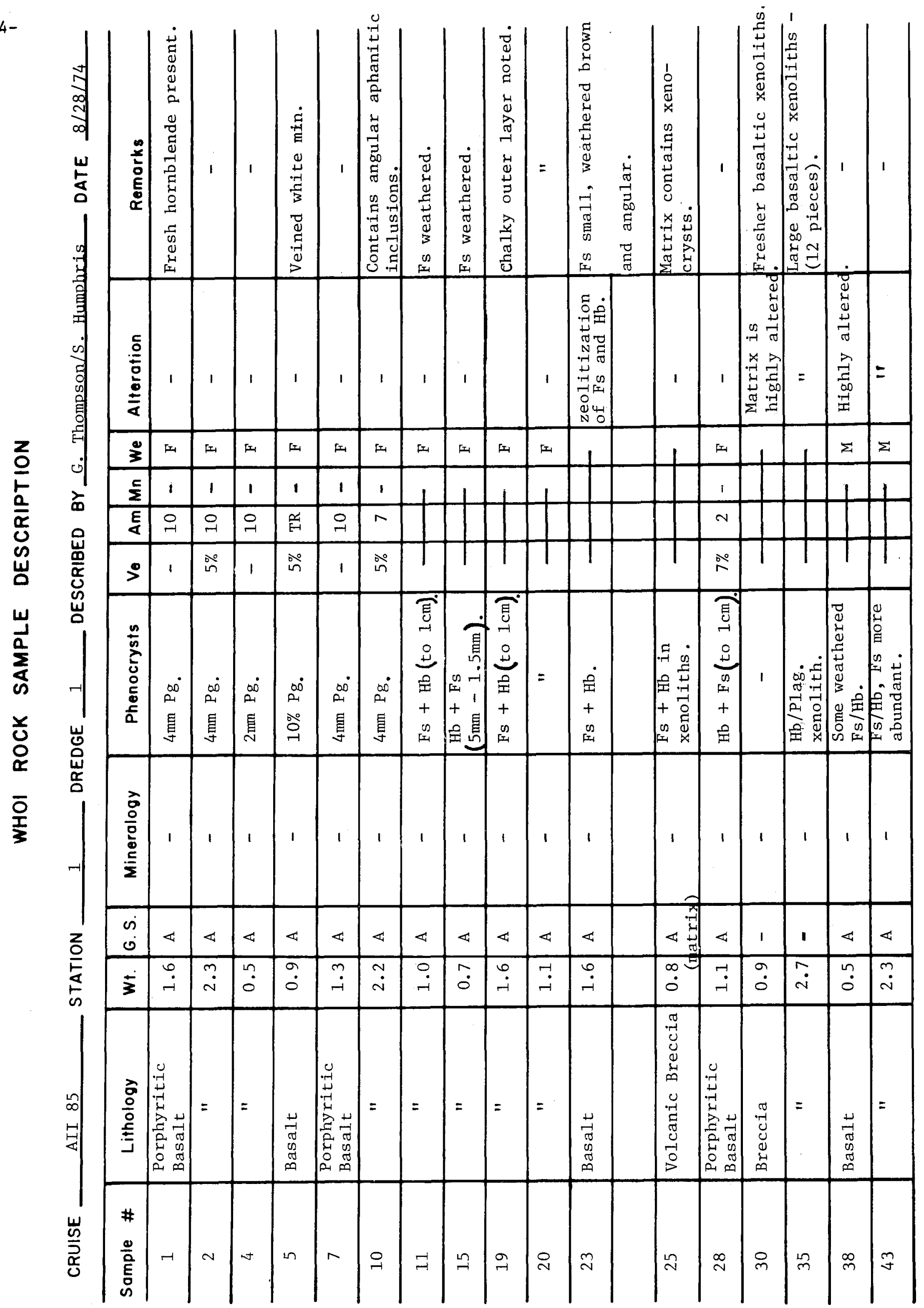




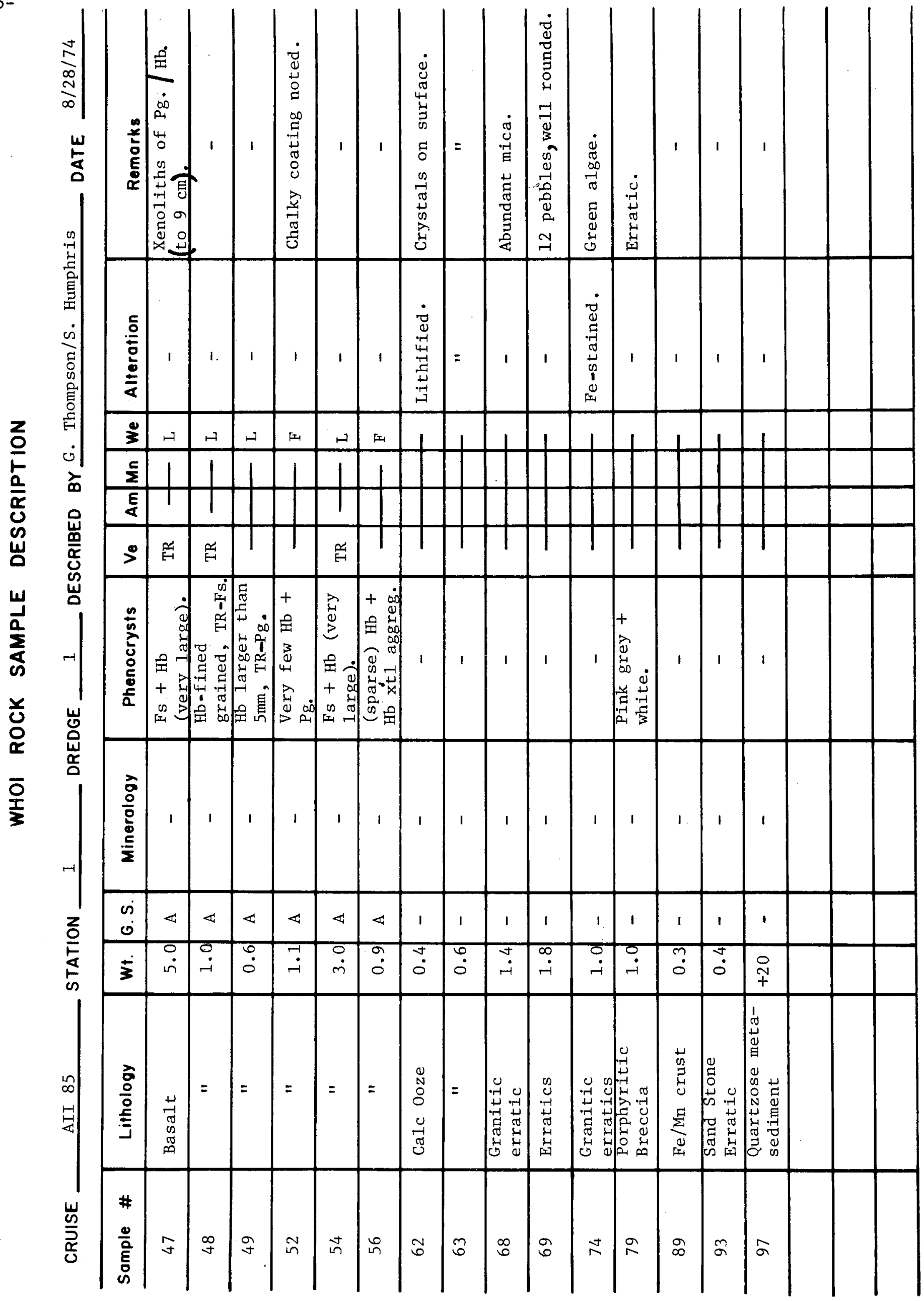




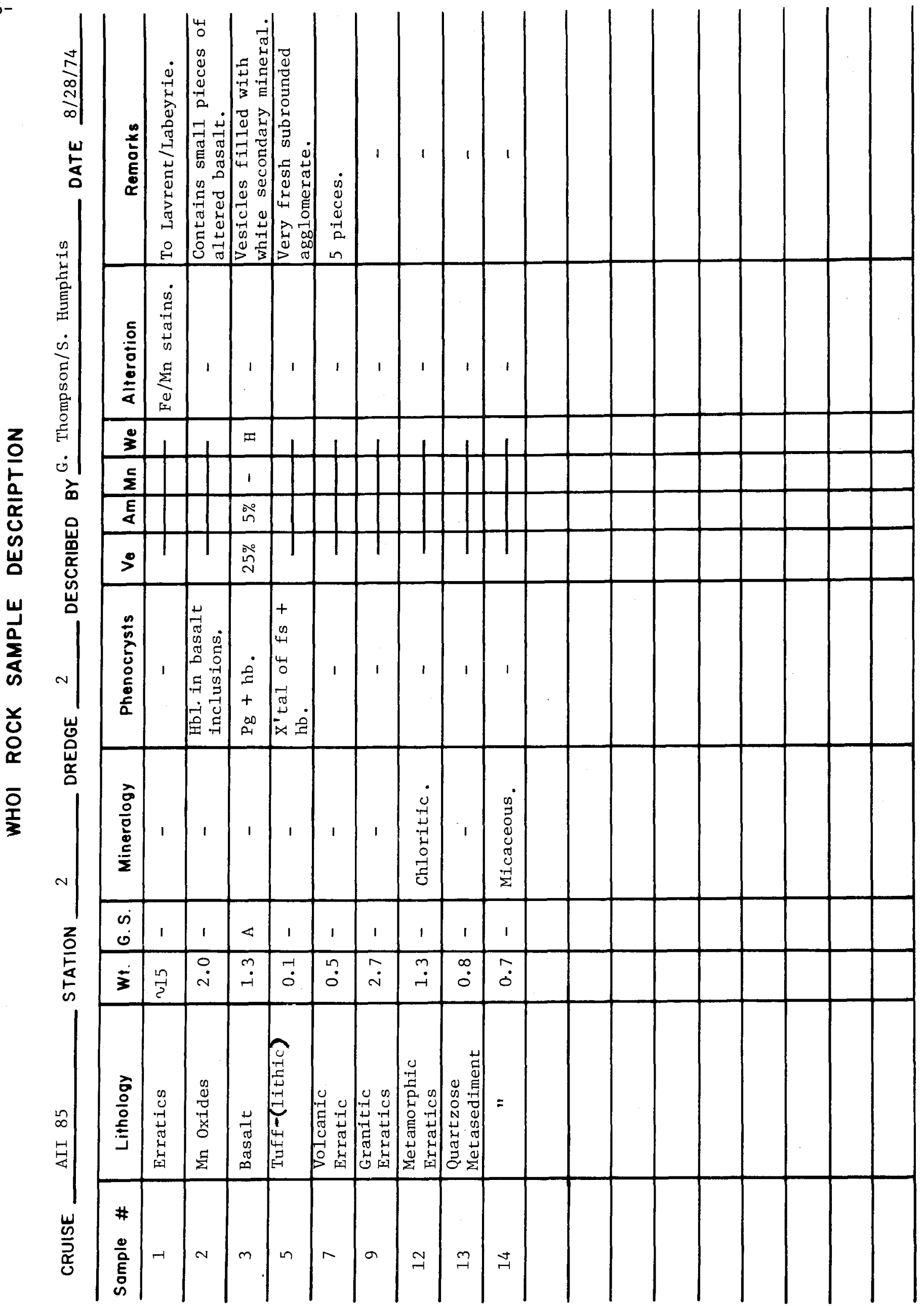




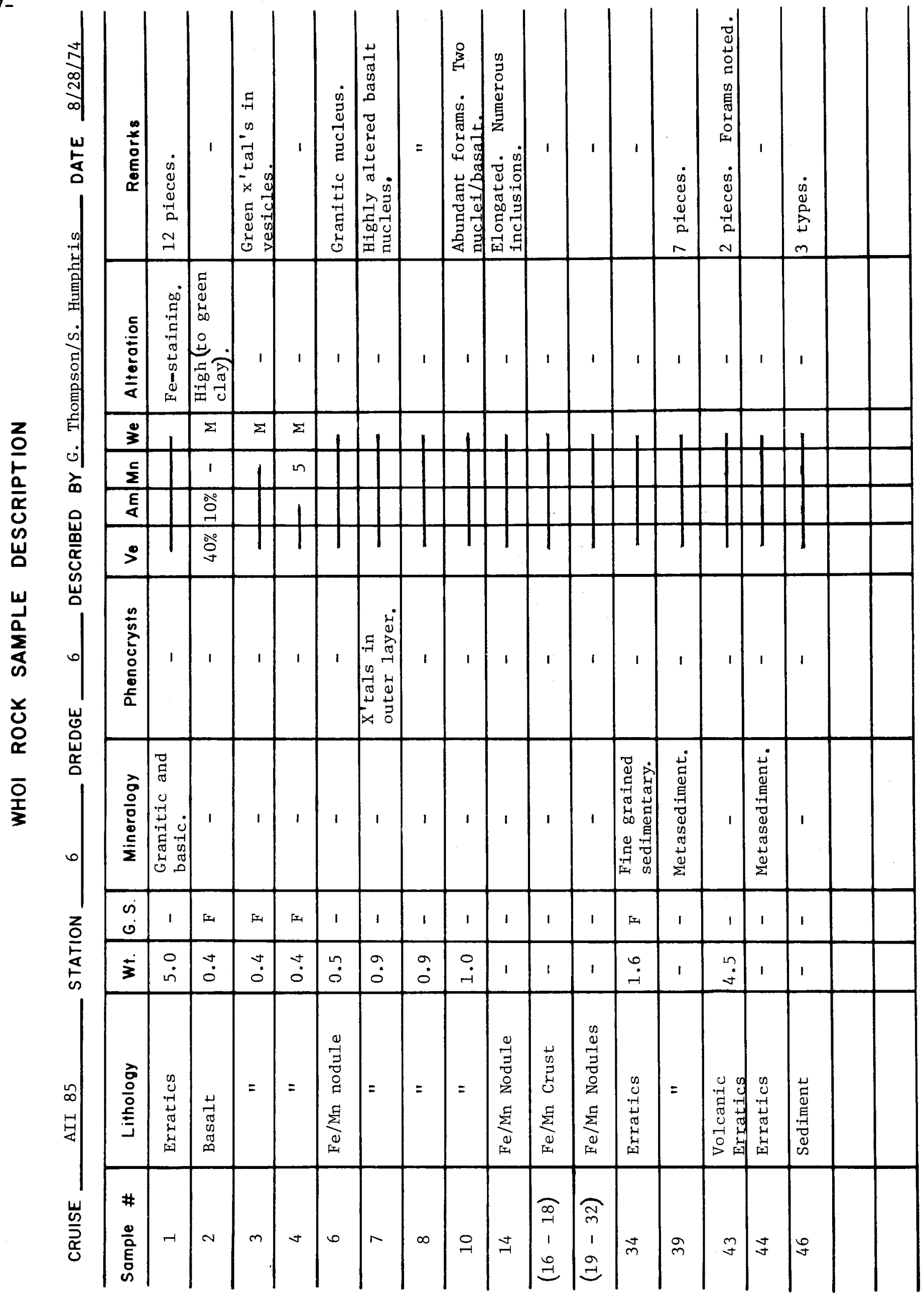




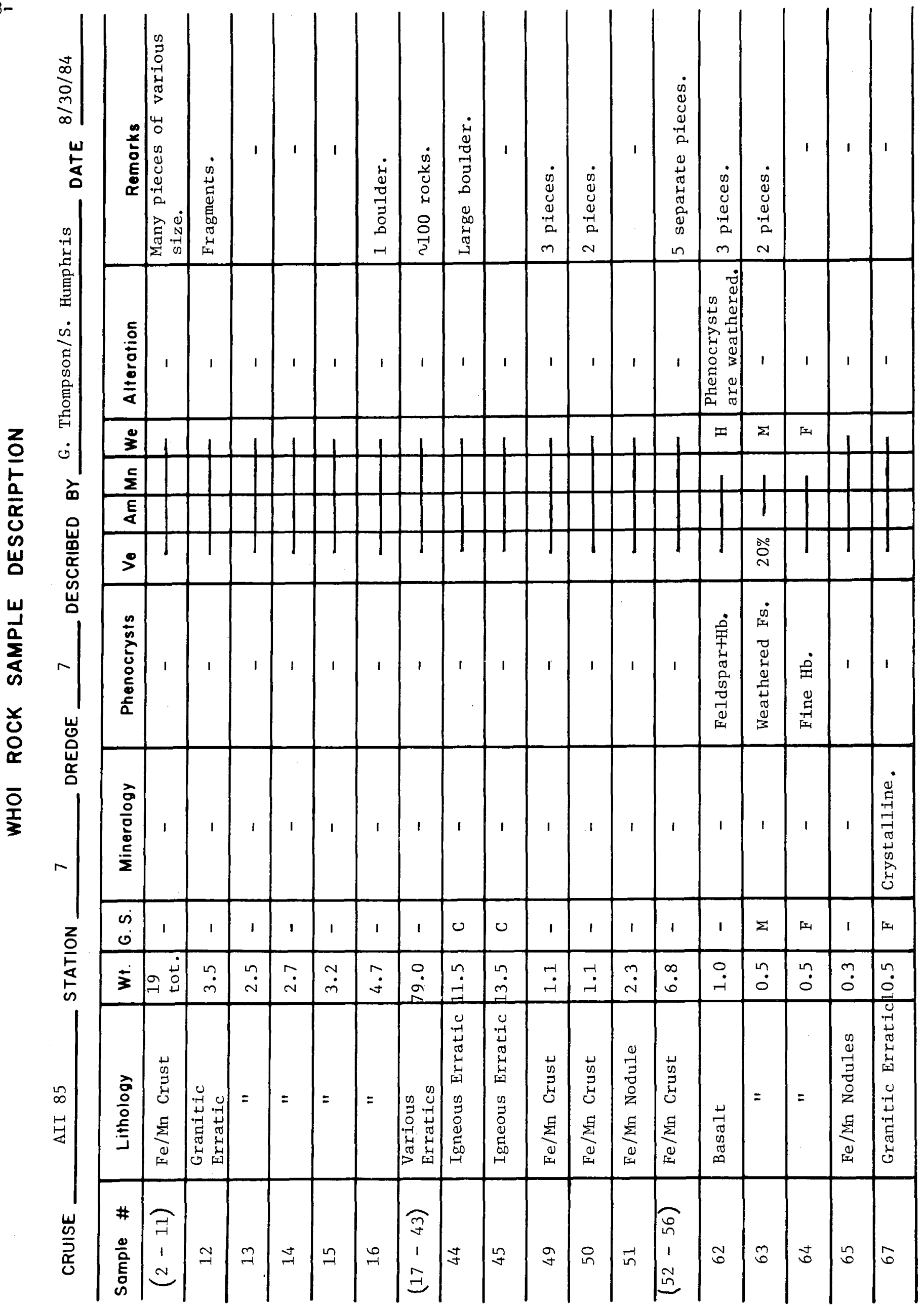




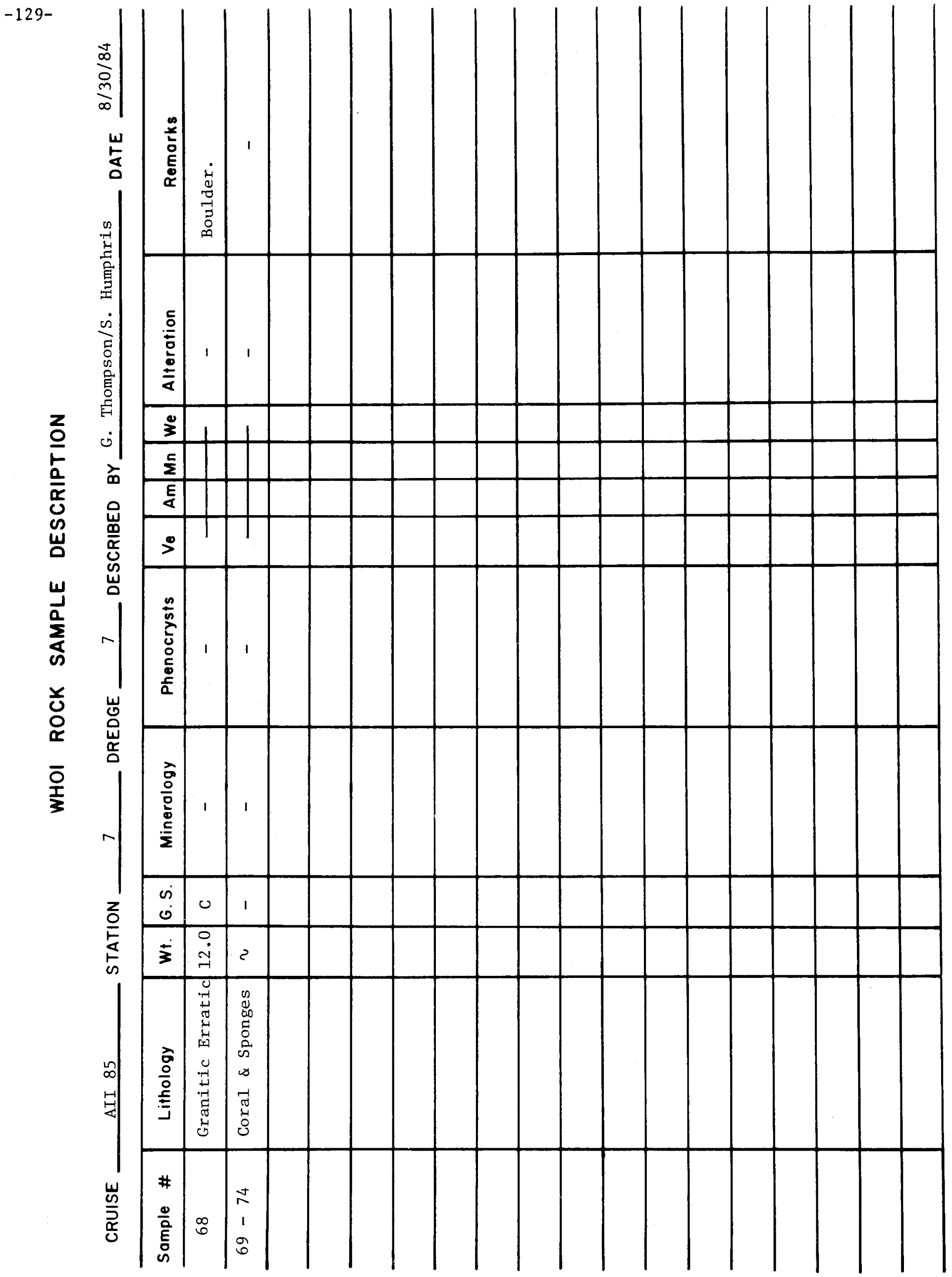




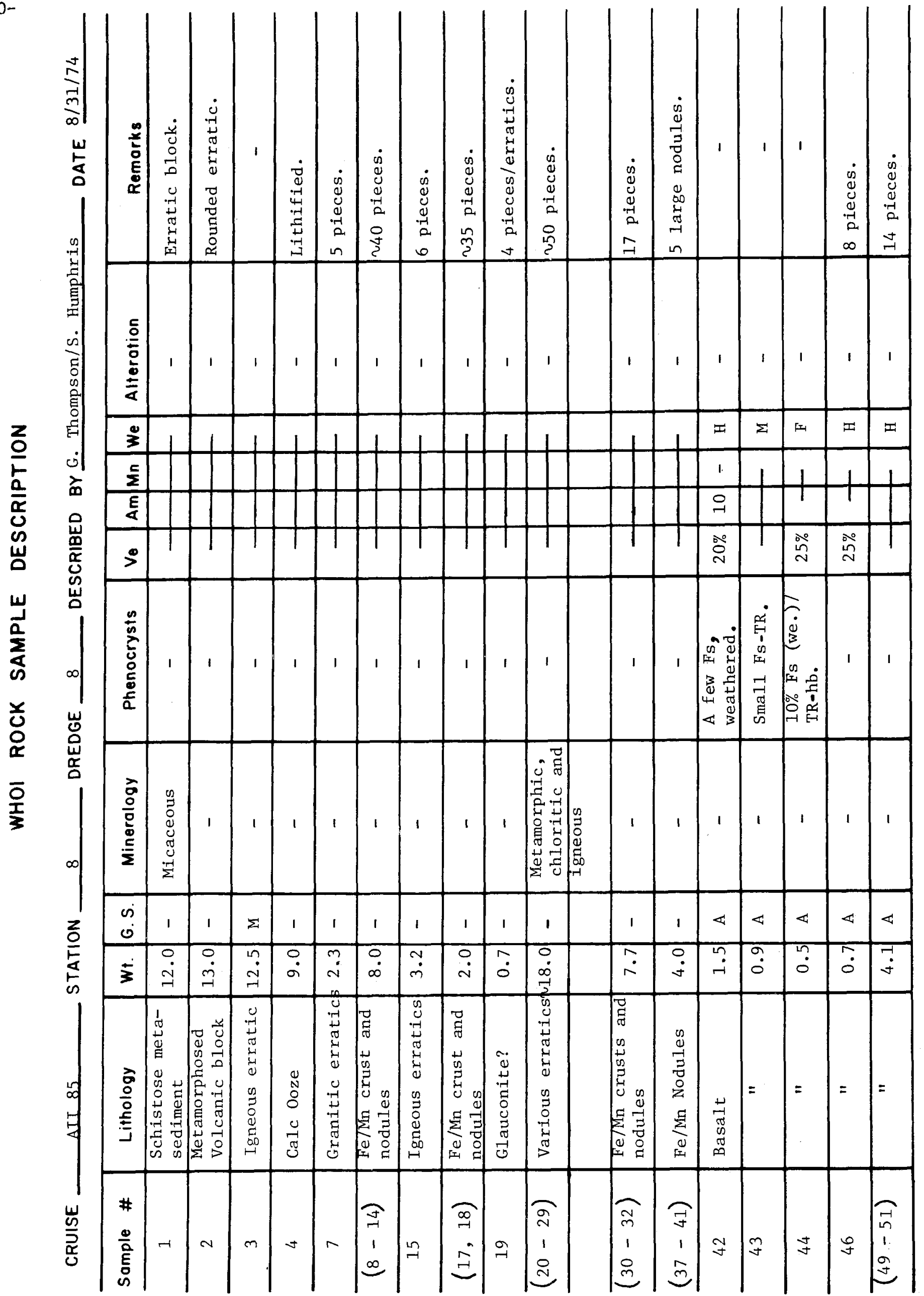




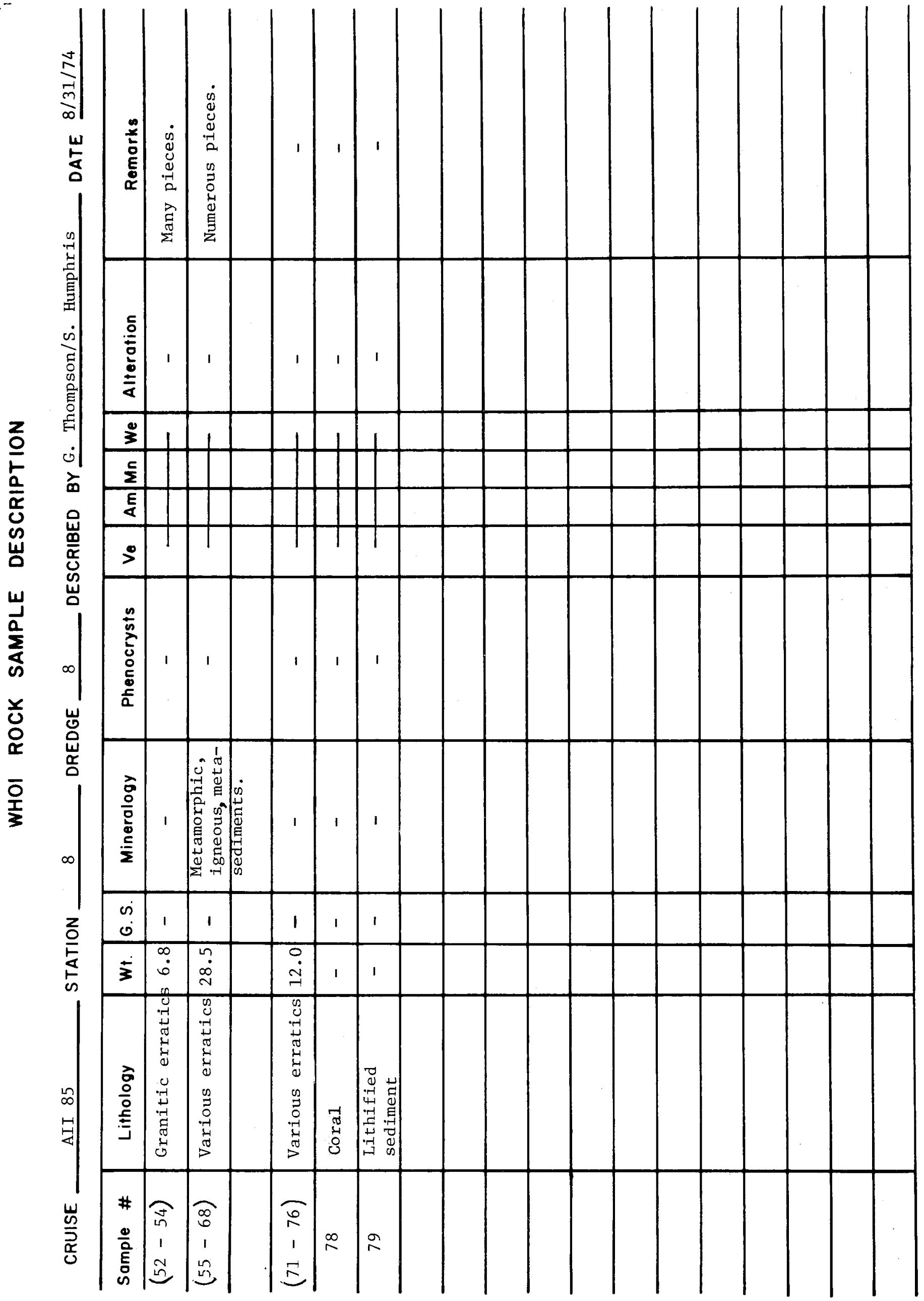




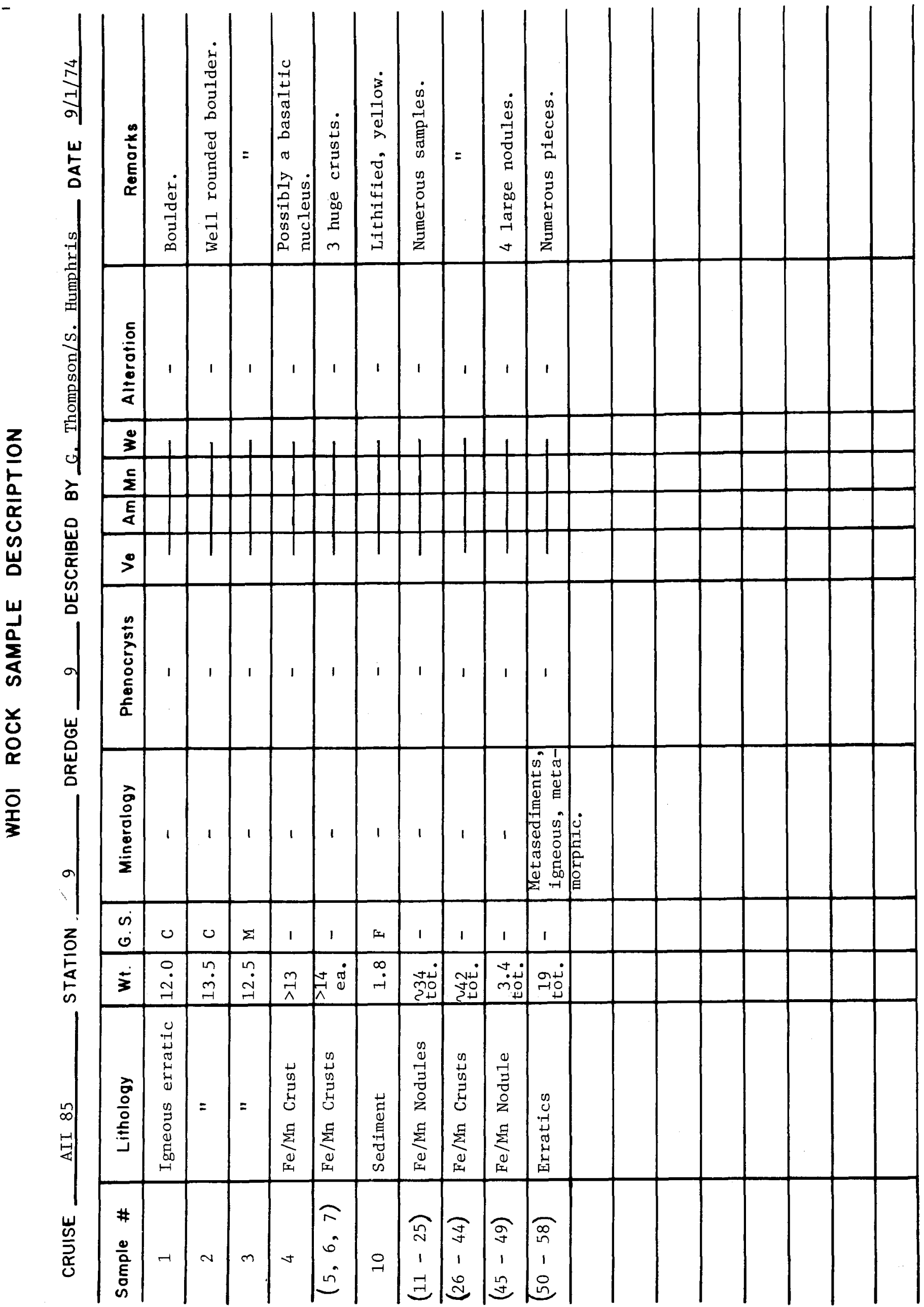




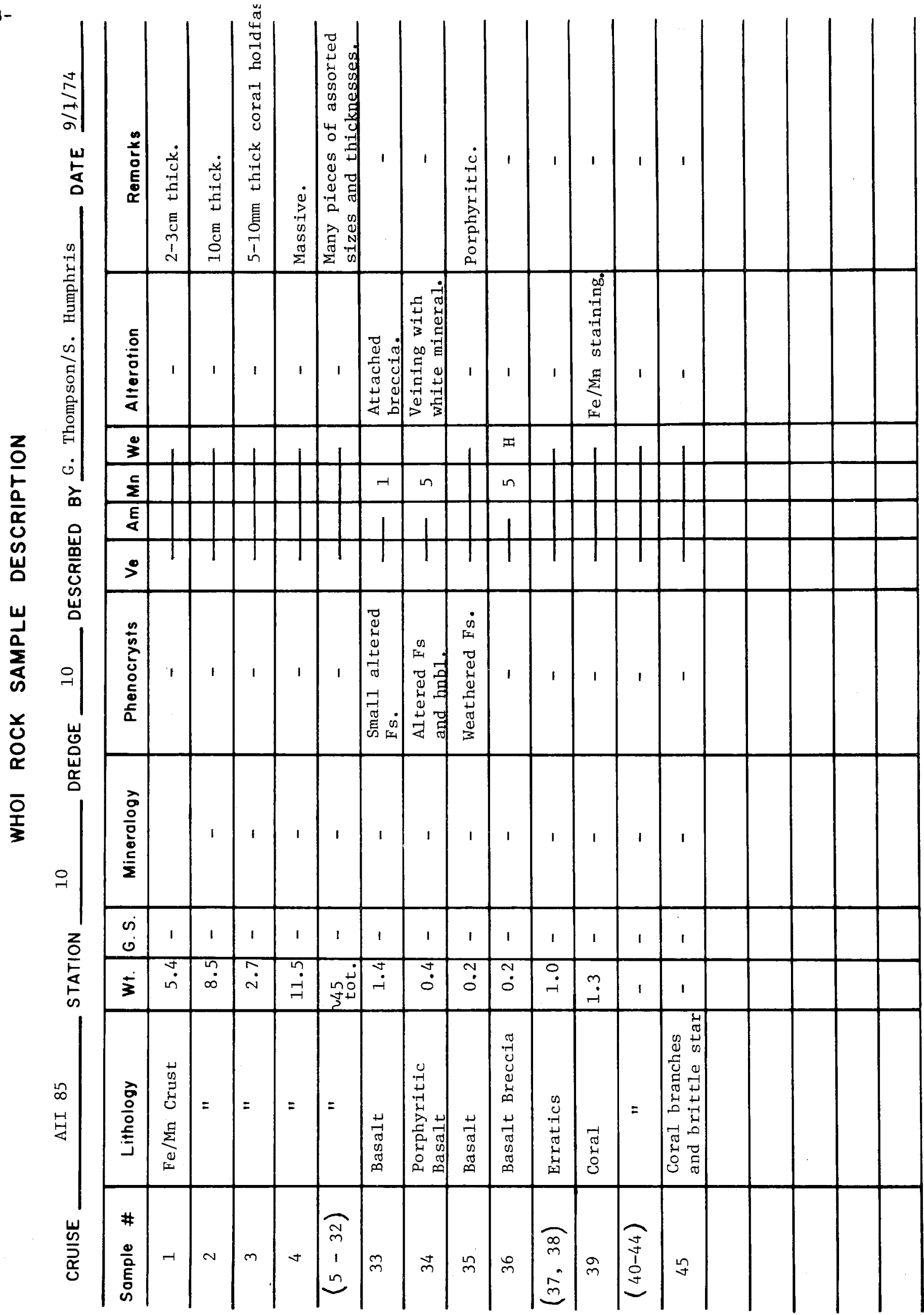




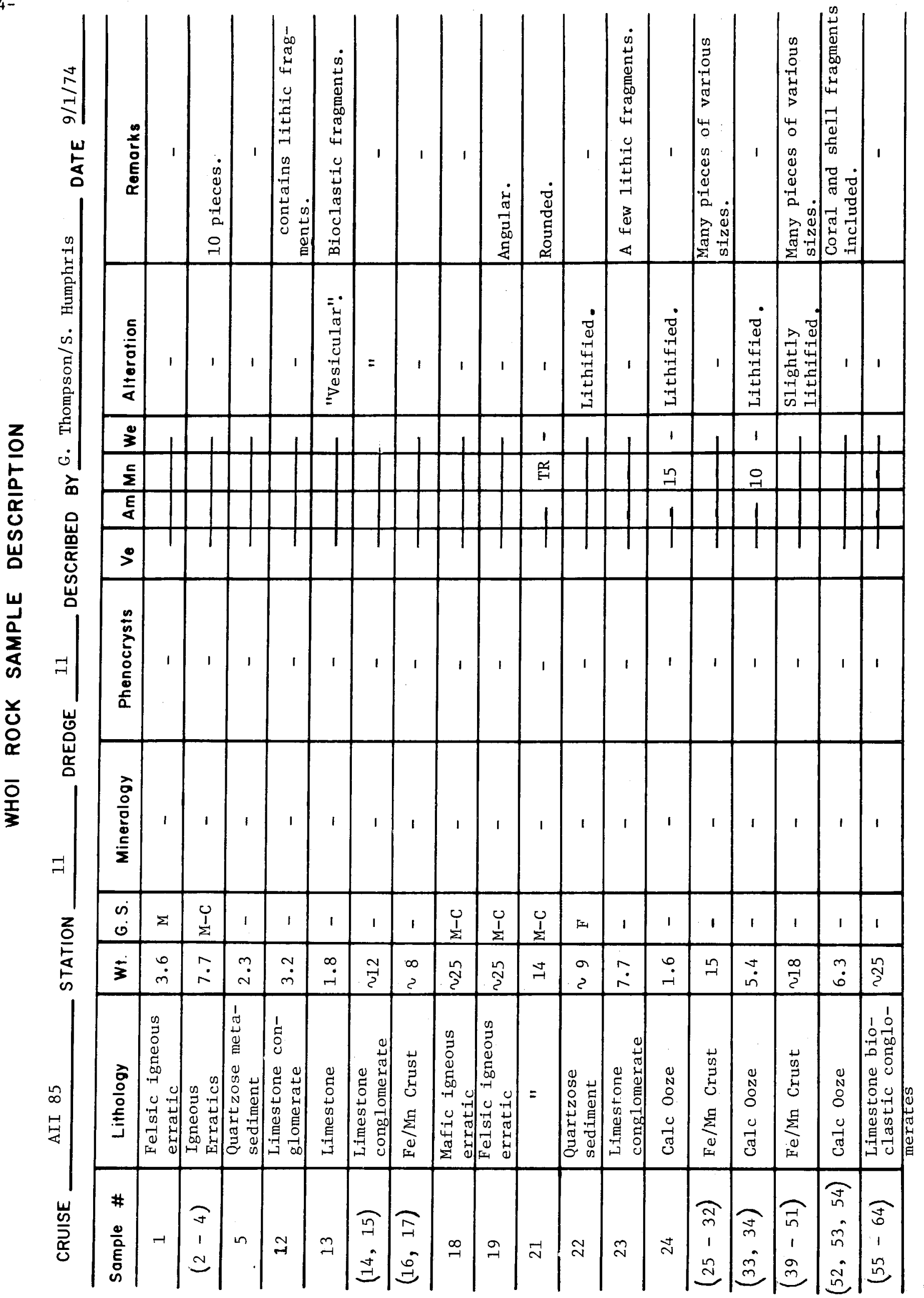




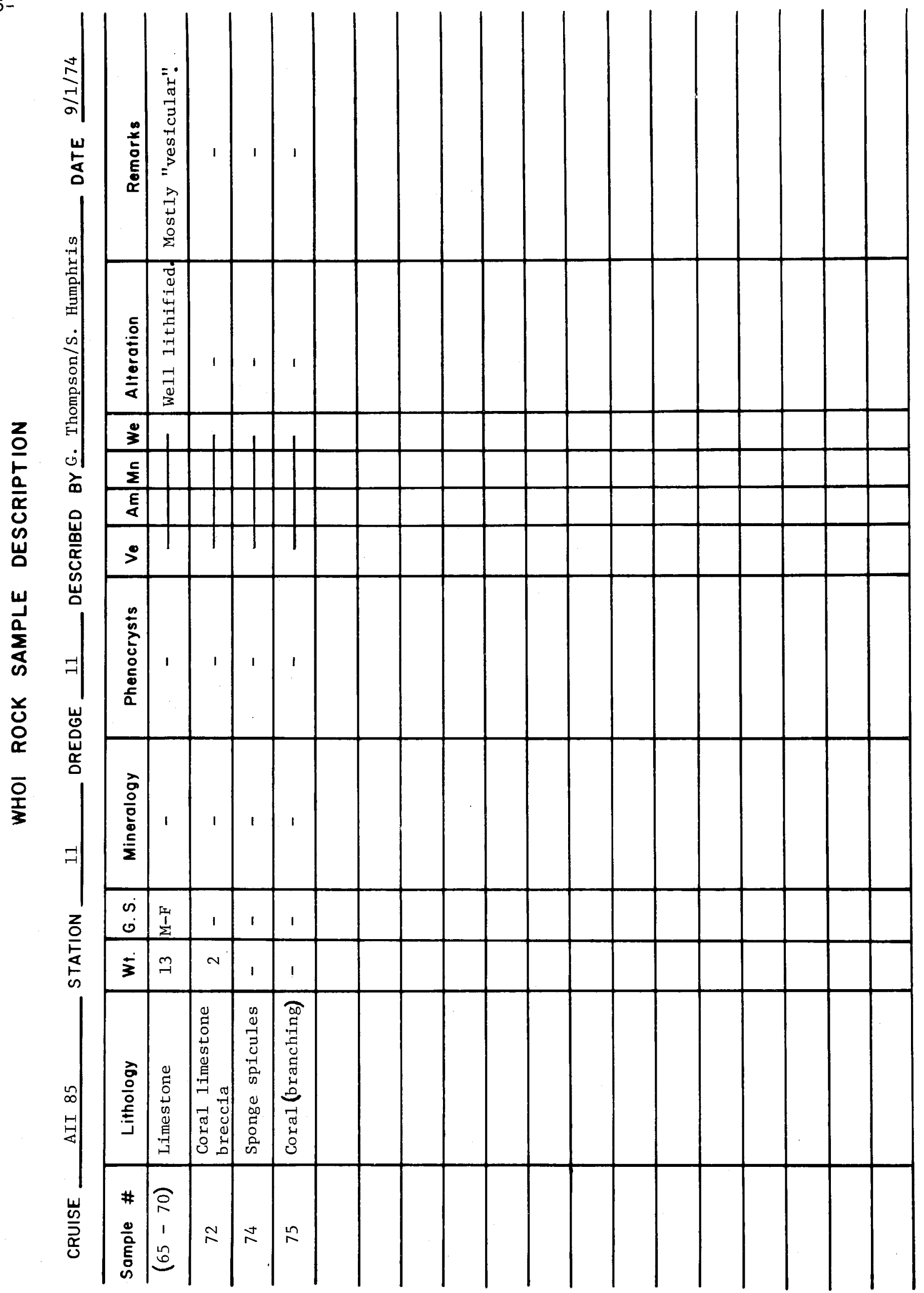




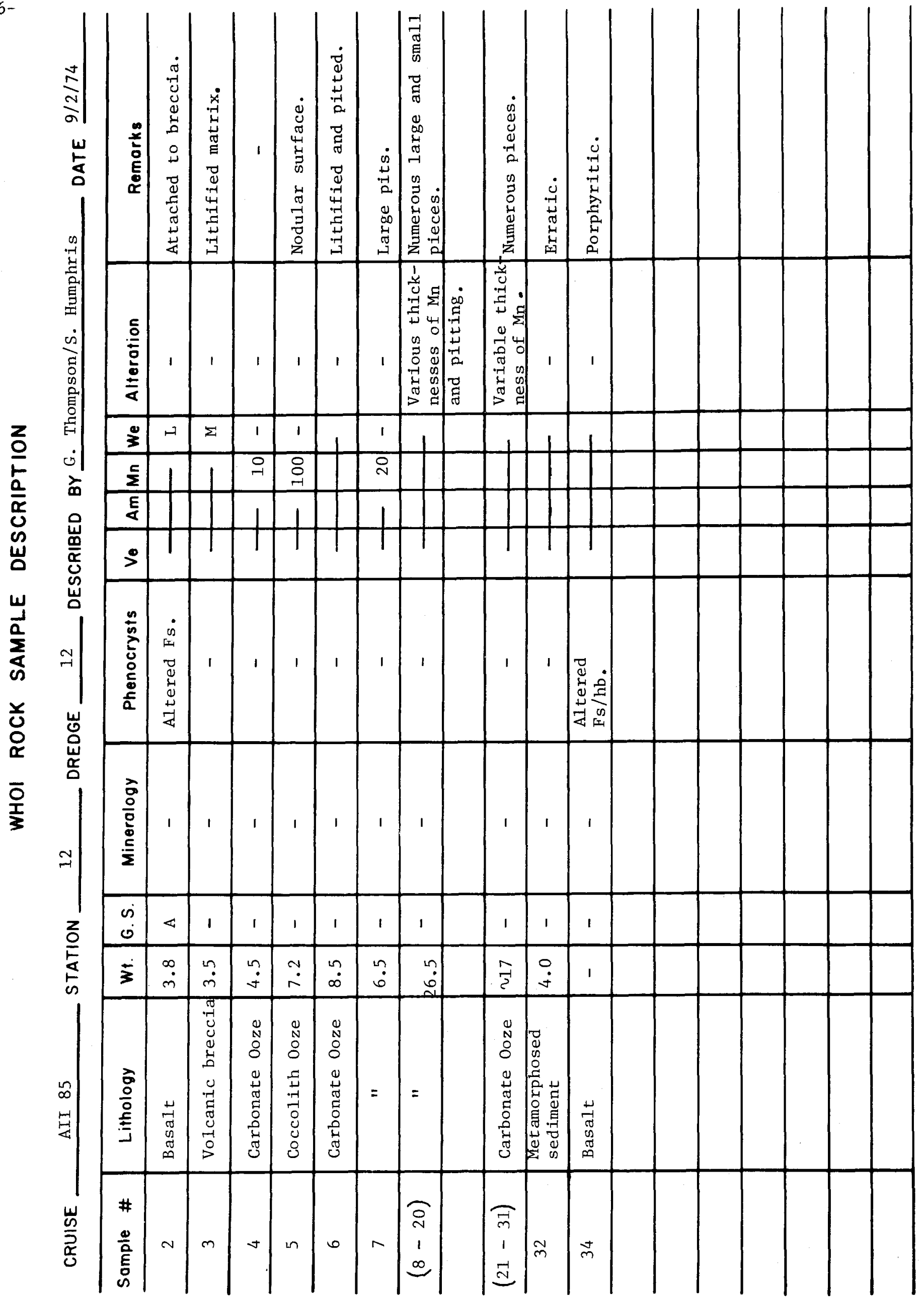



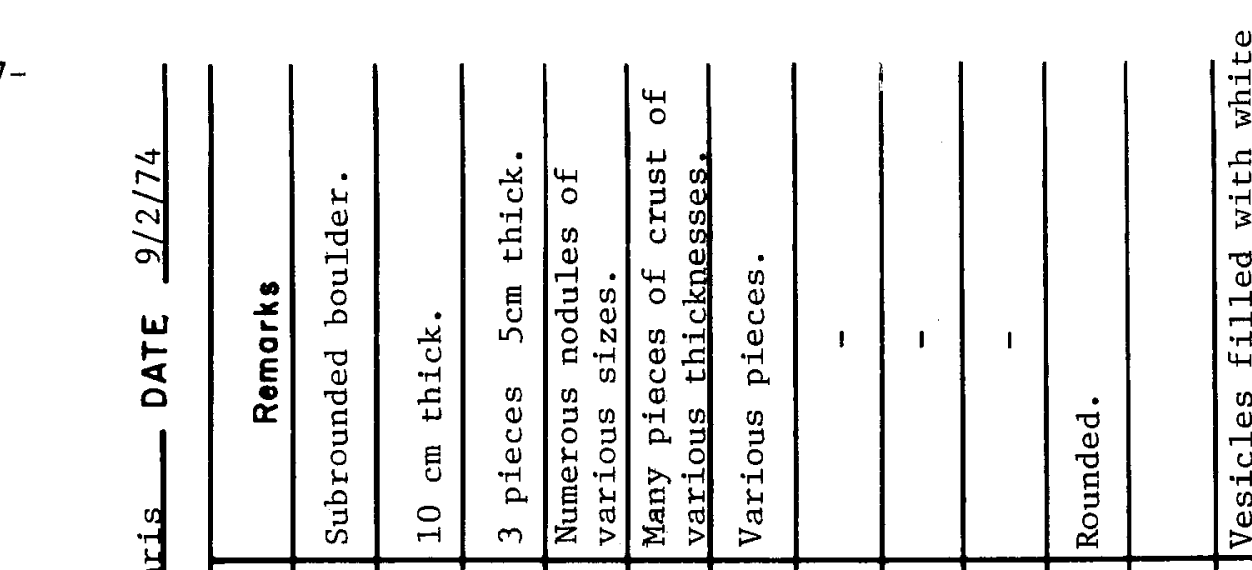


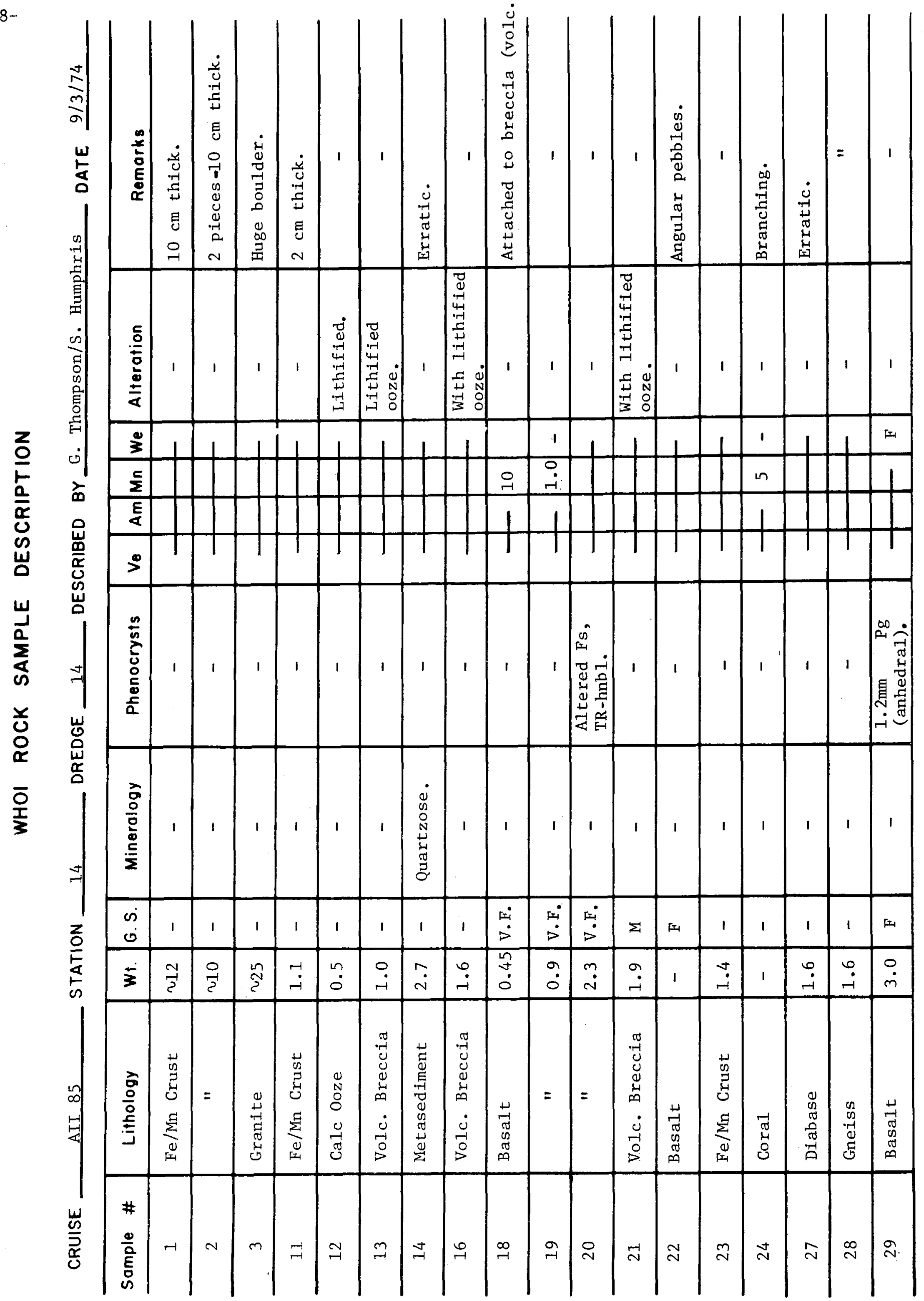




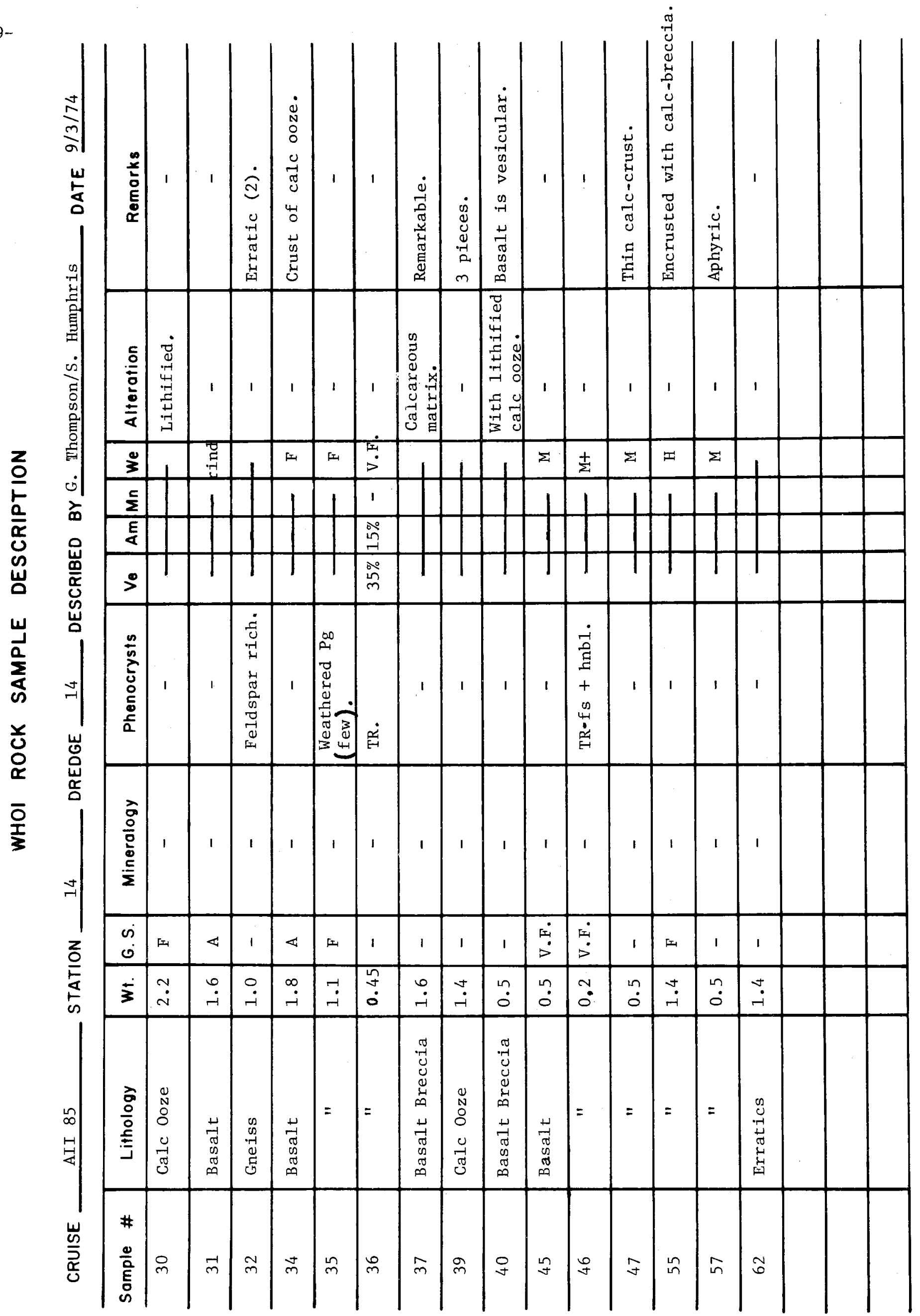




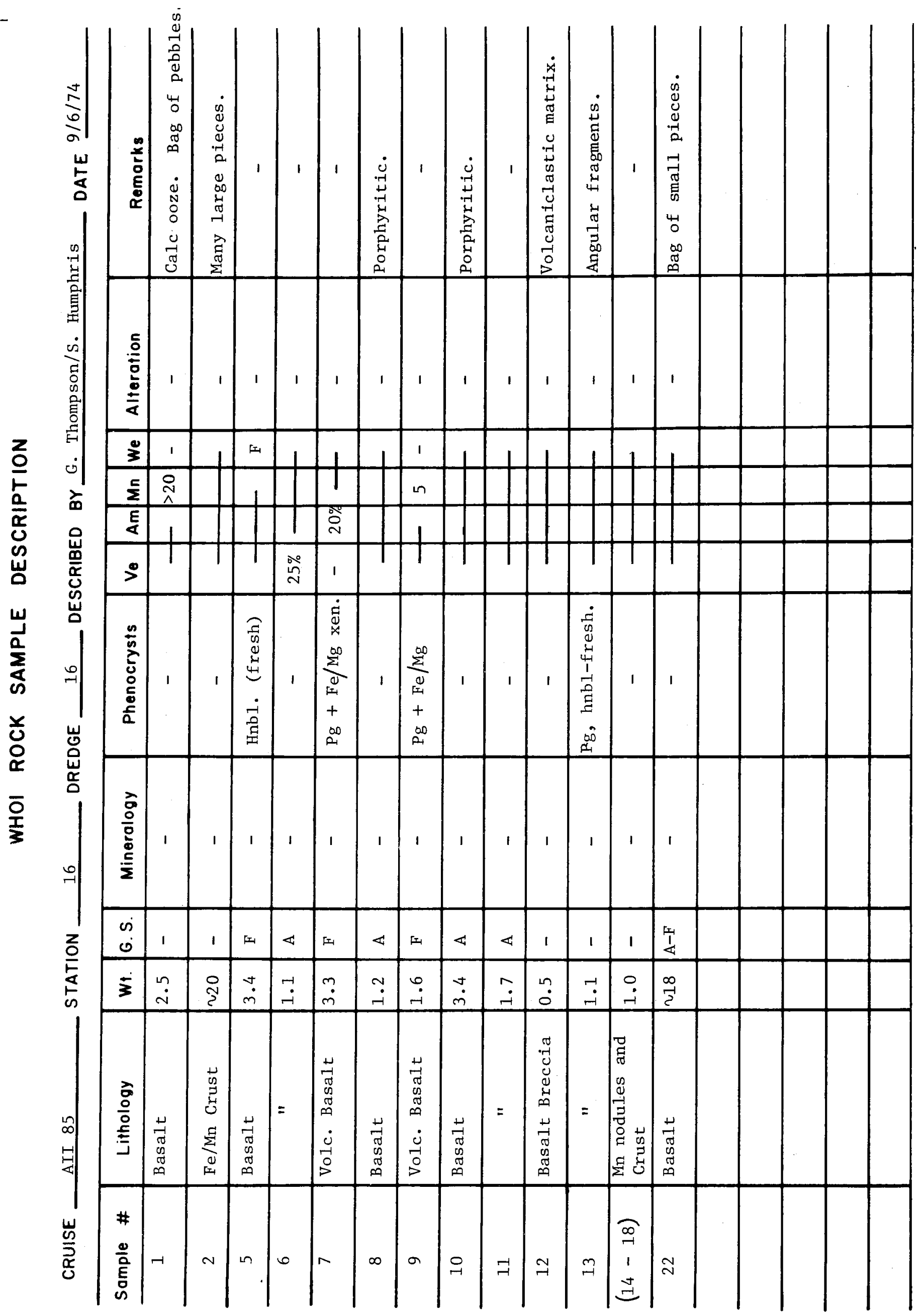




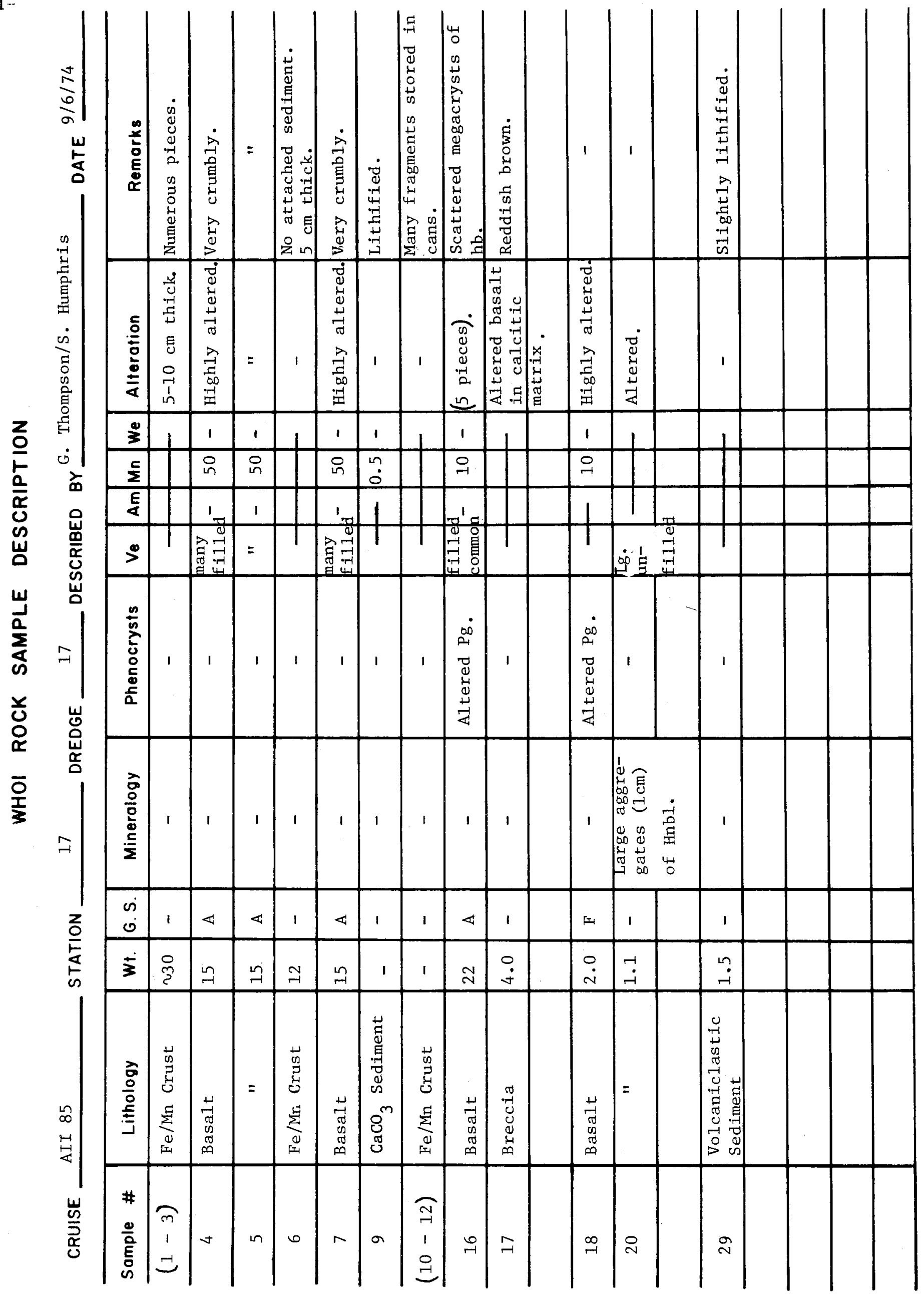




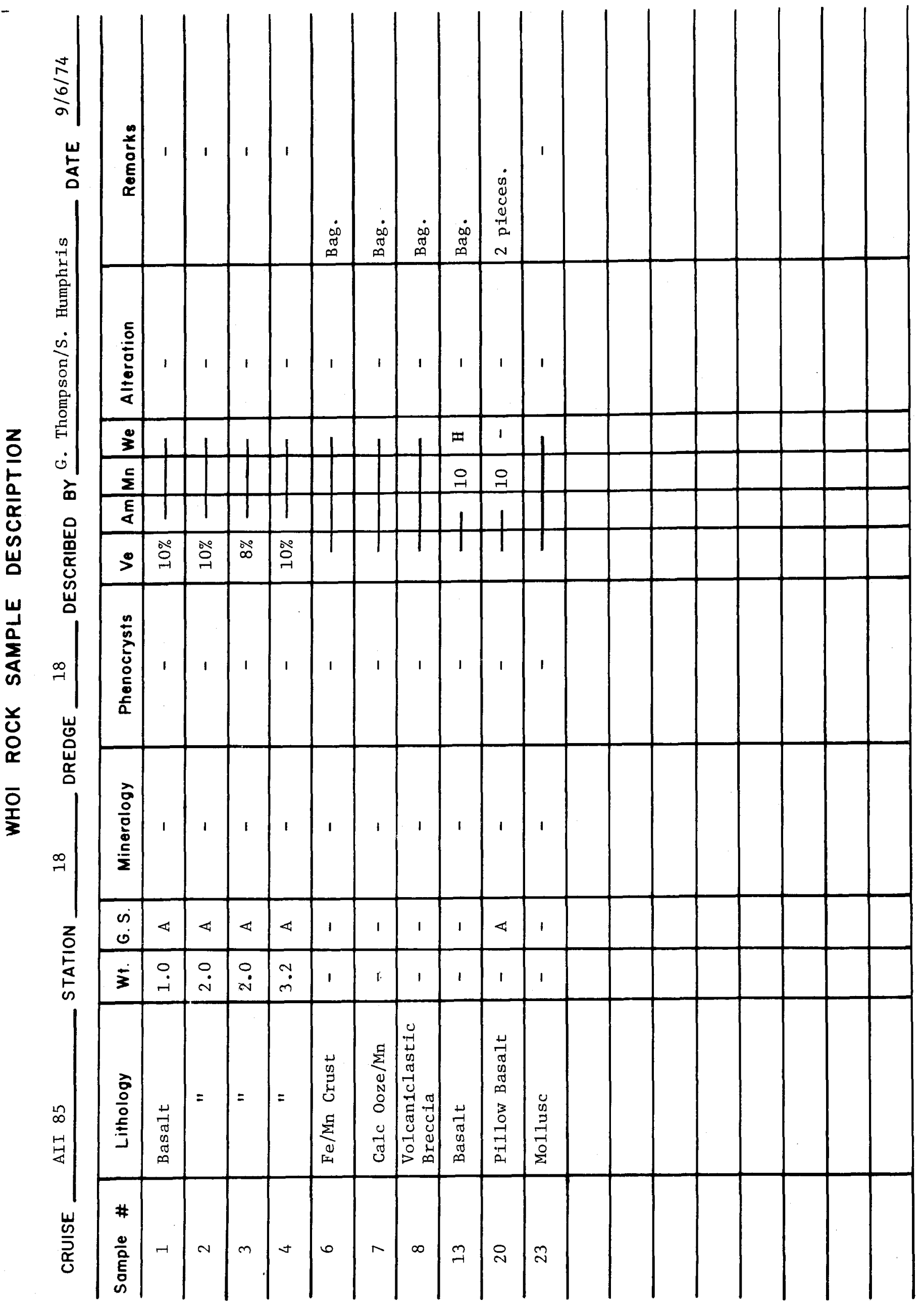




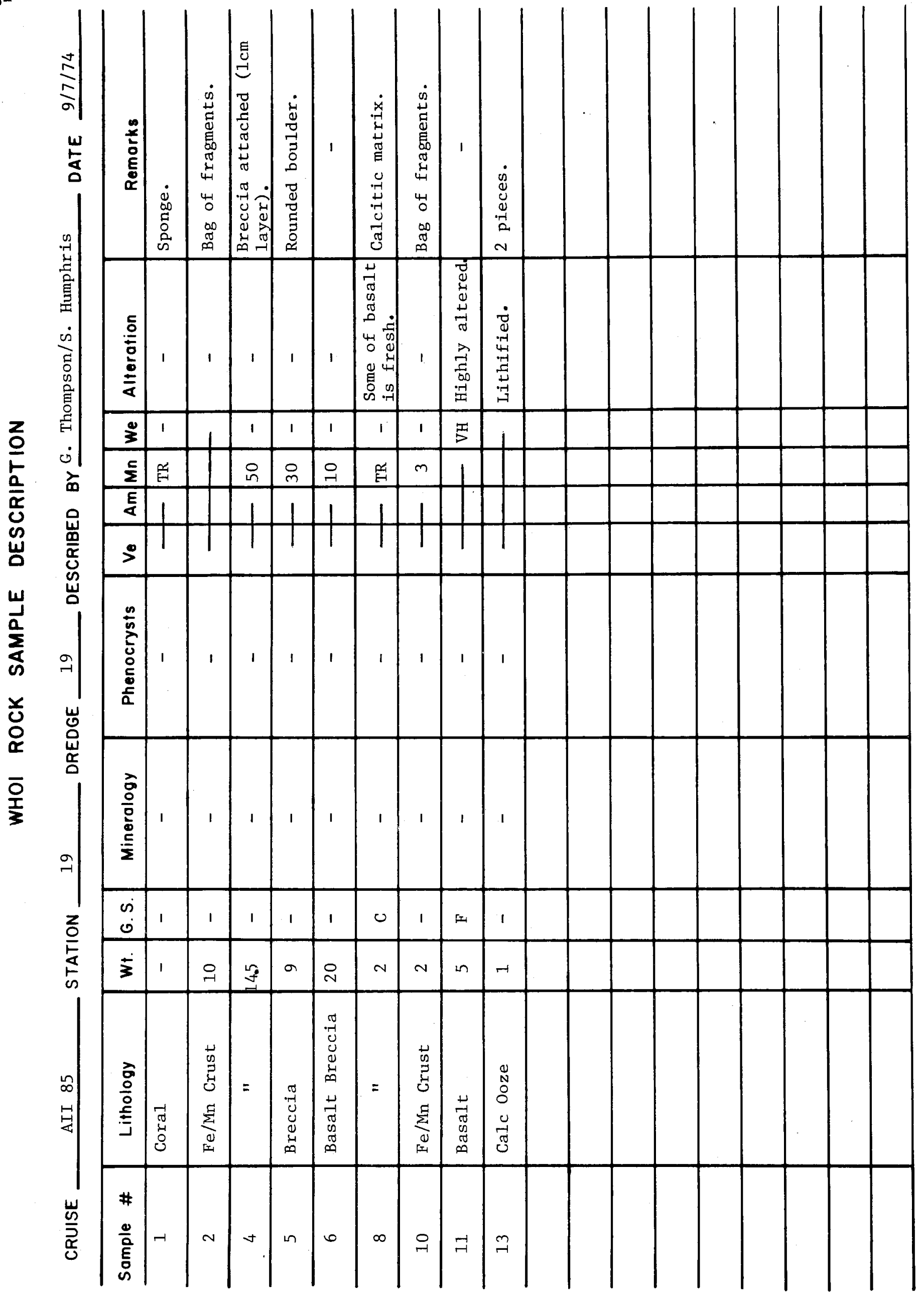




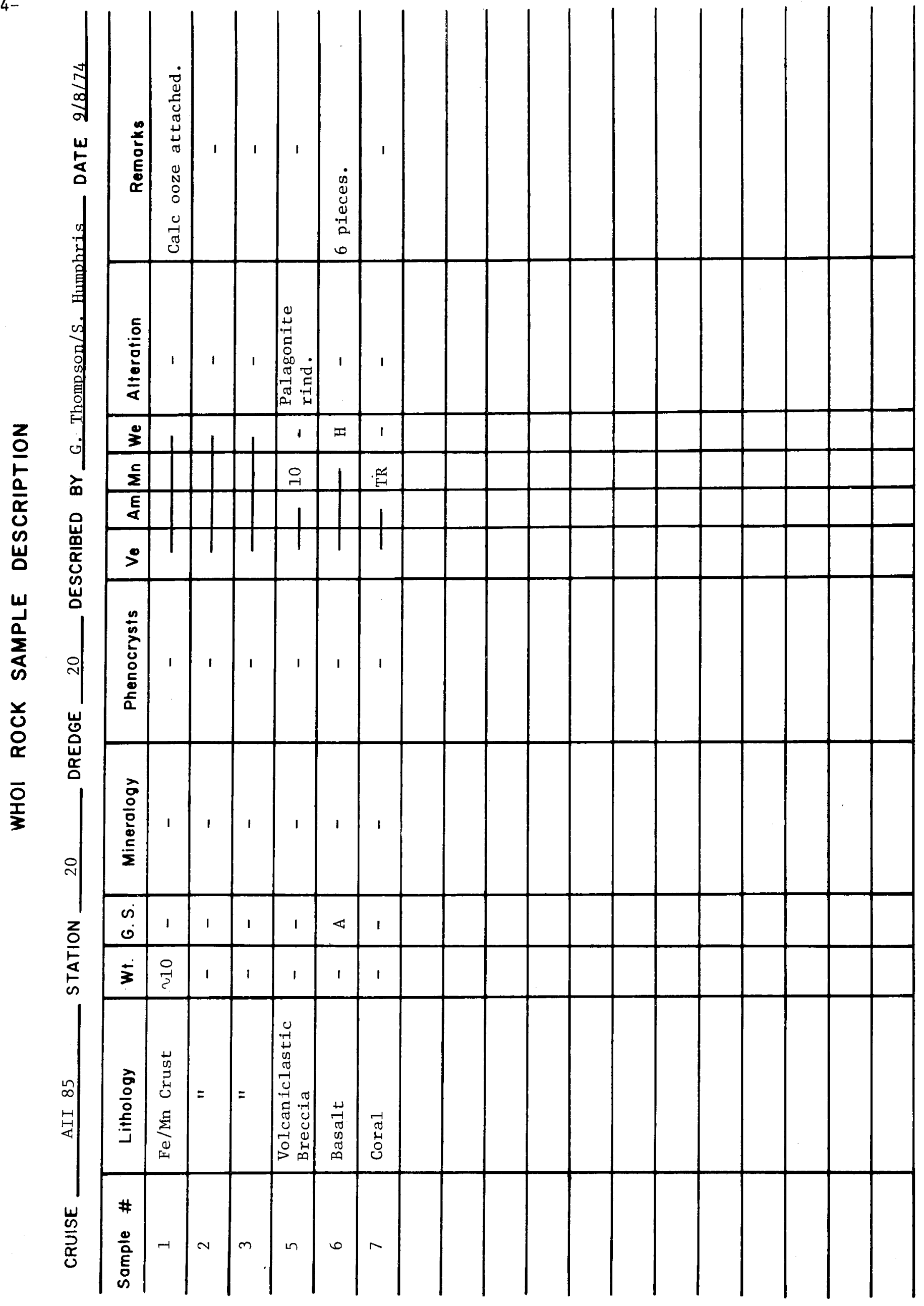




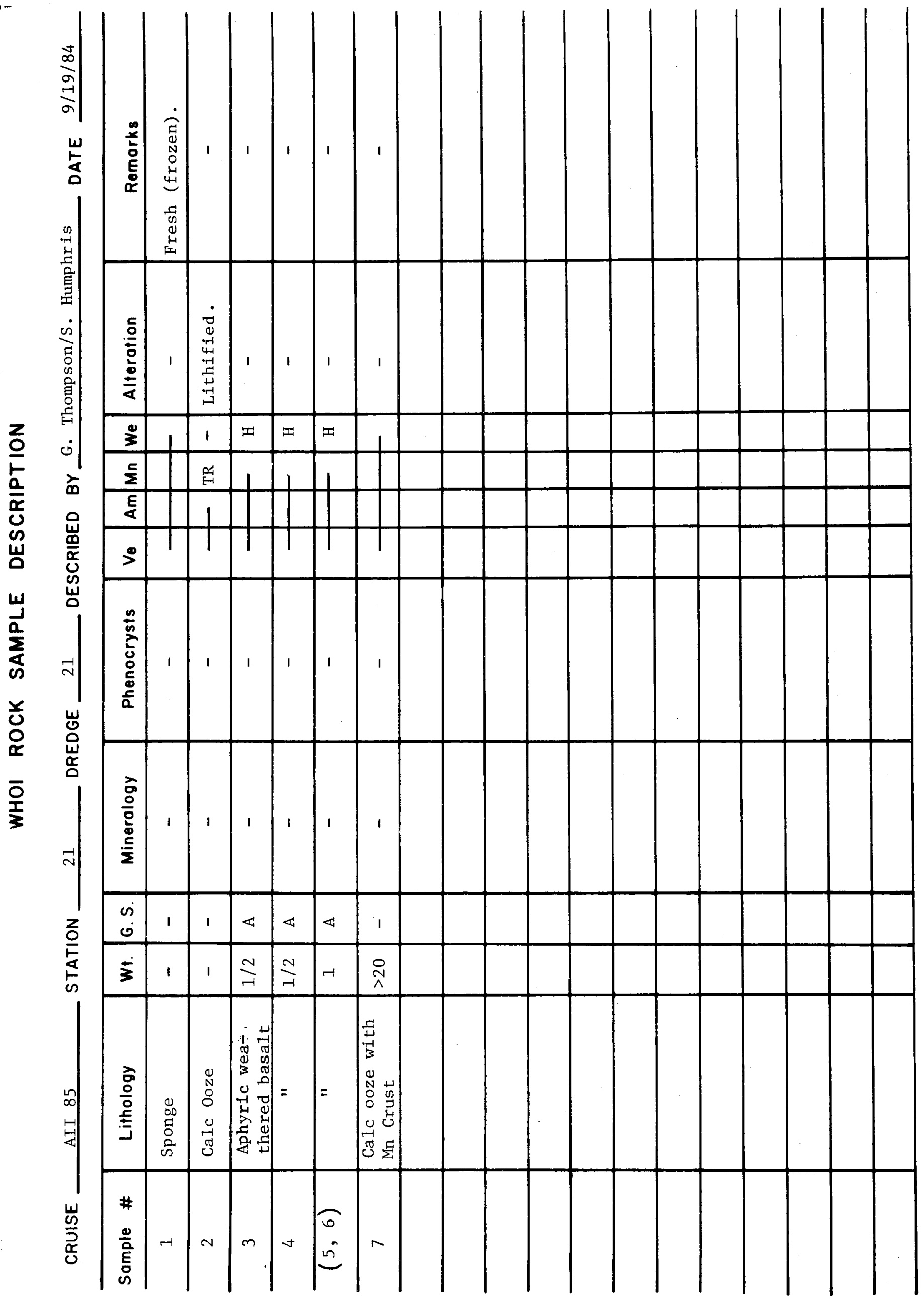




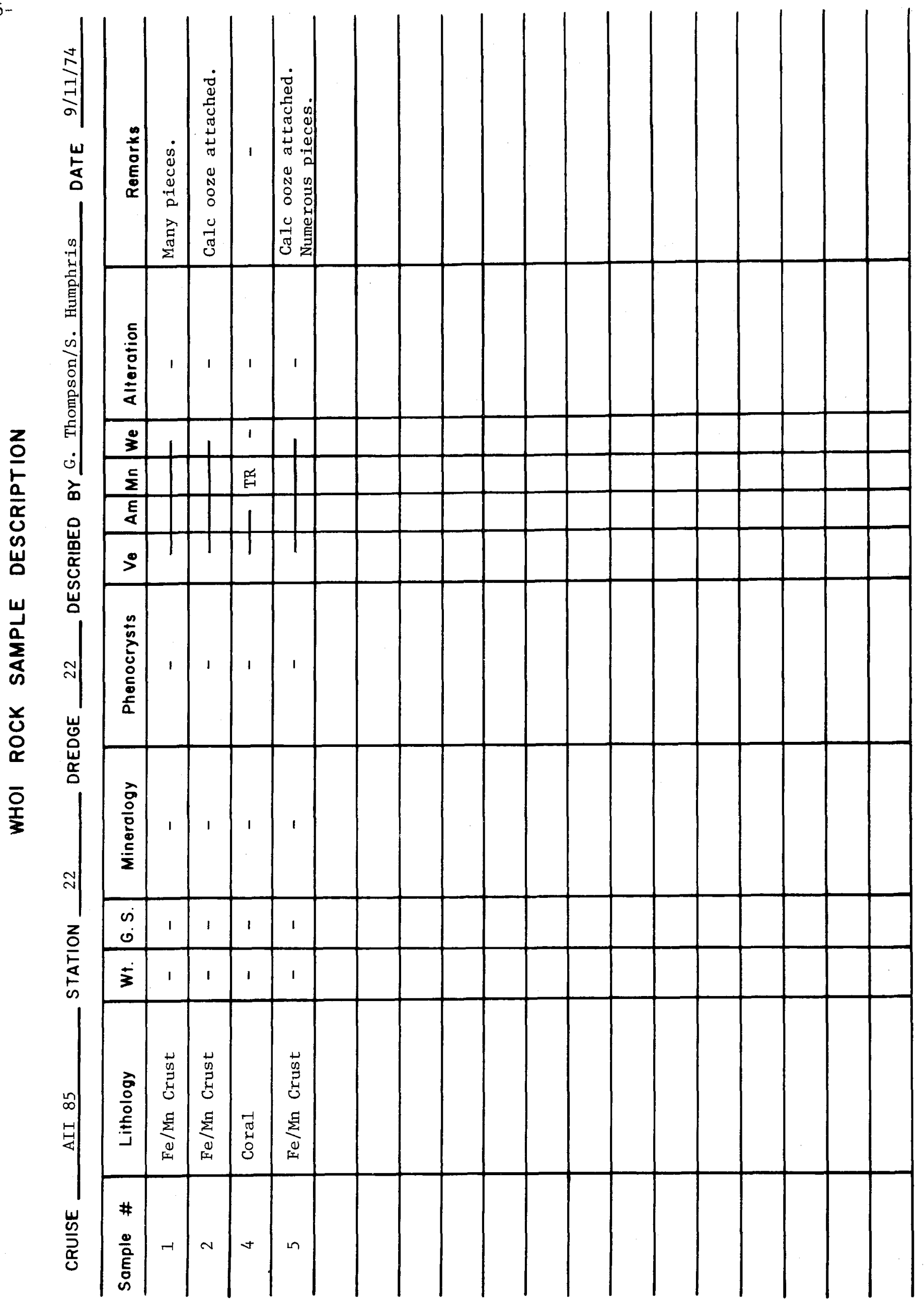




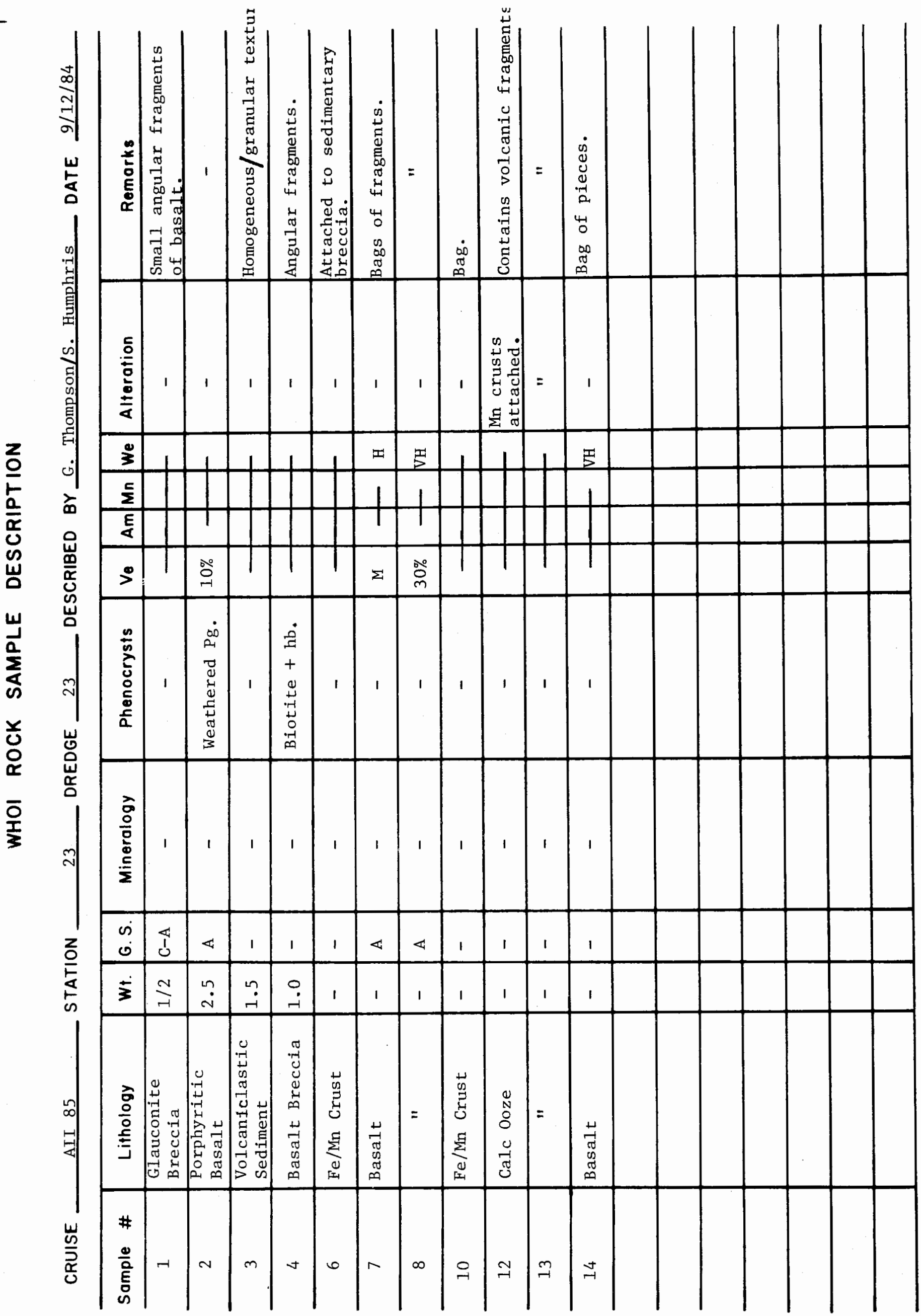




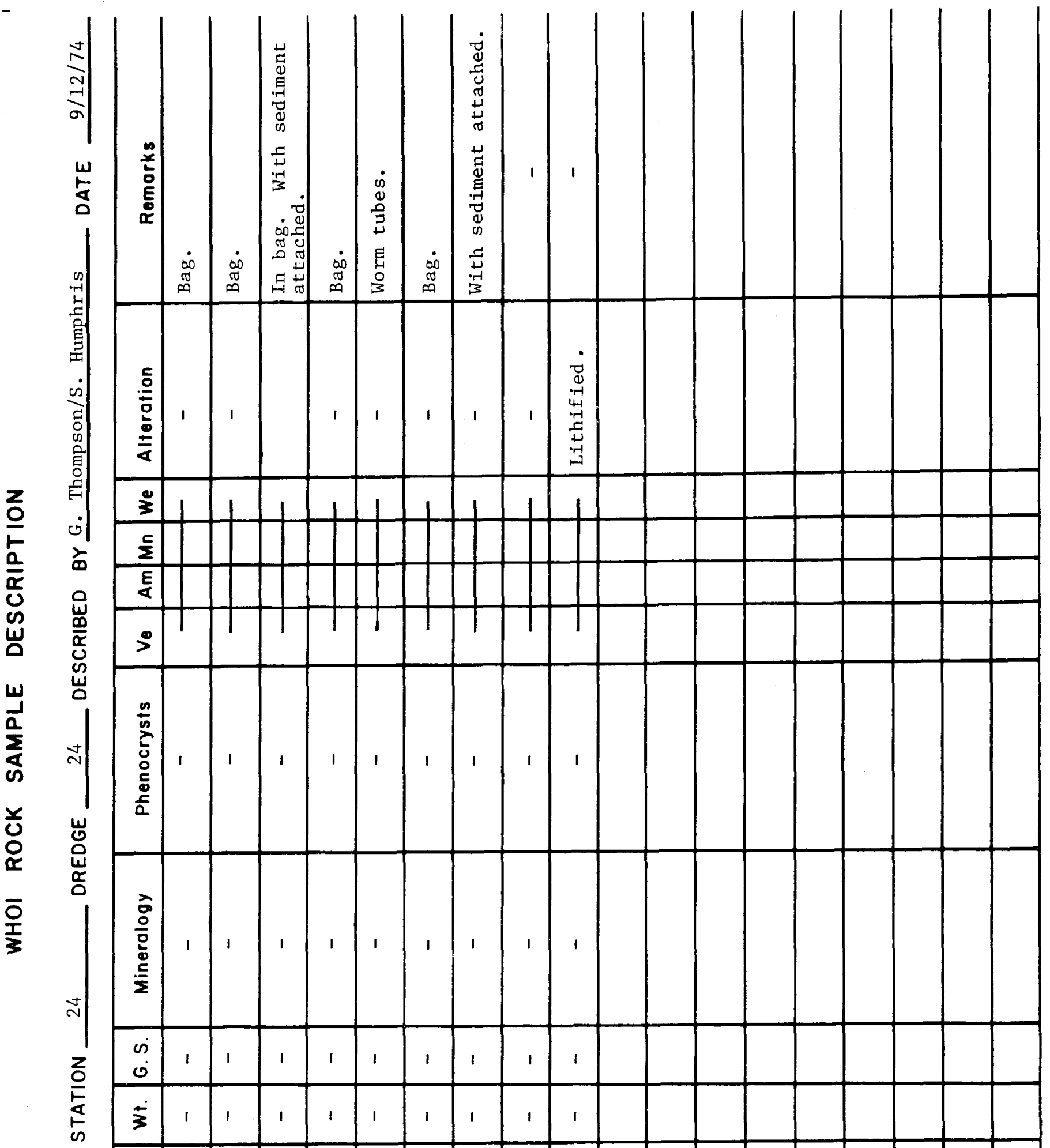

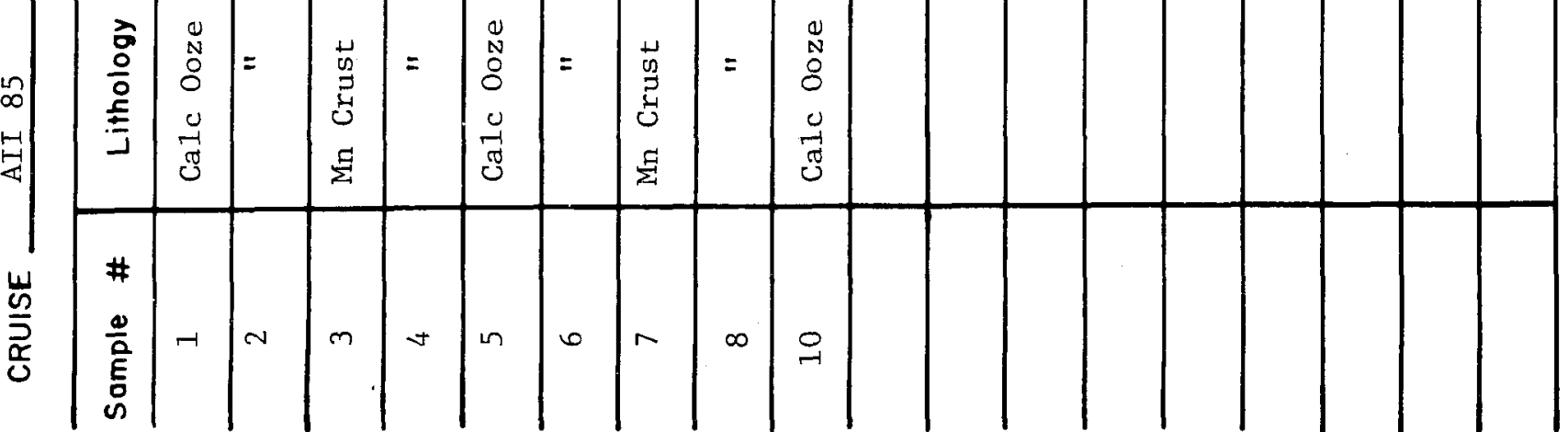




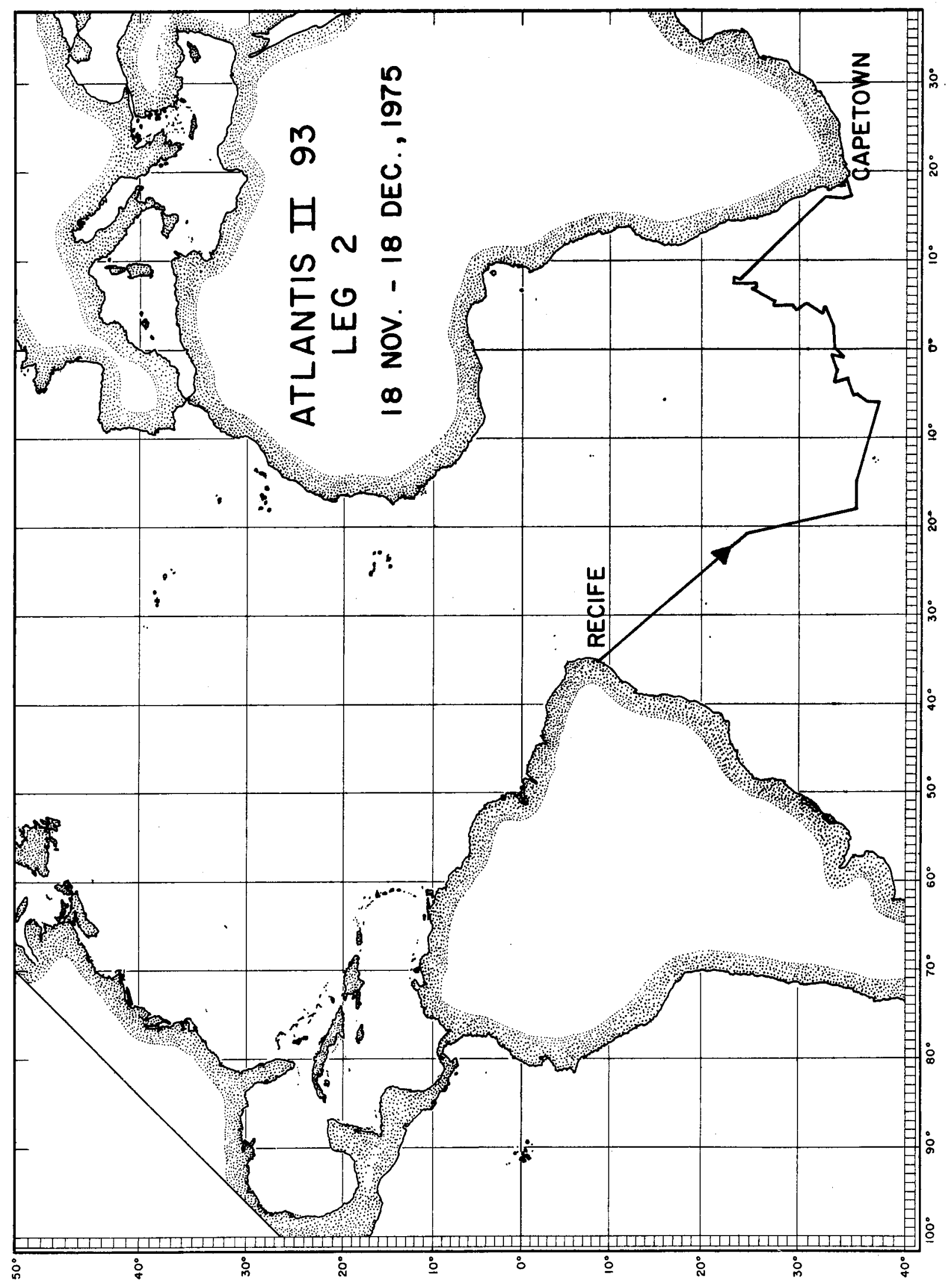




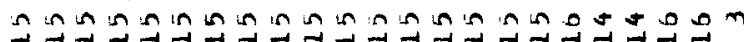

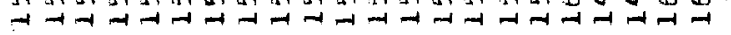

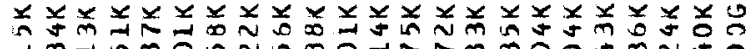

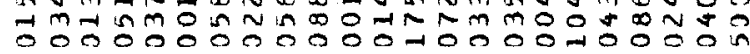

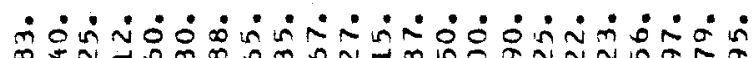
Nã

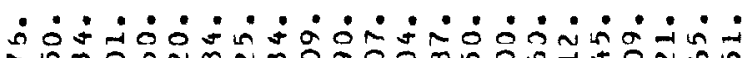

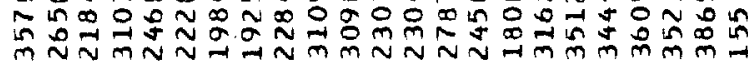

$\stackrel{x}{\theta} x$

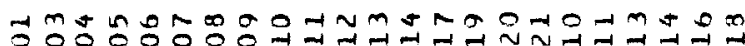

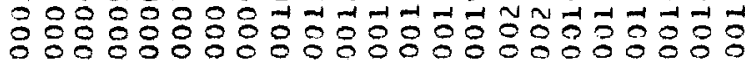

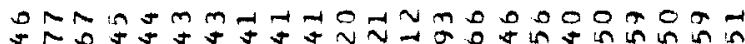

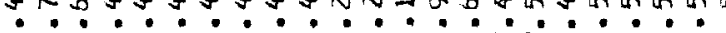

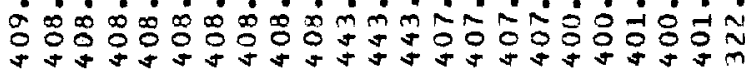

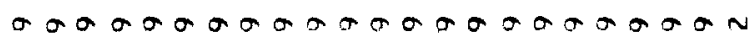

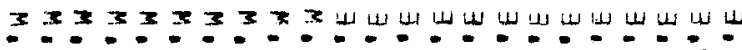

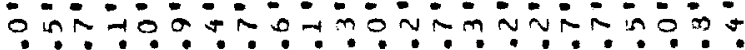

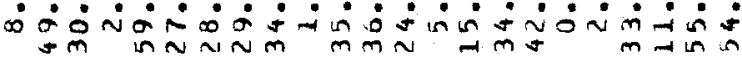

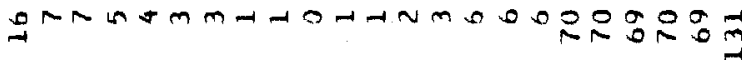

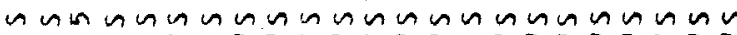
- mino obo in

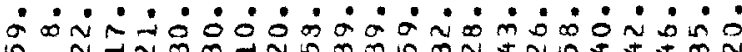
m

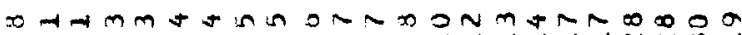

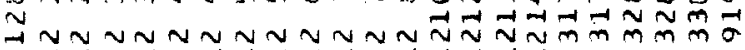

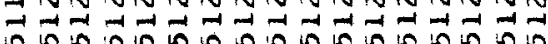

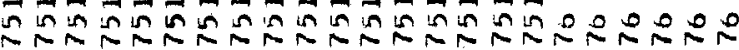

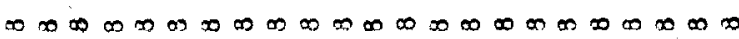

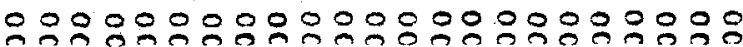

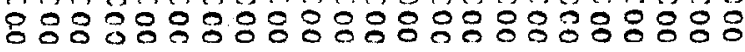

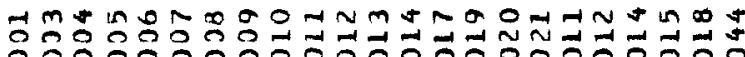

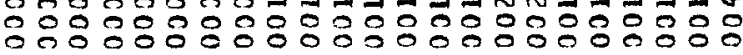
NNNNNNNNNNNNNNNNND00000\% กั้

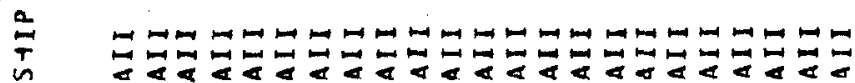




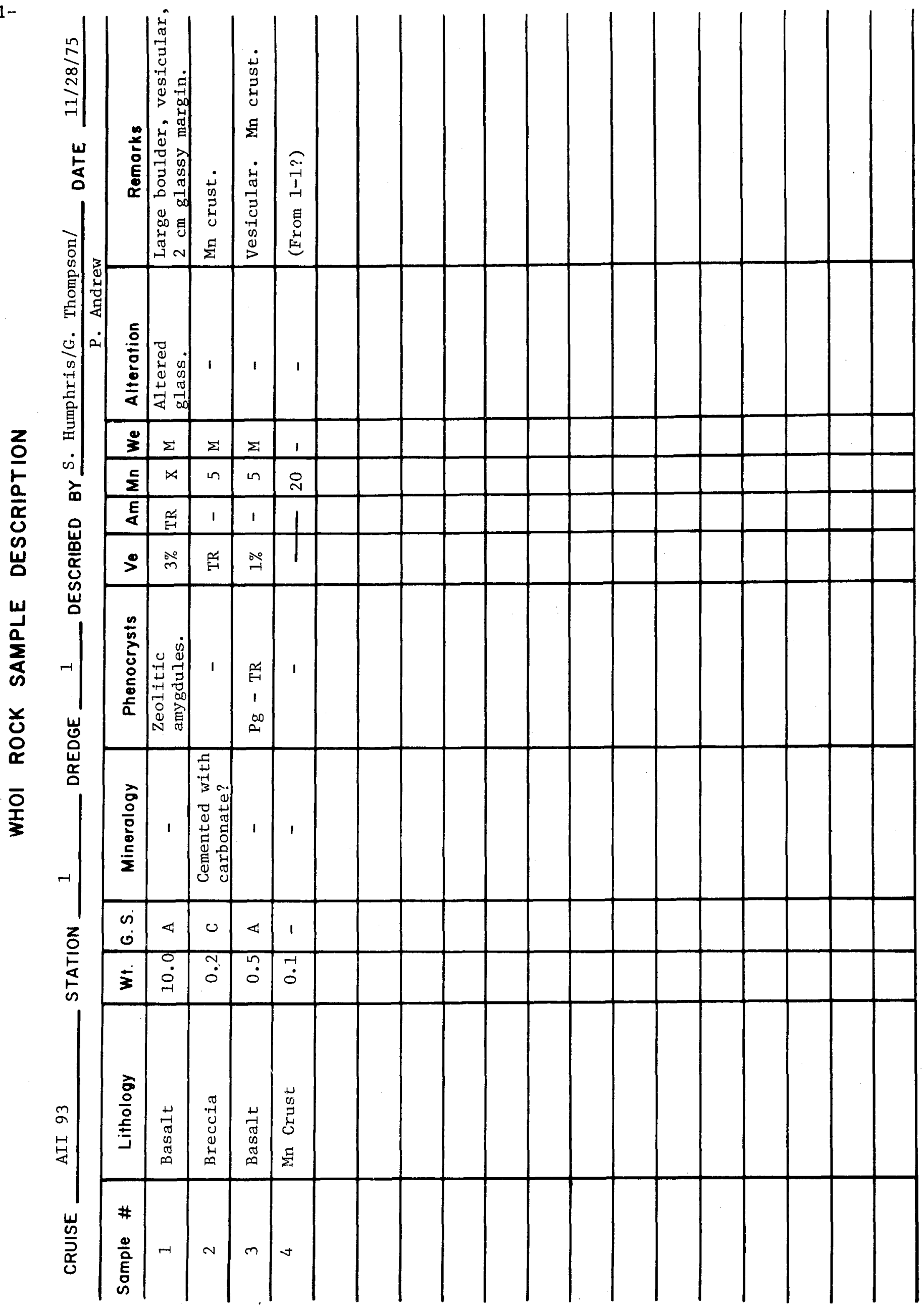




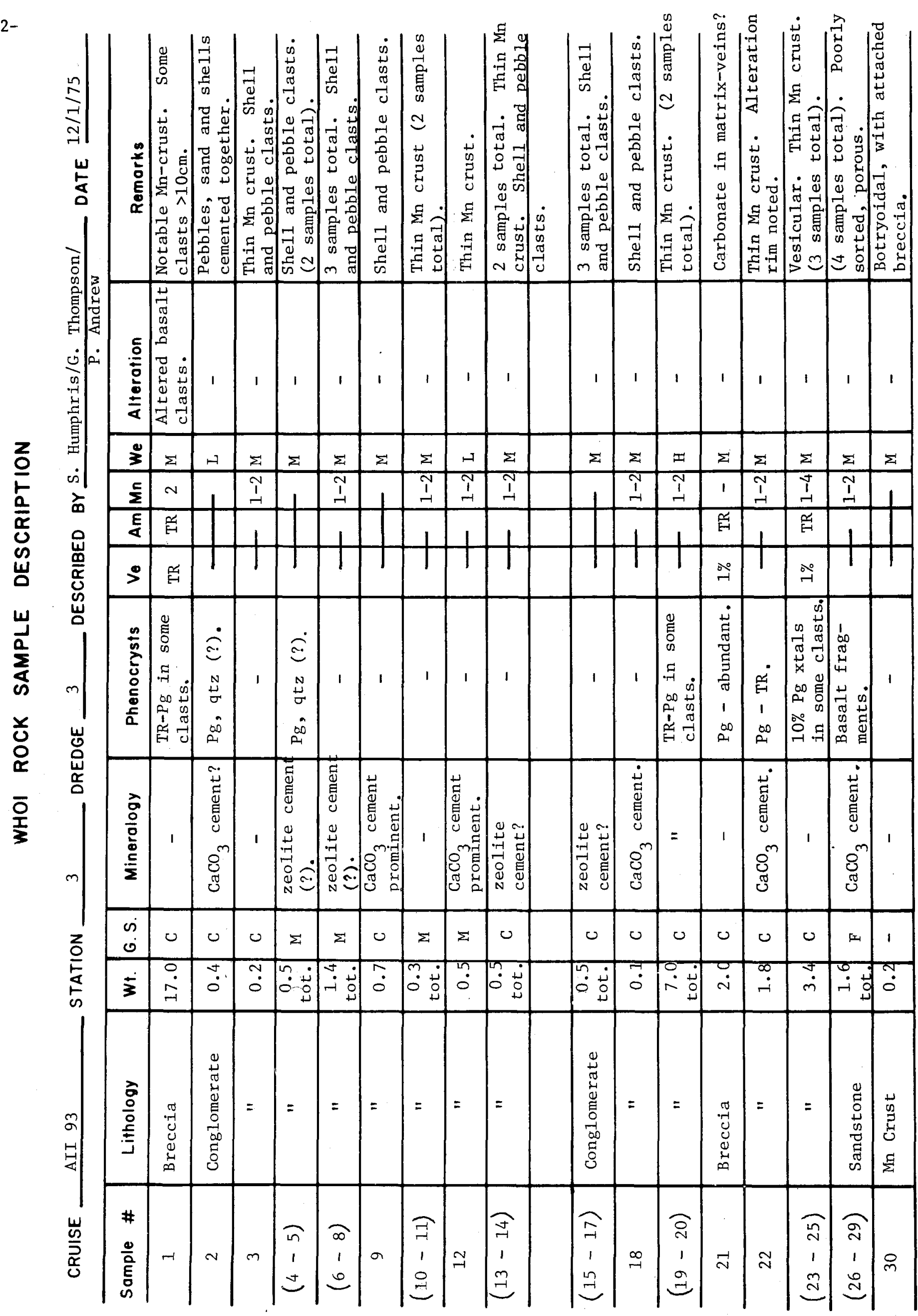




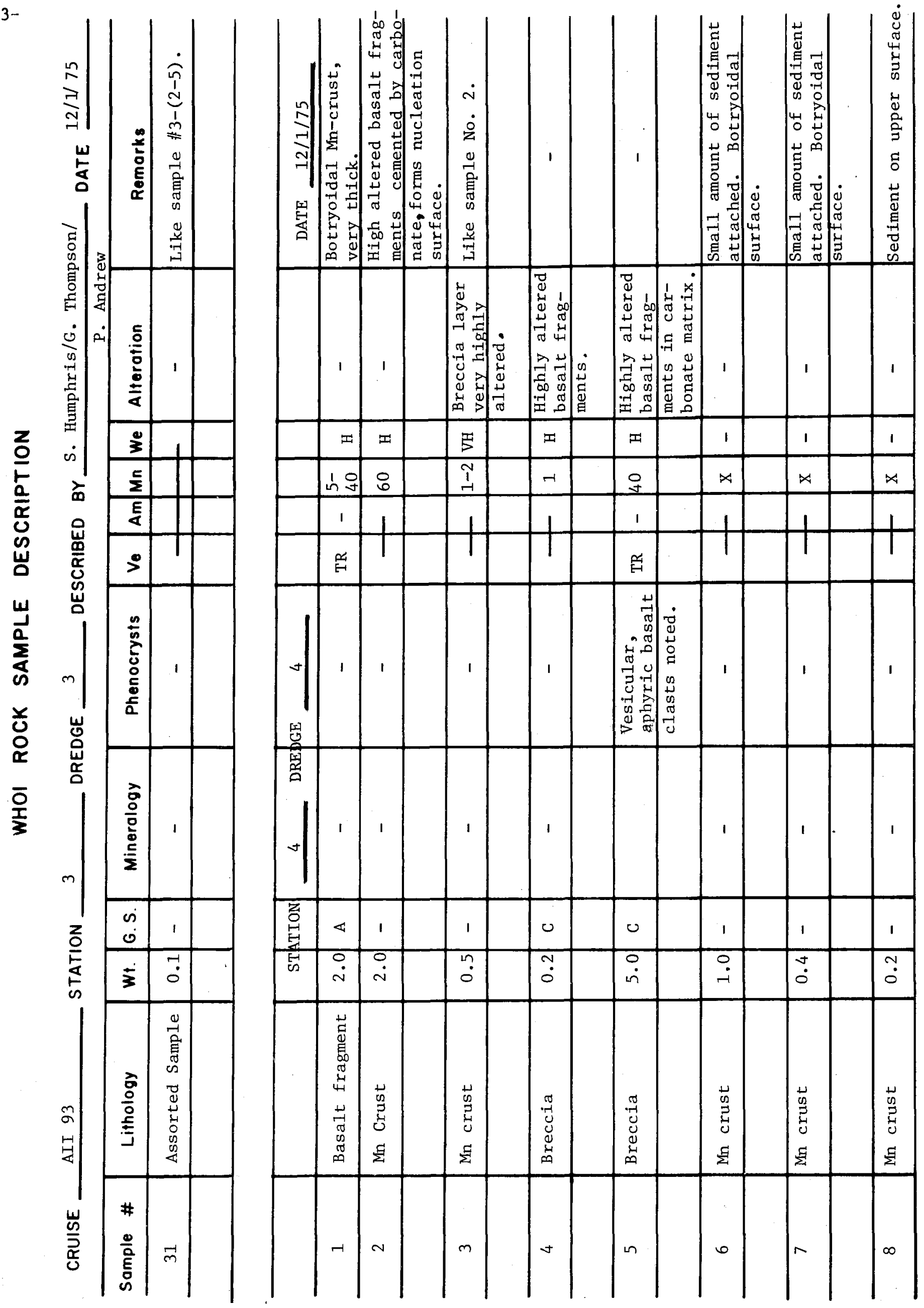




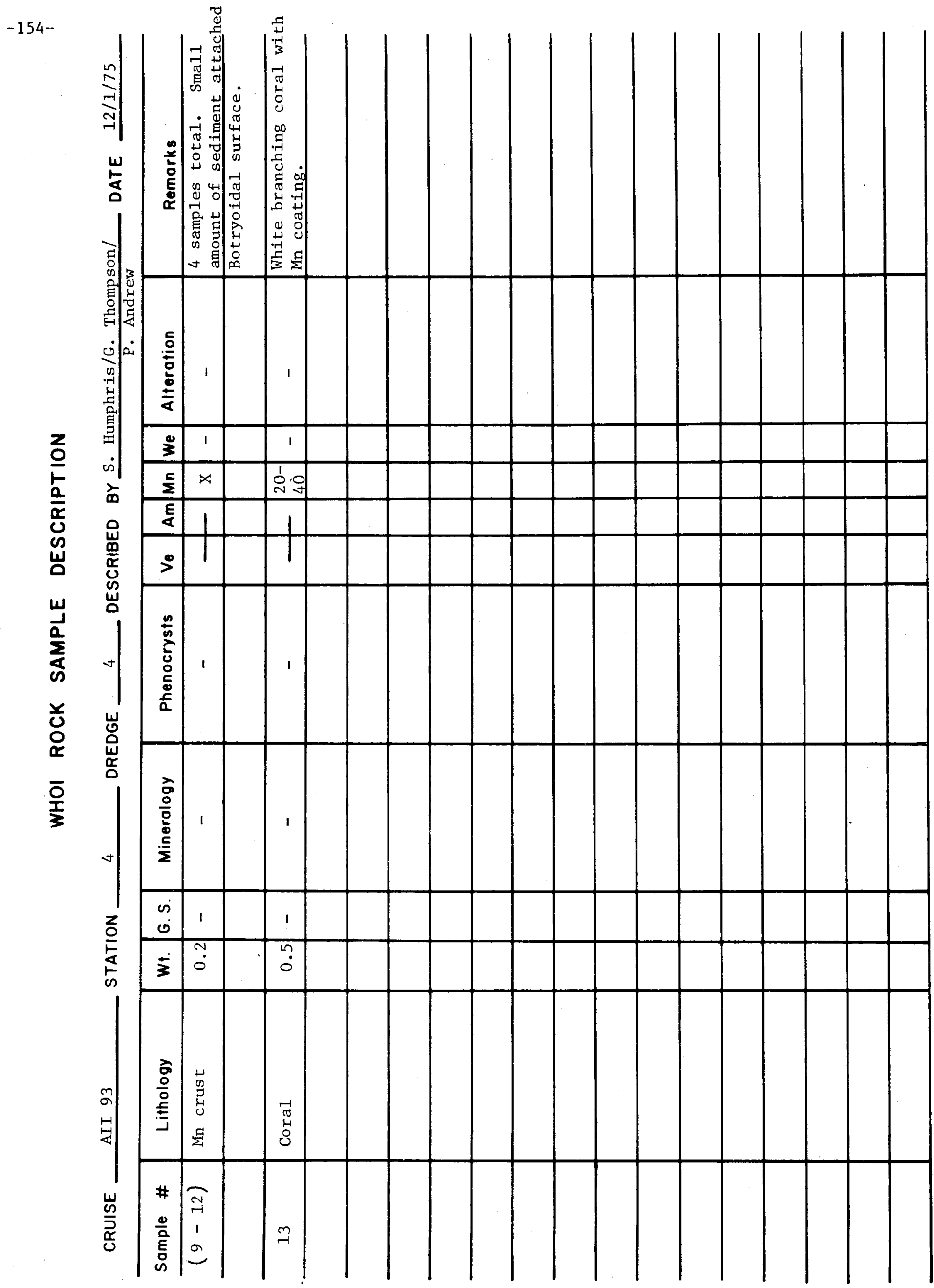


$-156$

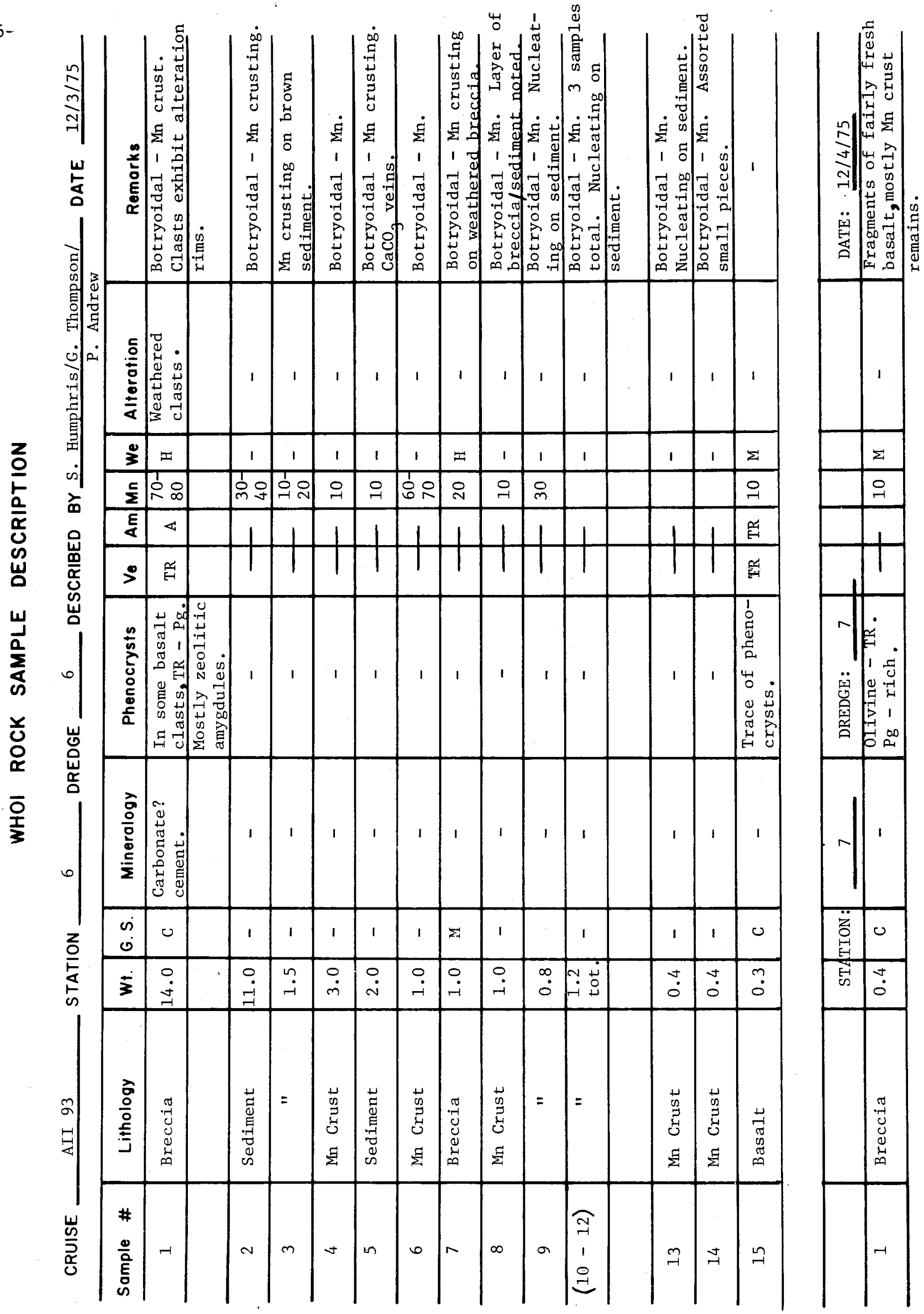


$-157-$

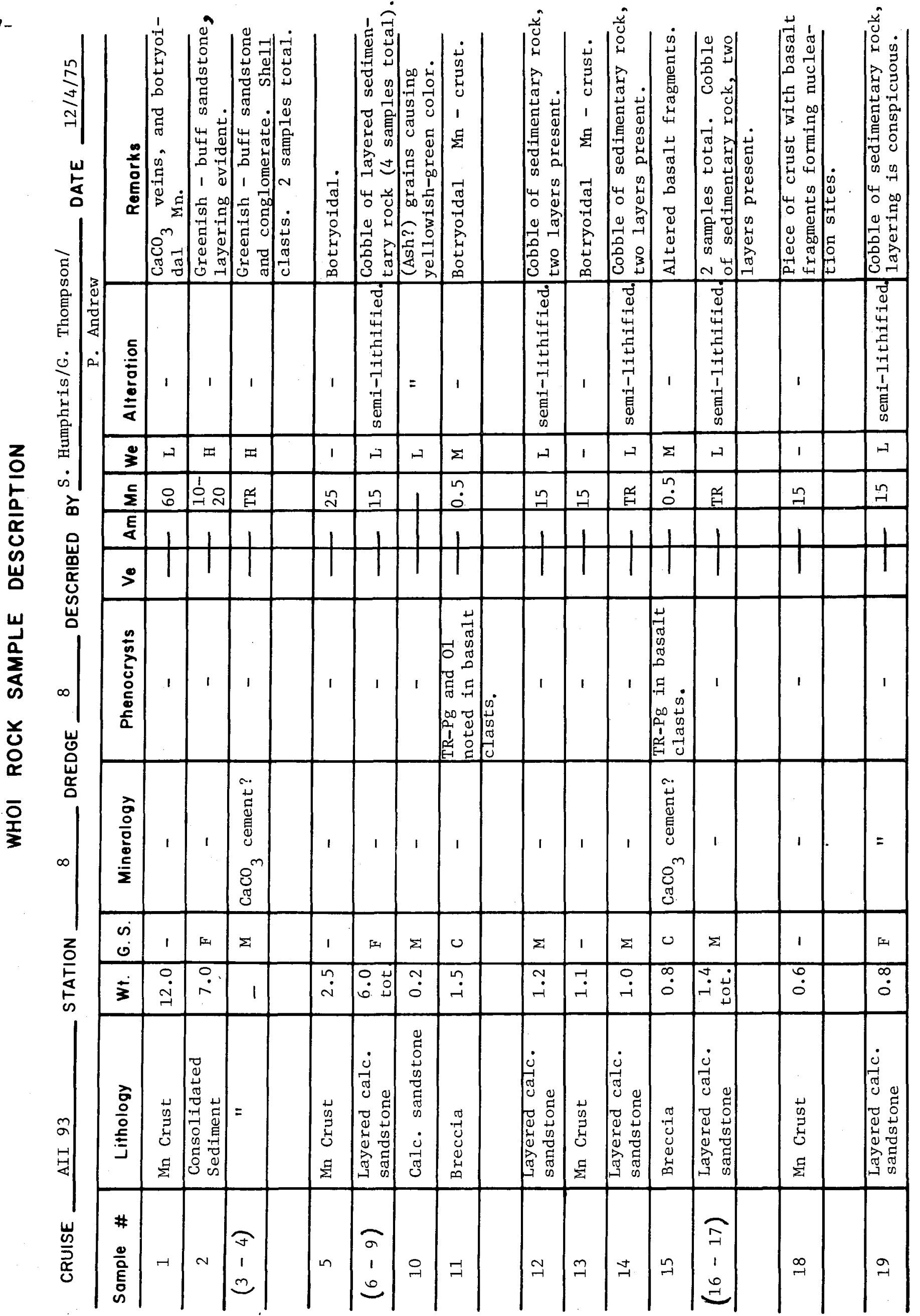


$-158-$

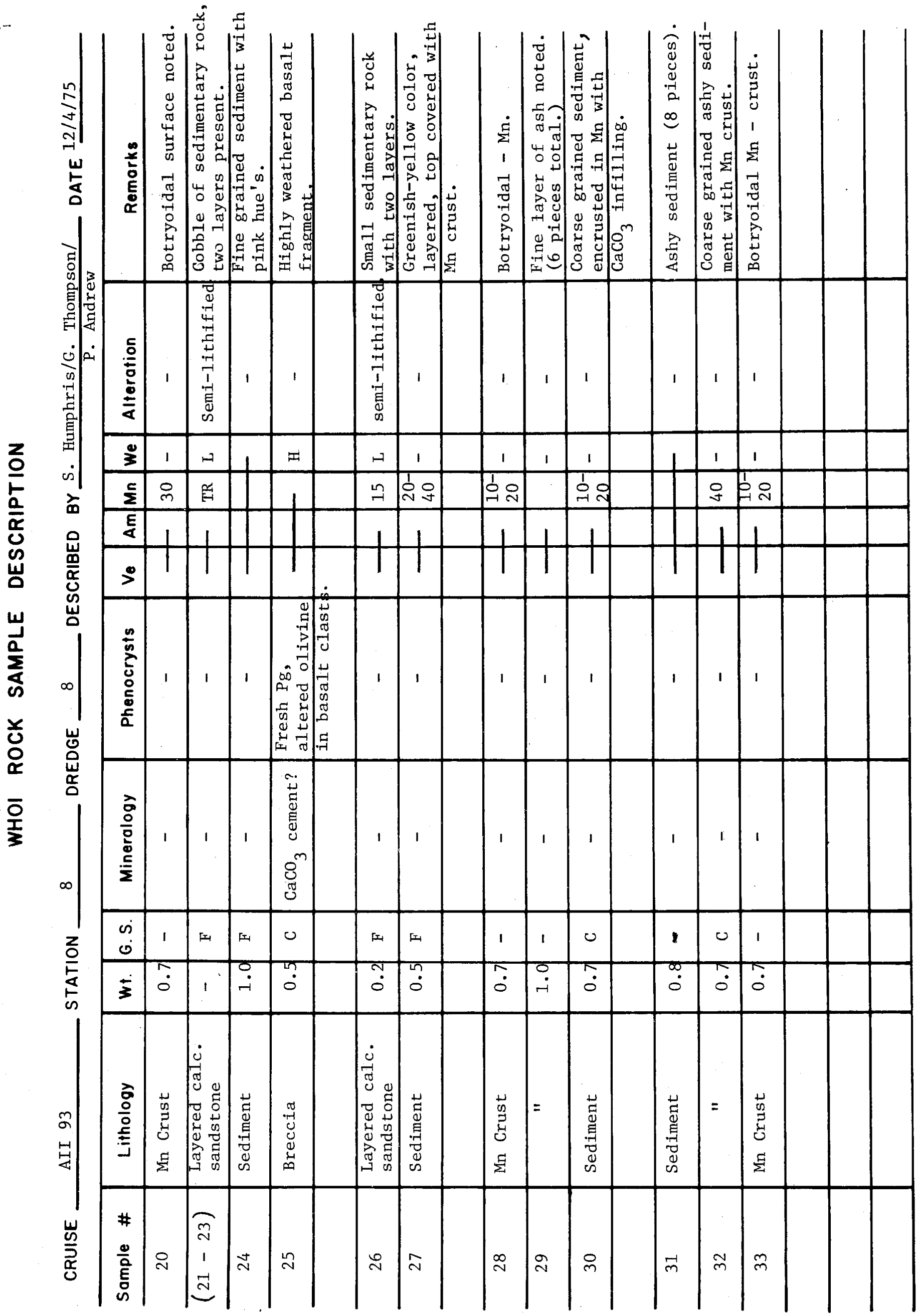




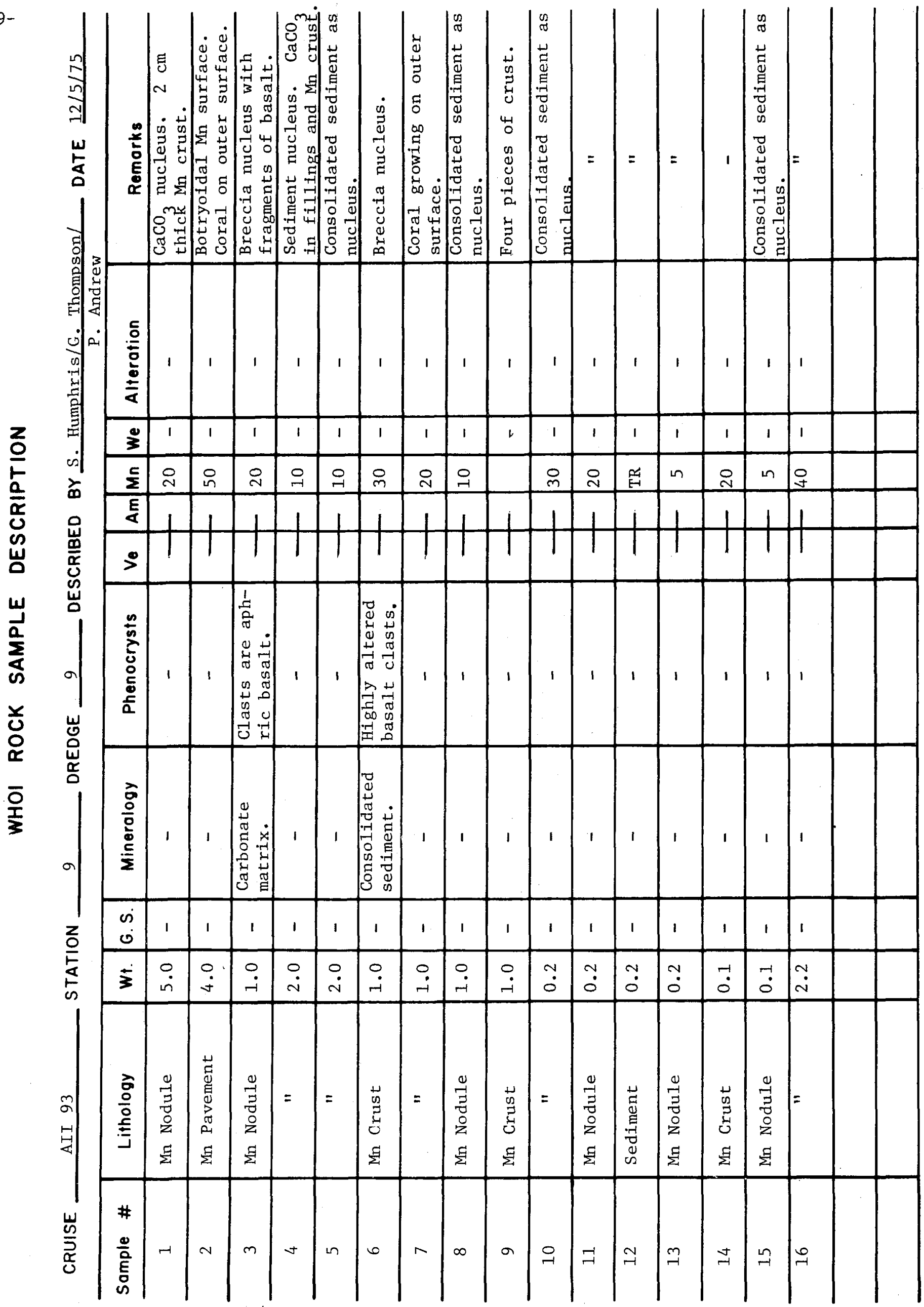




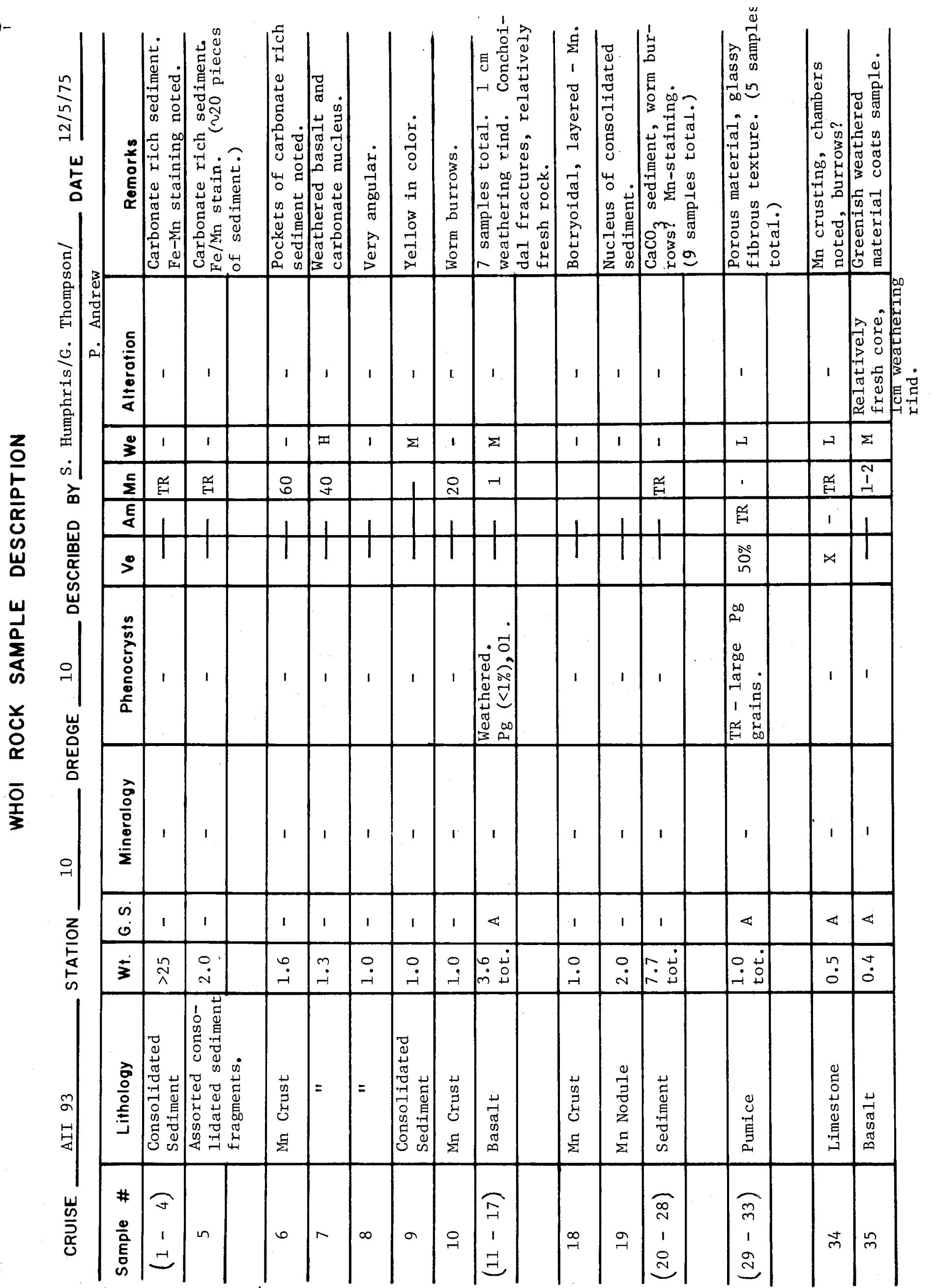


$-161-$

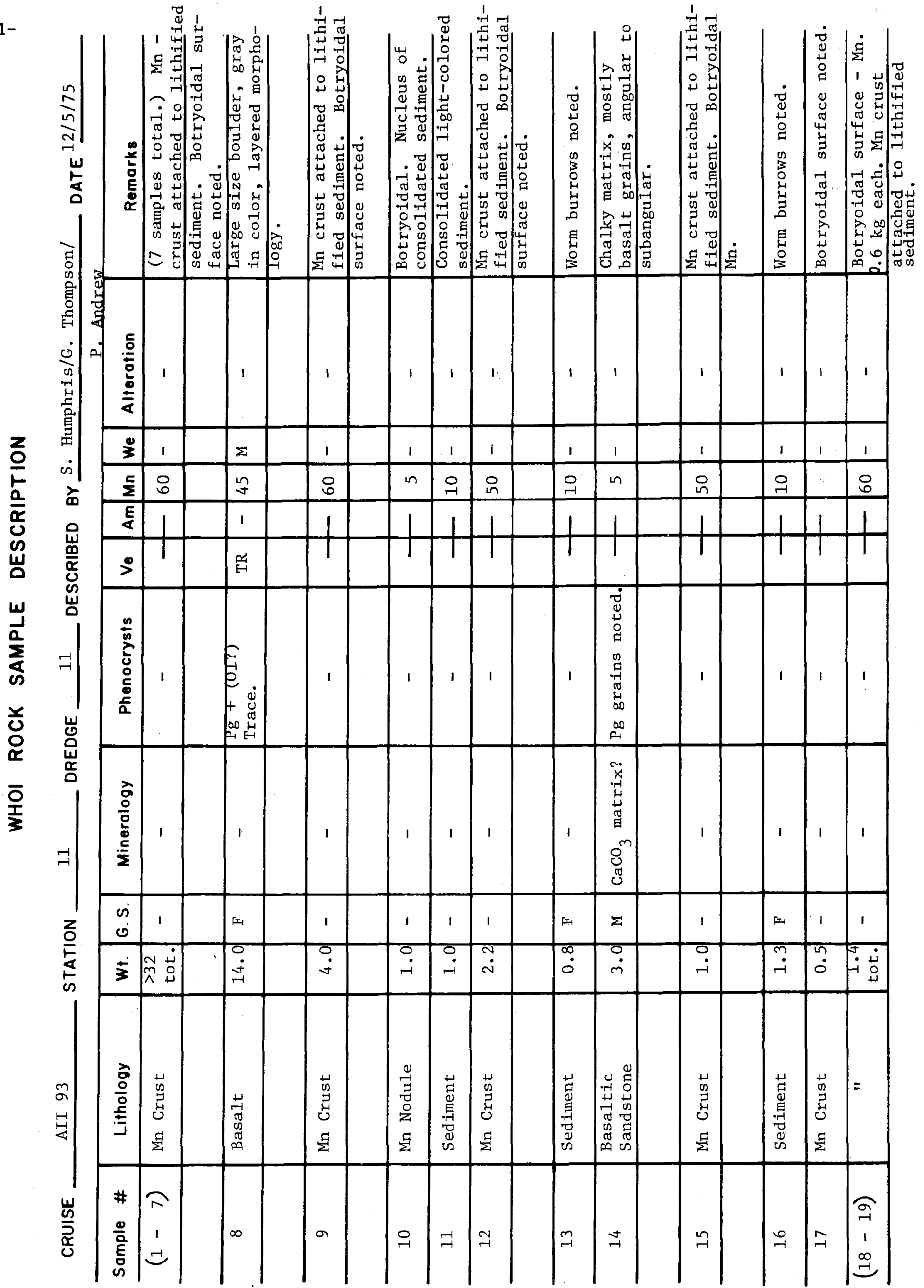




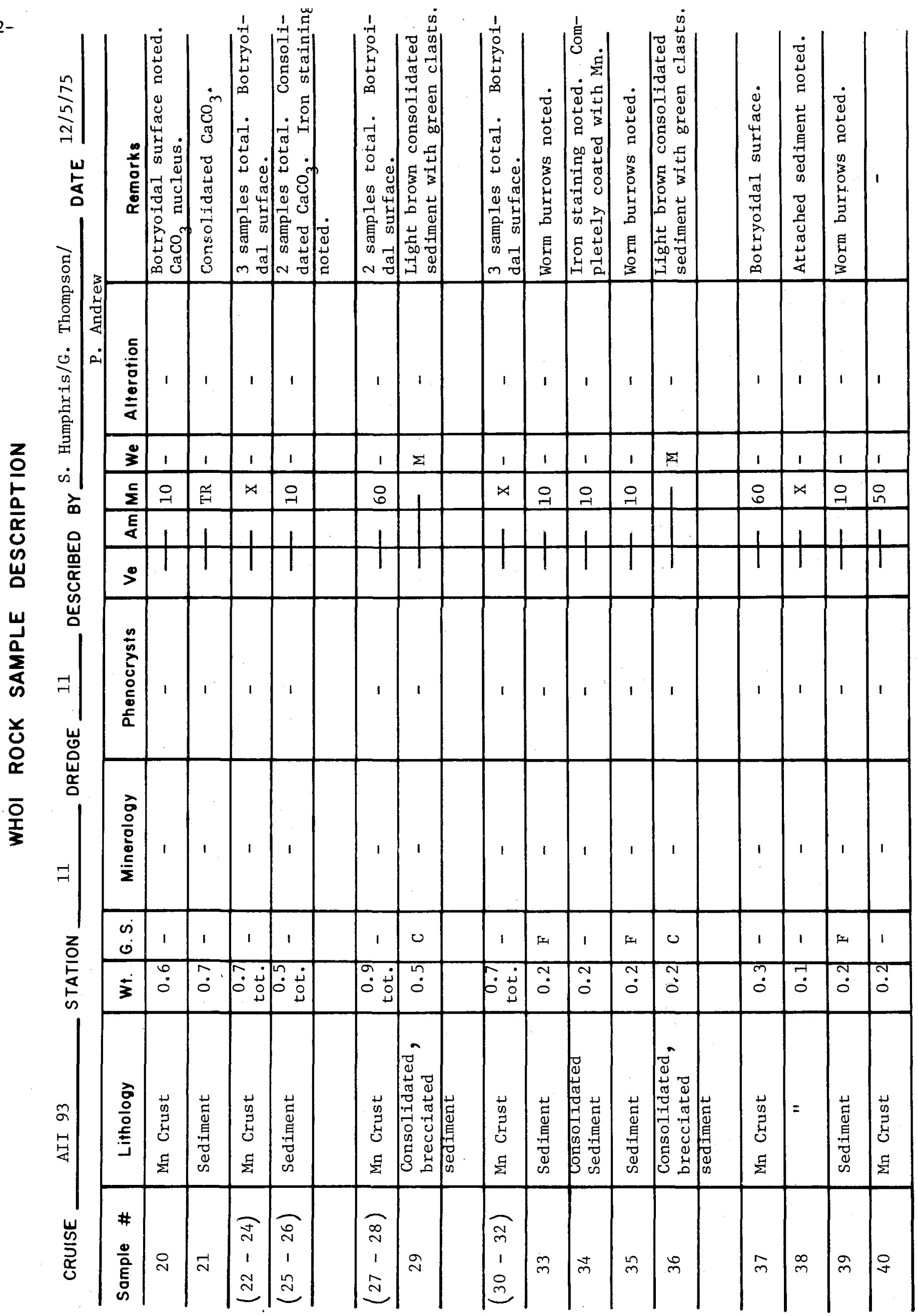




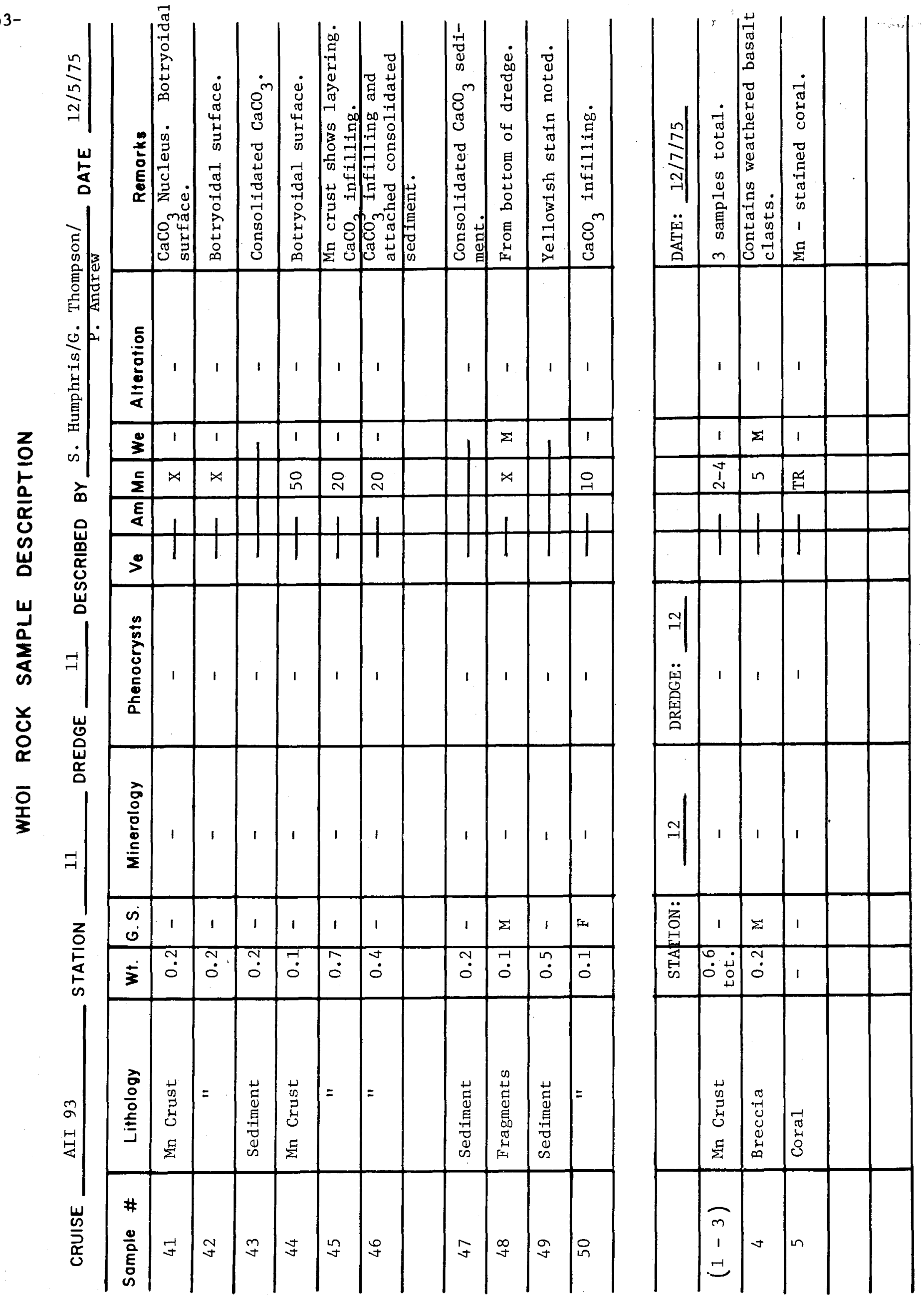




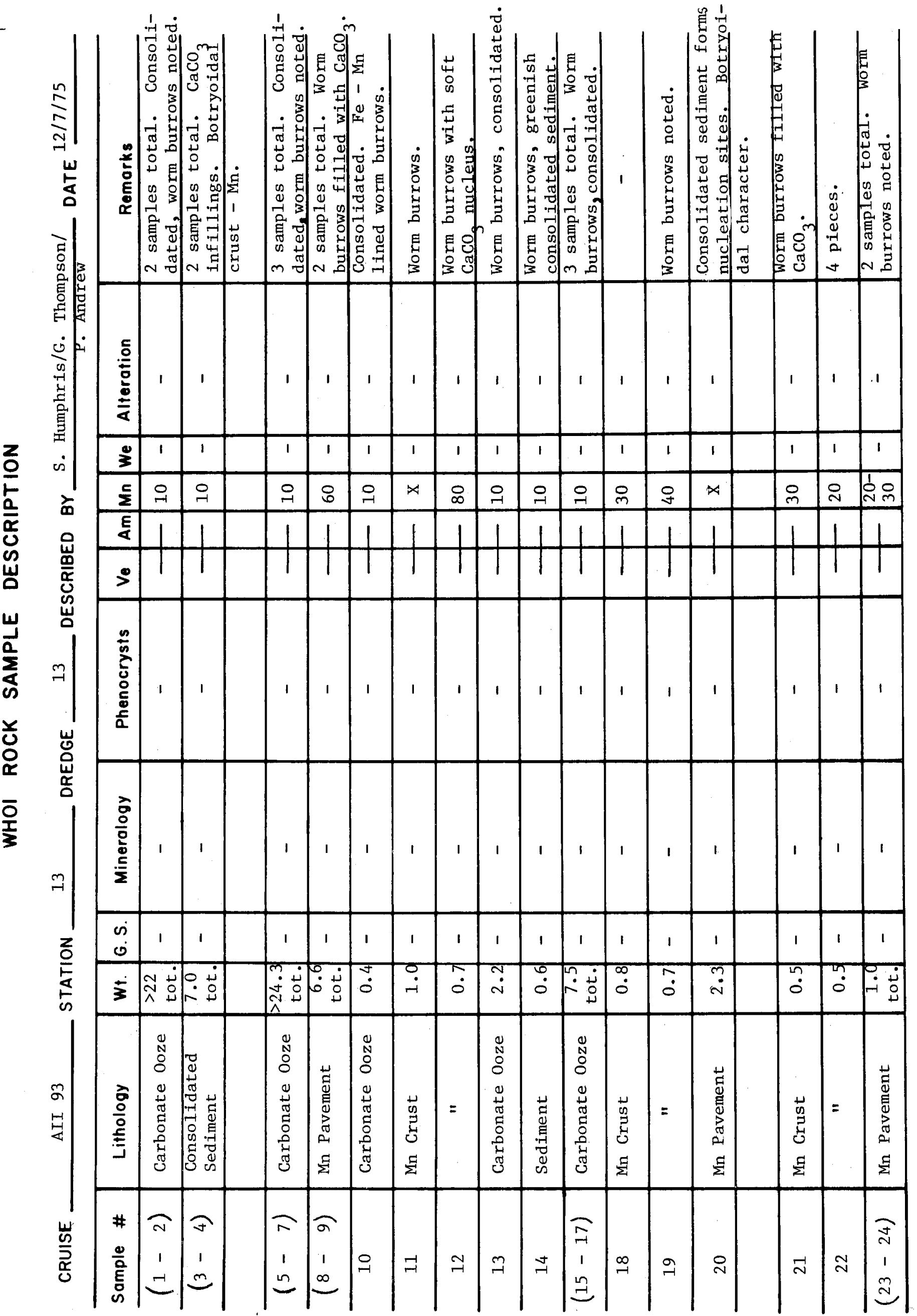


$-165-$

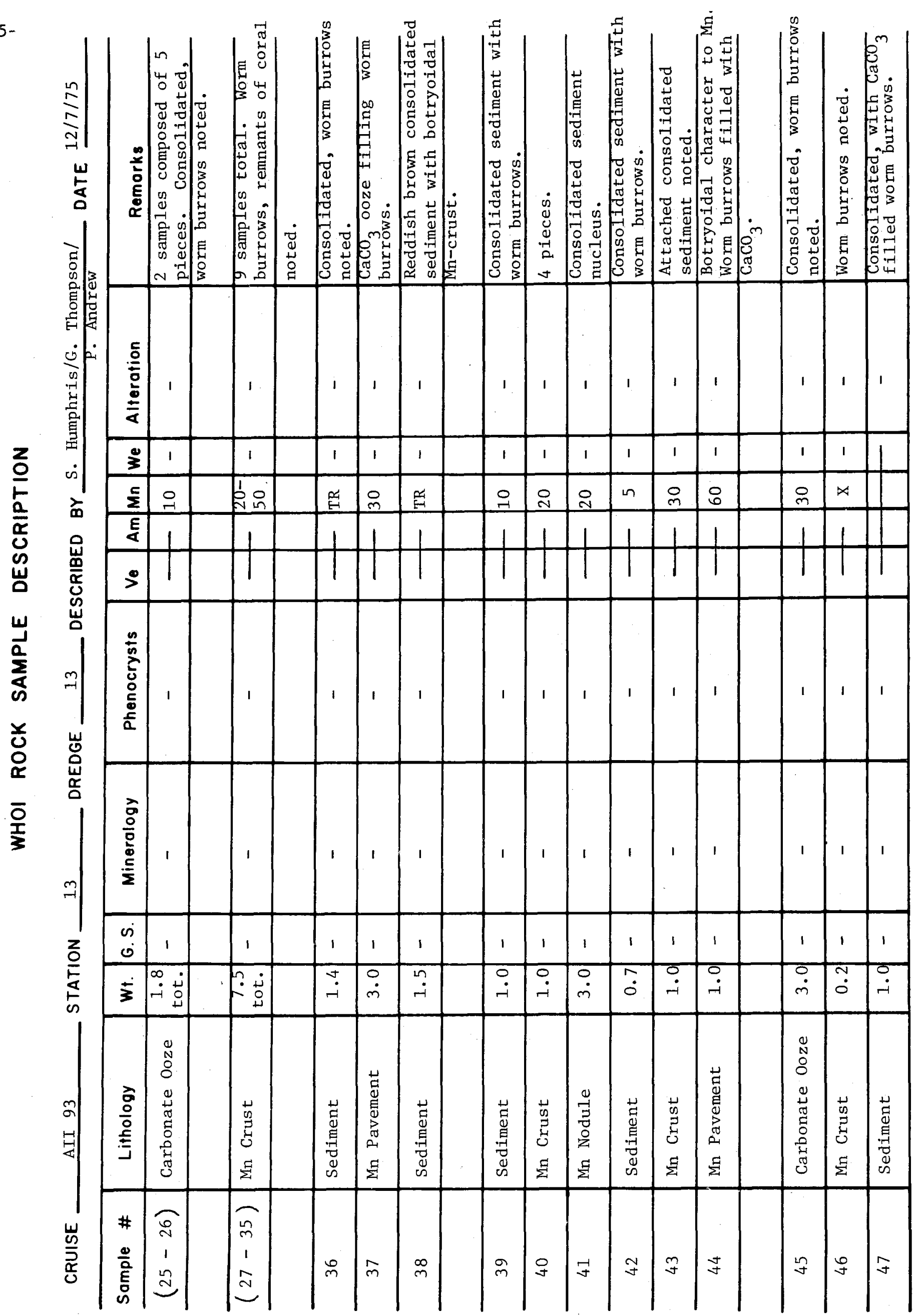




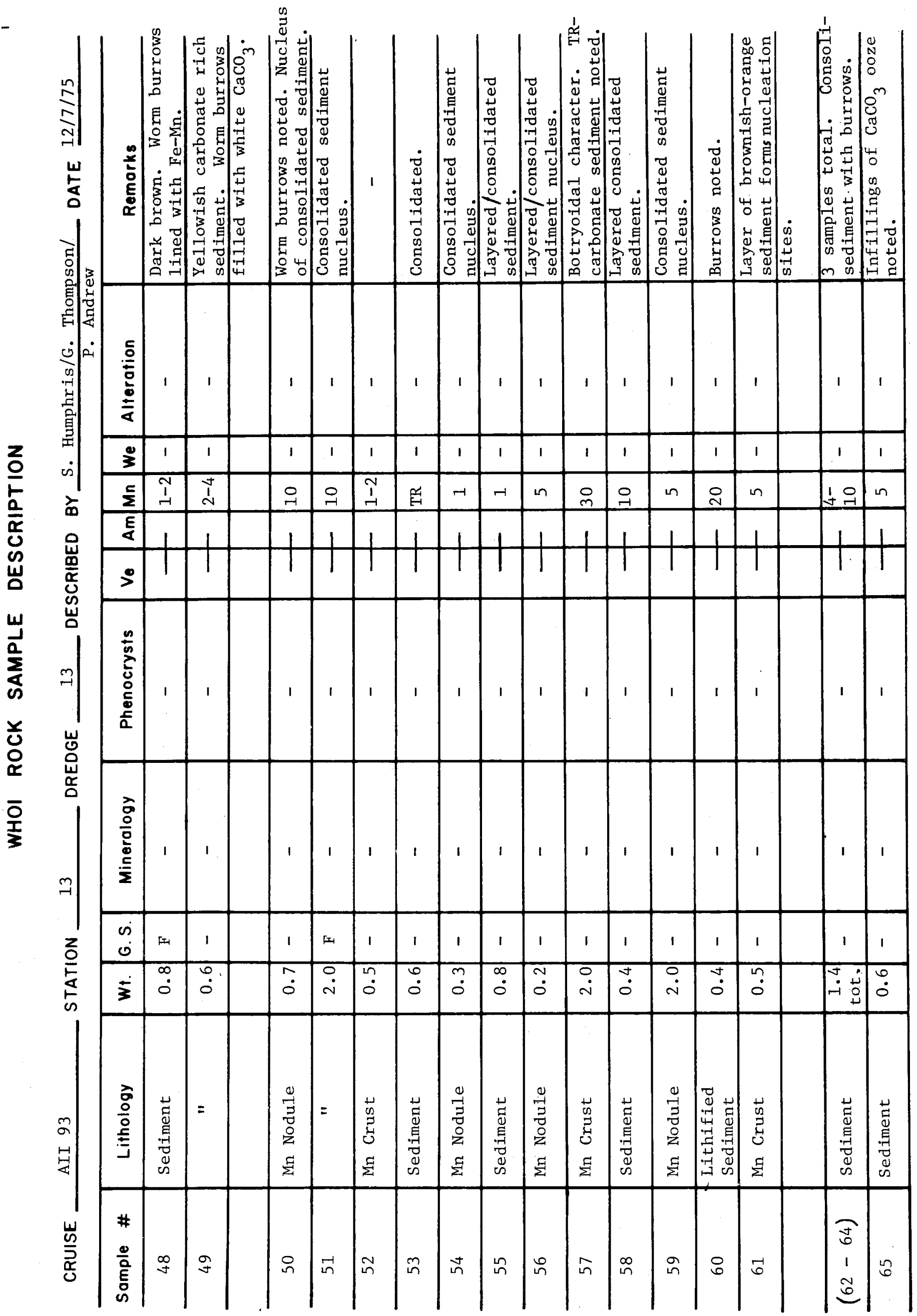




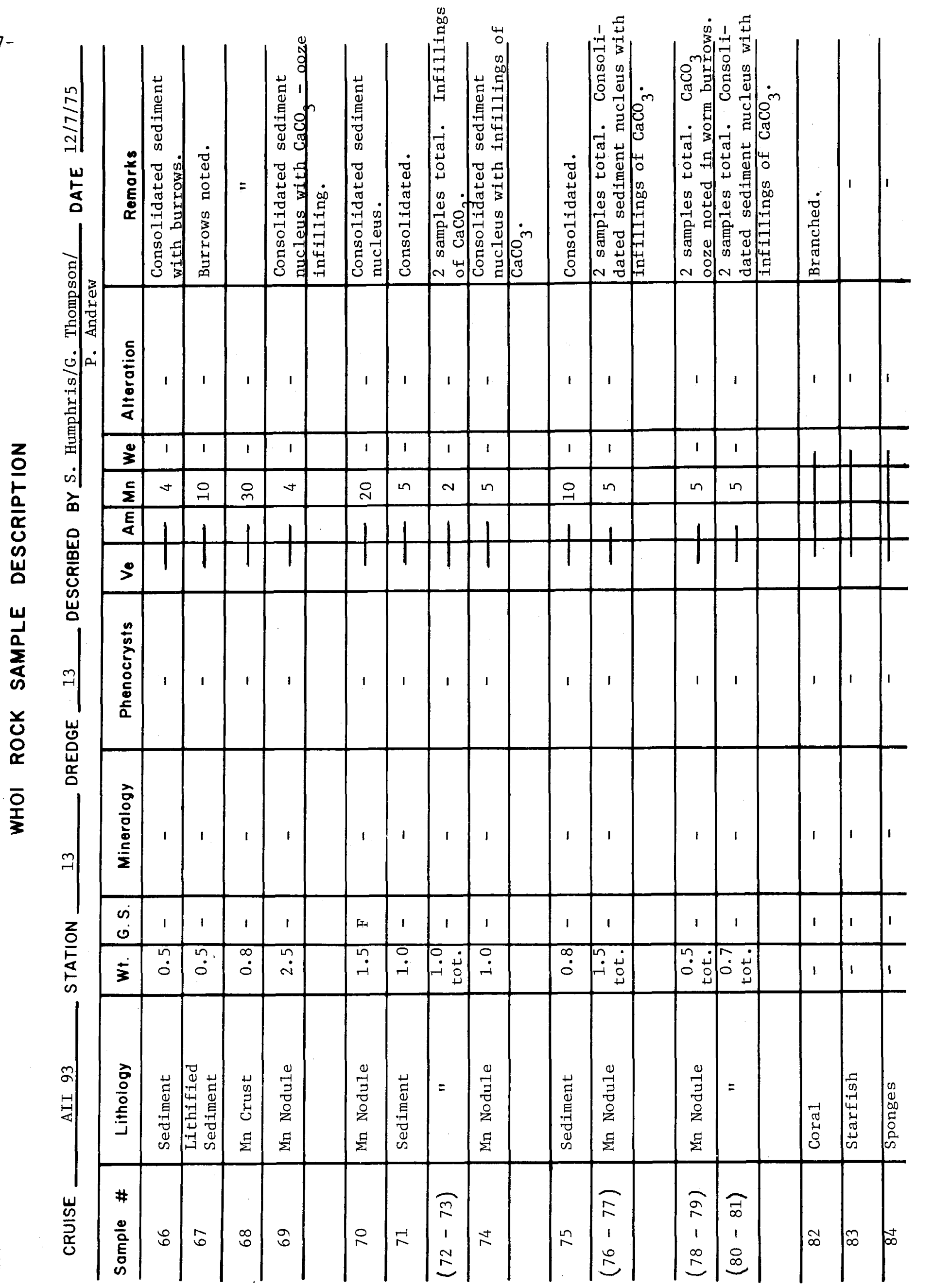




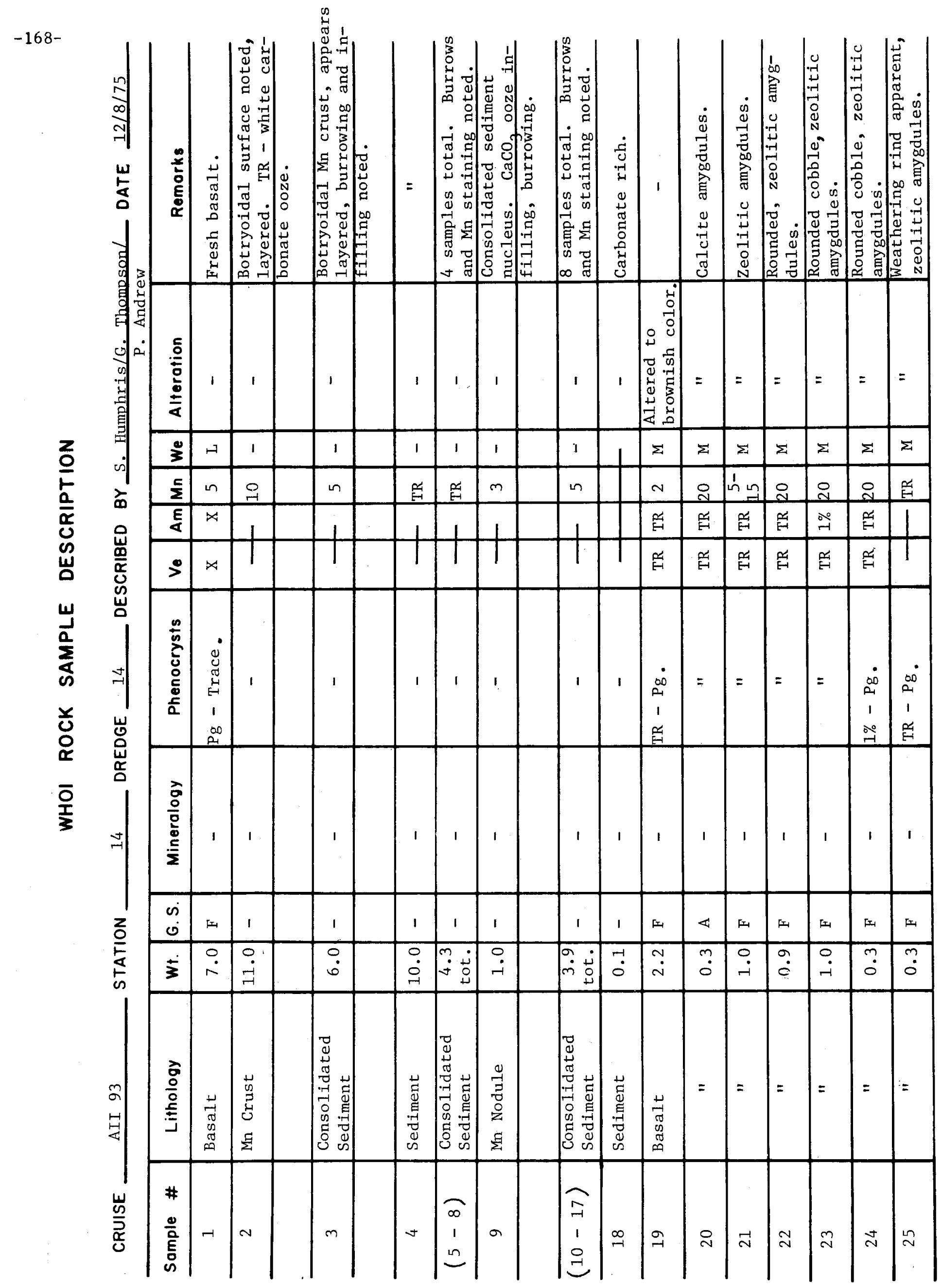




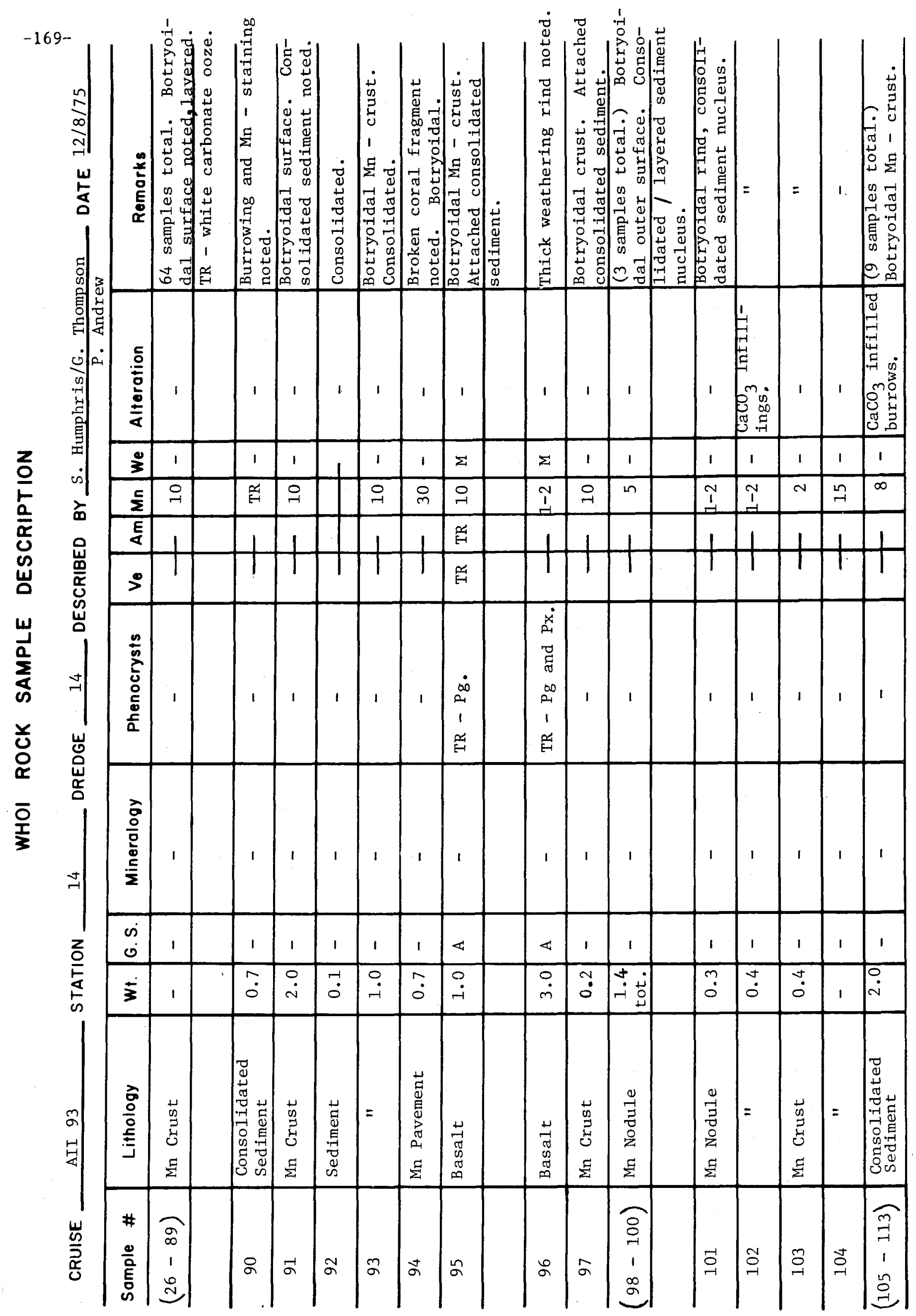


$-170$

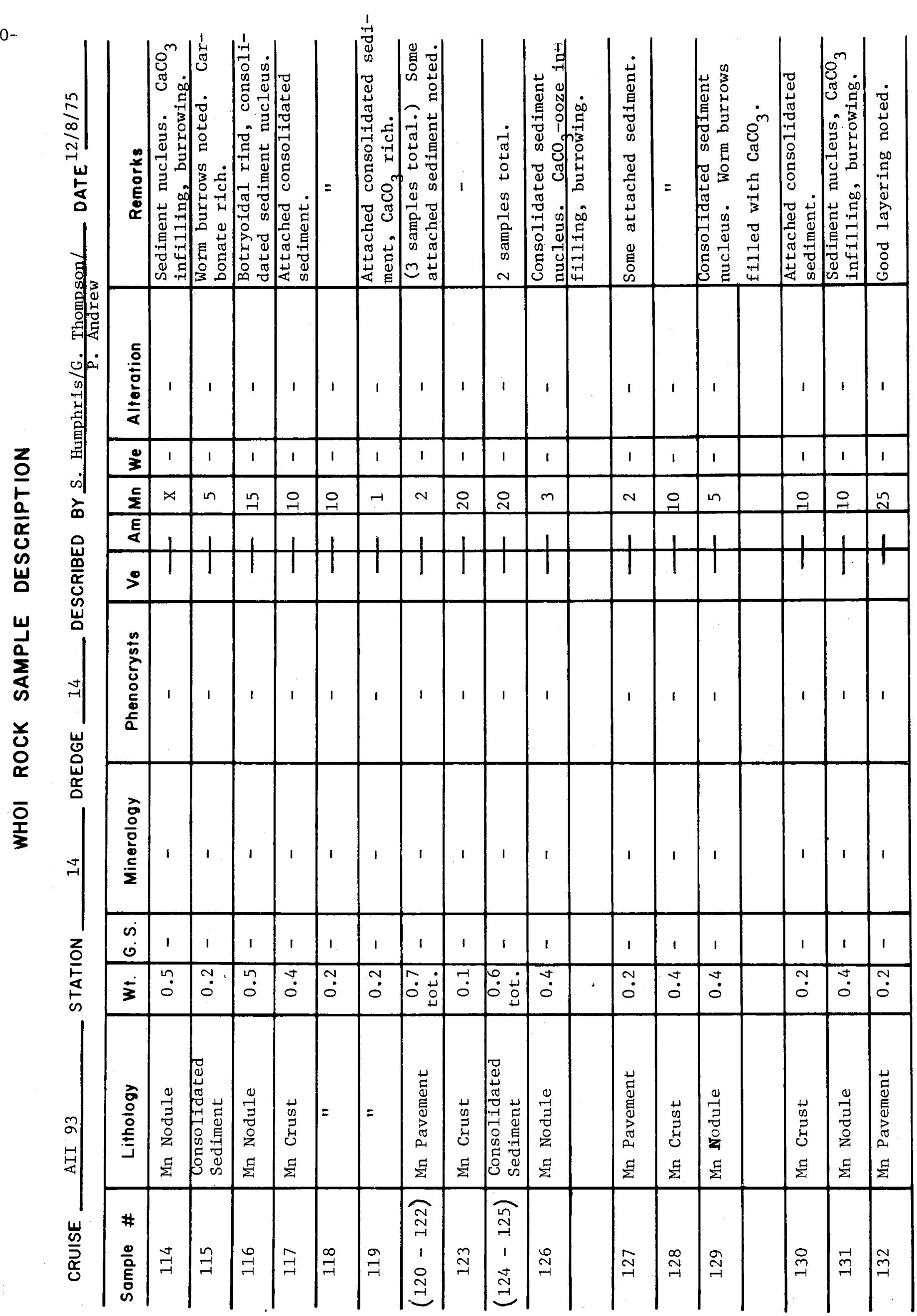




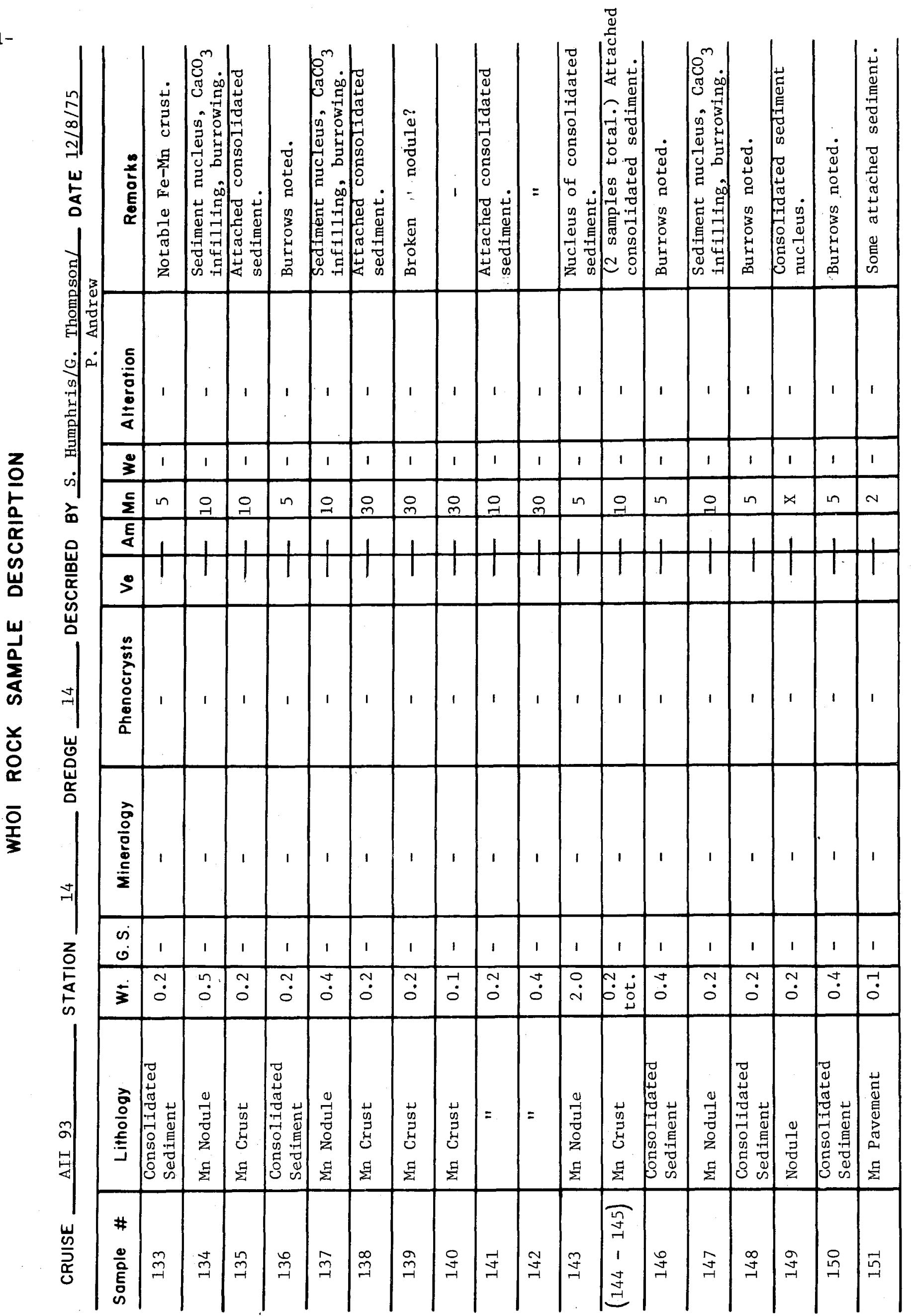


$-172-$

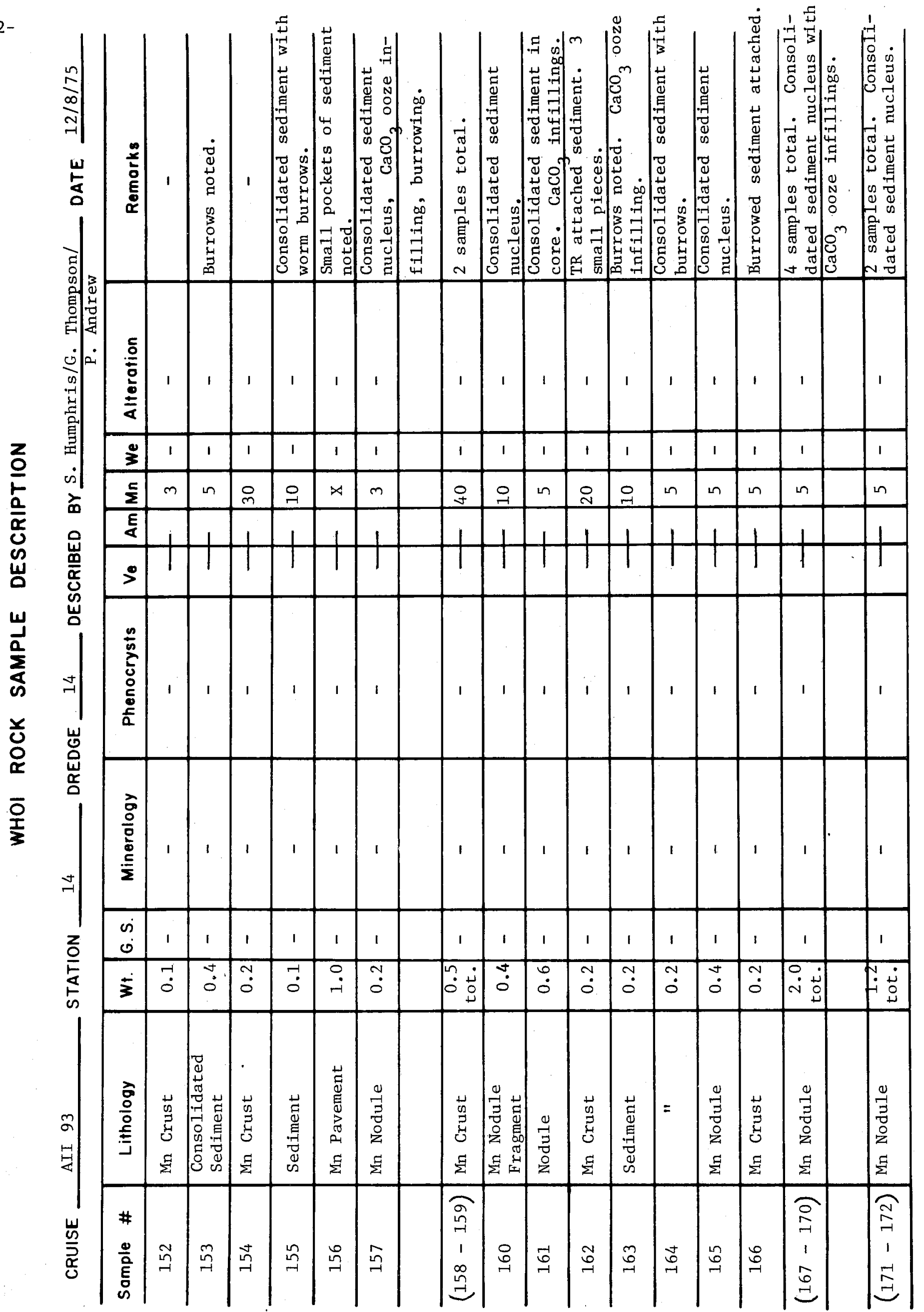




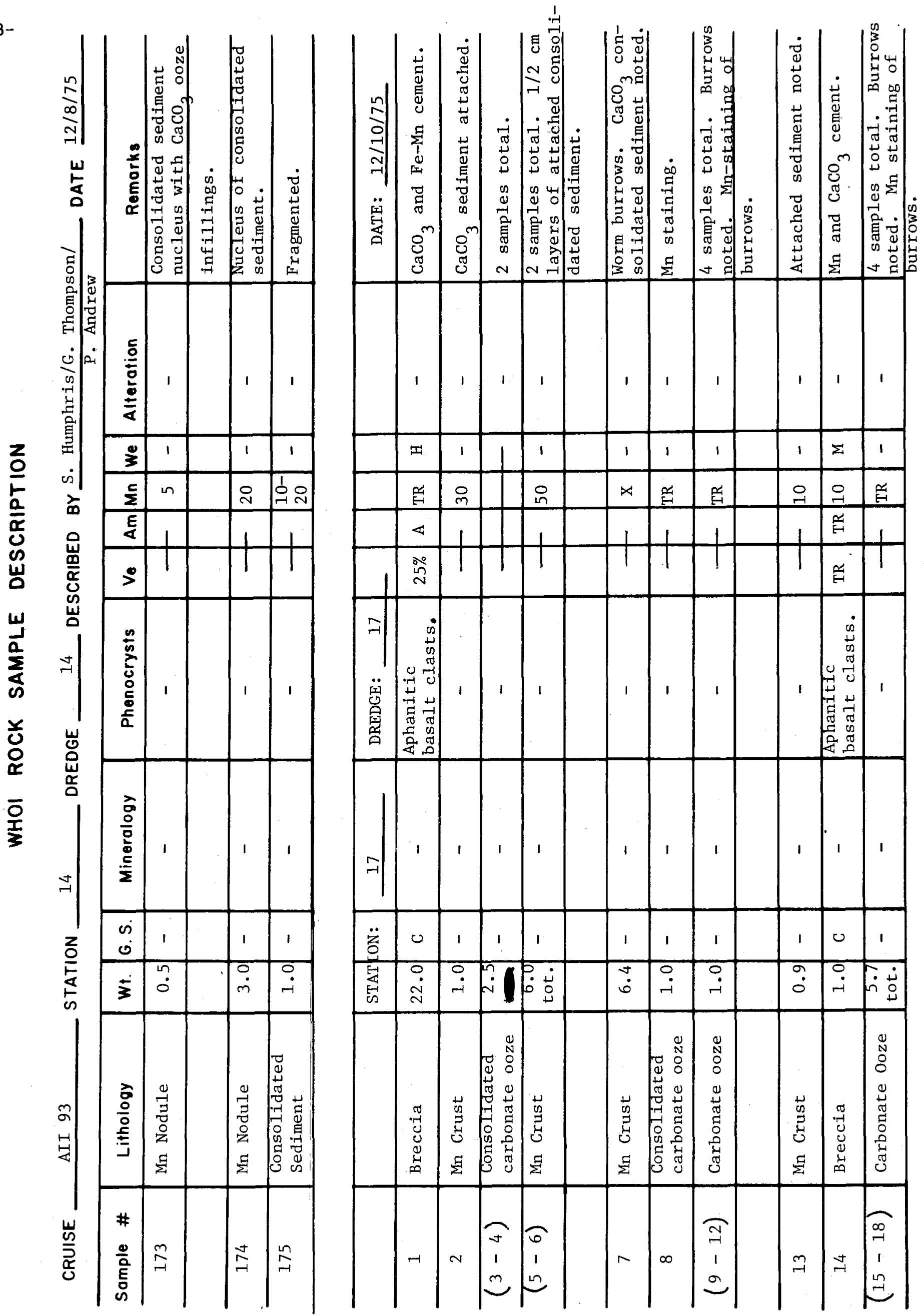


$-174$

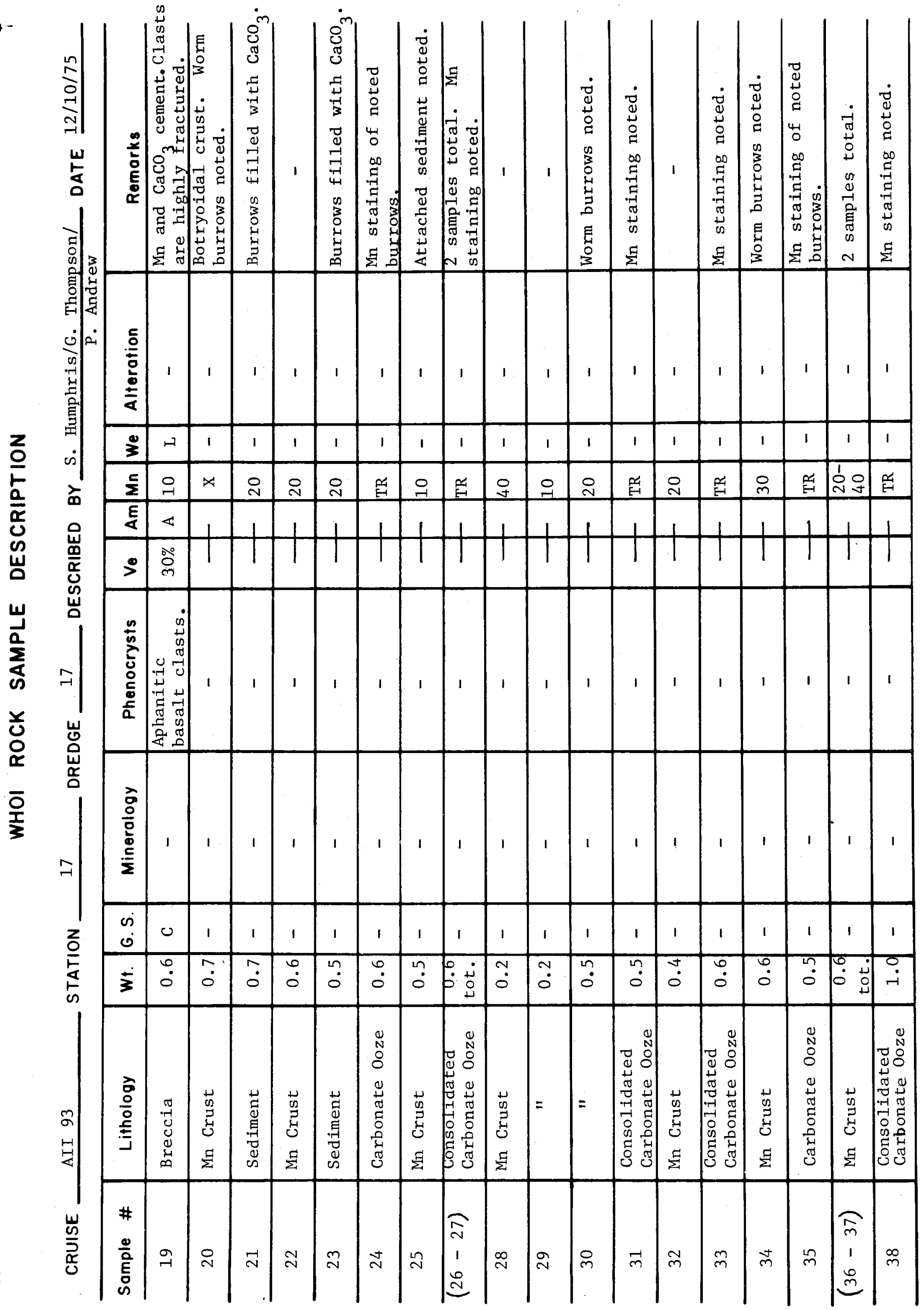




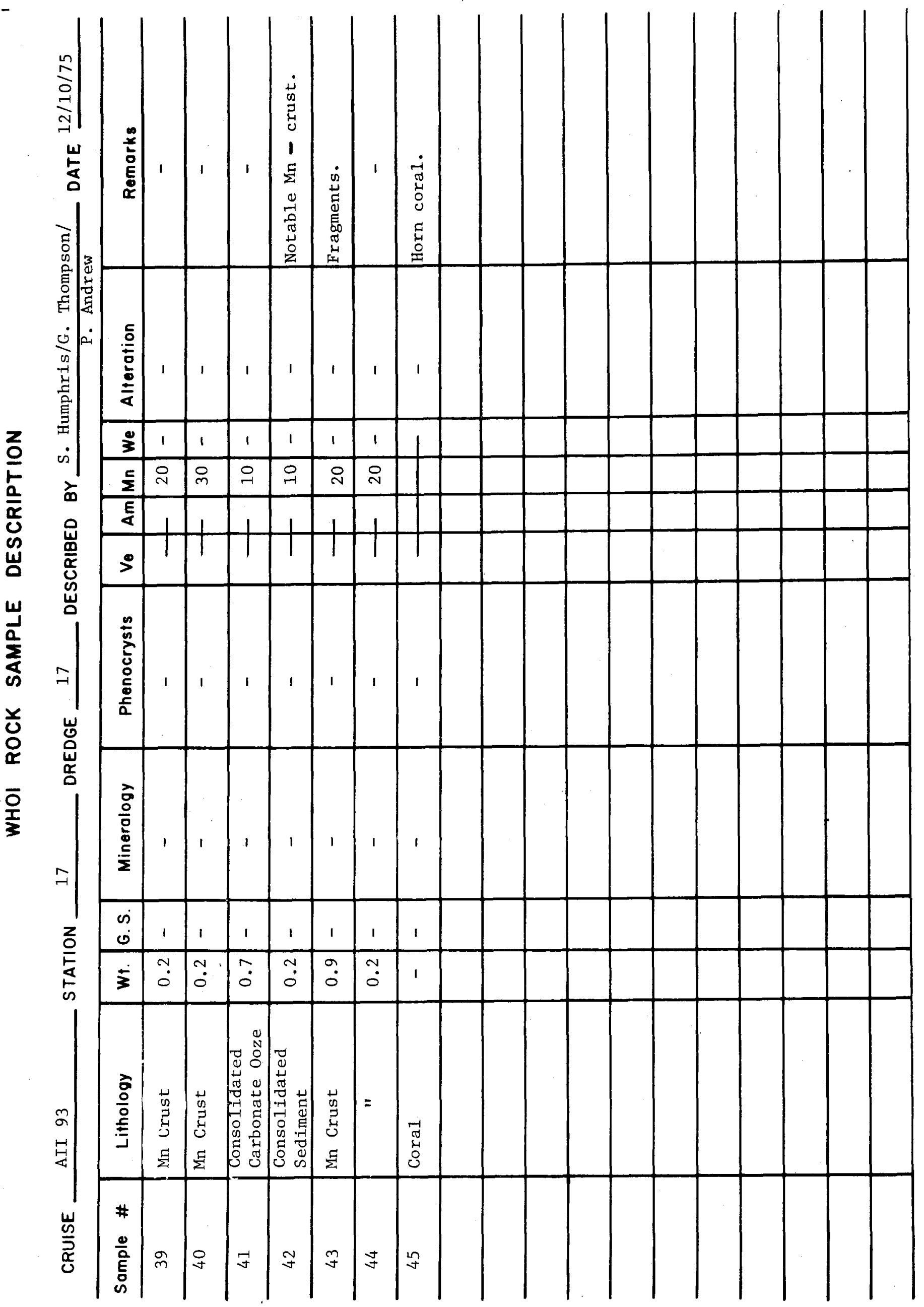




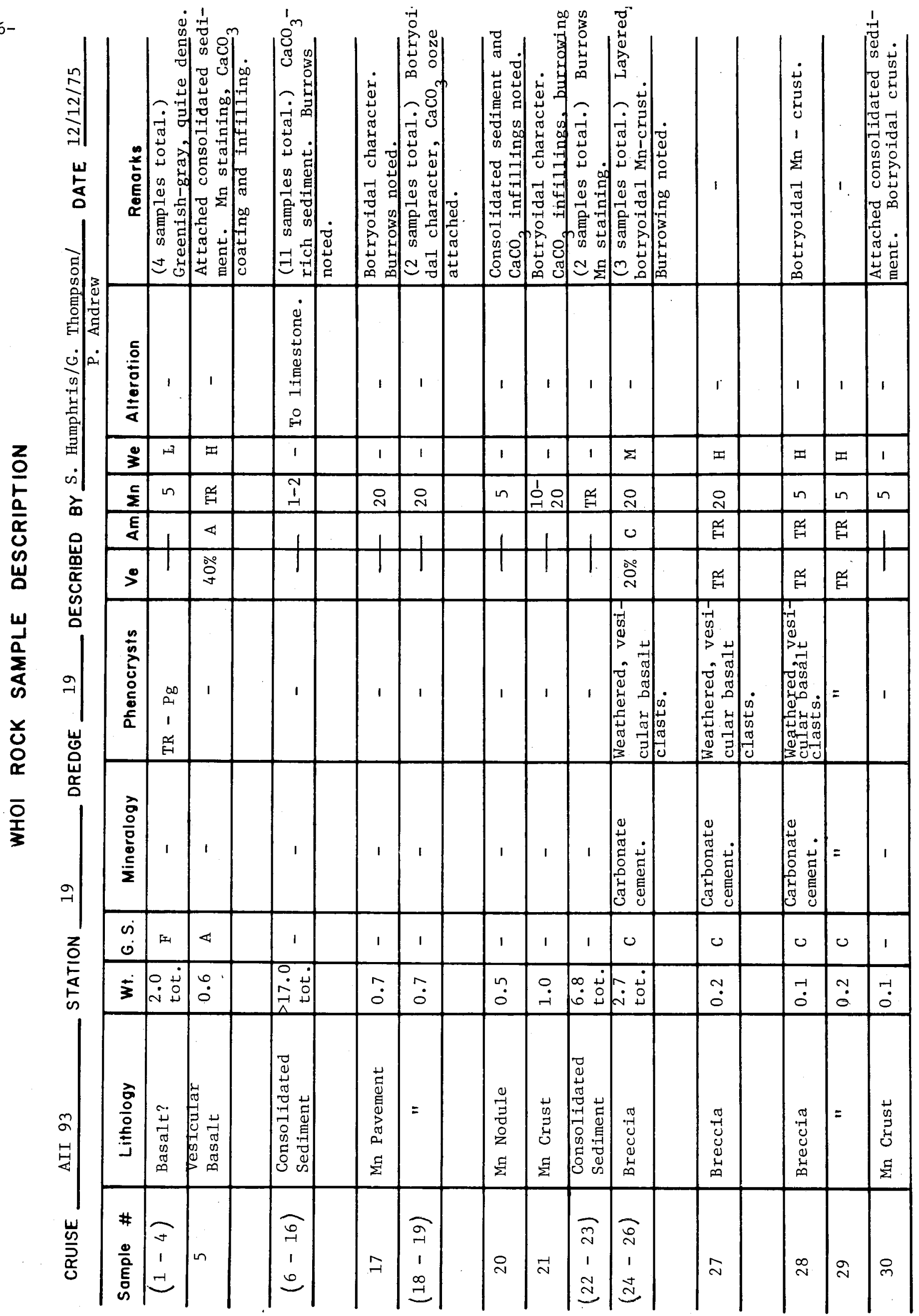


$-177-$

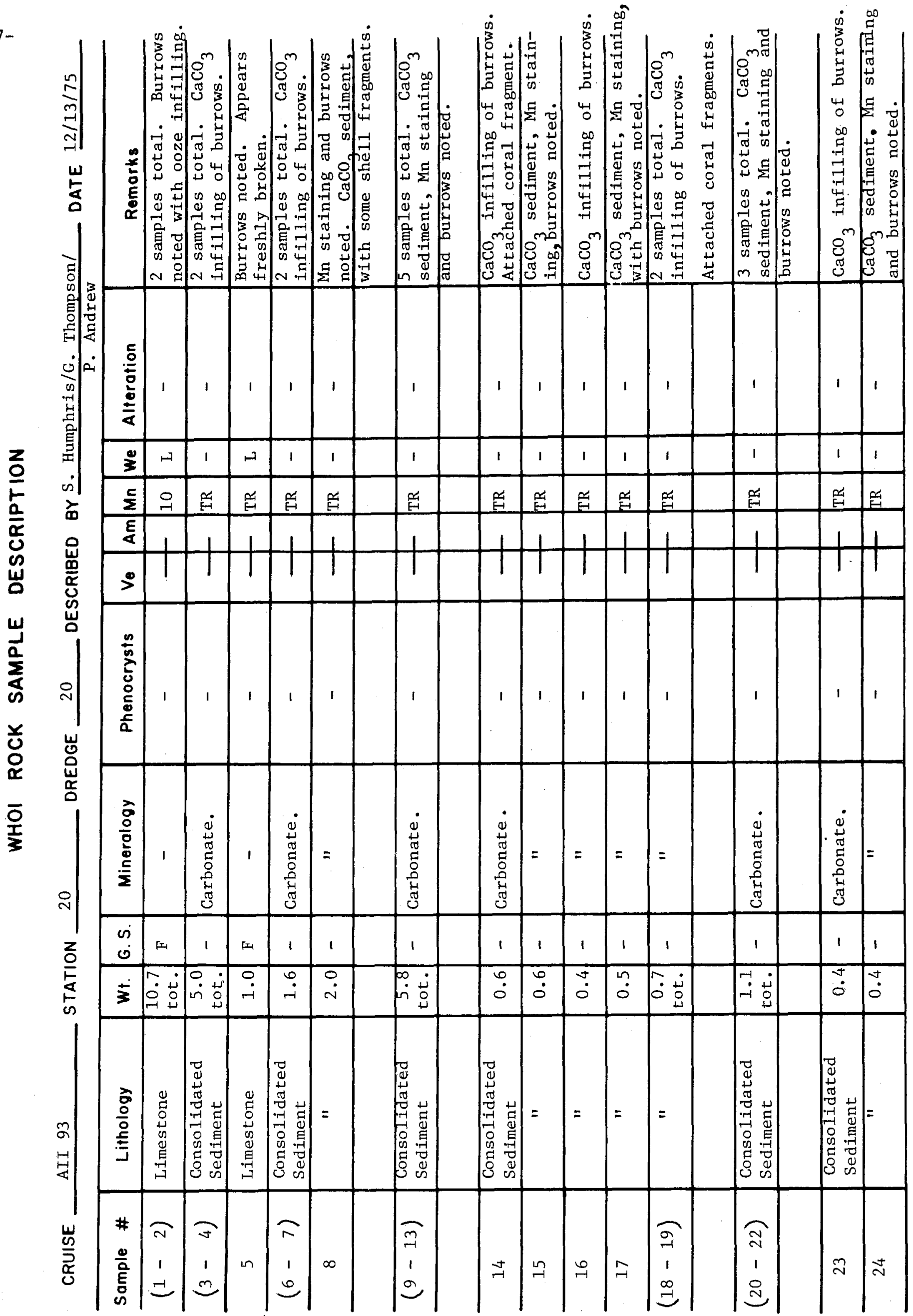


$-178$

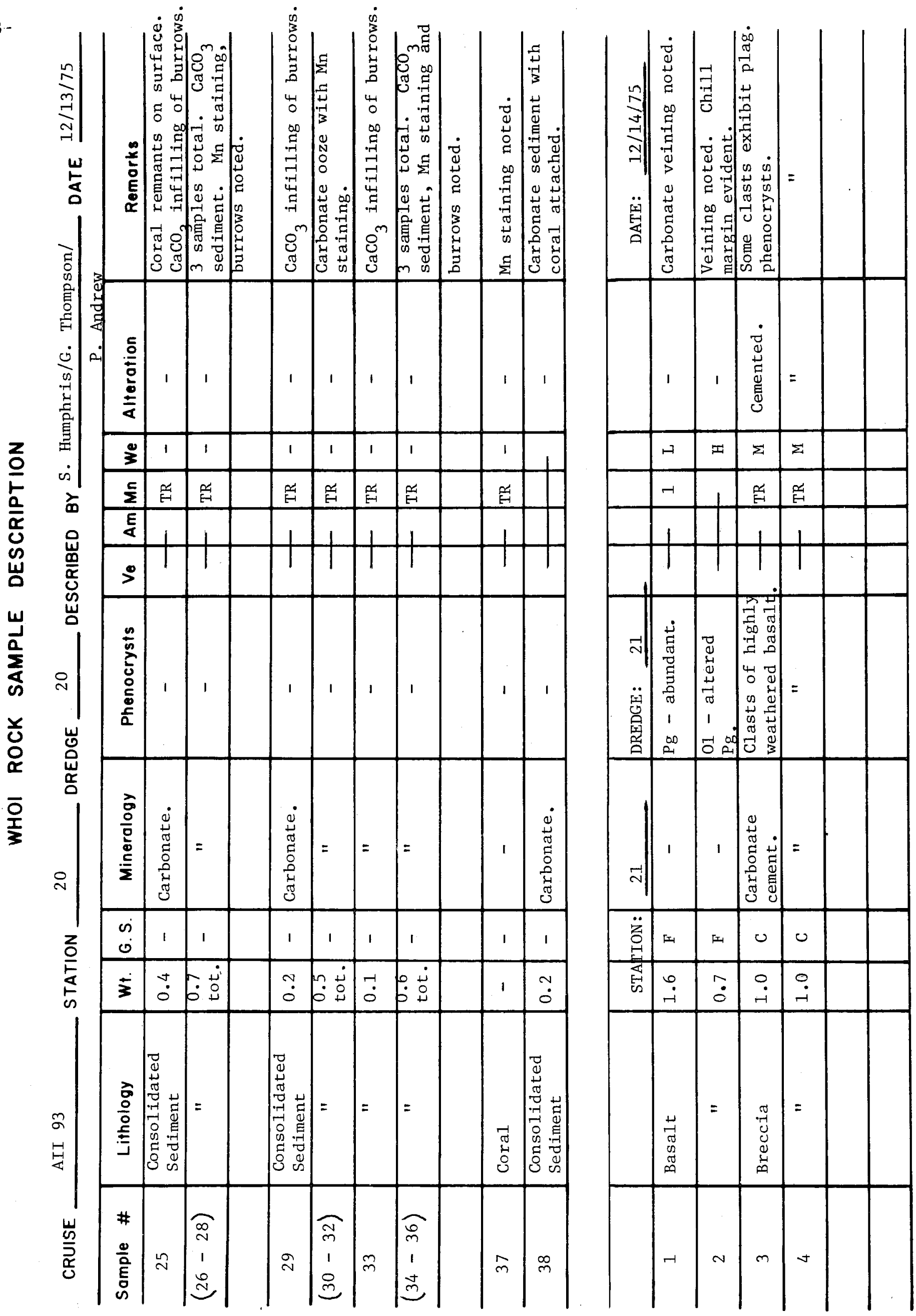




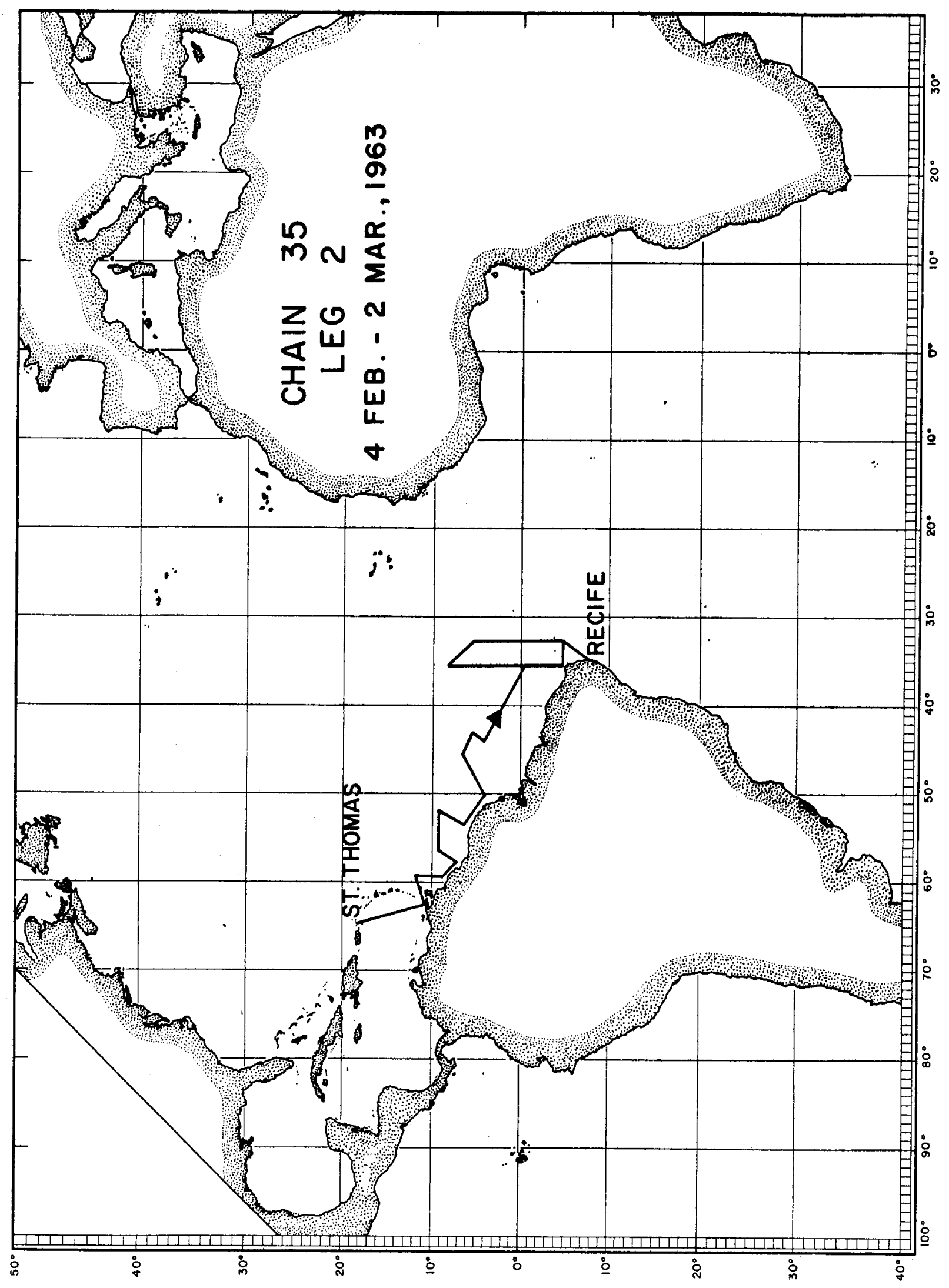




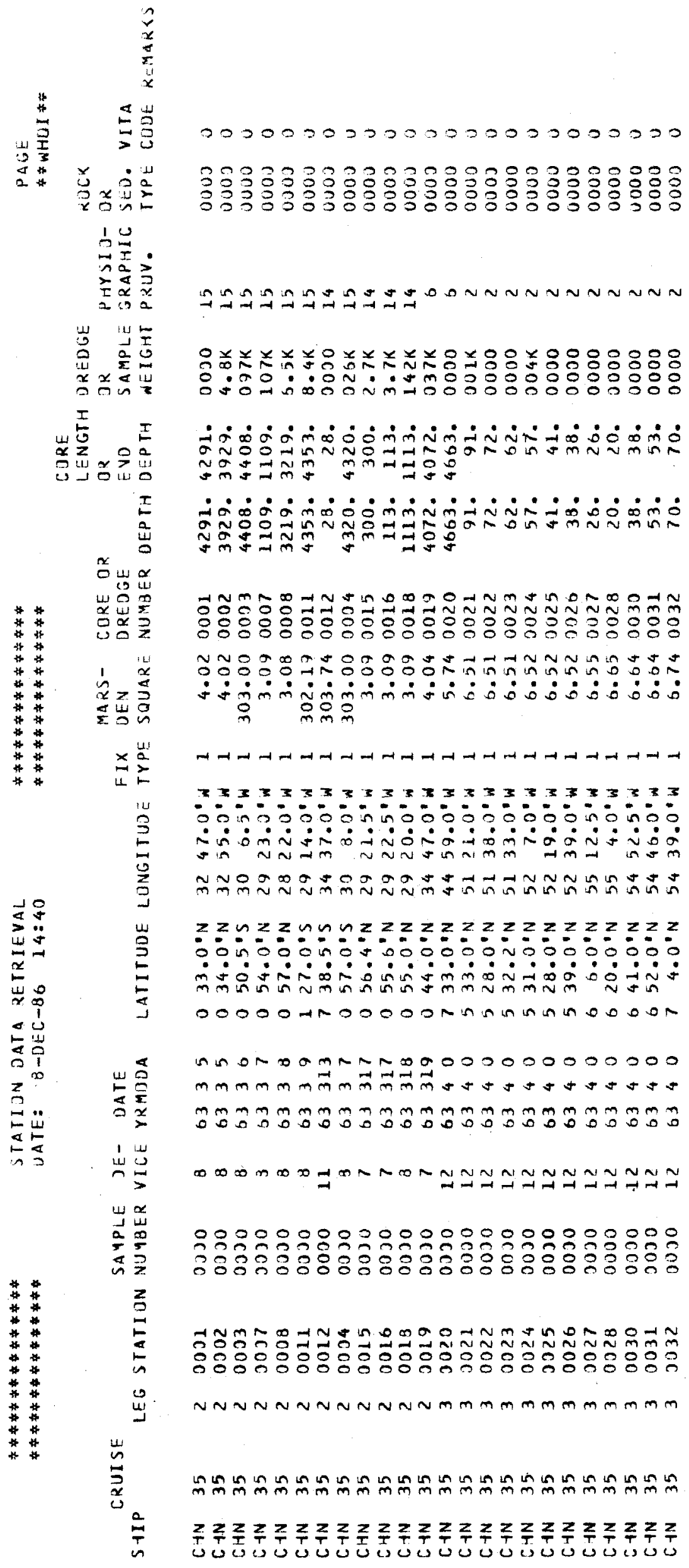




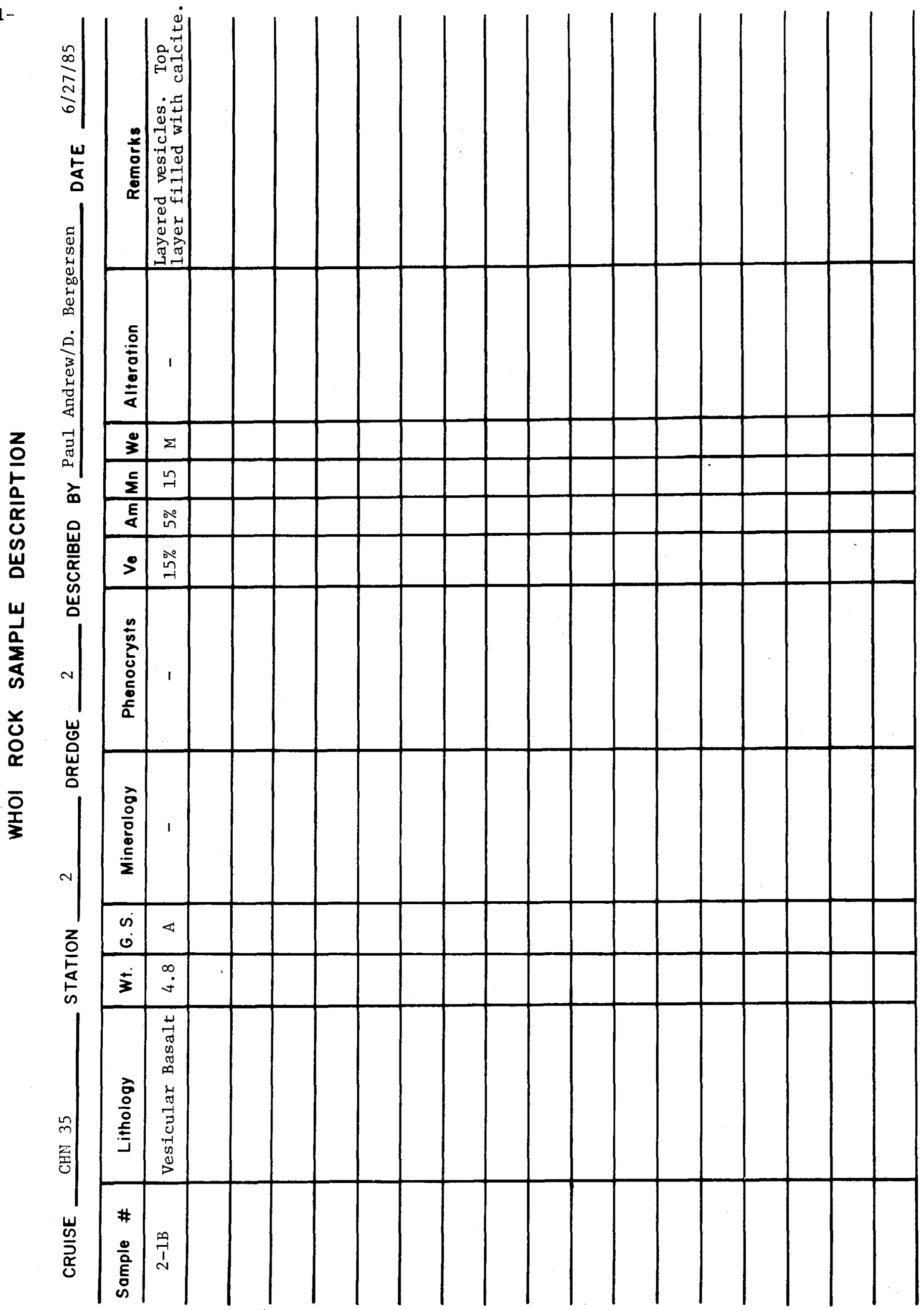




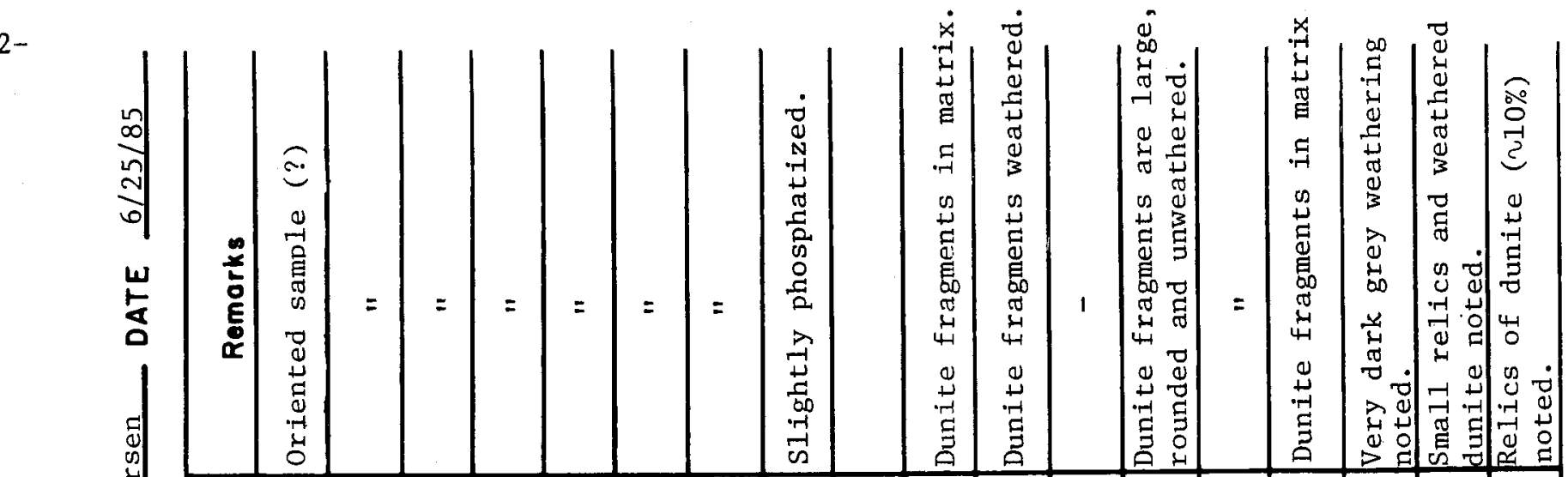

음

$\frac{2}{0}$

山岀

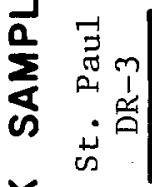

\begin{tabular}{l} 
U \\
0 \\
0 \\
\hline
\end{tabular}

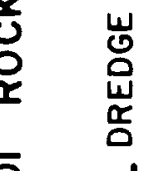

올

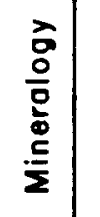

$\frac{n}{5}$
$\frac{2}{2}$
0
$\frac{0}{0}$
$\frac{c}{\alpha}$
$\frac{c}{2}$

ํㅗㅎำ

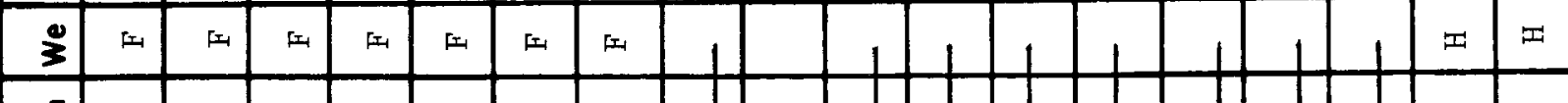

E

$\$$

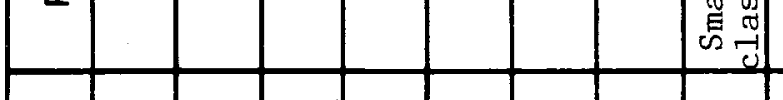

즘

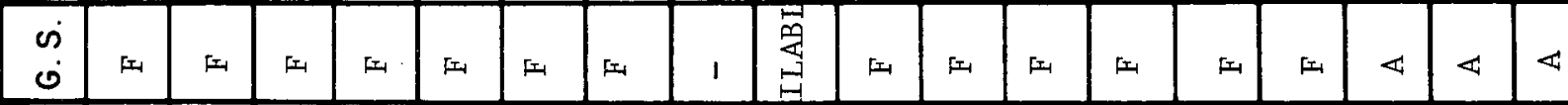

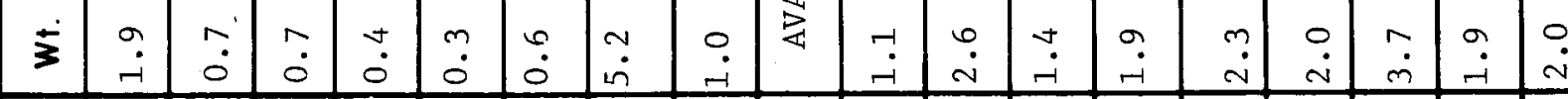

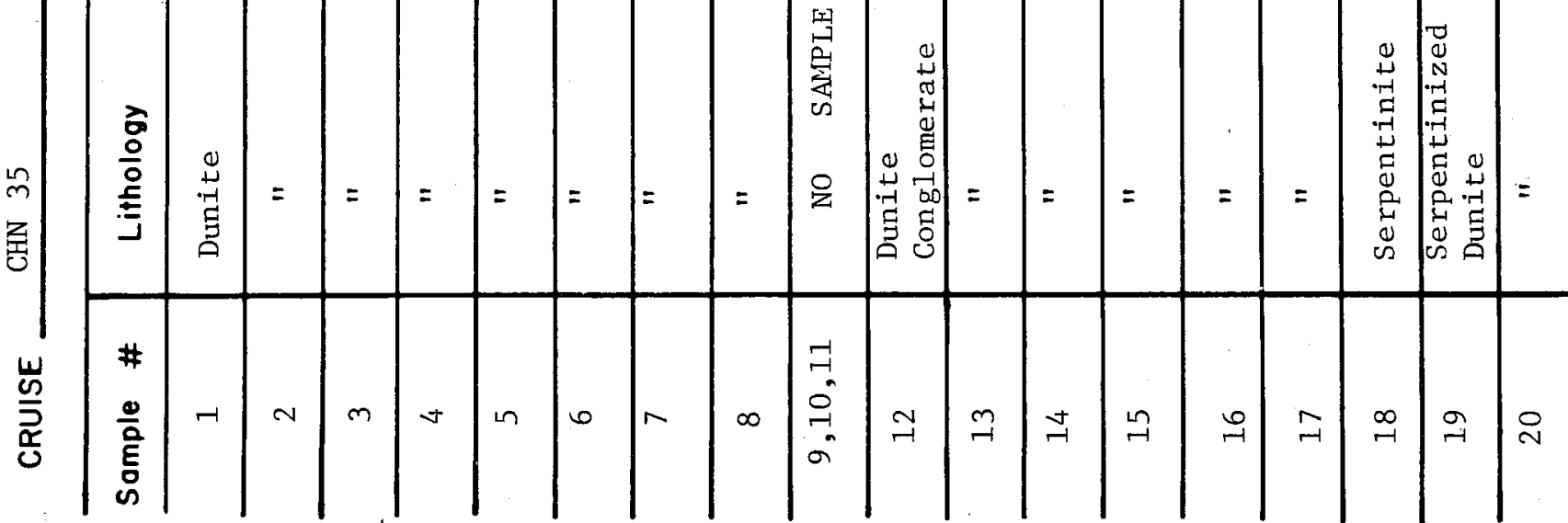


$-183$

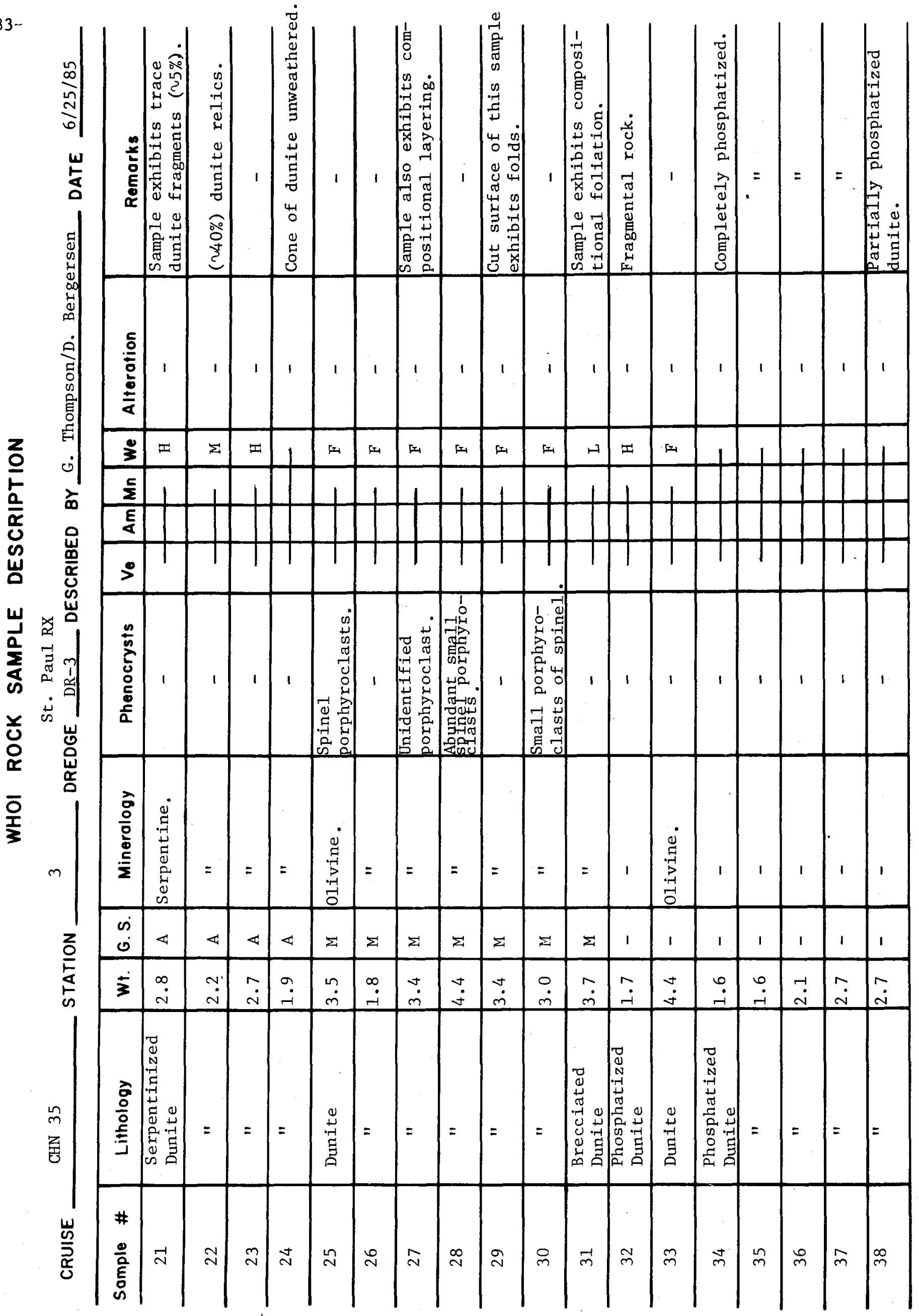




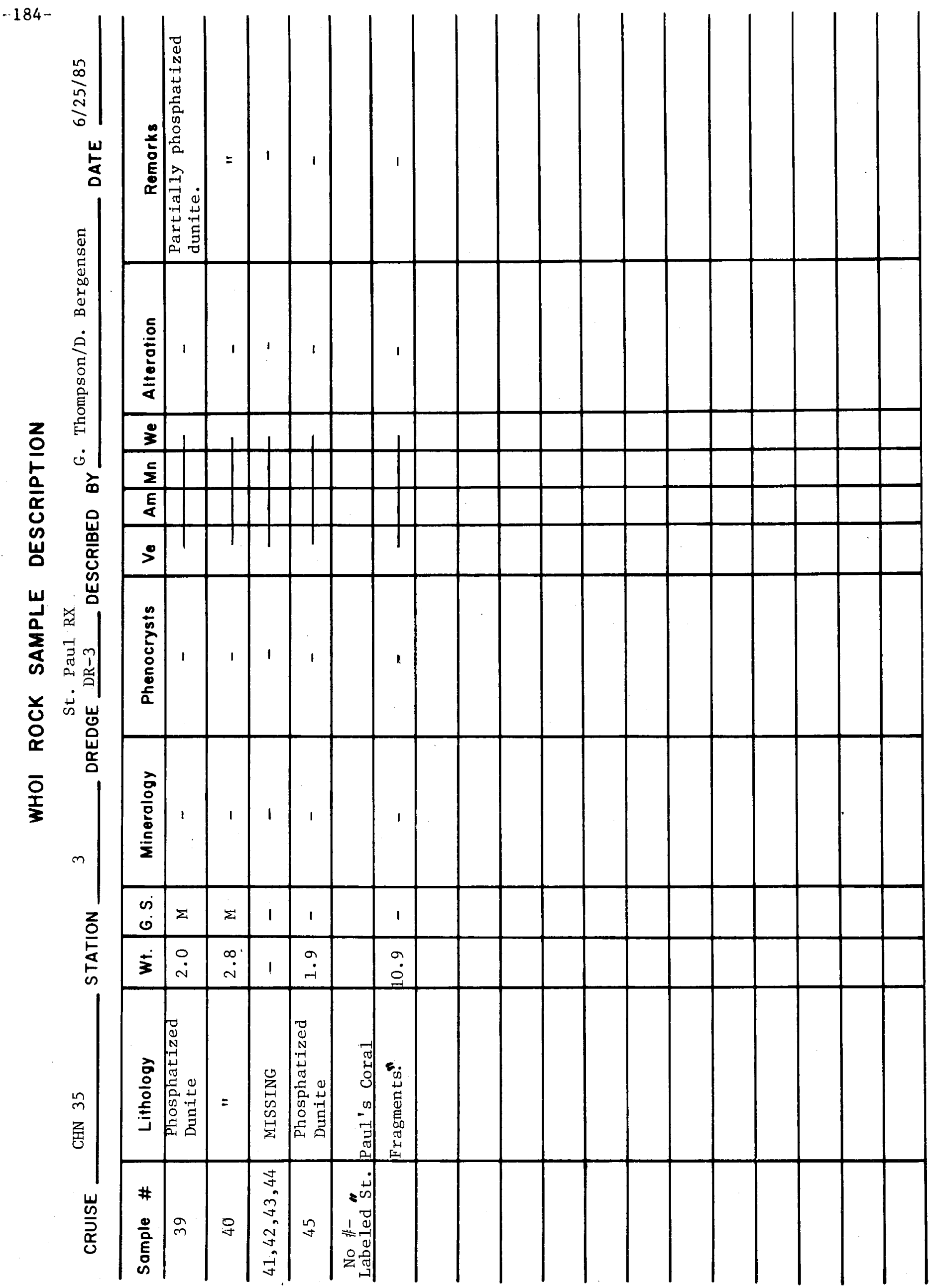




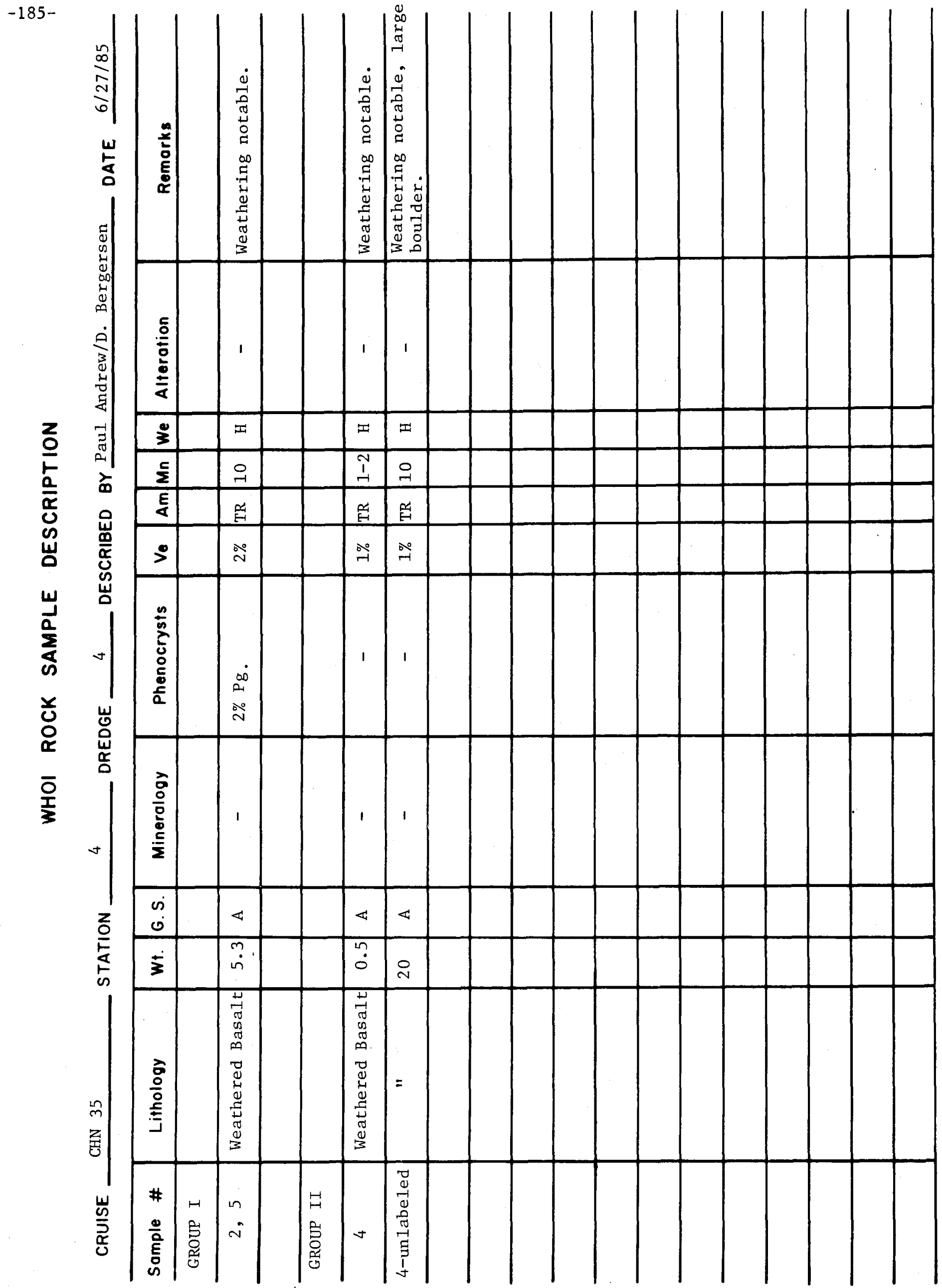




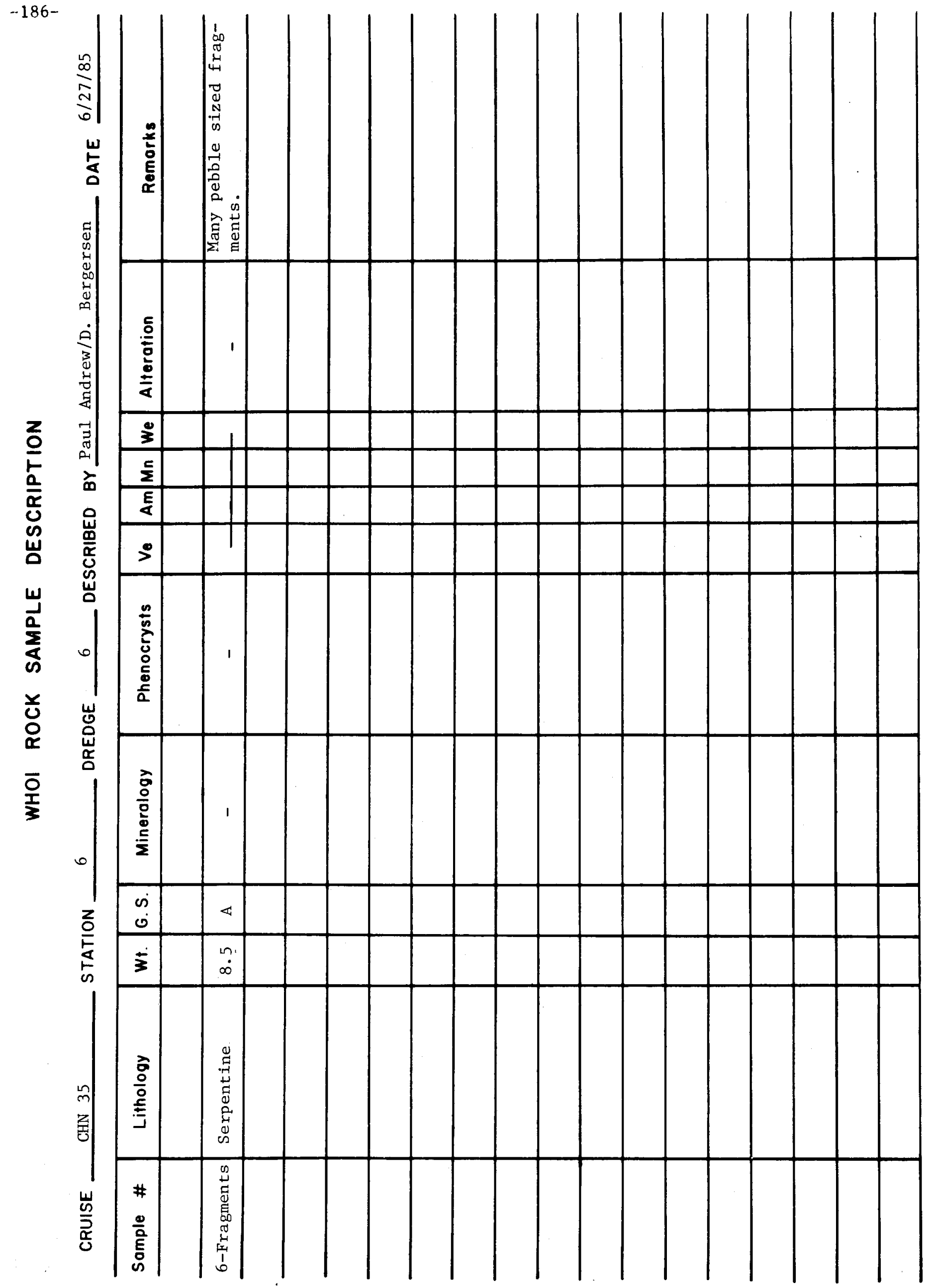




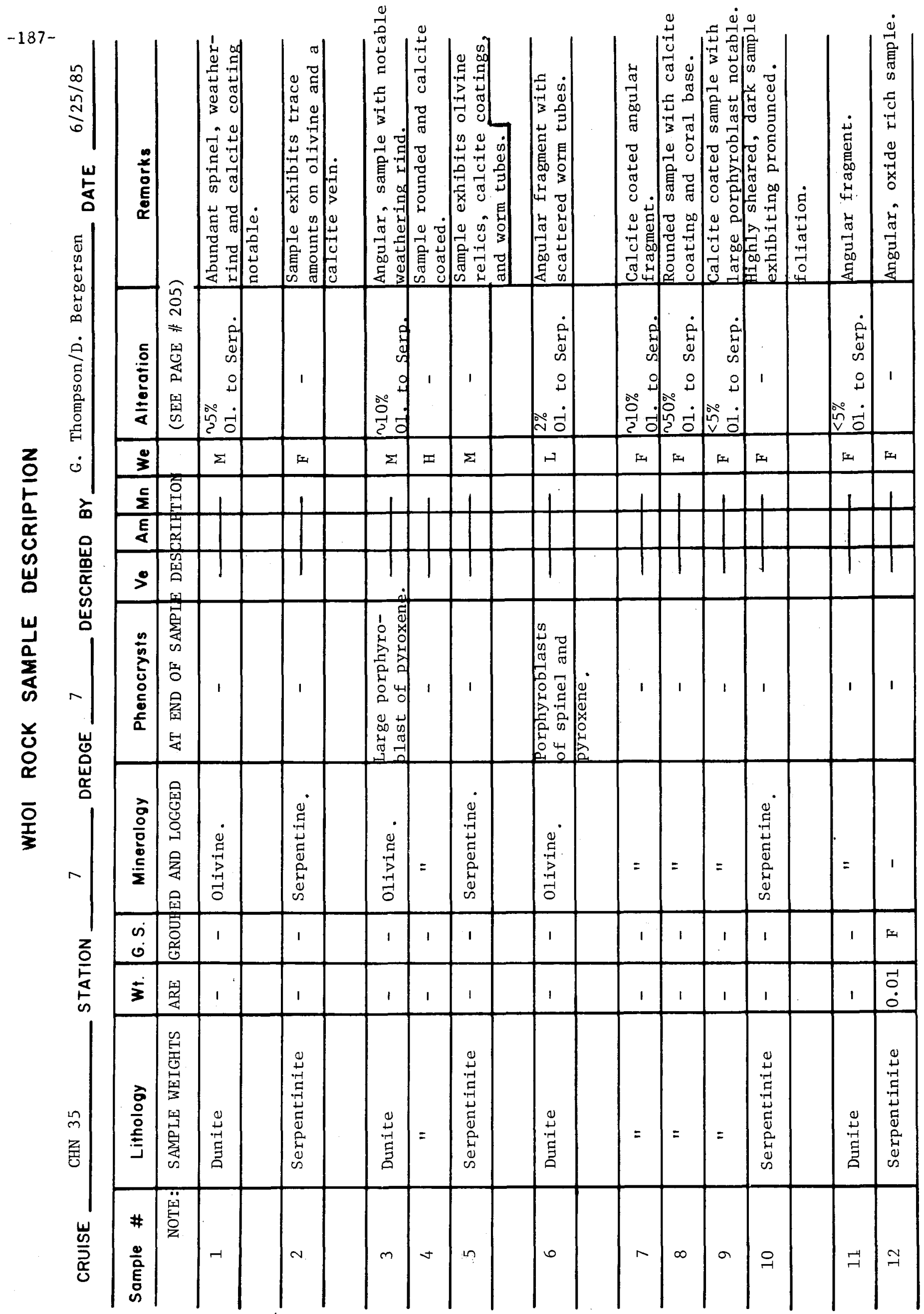




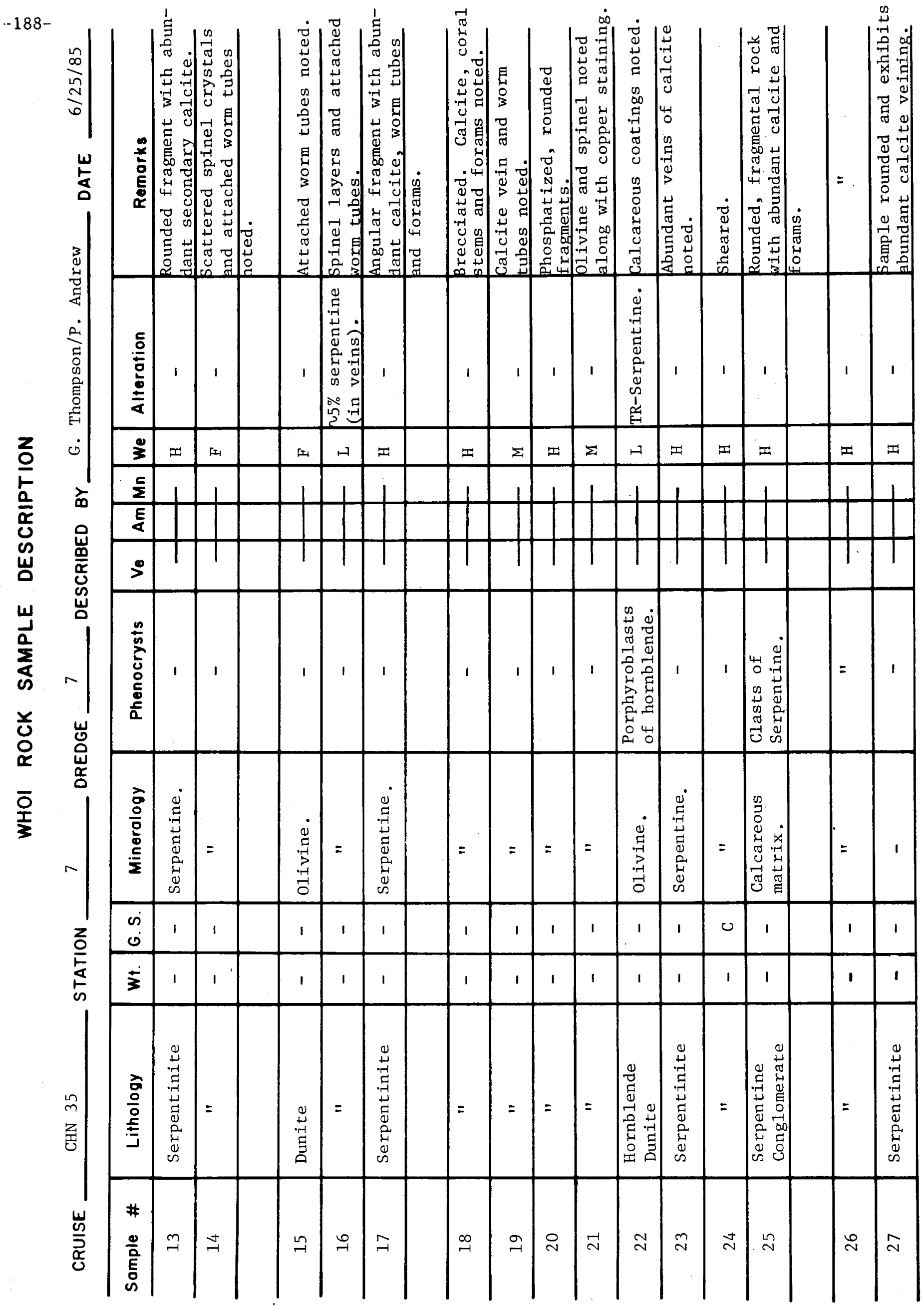




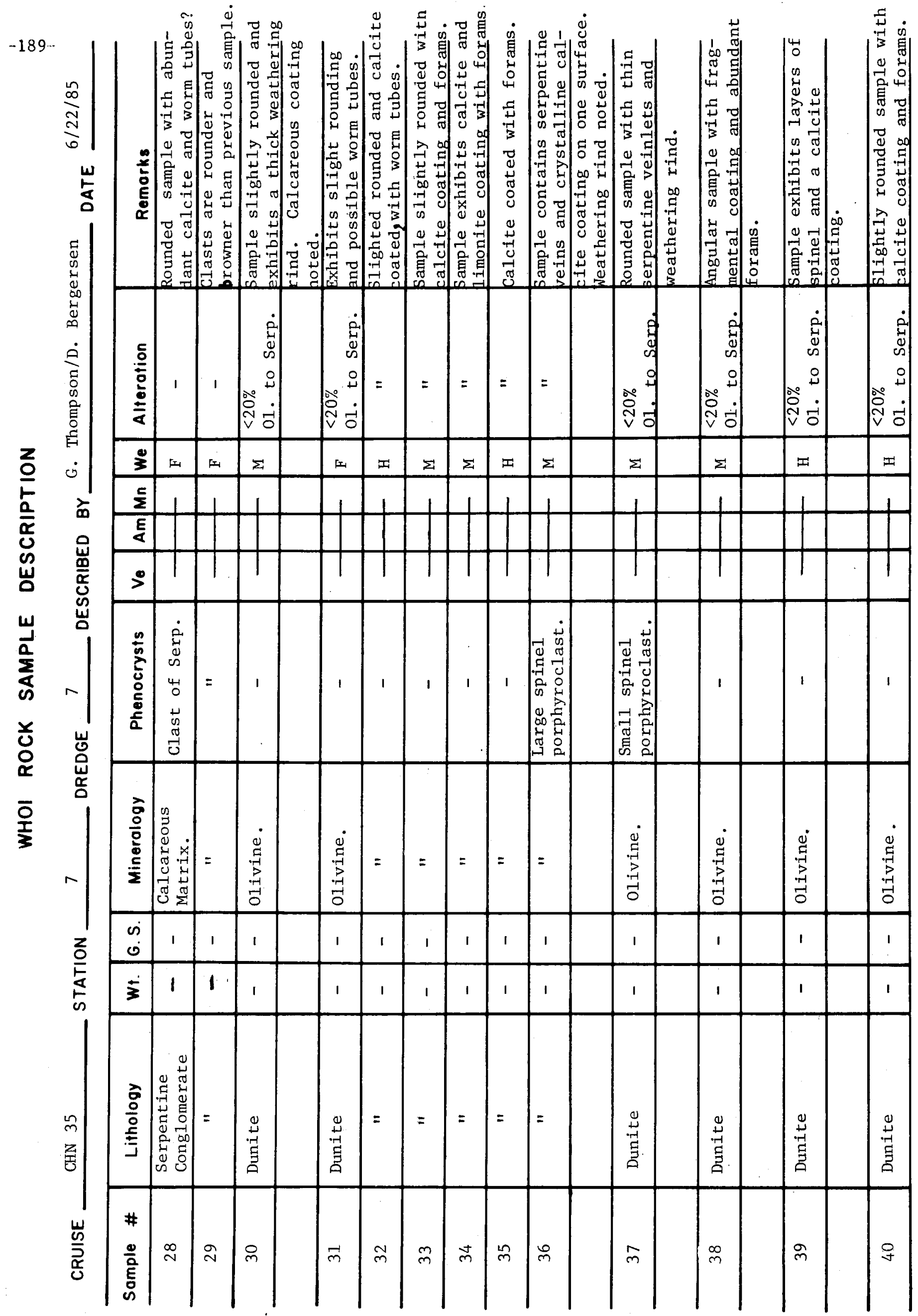




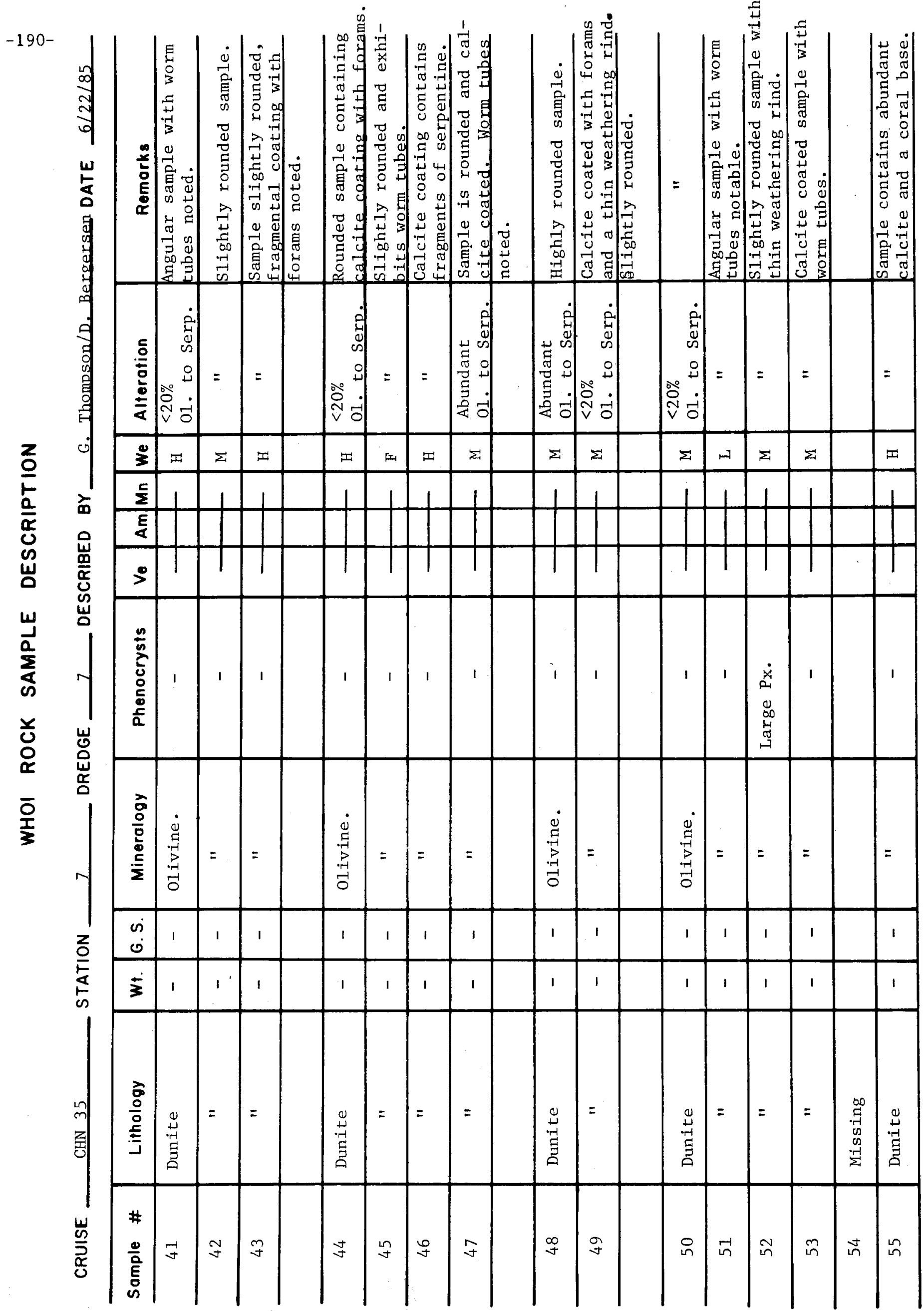




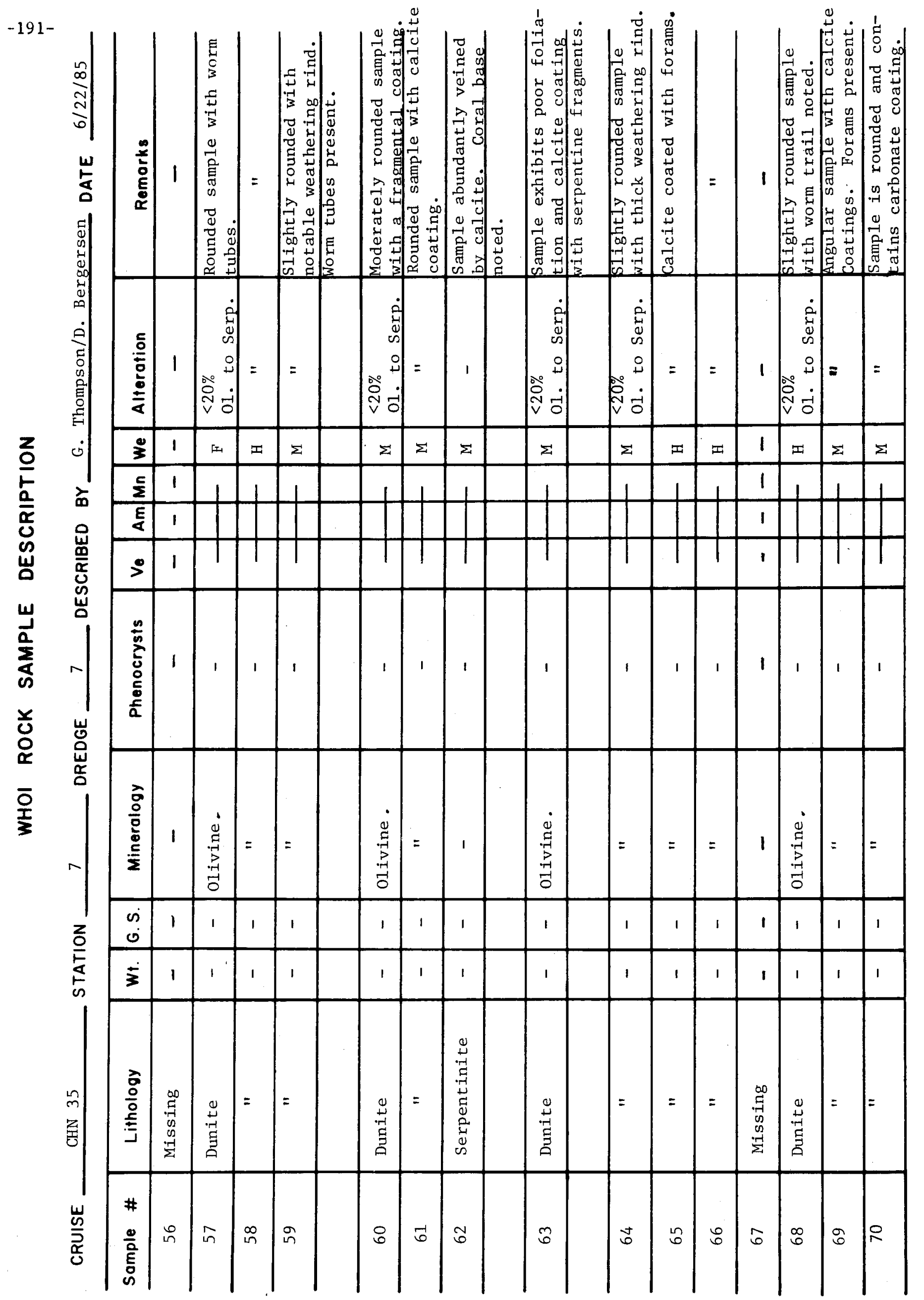




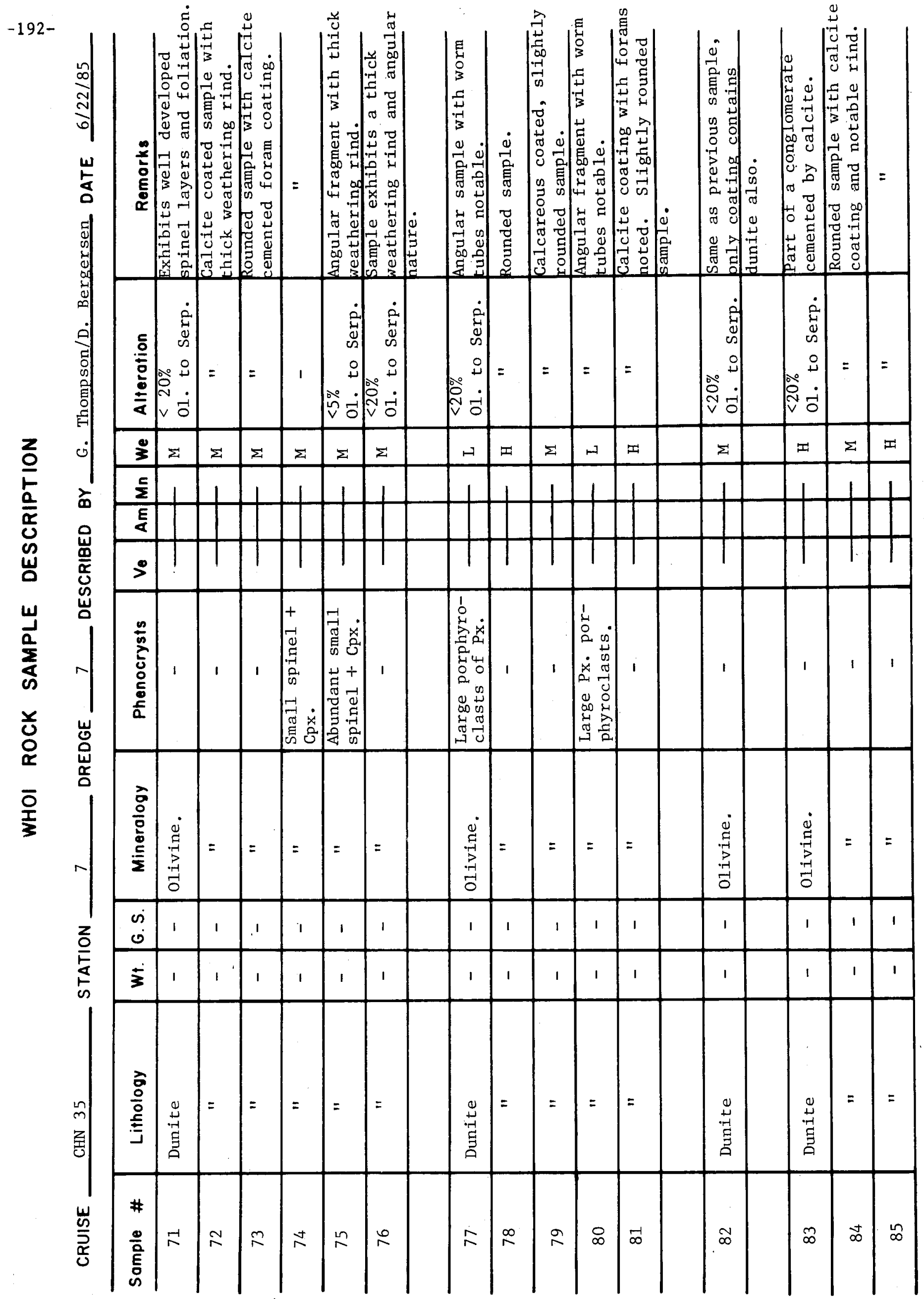




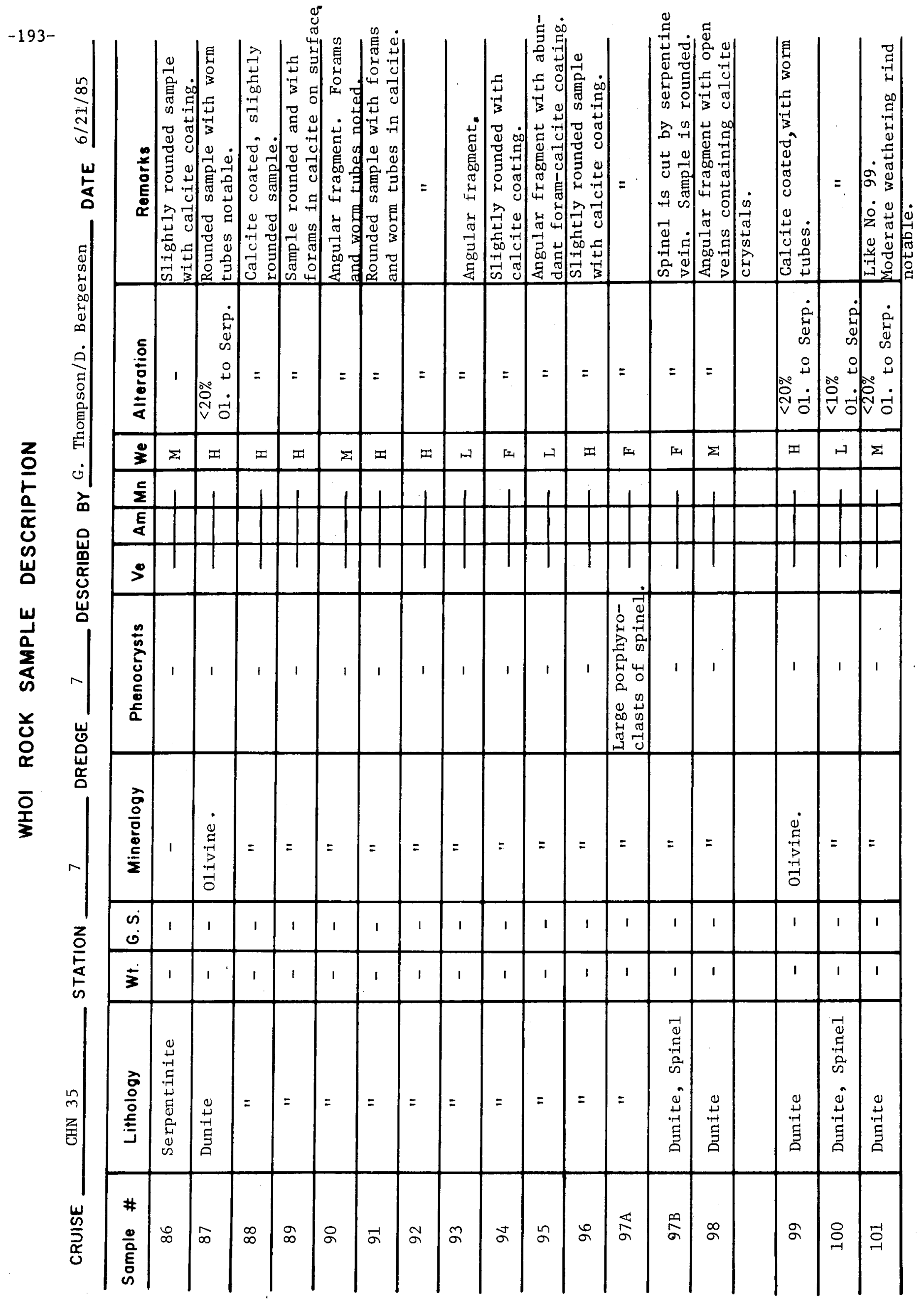




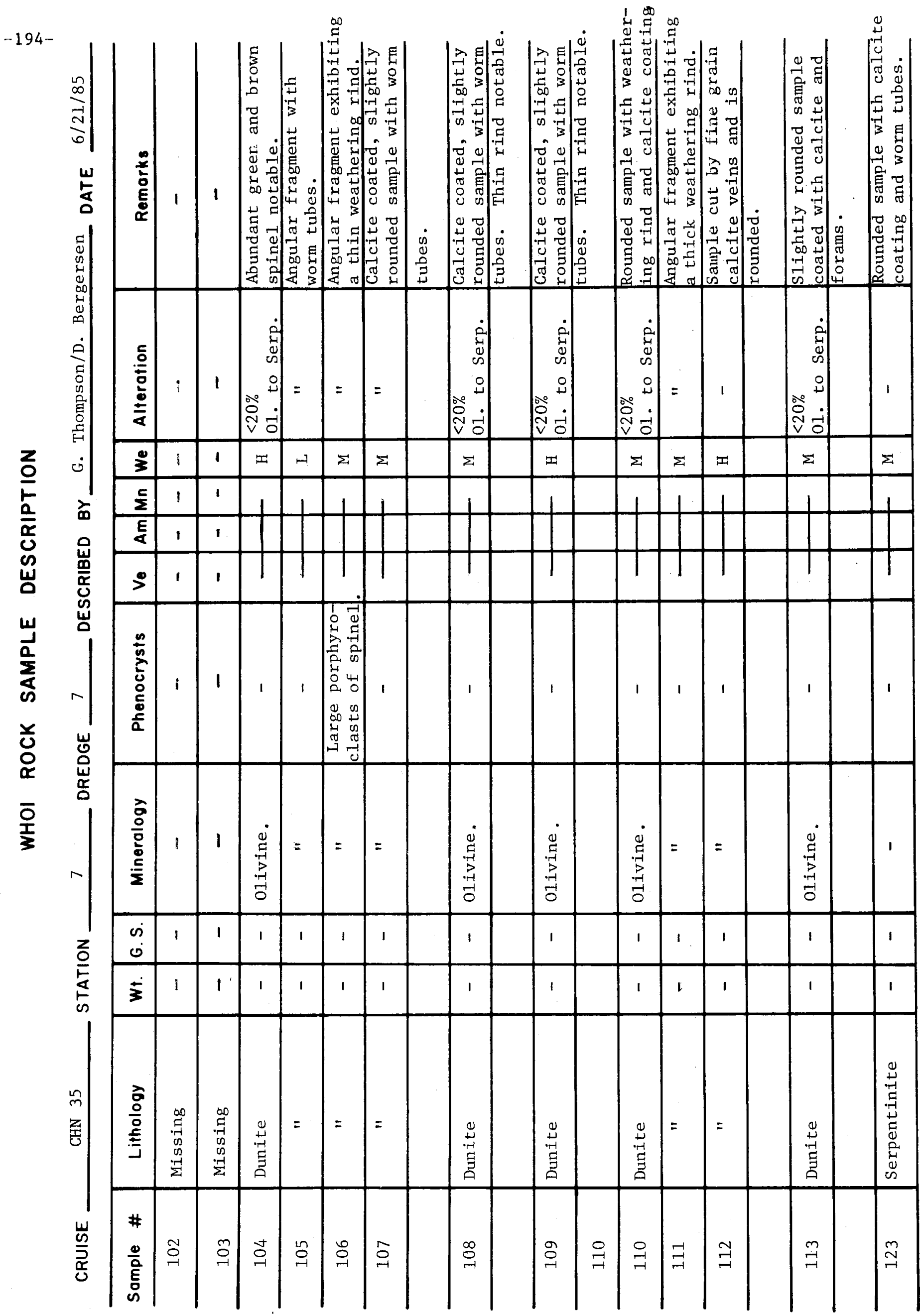




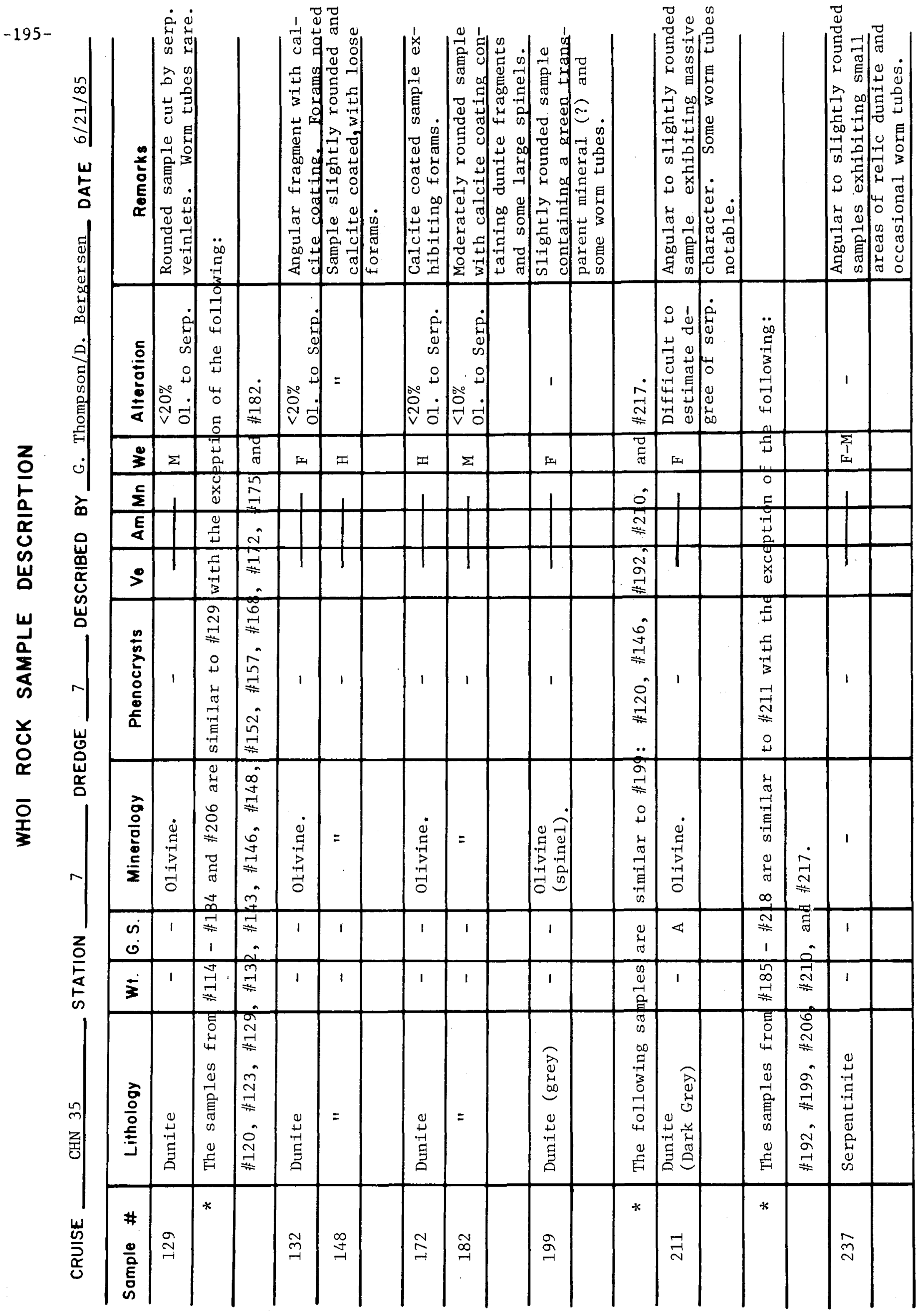




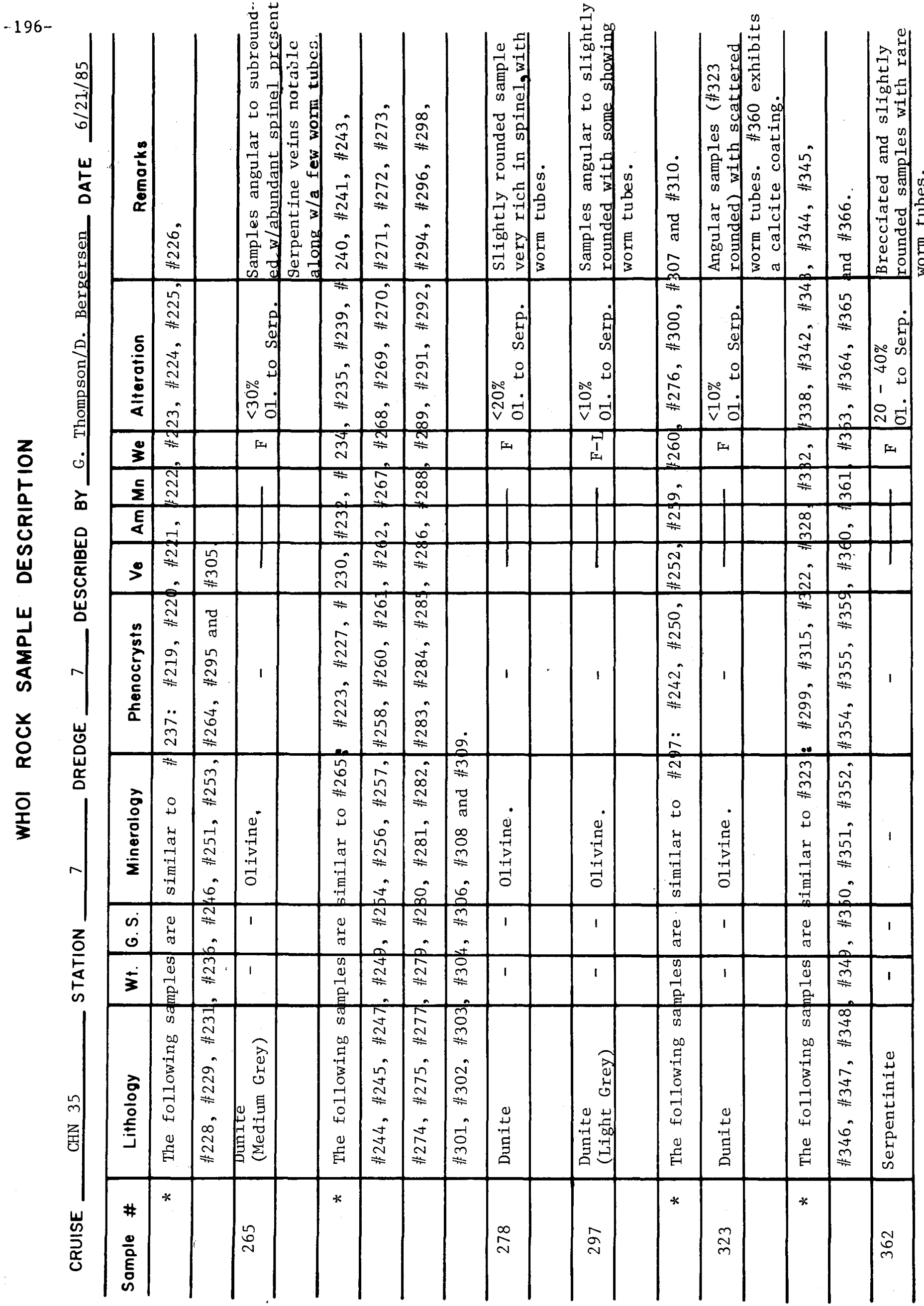




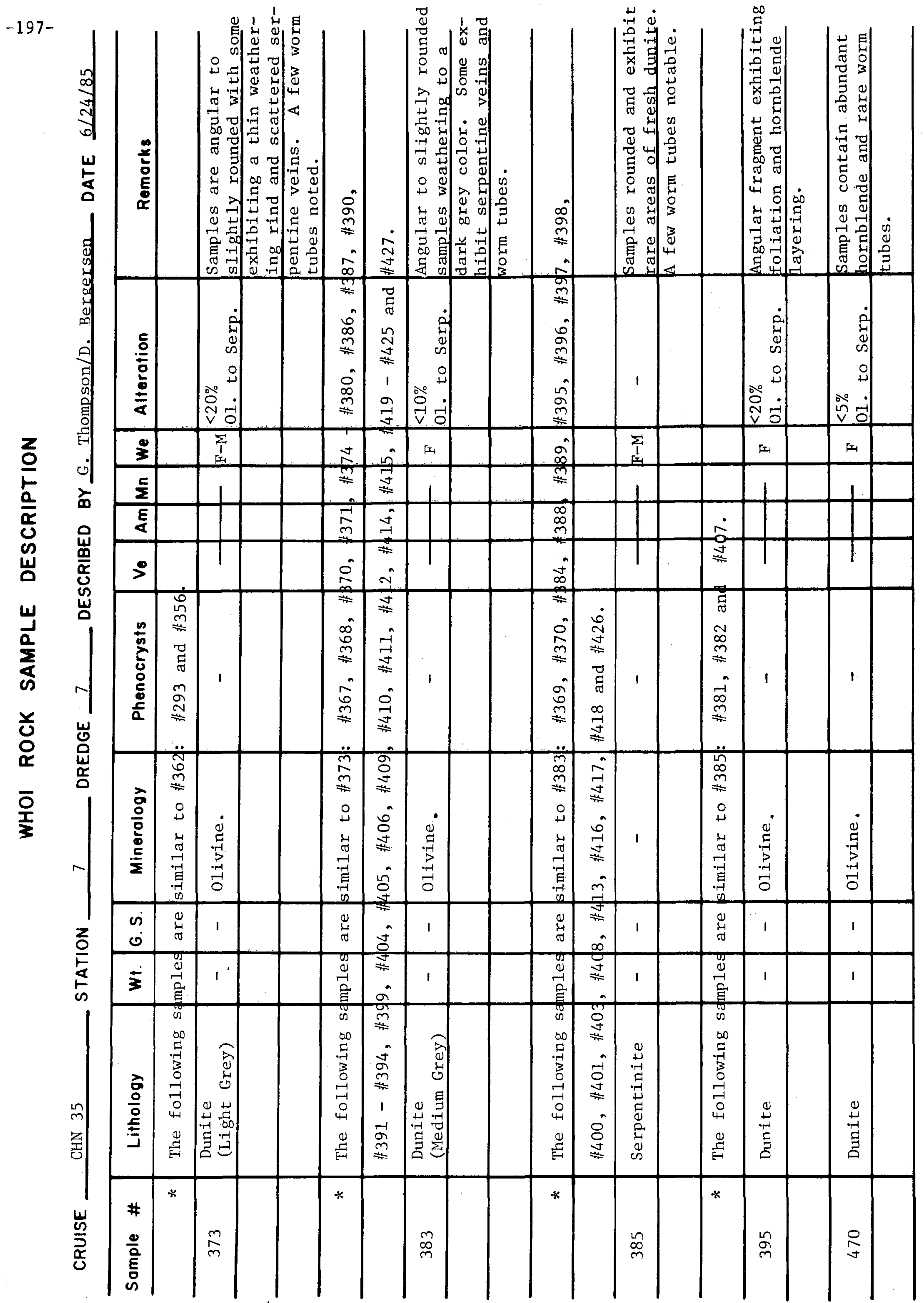




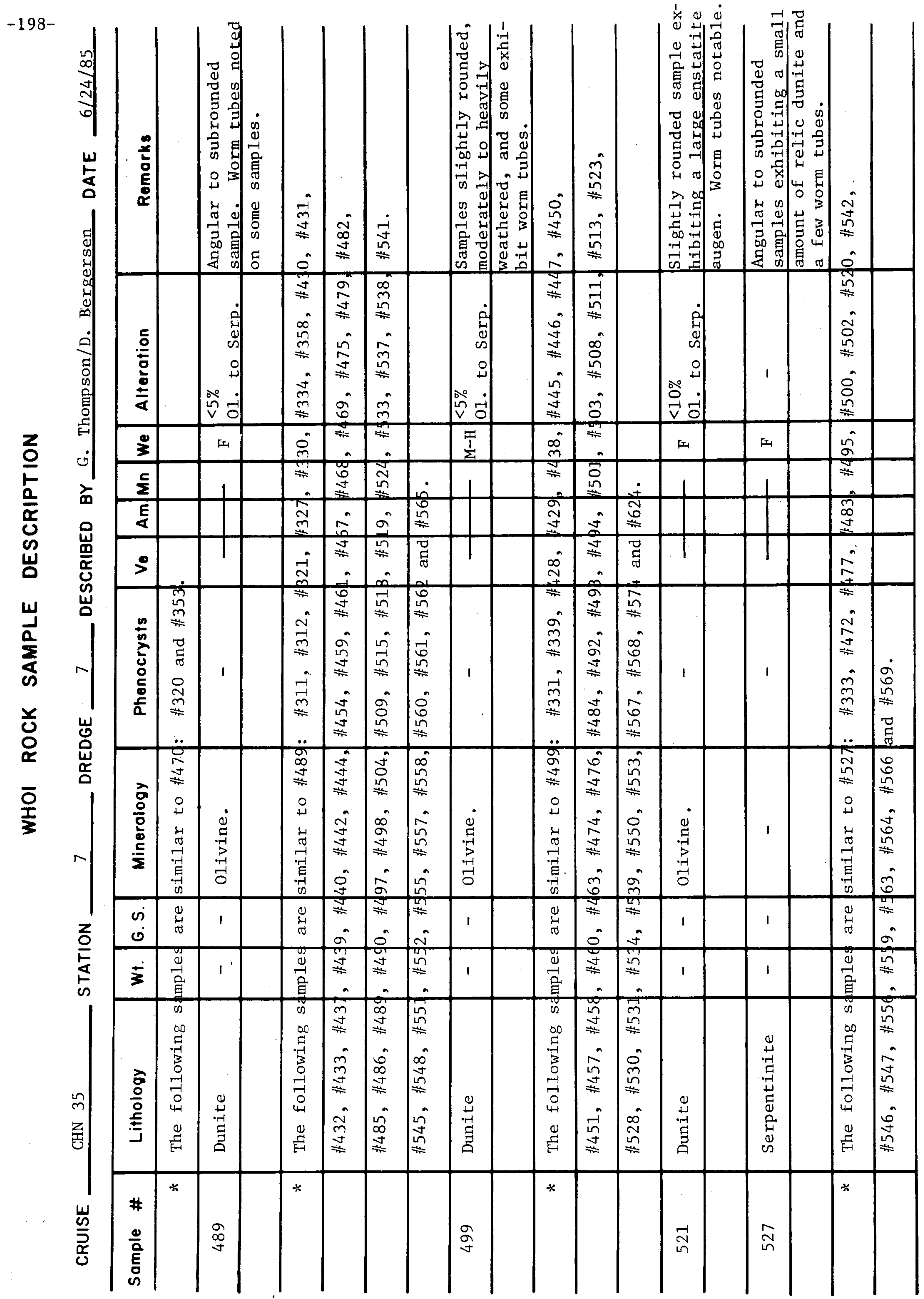




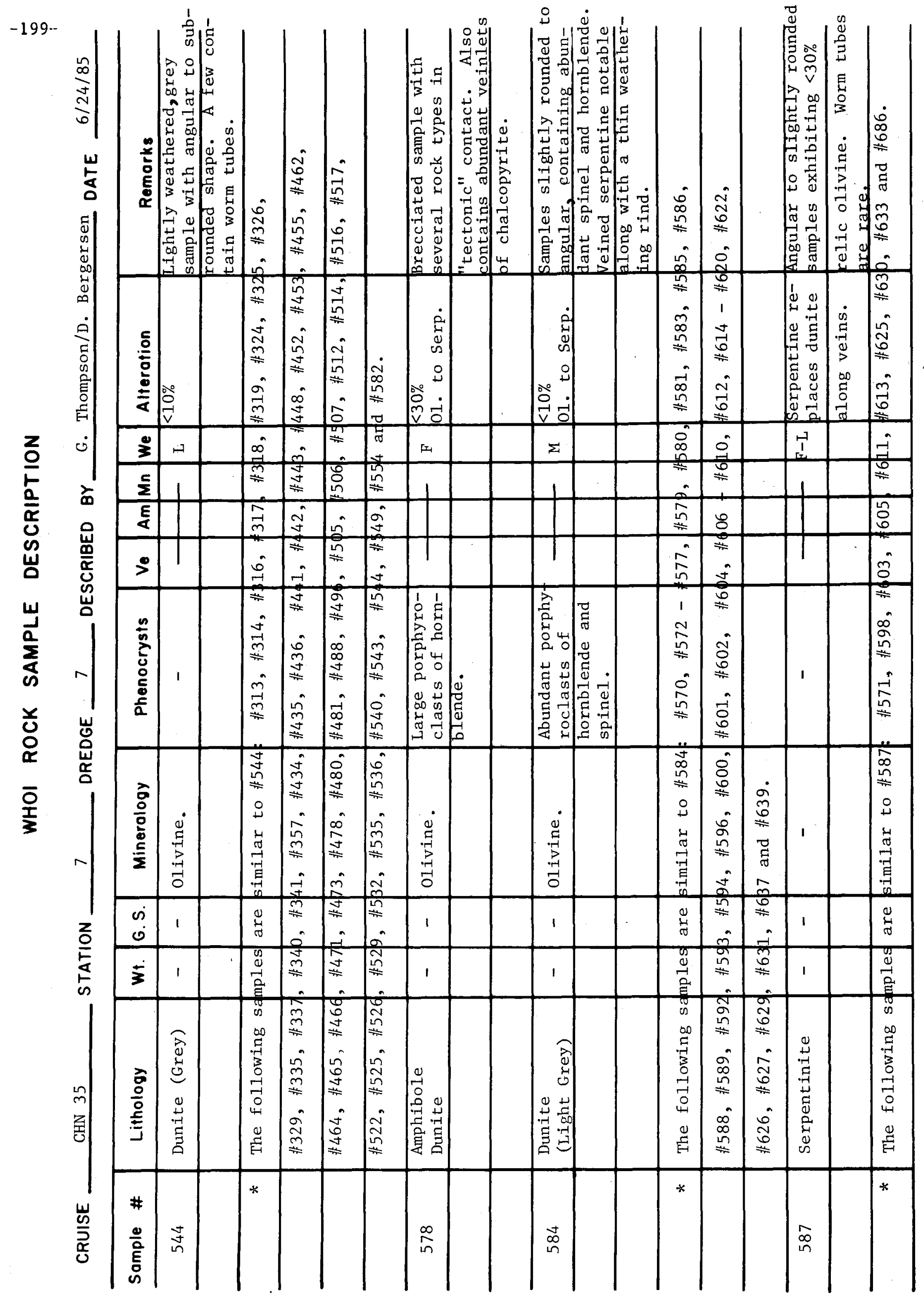




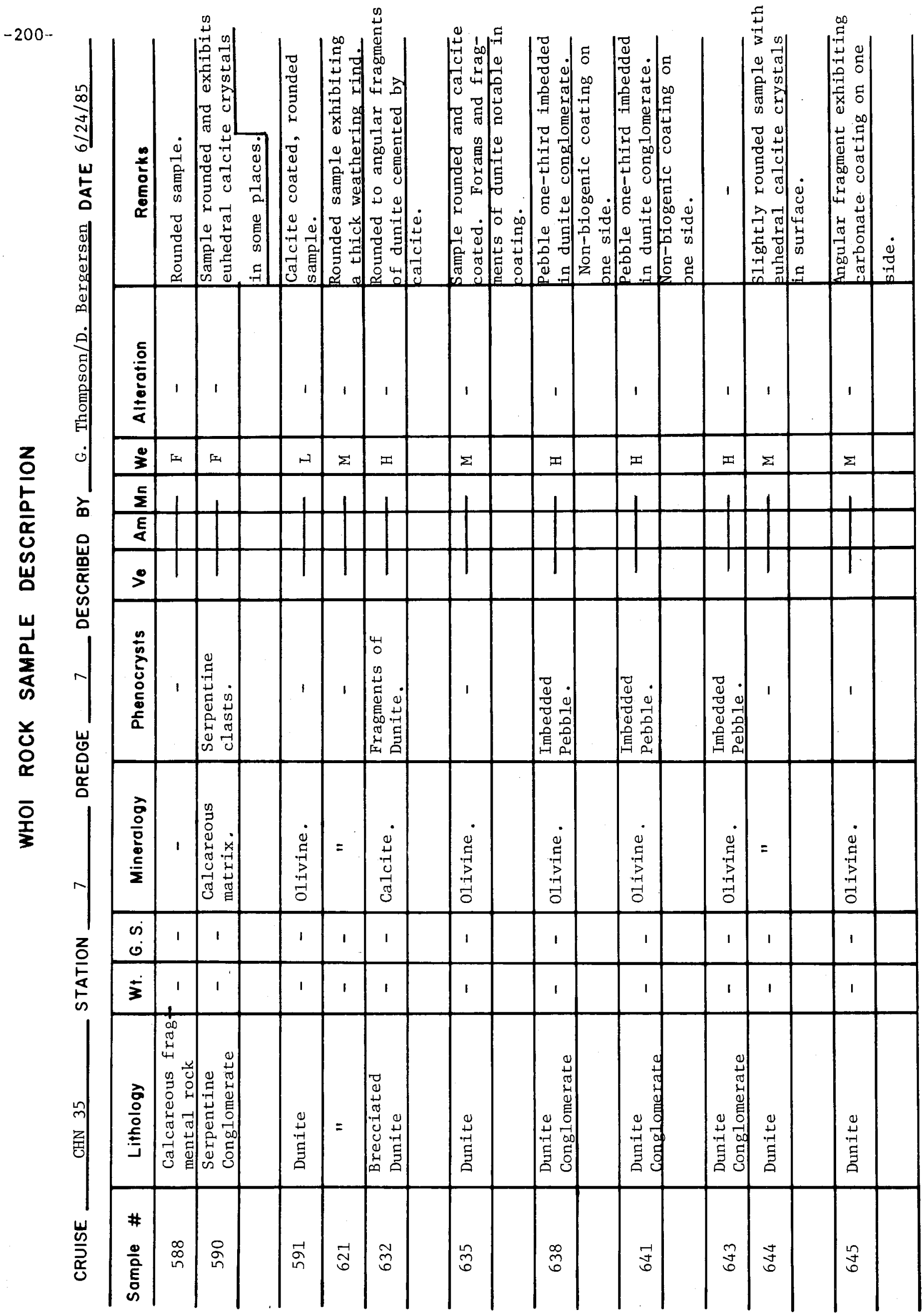




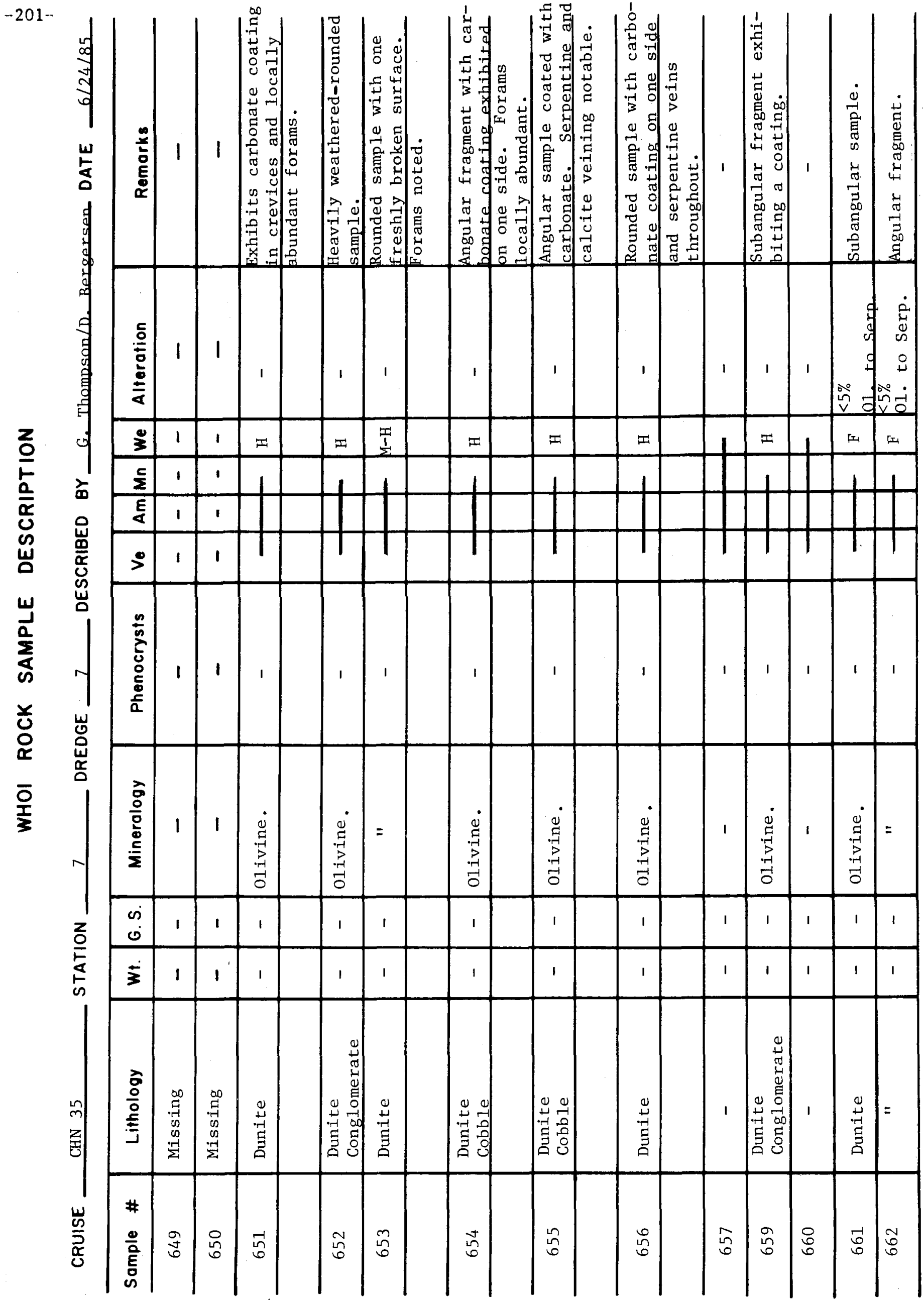




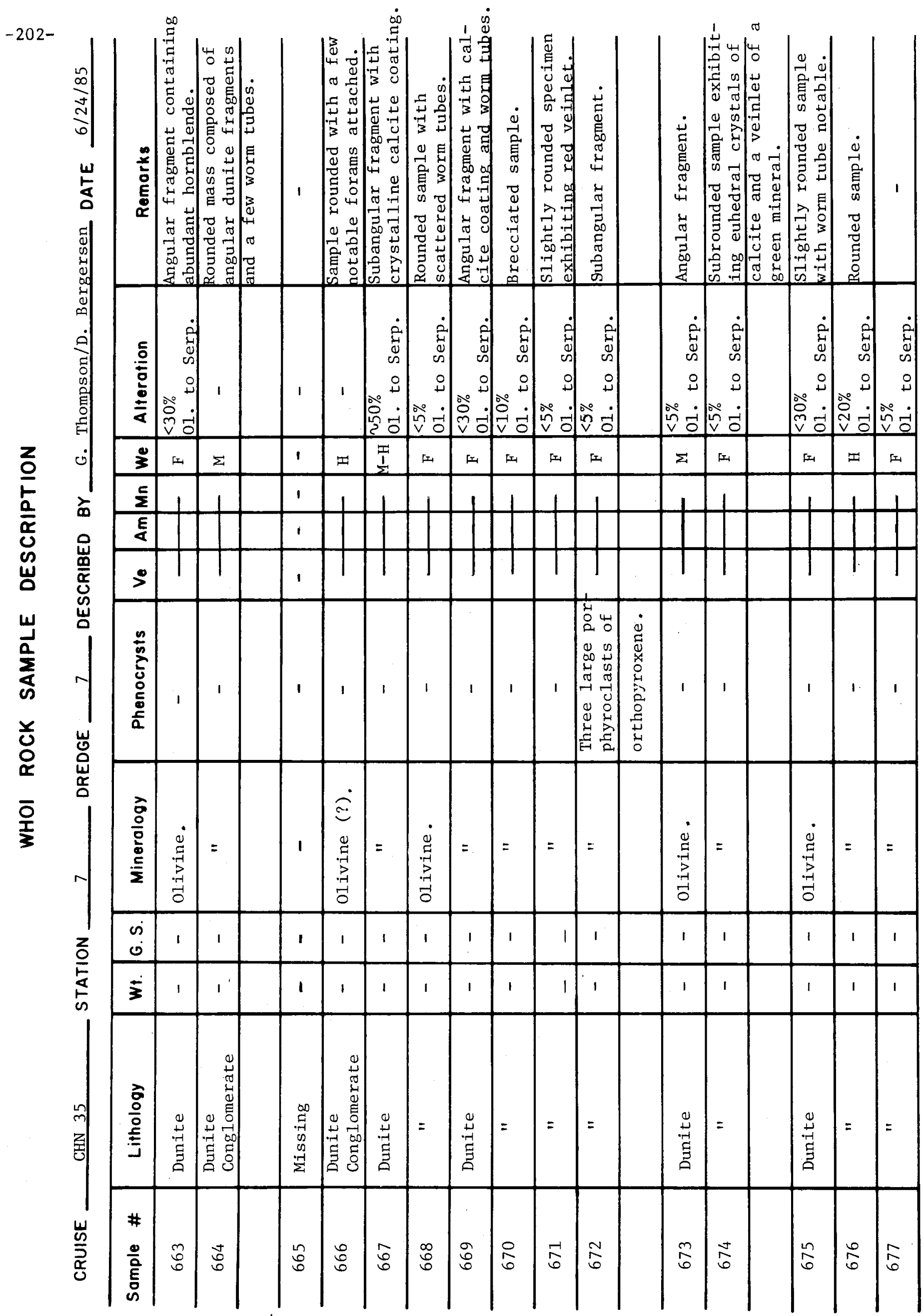




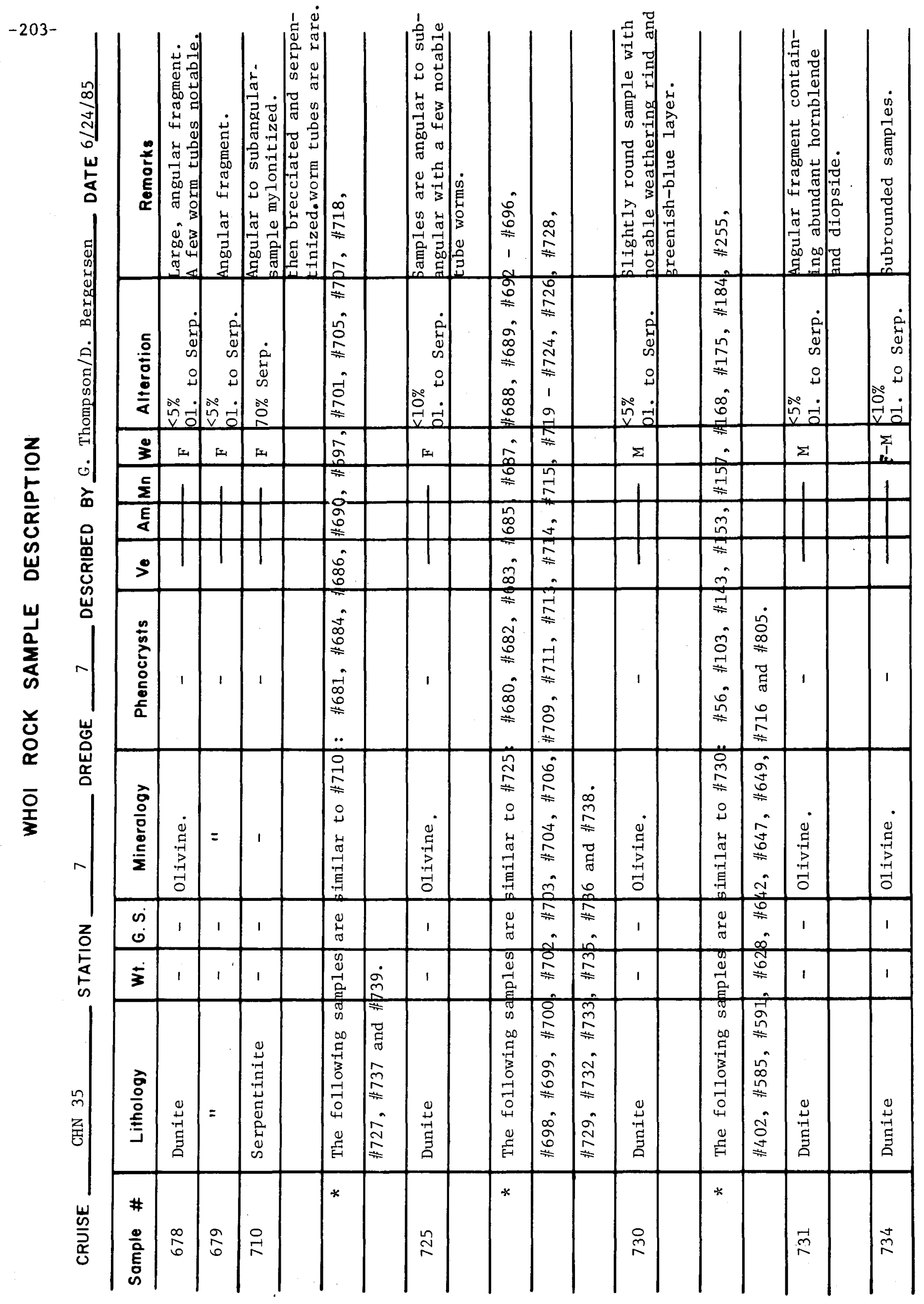




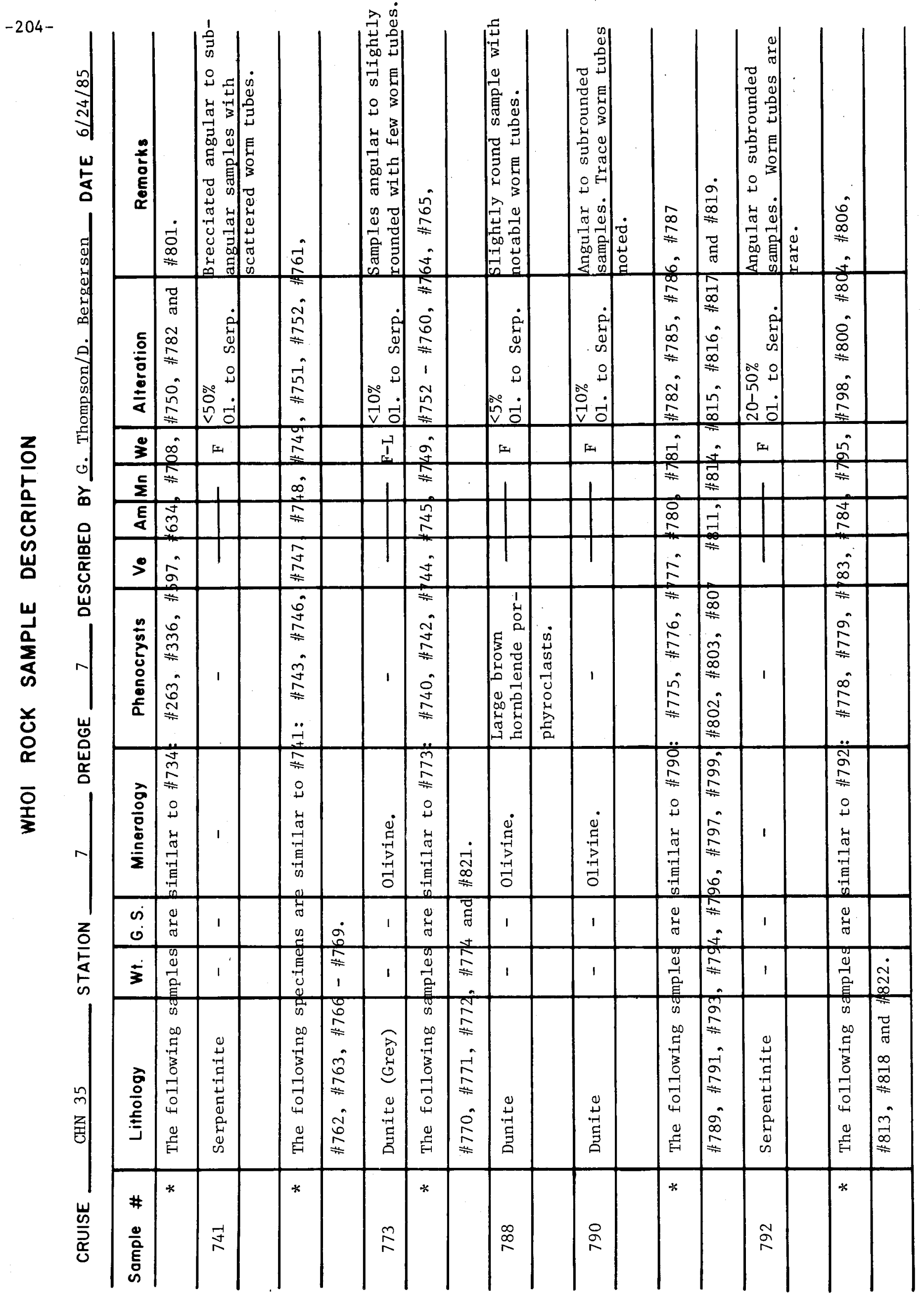


$-205-$

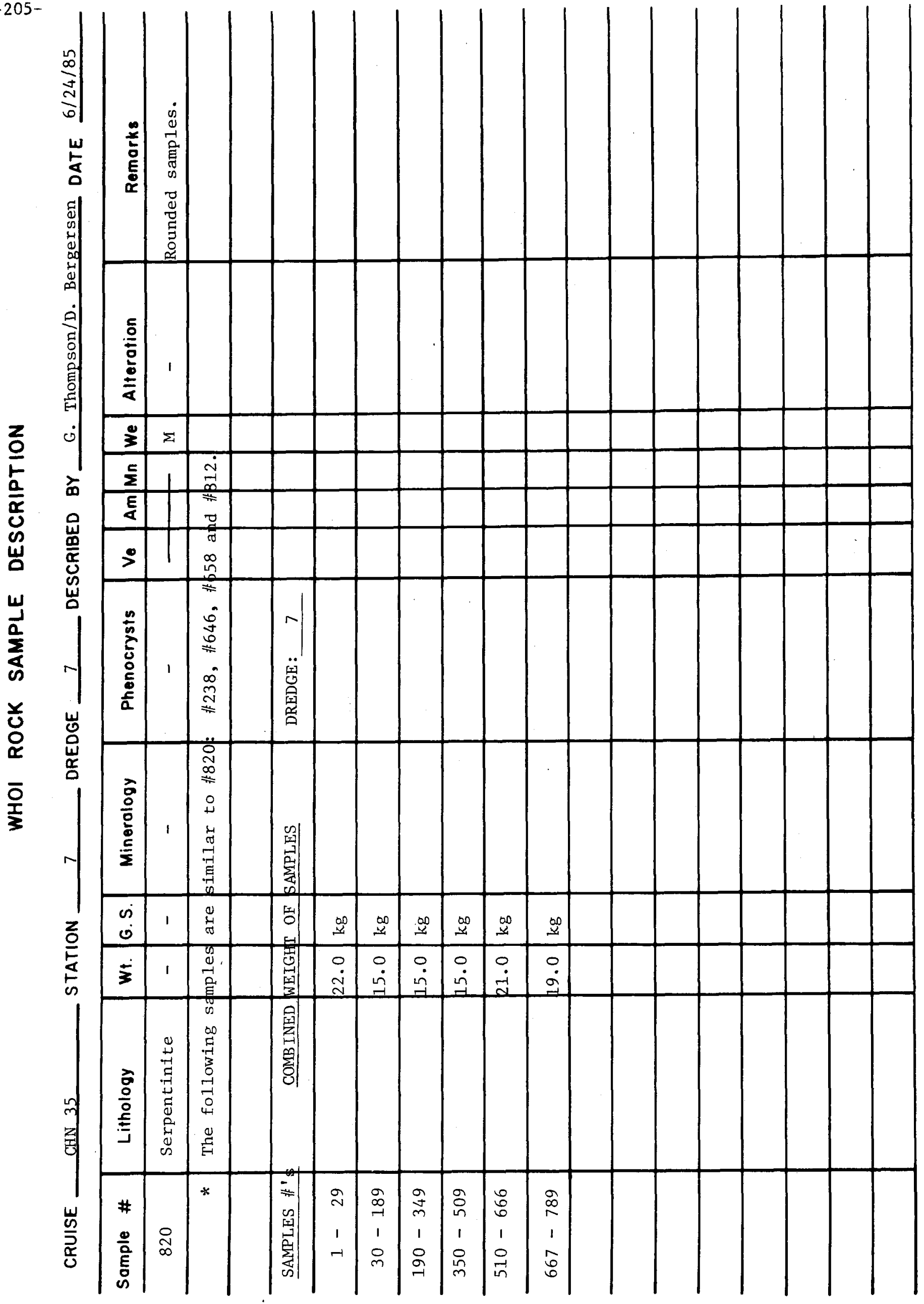




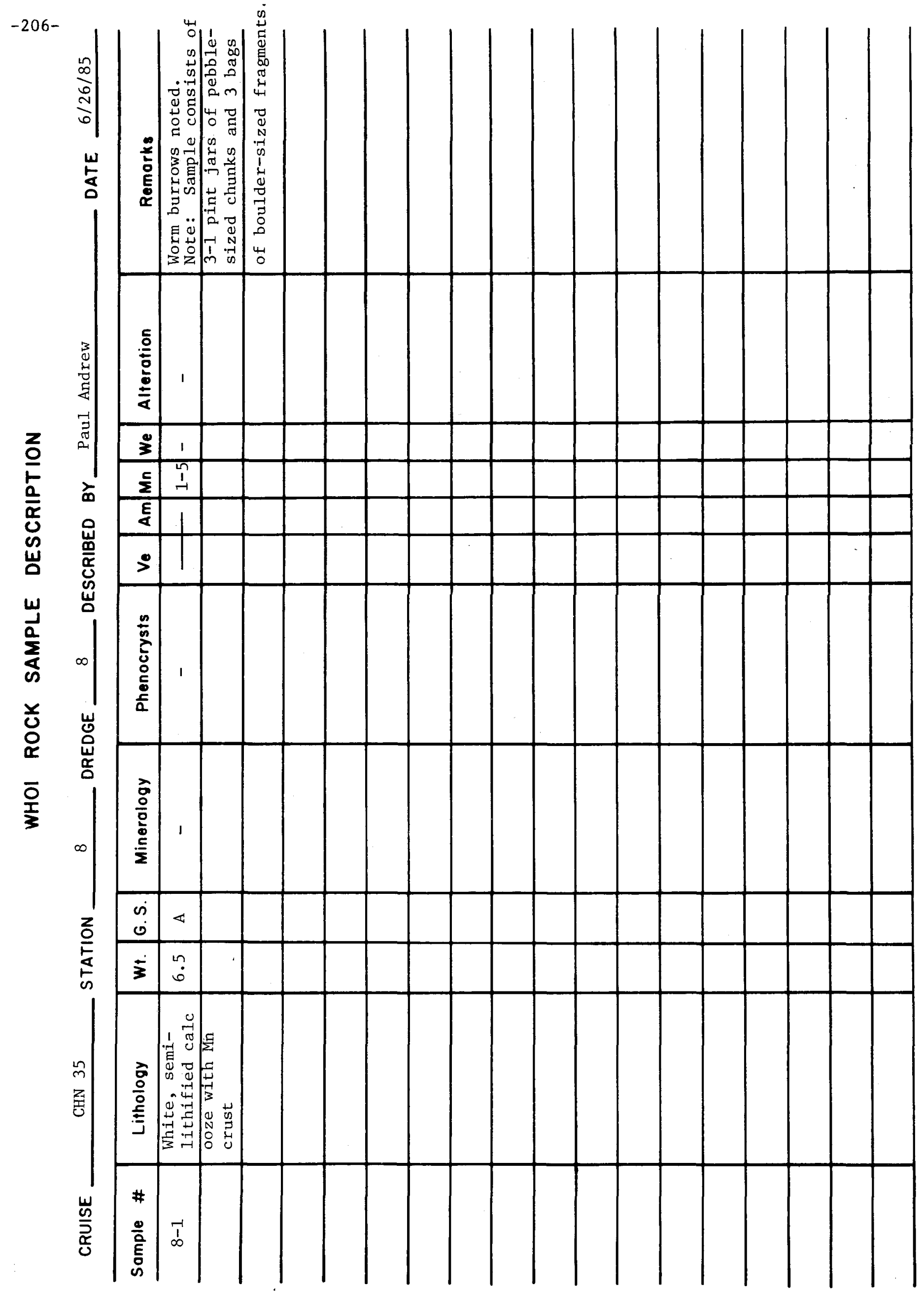




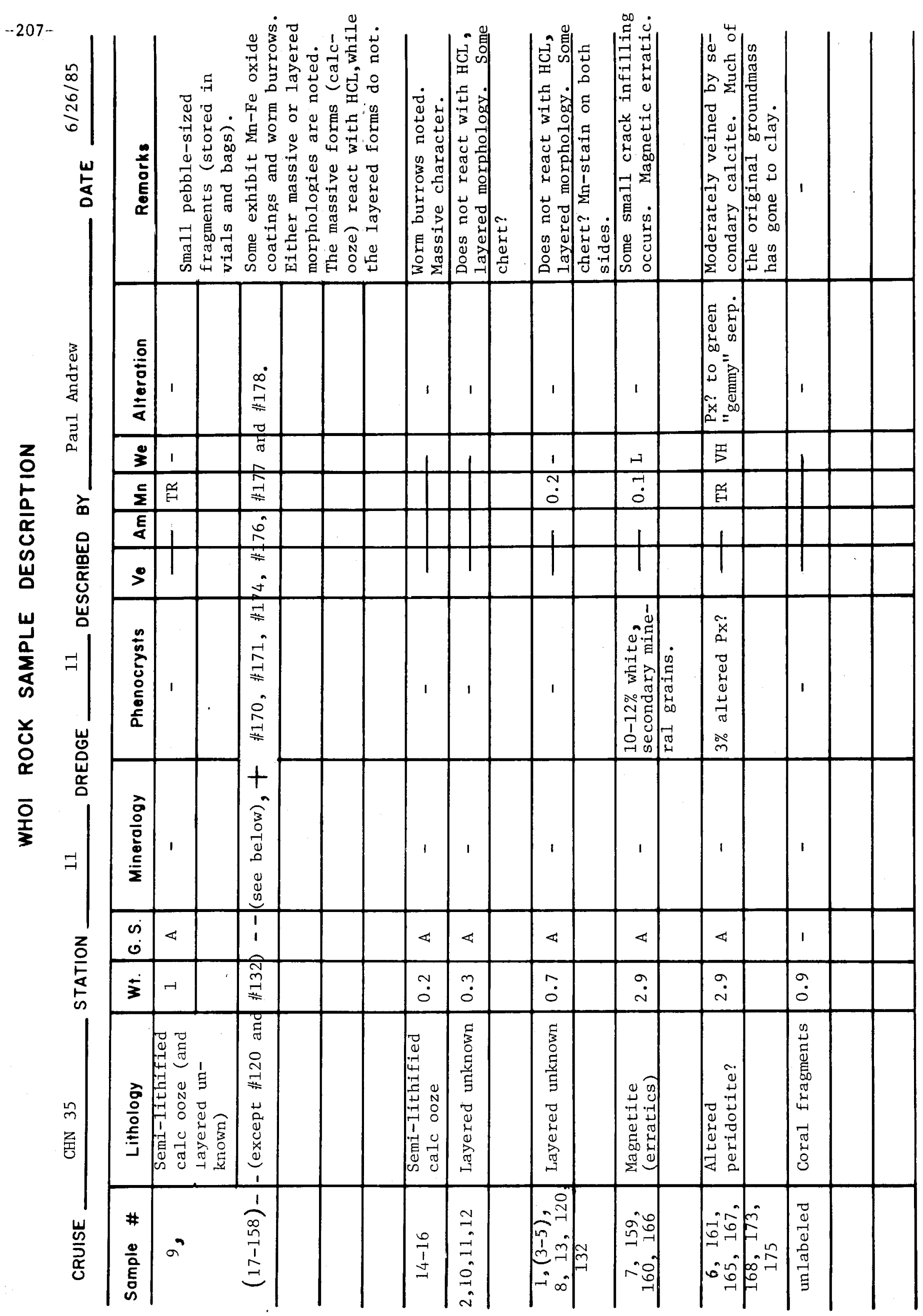




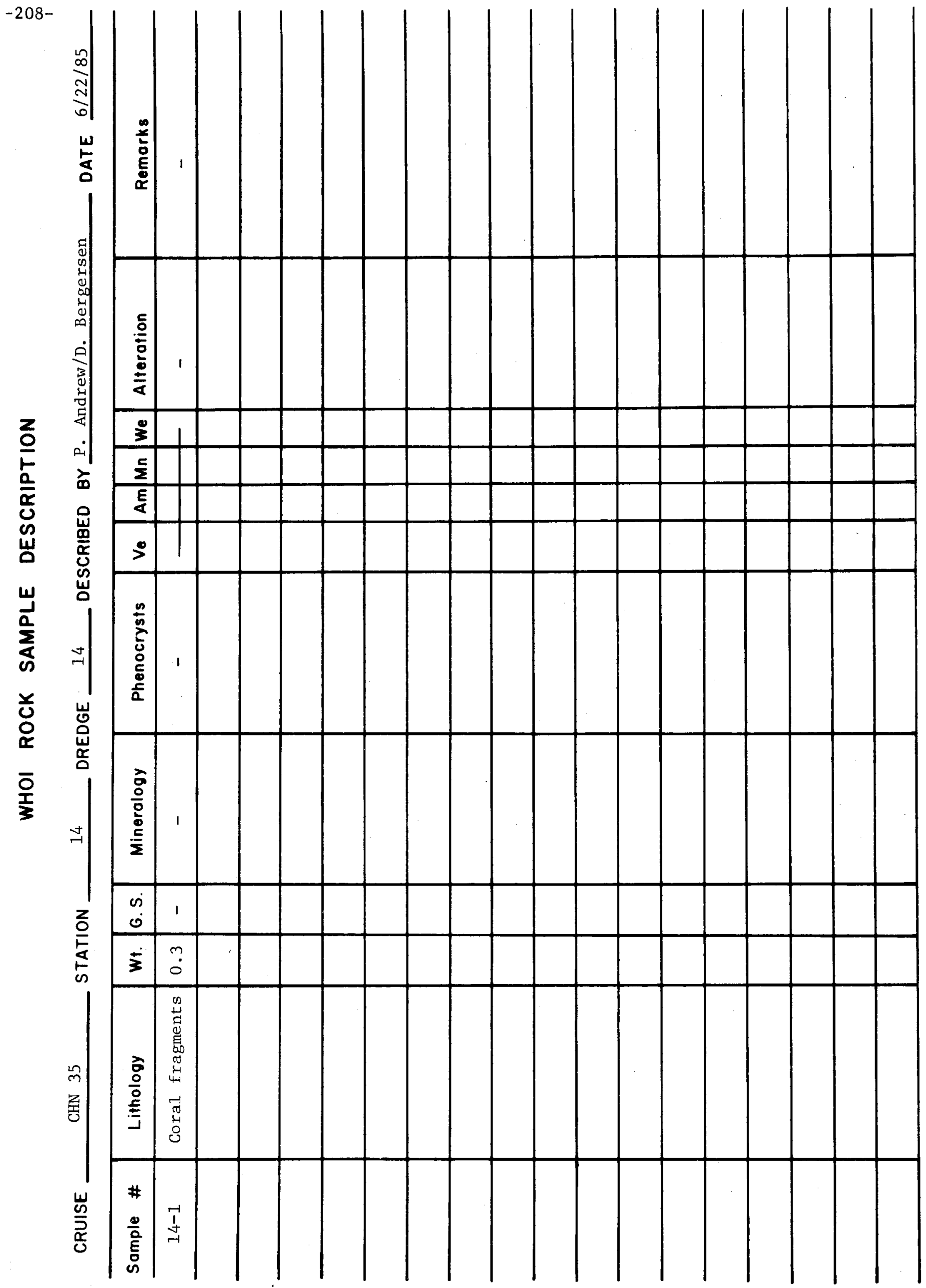




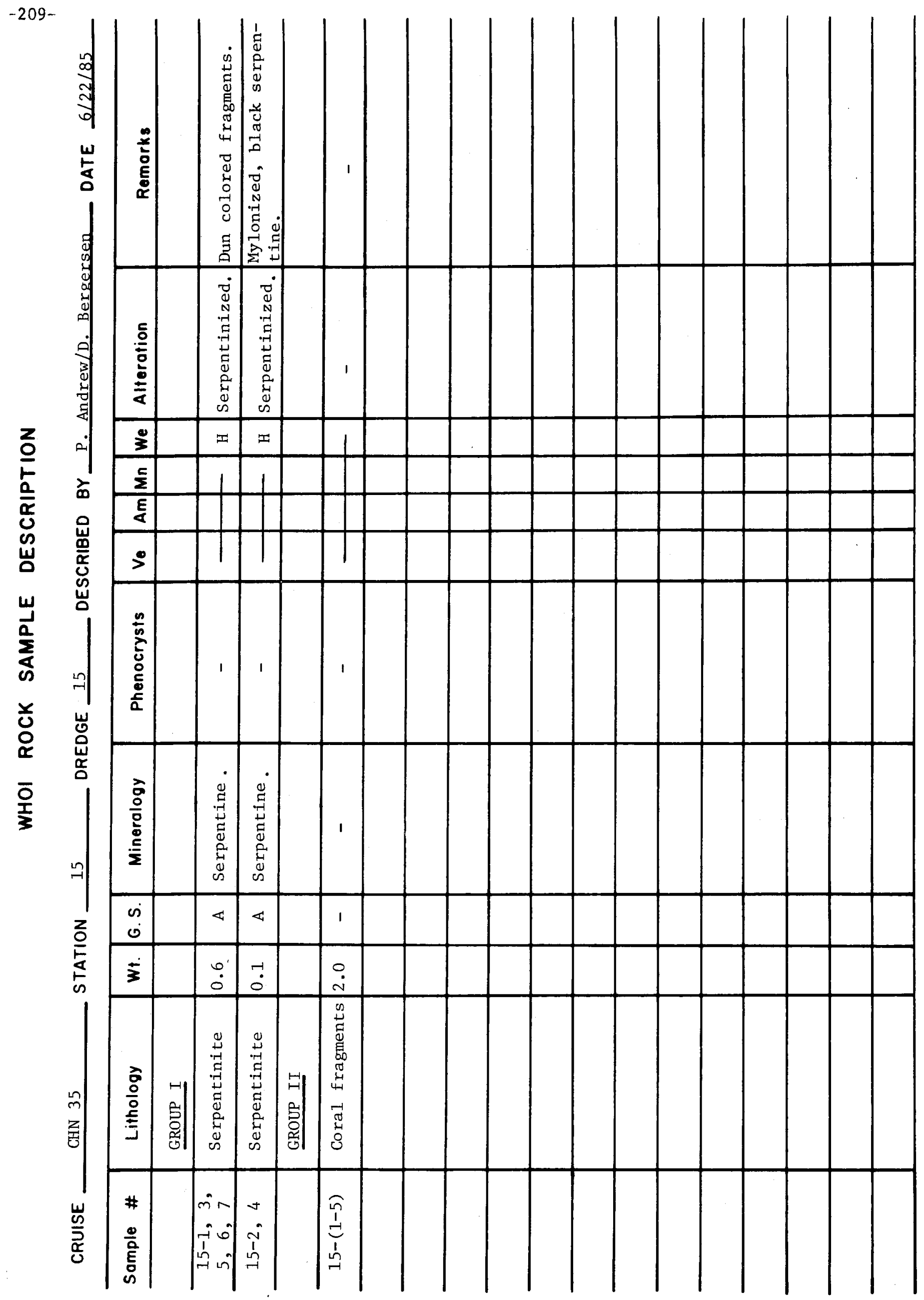




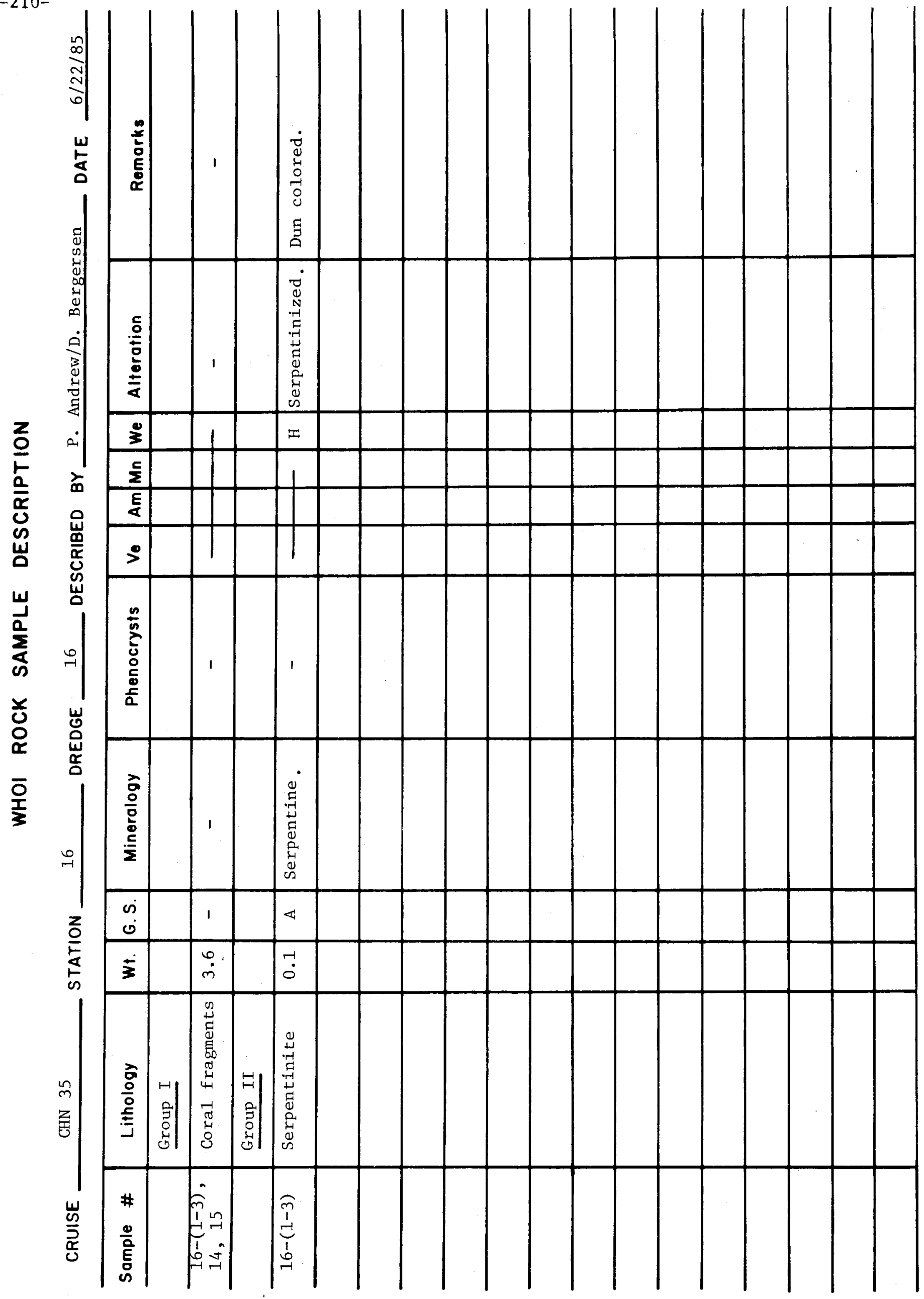




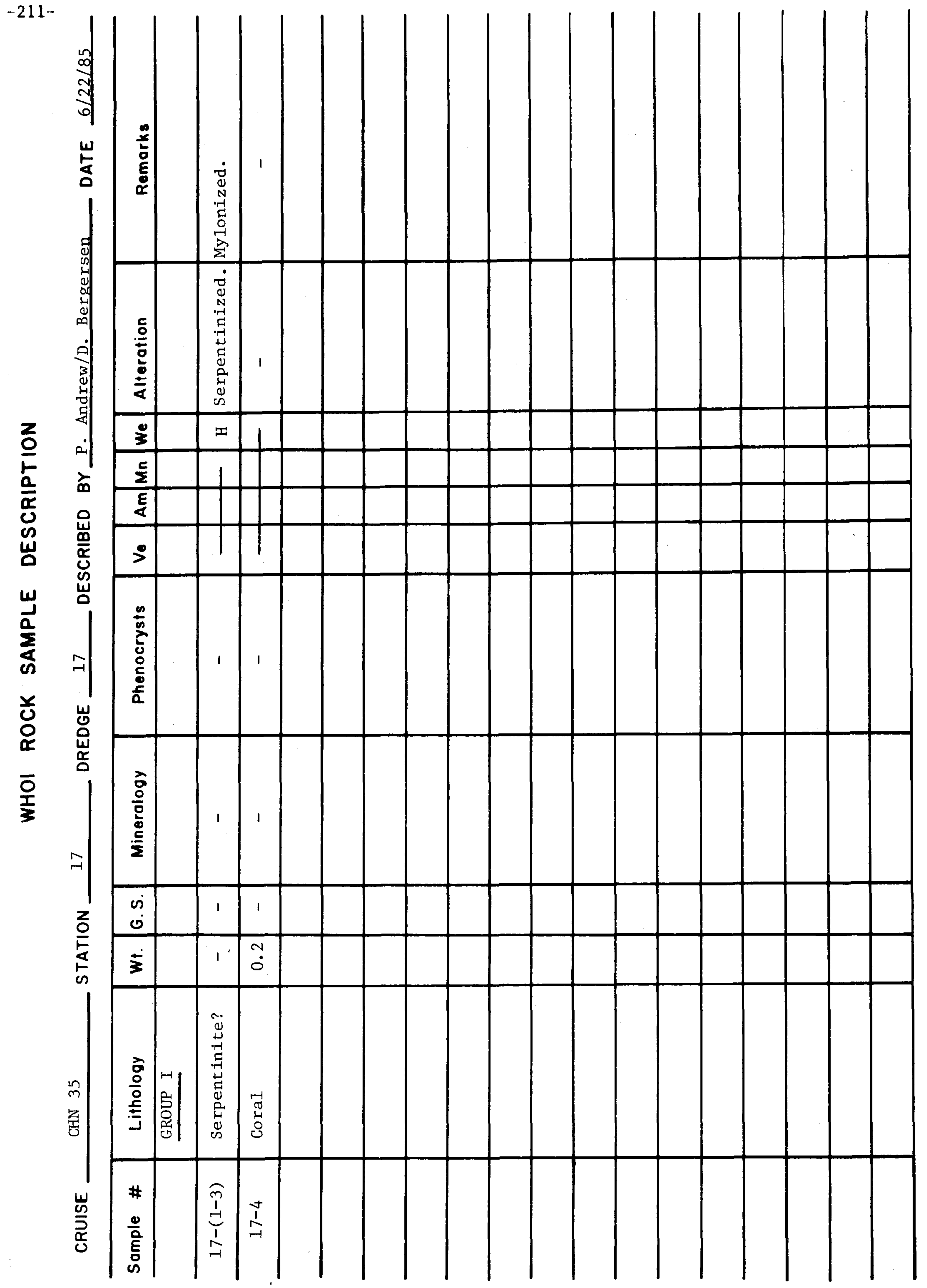




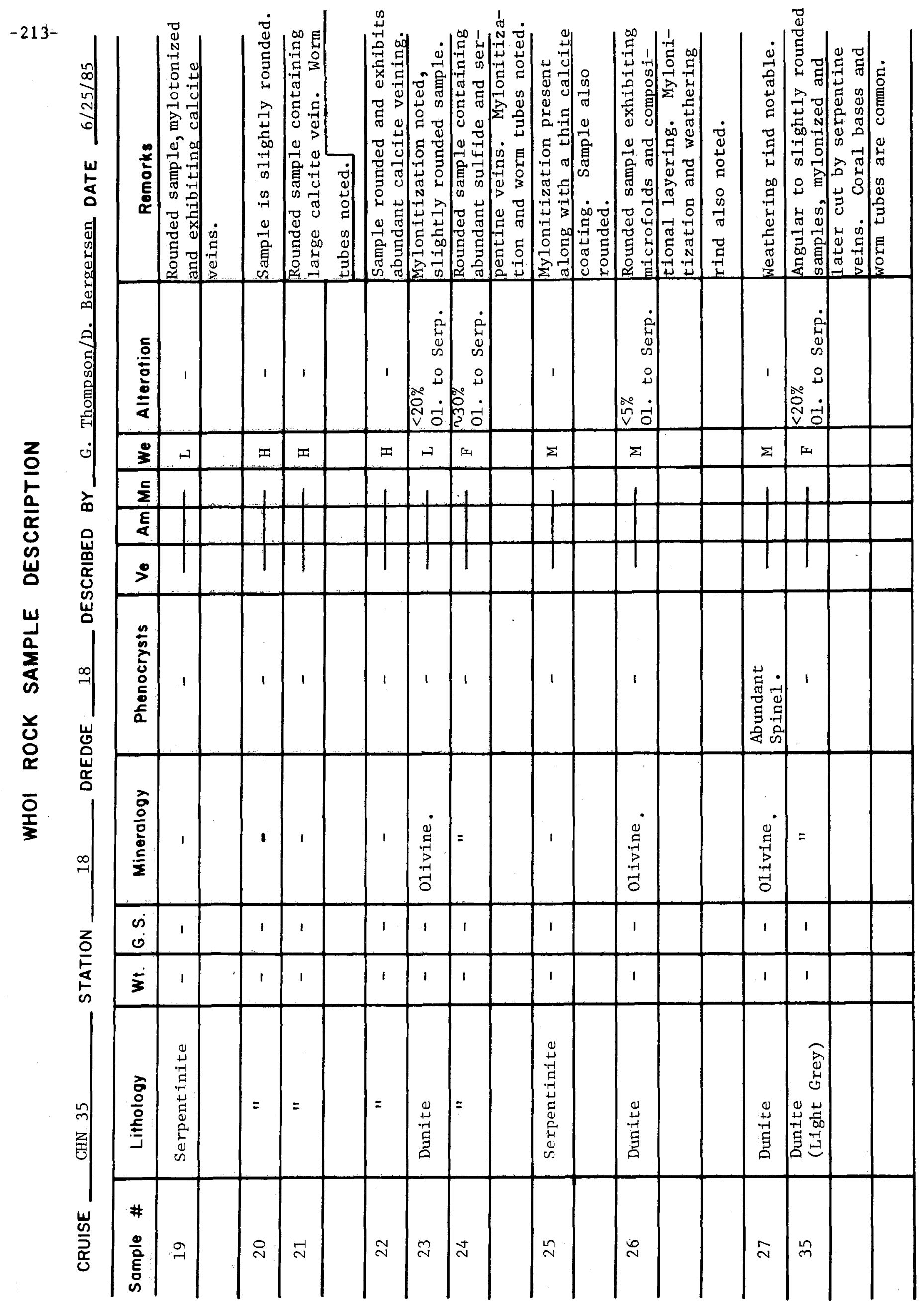




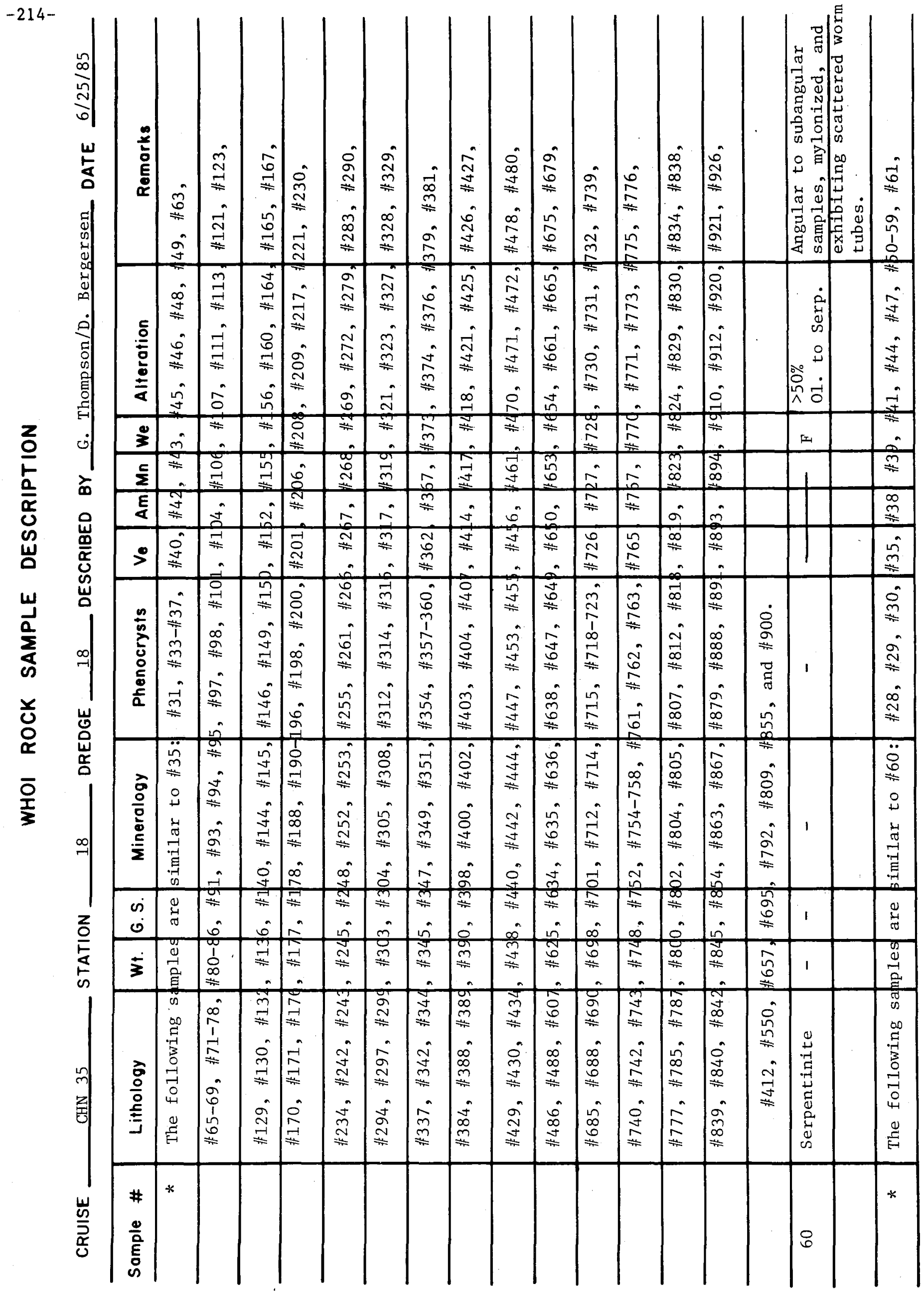




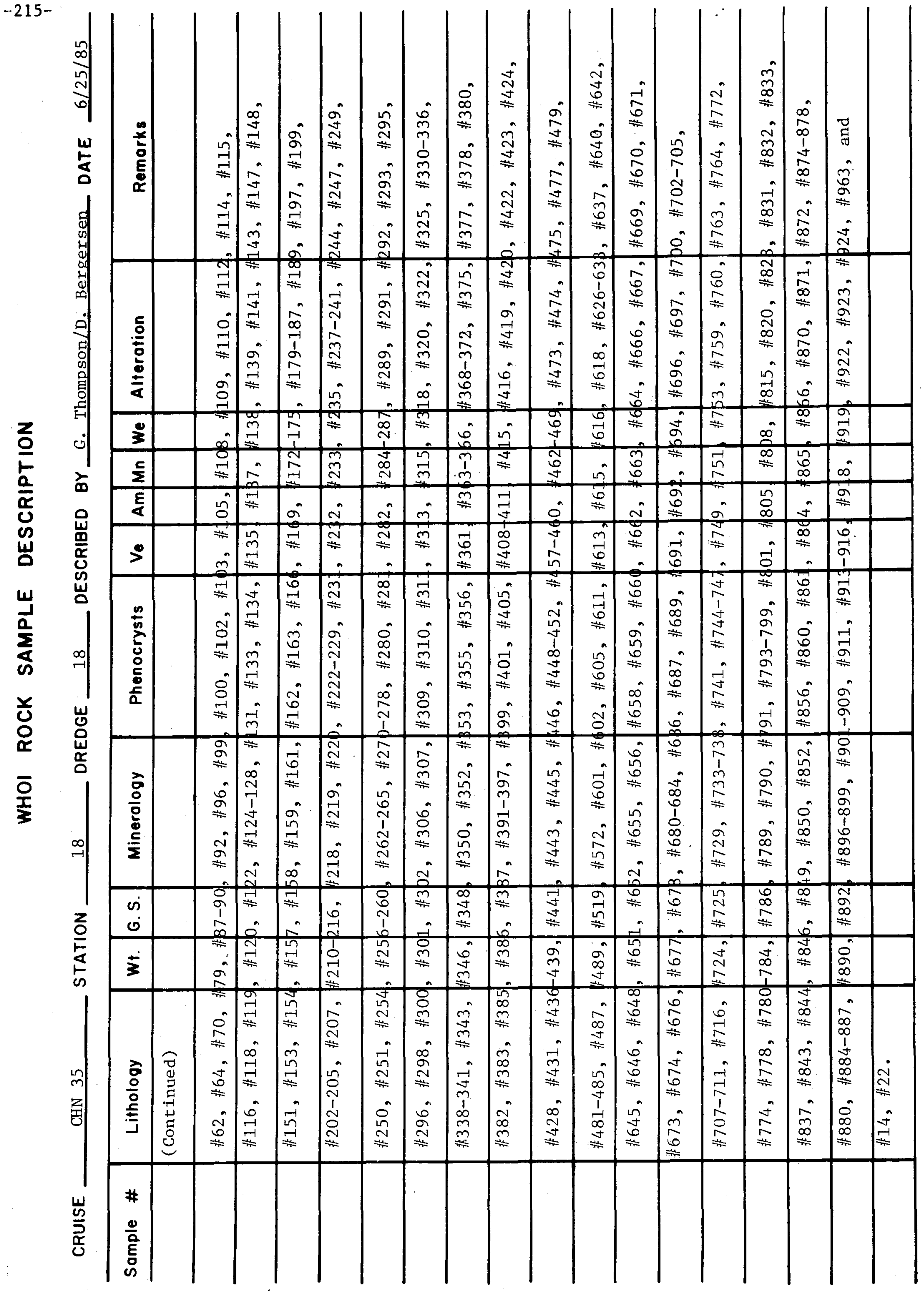




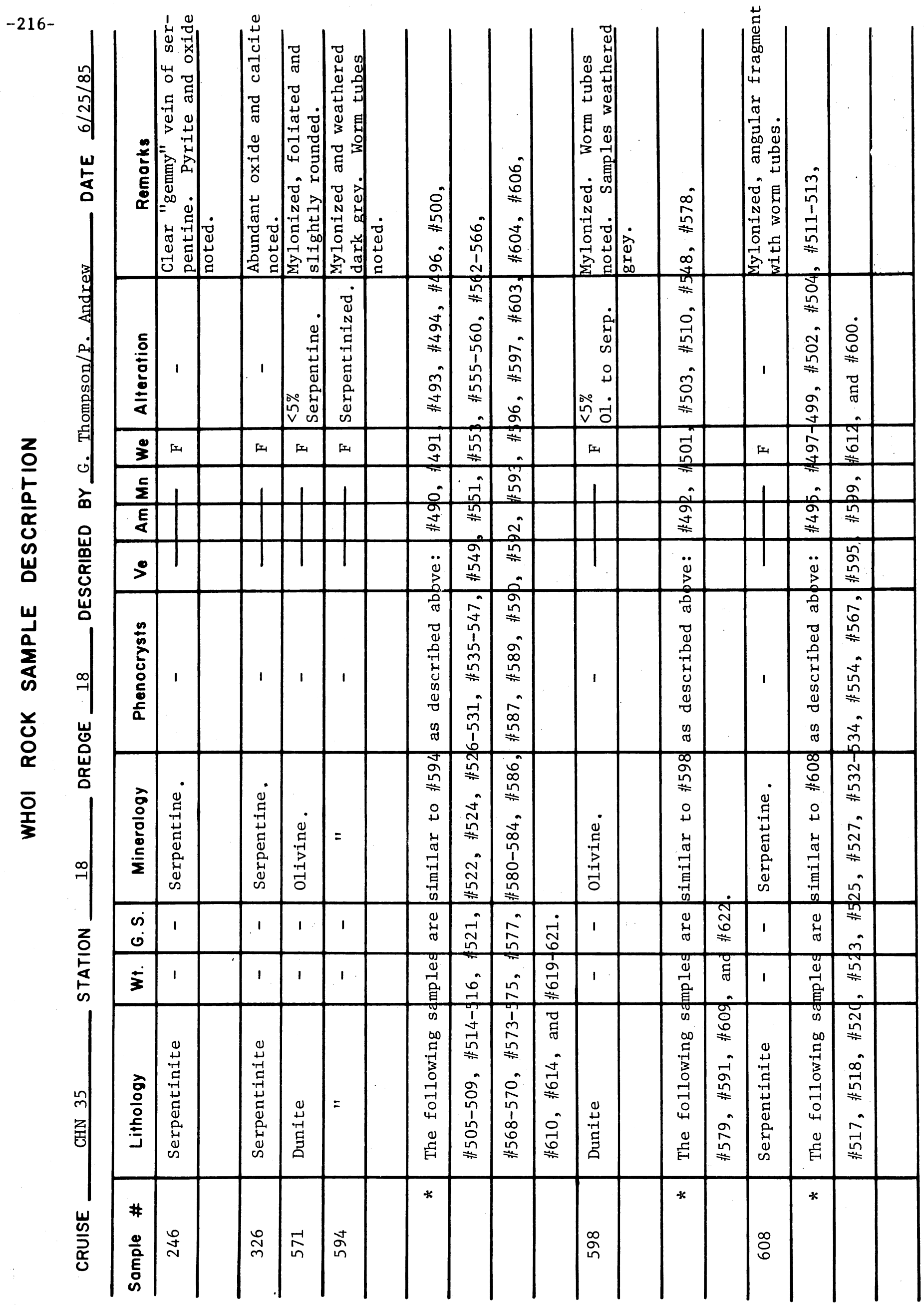




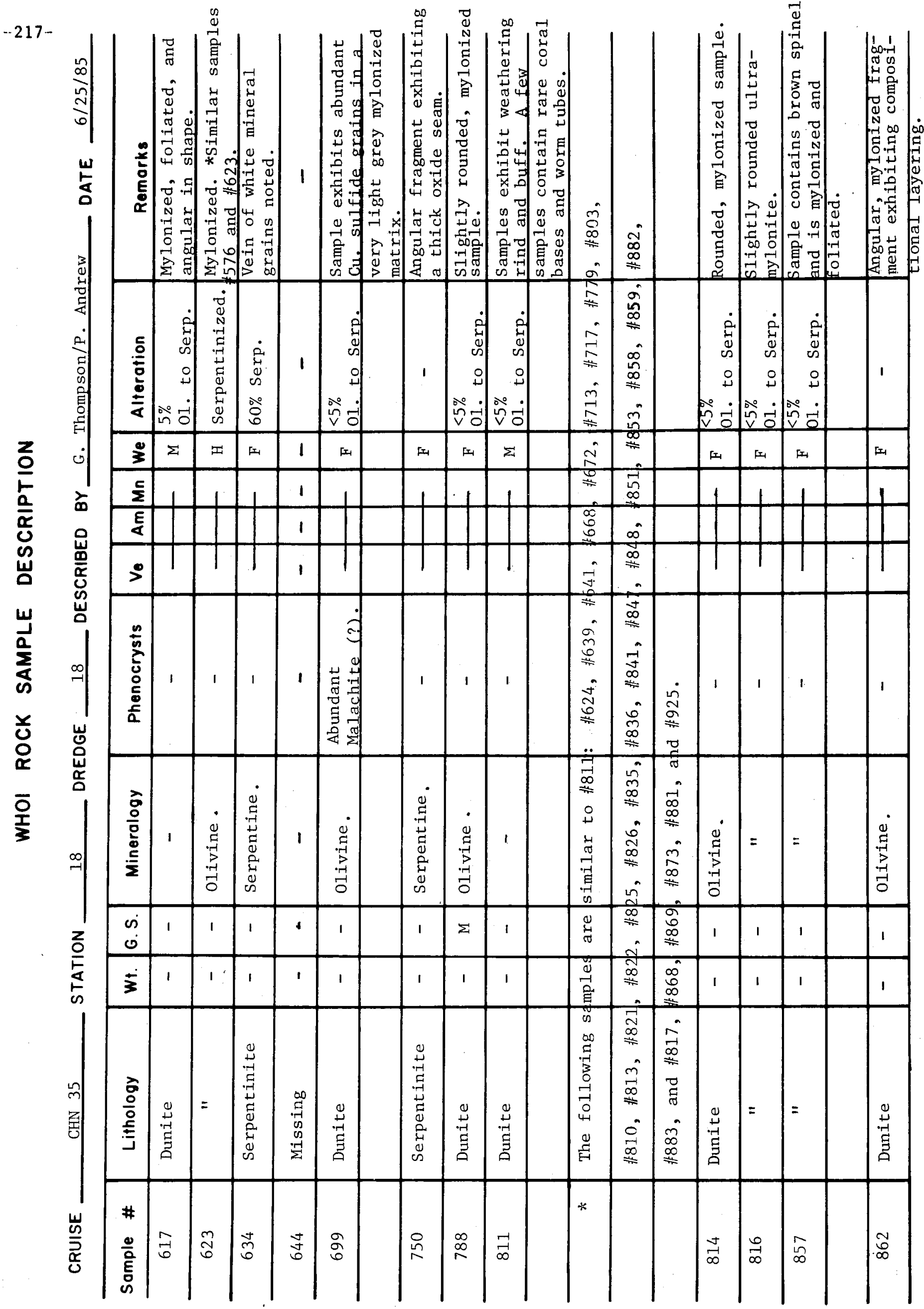




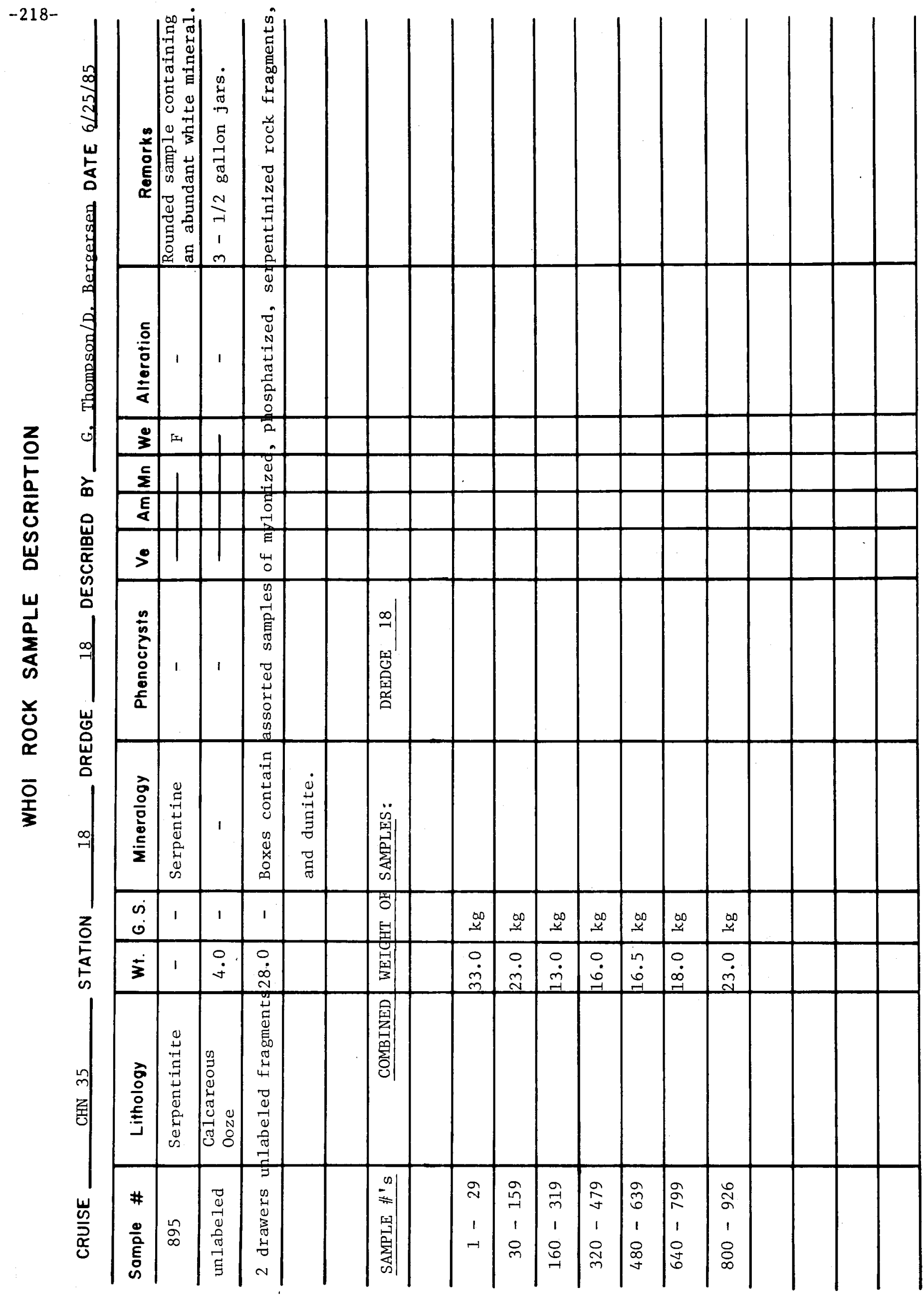




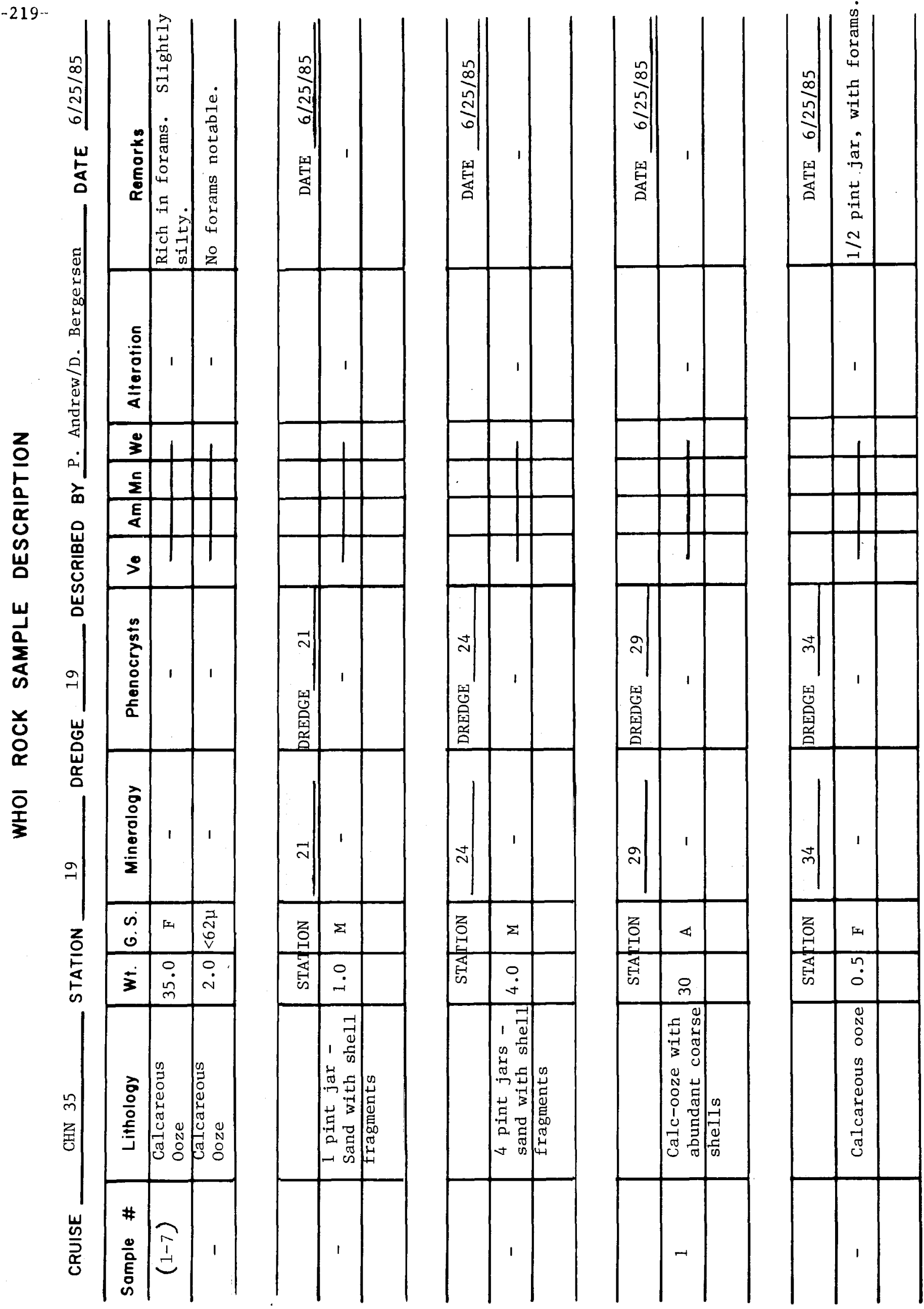




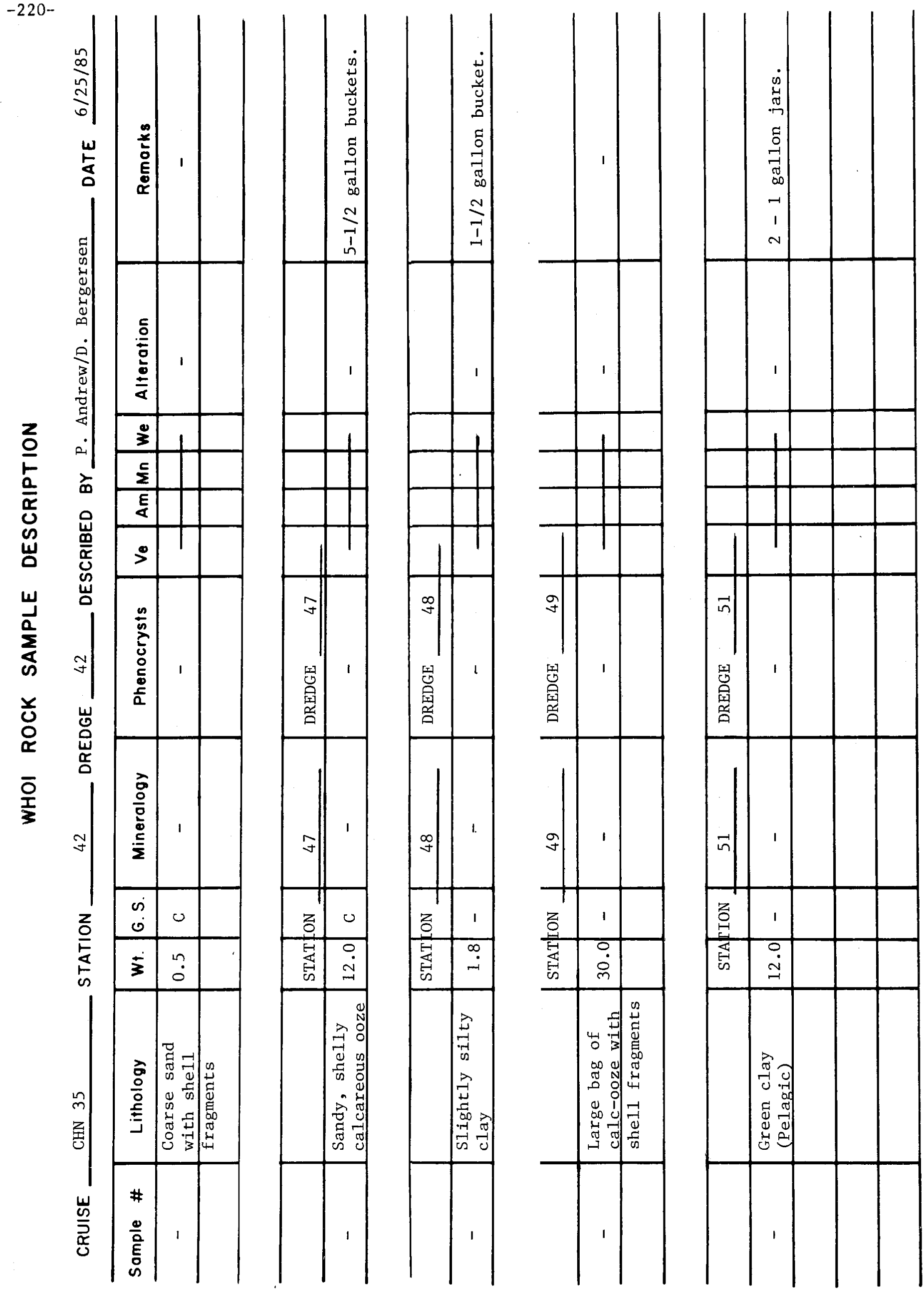




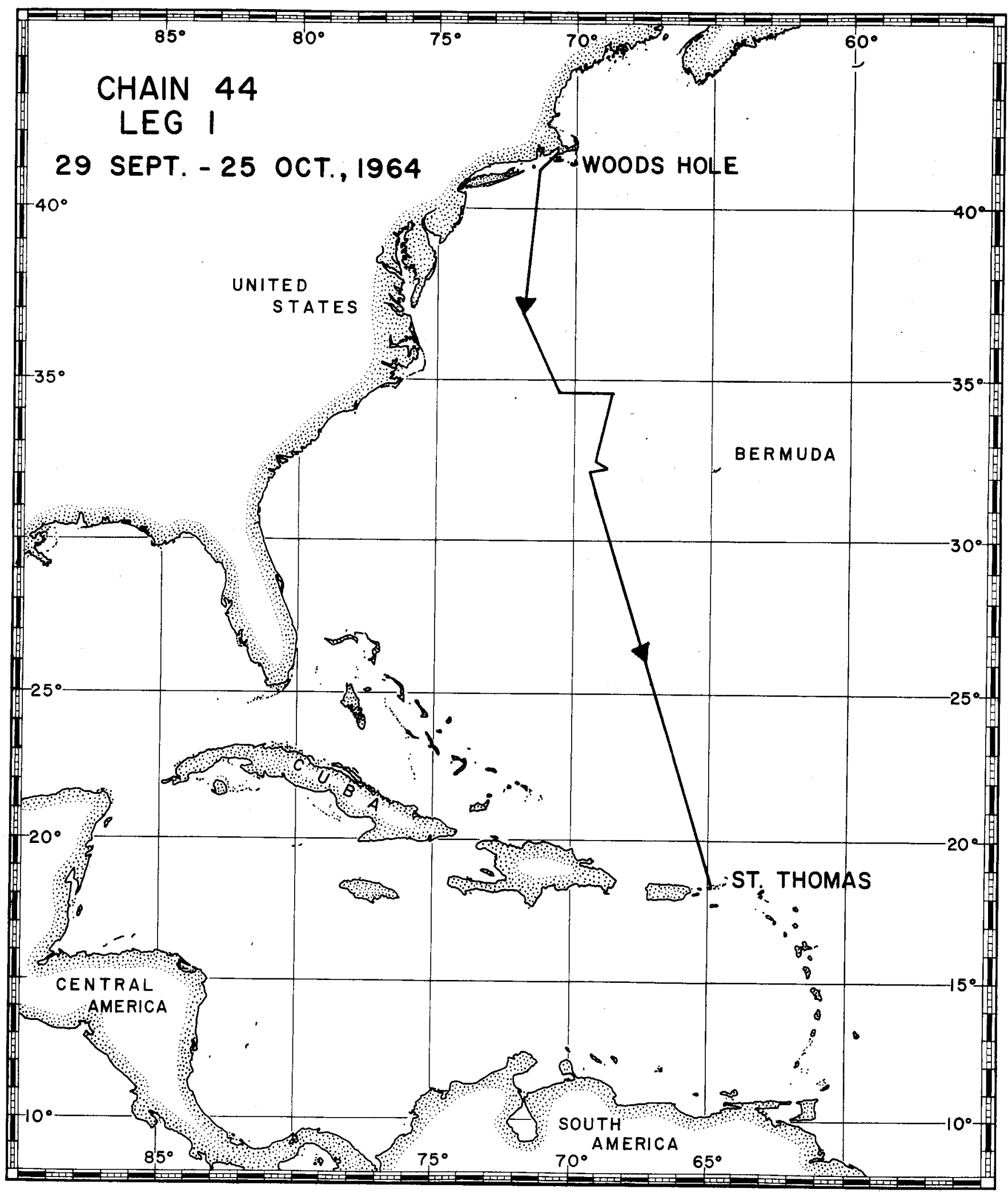




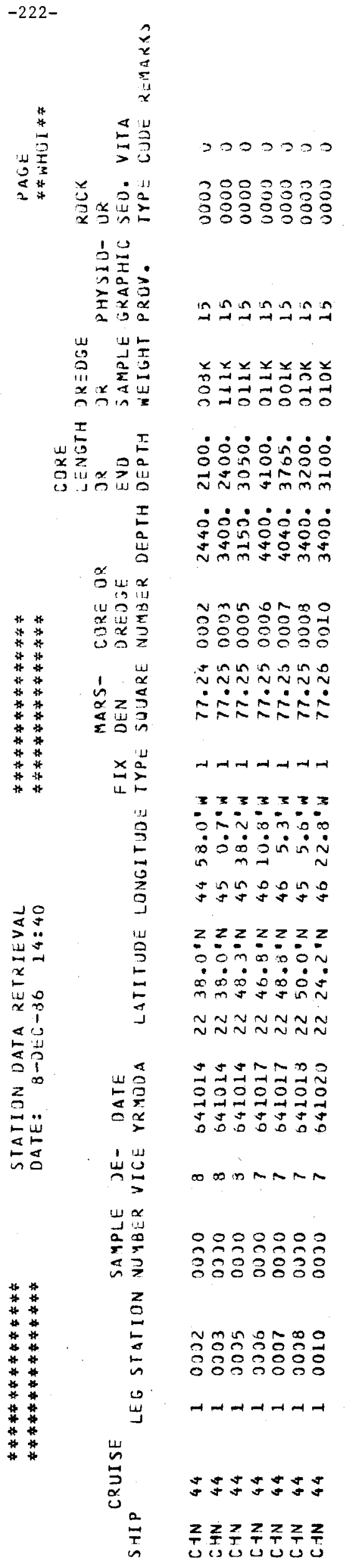




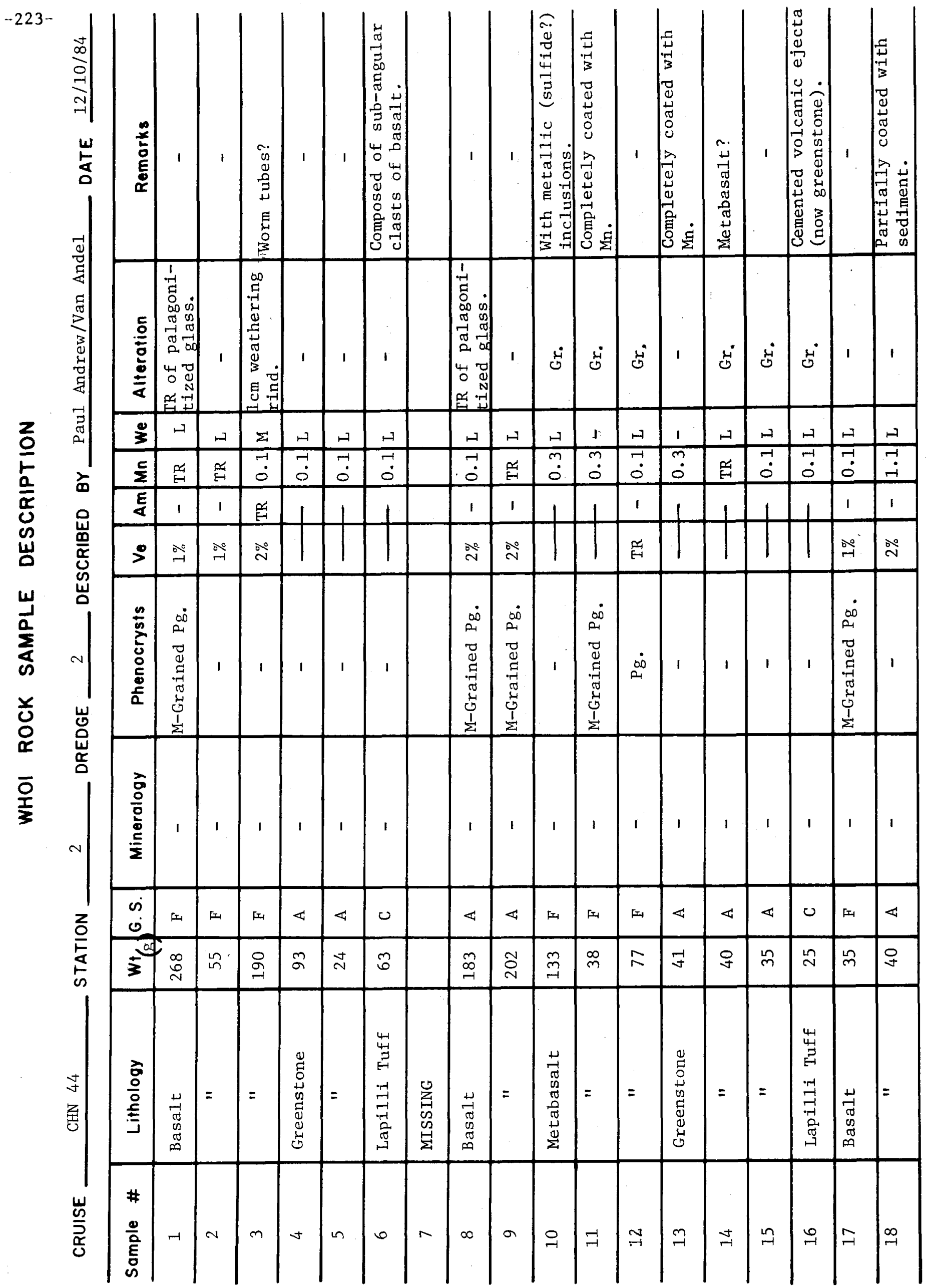




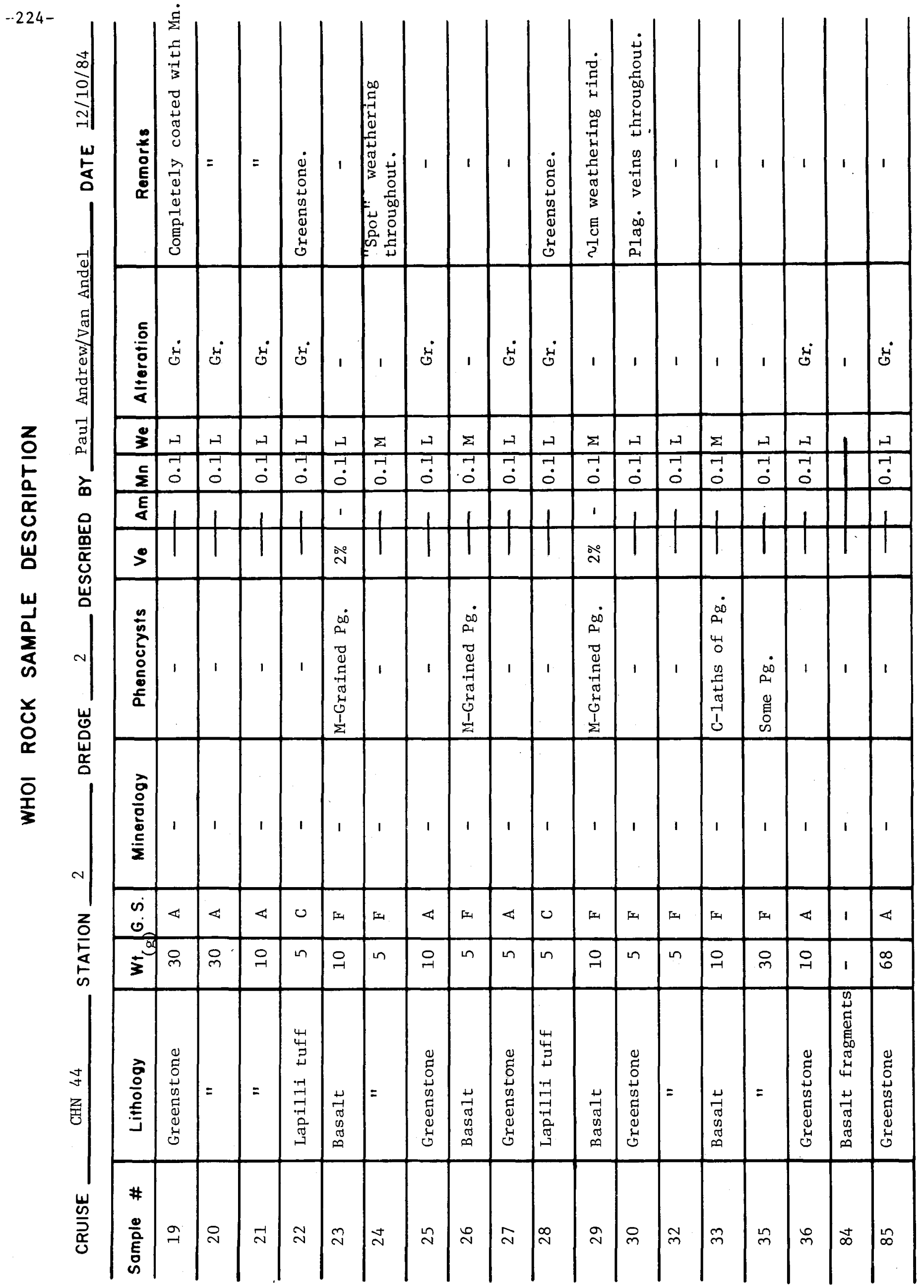




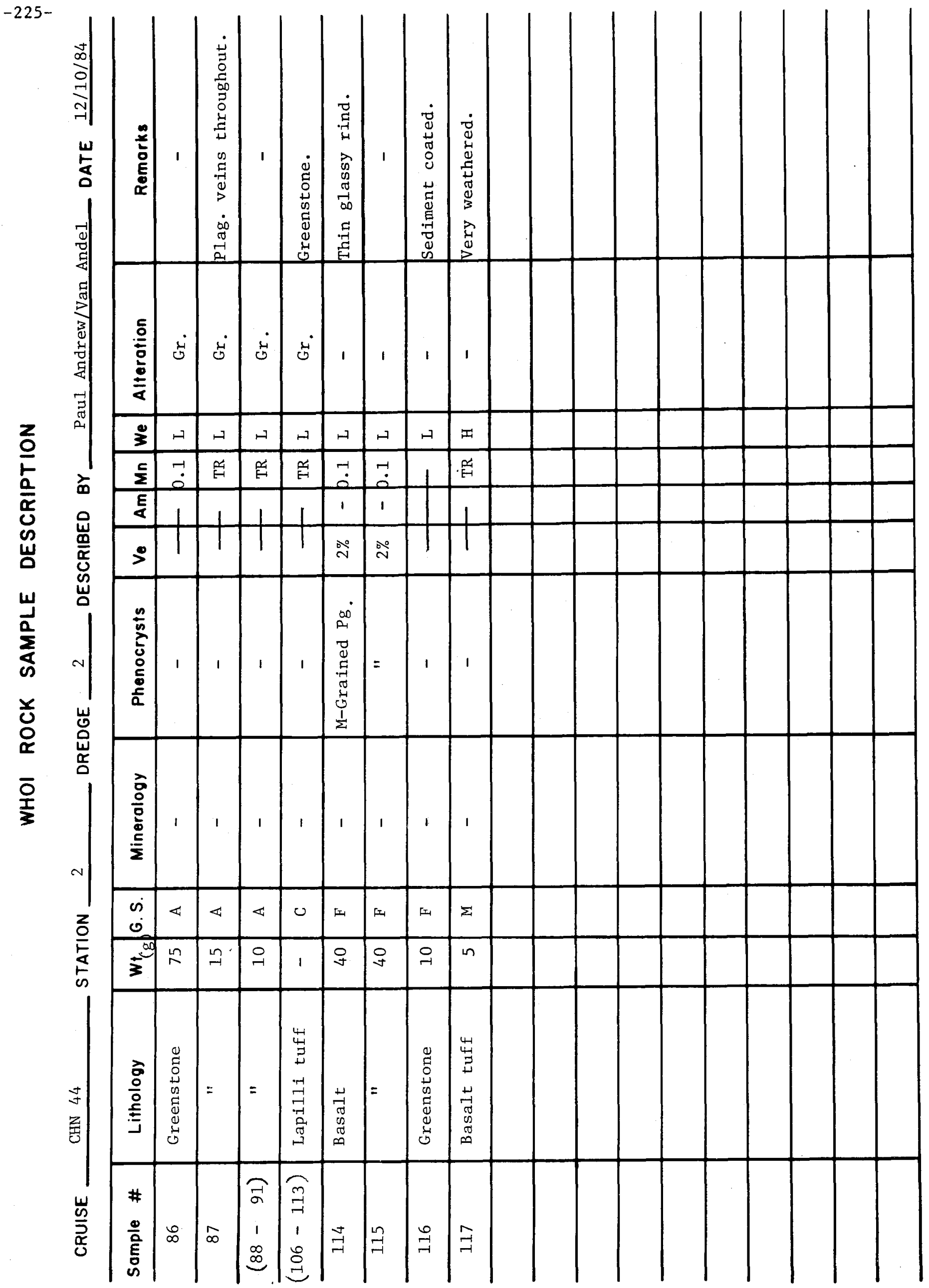




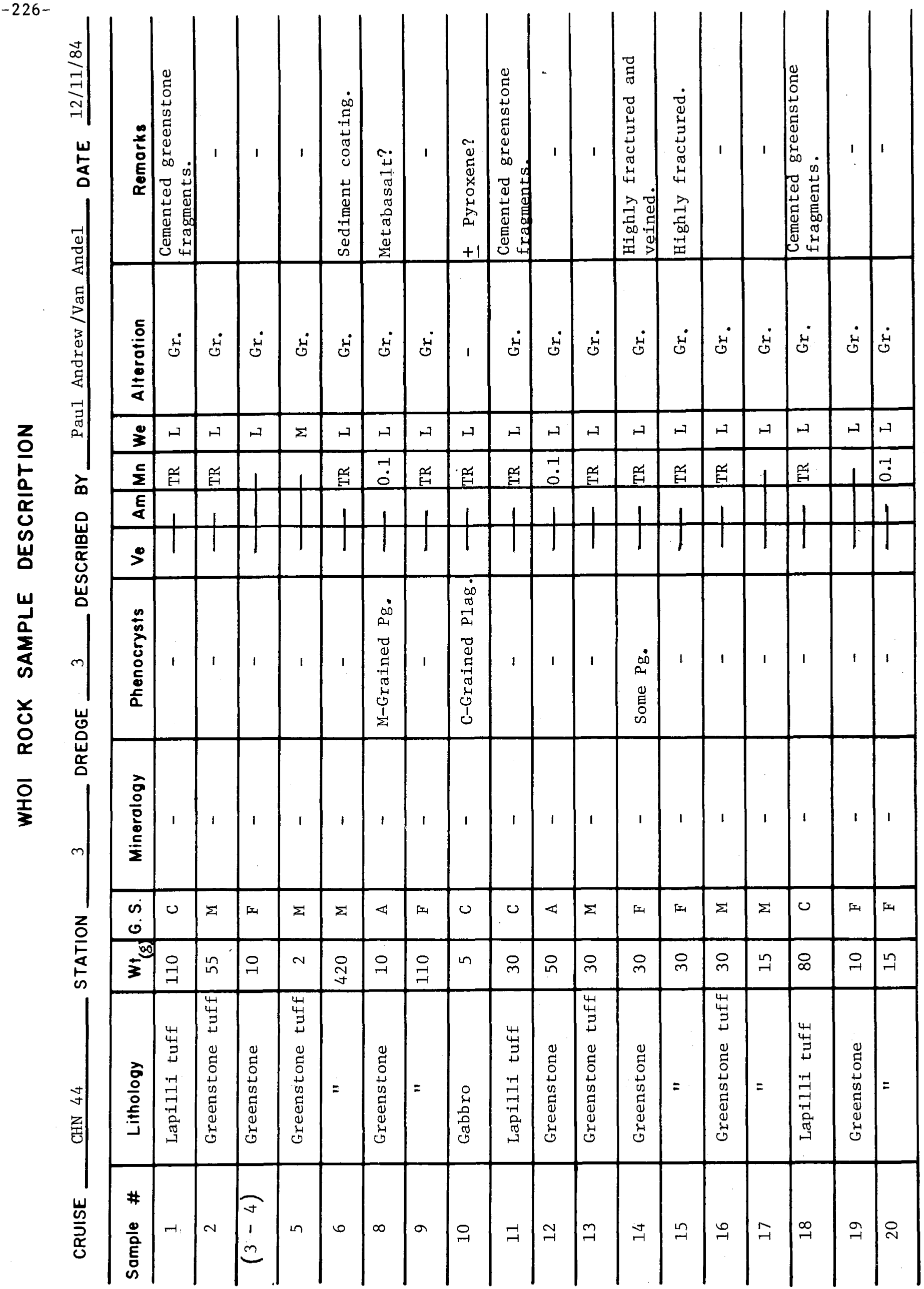




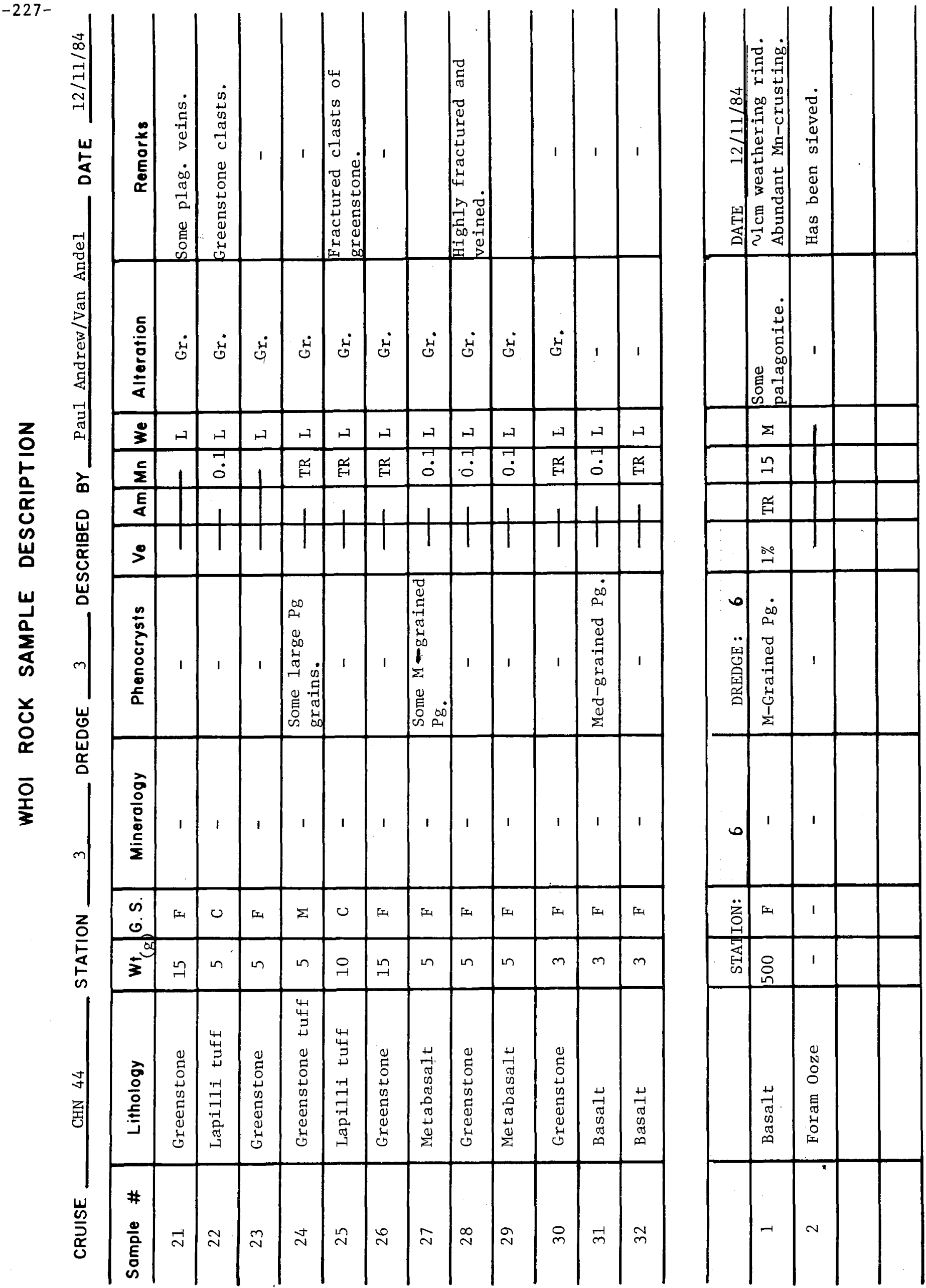



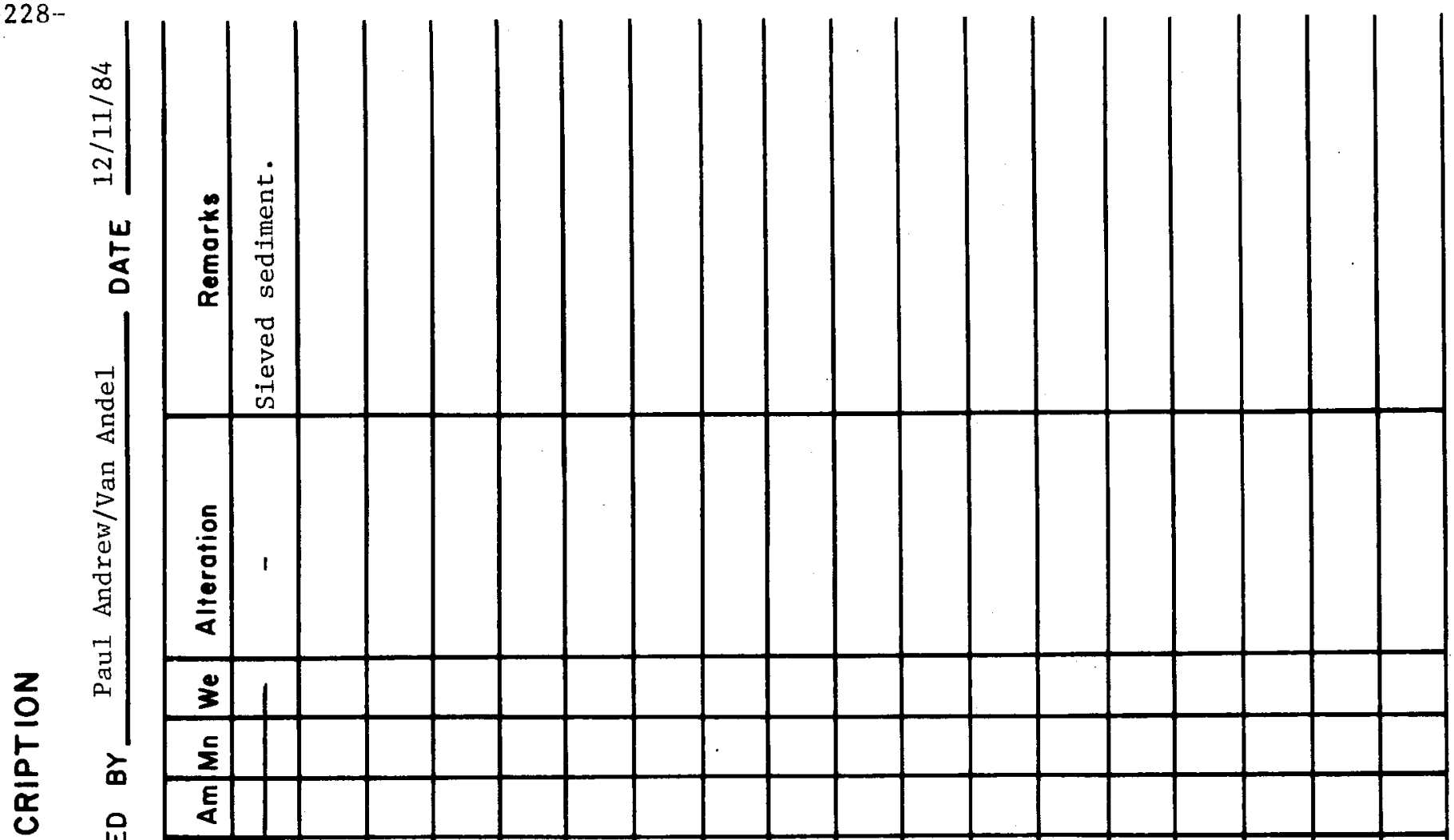

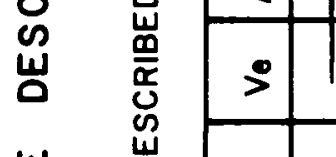

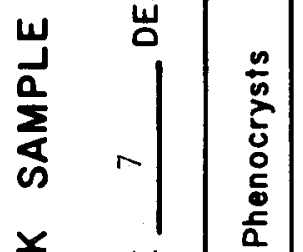

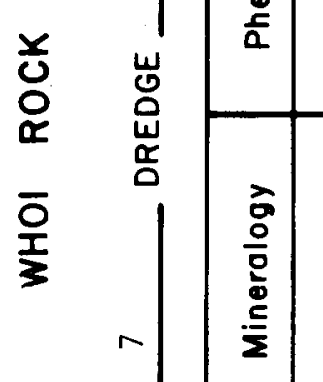

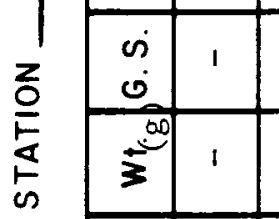

范

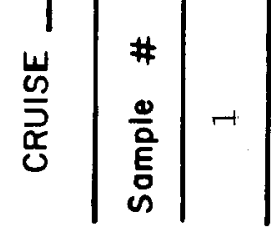




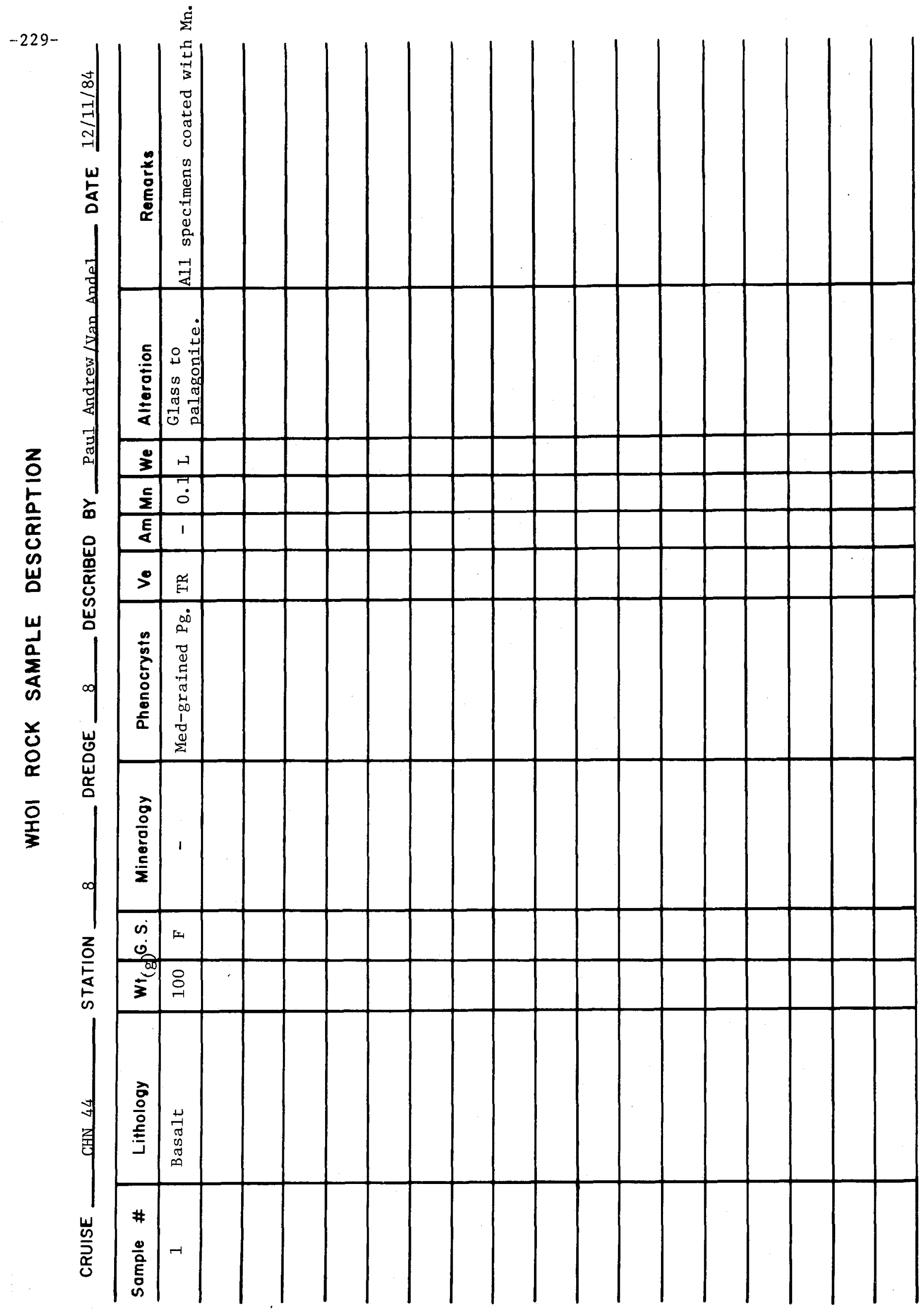




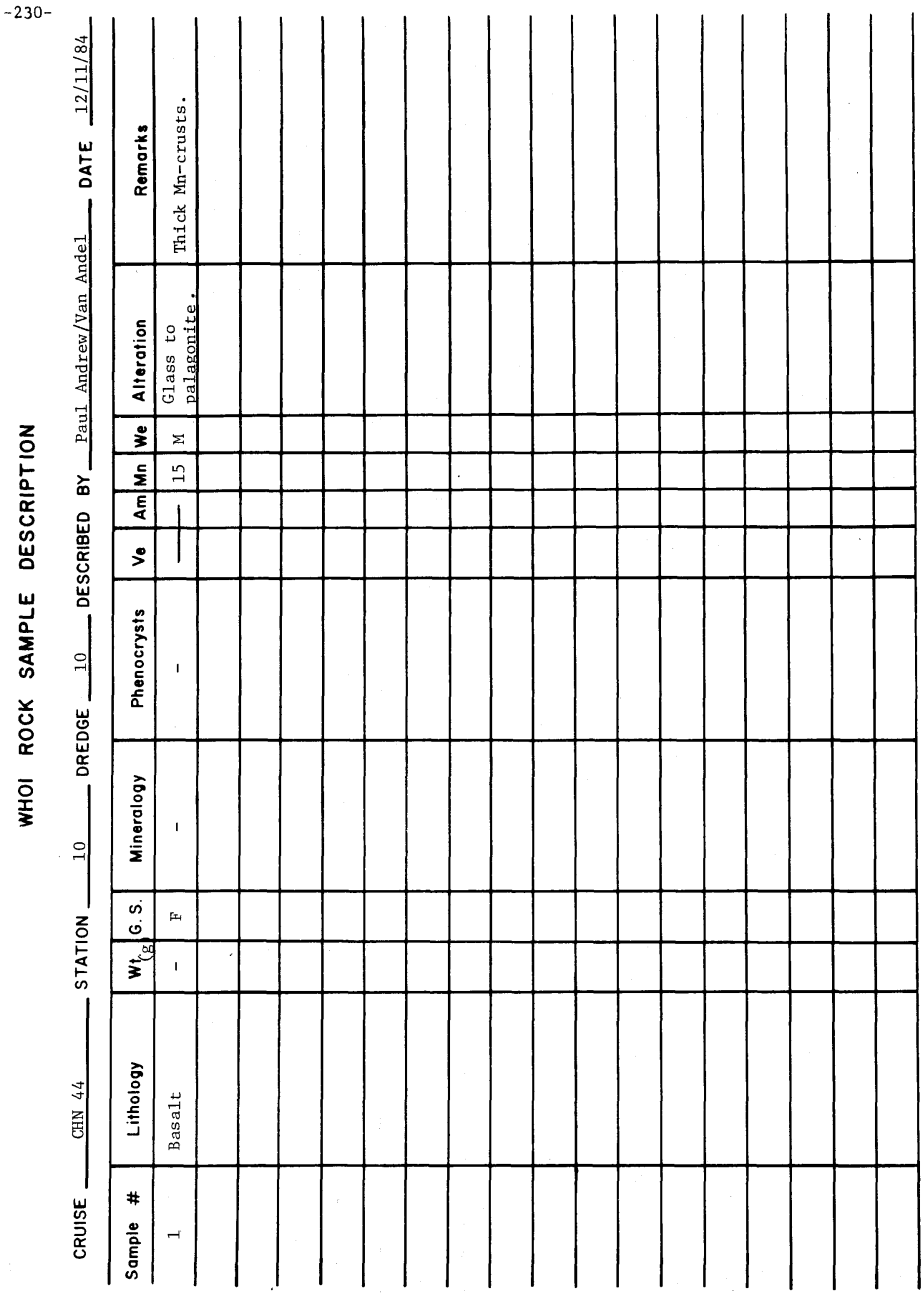




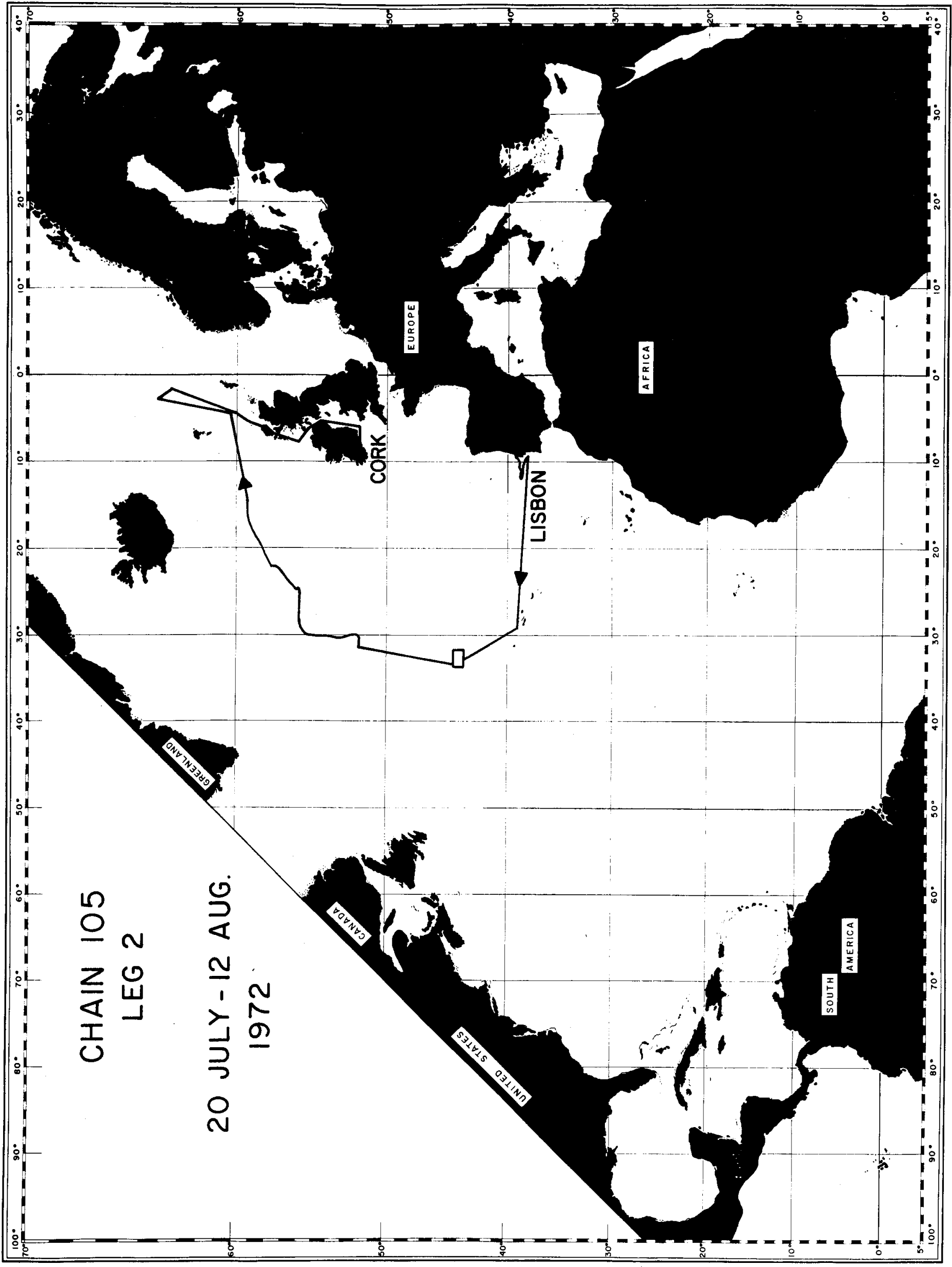




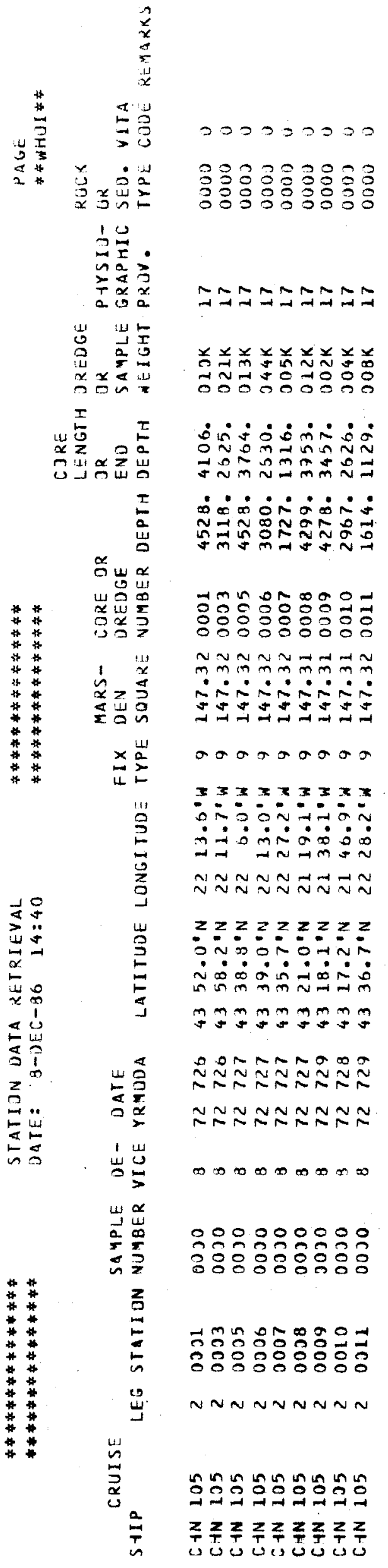




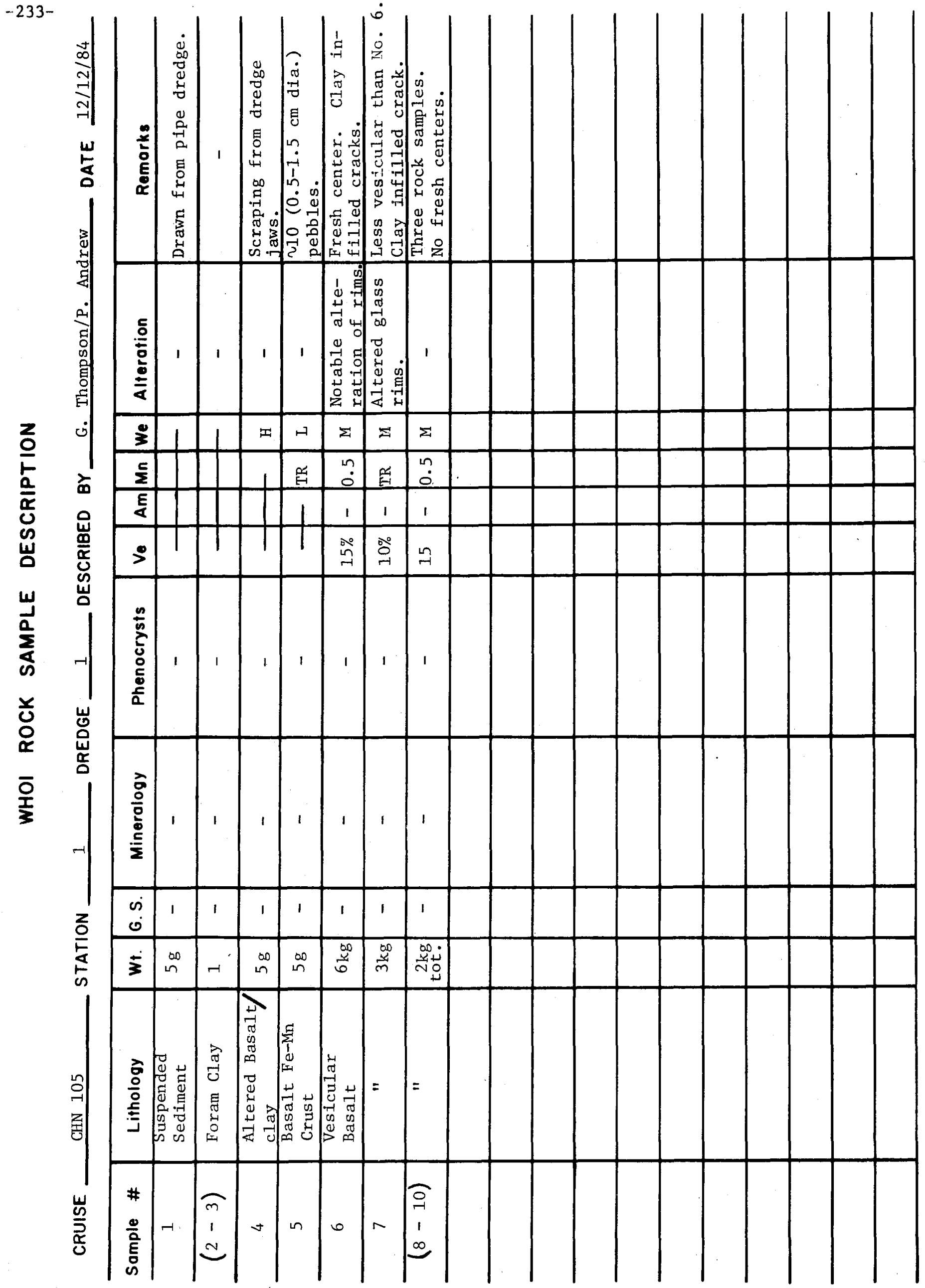




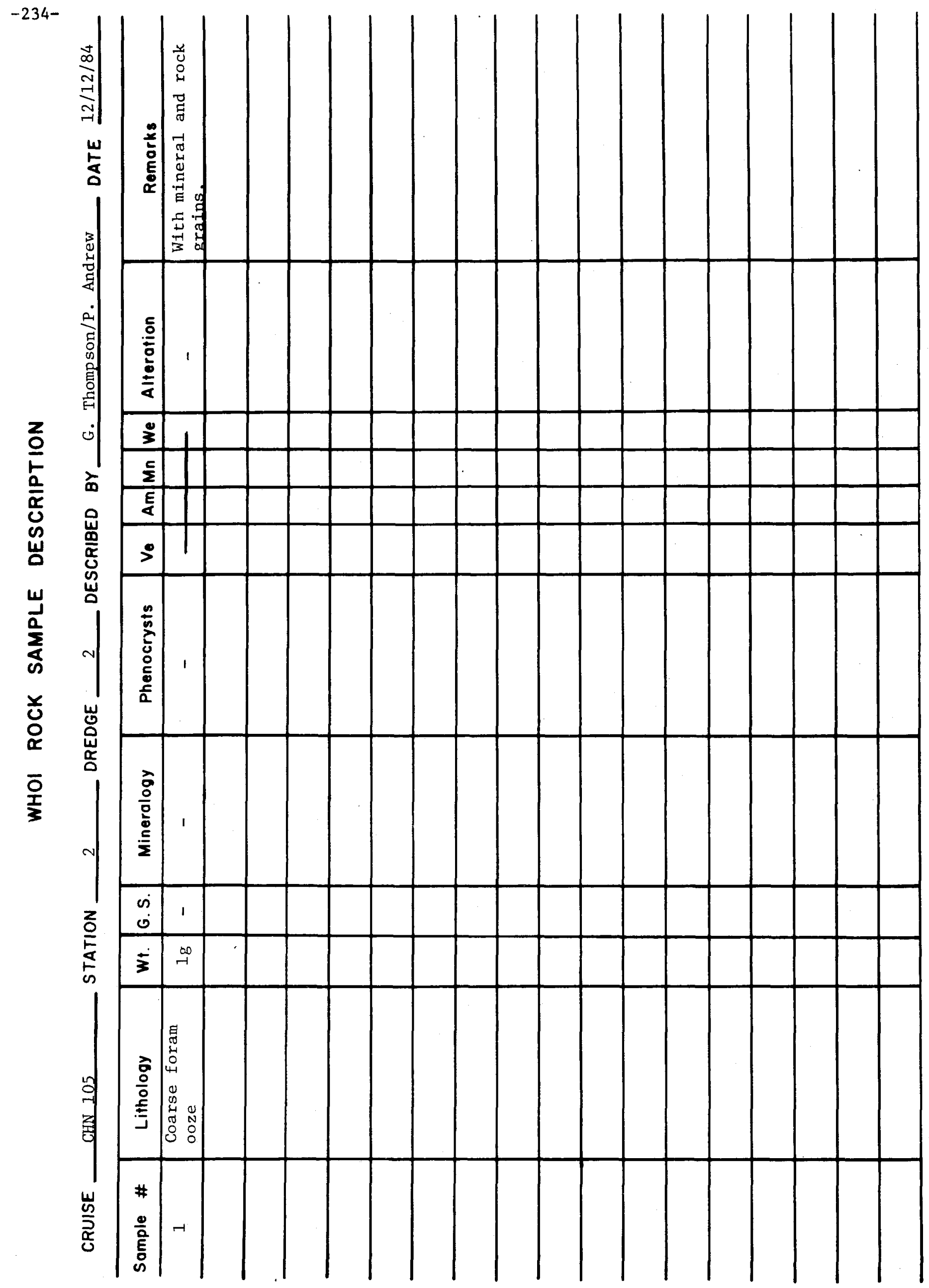




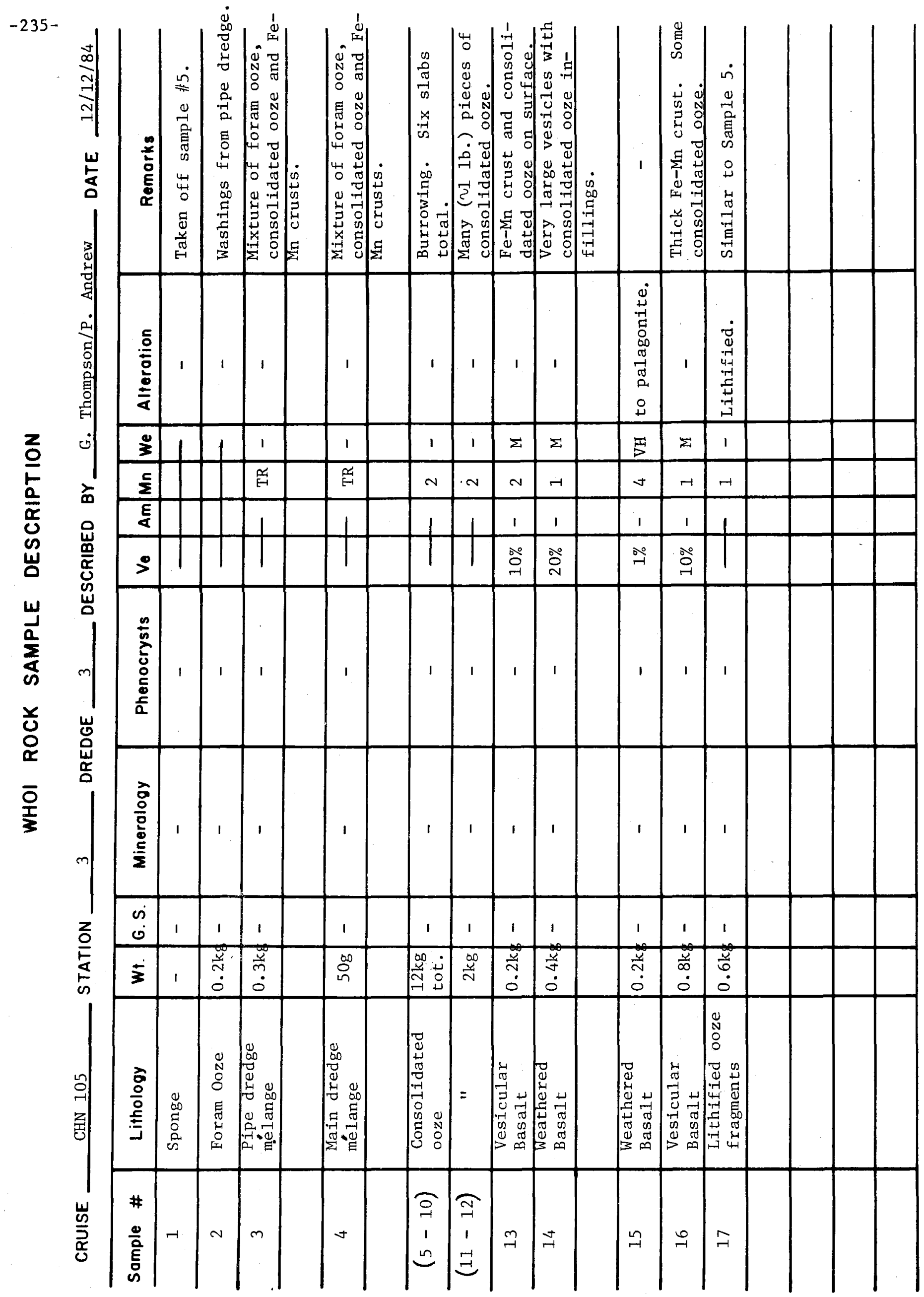




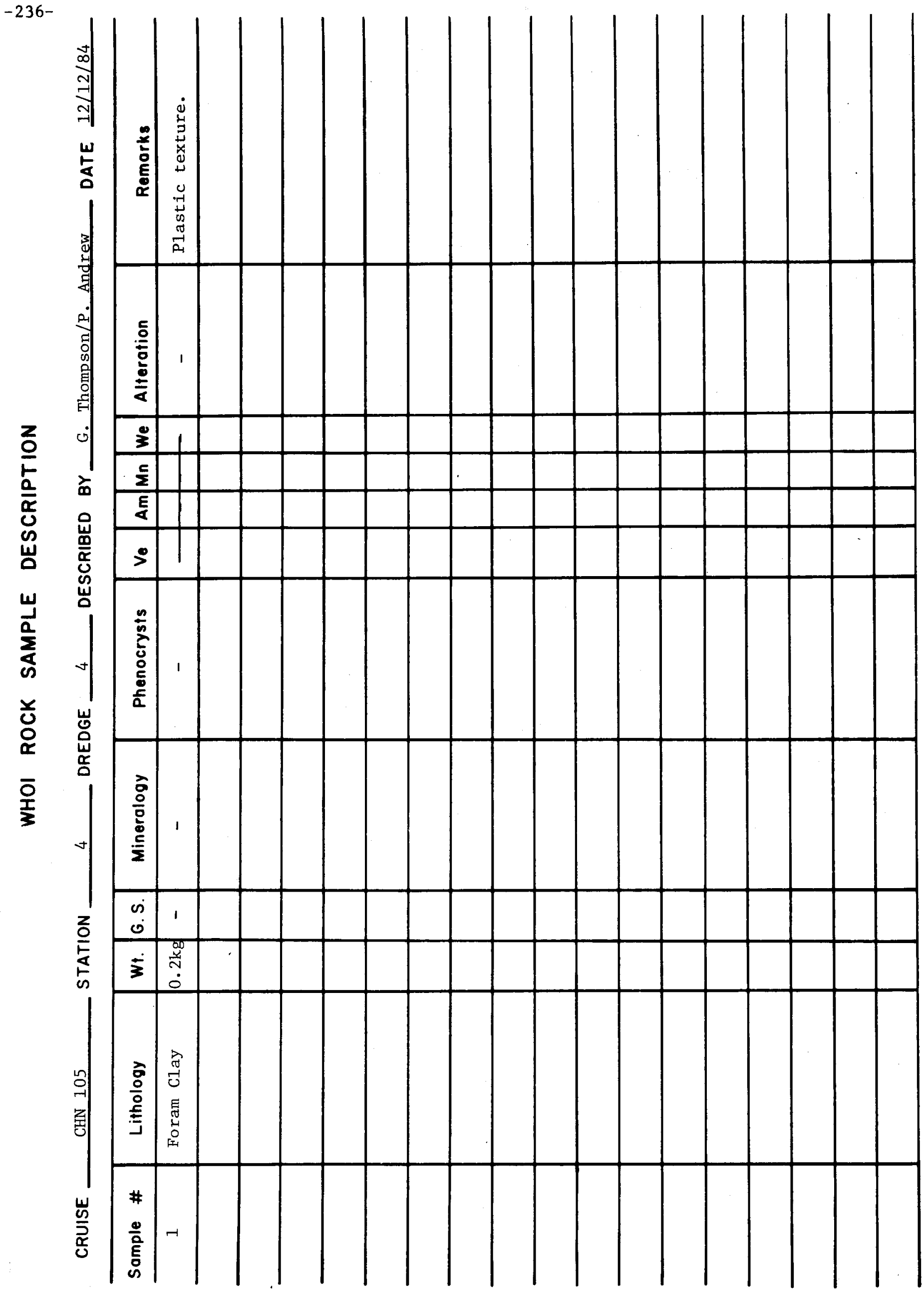




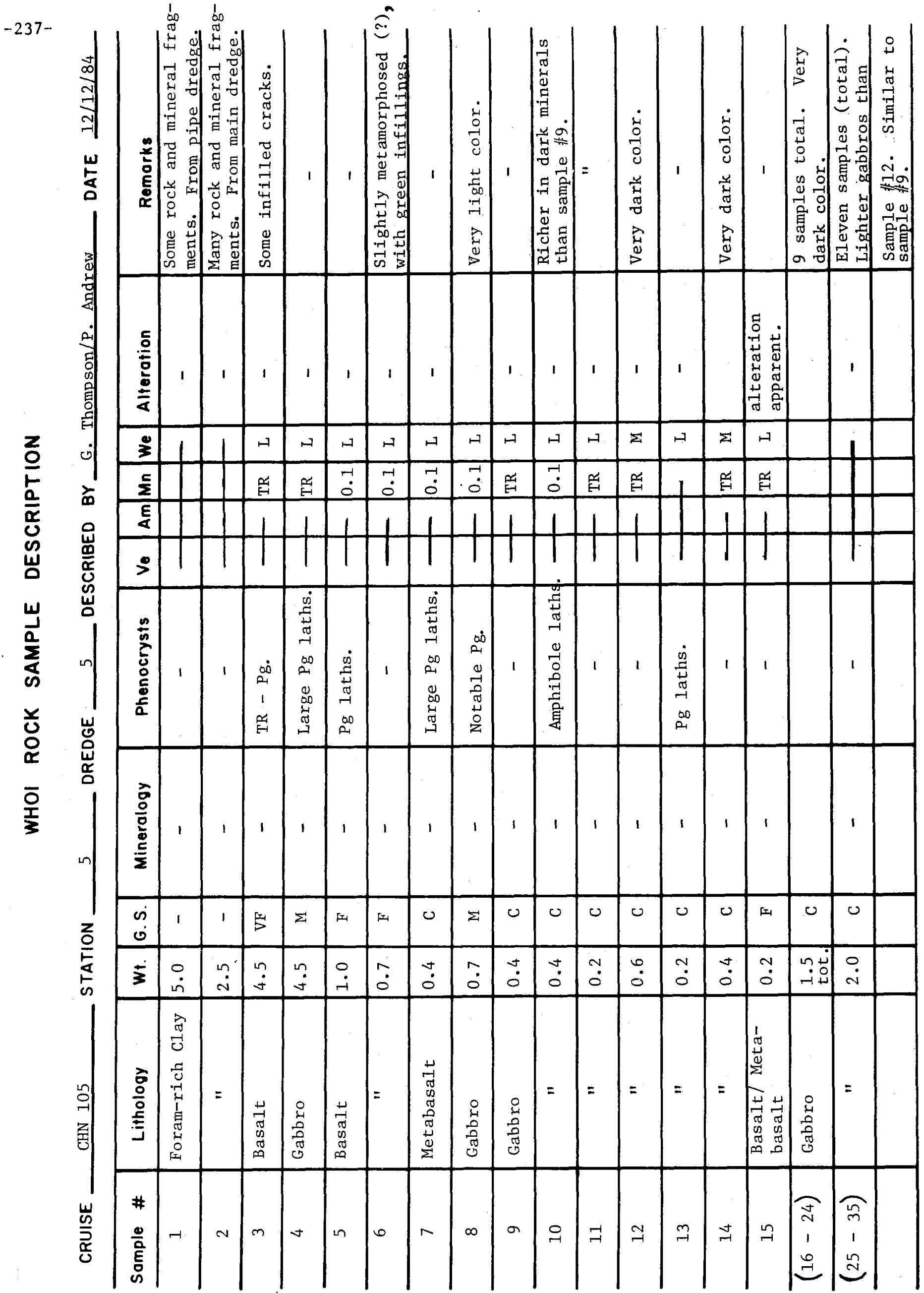




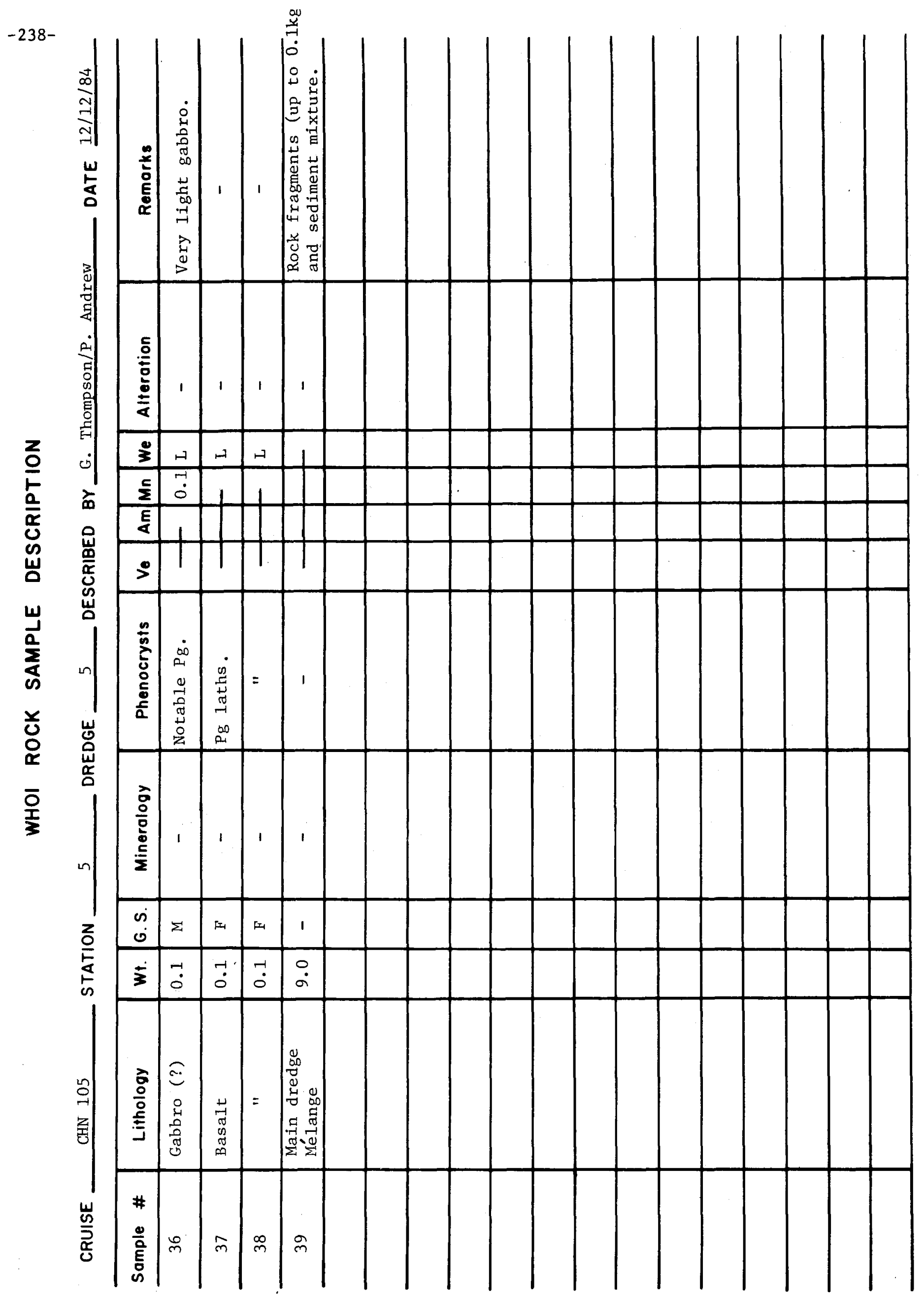




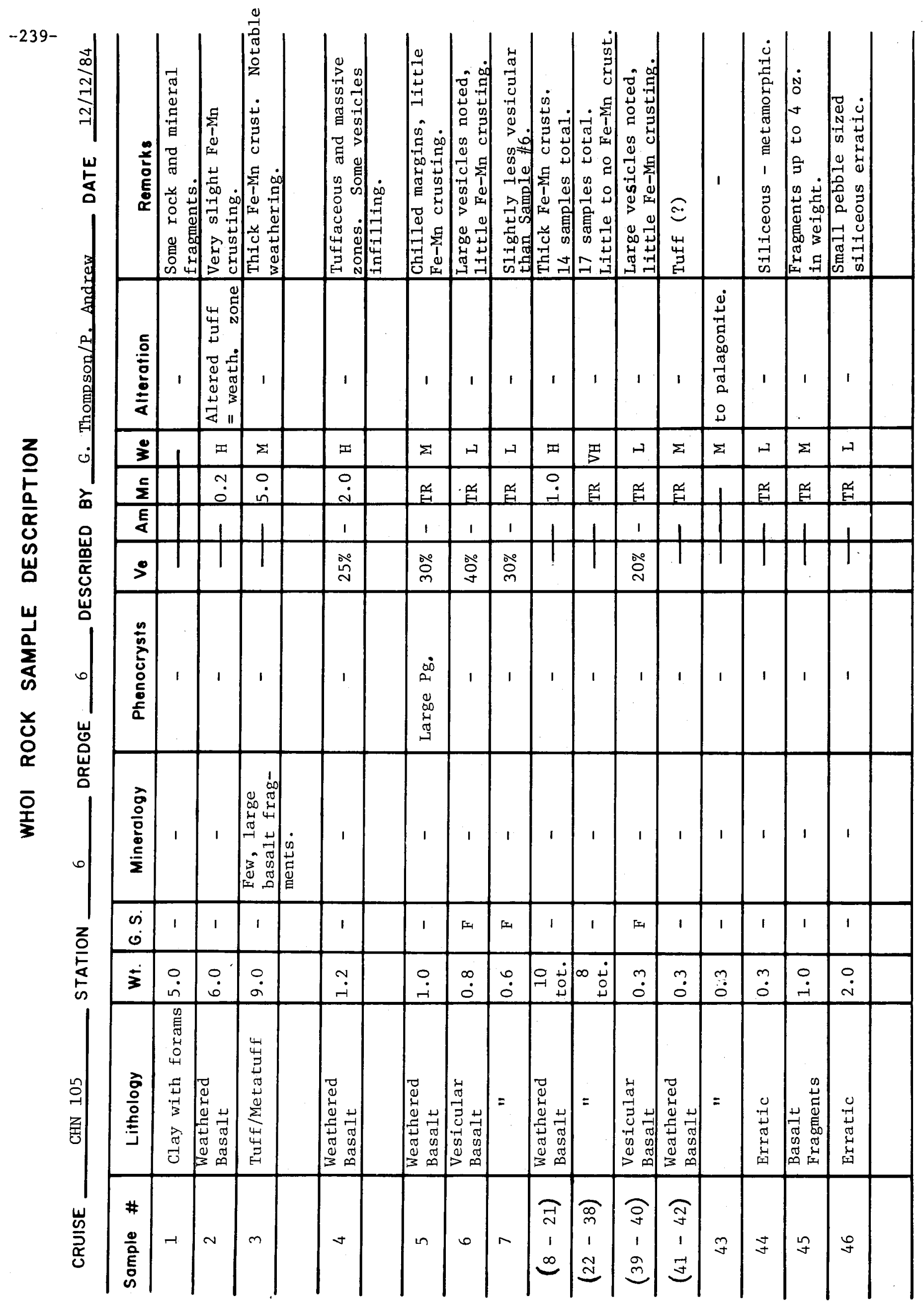




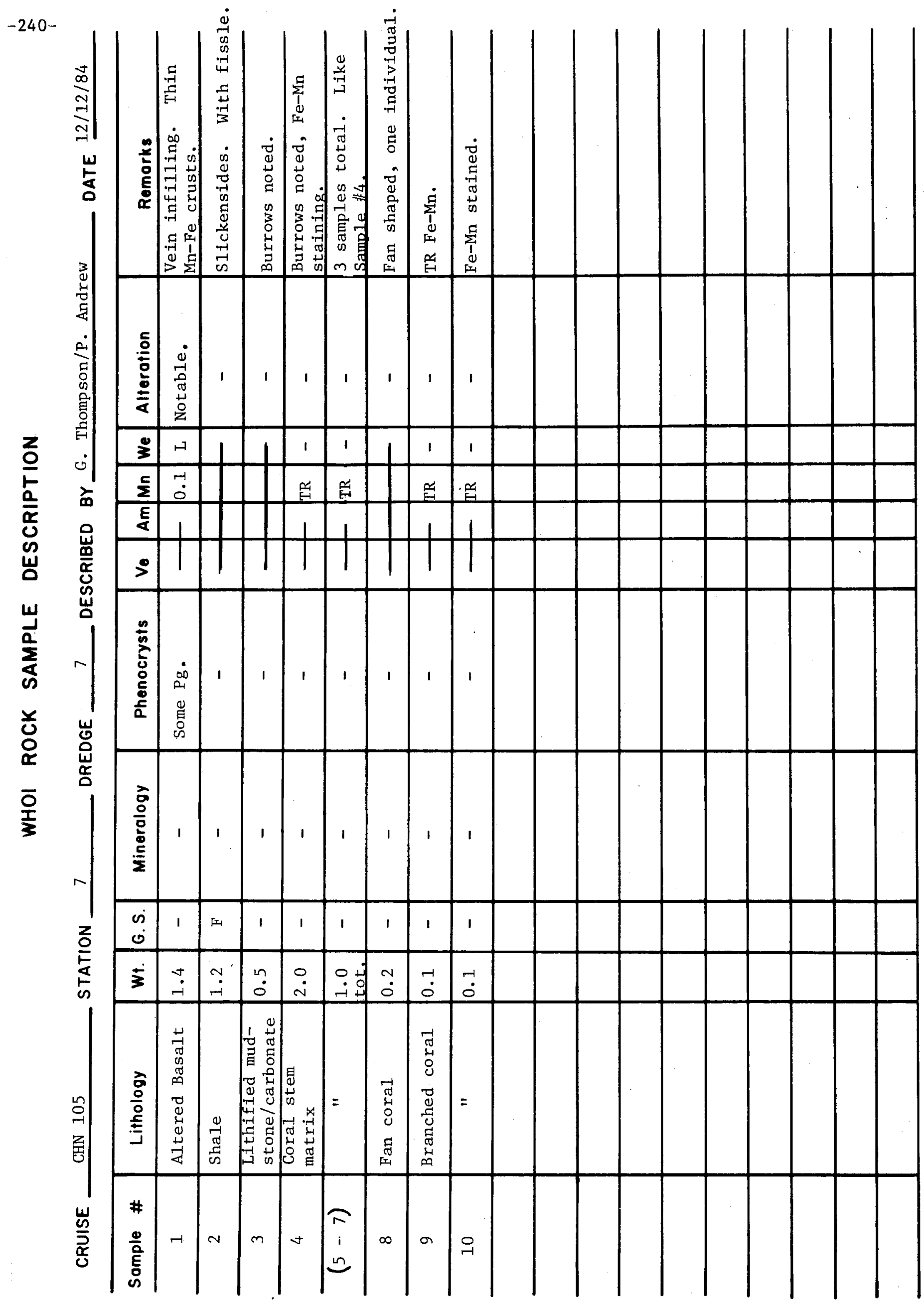




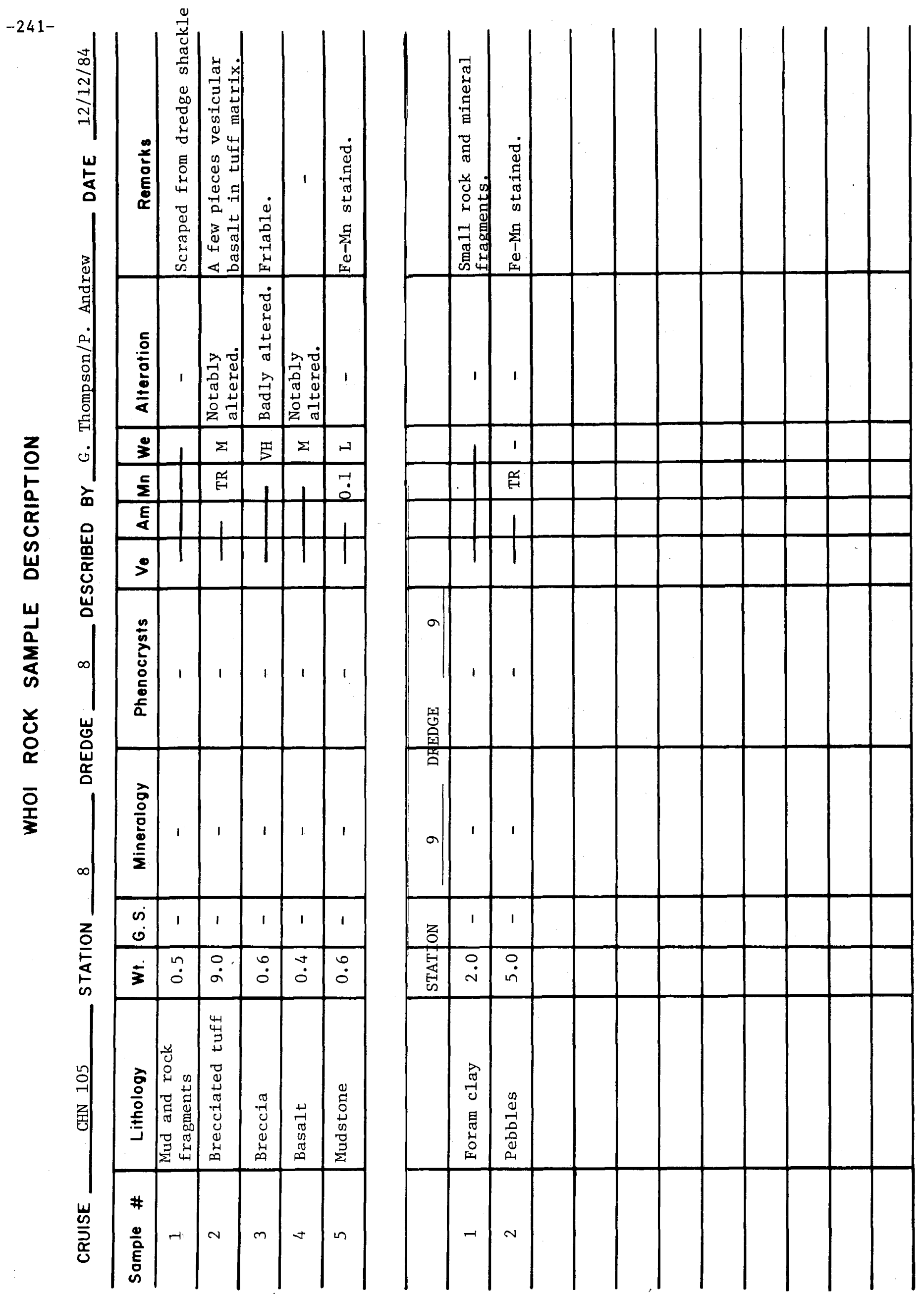




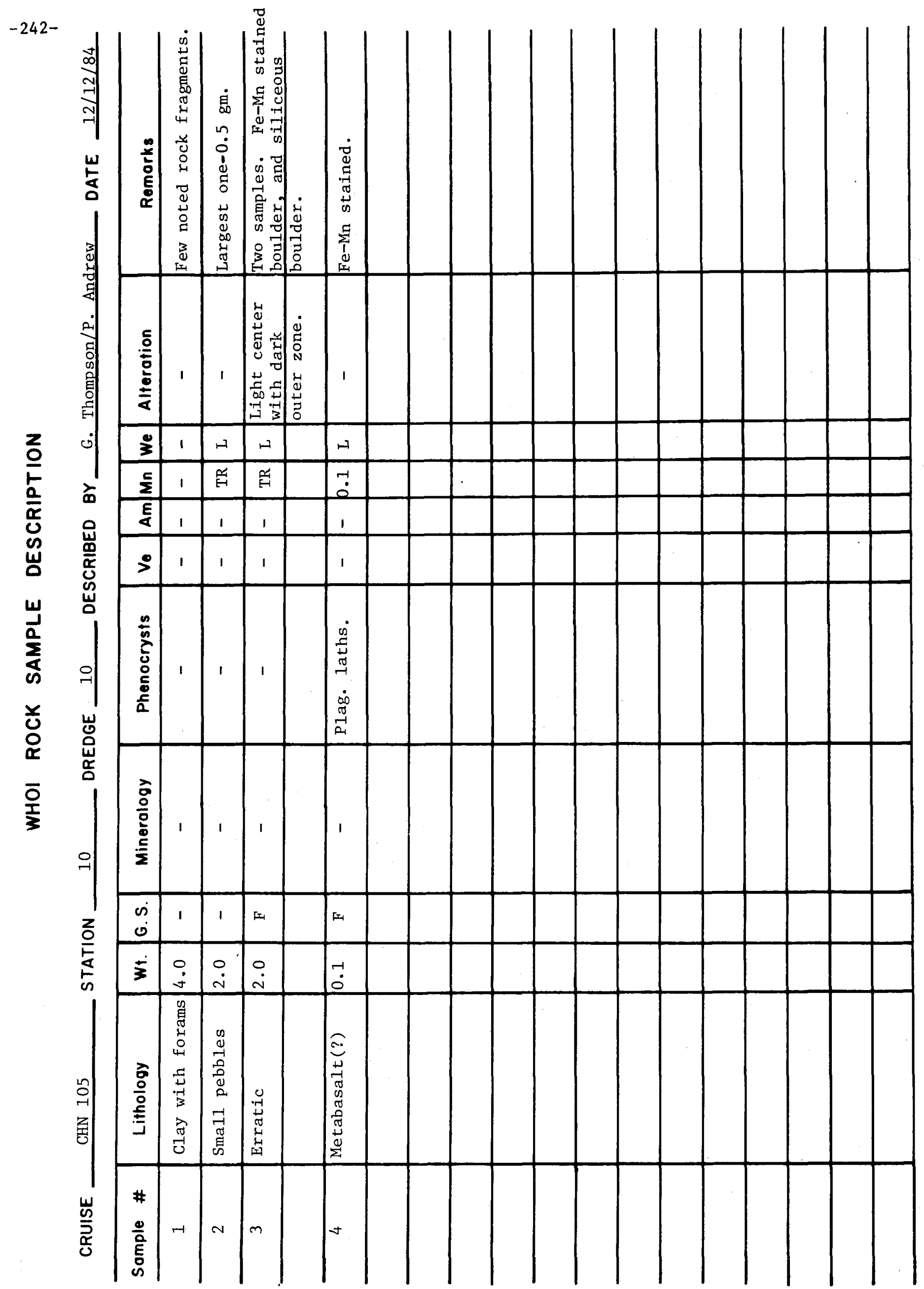




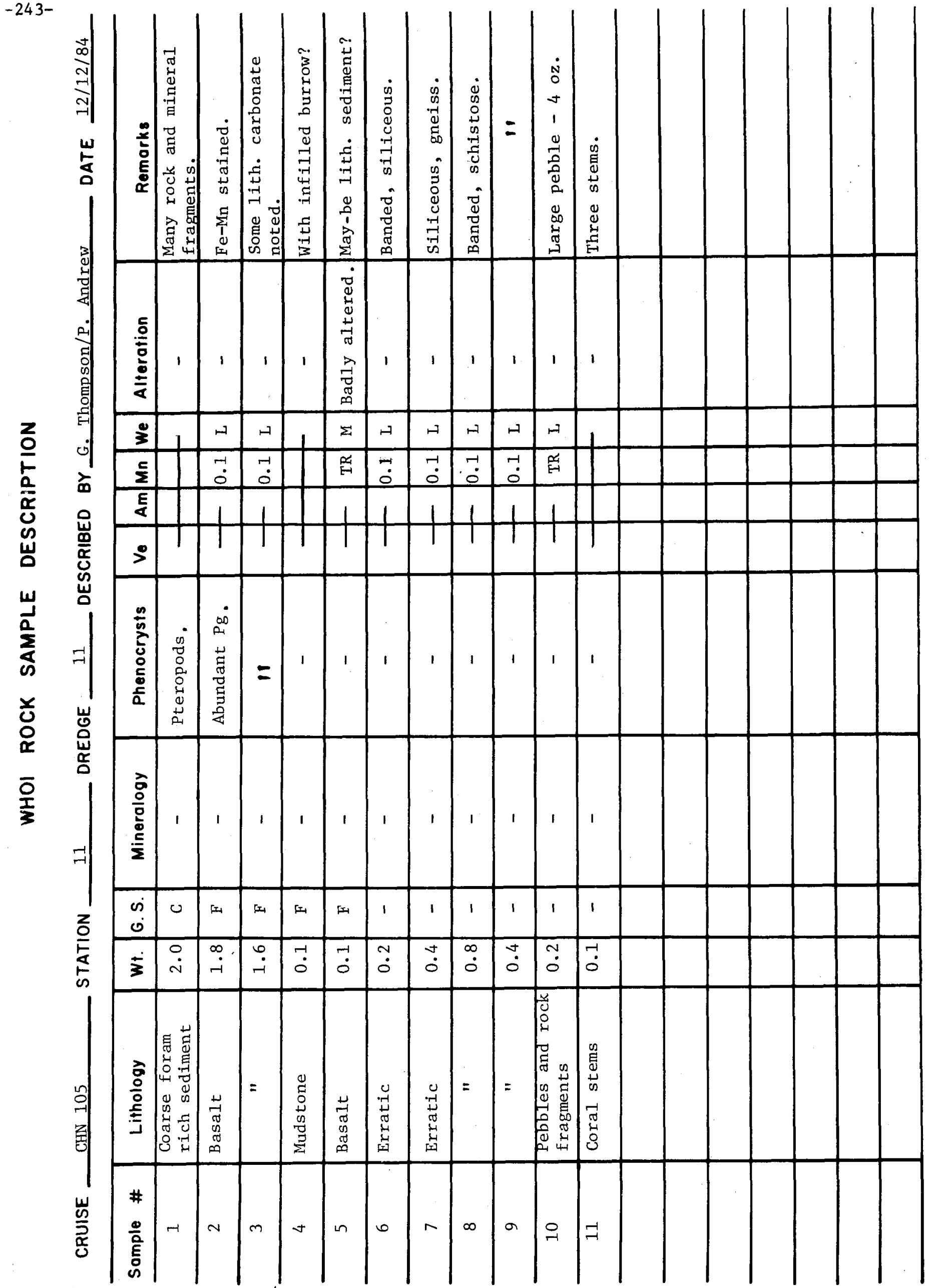




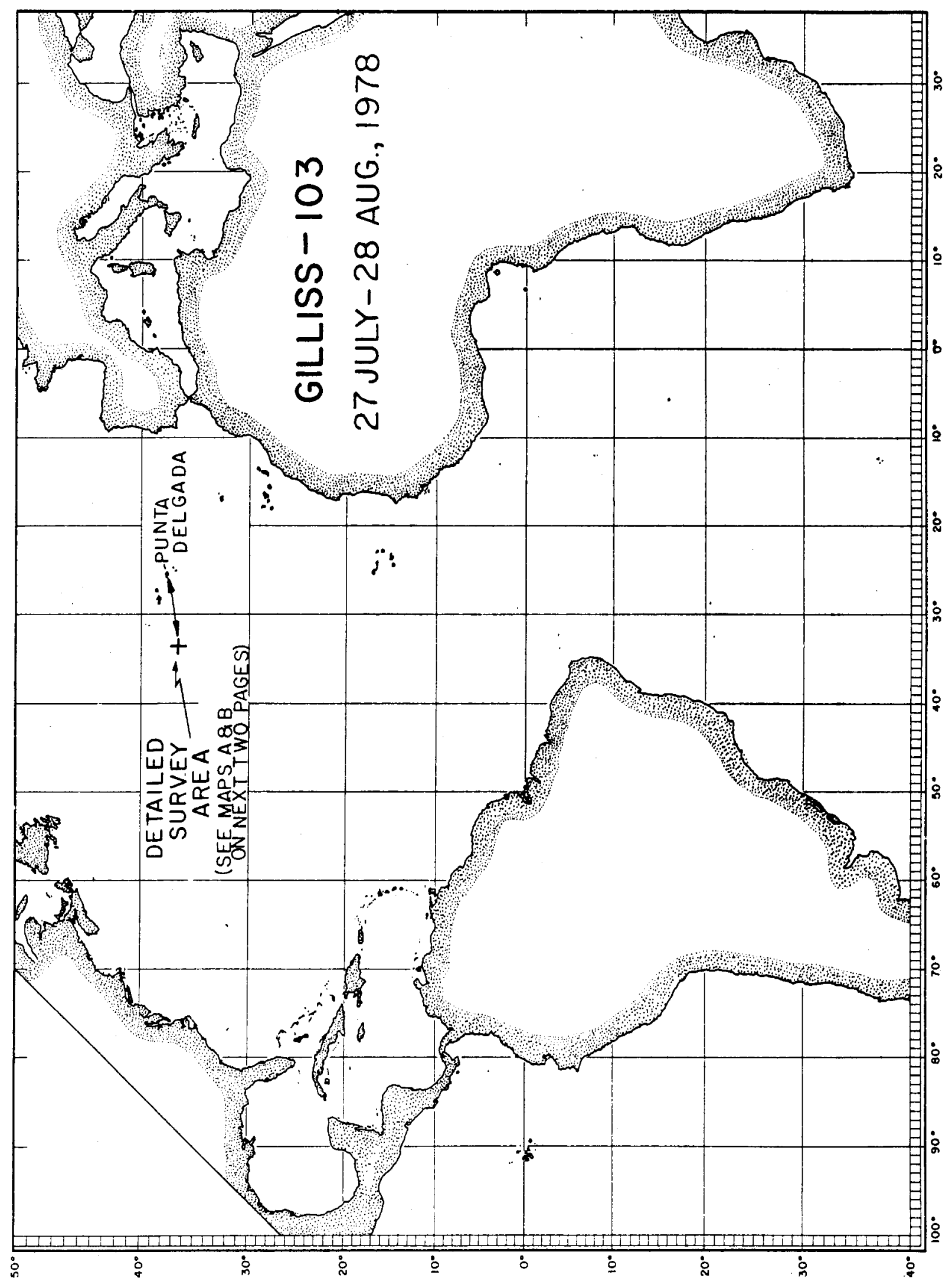




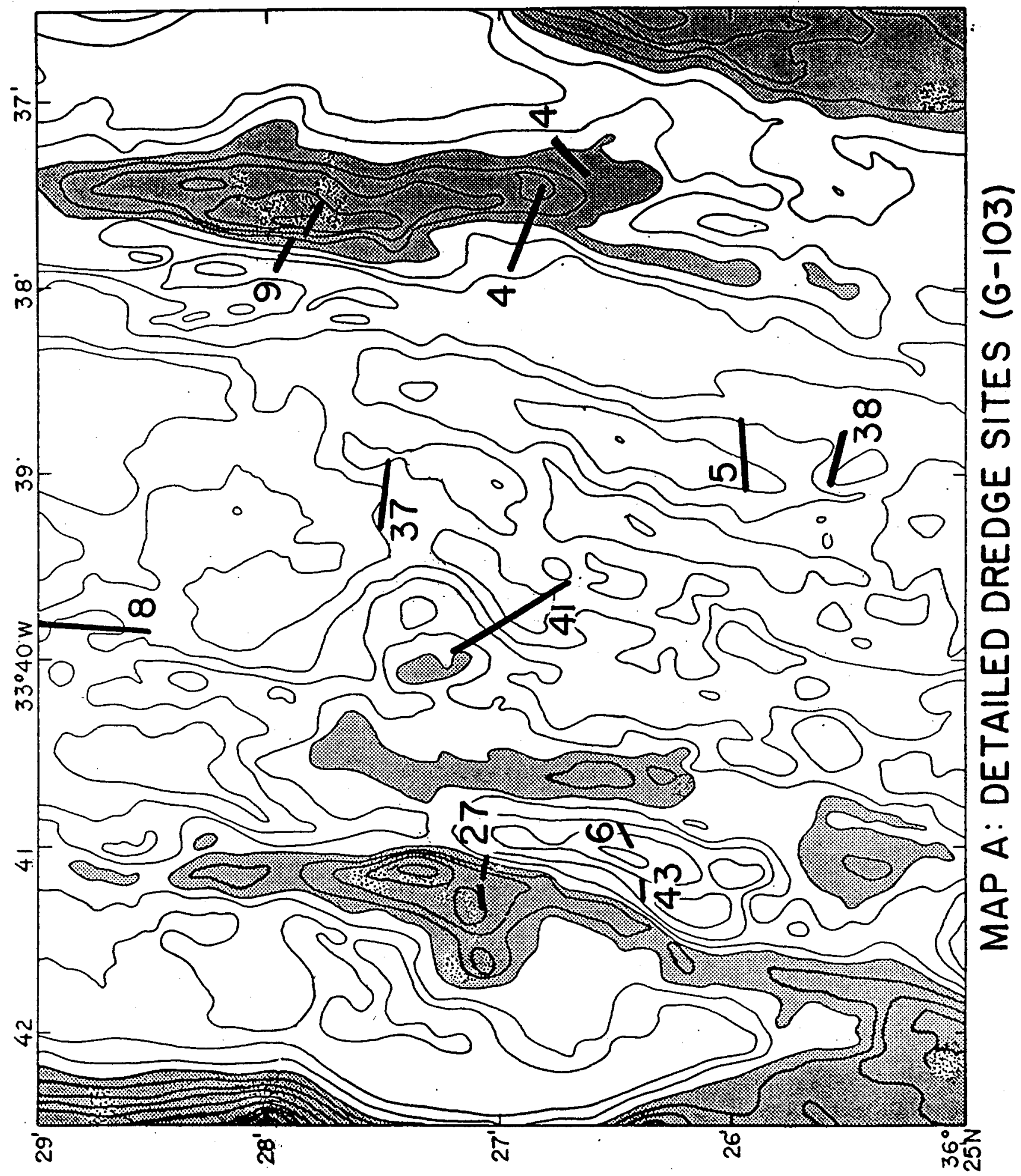




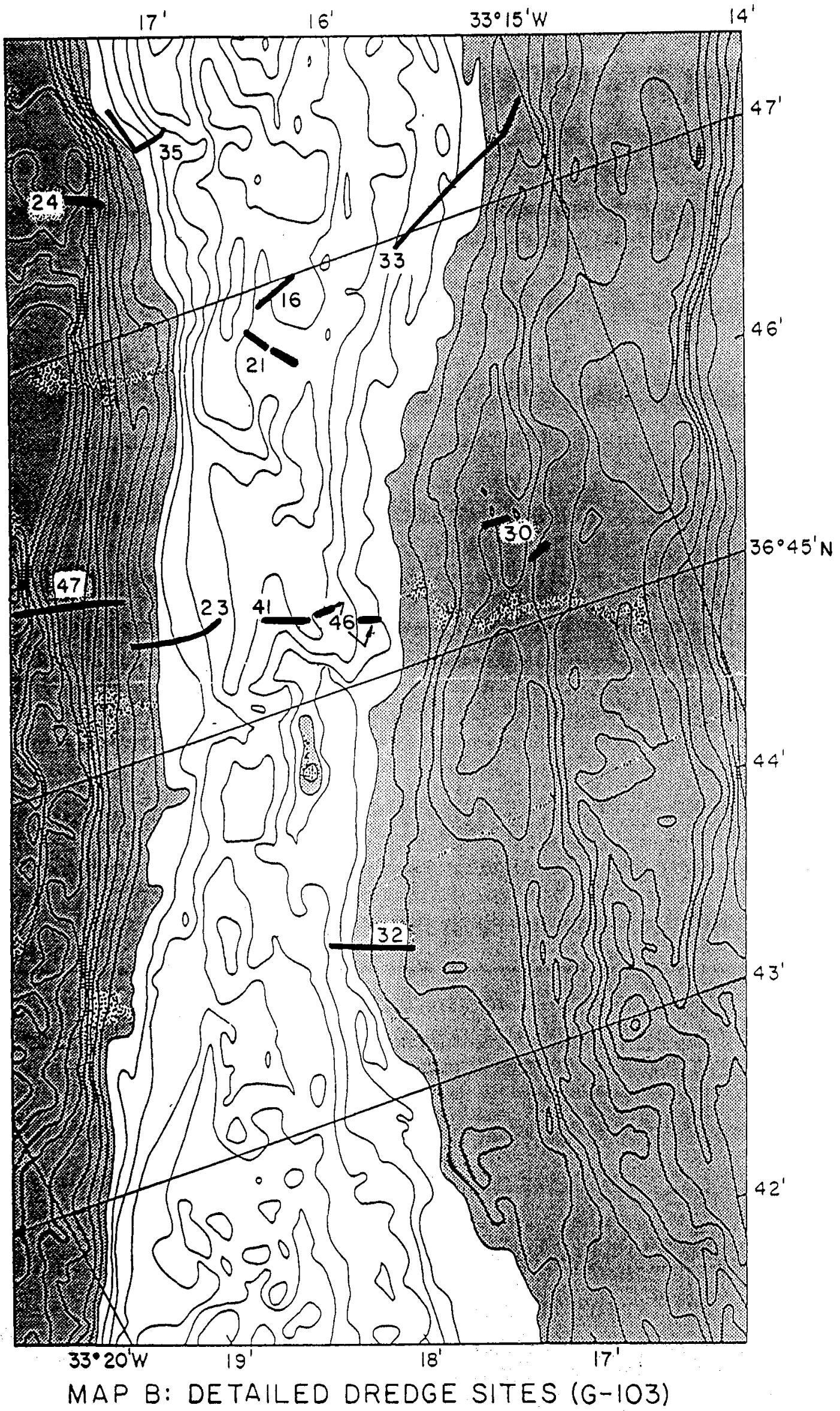




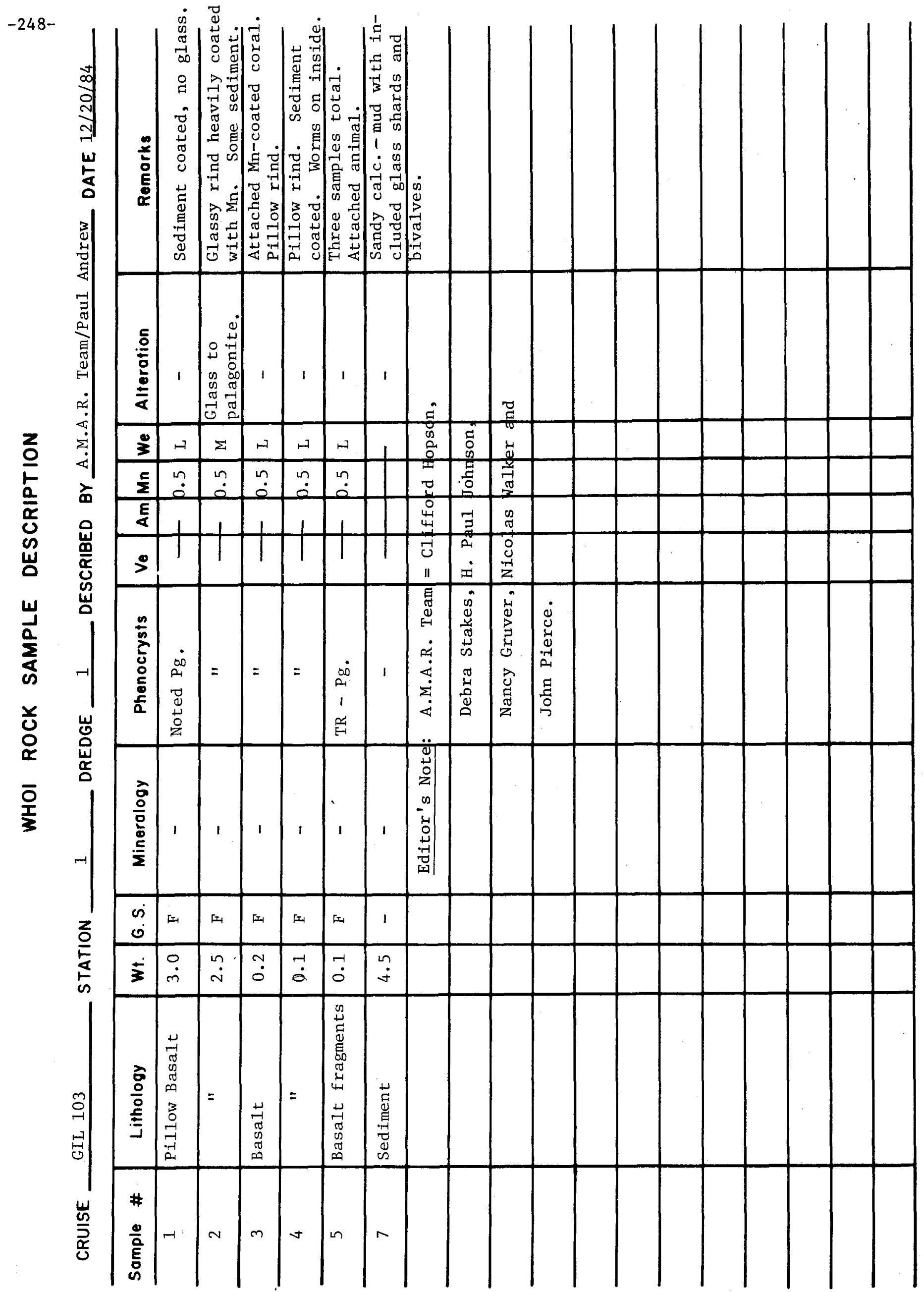




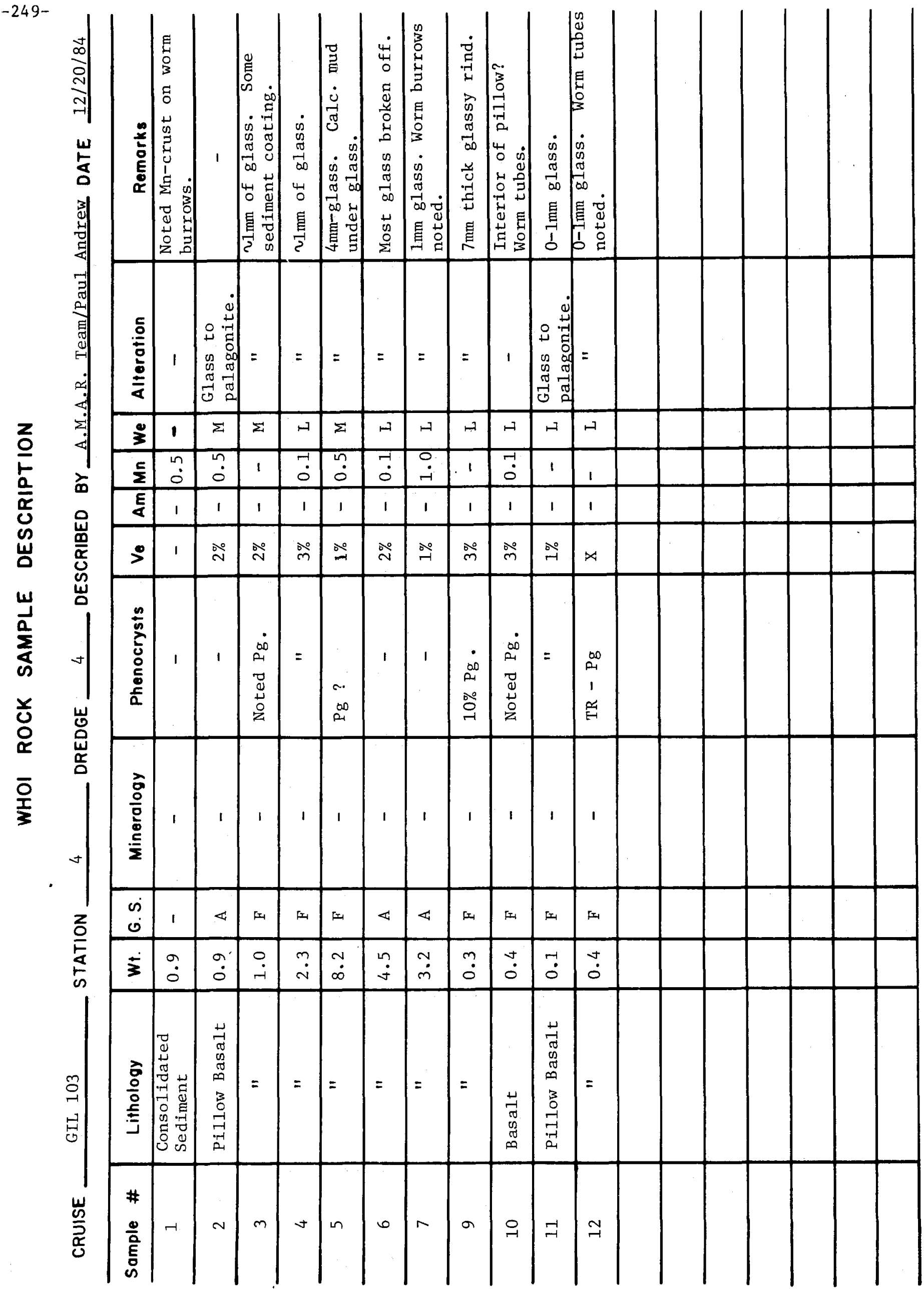




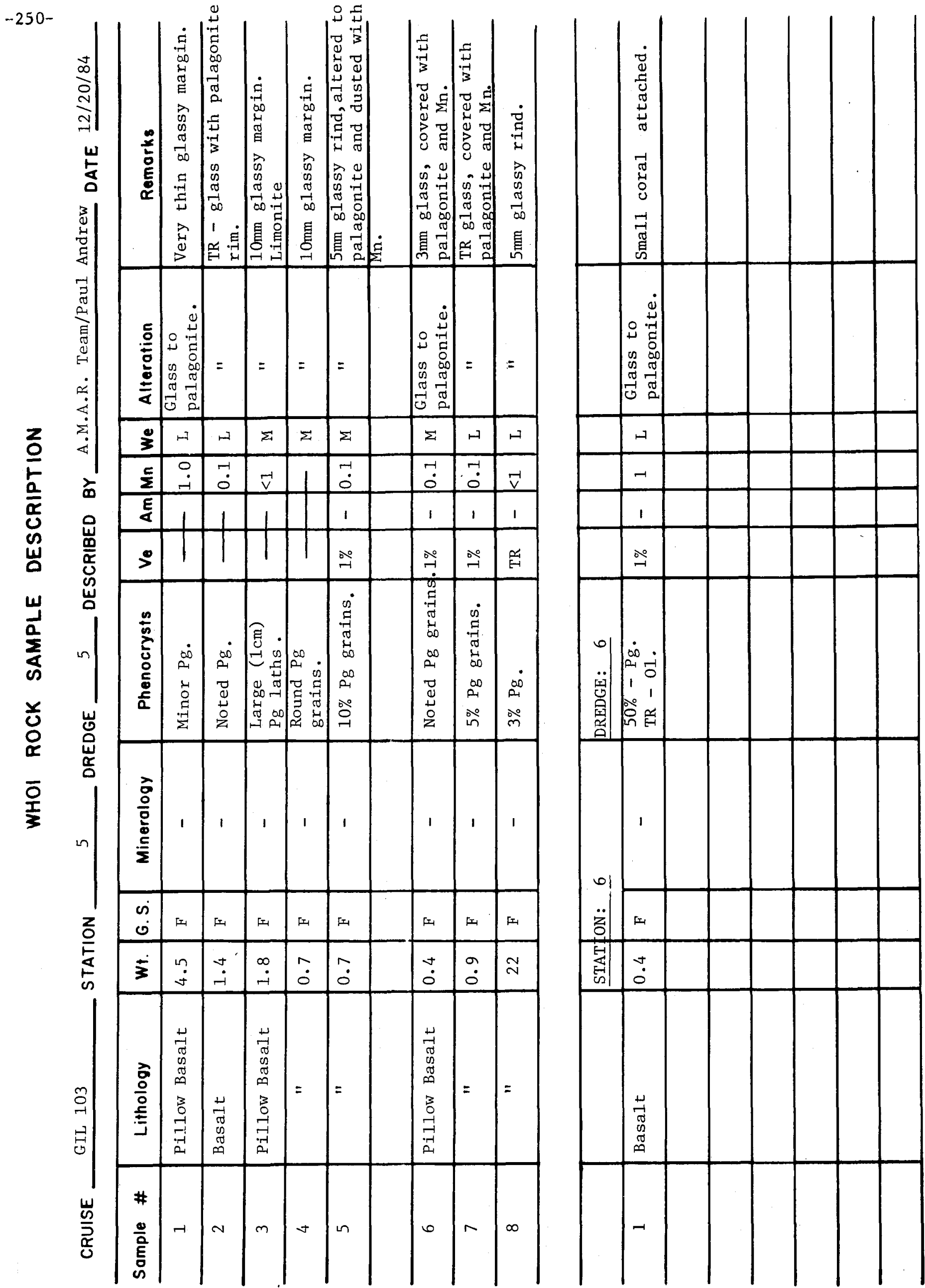




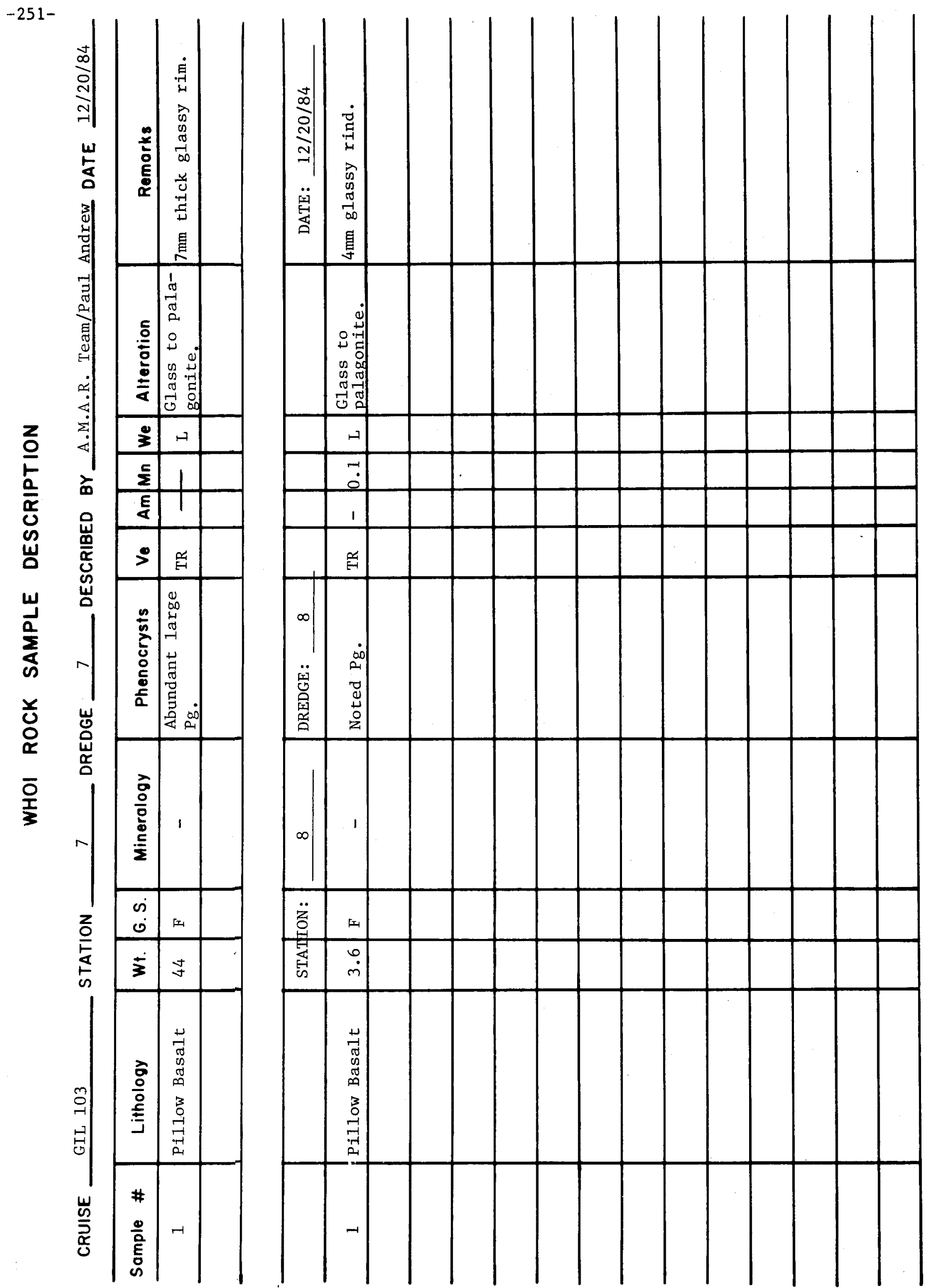




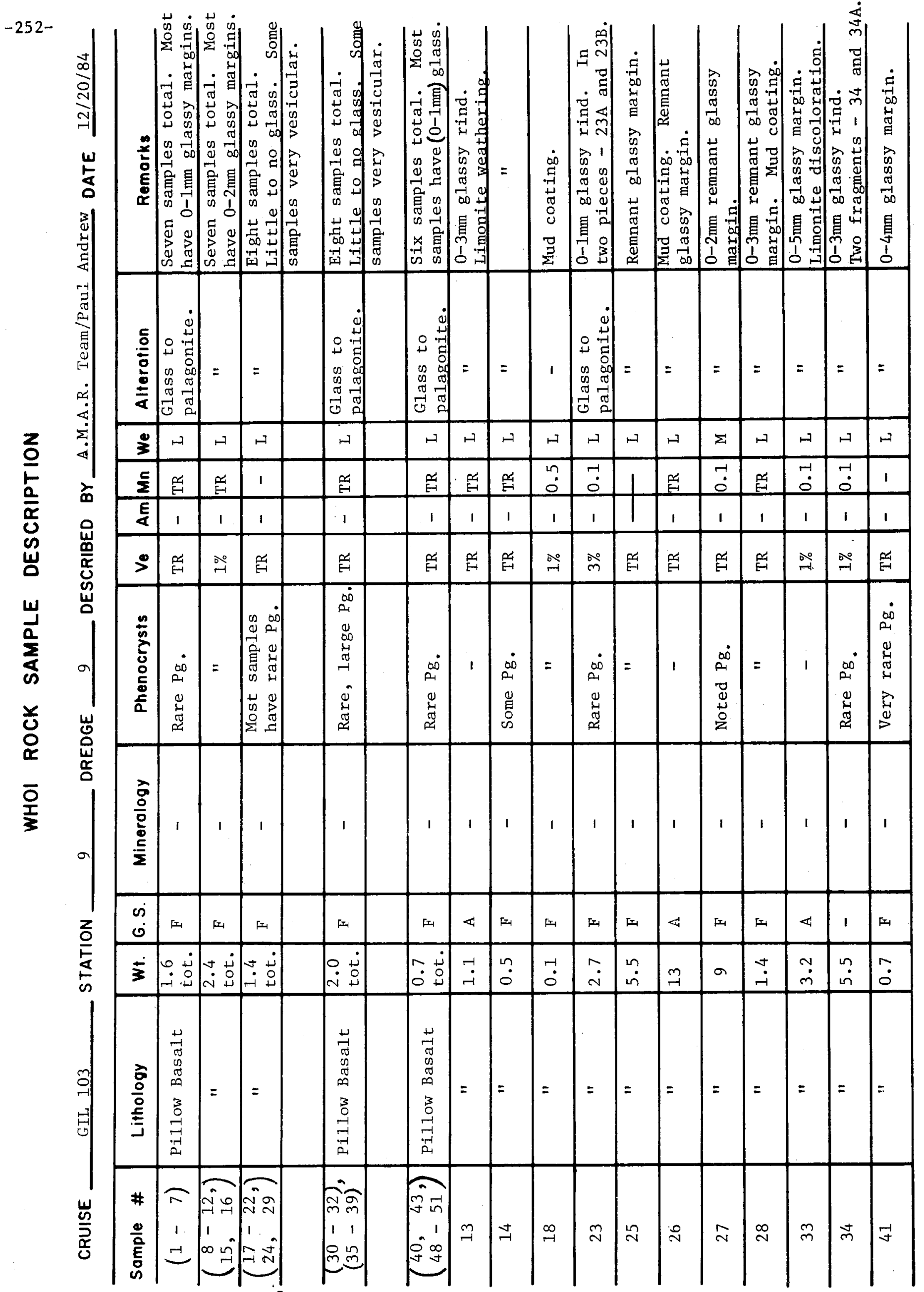




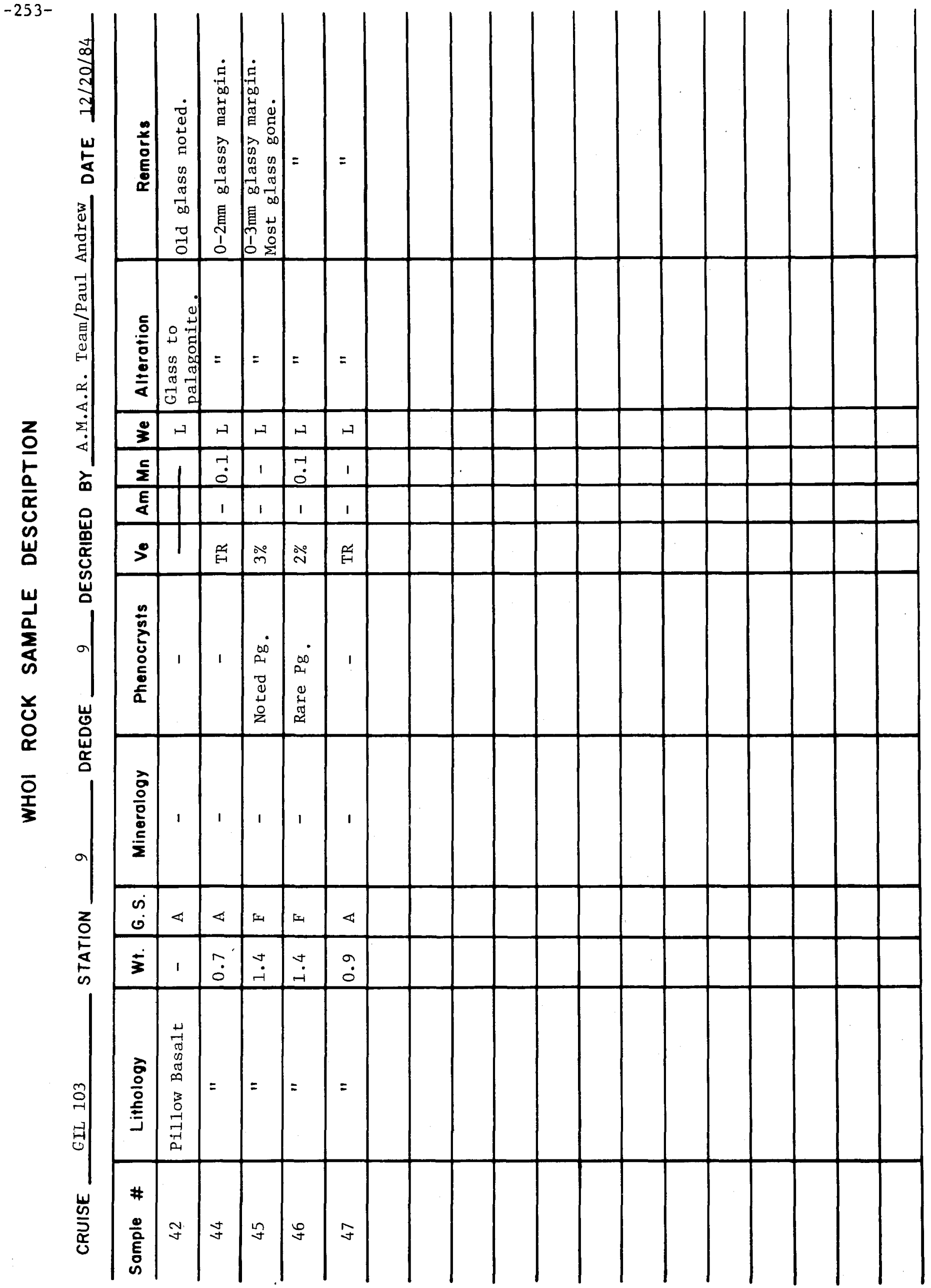




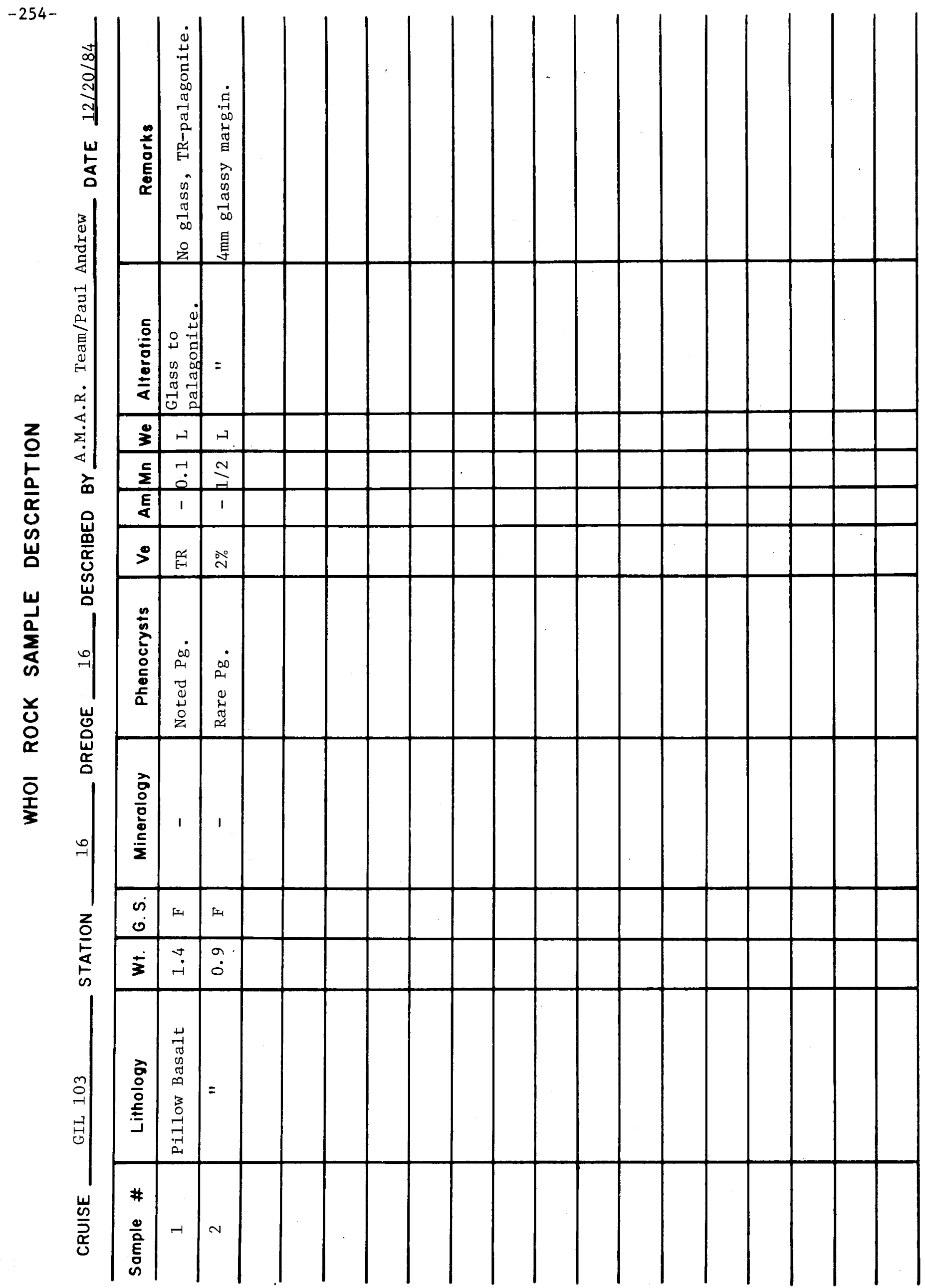




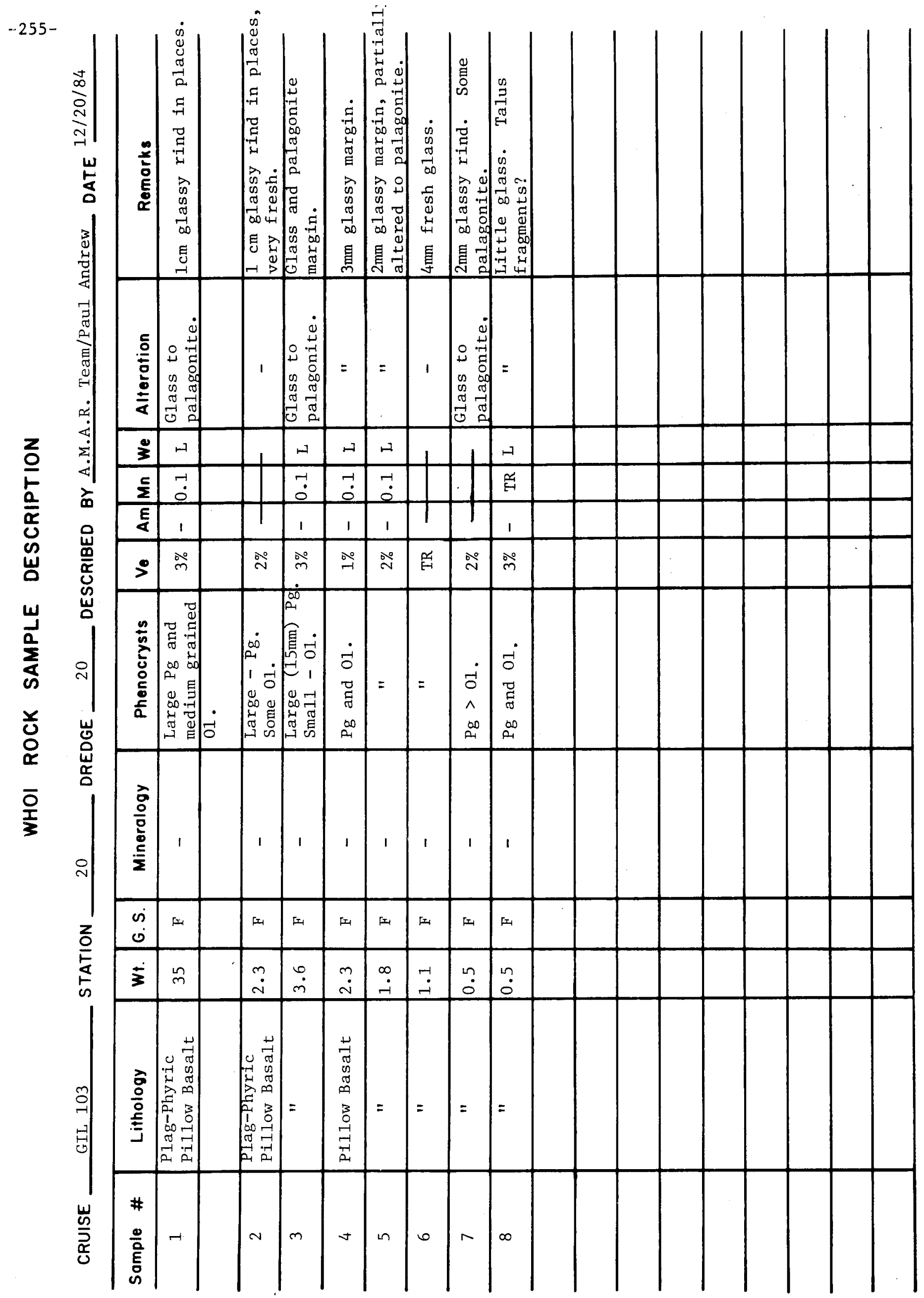




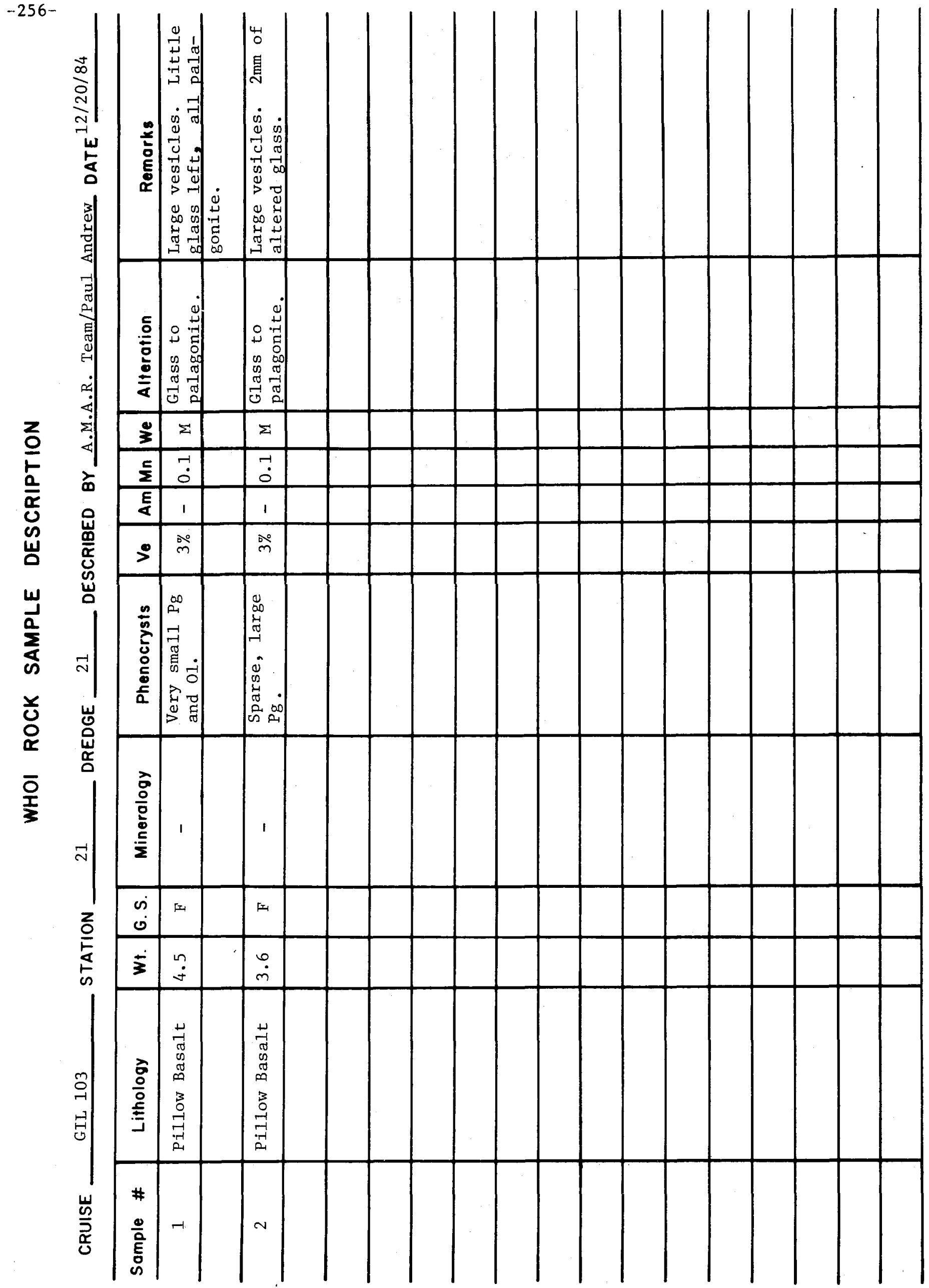




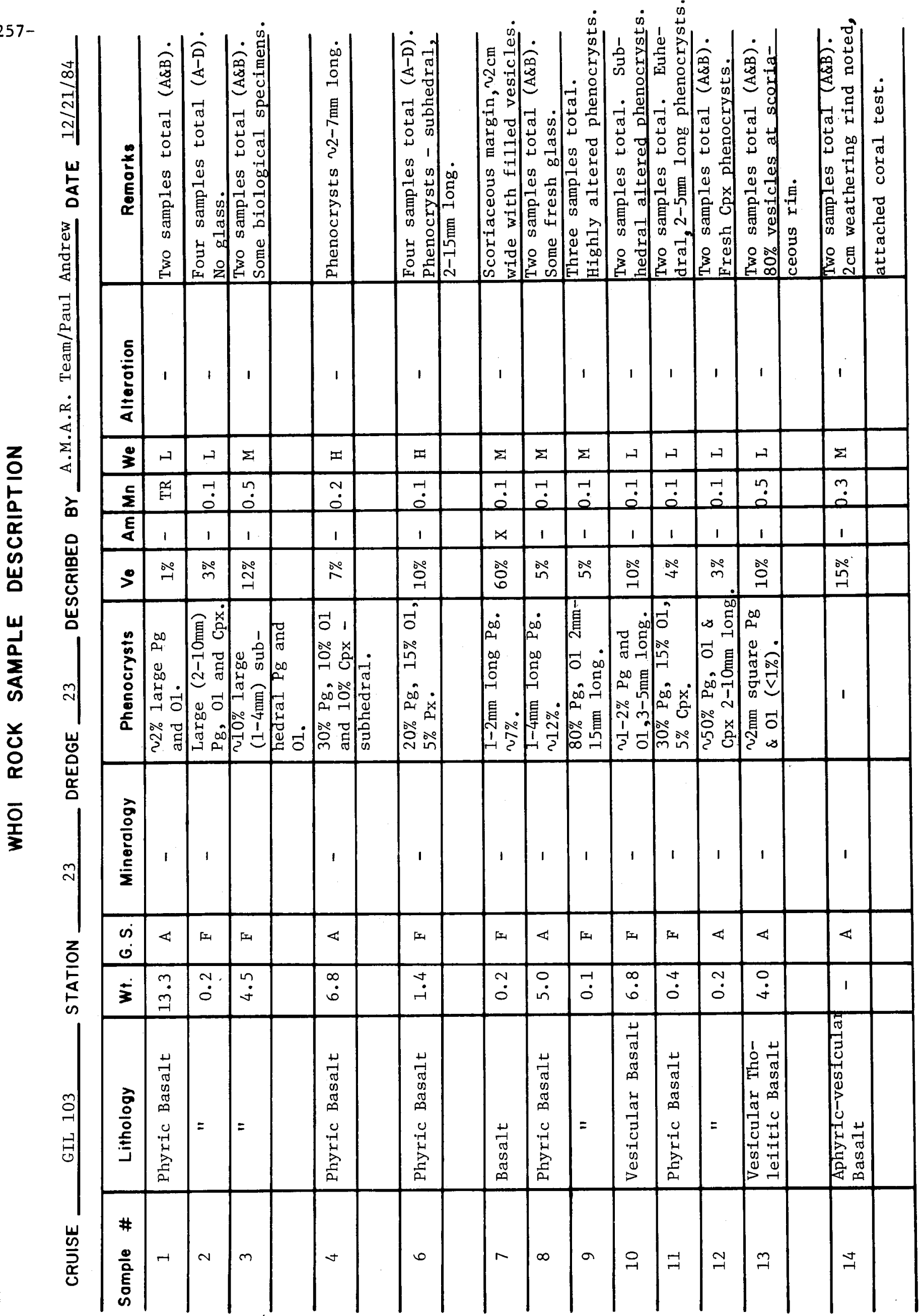




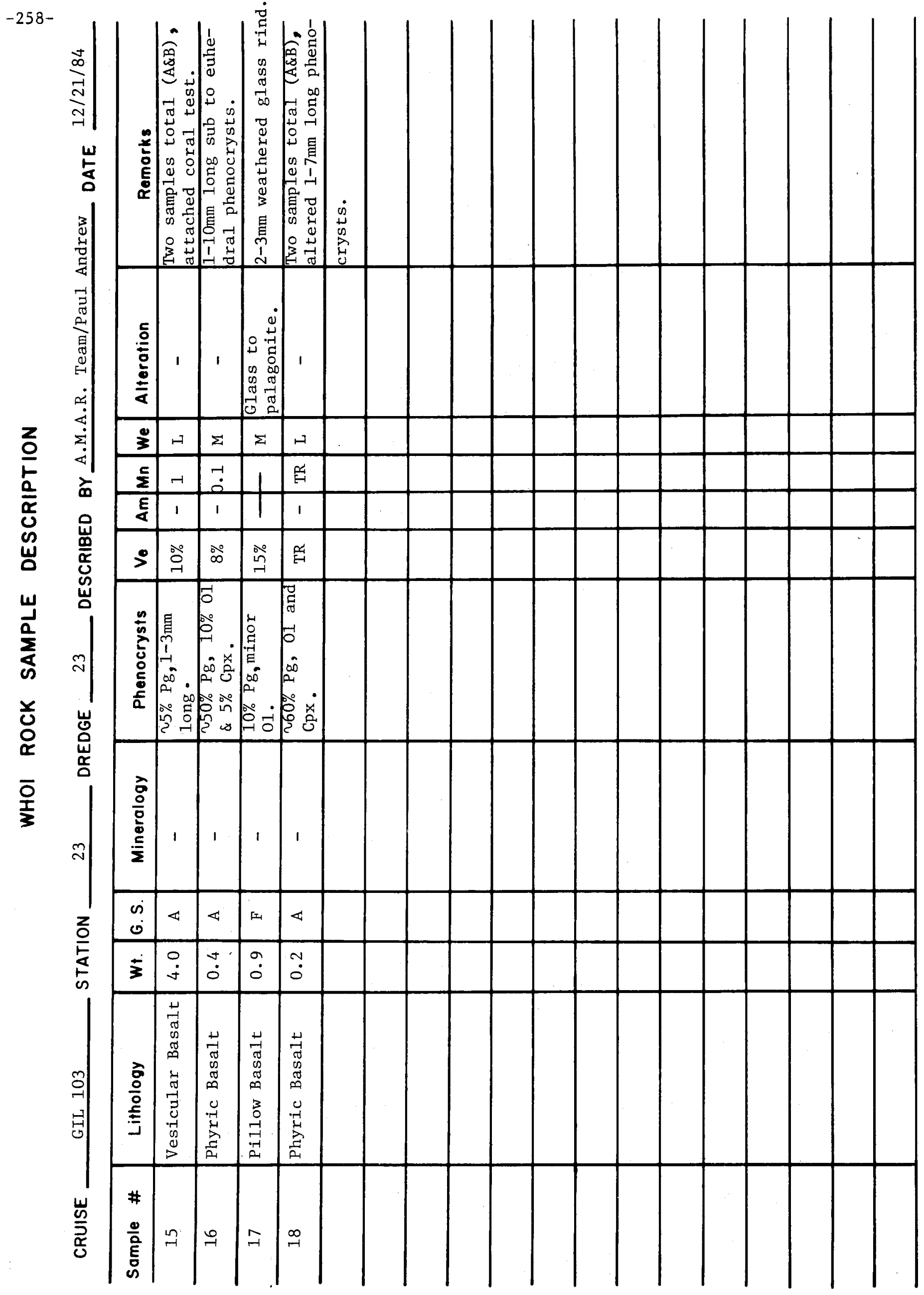




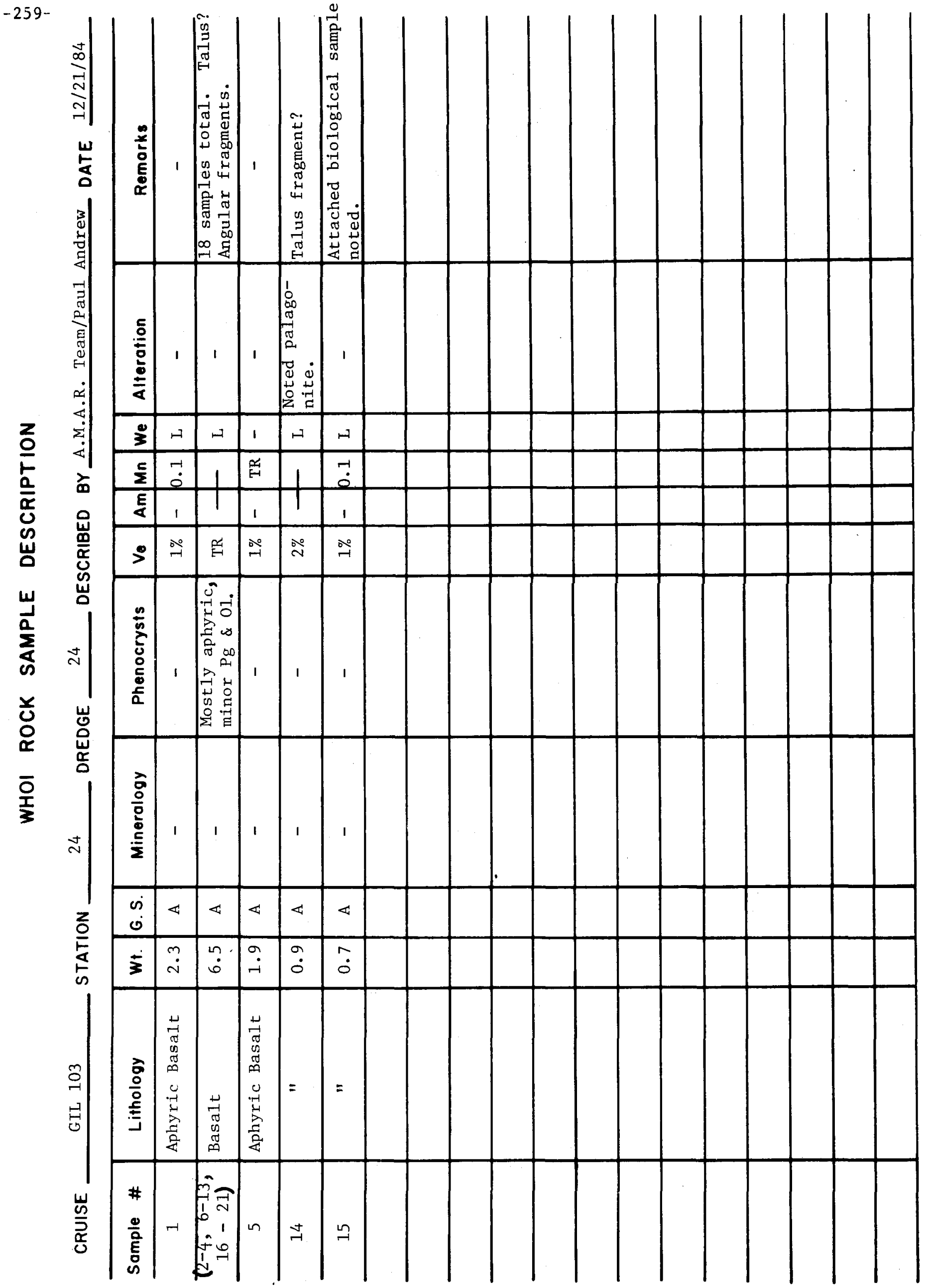




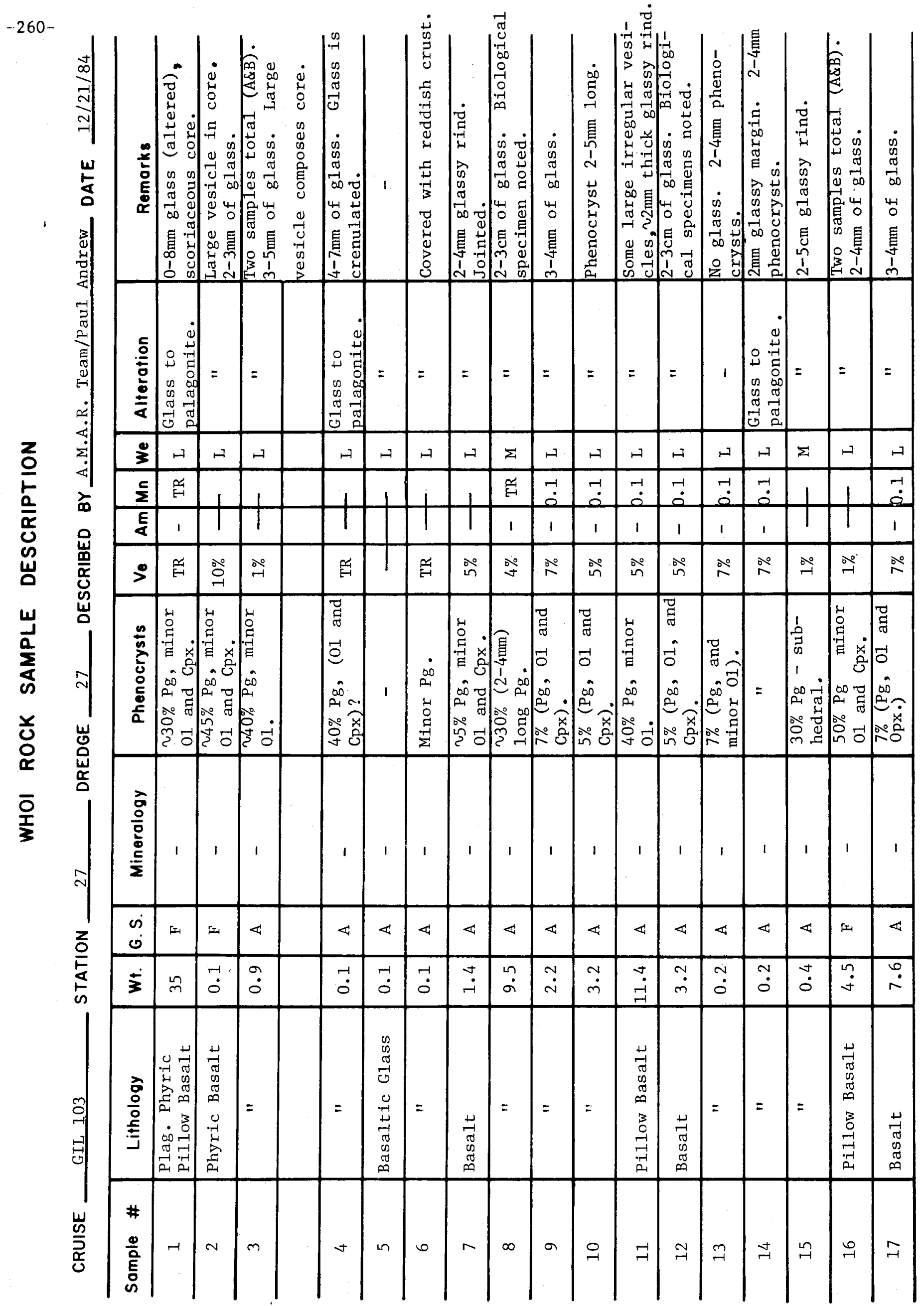




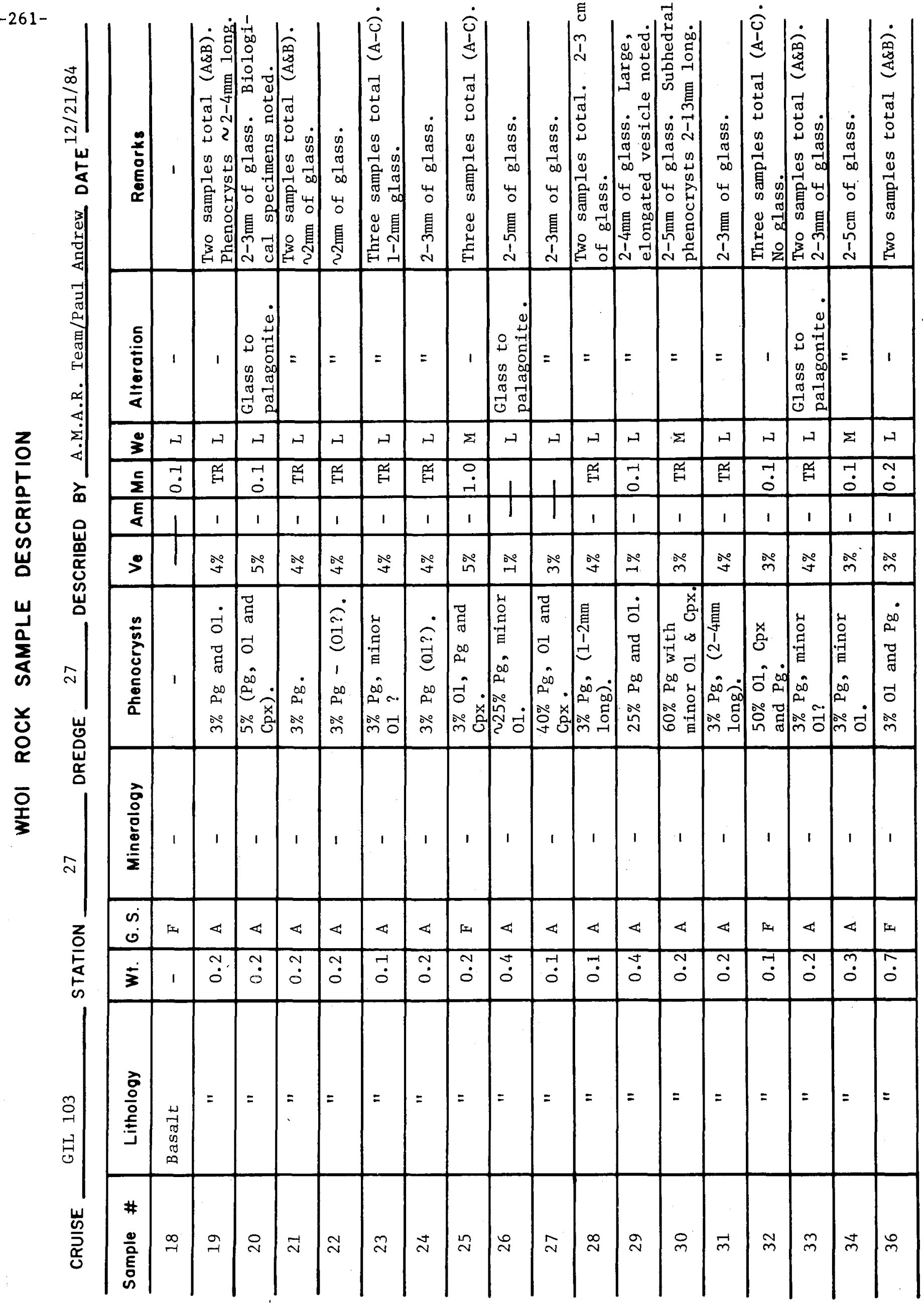




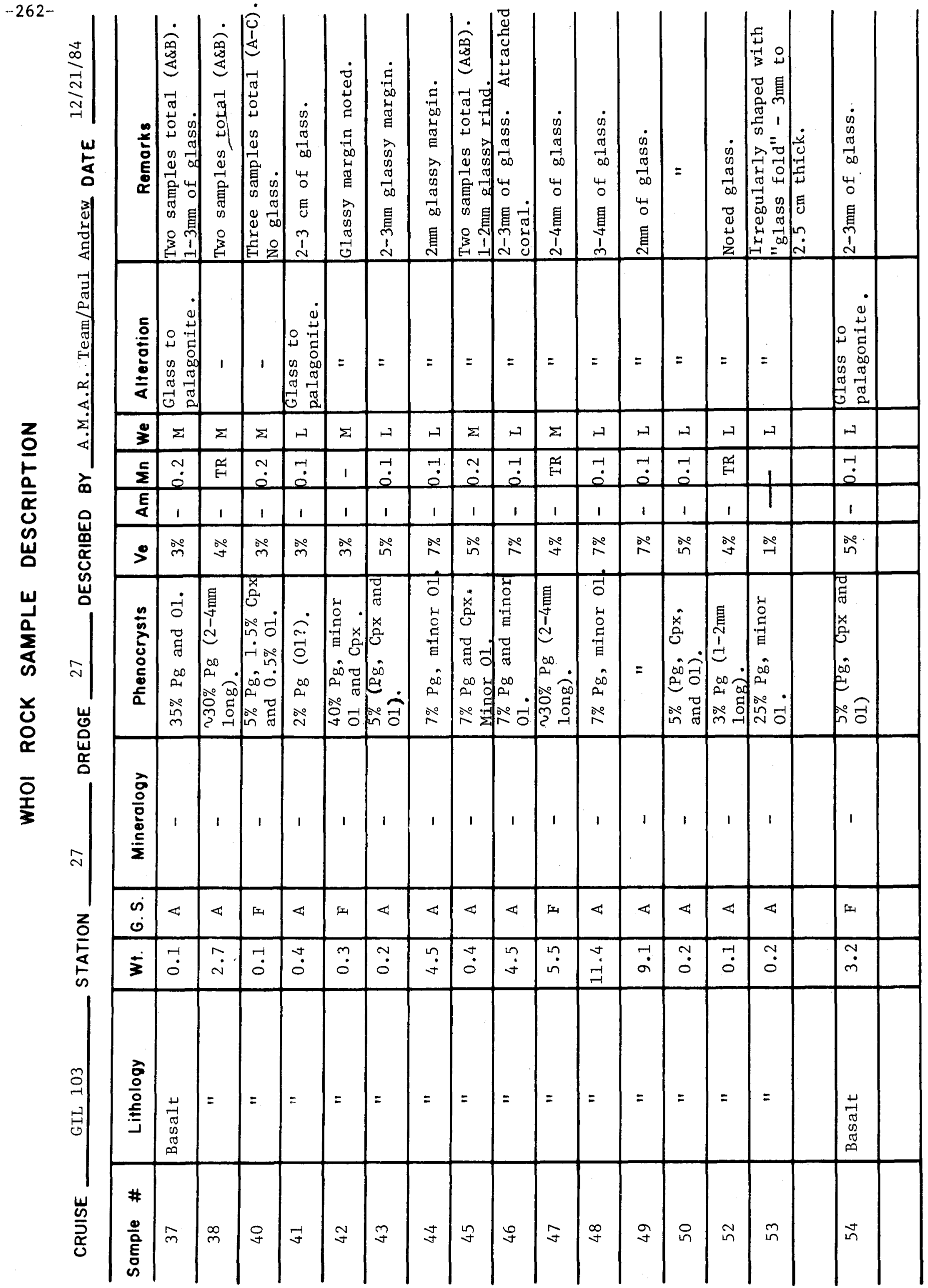




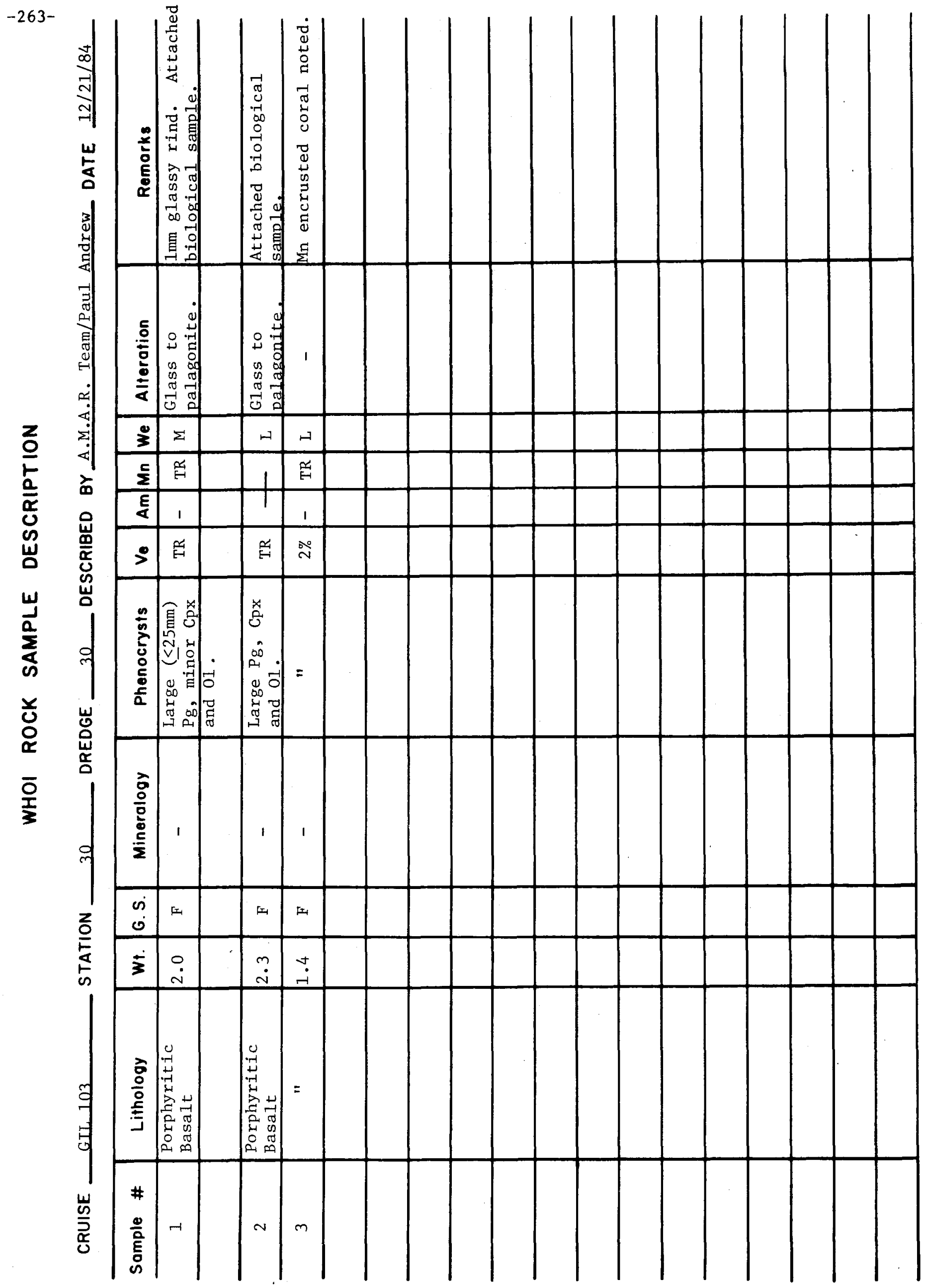




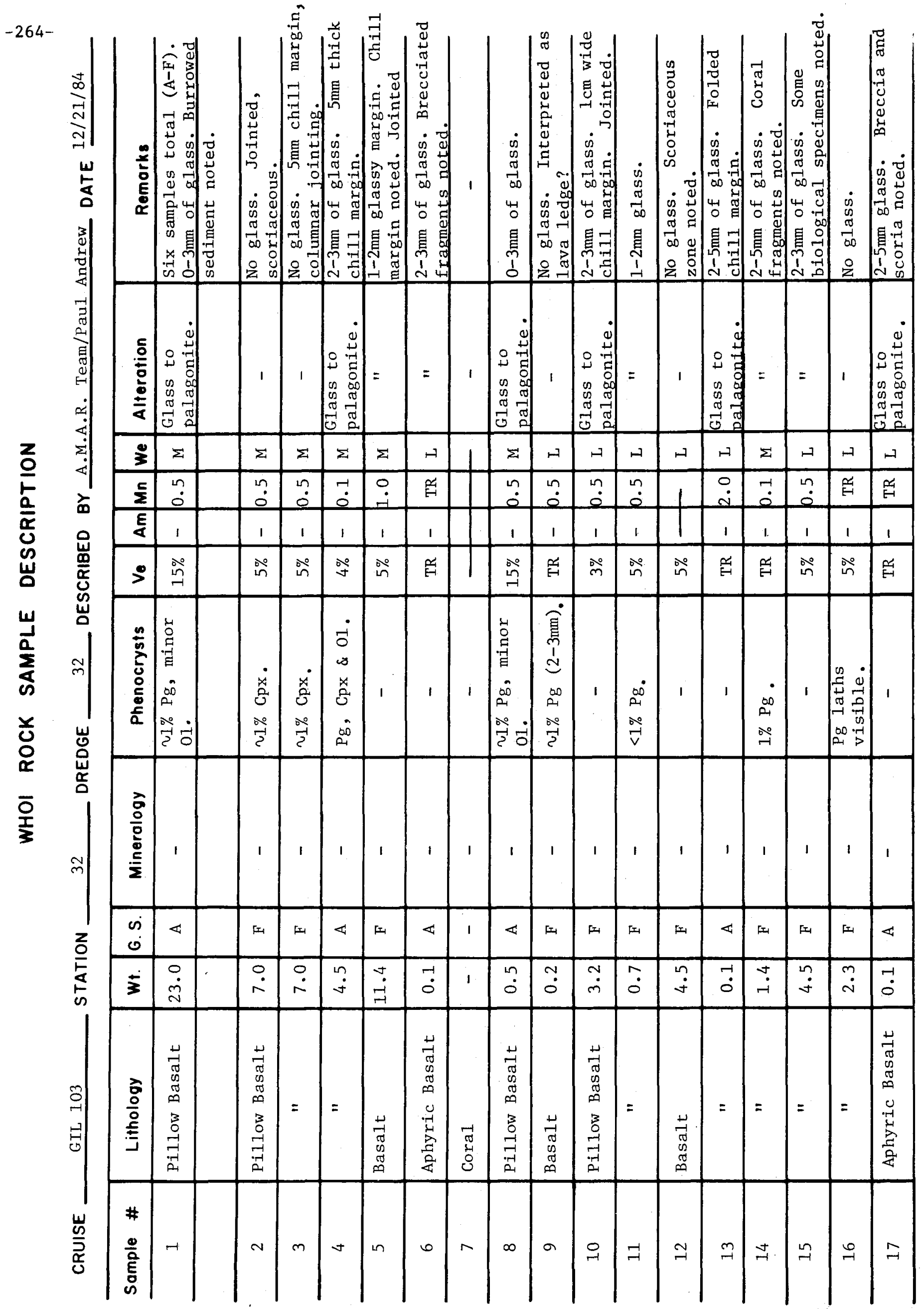




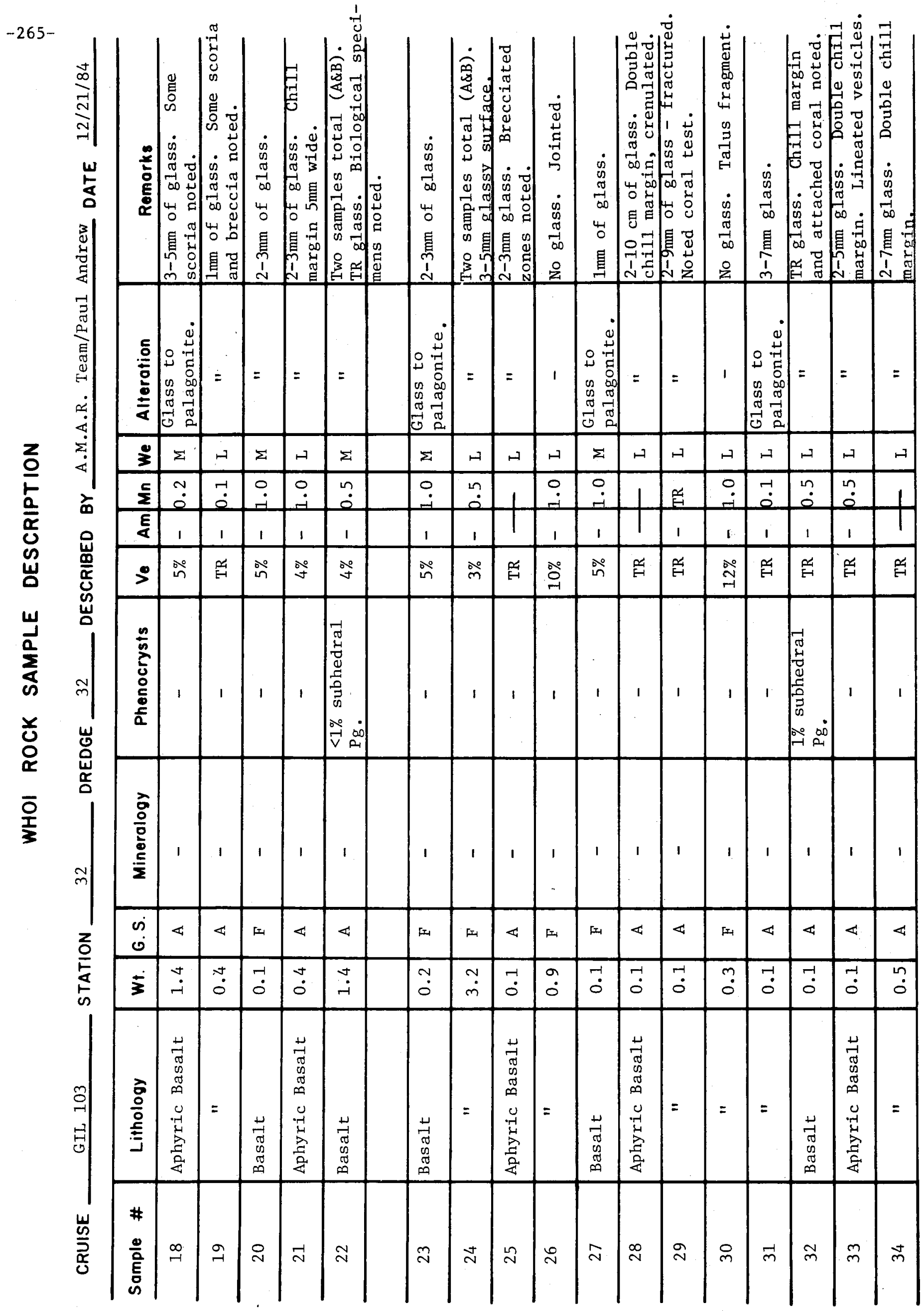




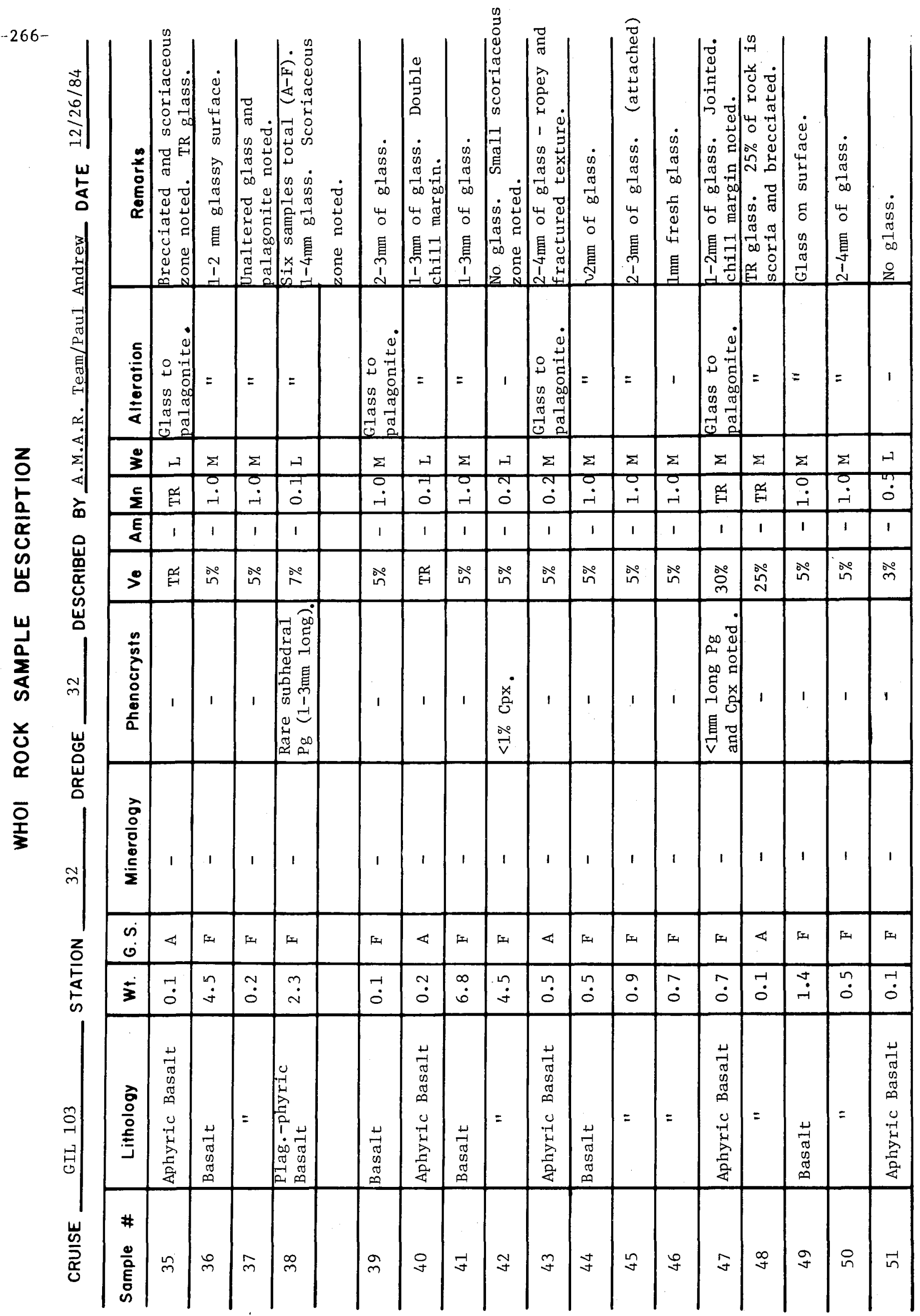




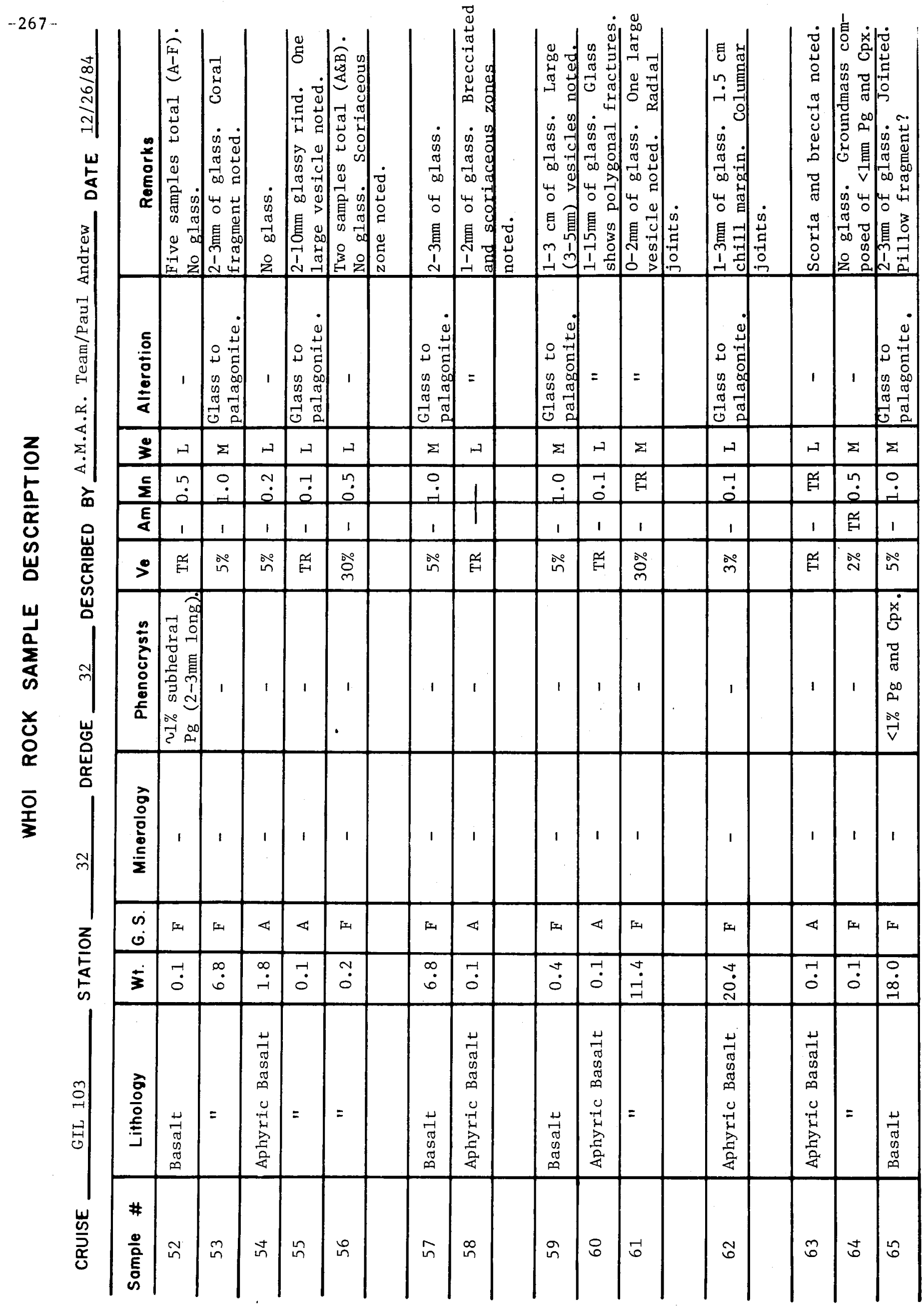




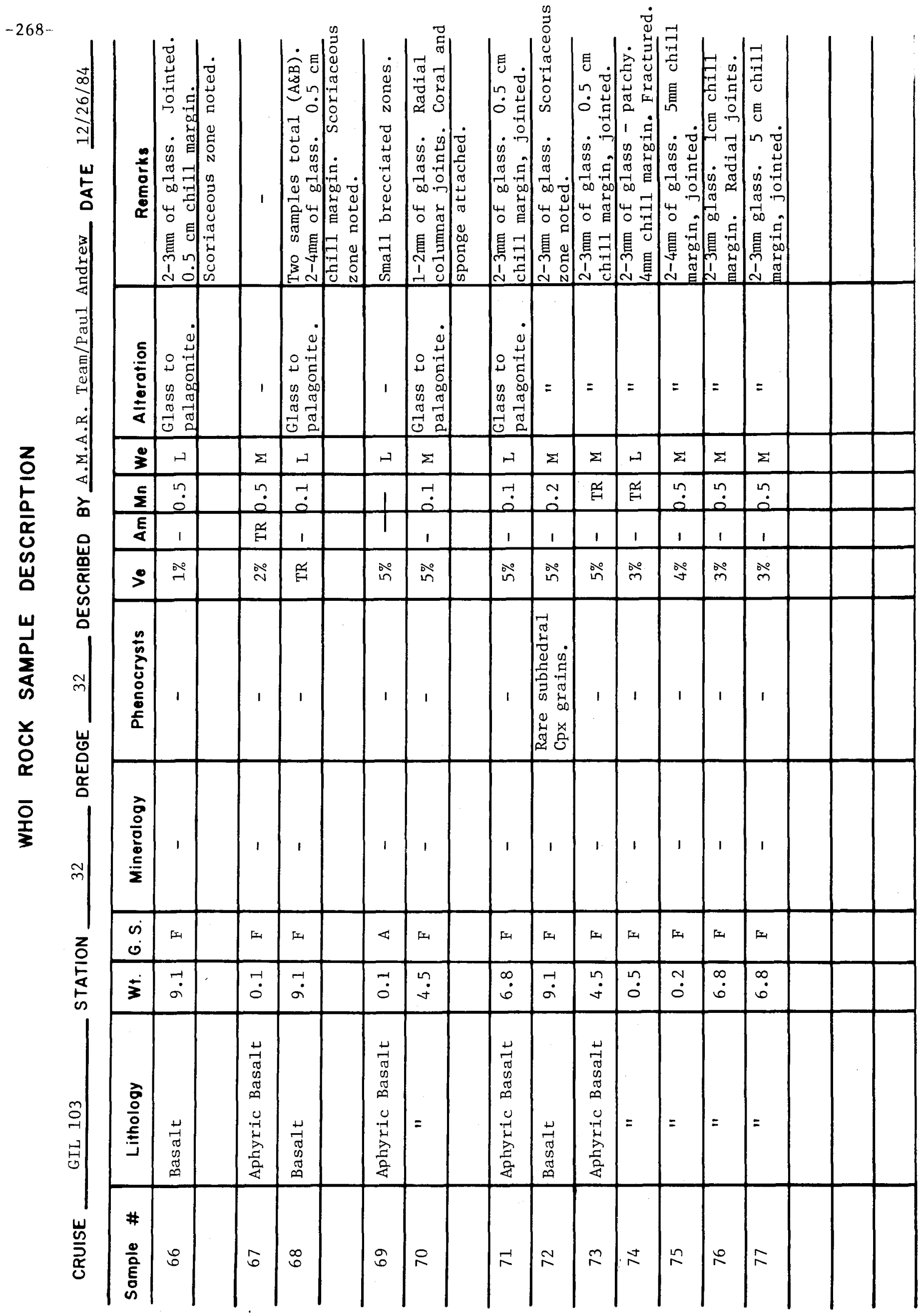




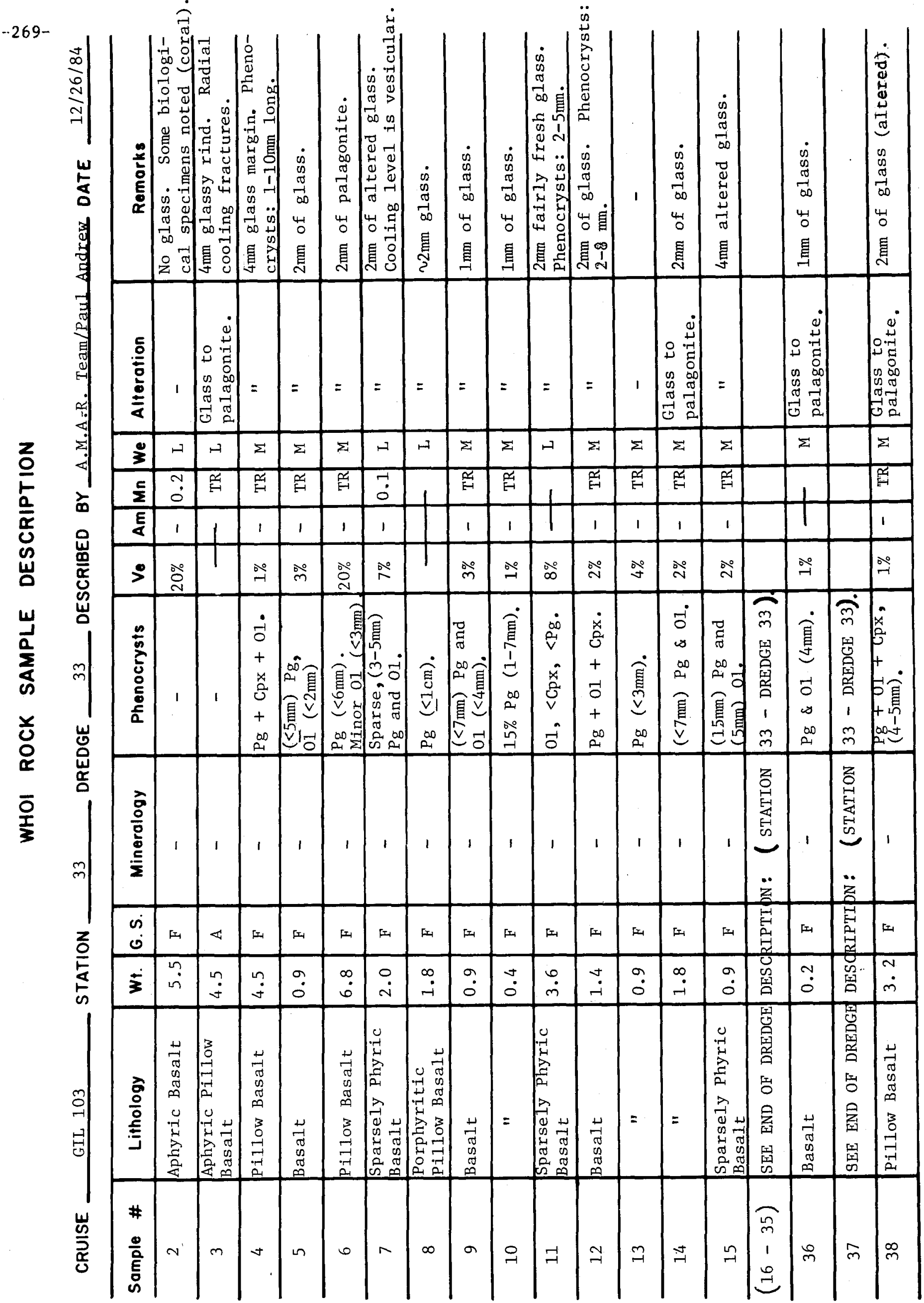




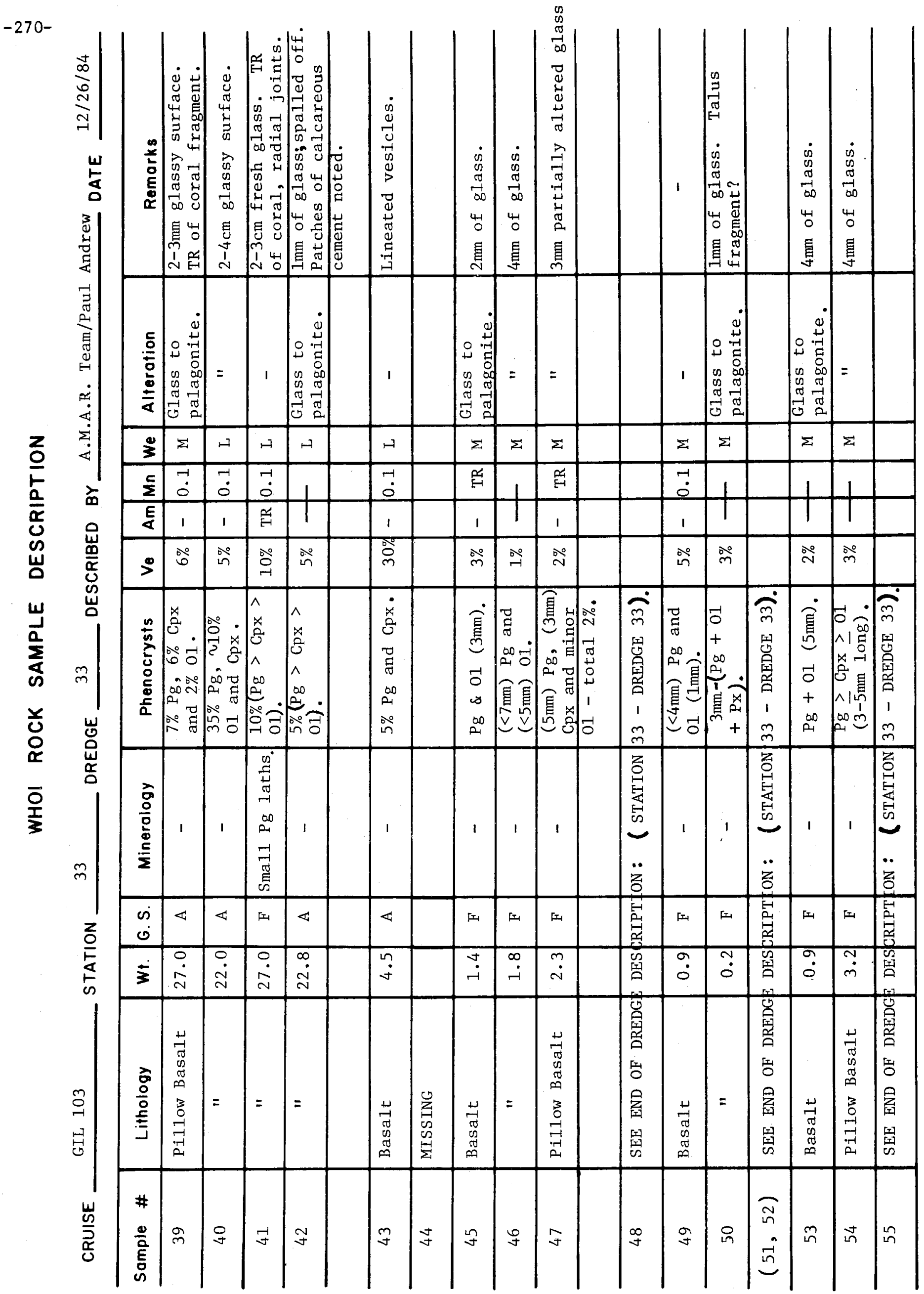




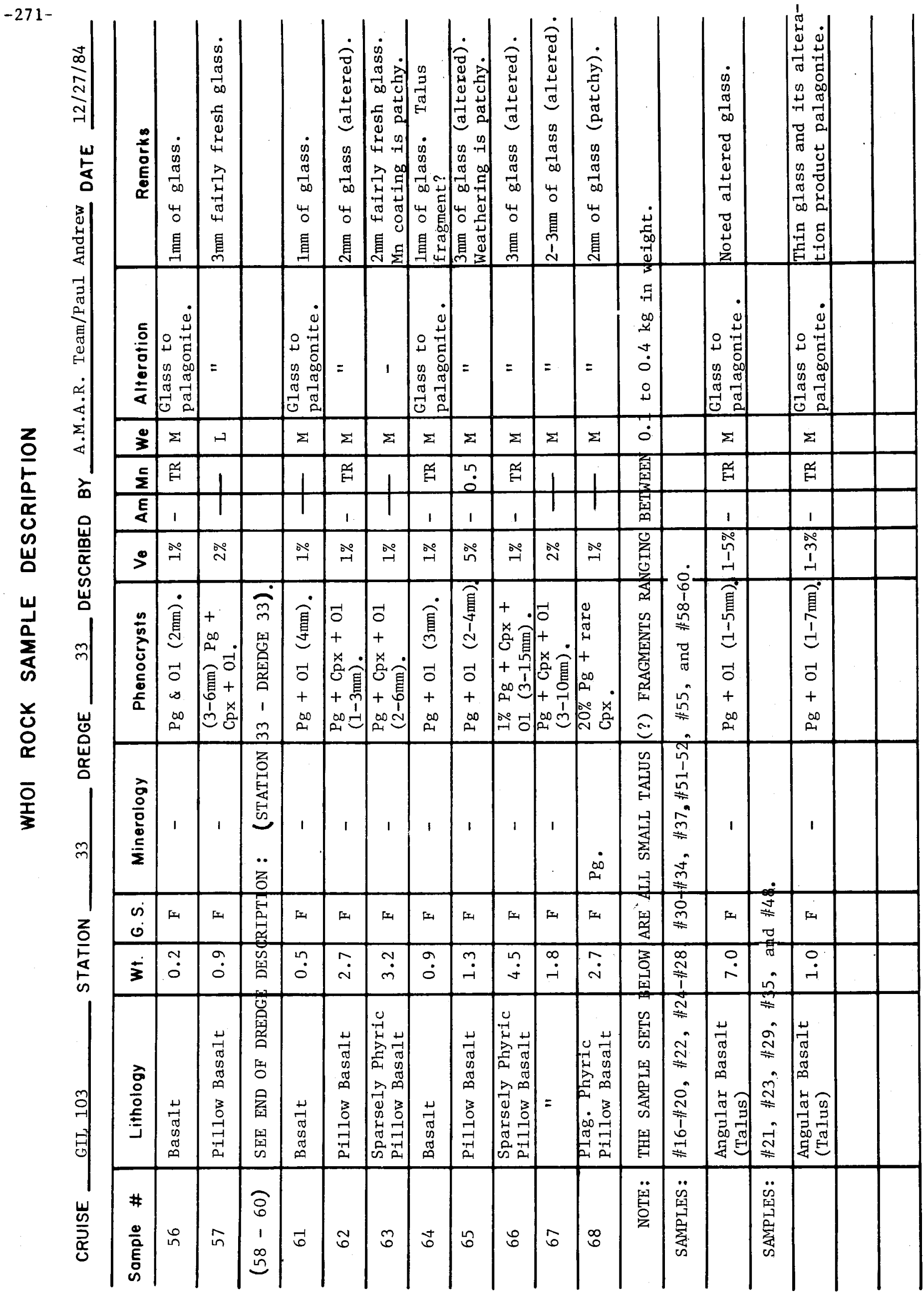




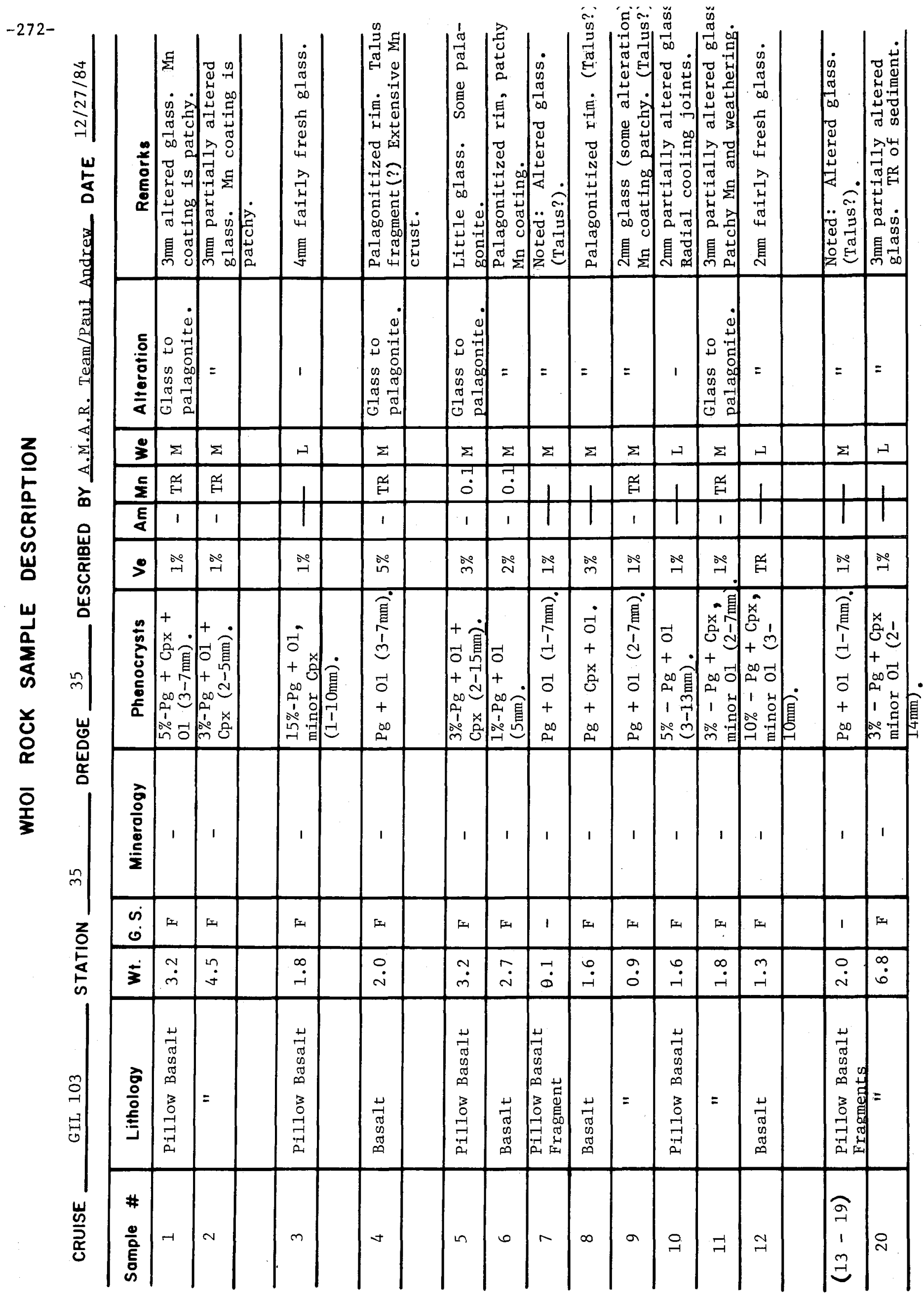




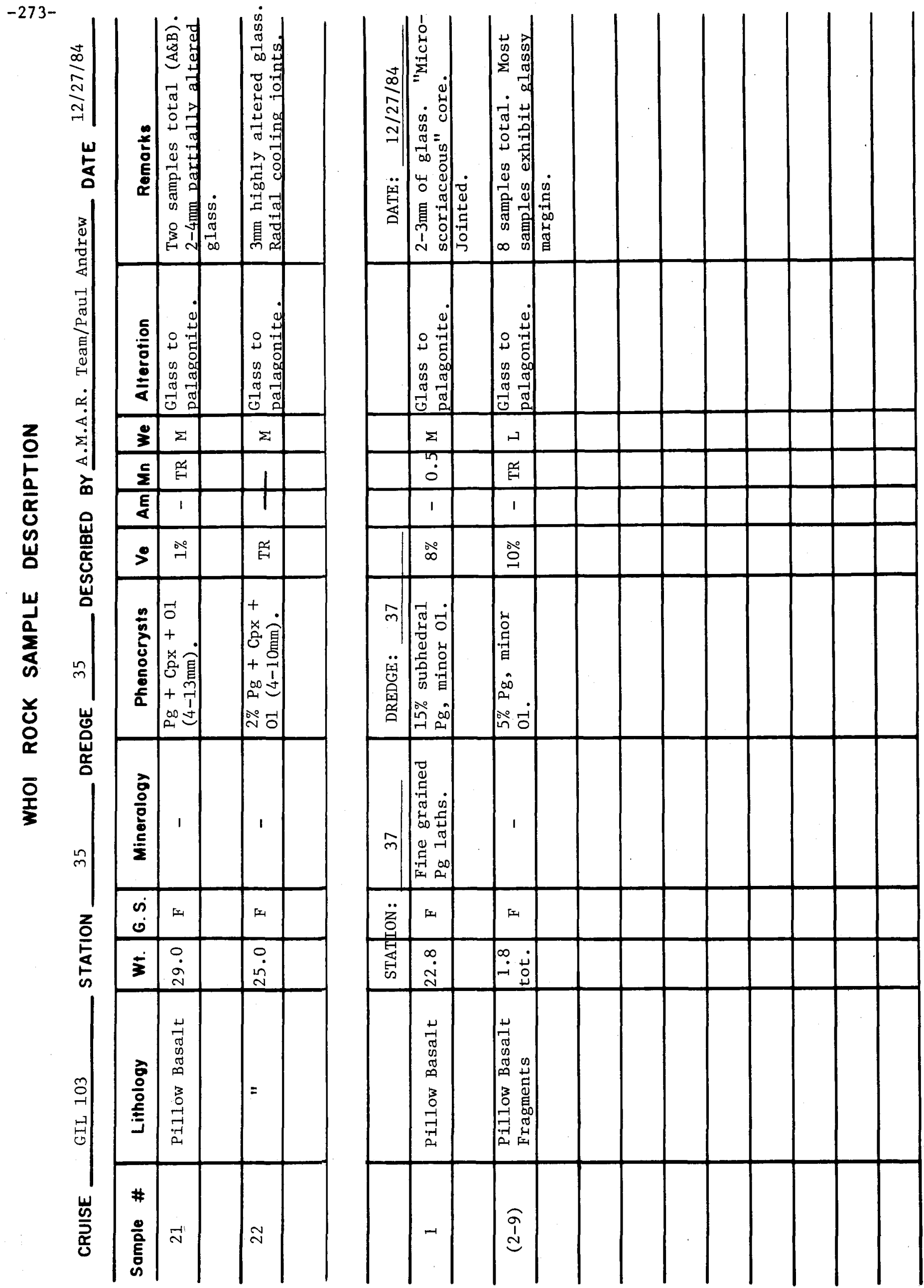




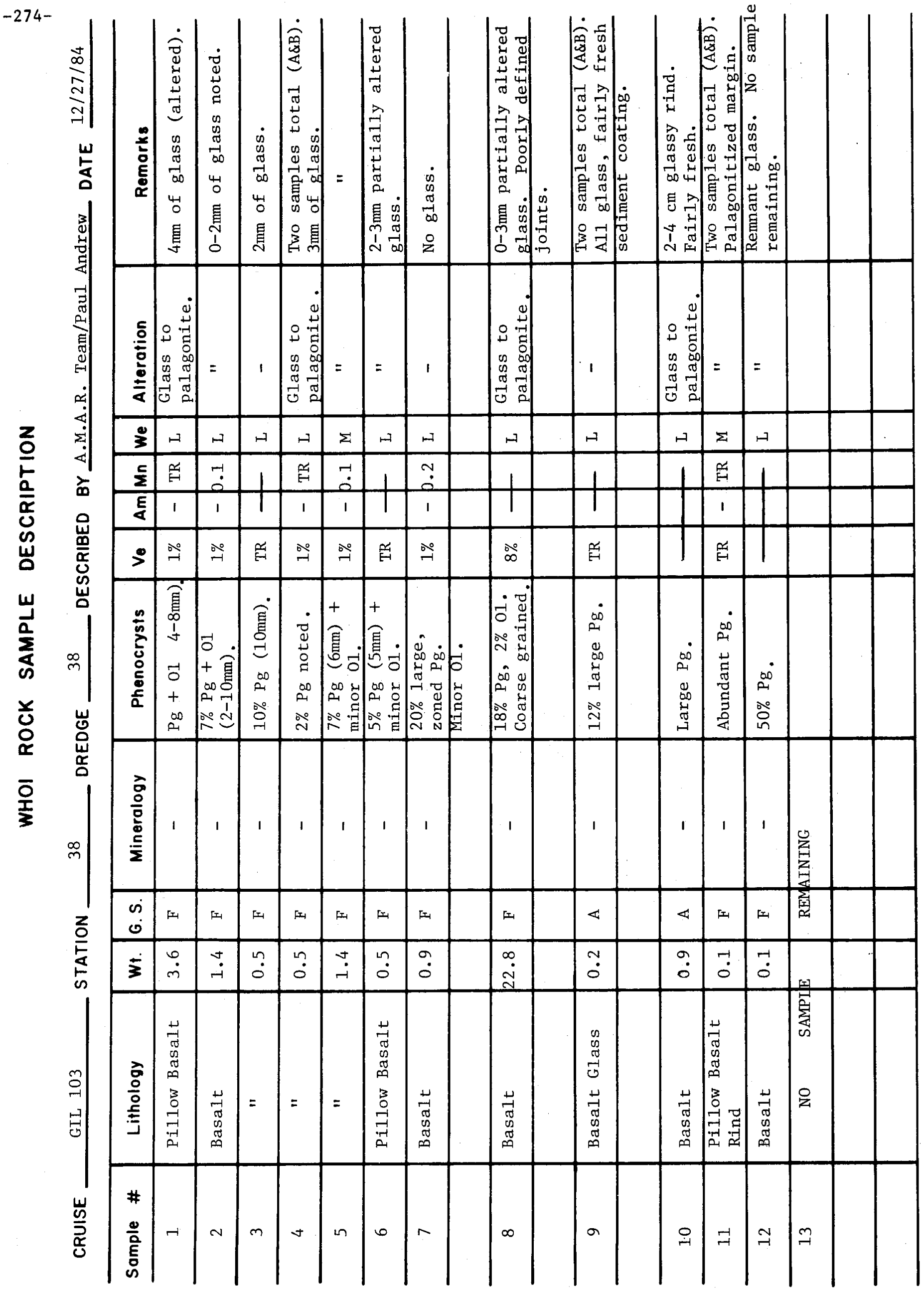




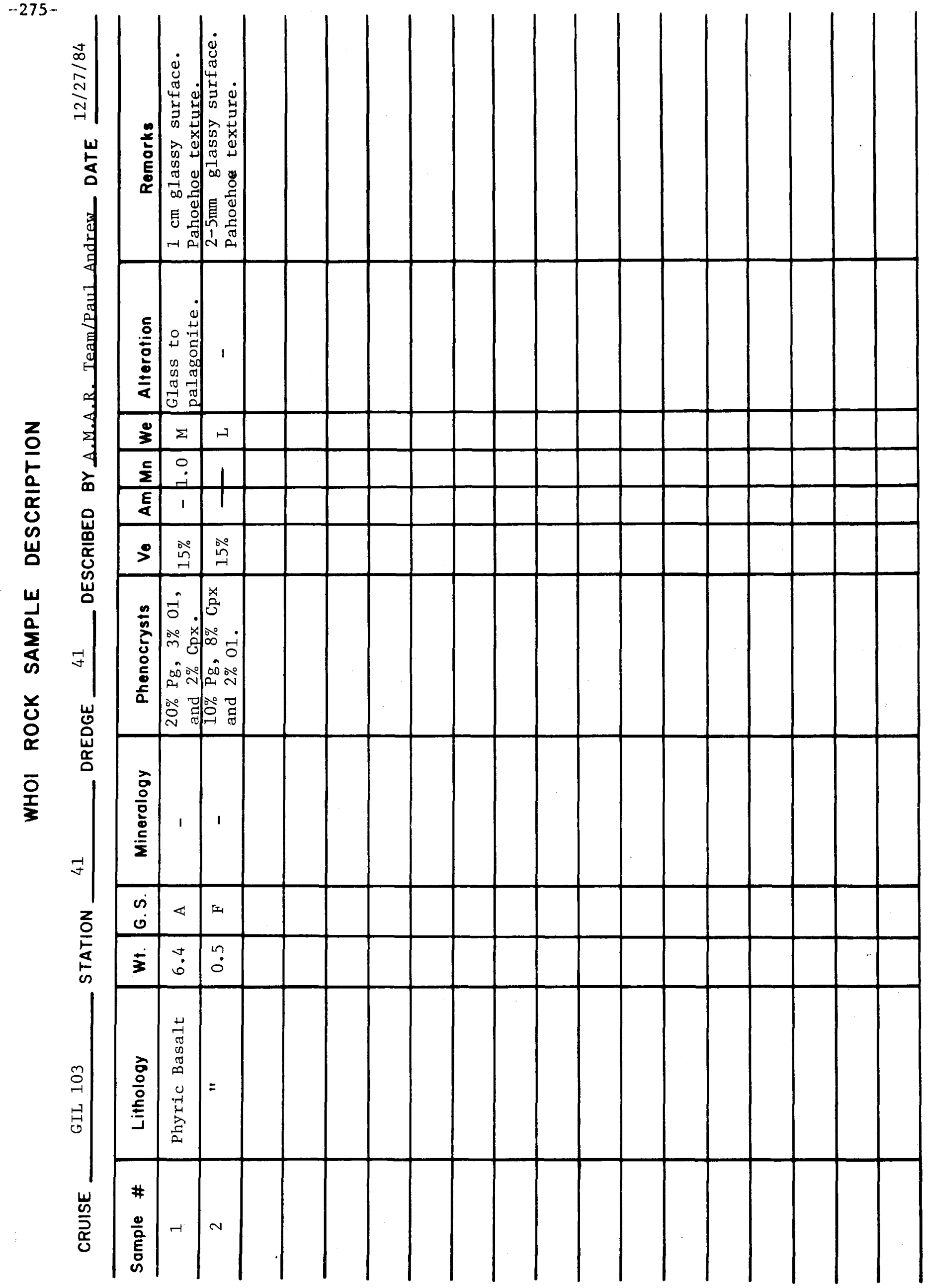




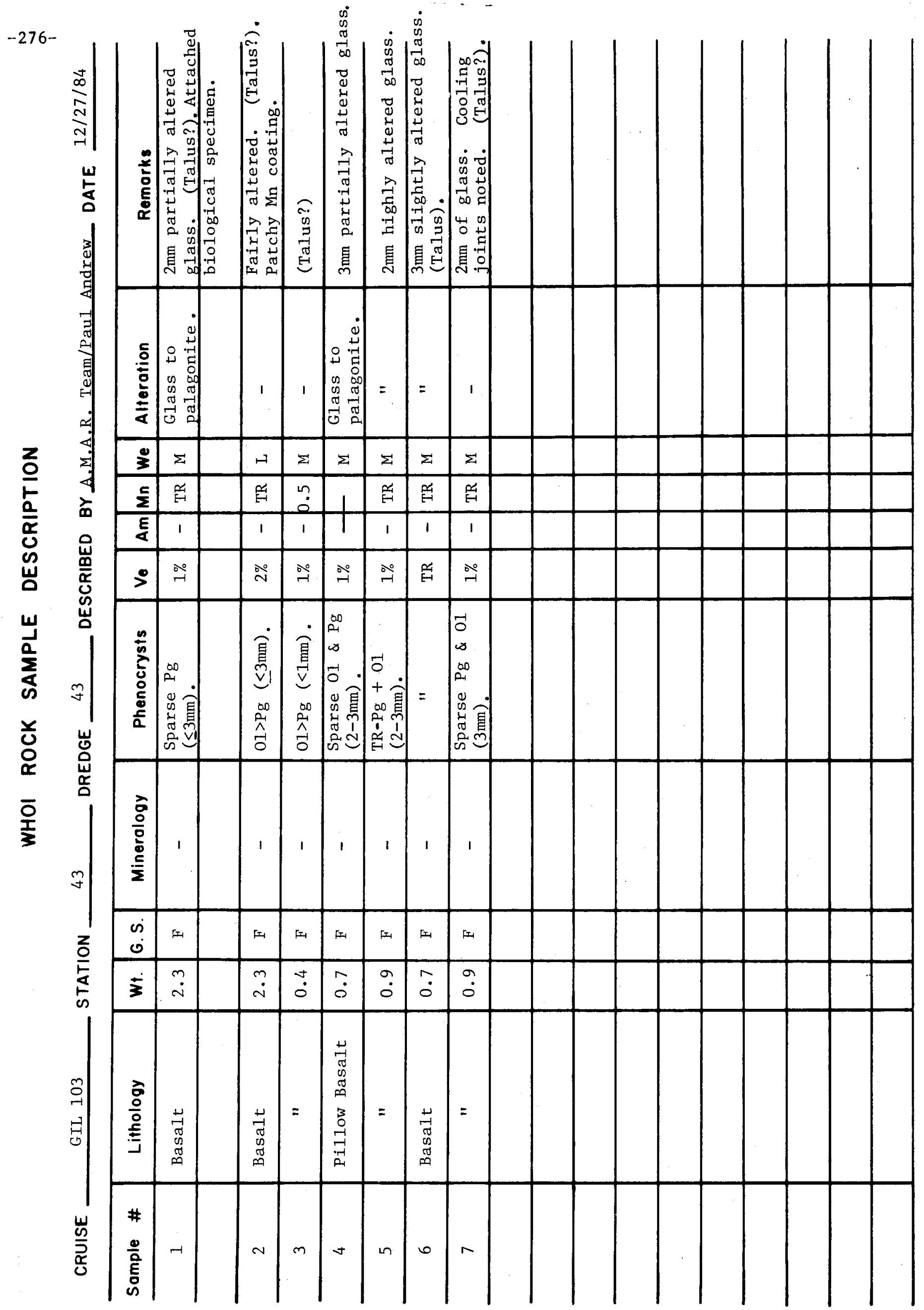




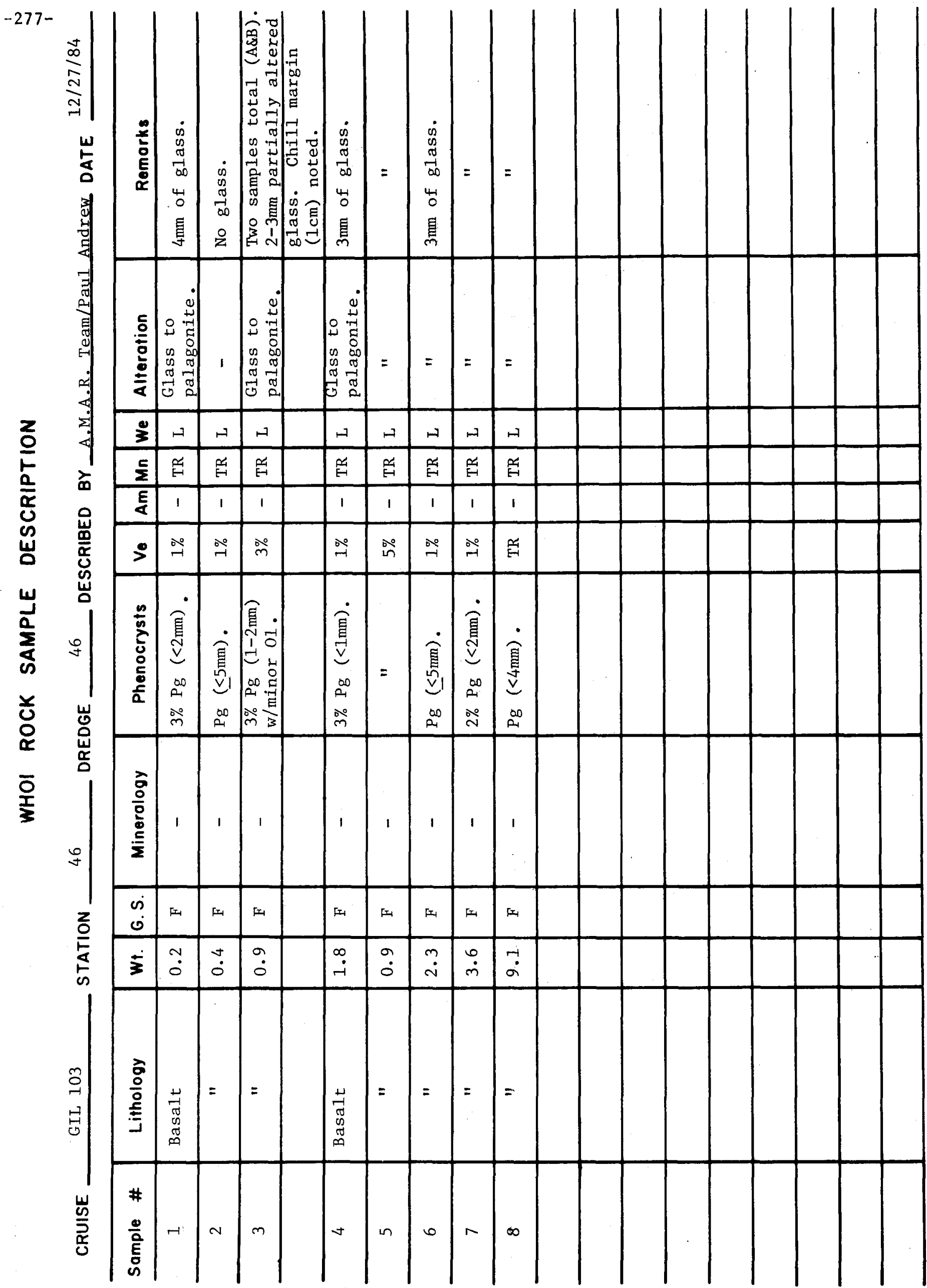




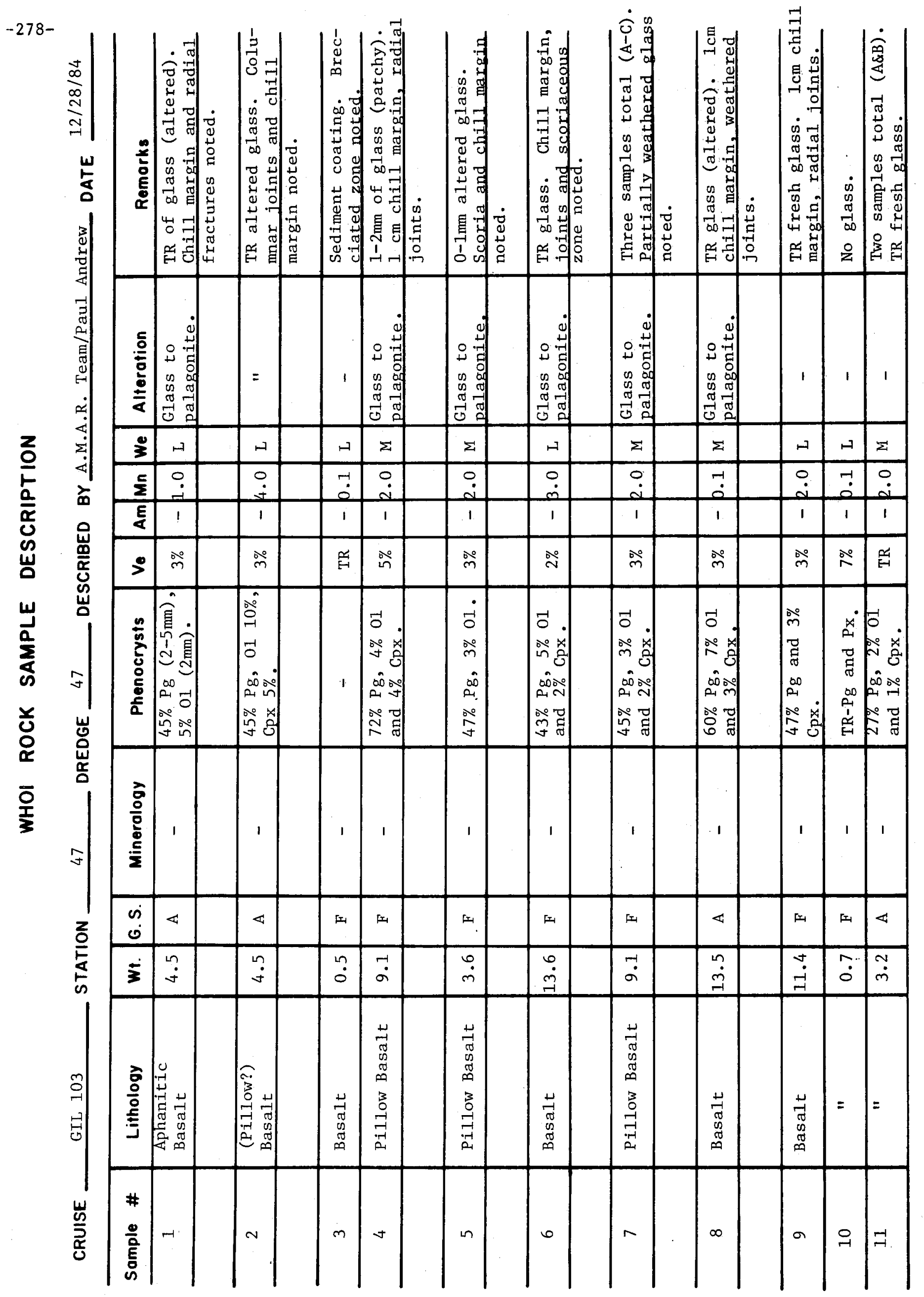




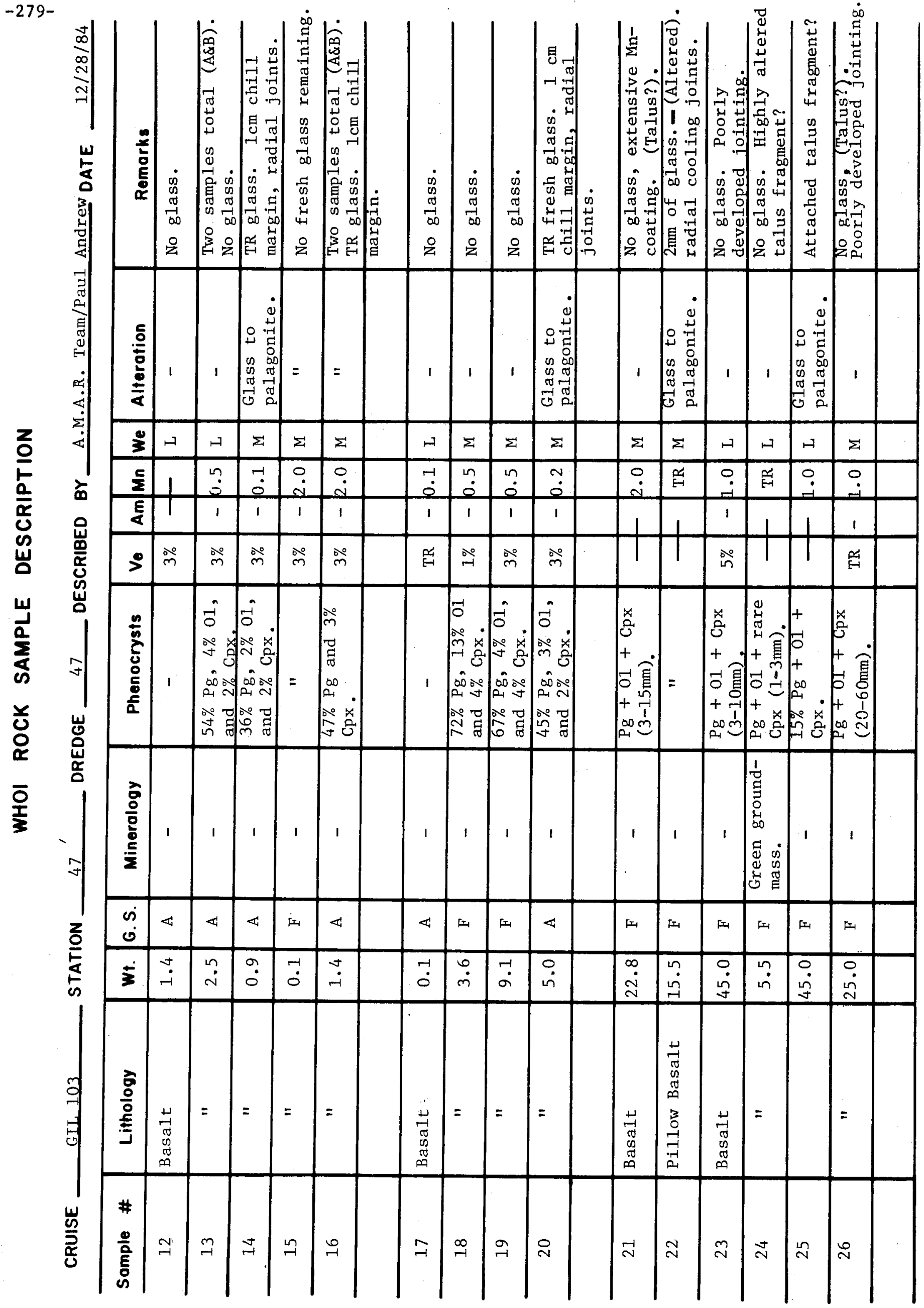




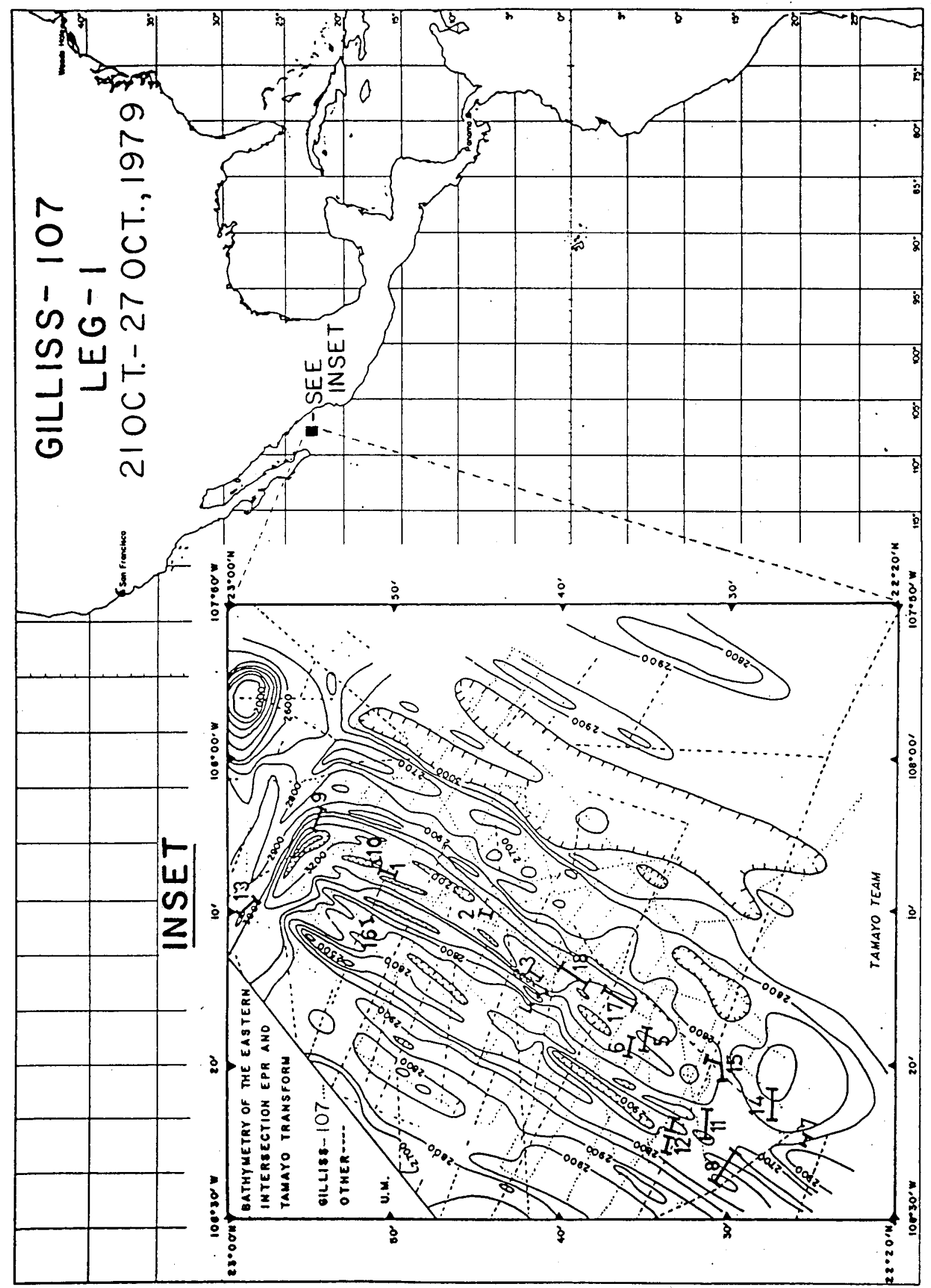




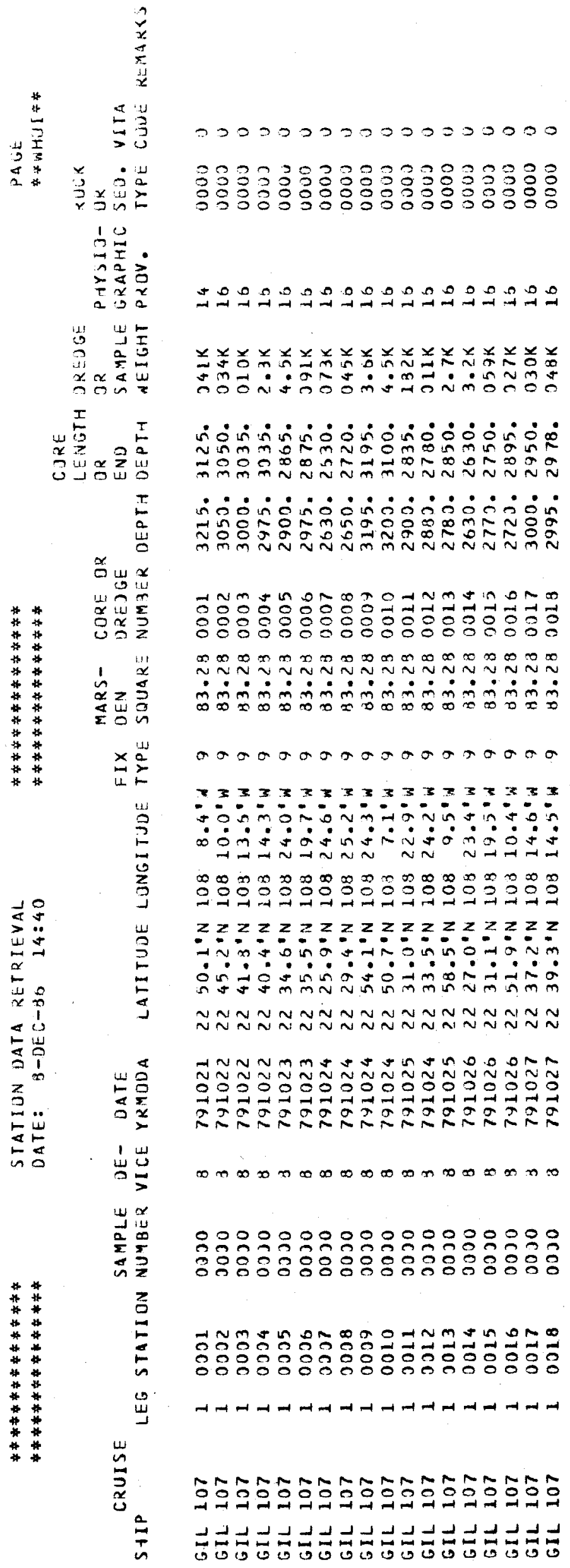




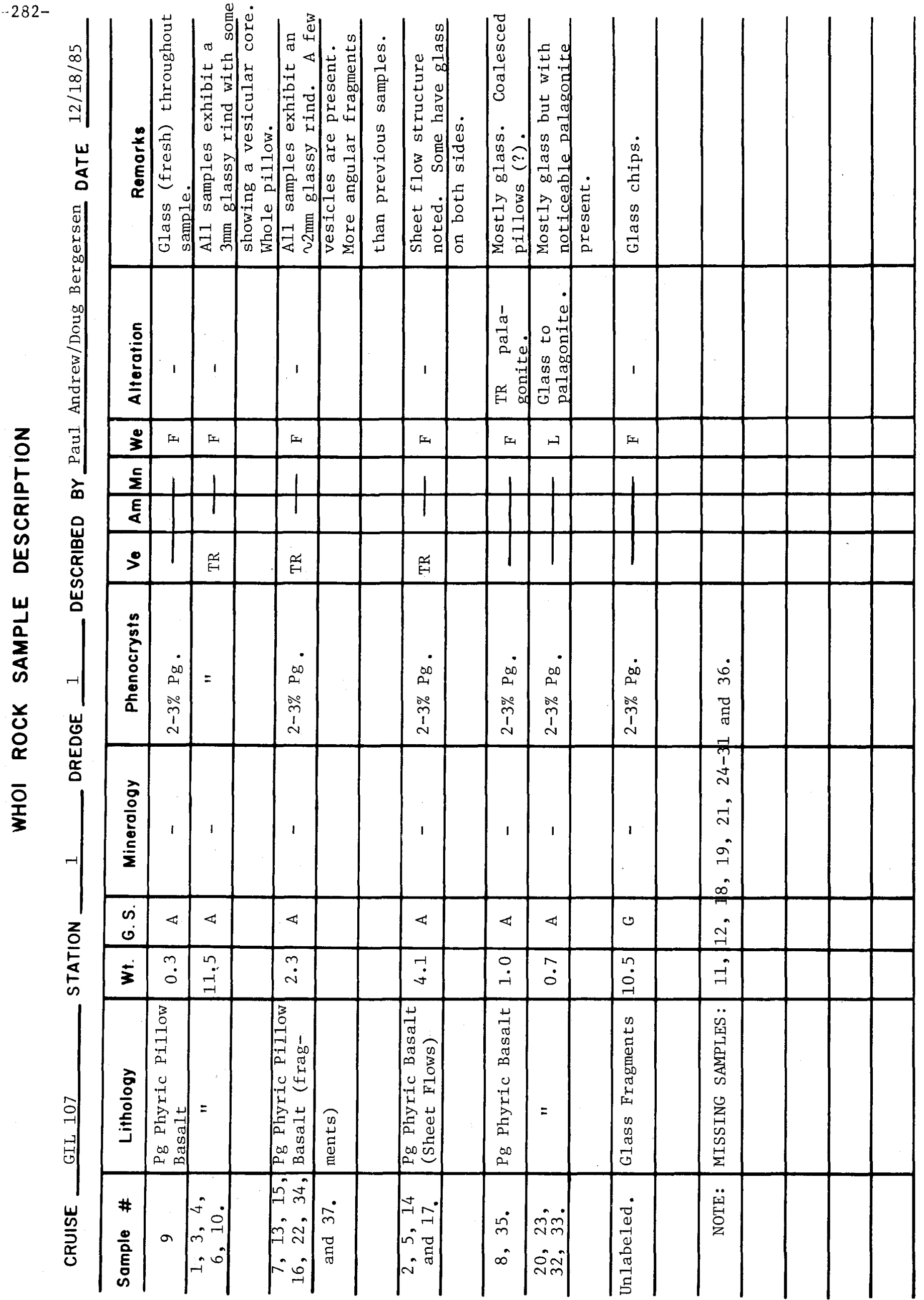




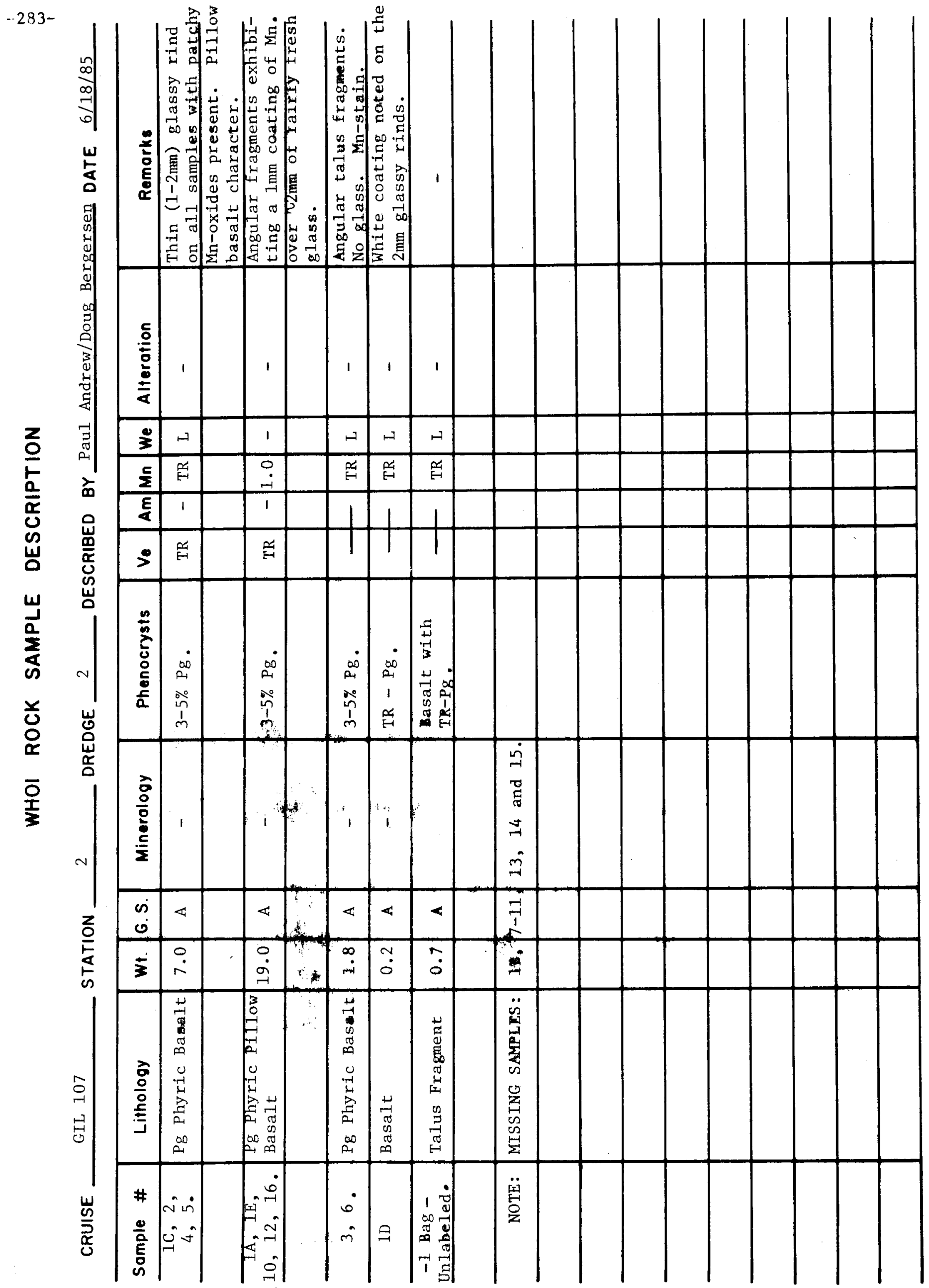




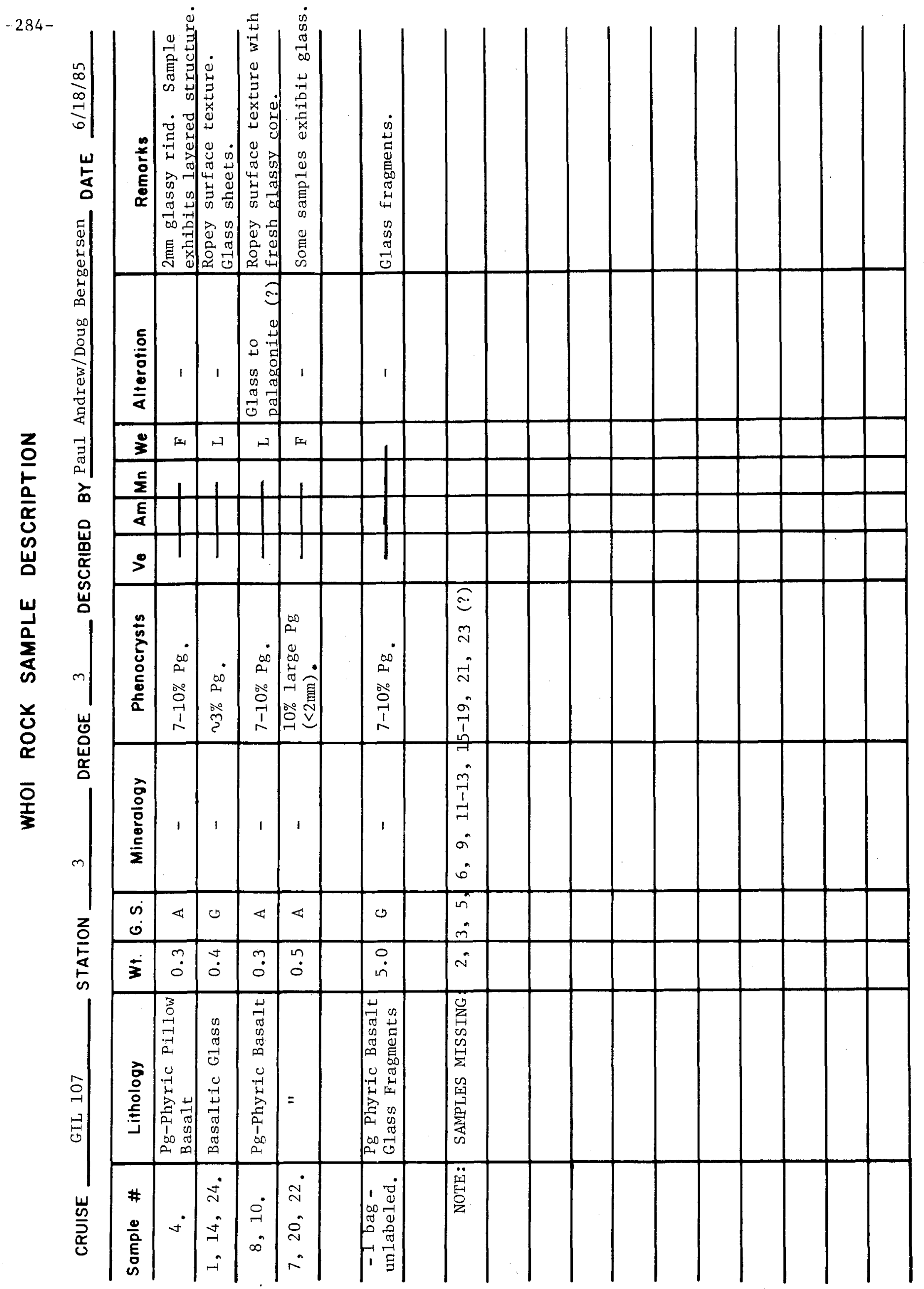




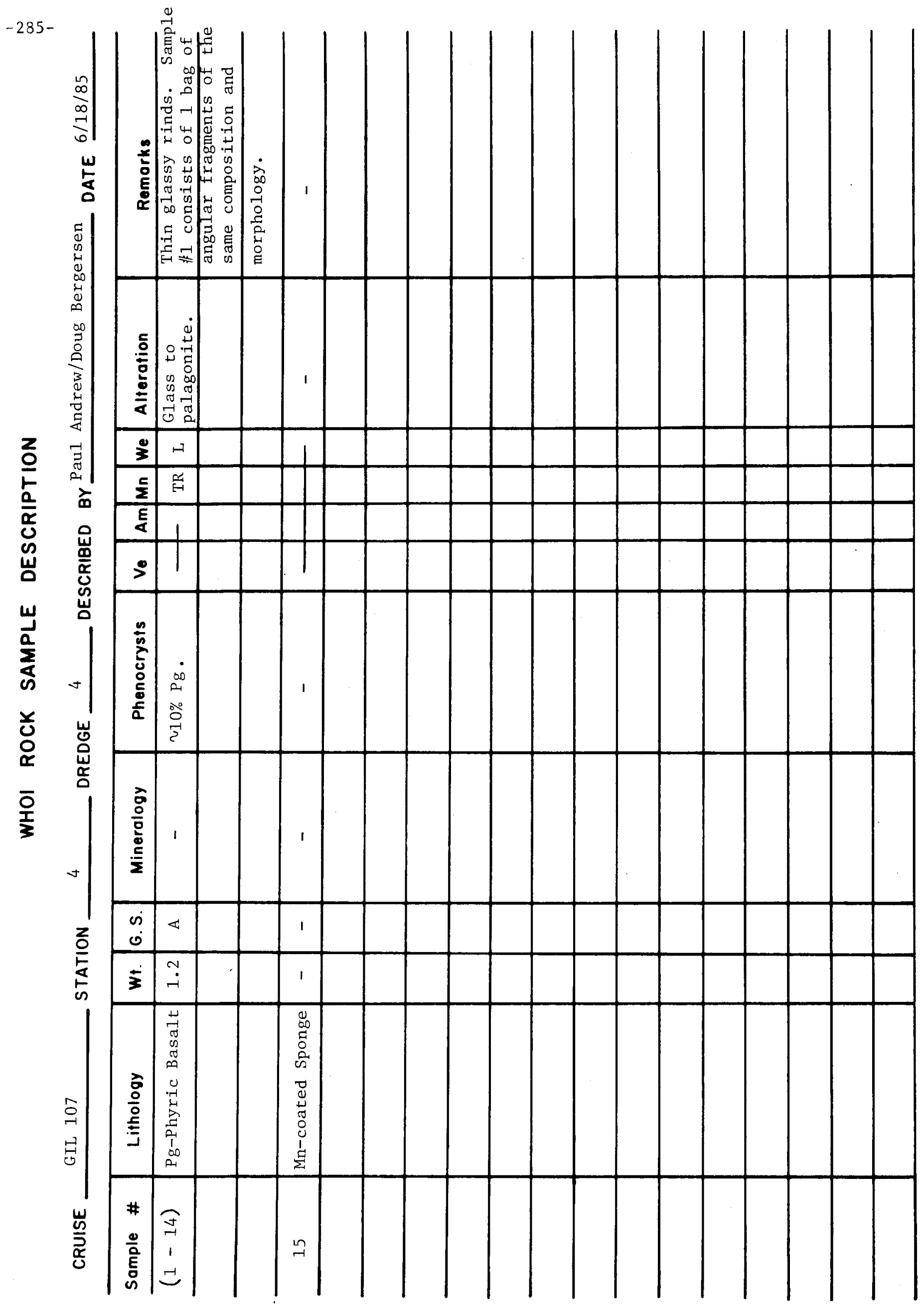




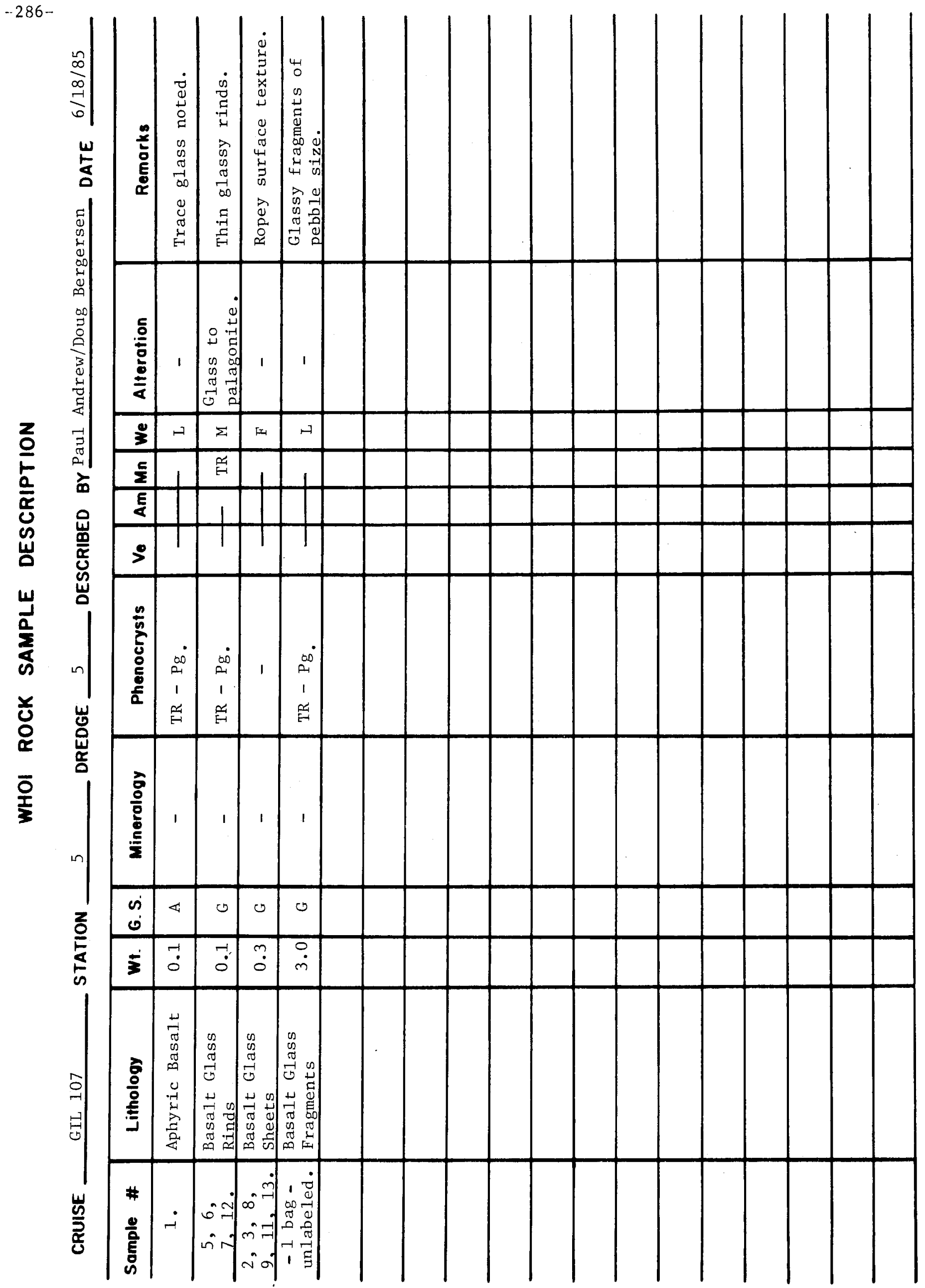




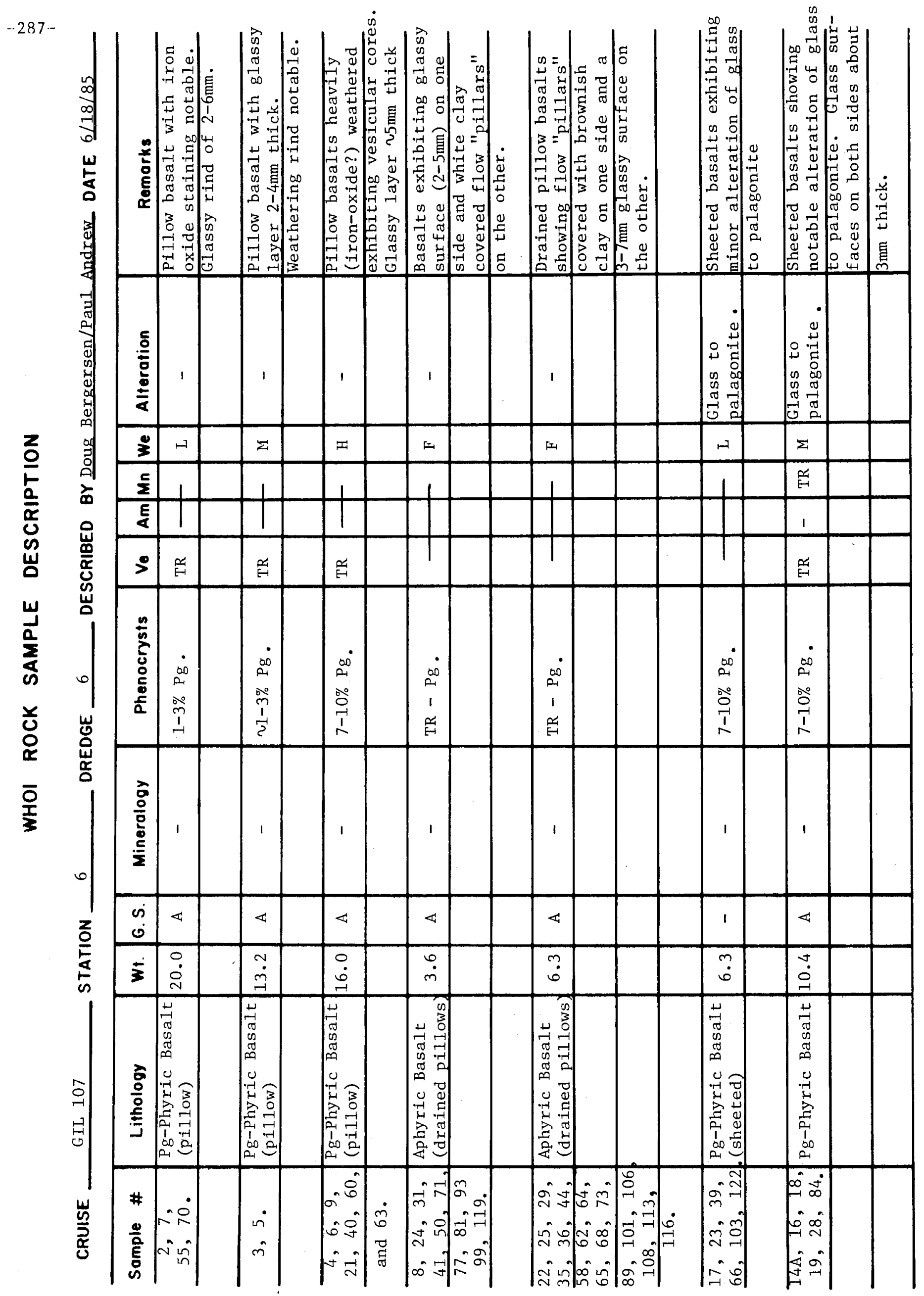




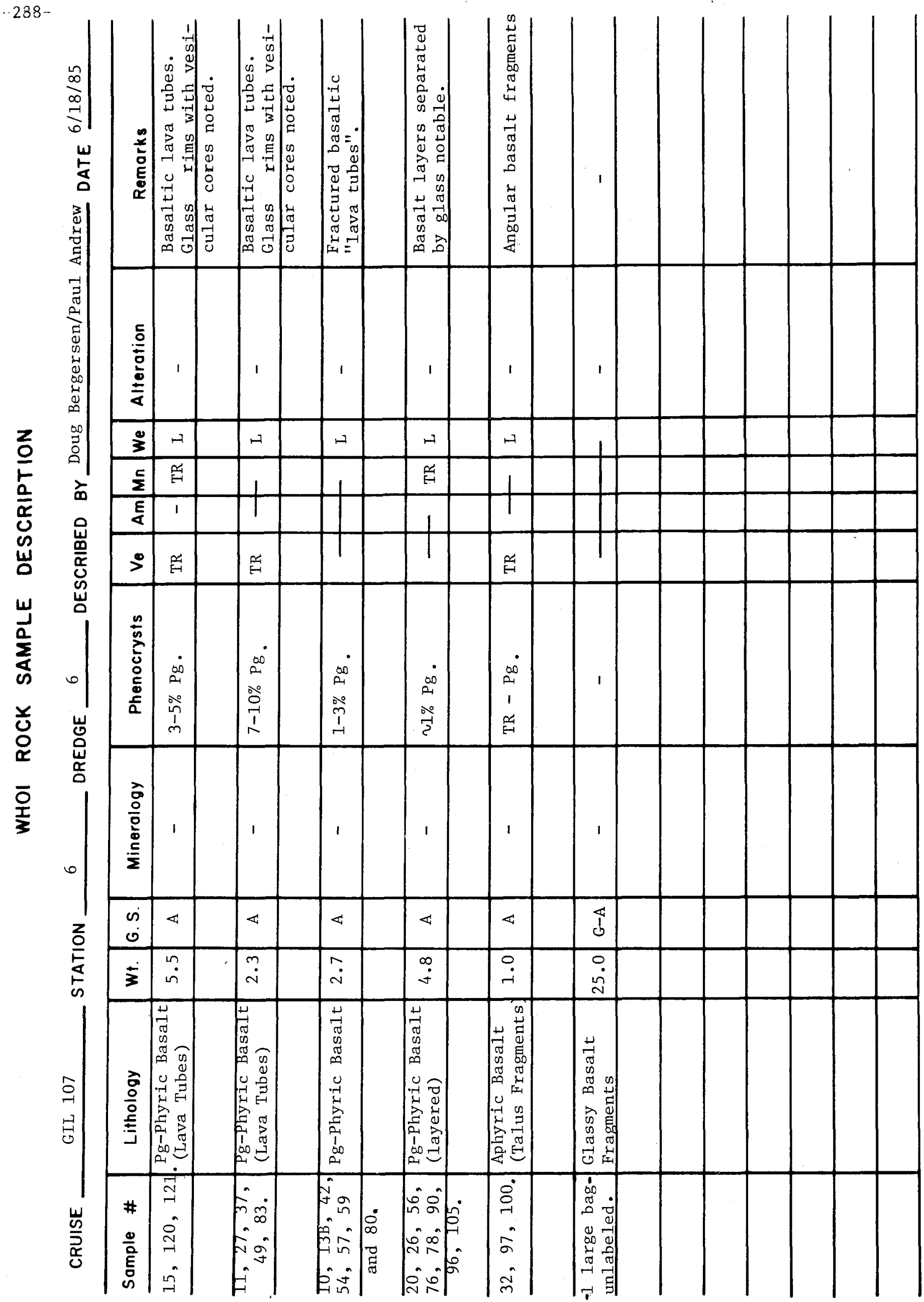




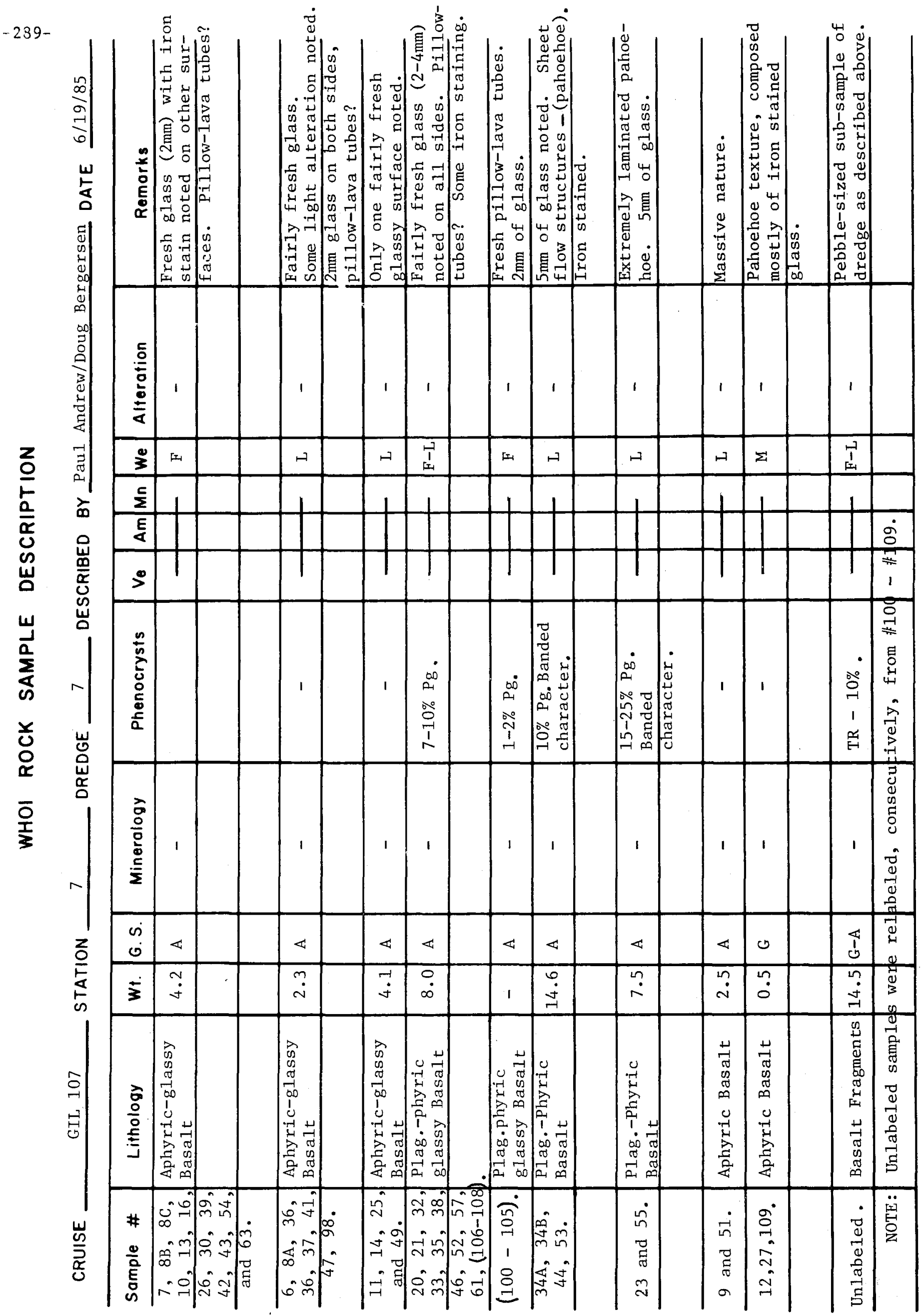




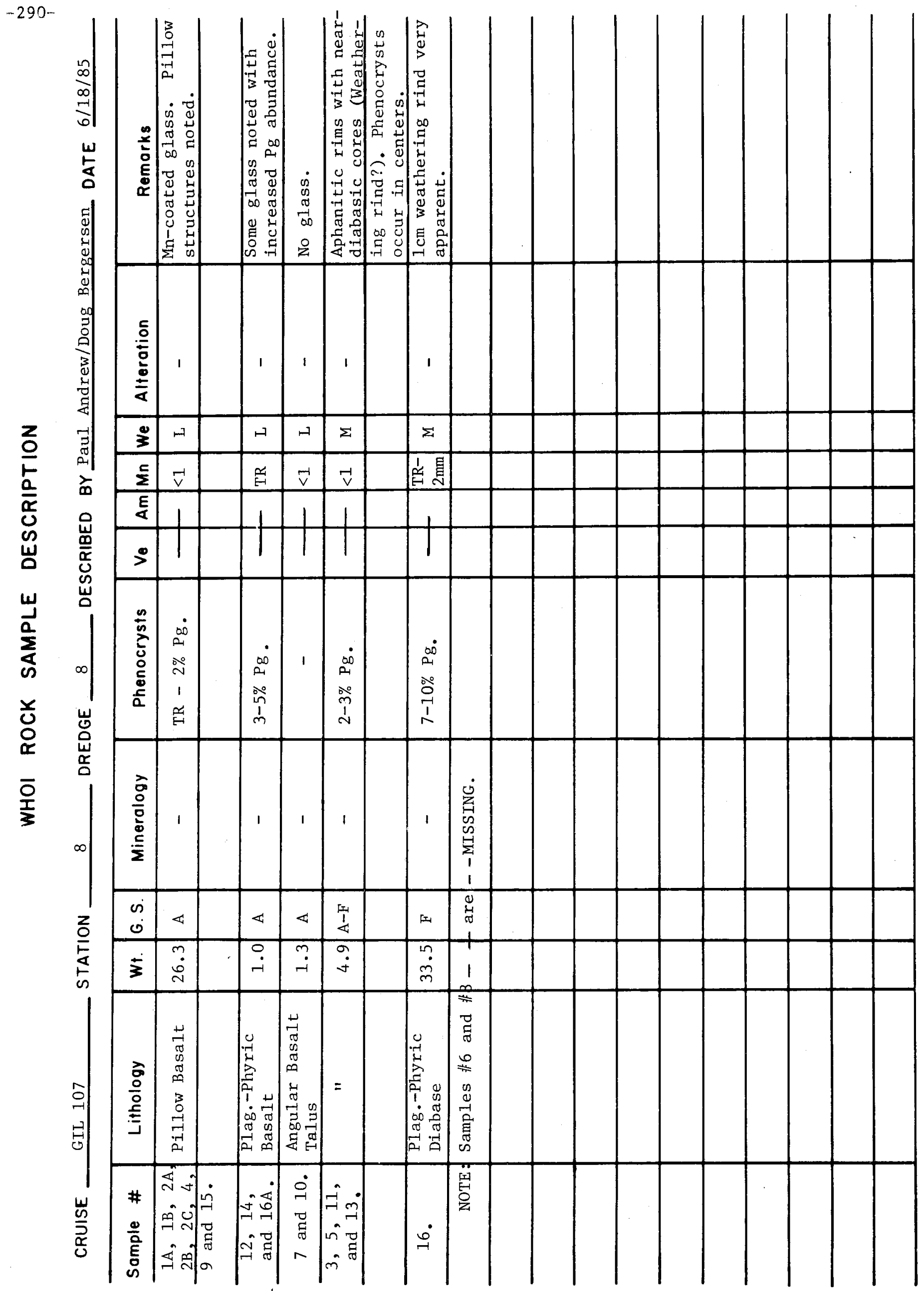




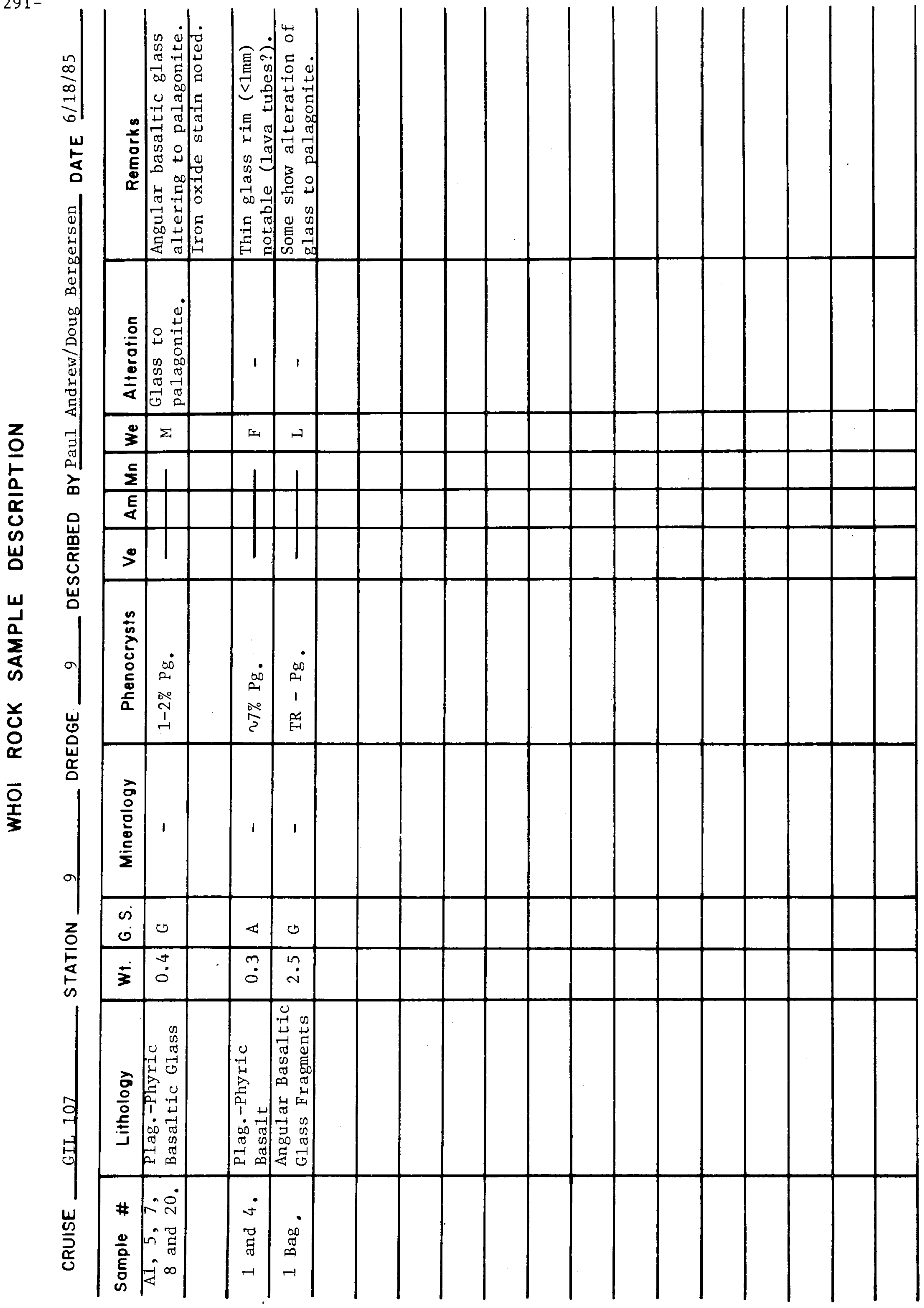




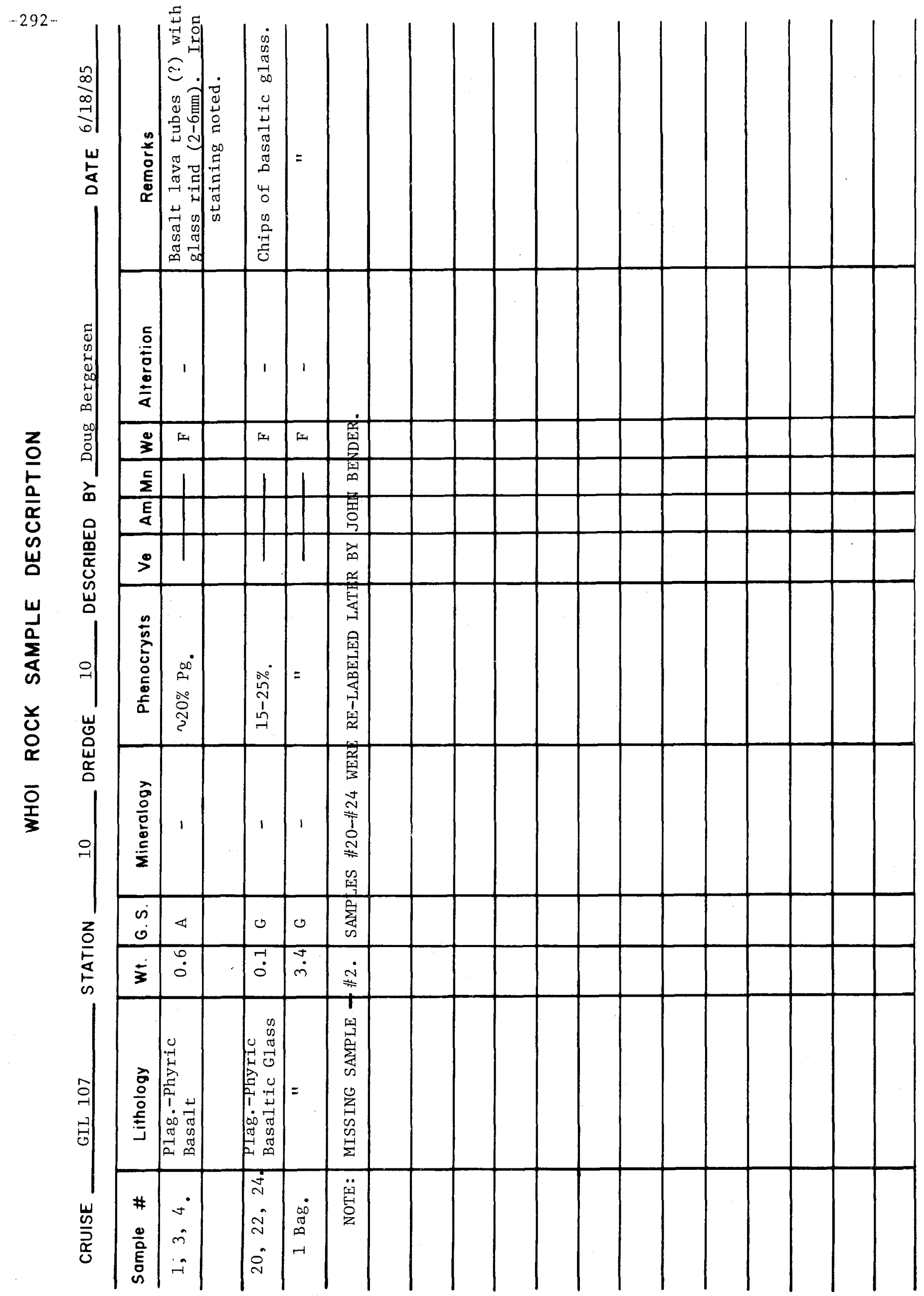




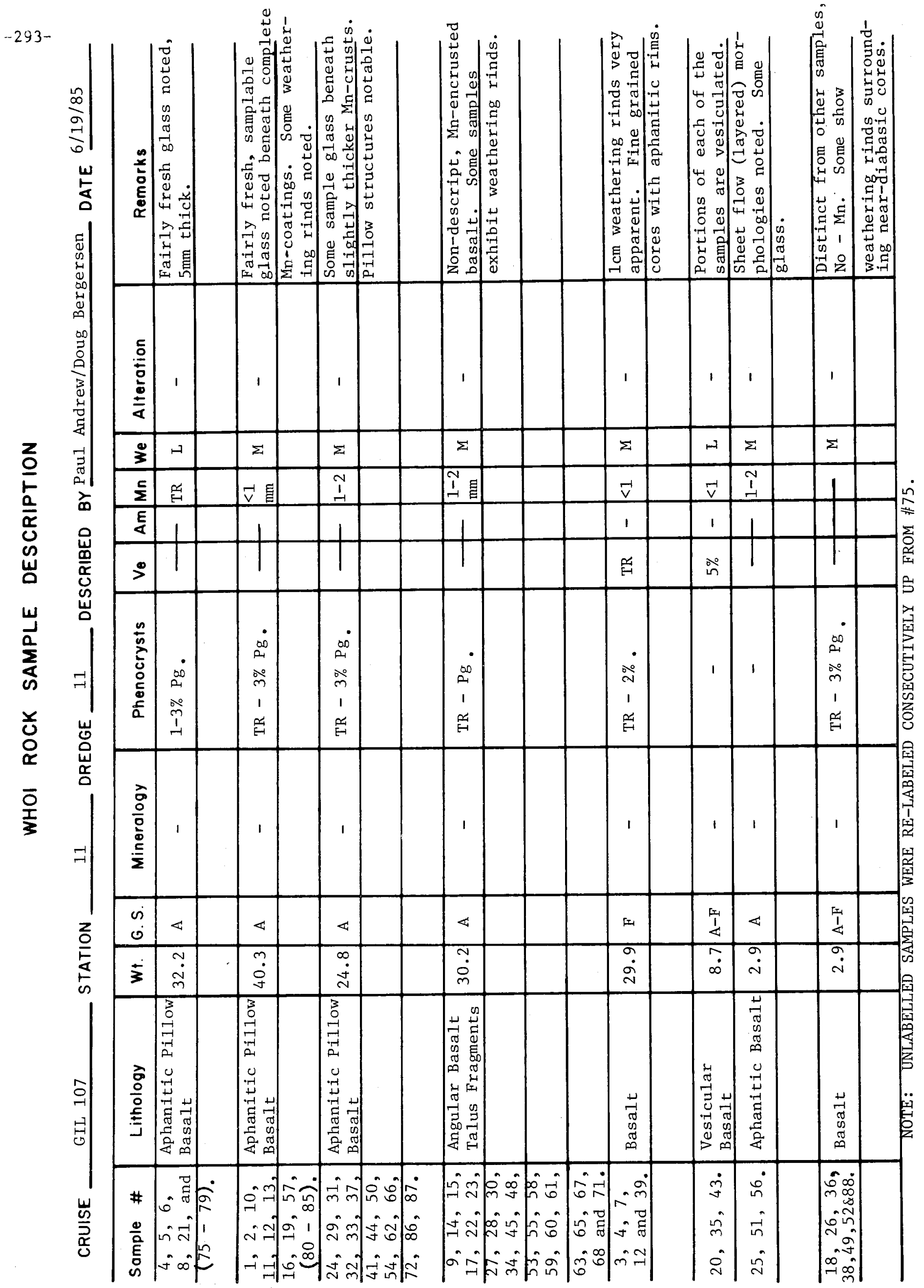




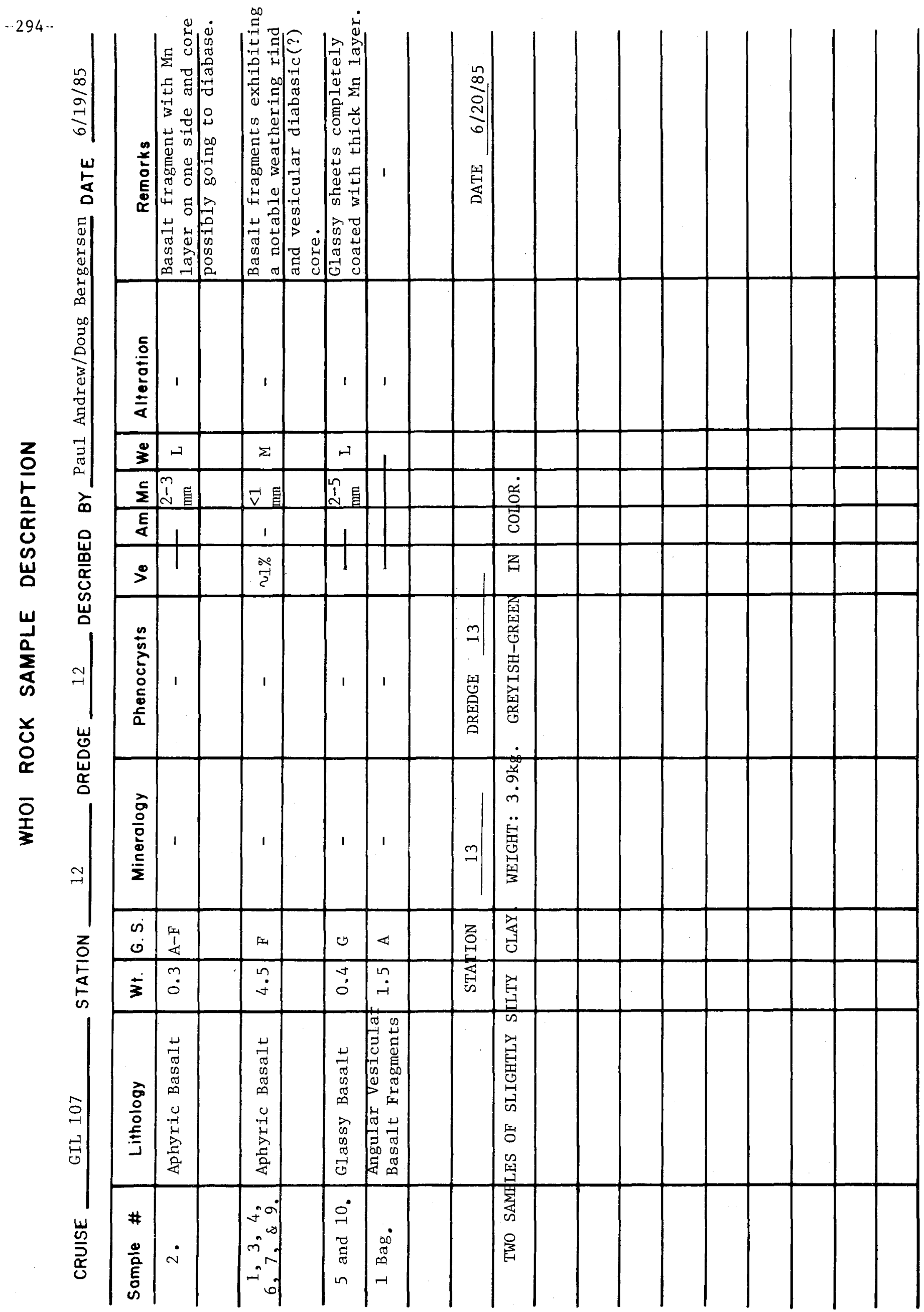




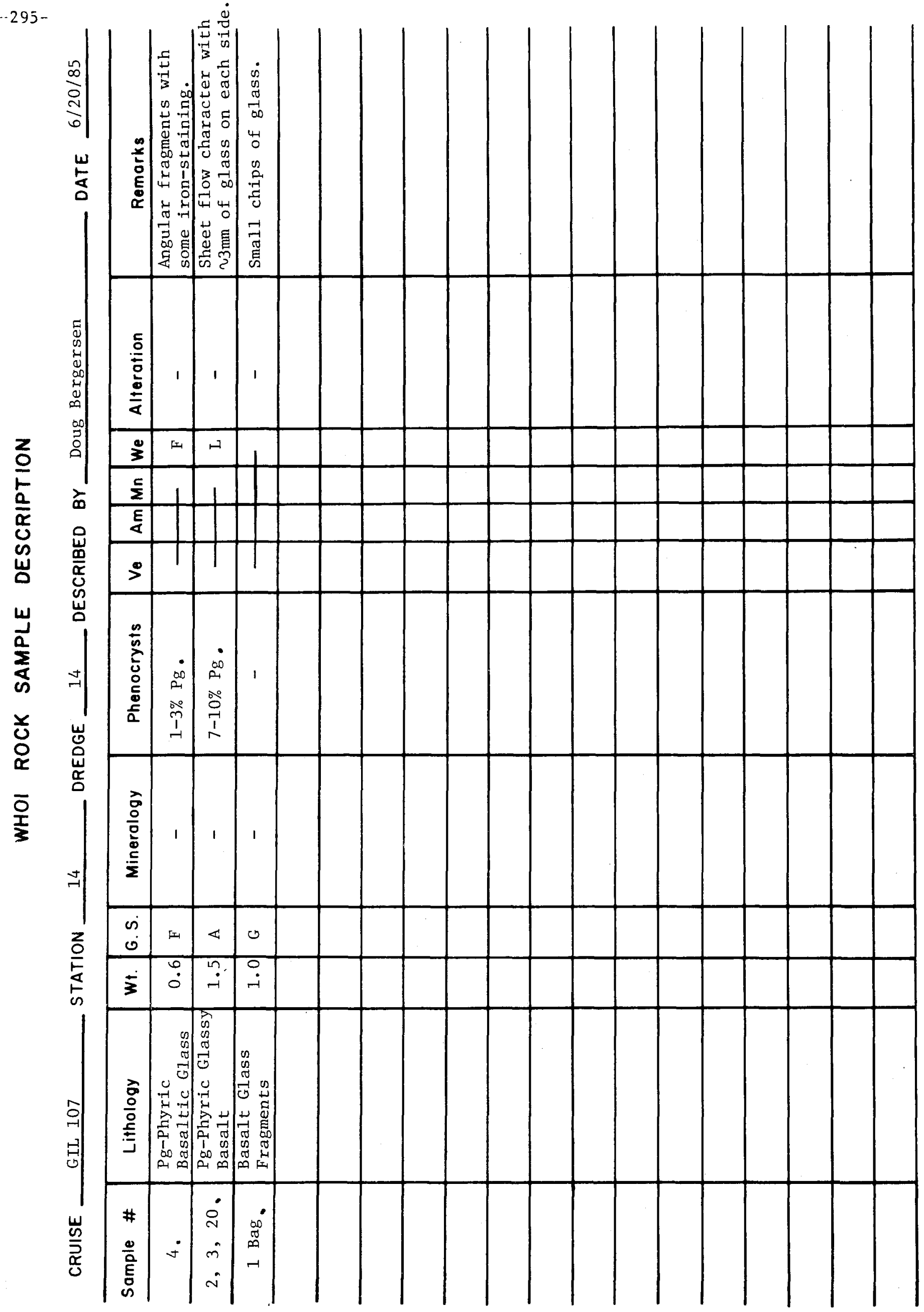




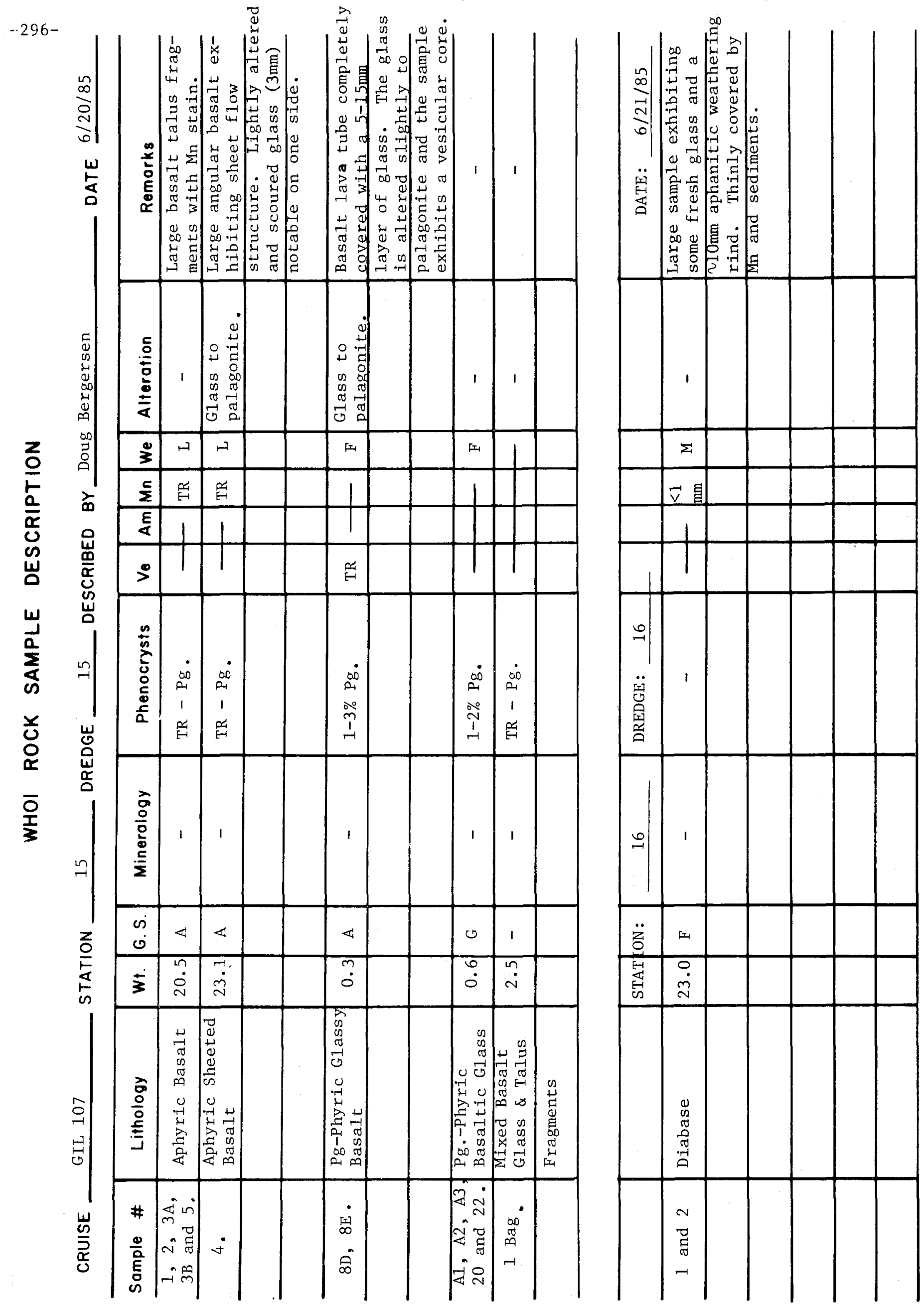




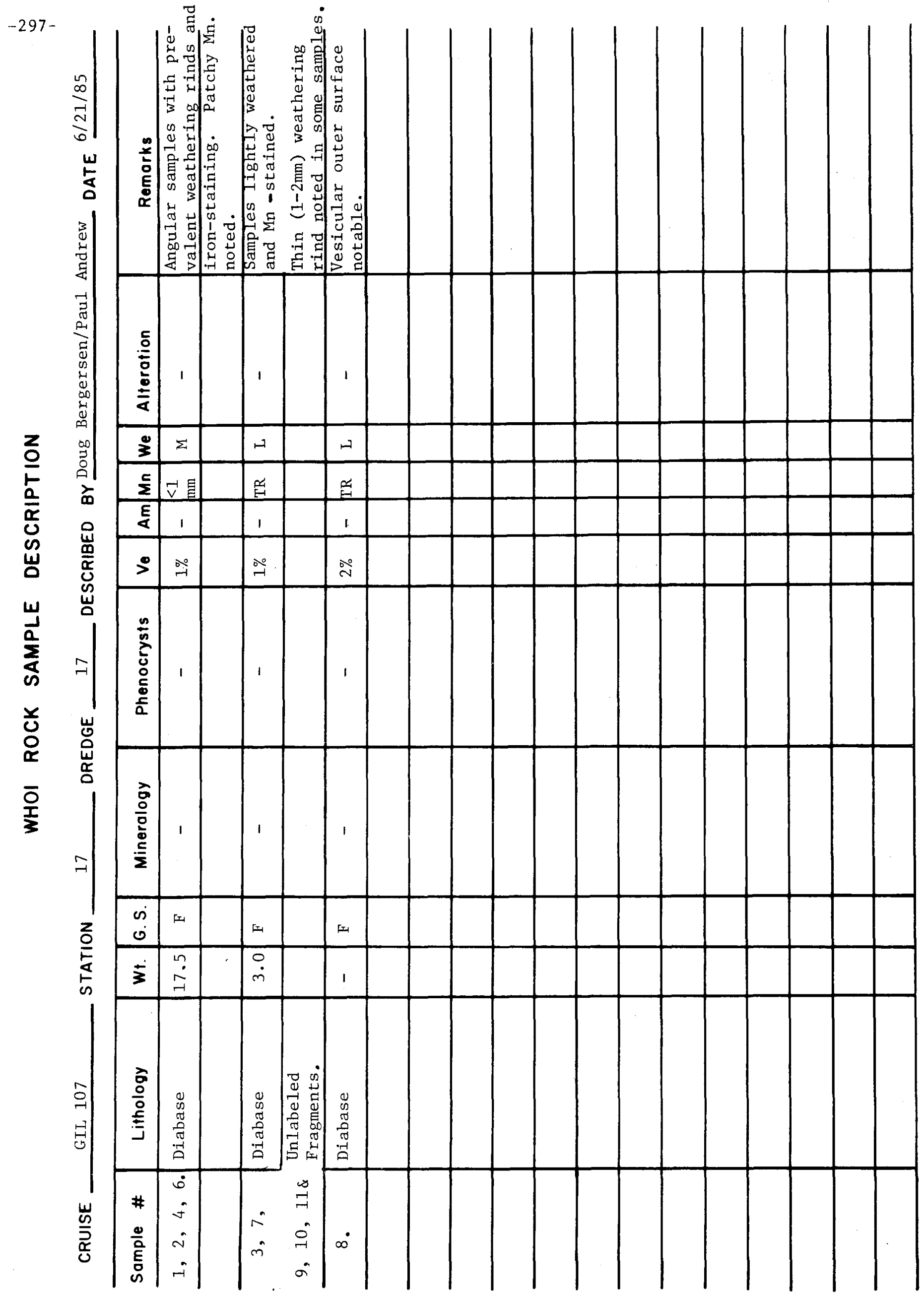




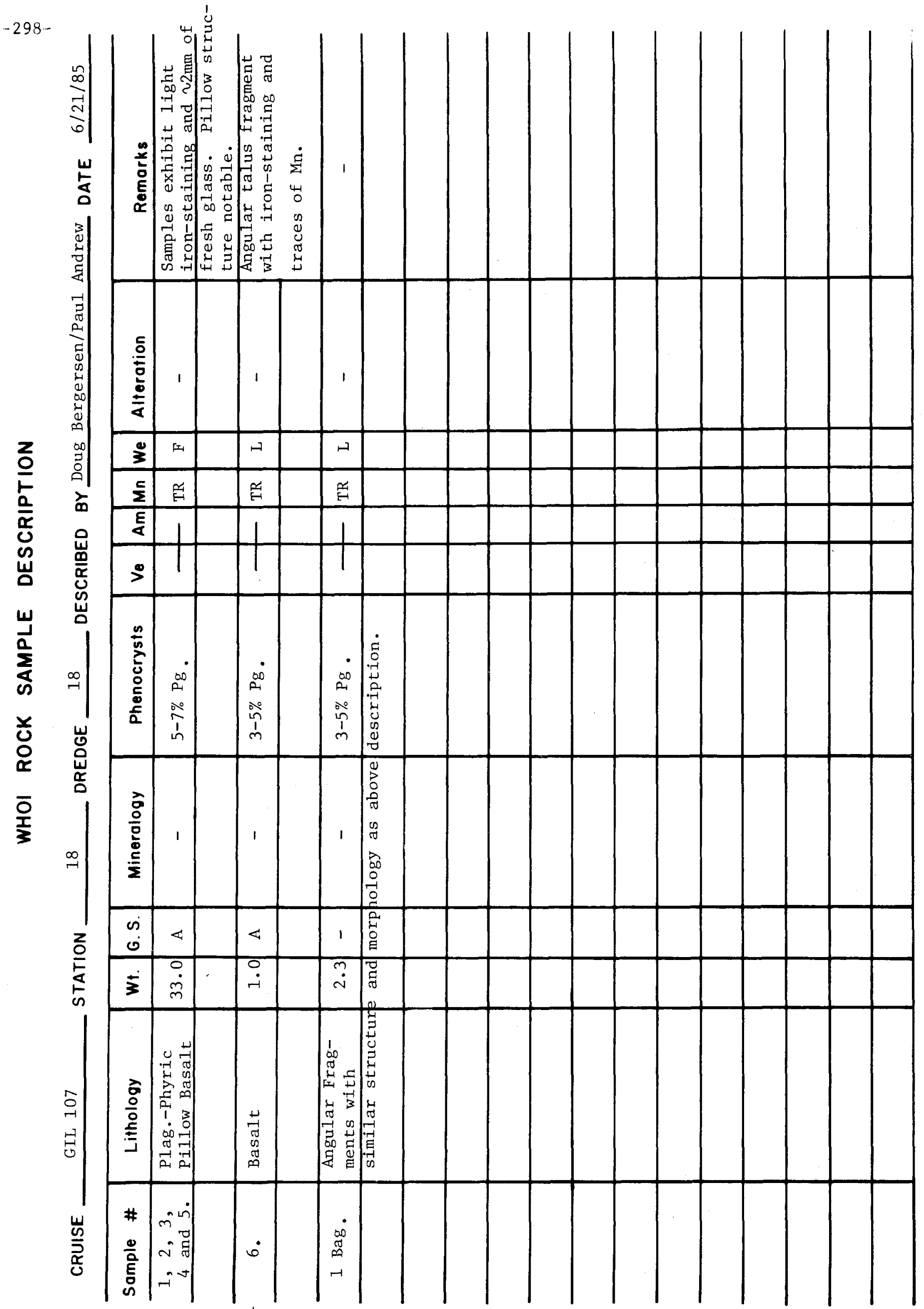


ARA ISLAS ORCADAS $11 / 76$

LEG I

18 NOVEMBER - 23 DECEMBER

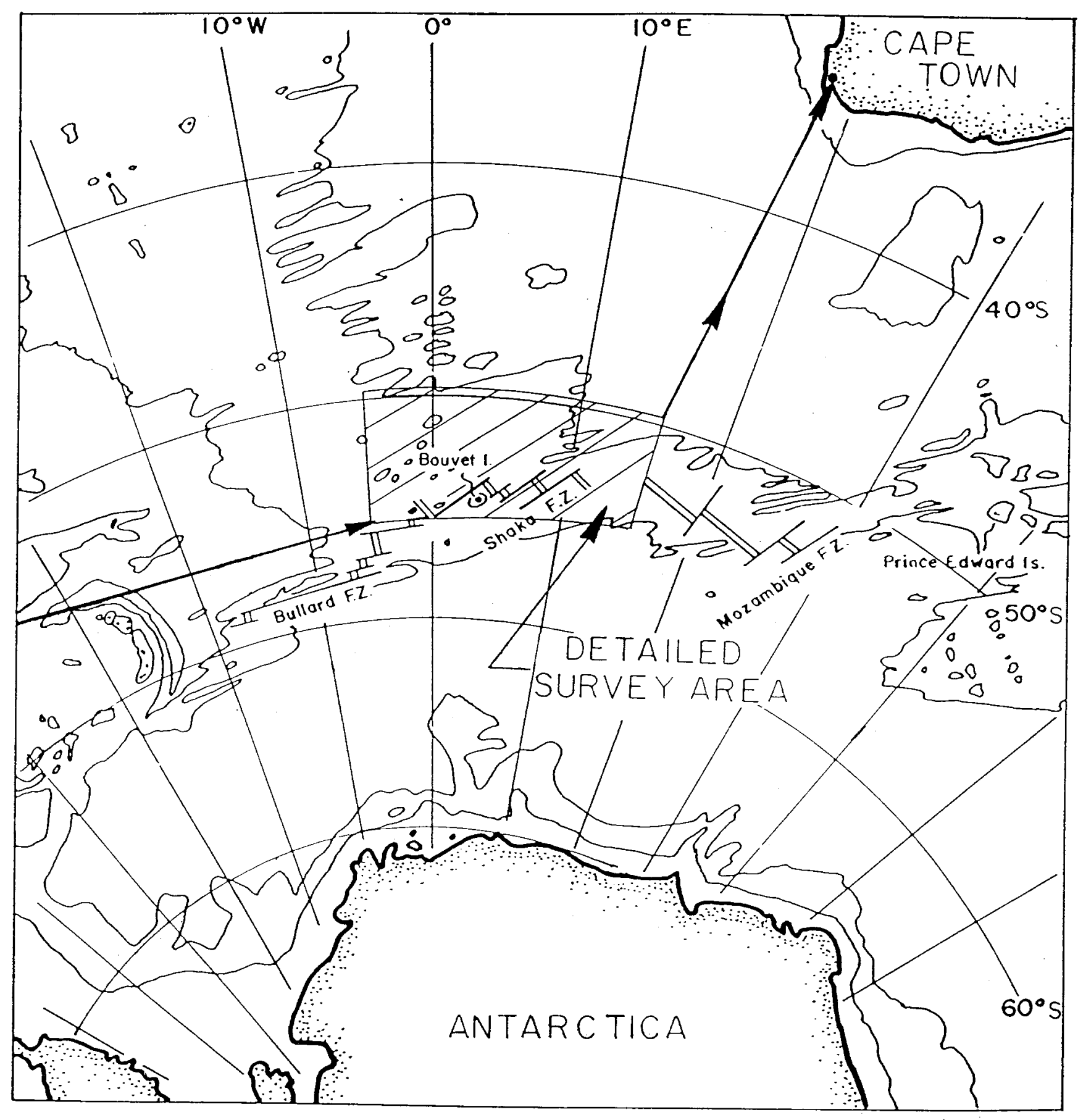




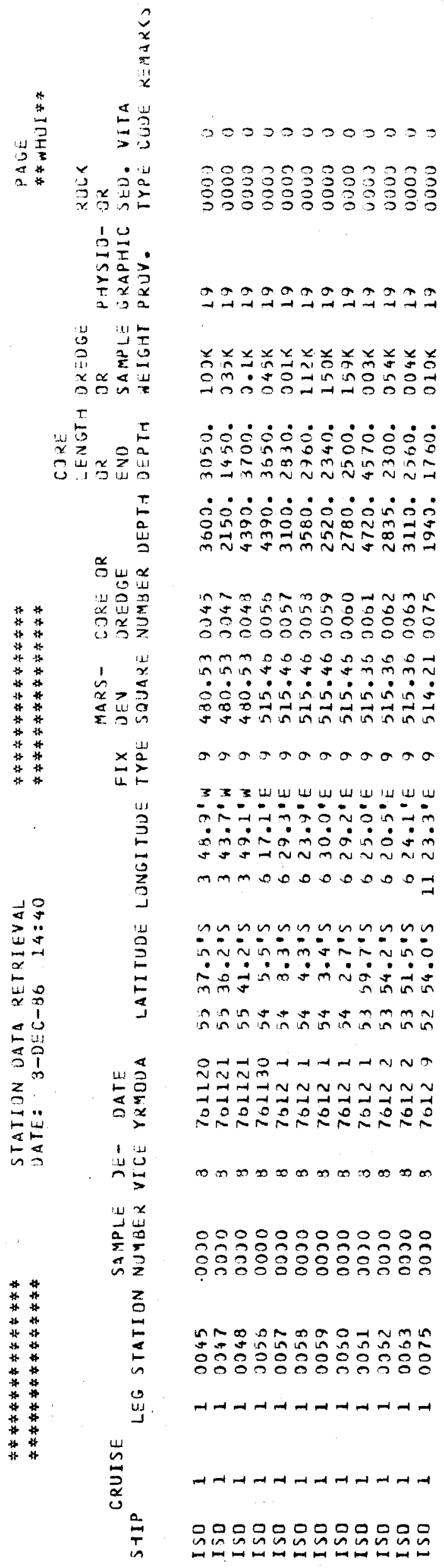




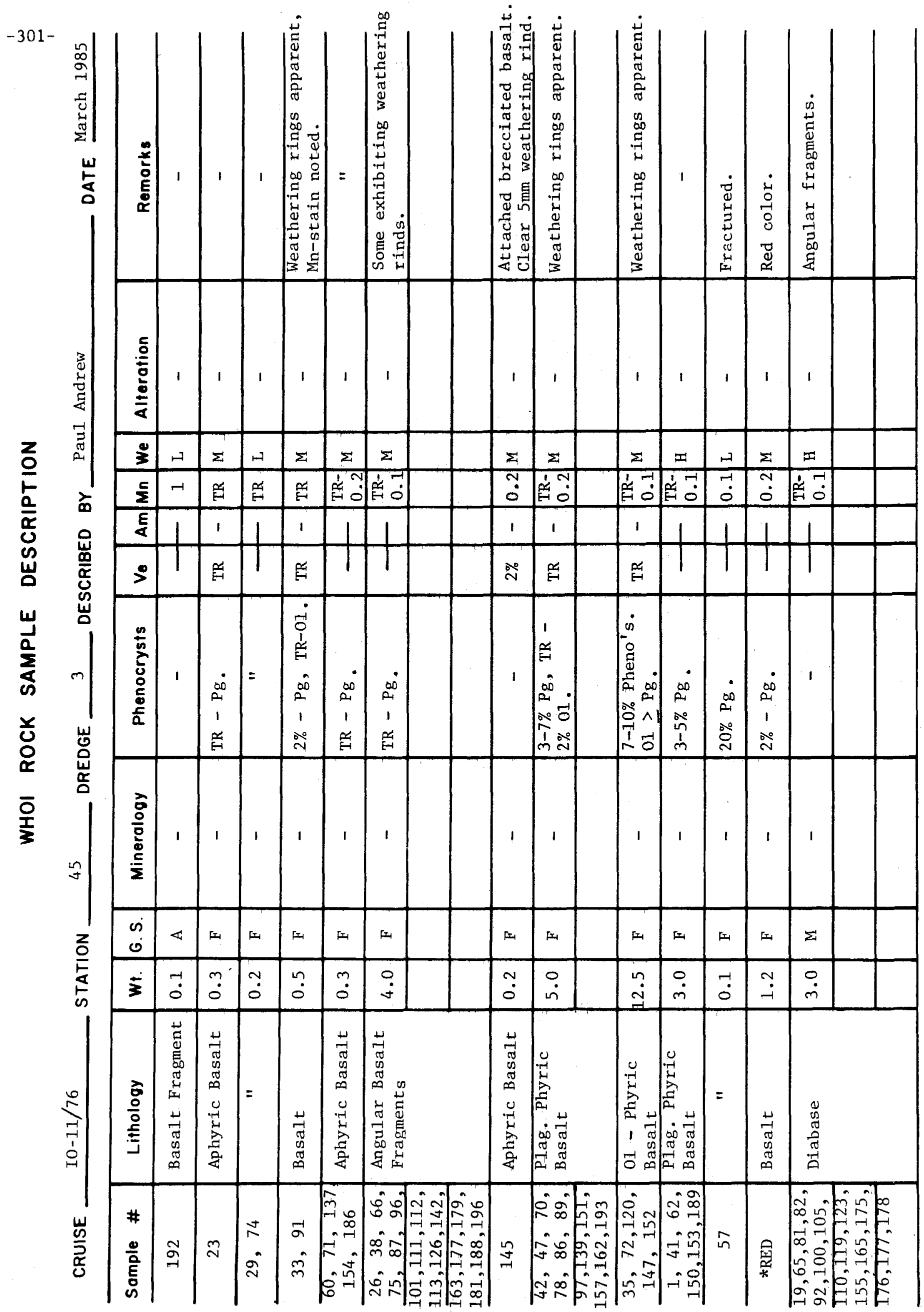




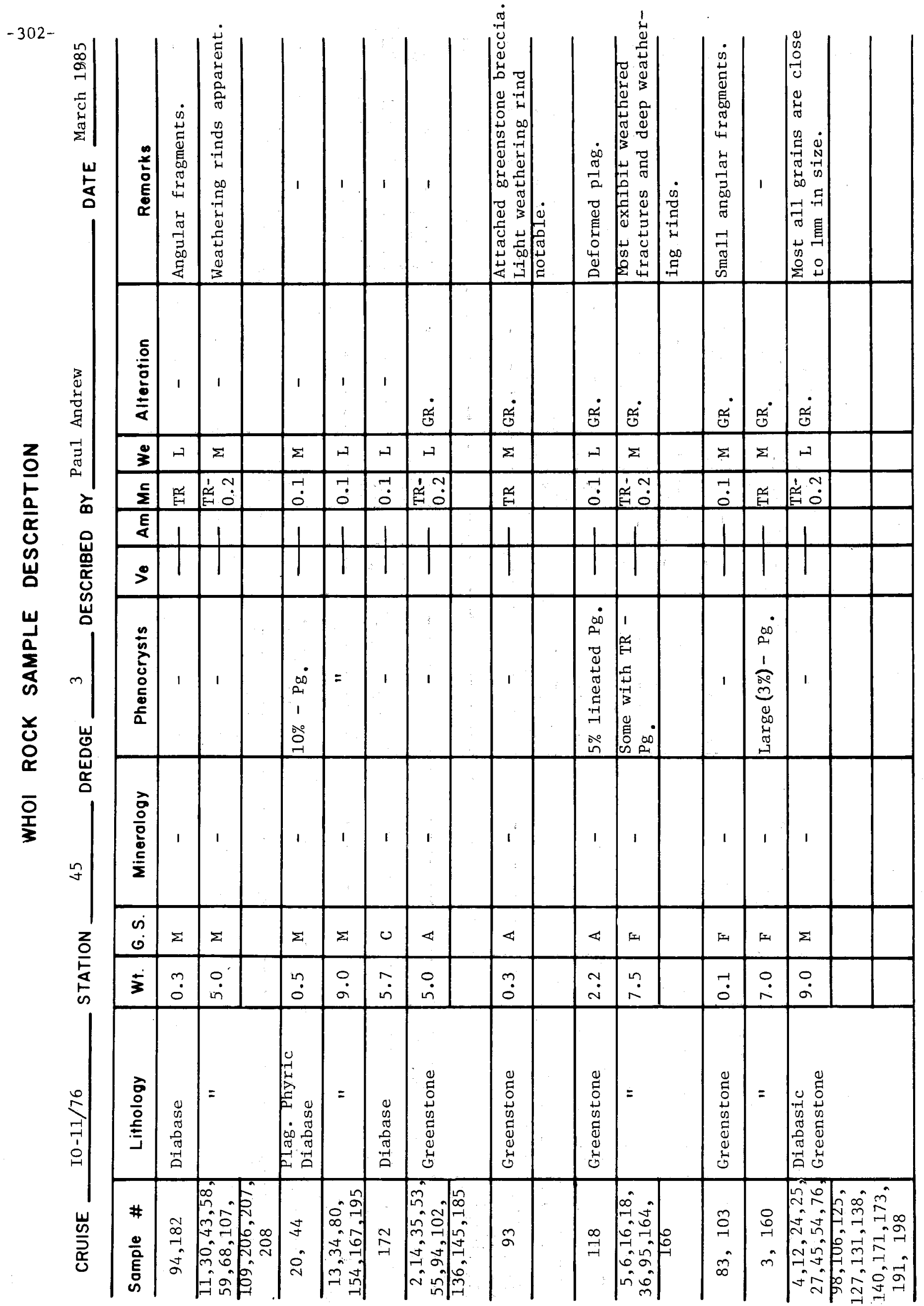




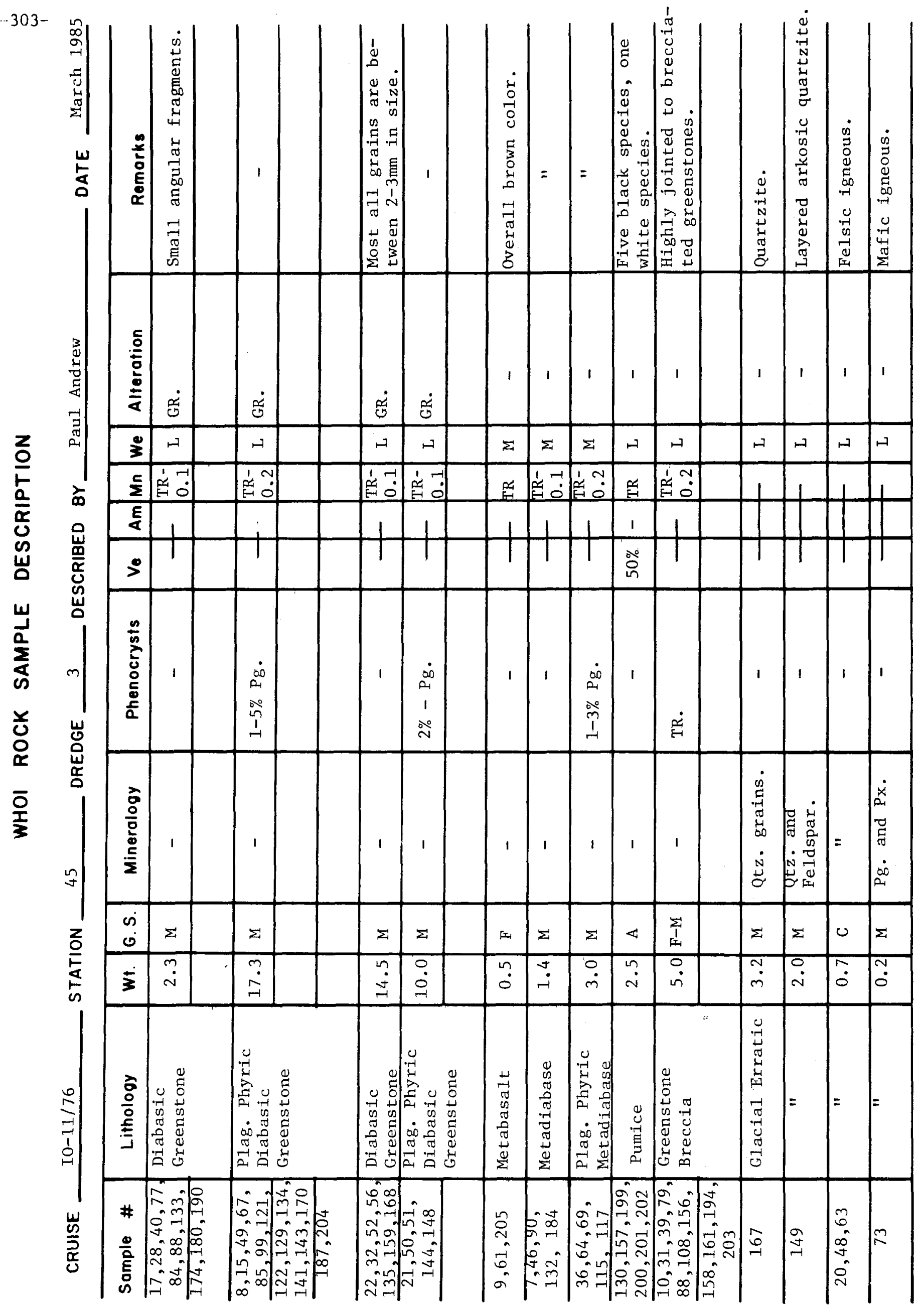




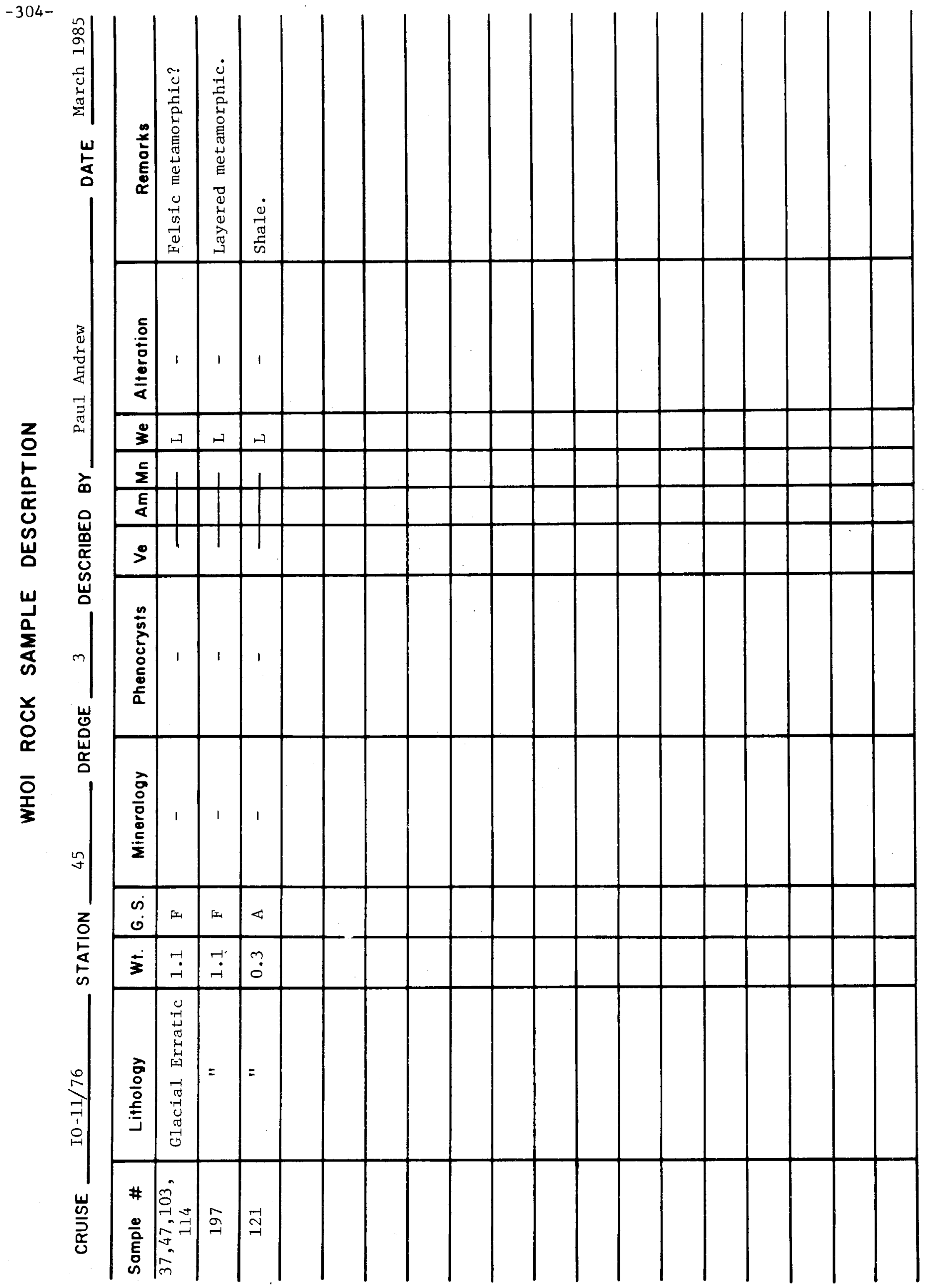




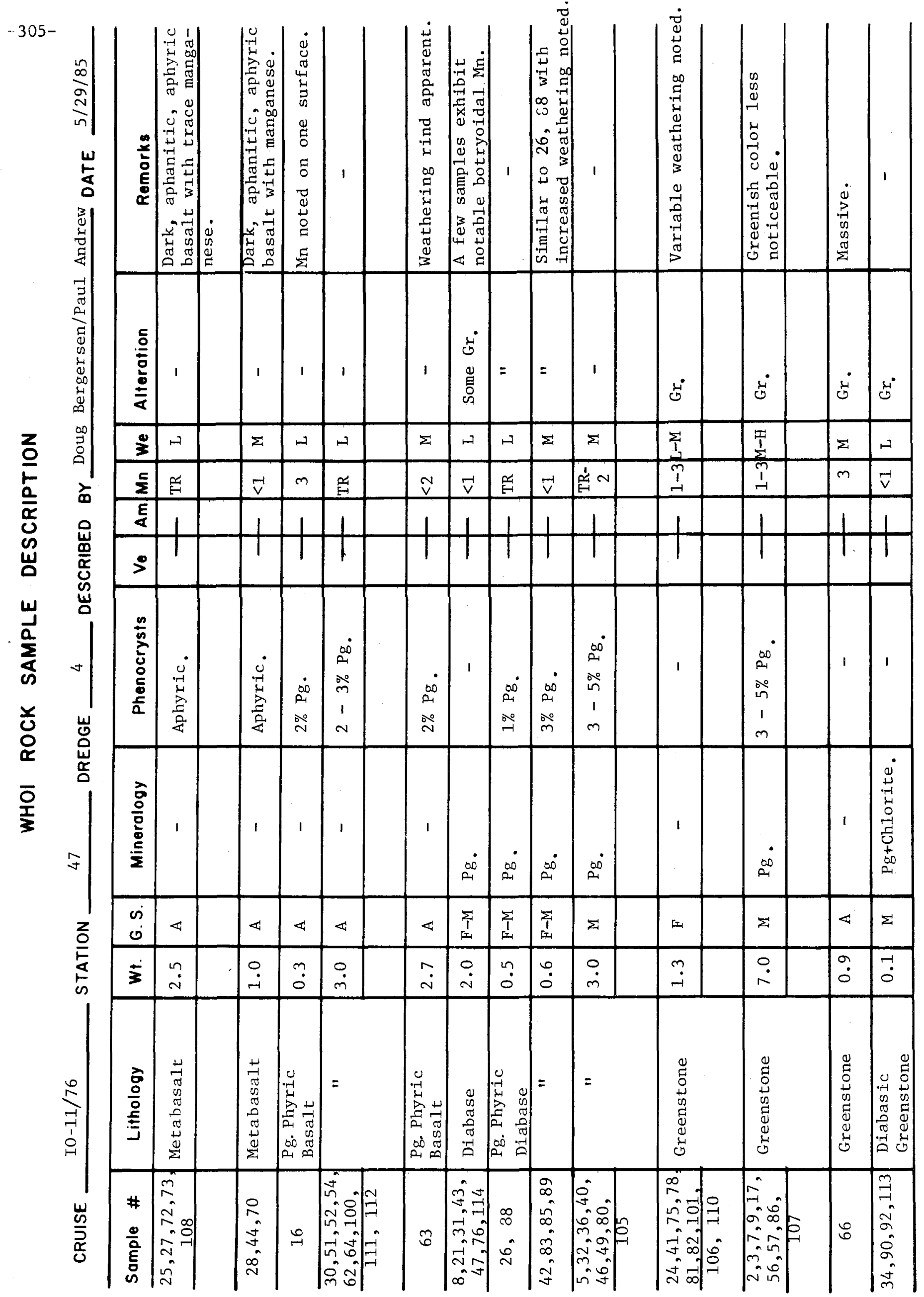




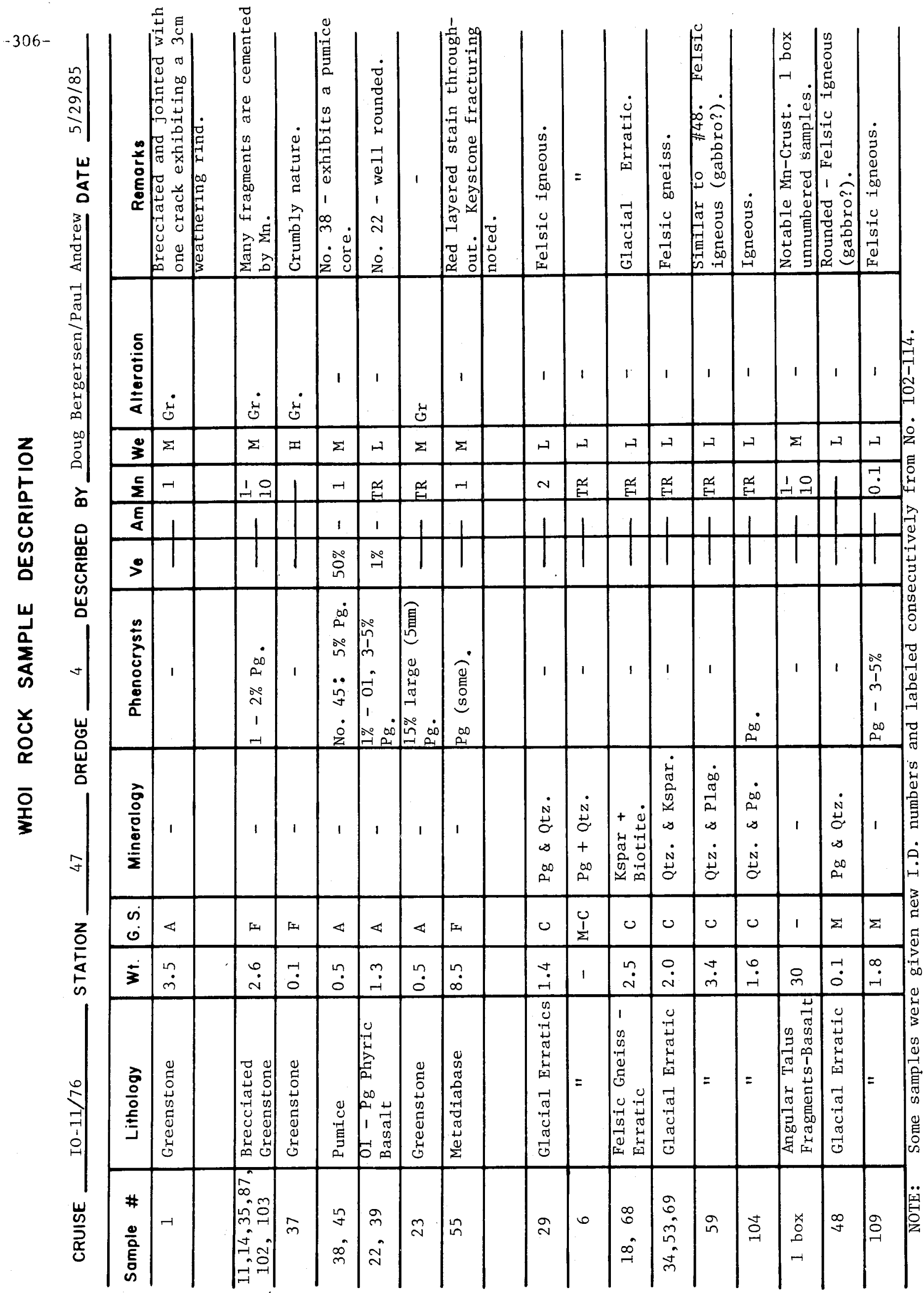




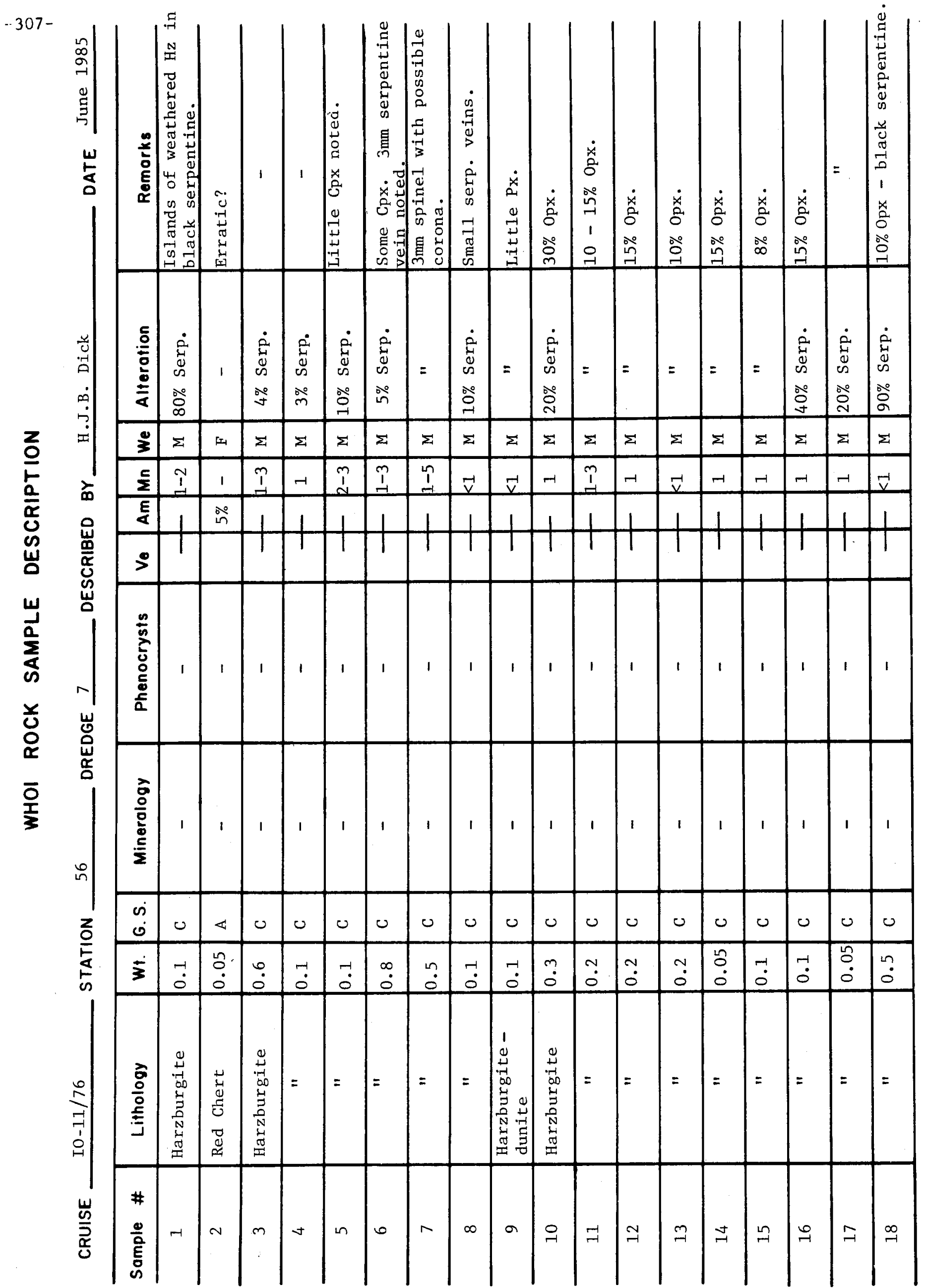




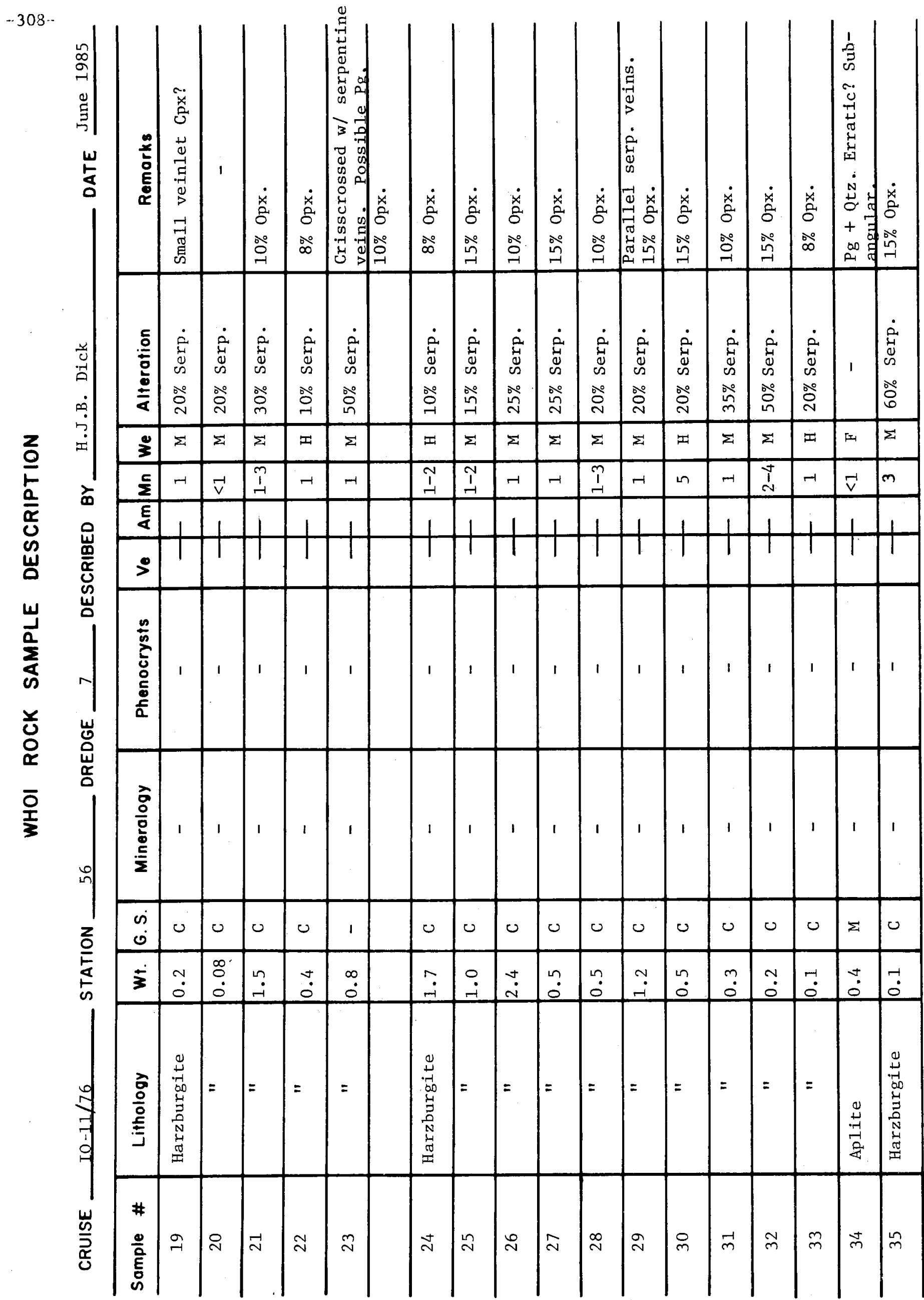




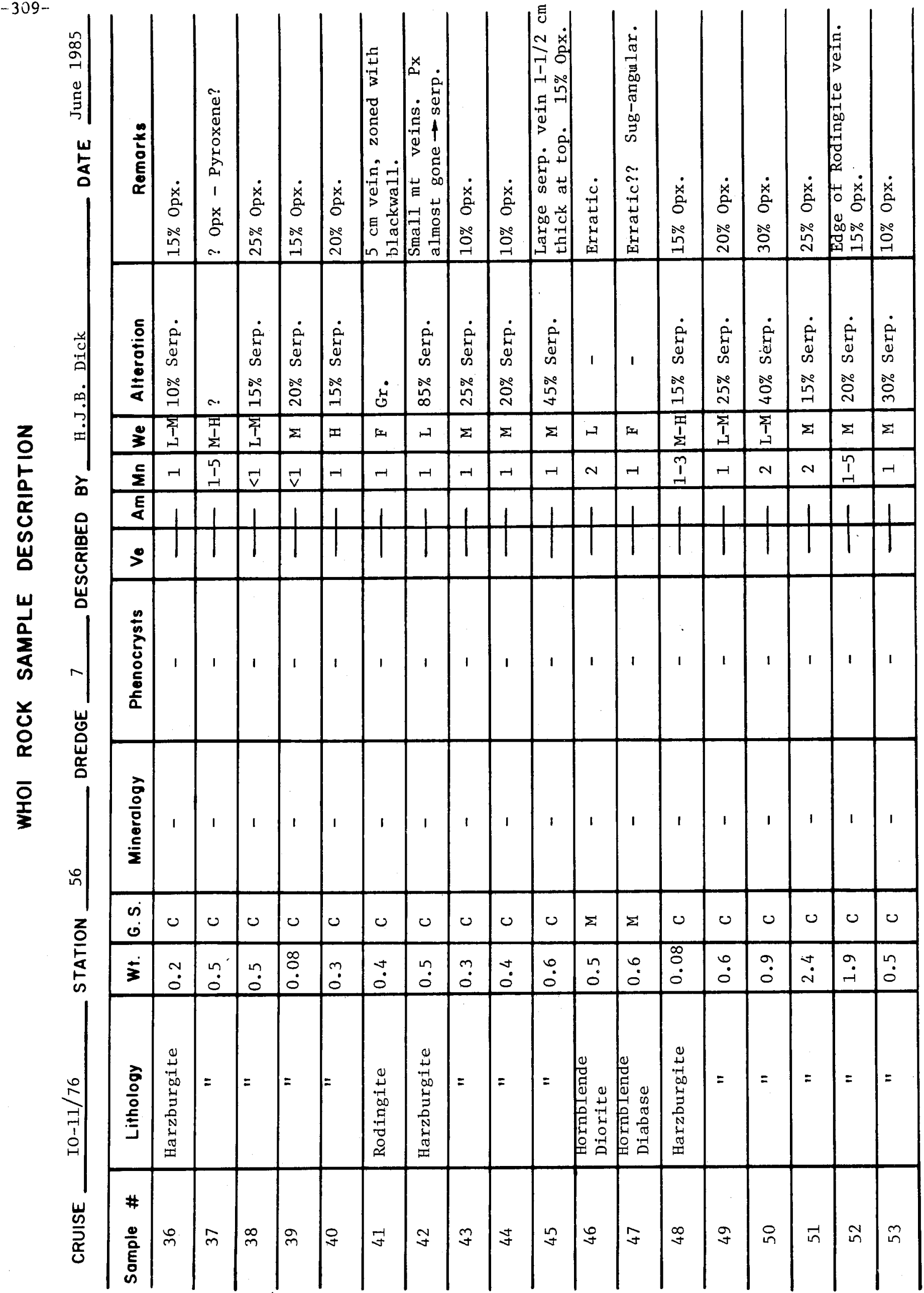




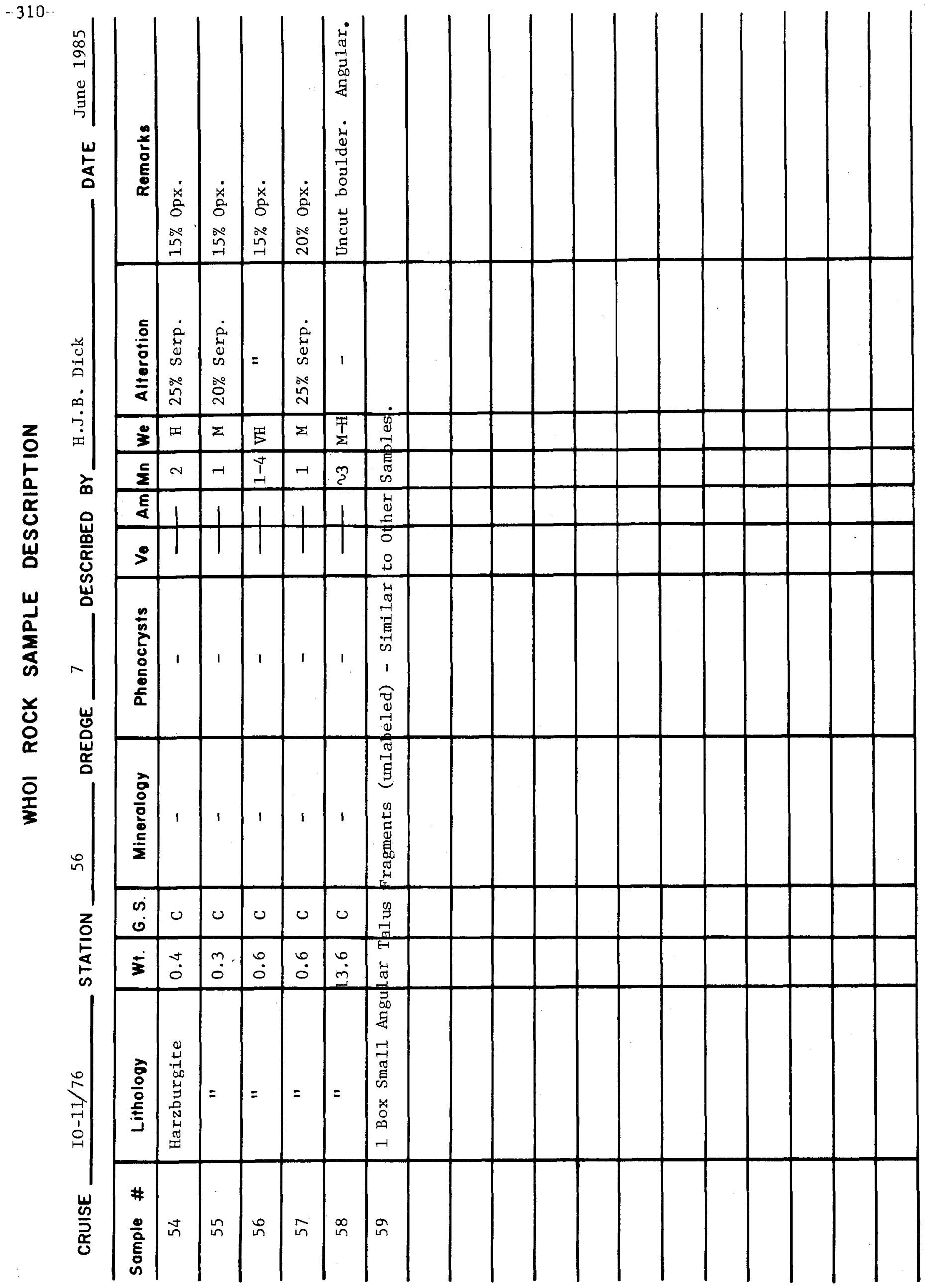




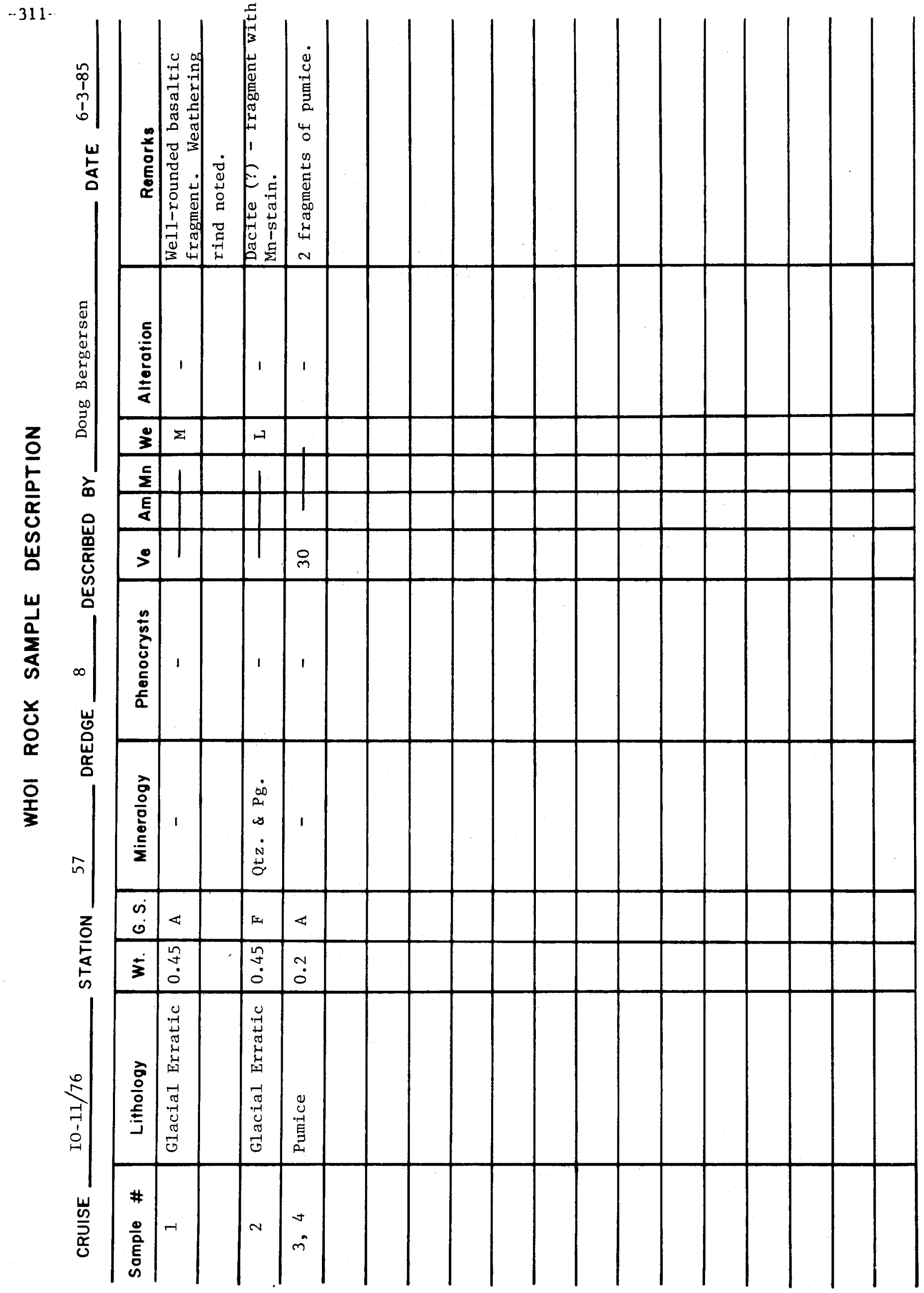



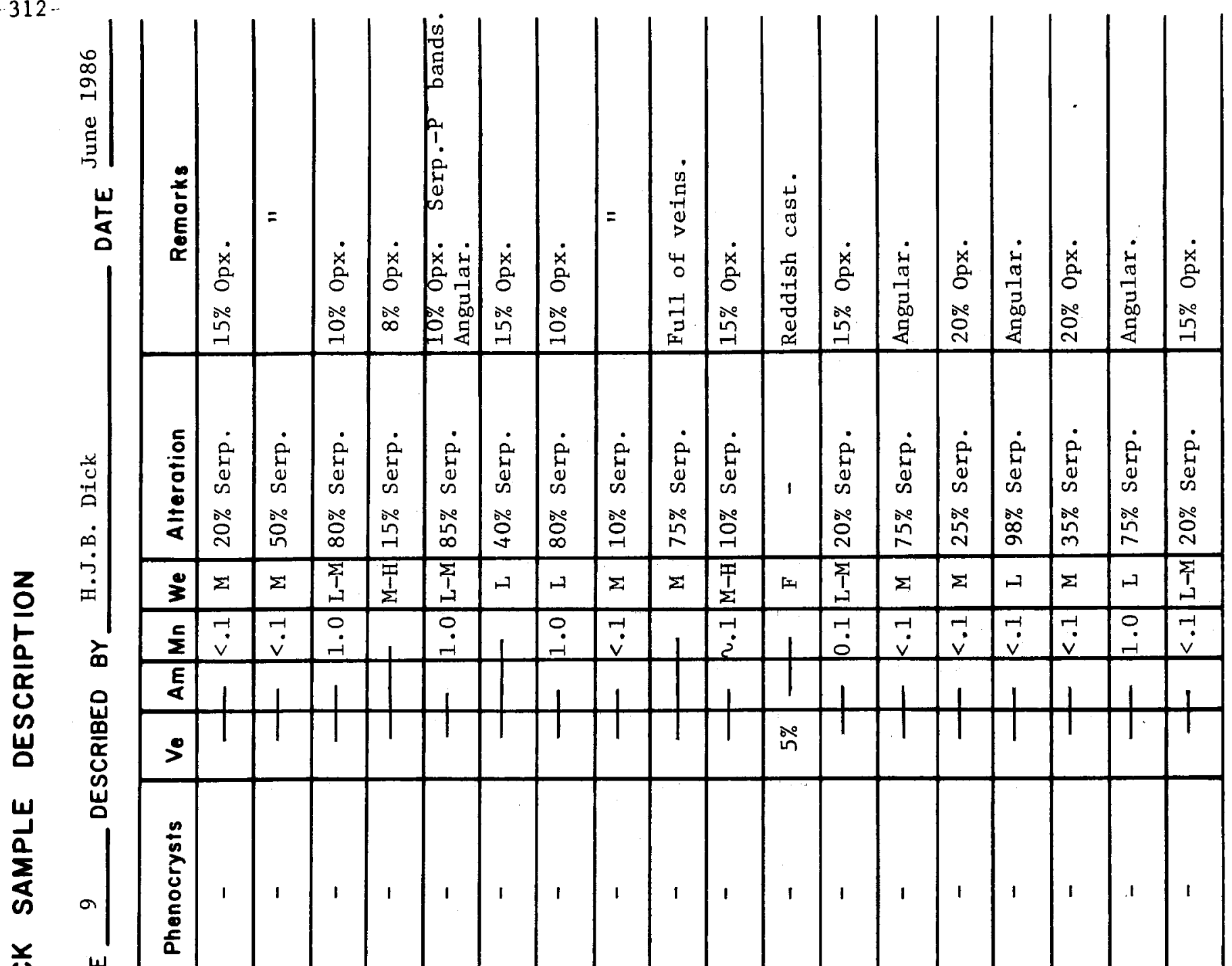

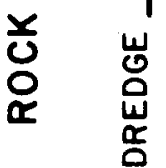

운

in

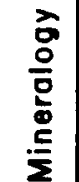

咅

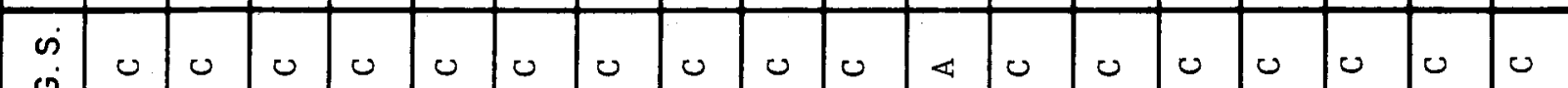

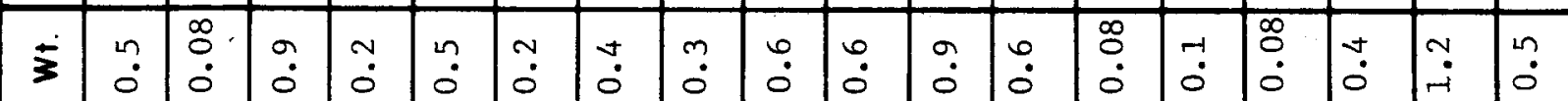

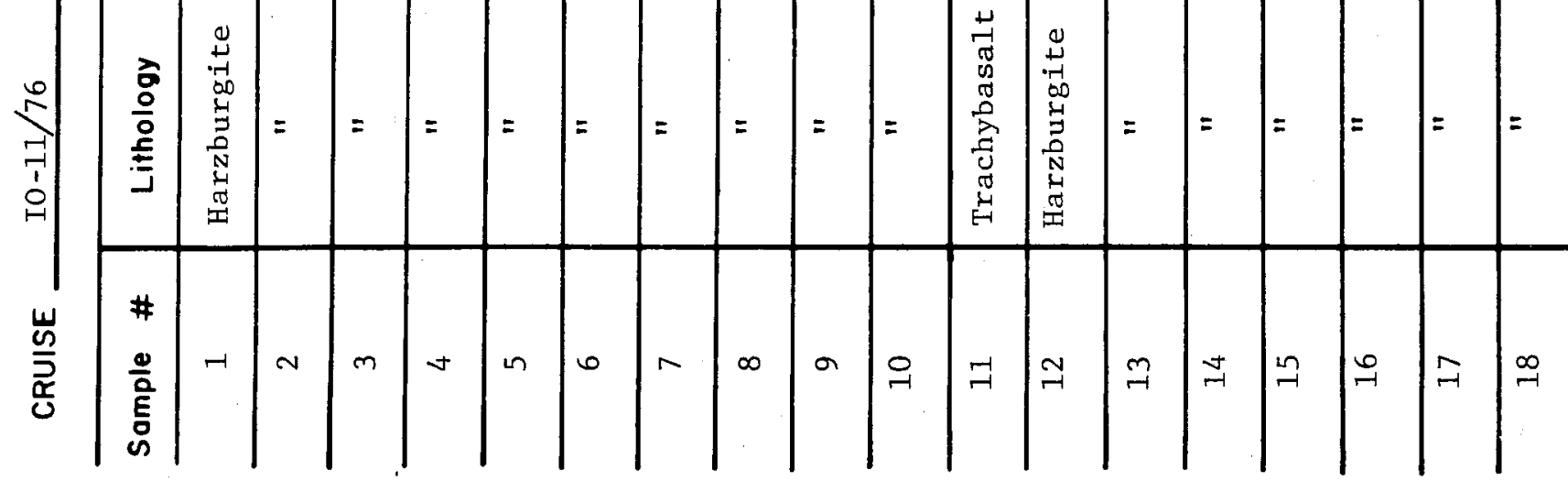



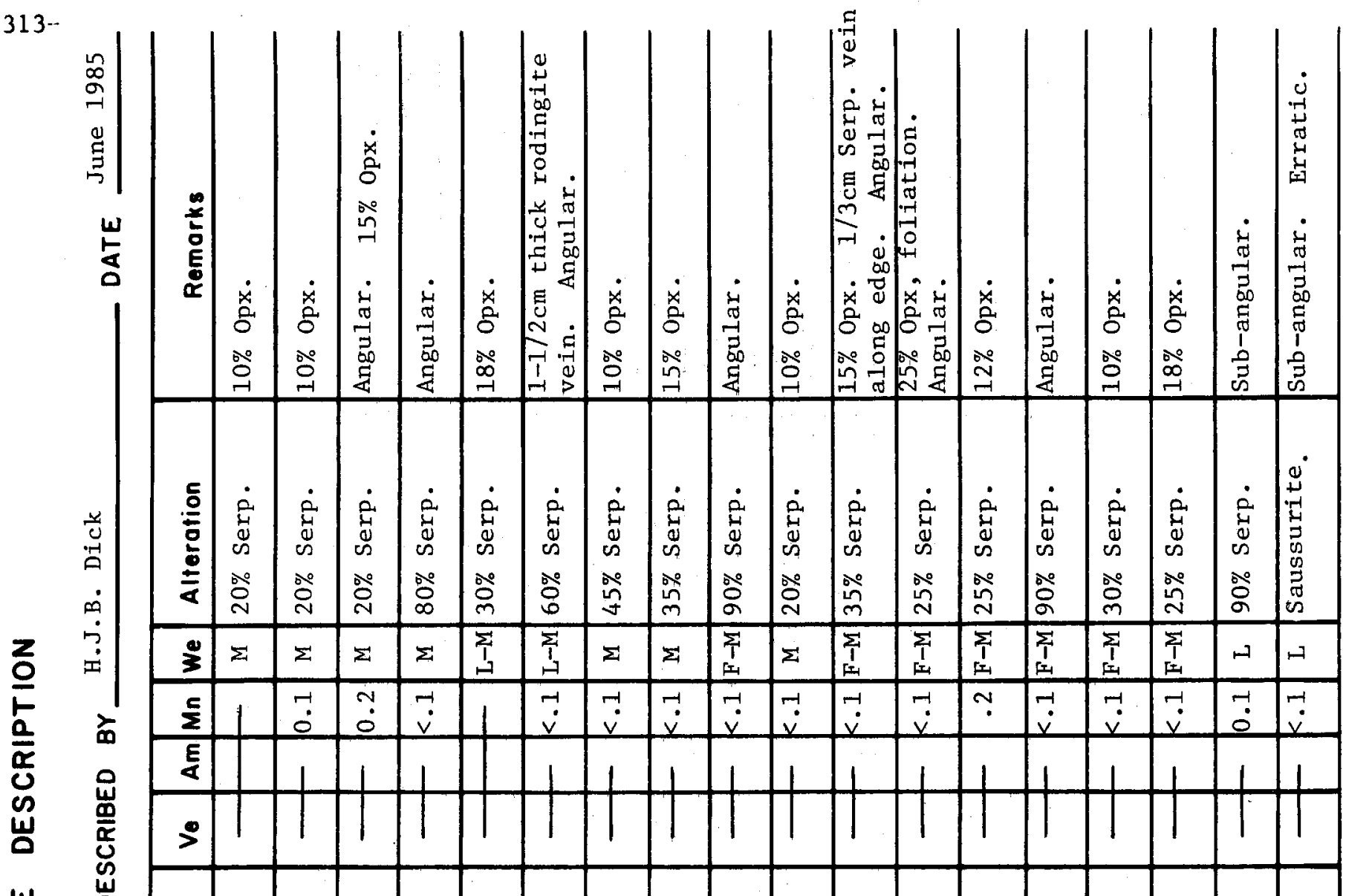

列

$\stackrel{\infty}{n}$

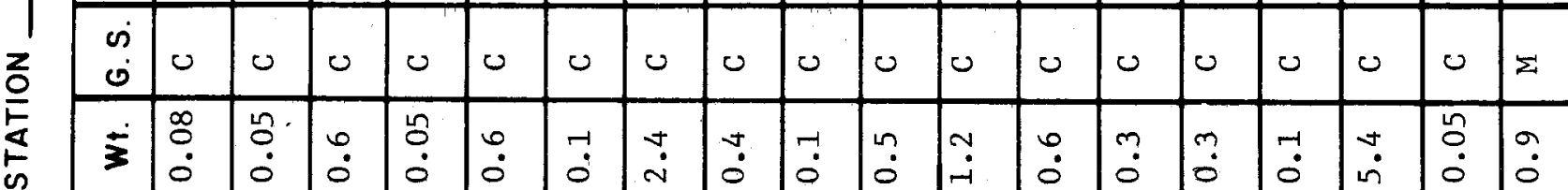

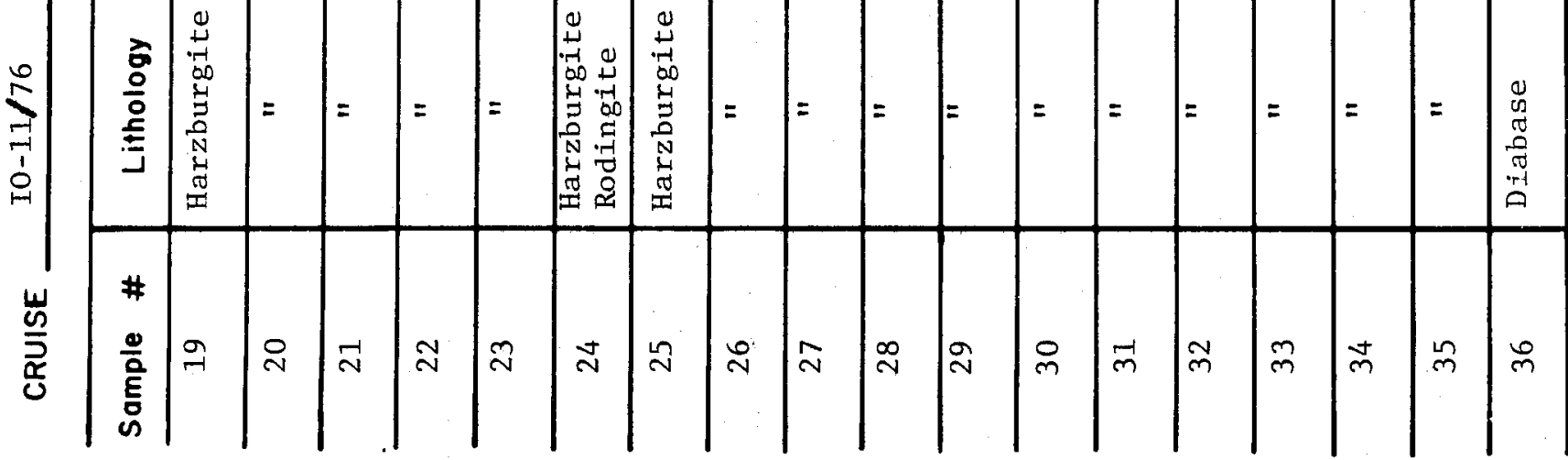




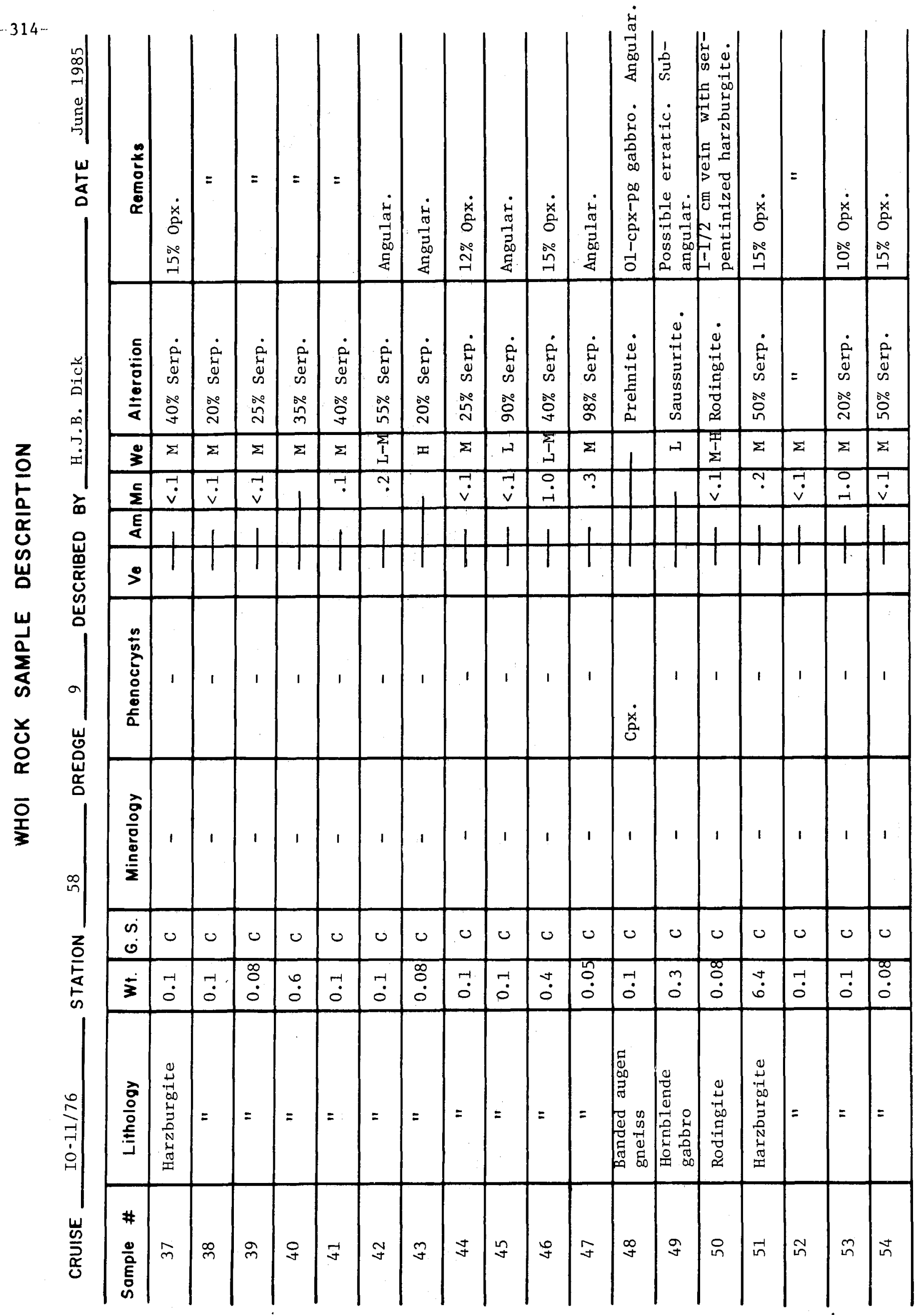




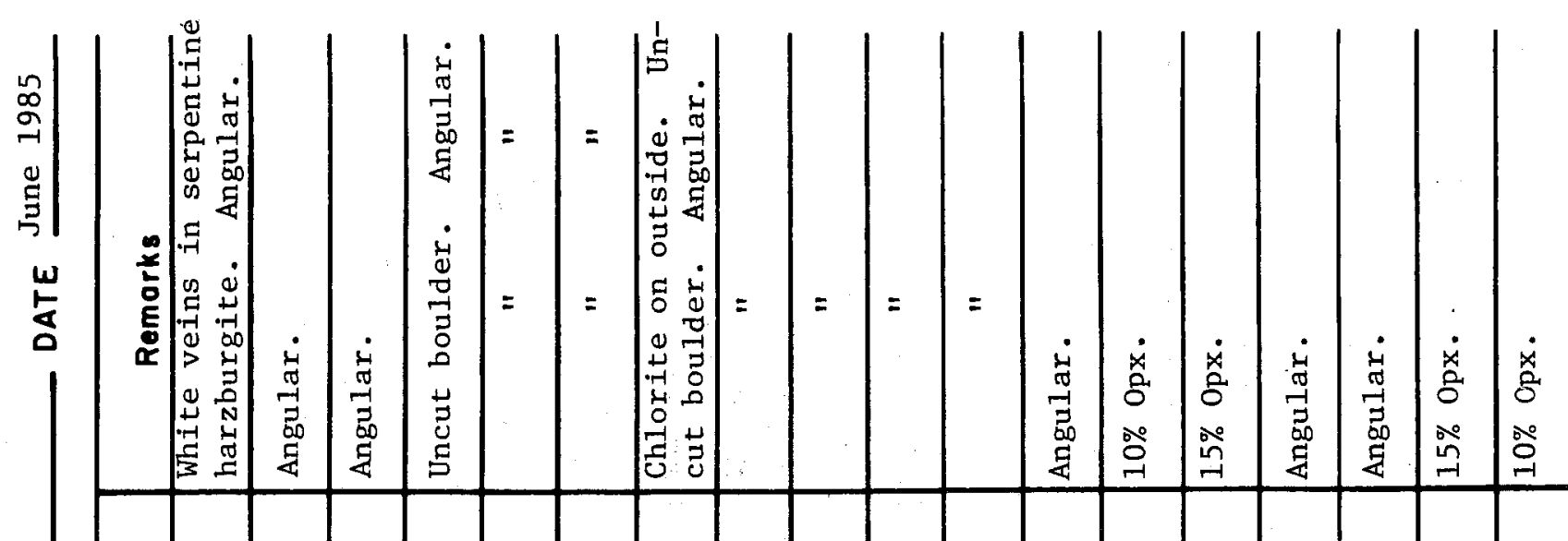

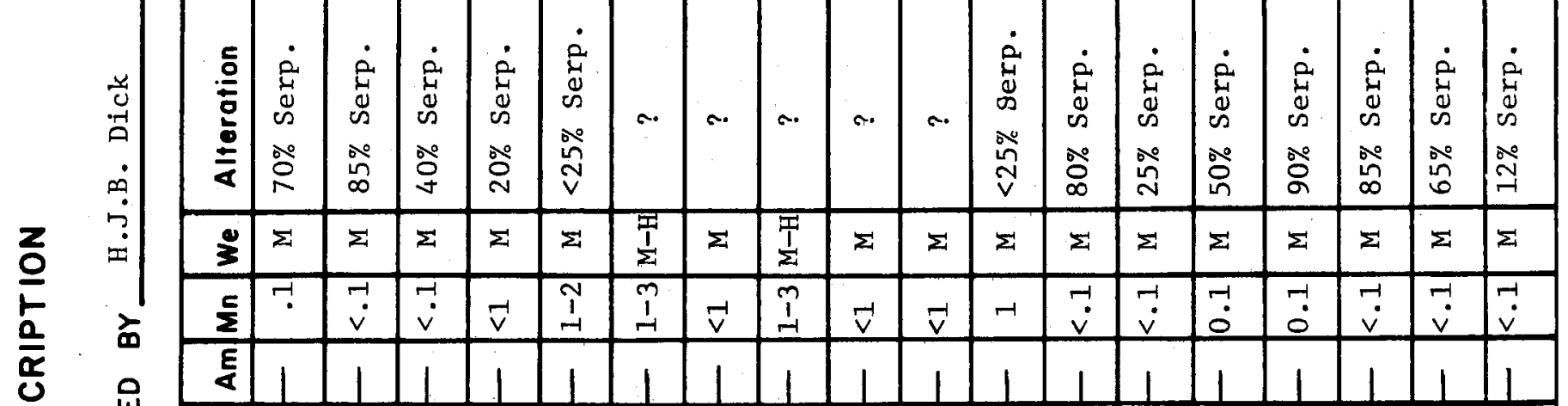
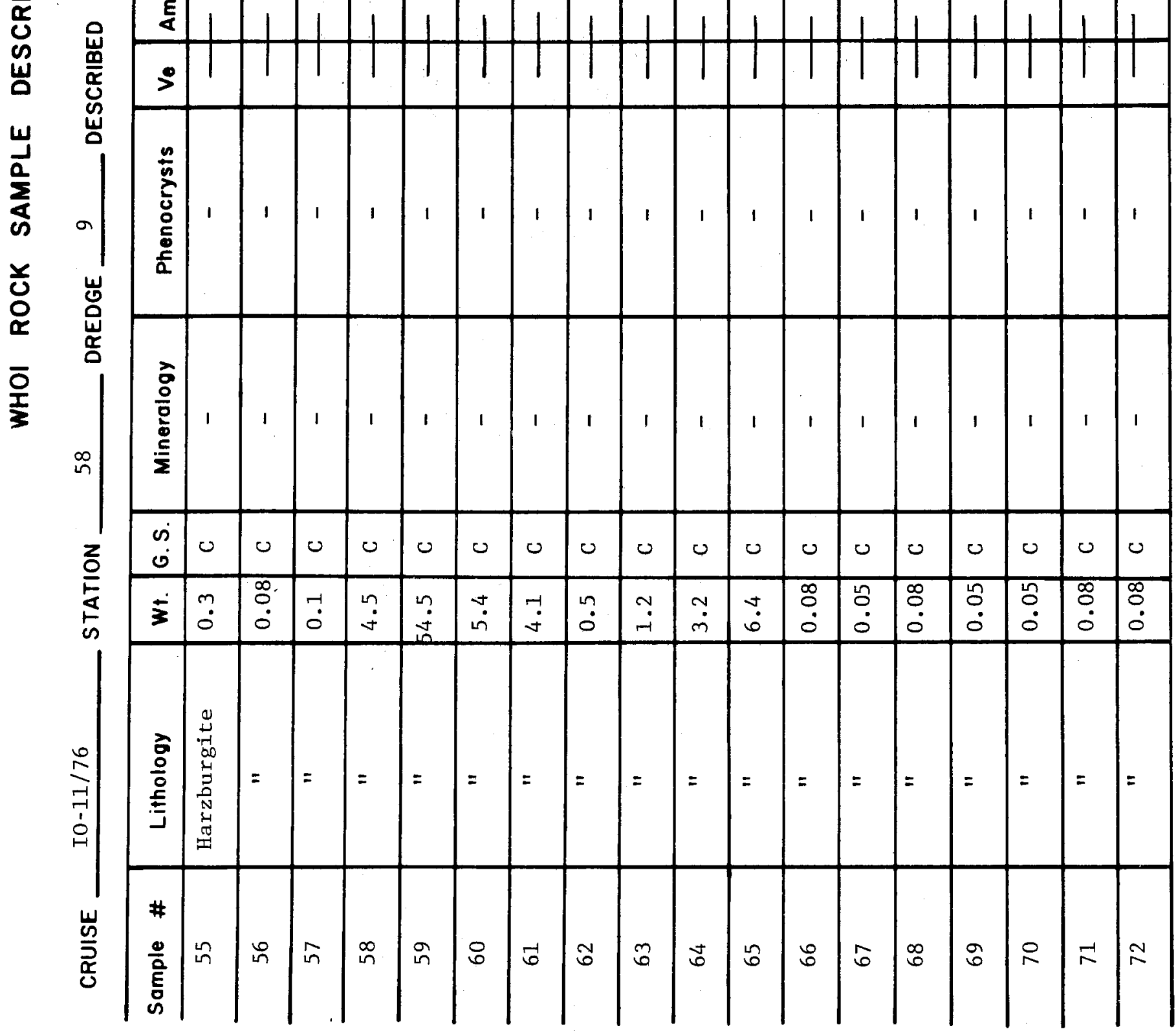


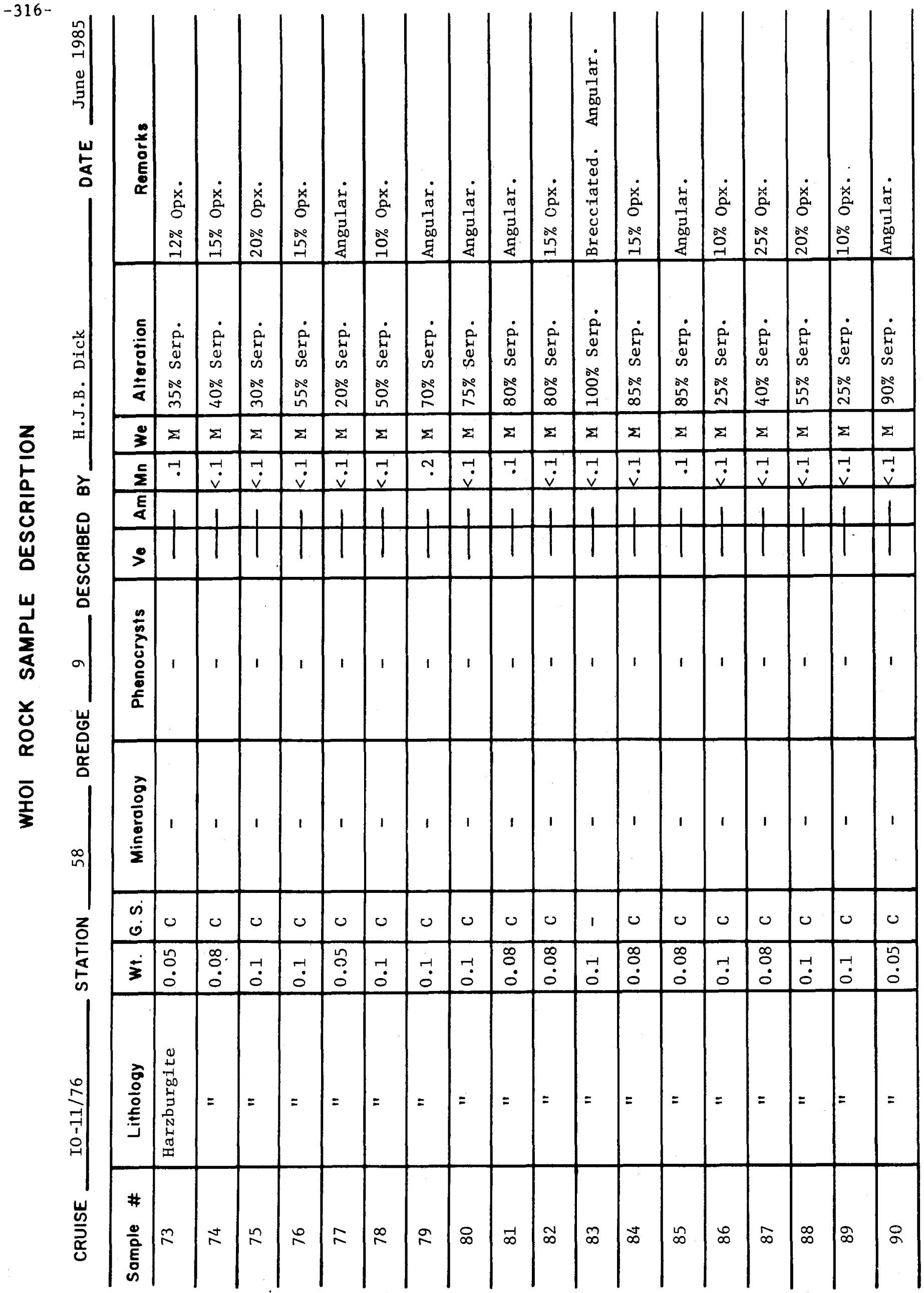




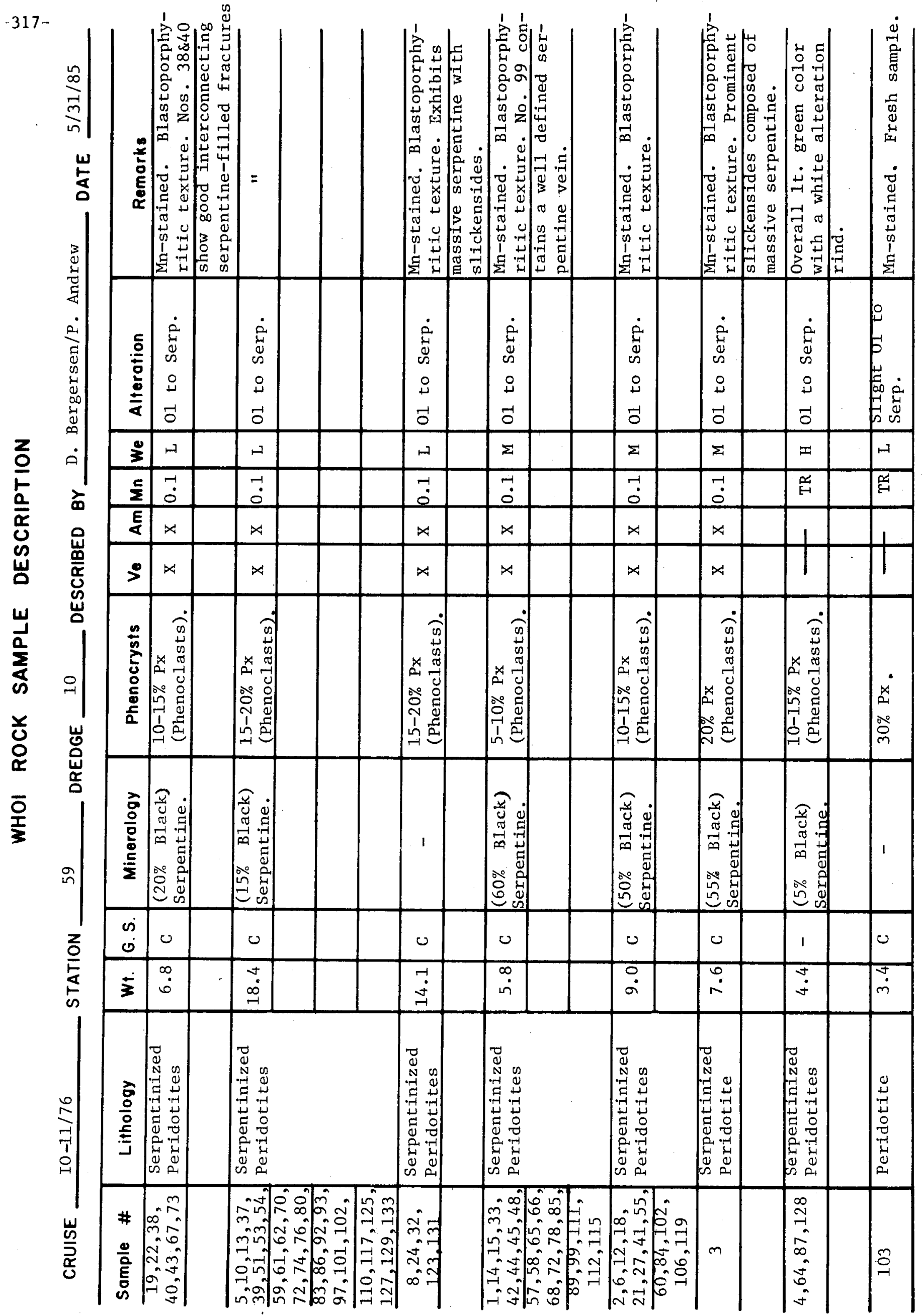




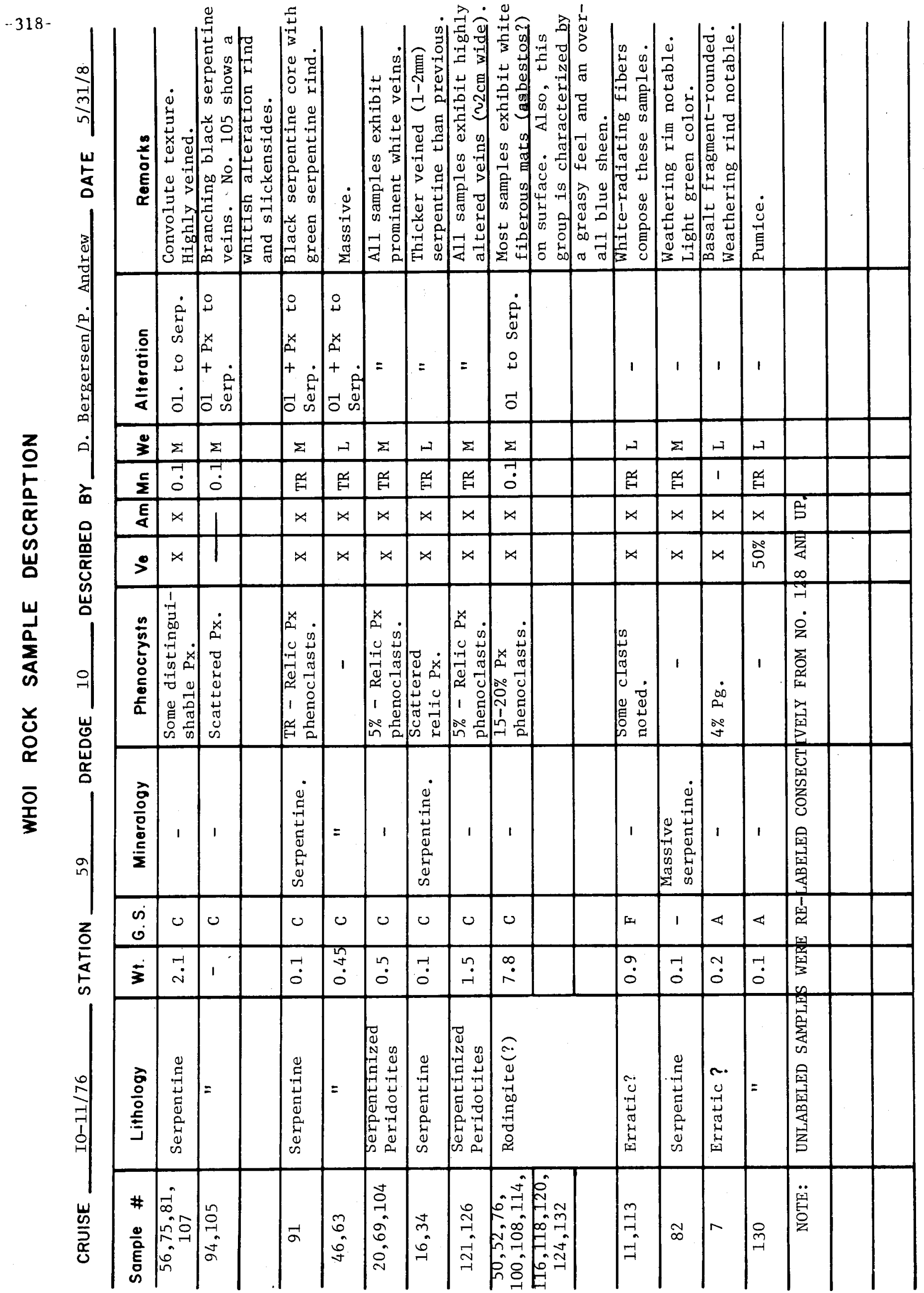




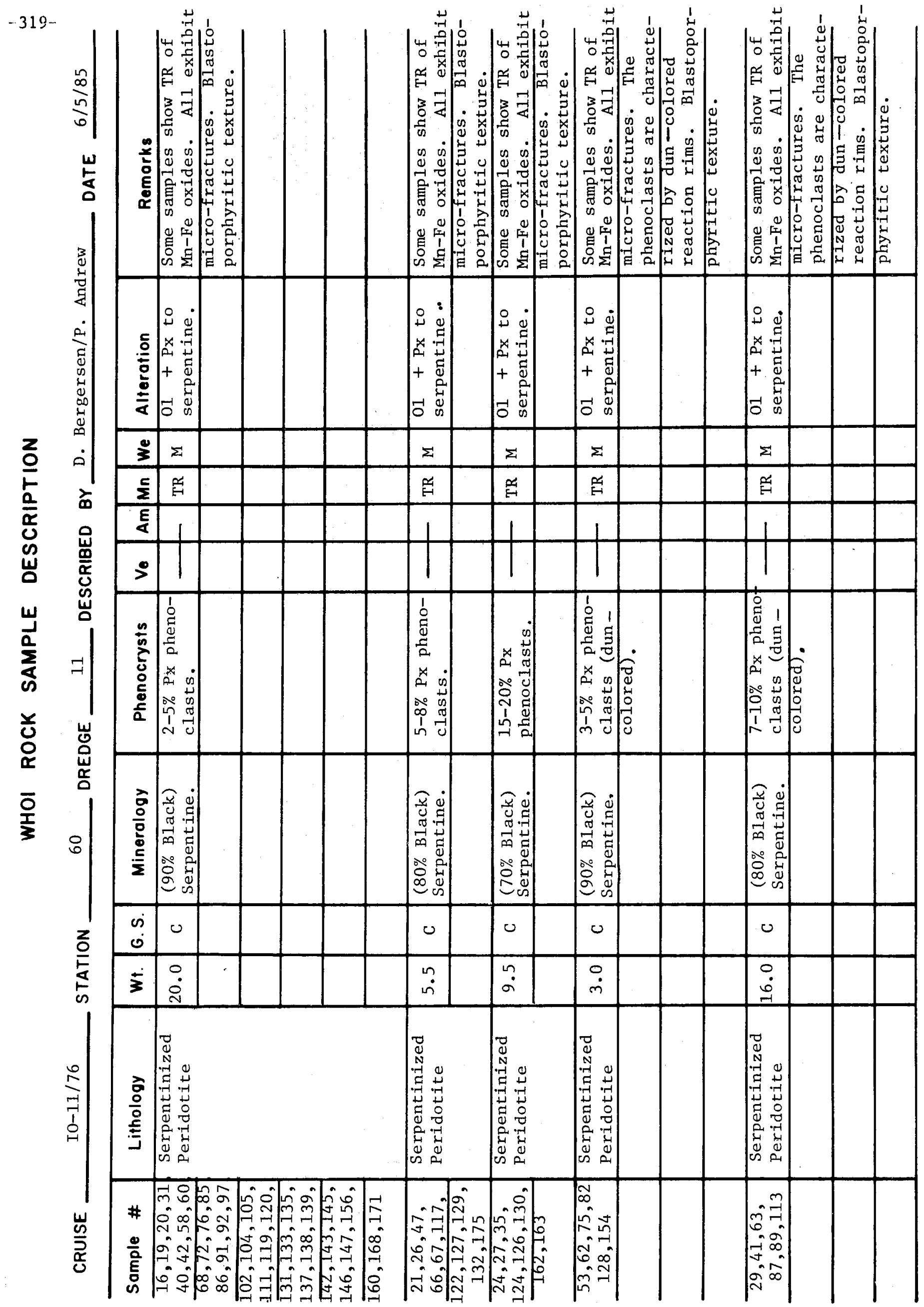




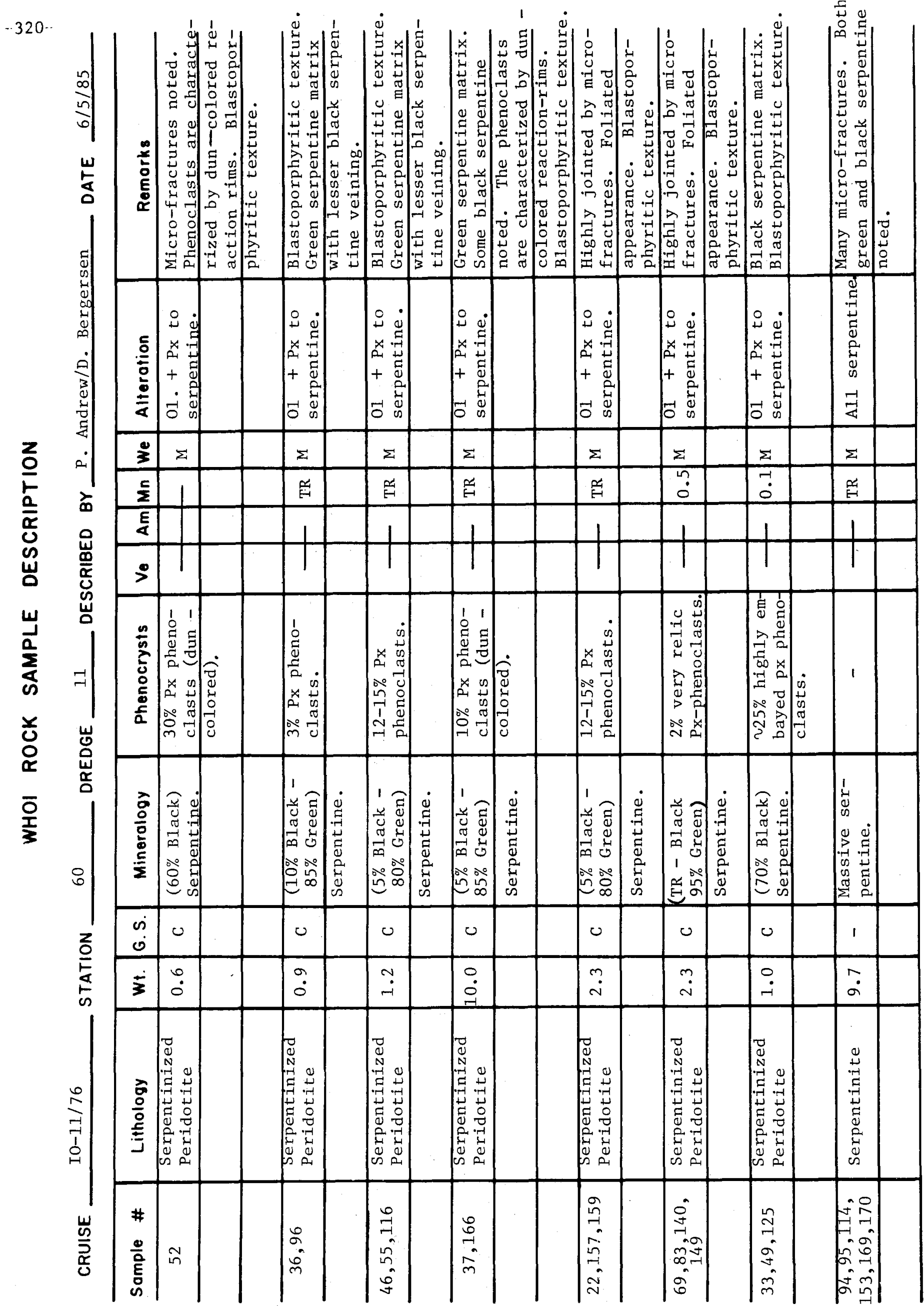




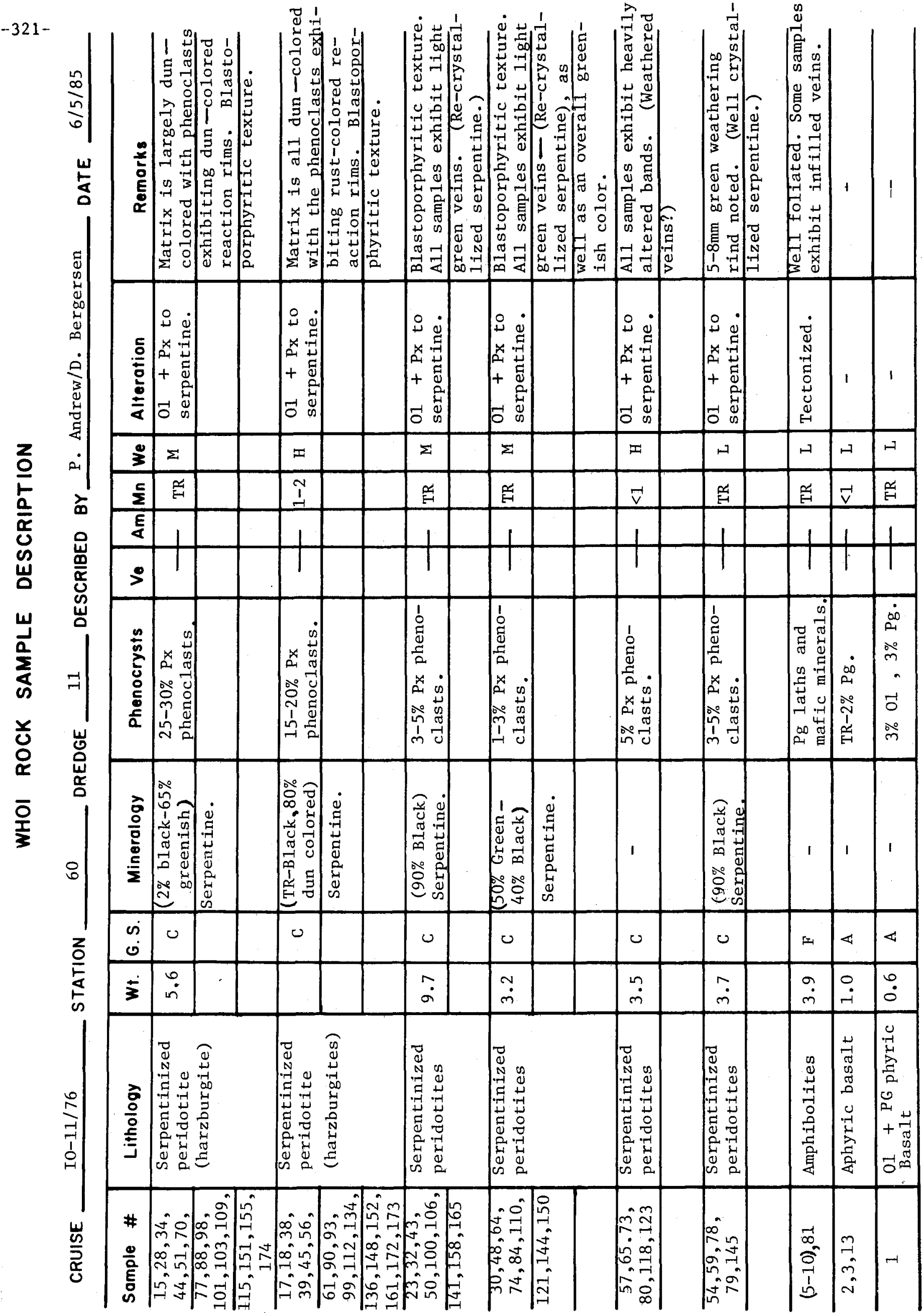




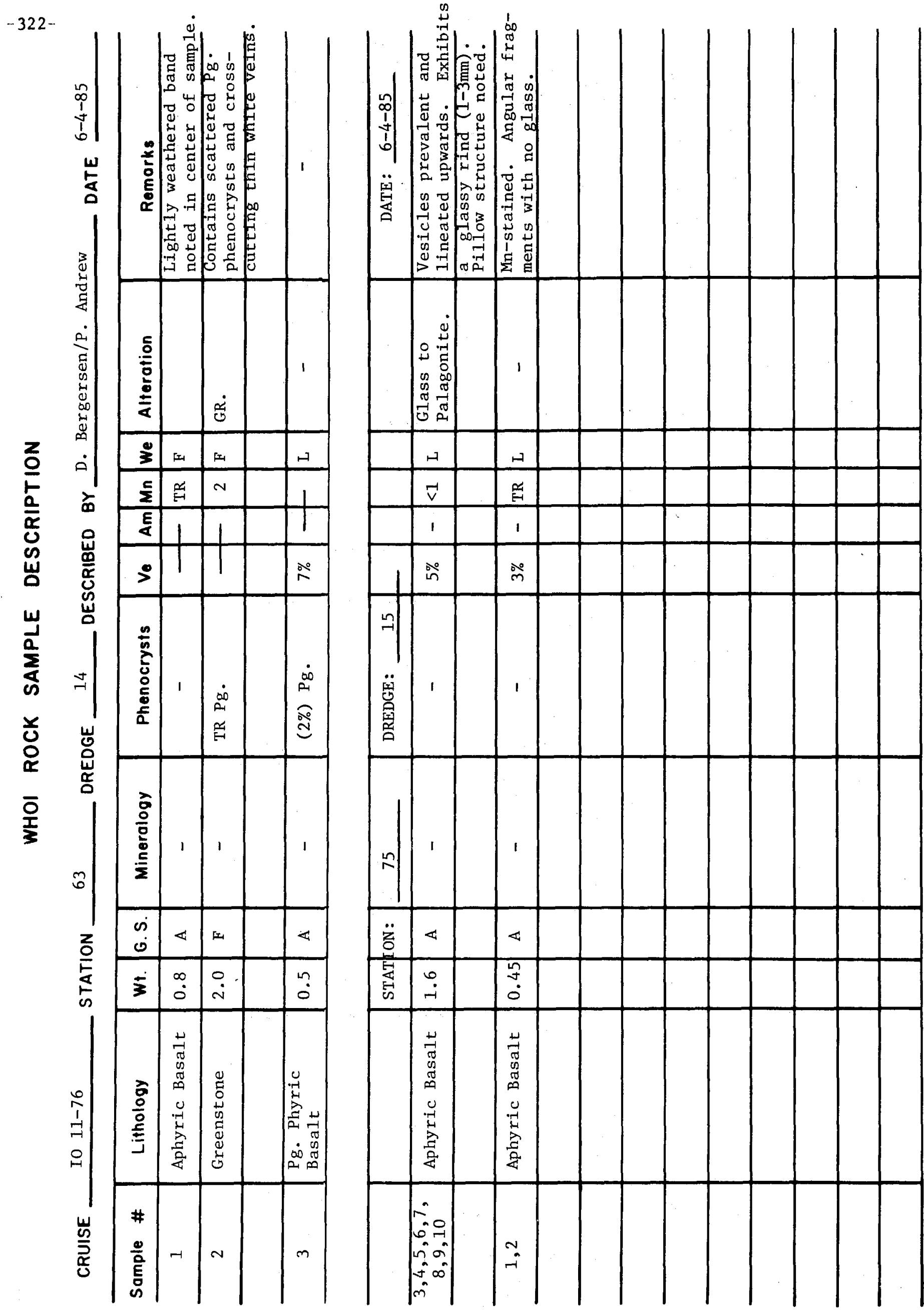




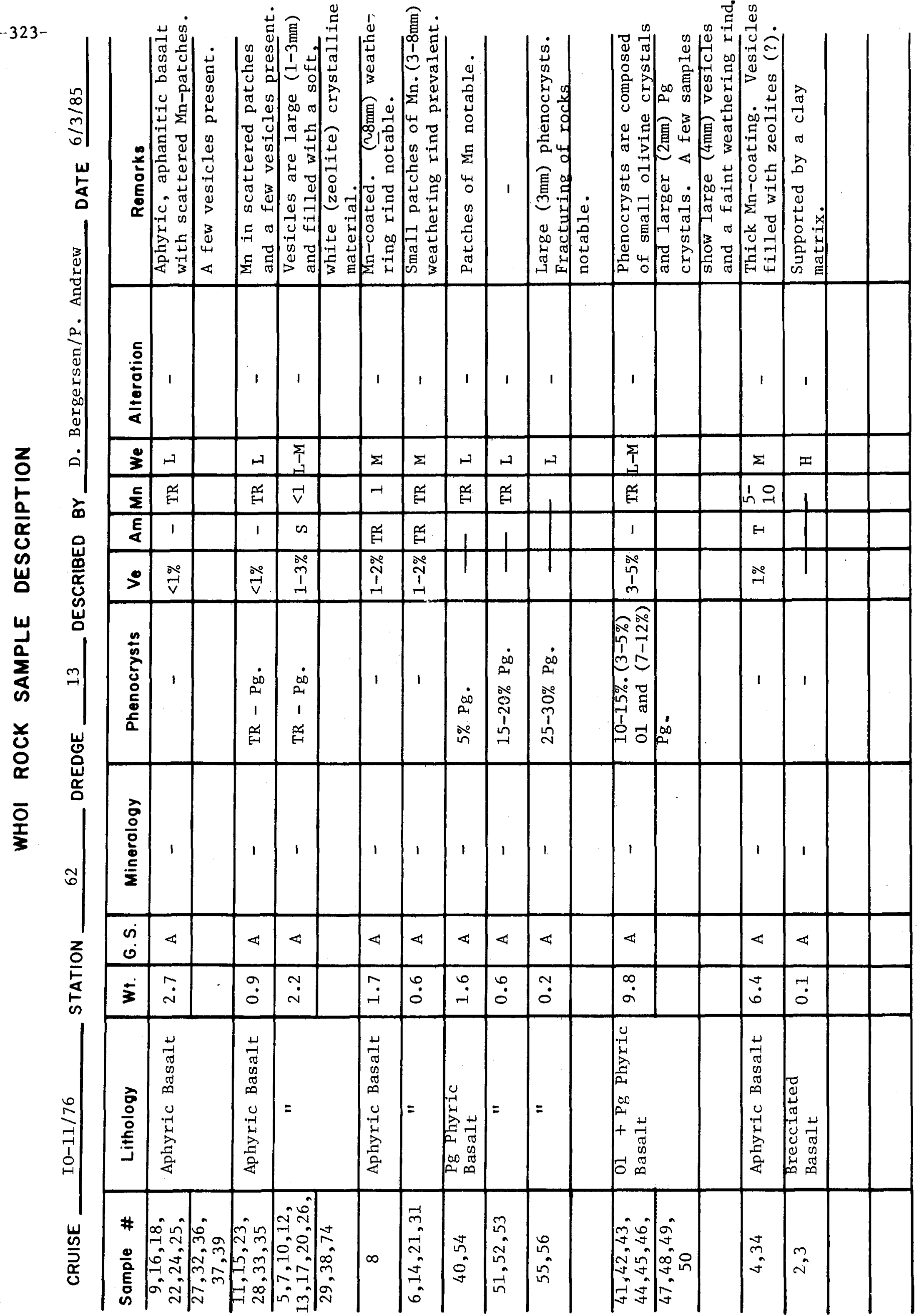




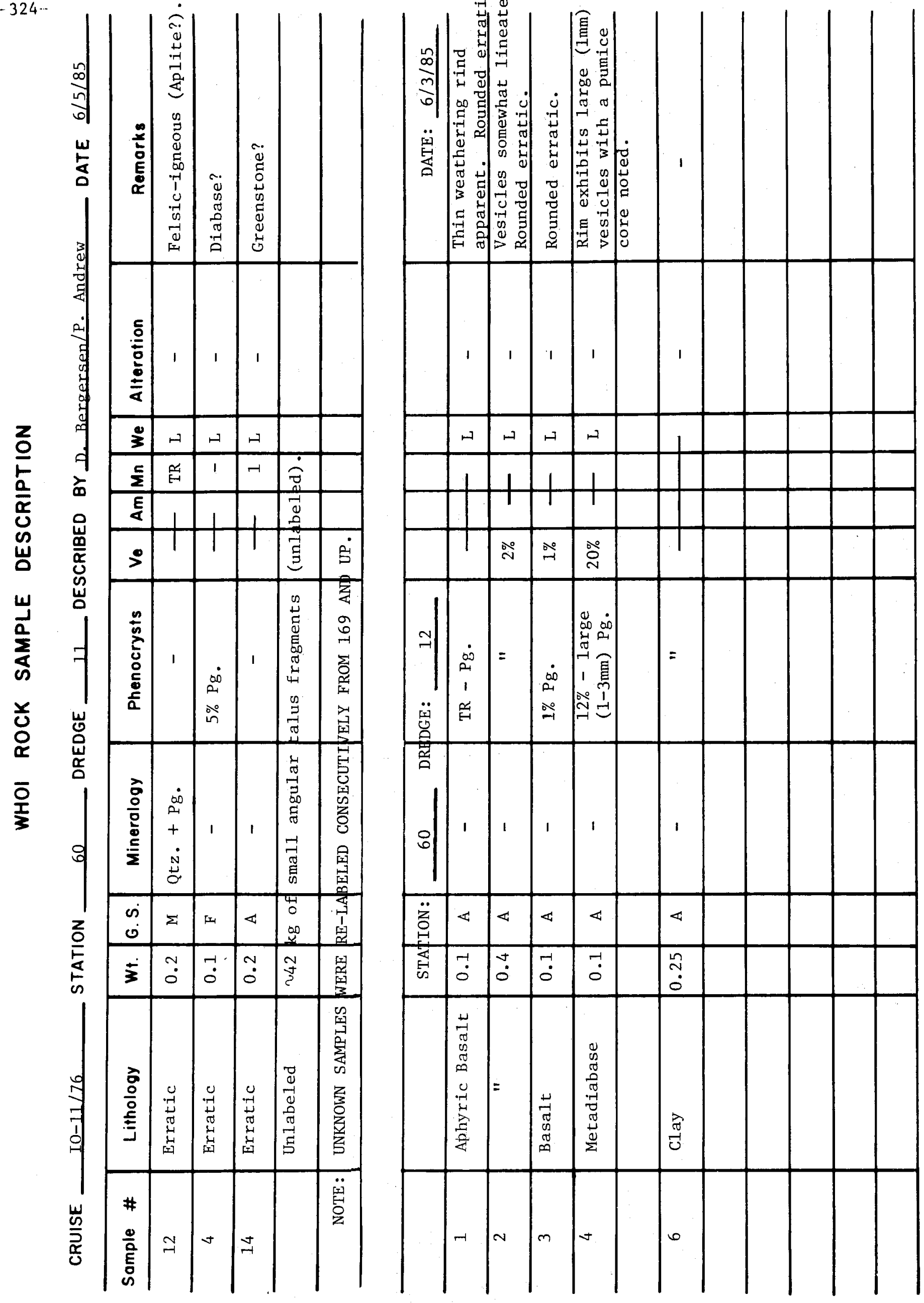




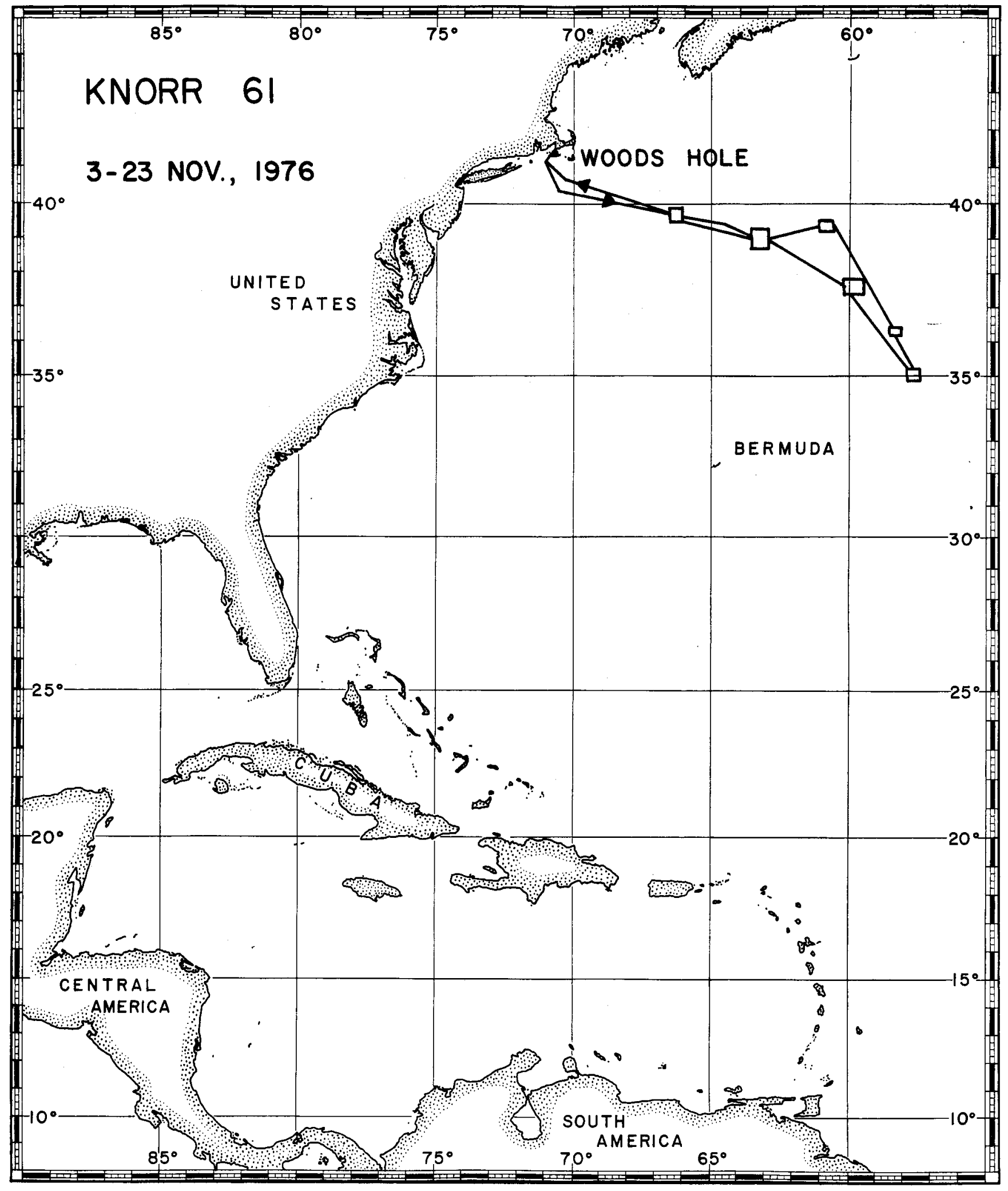




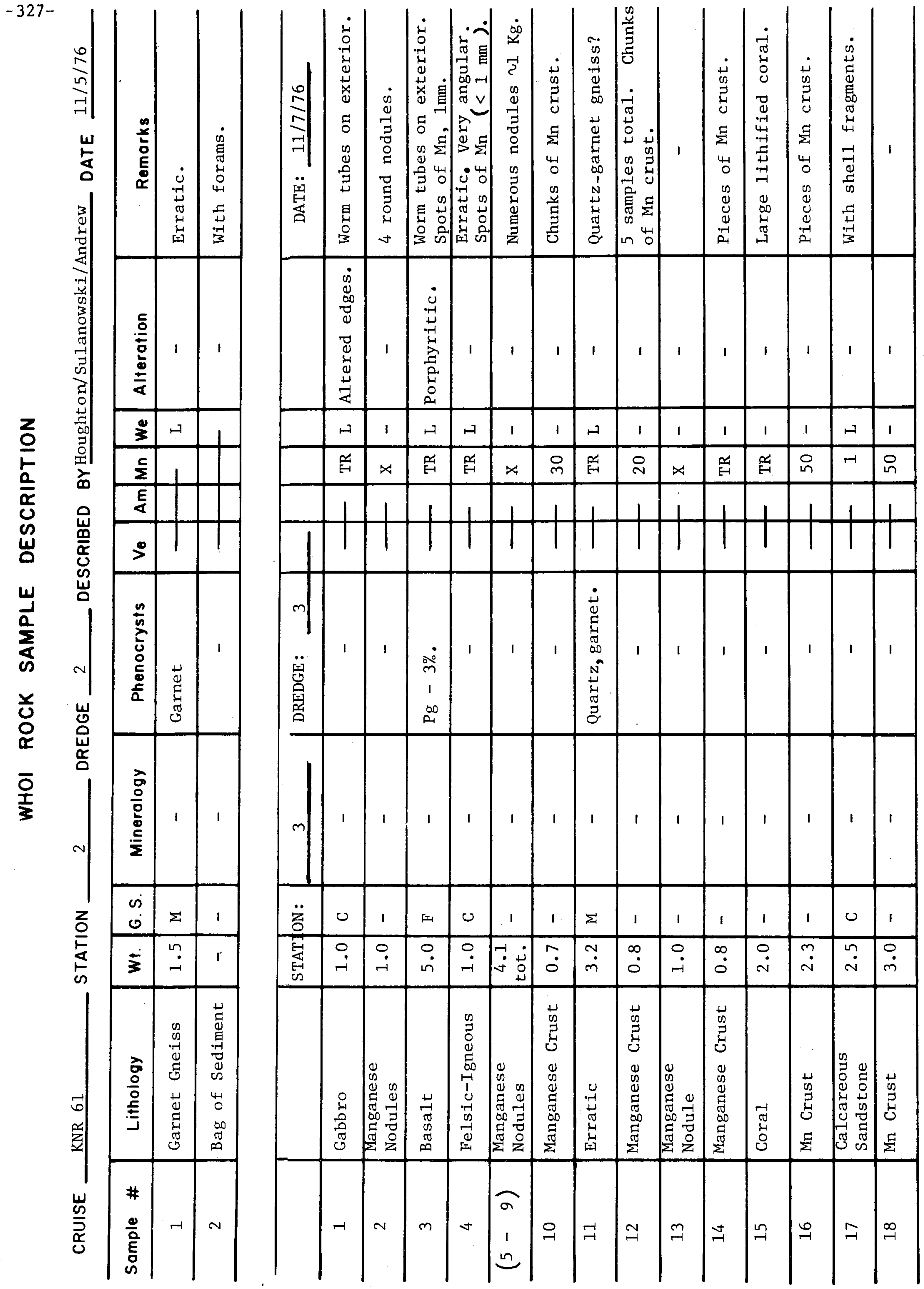



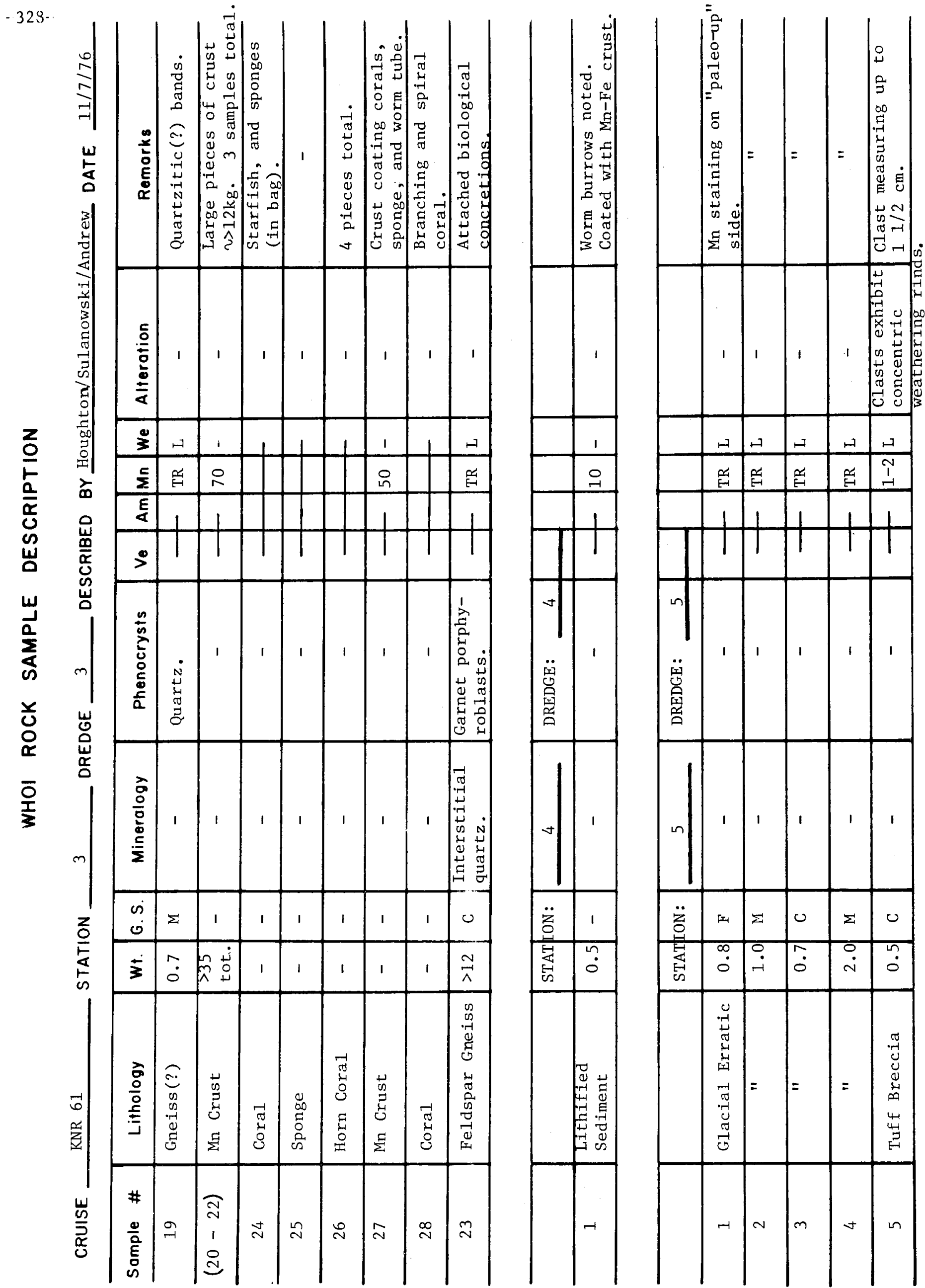


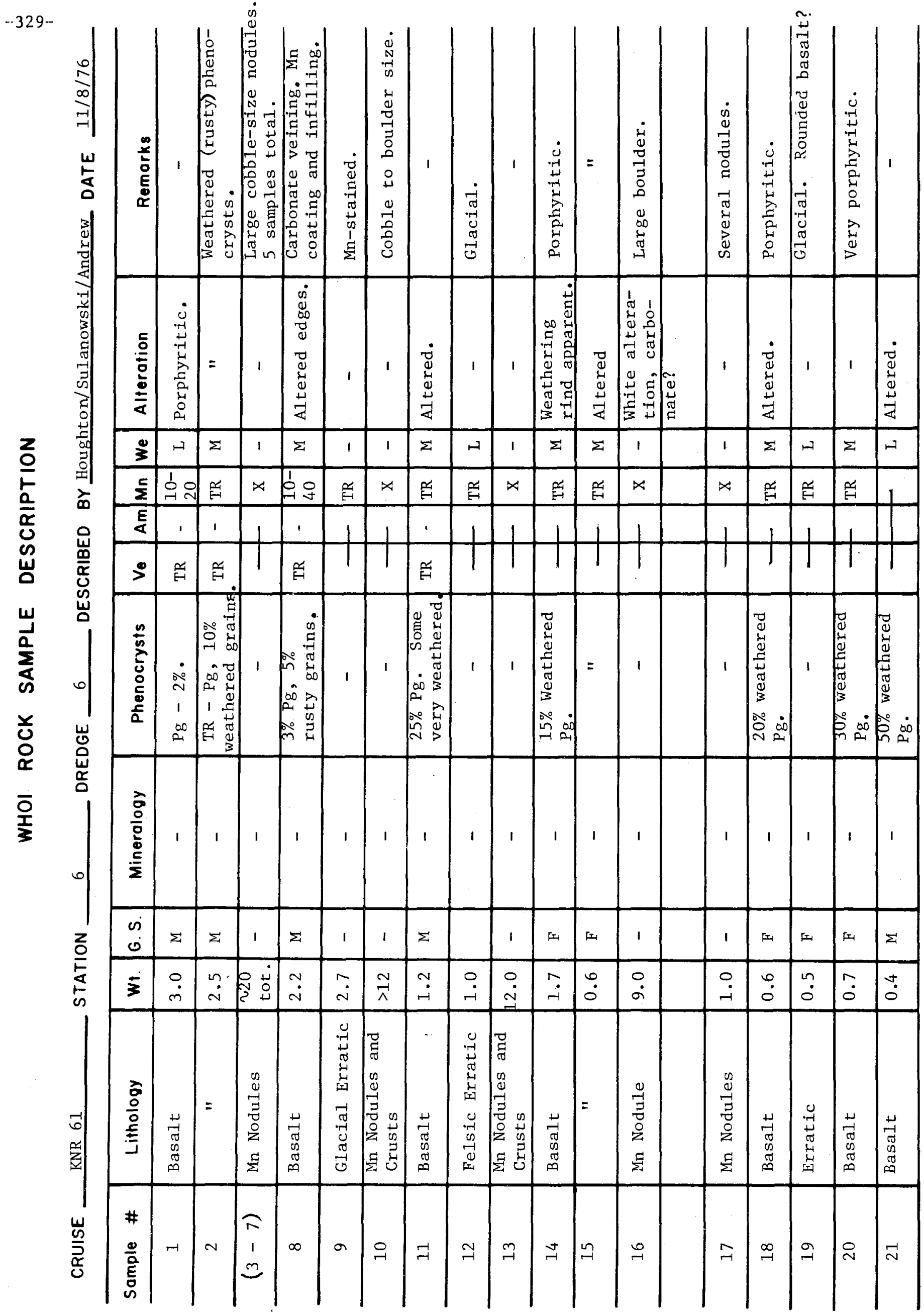




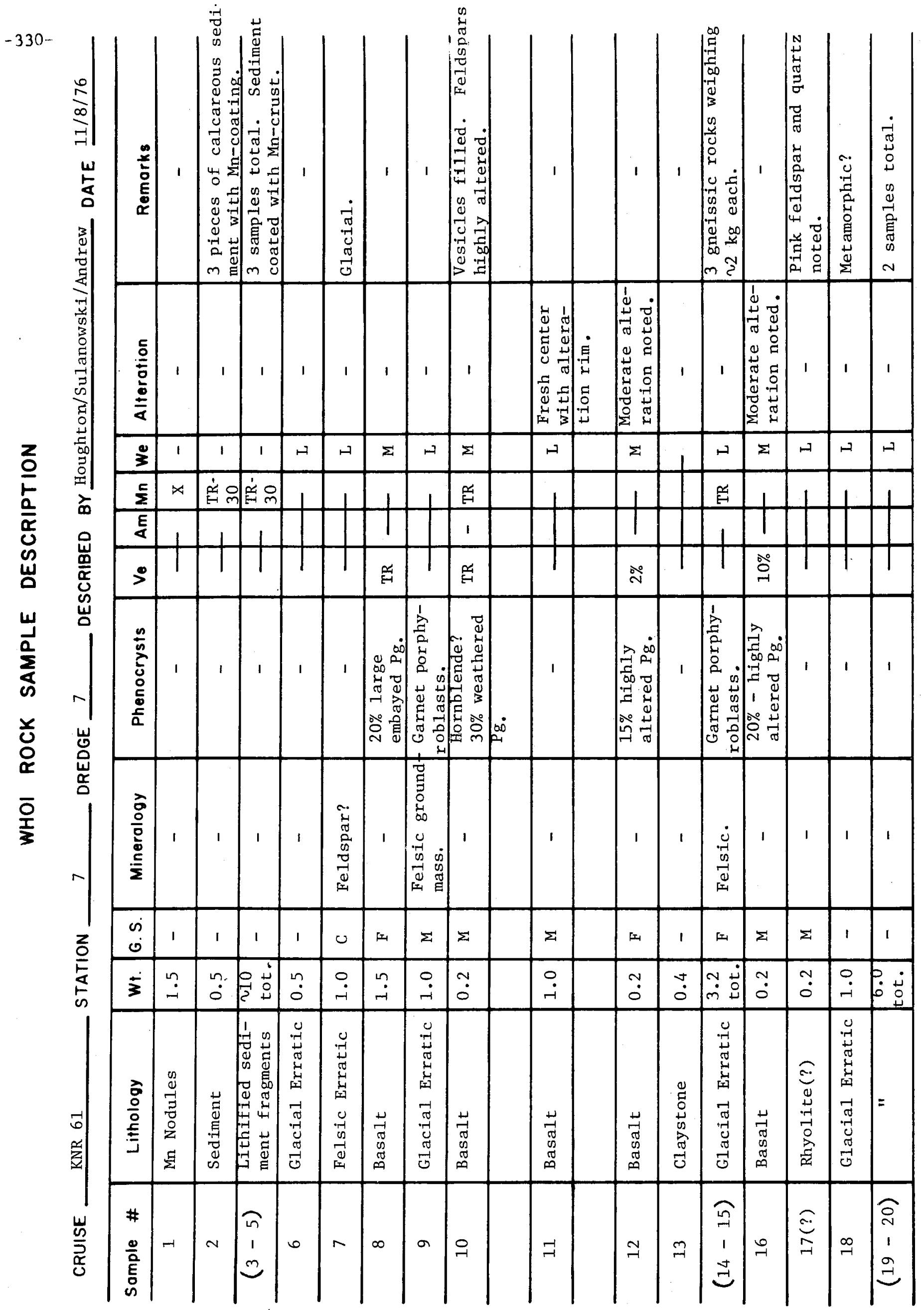




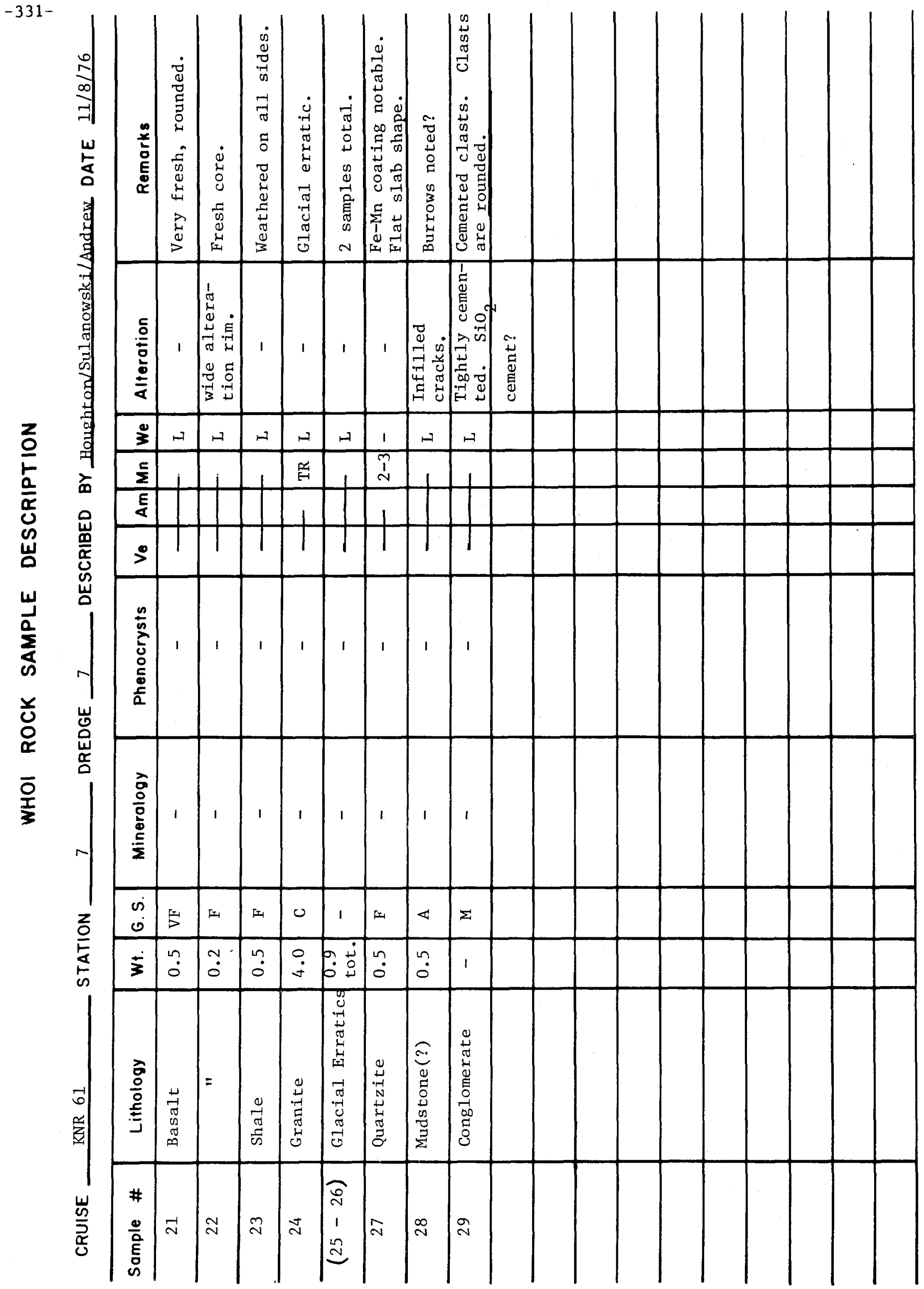




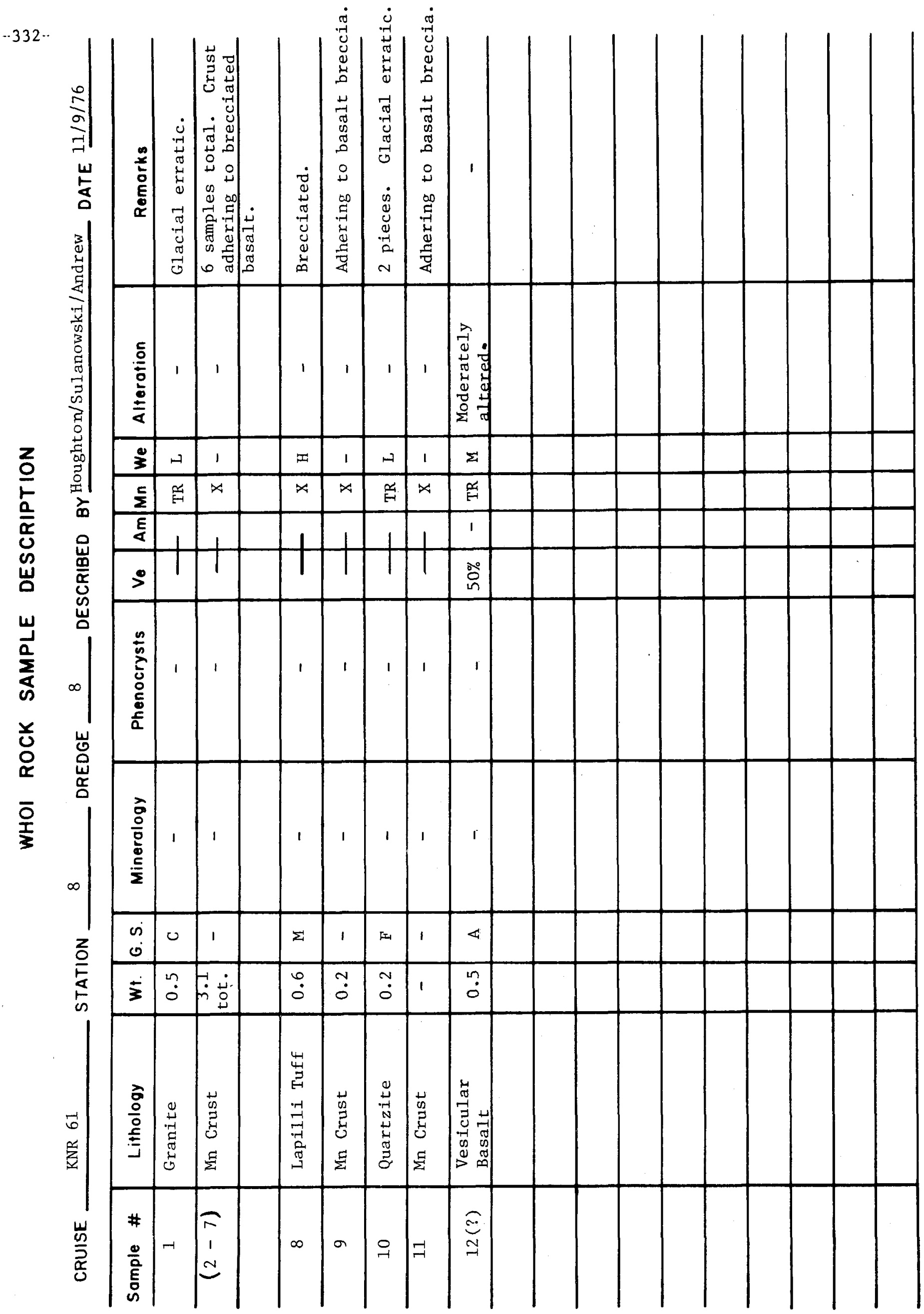




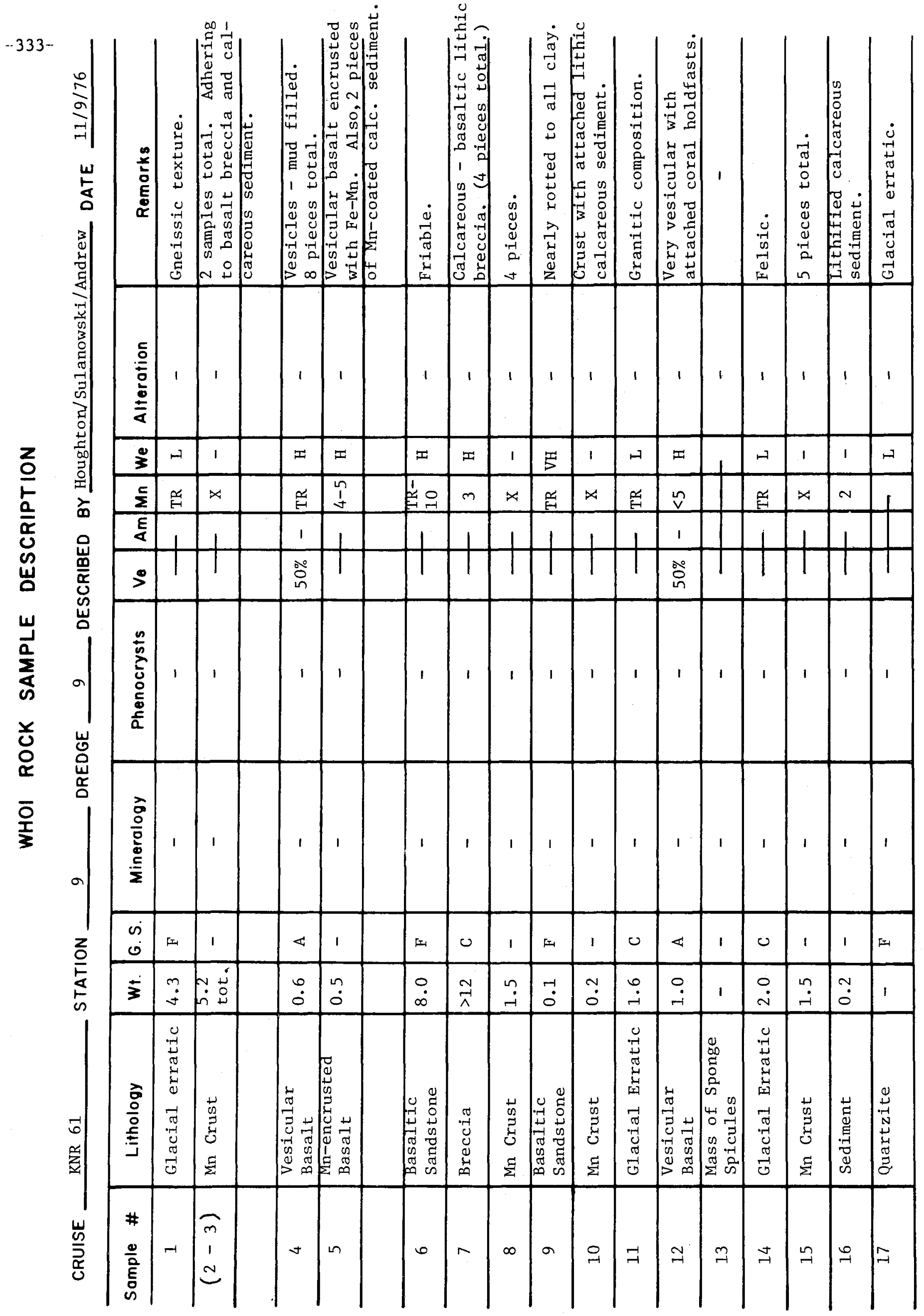




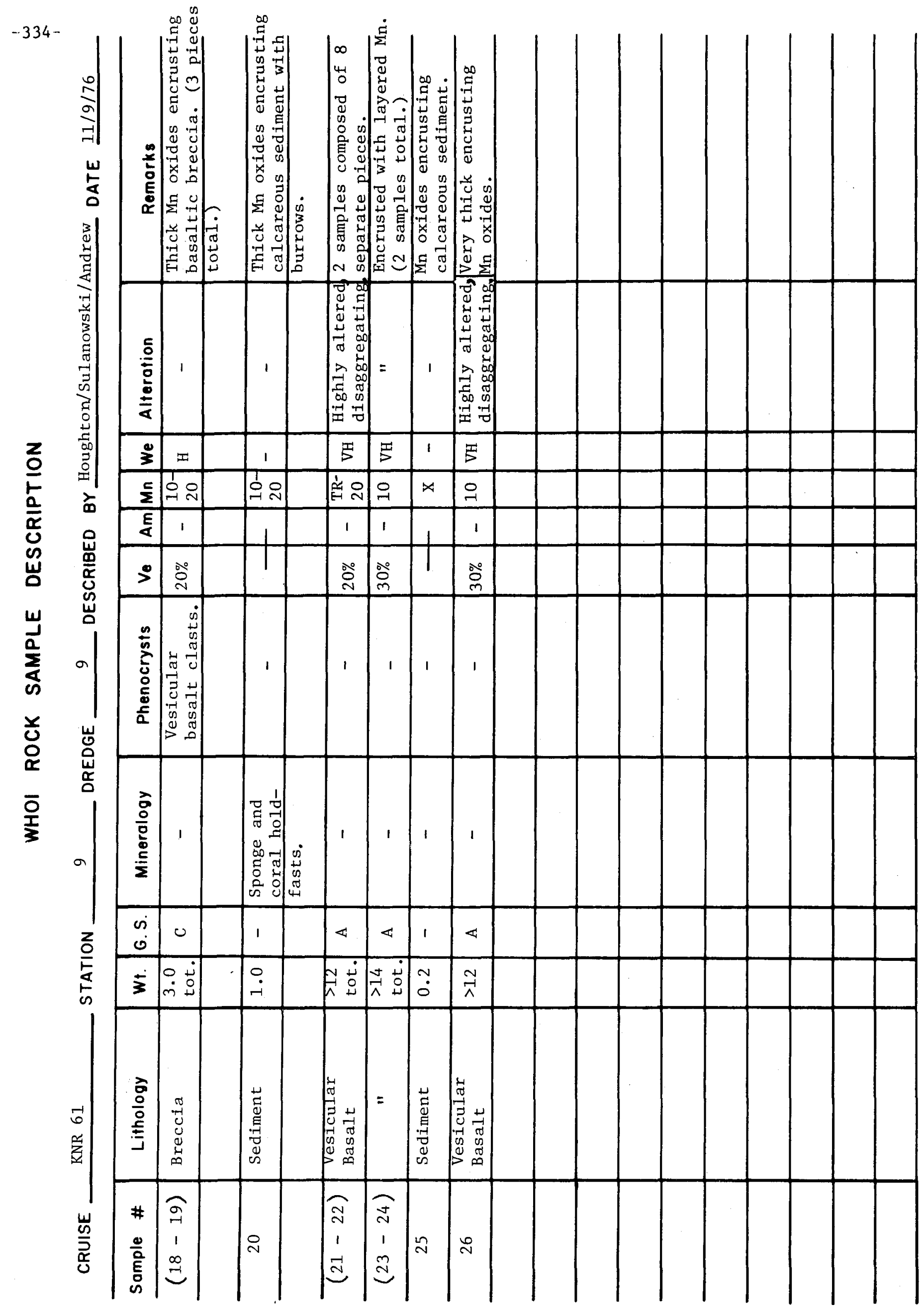




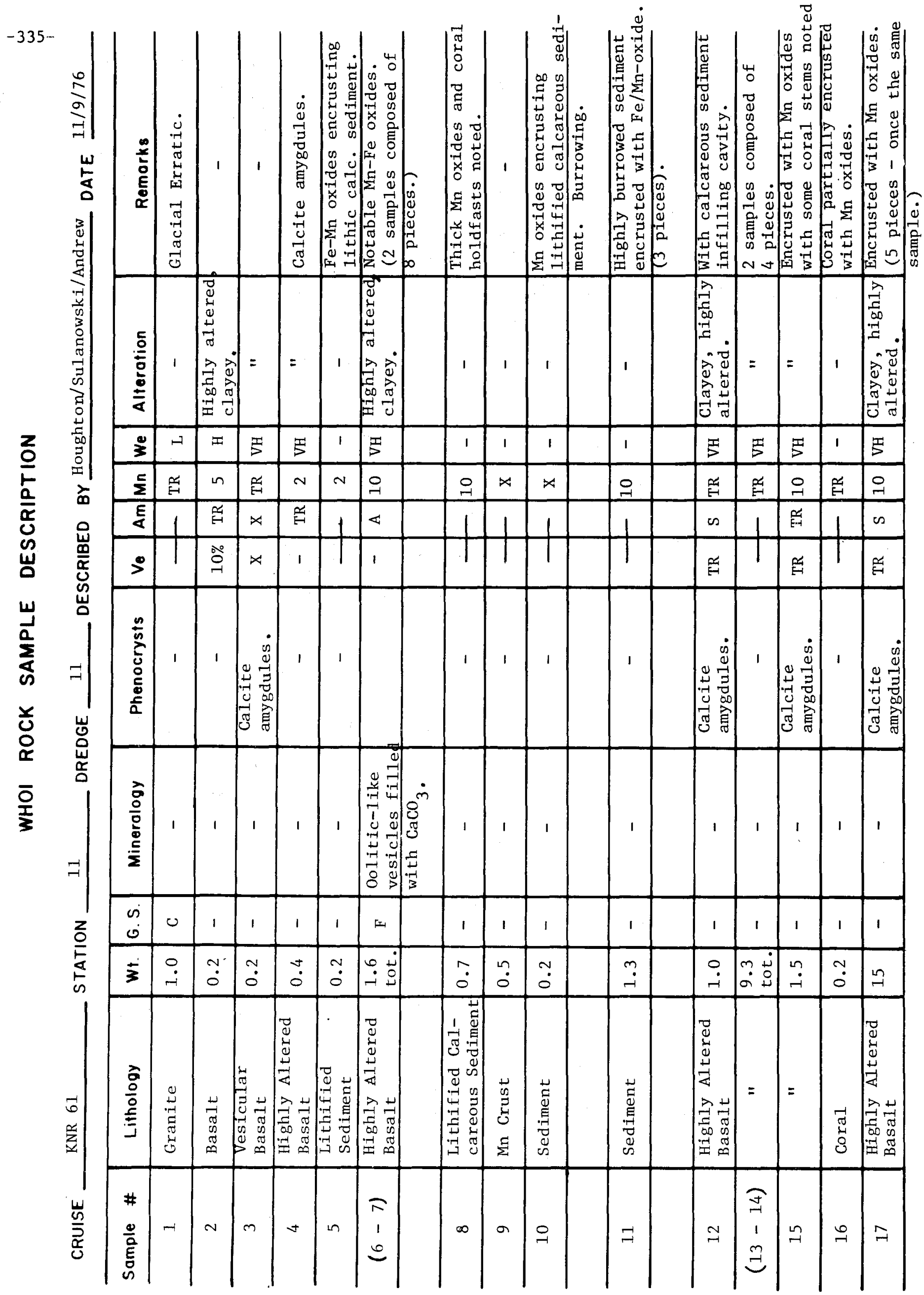




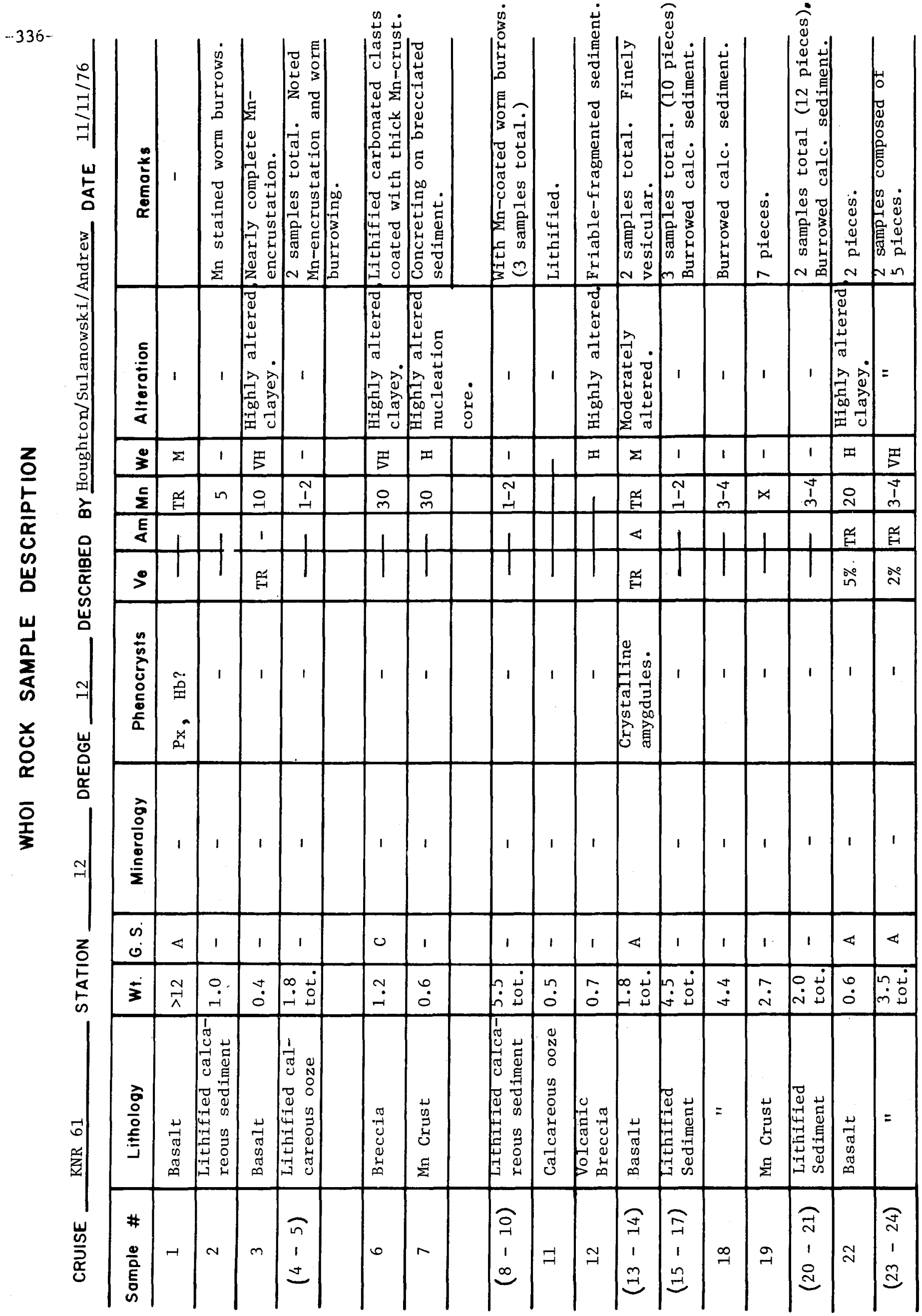




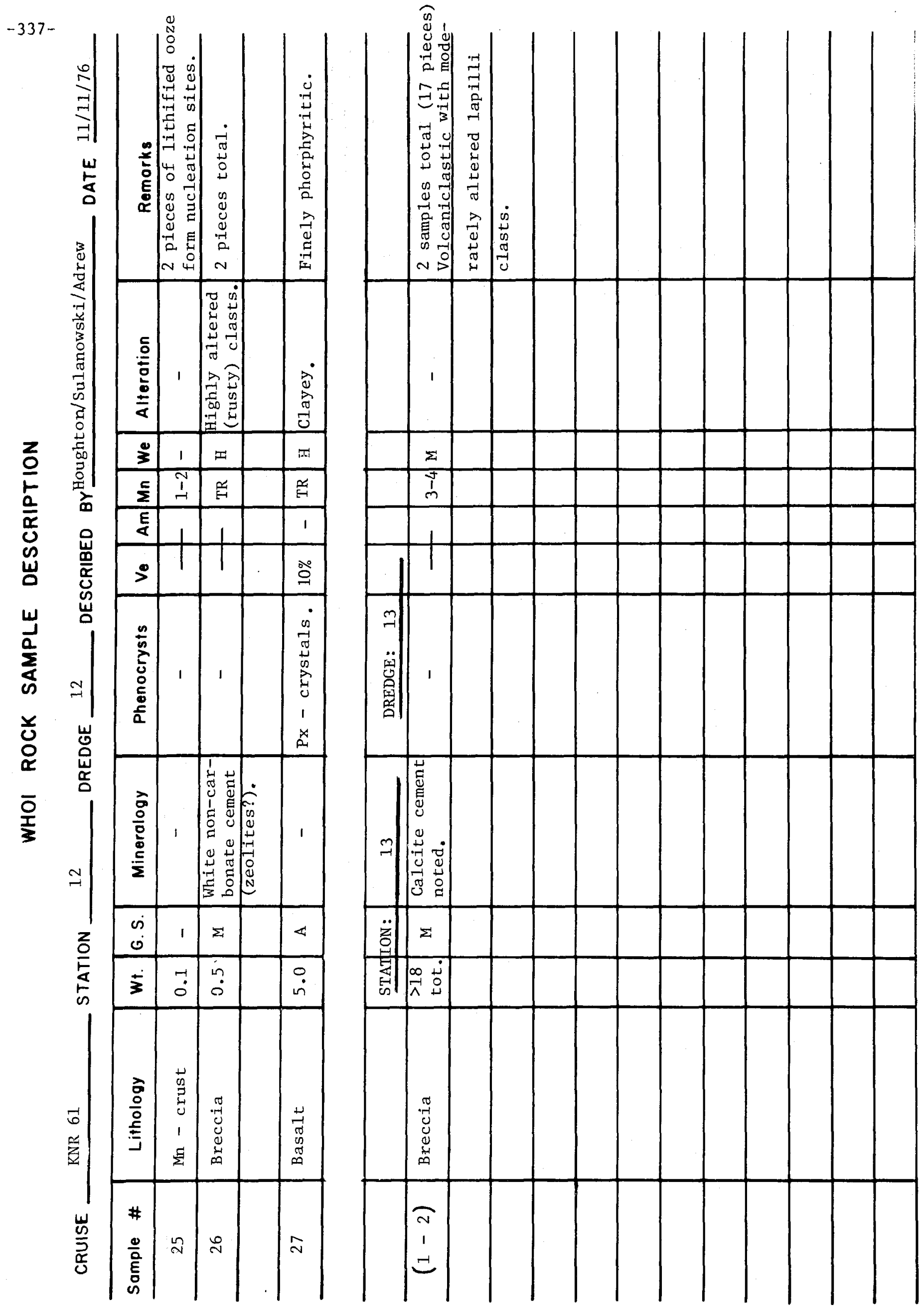




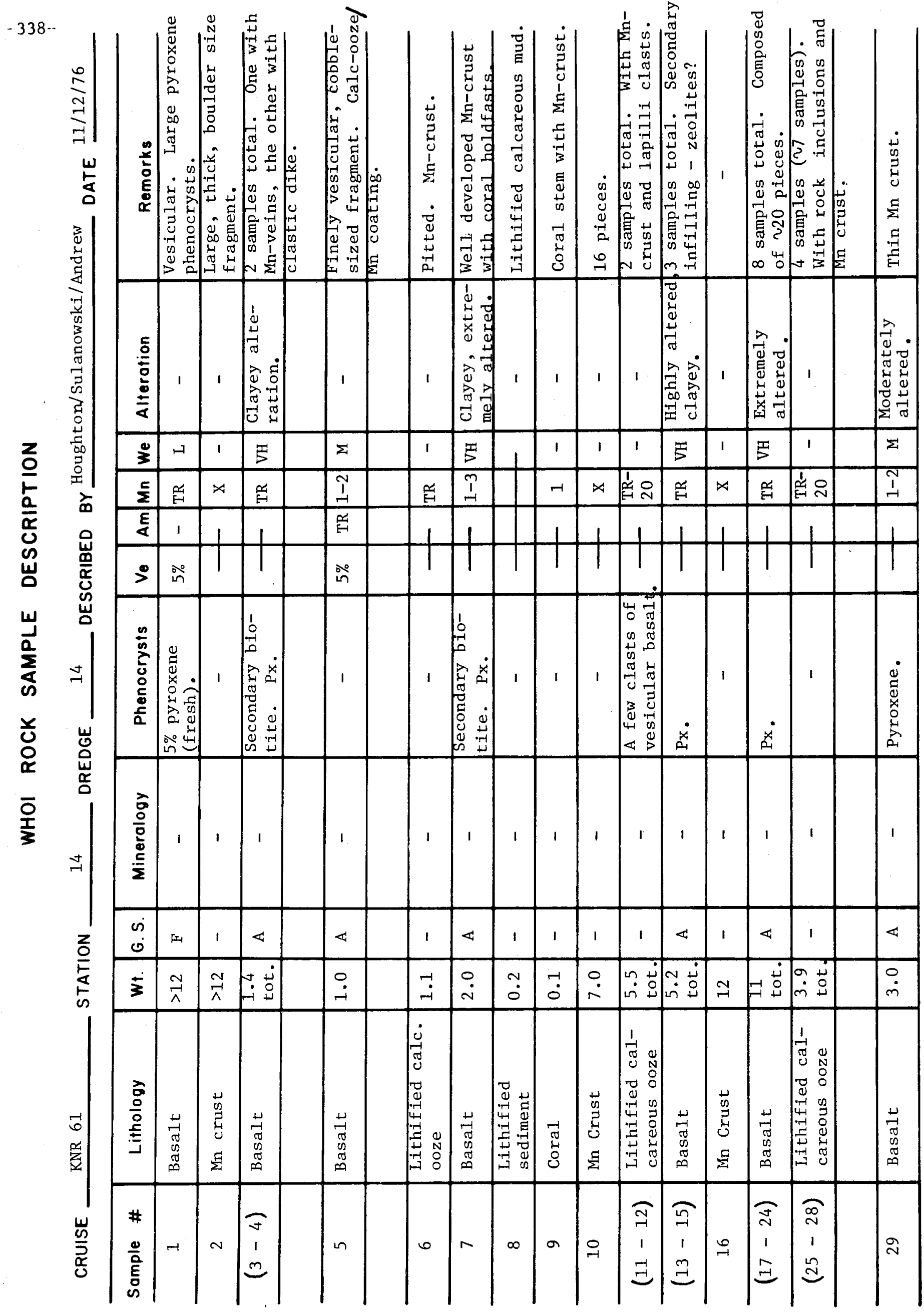




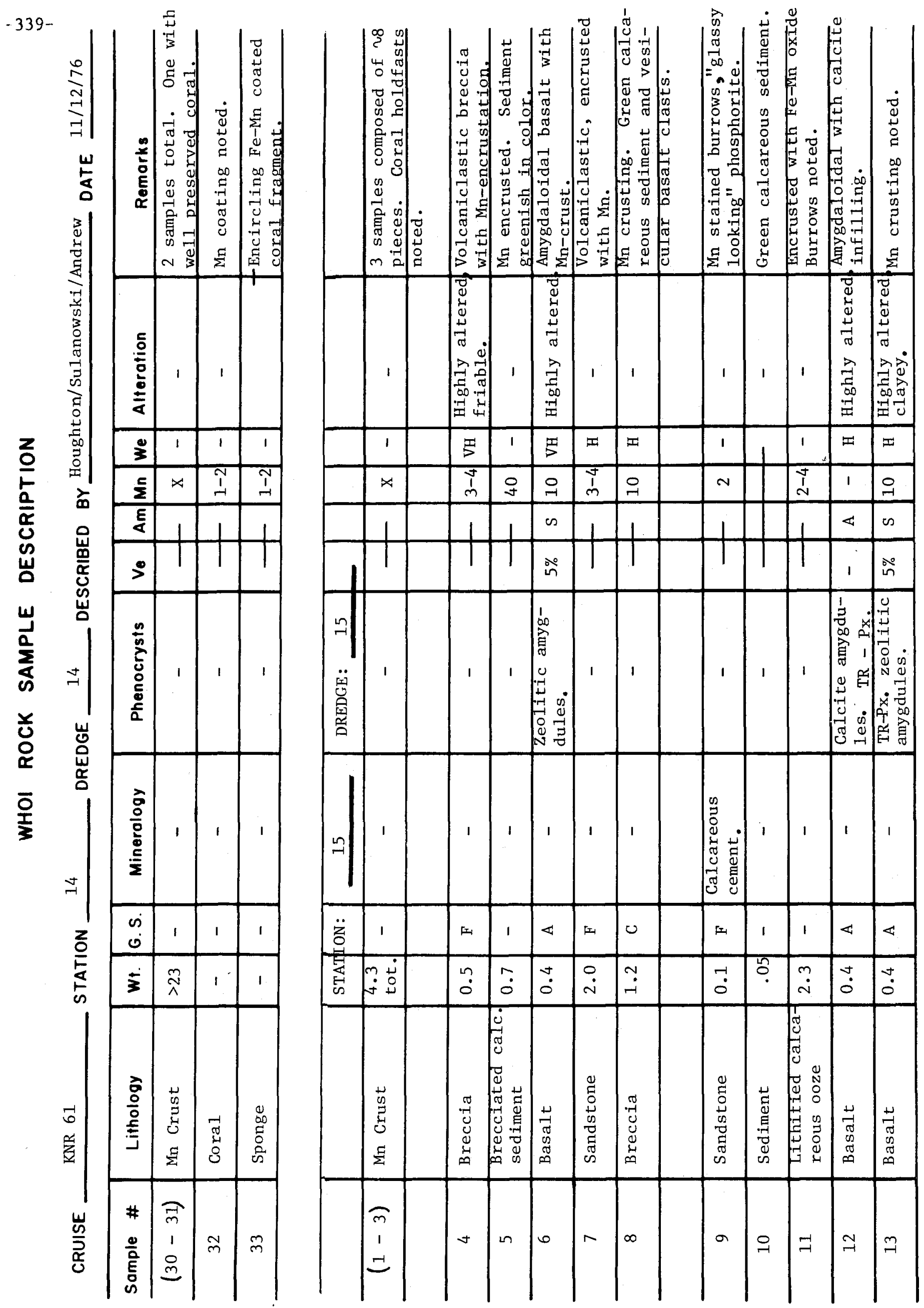




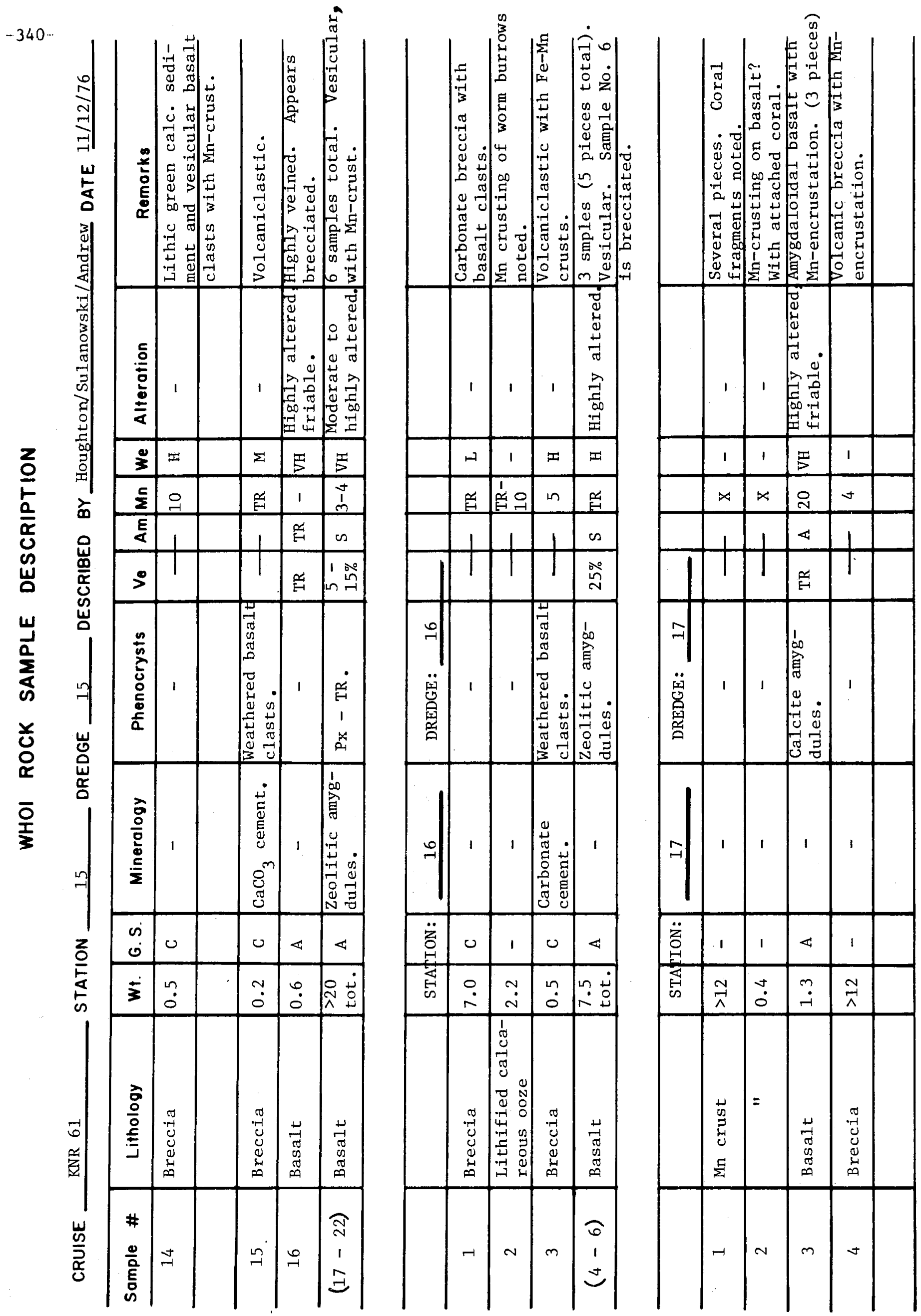




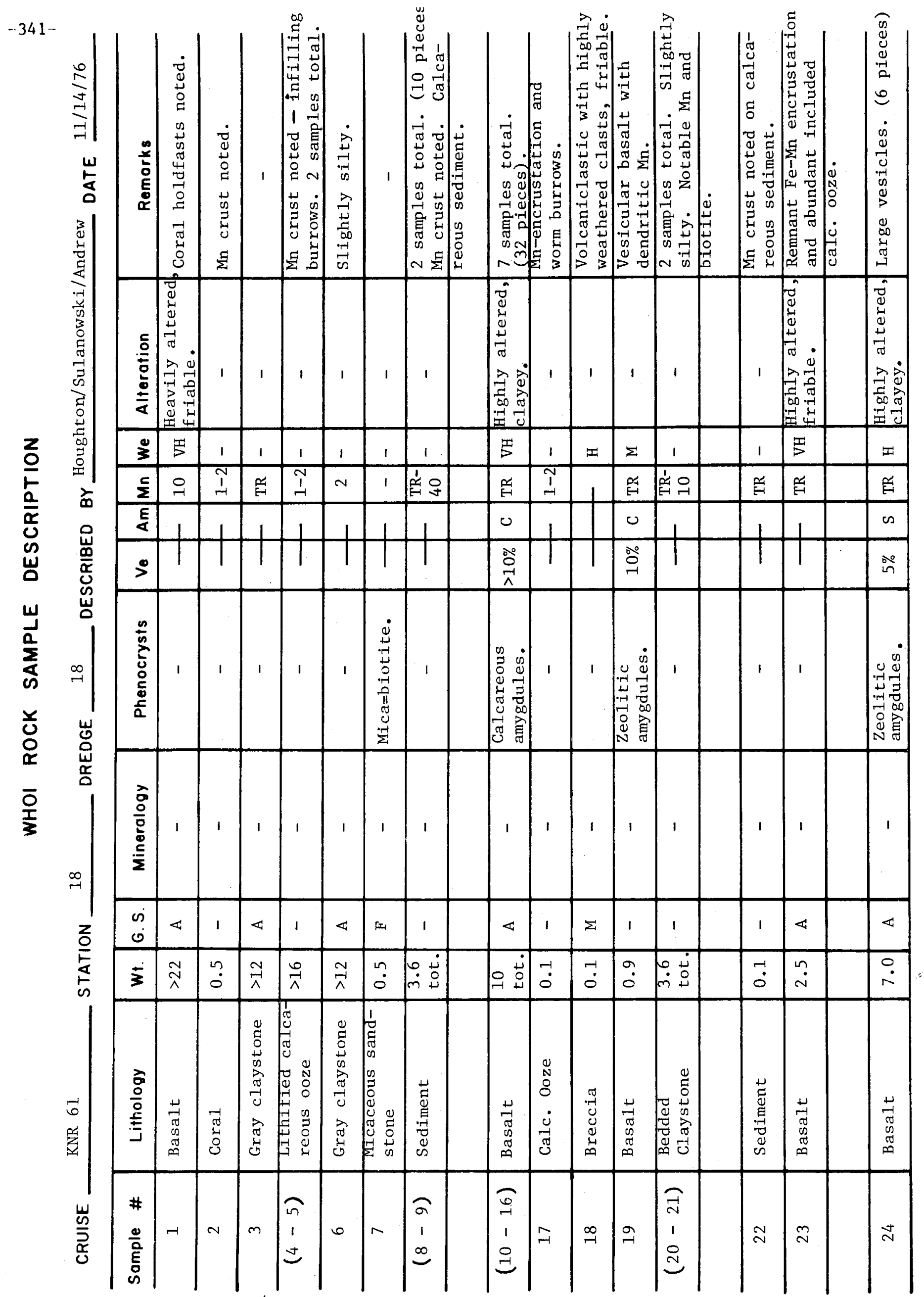




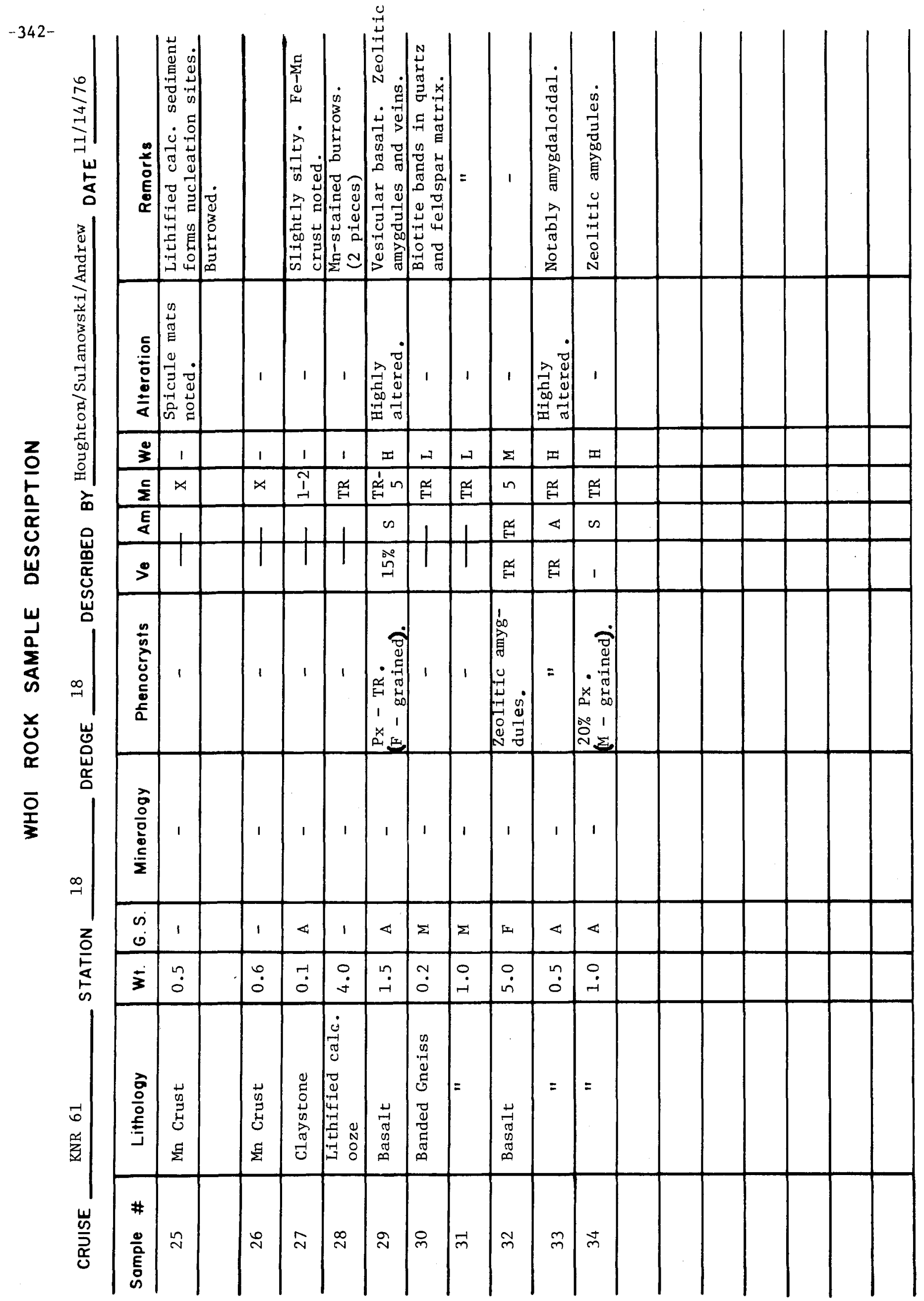




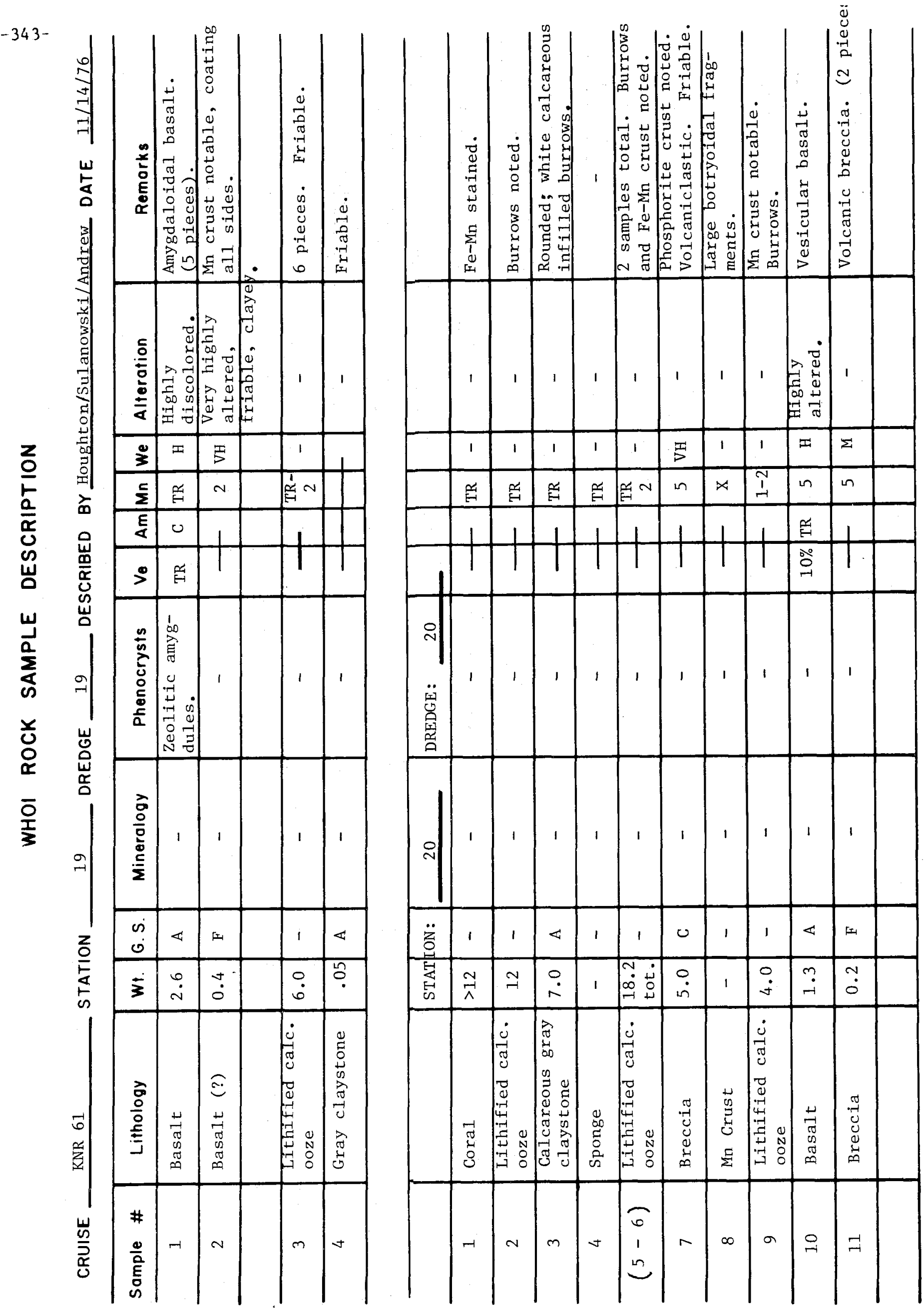




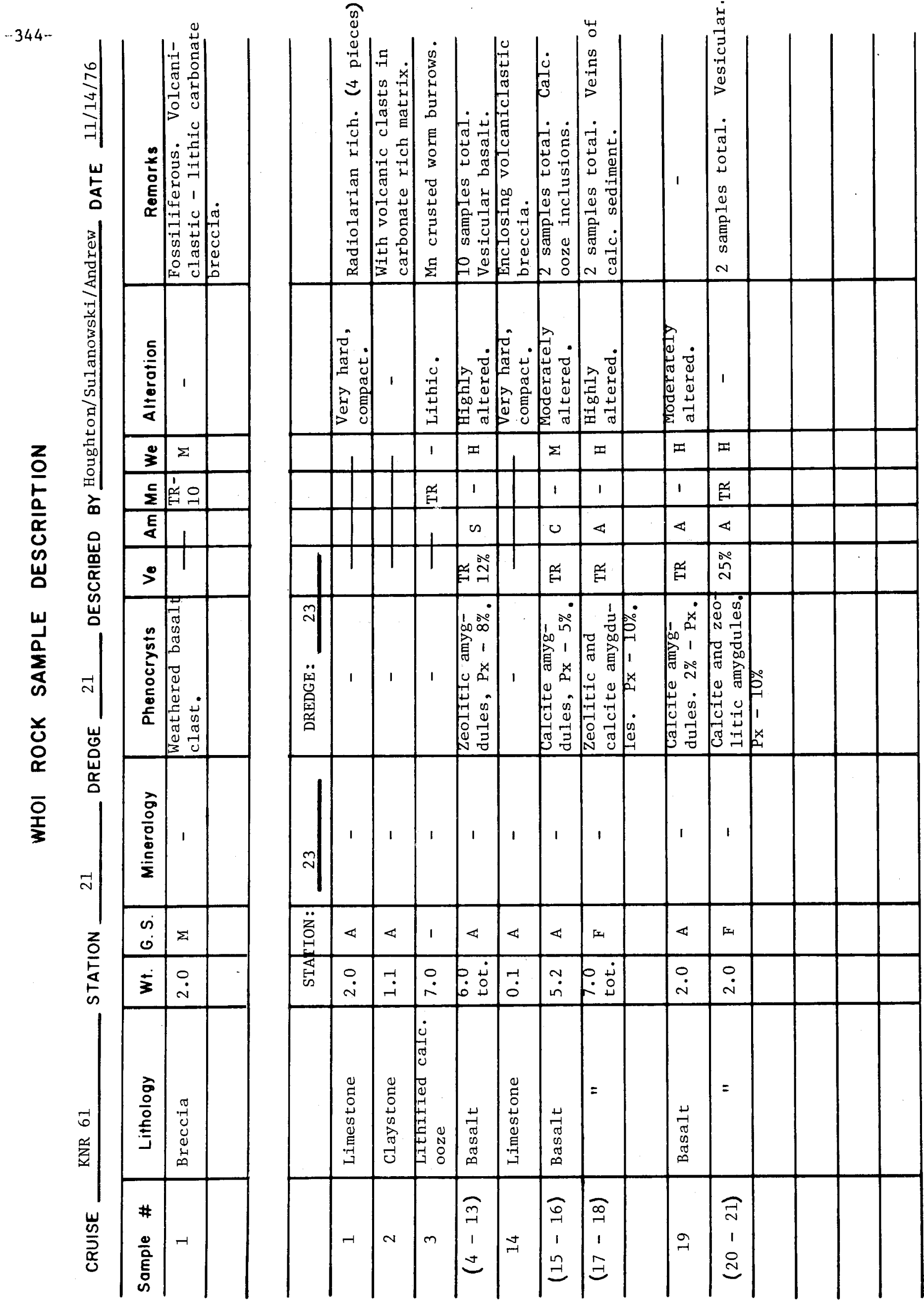




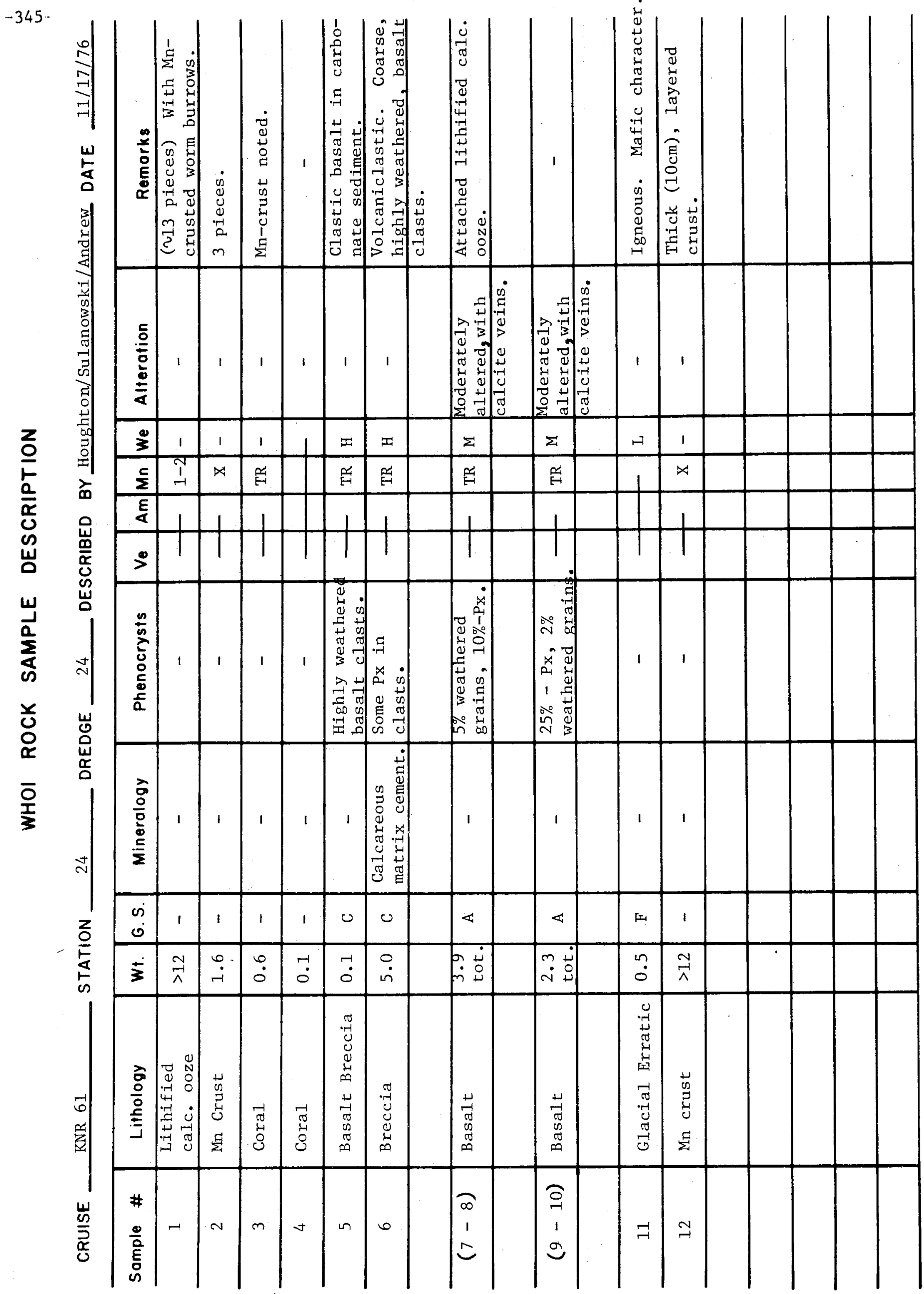




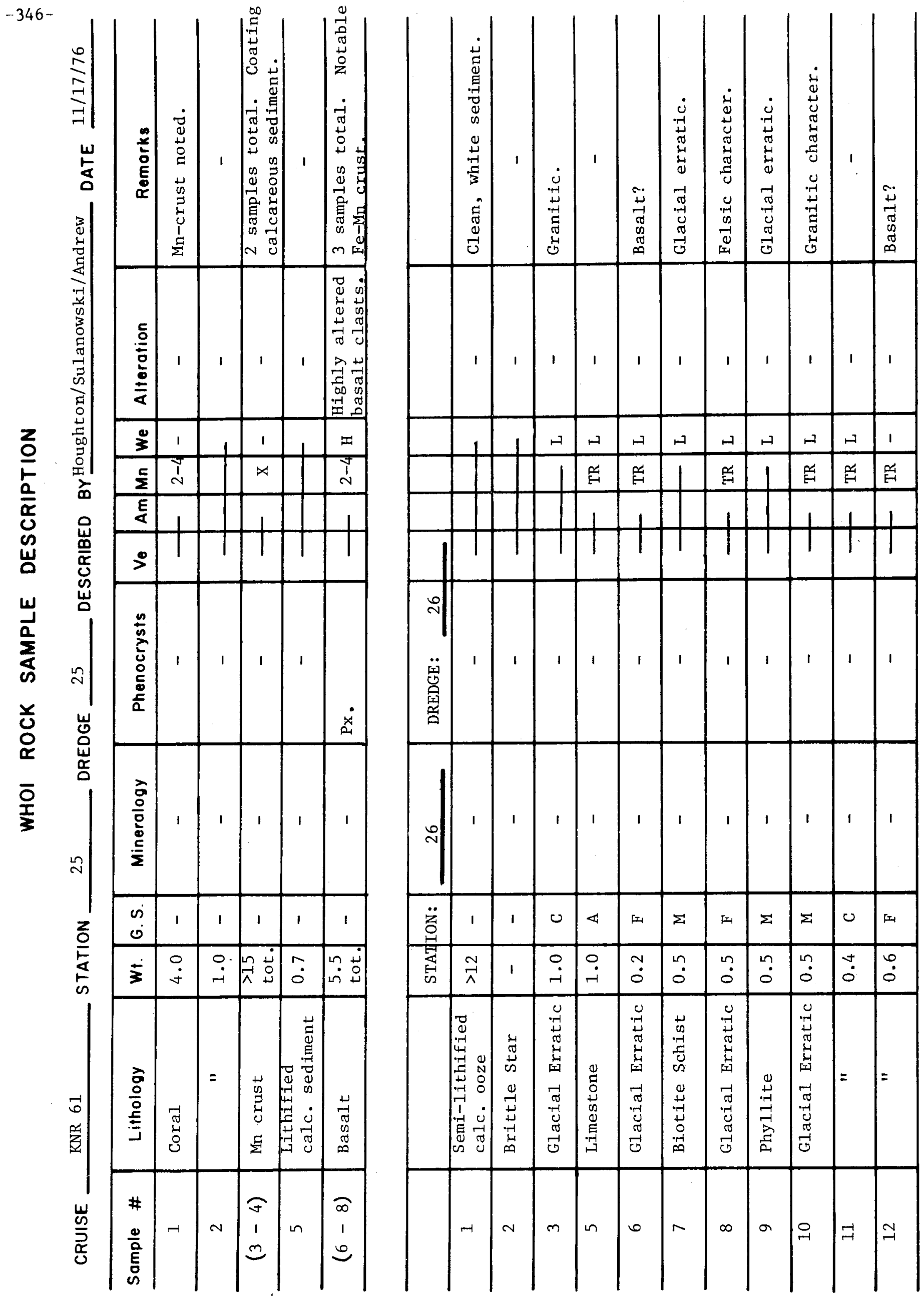




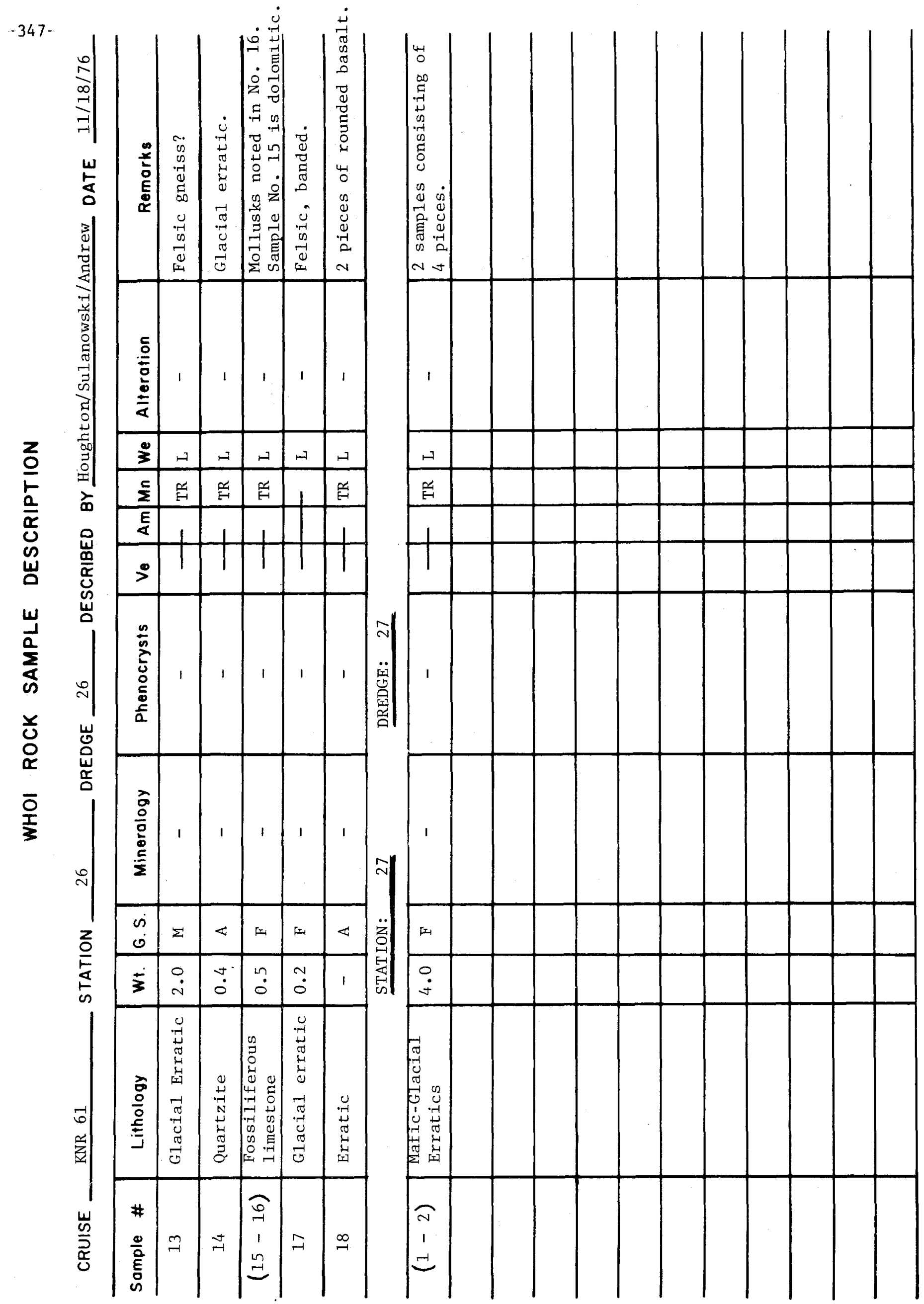




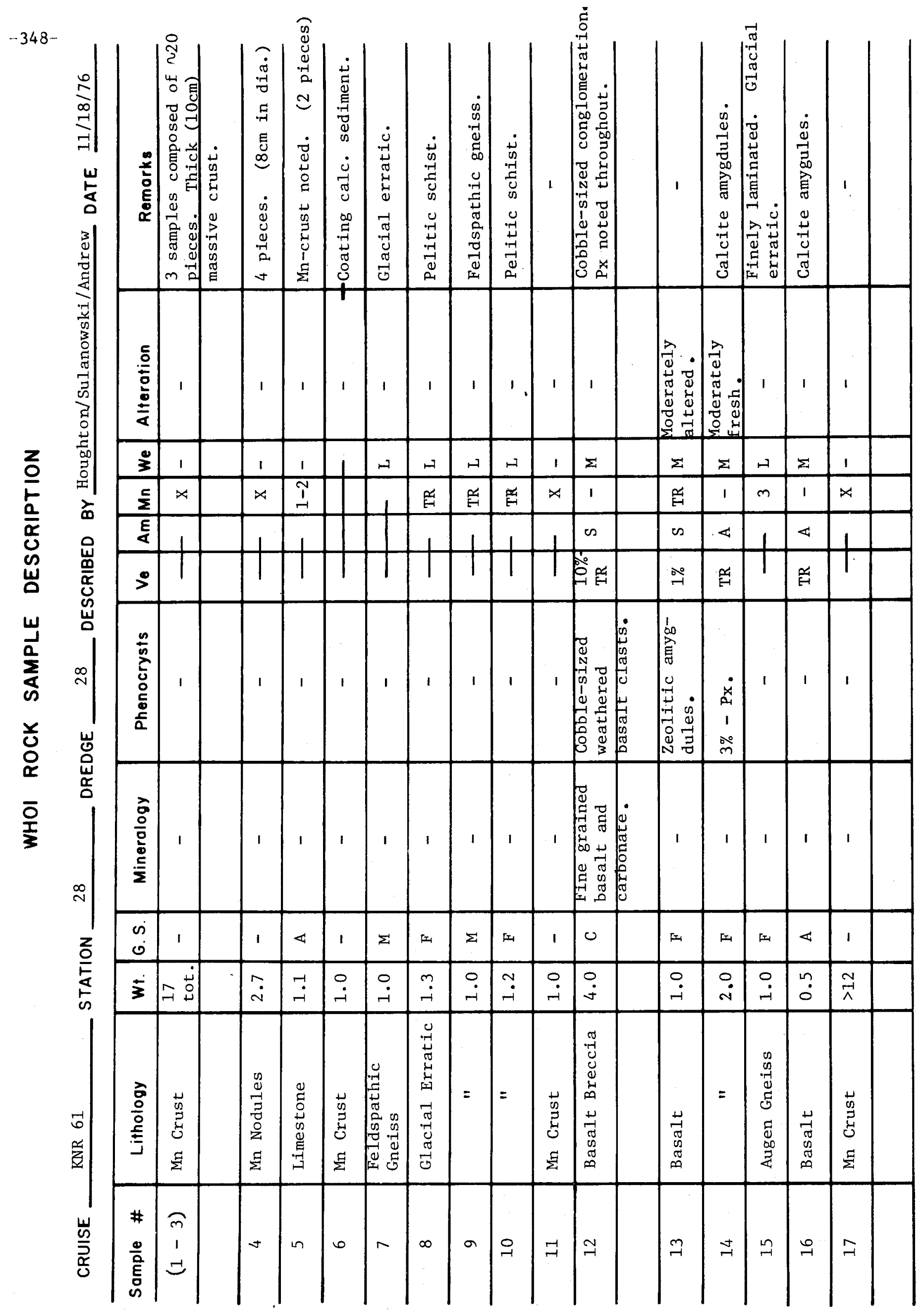



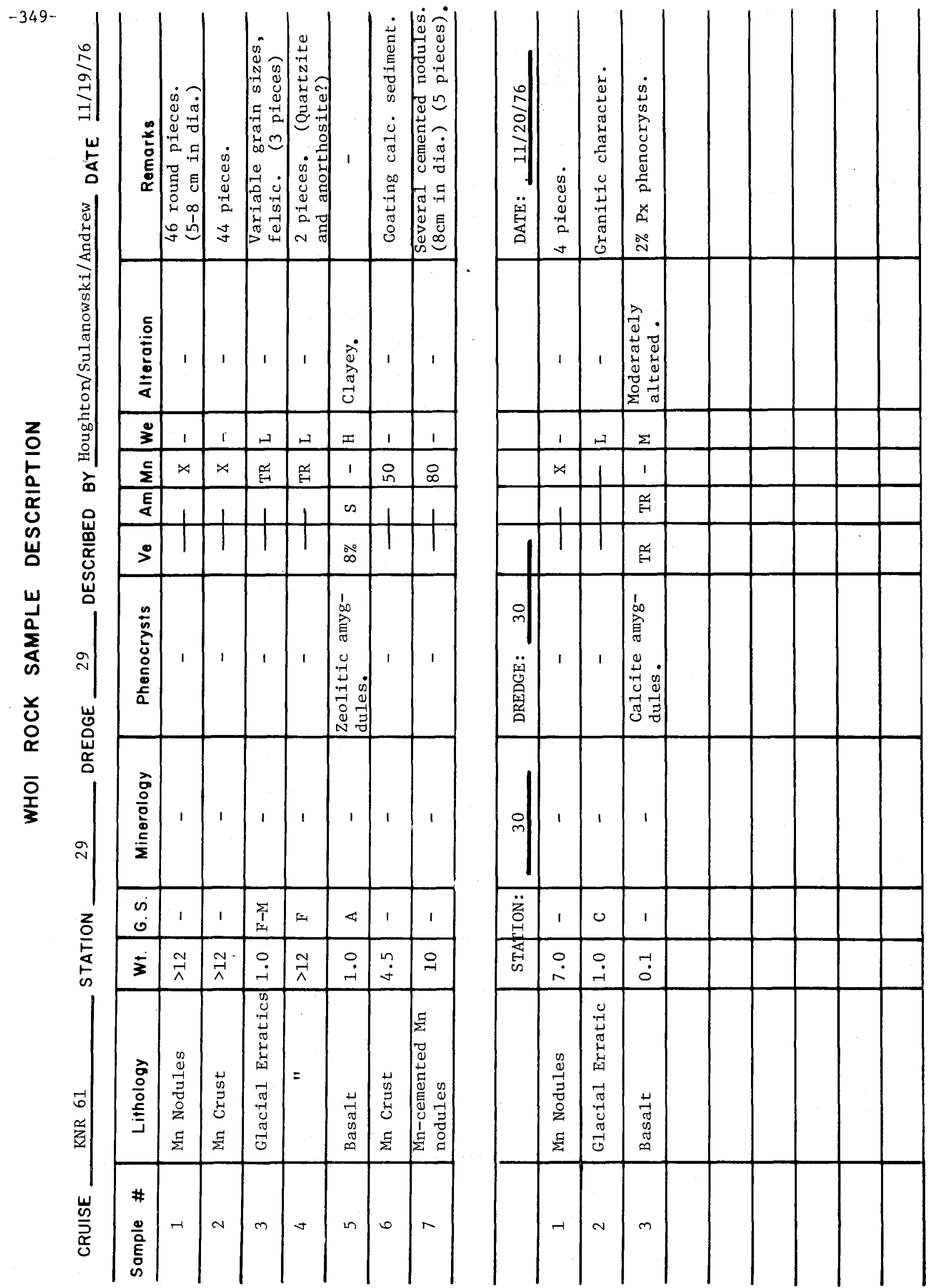


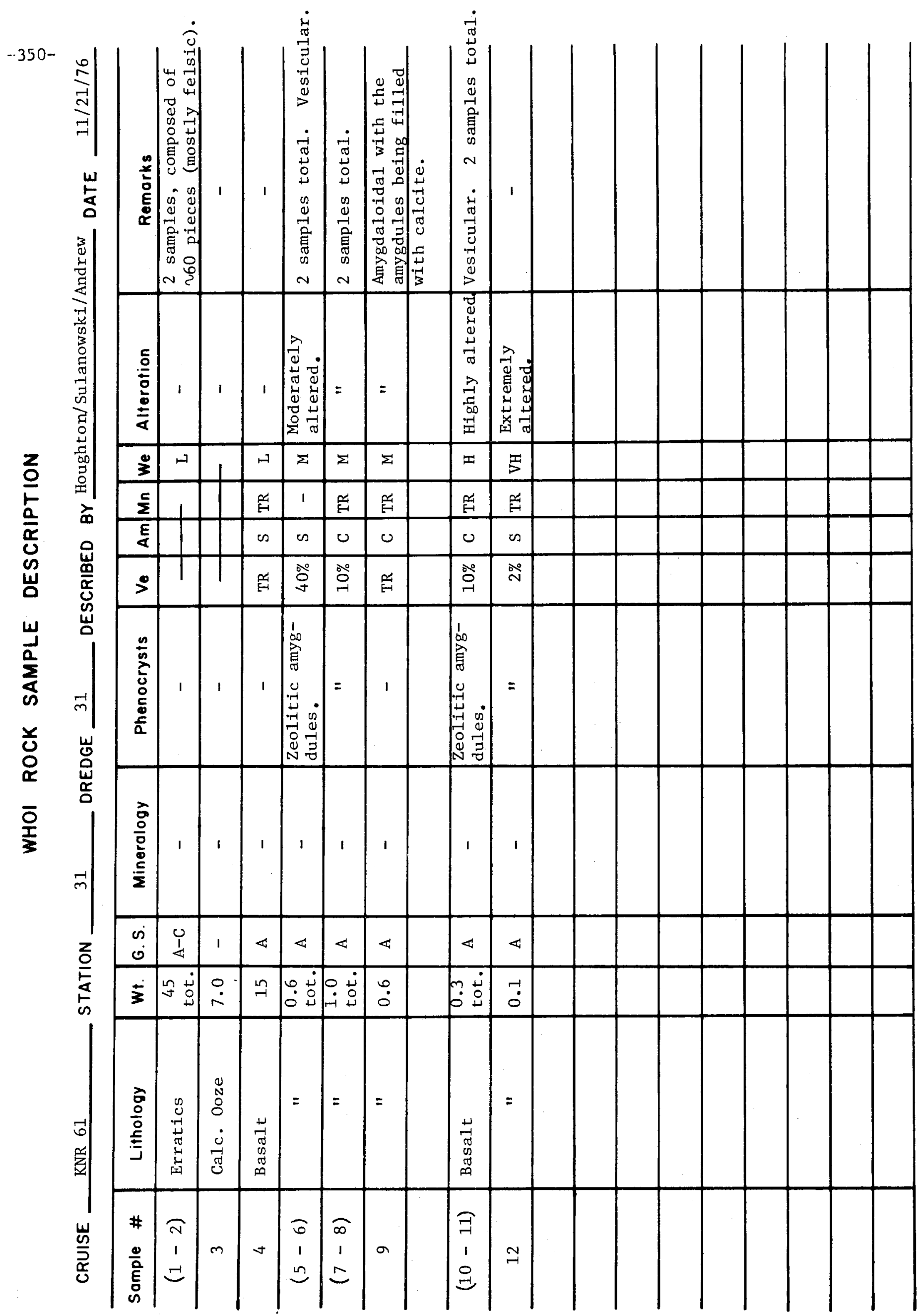




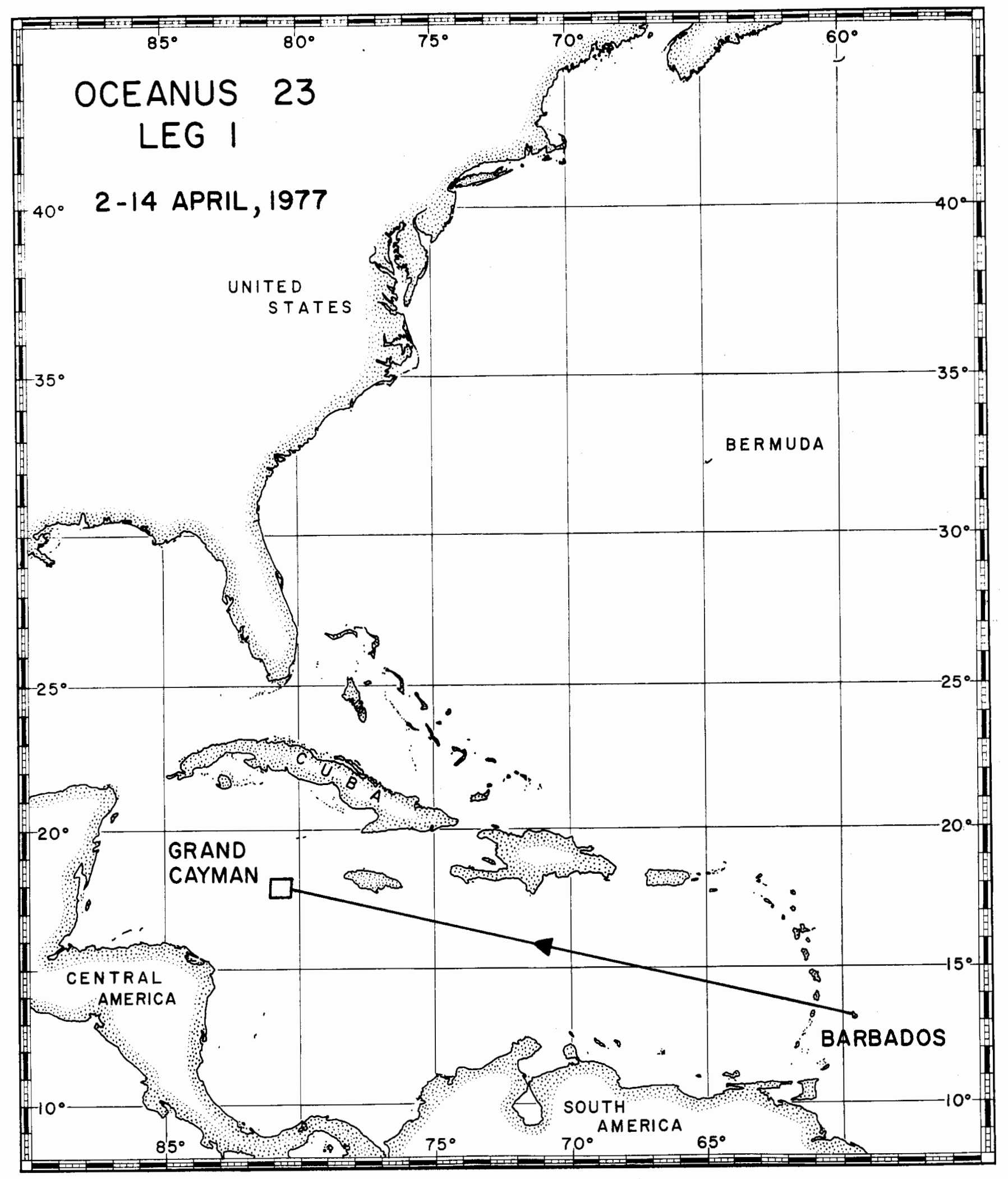




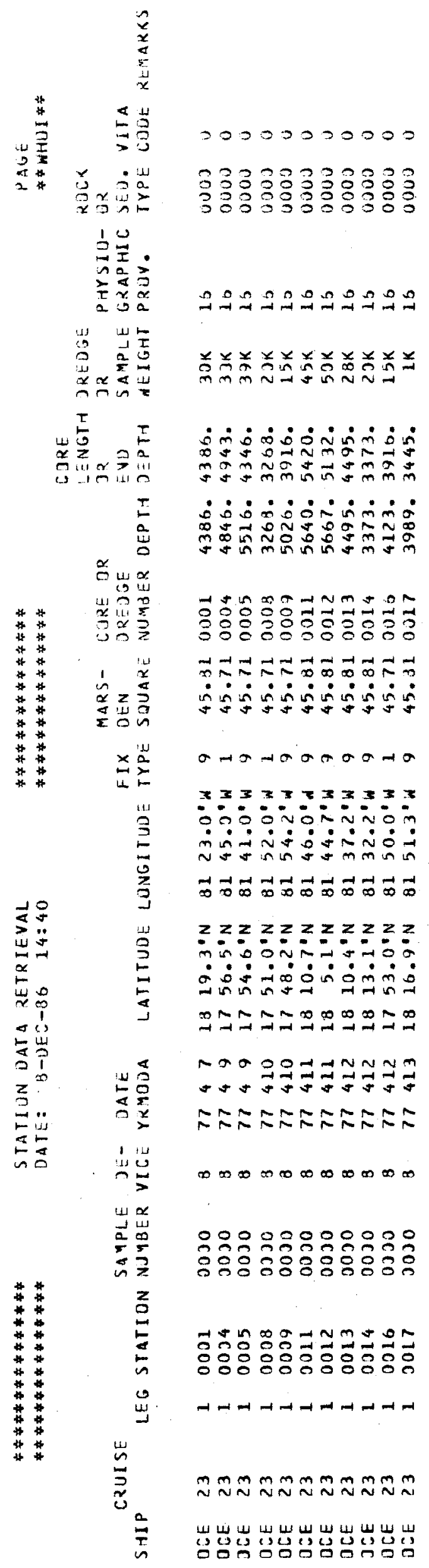




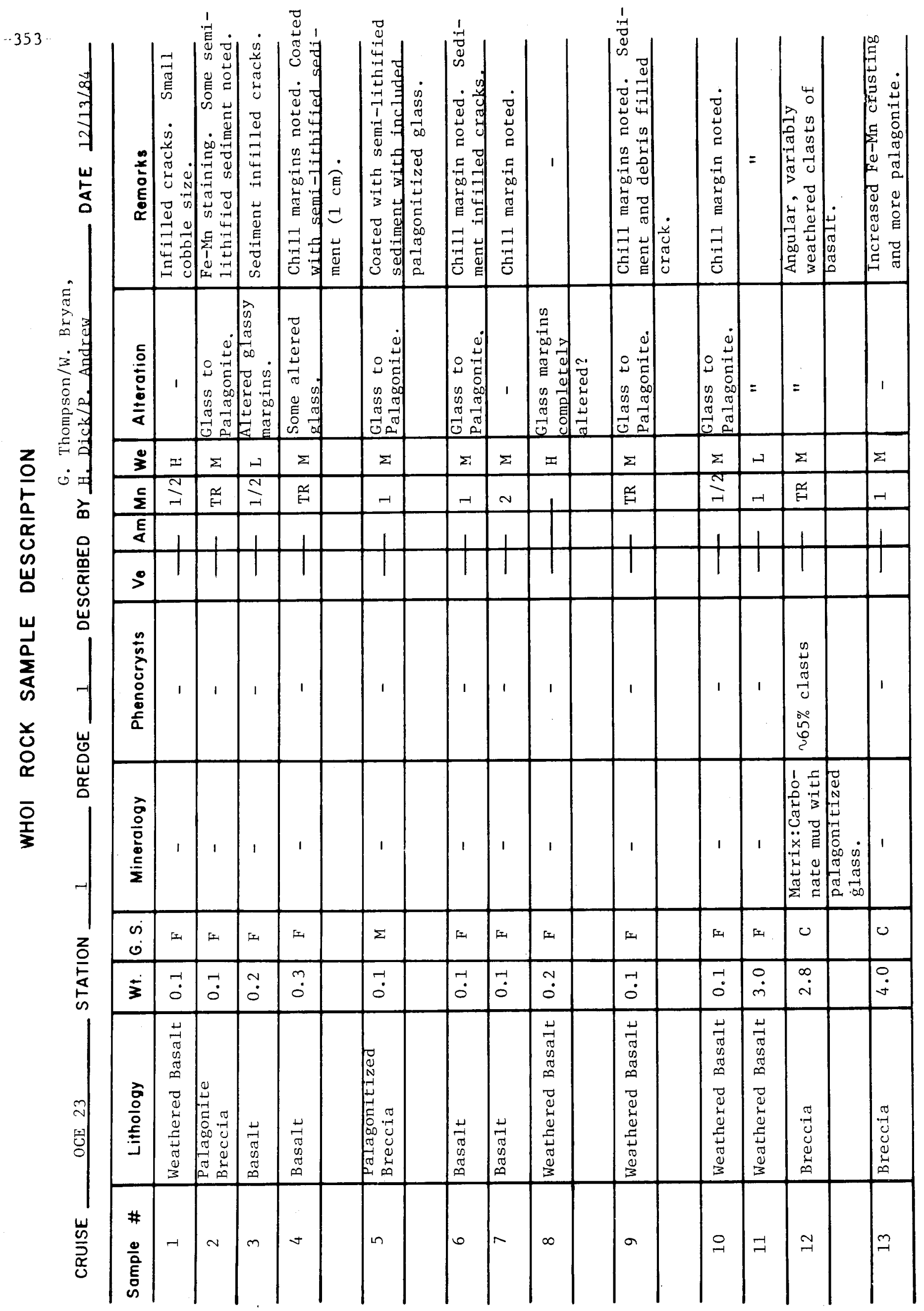




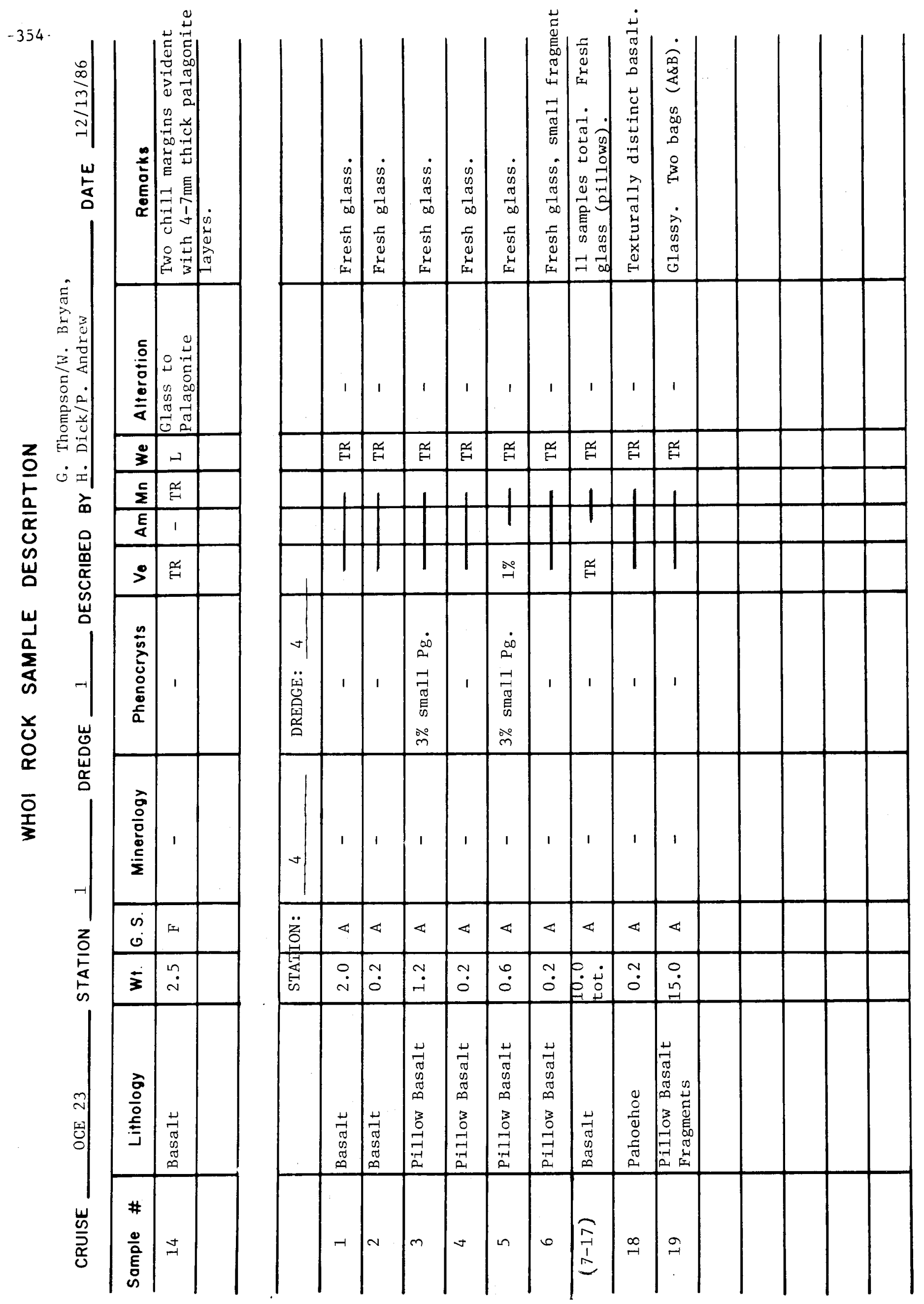




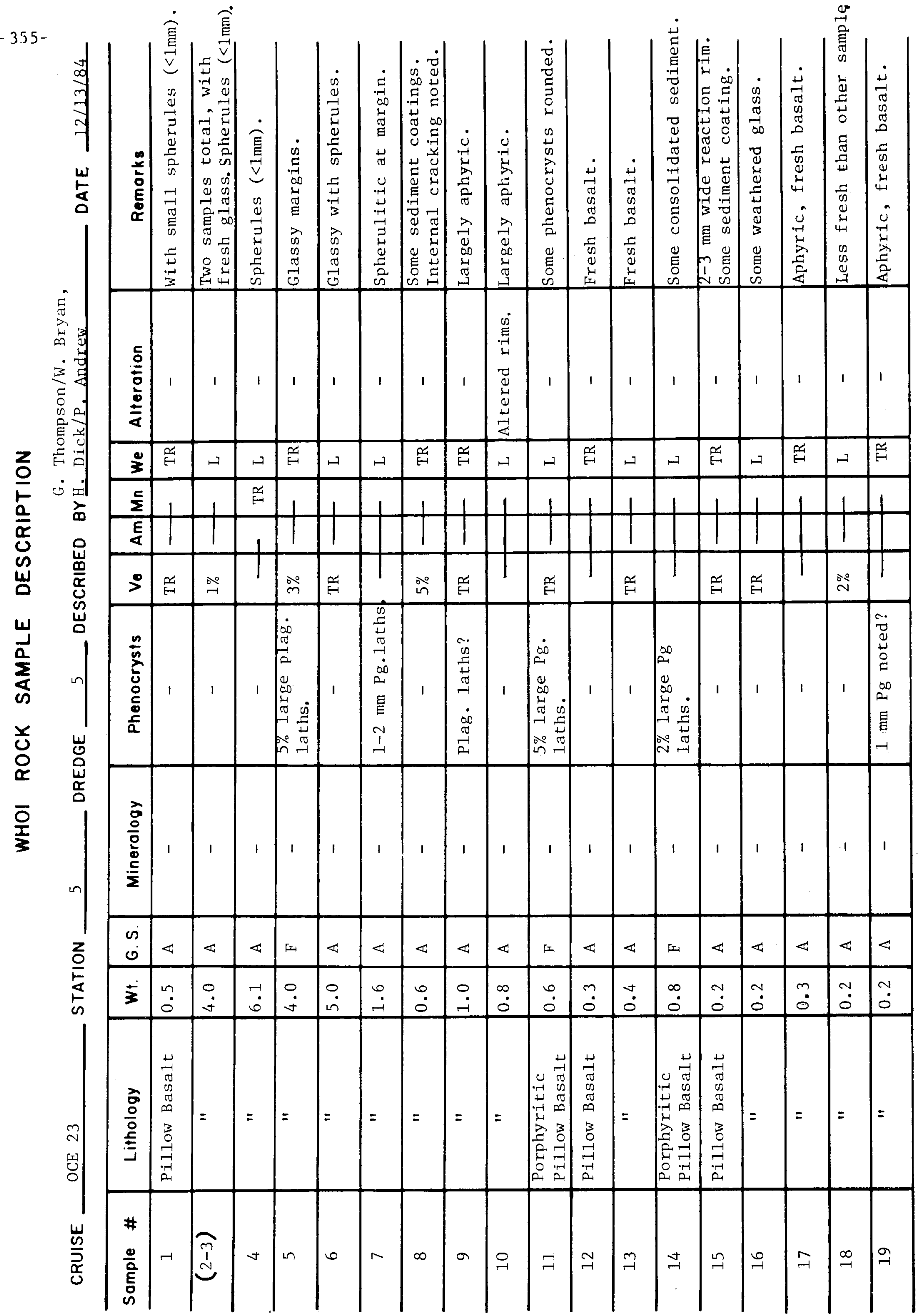




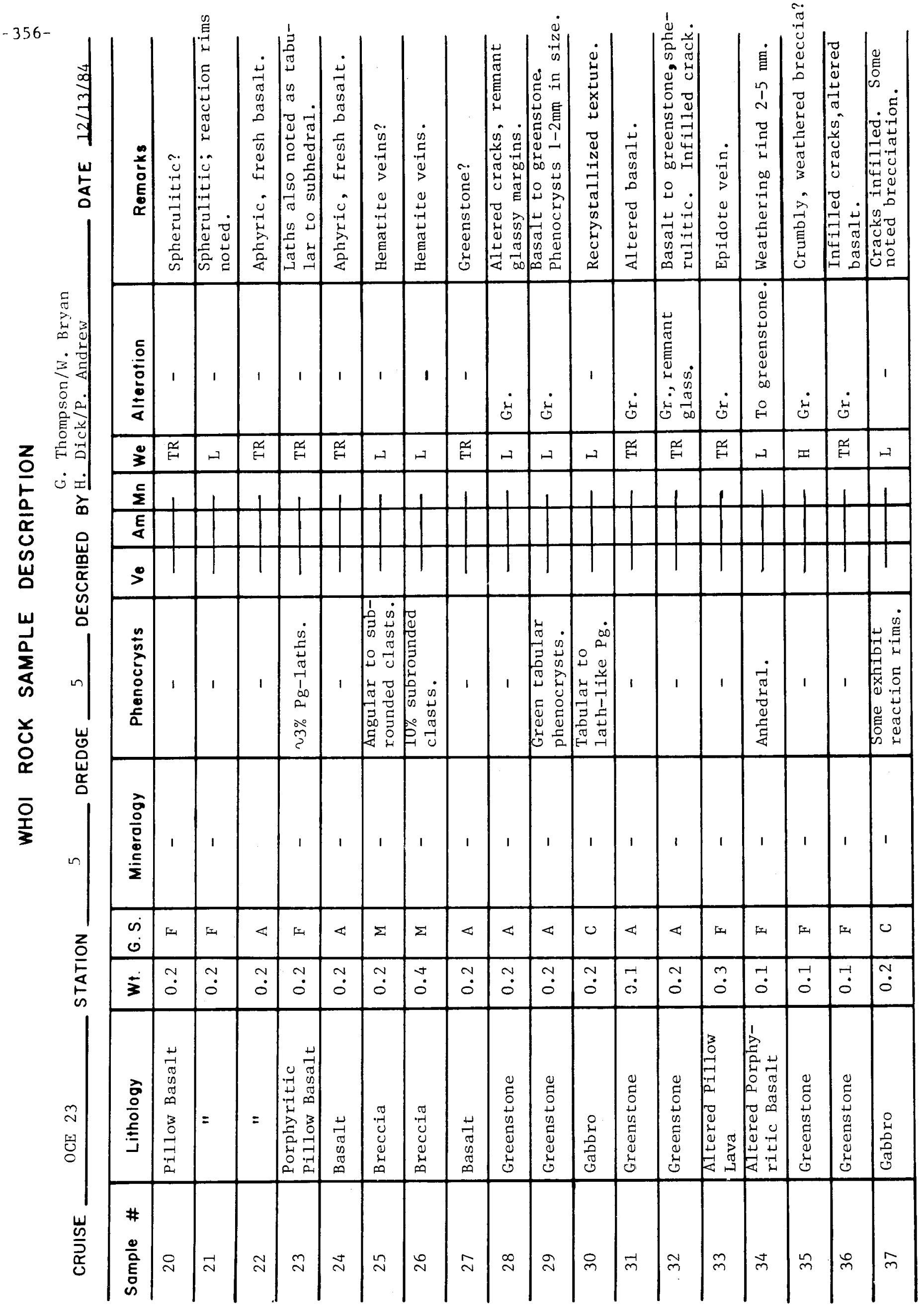




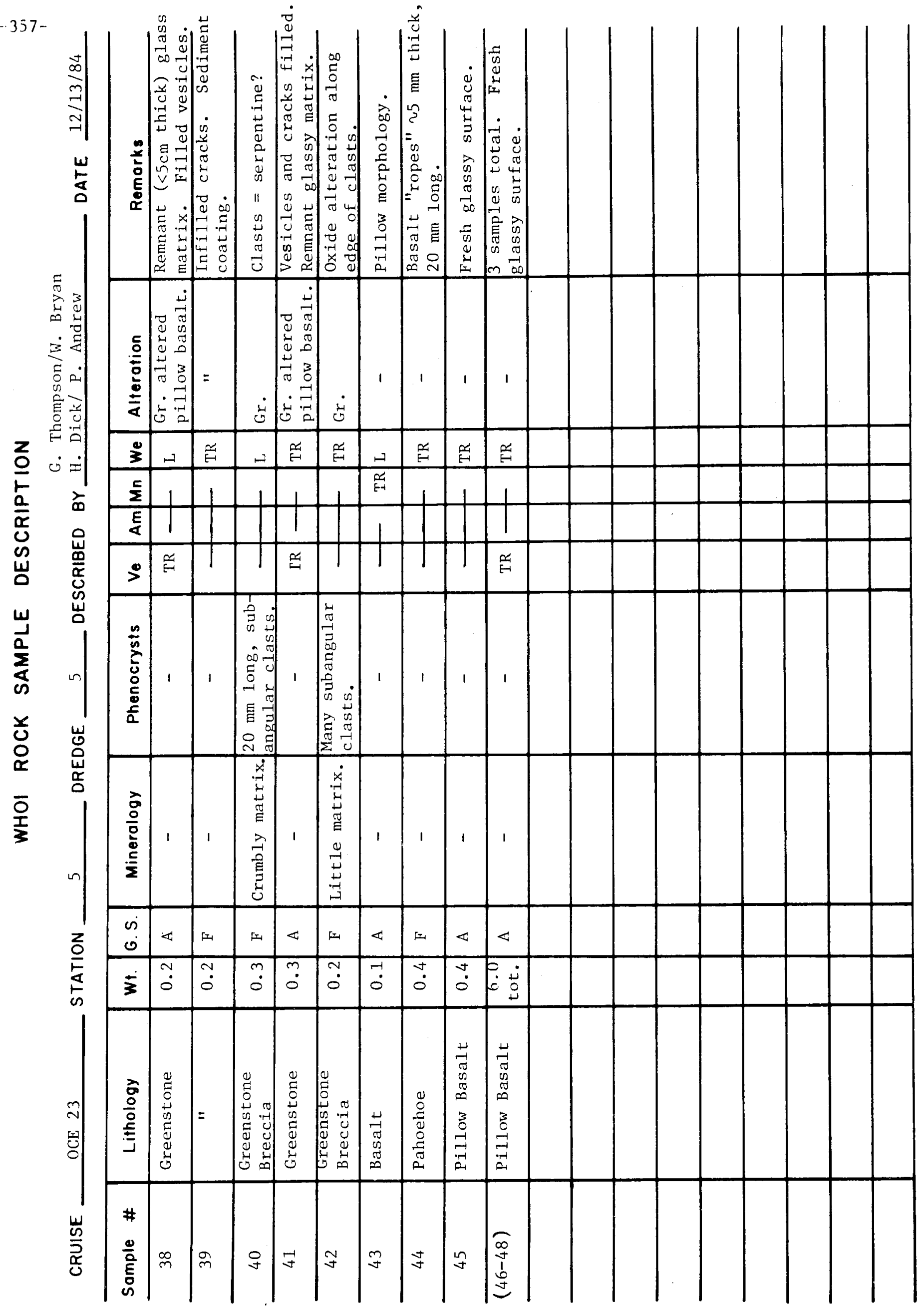




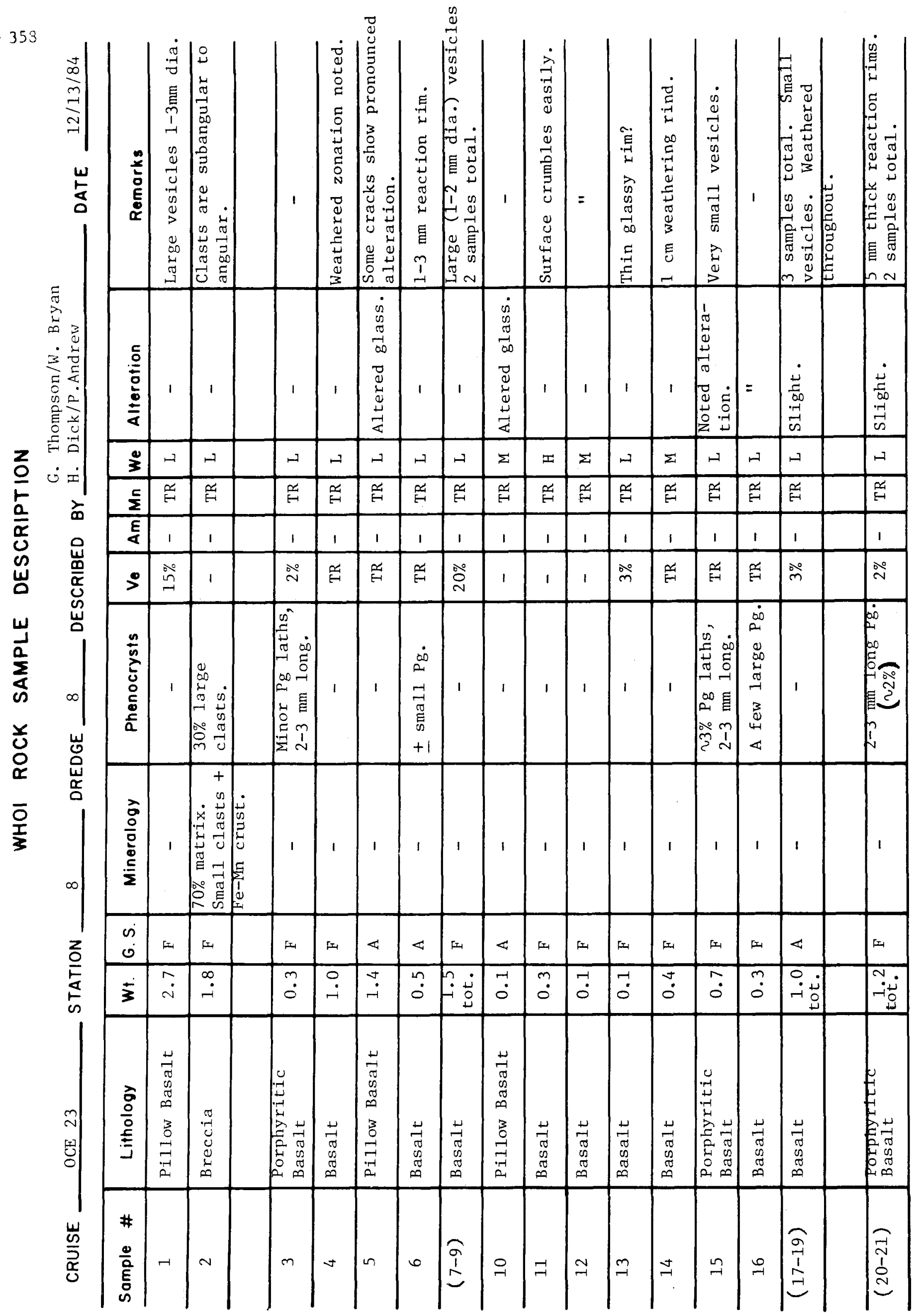




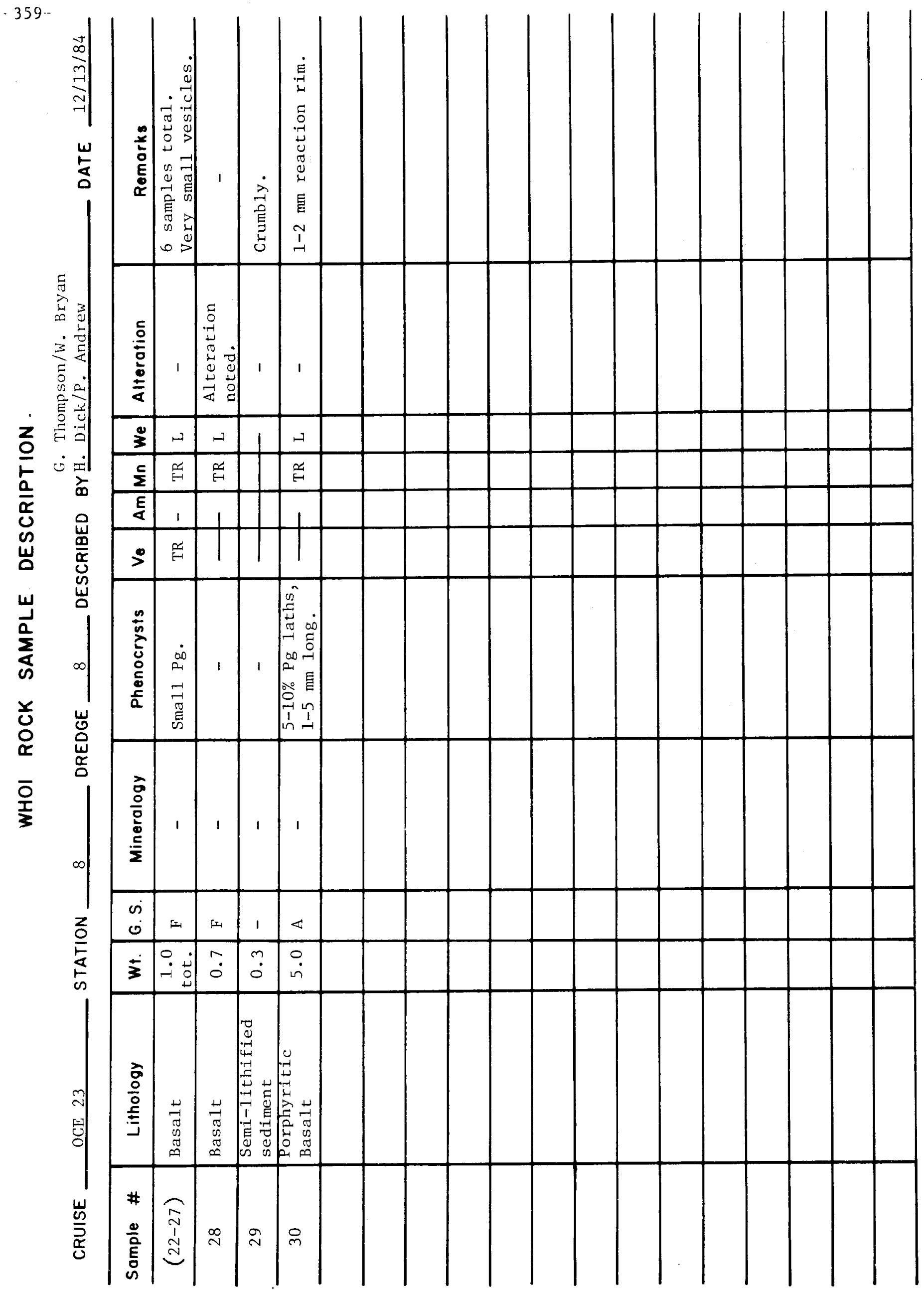




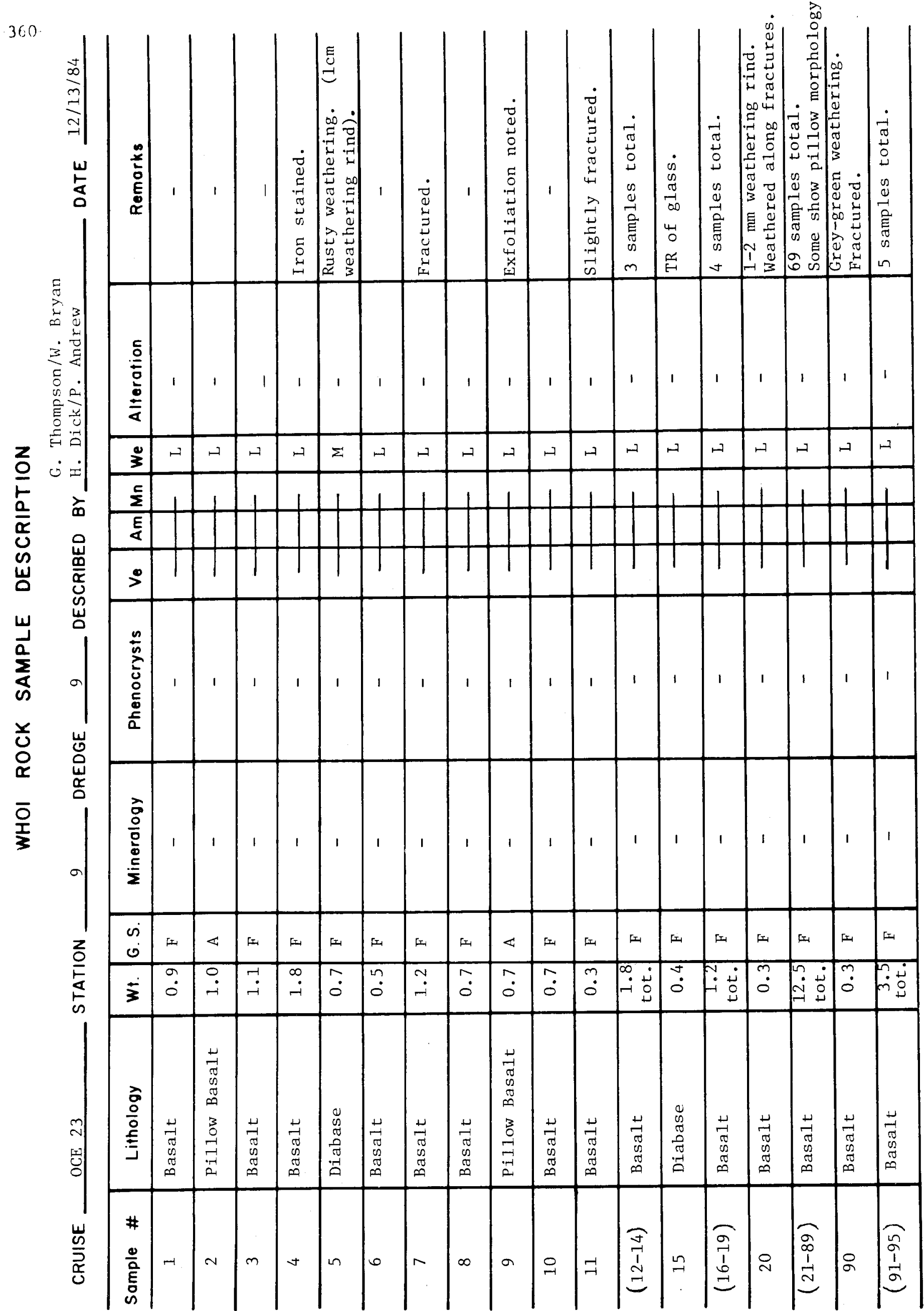




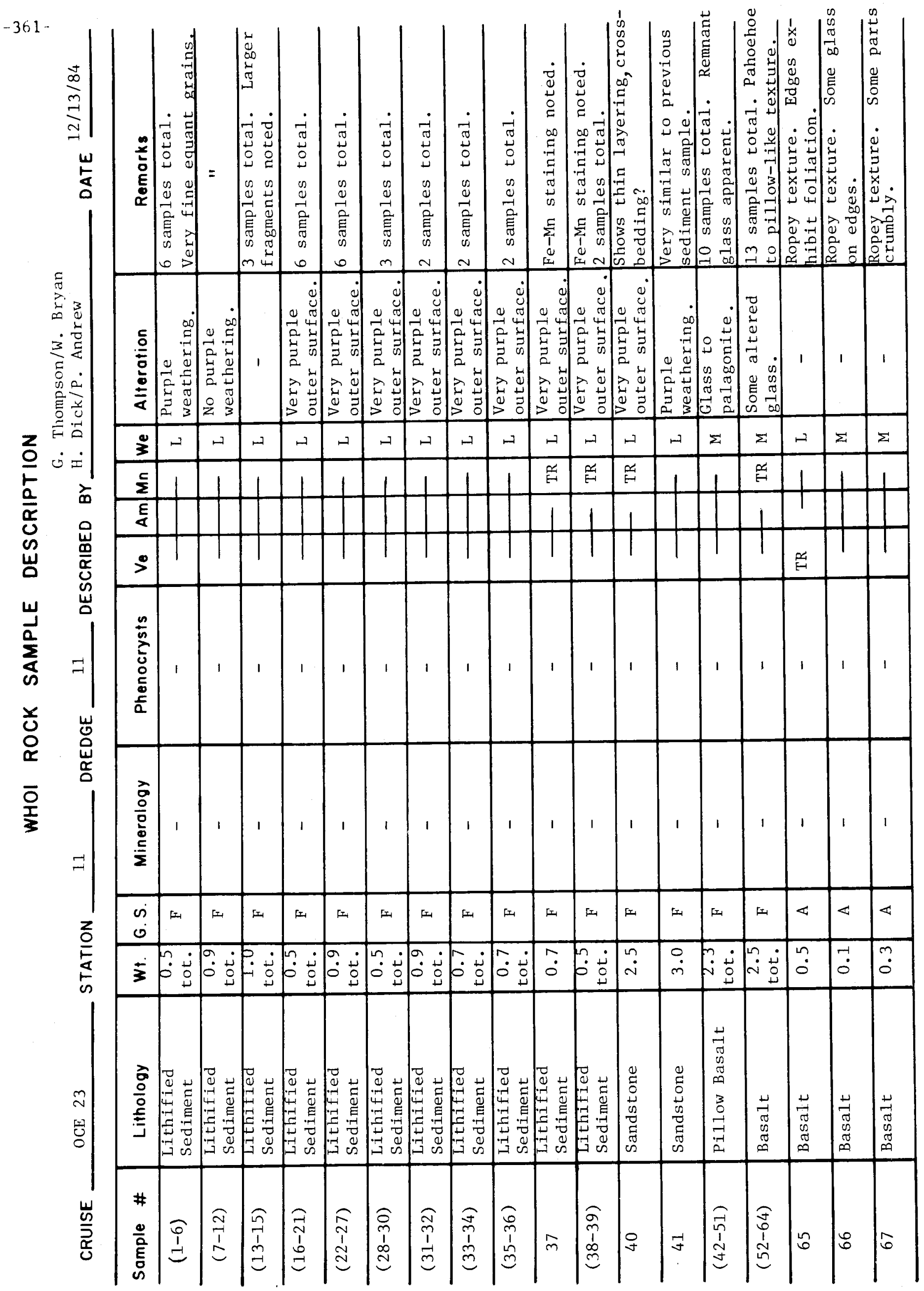




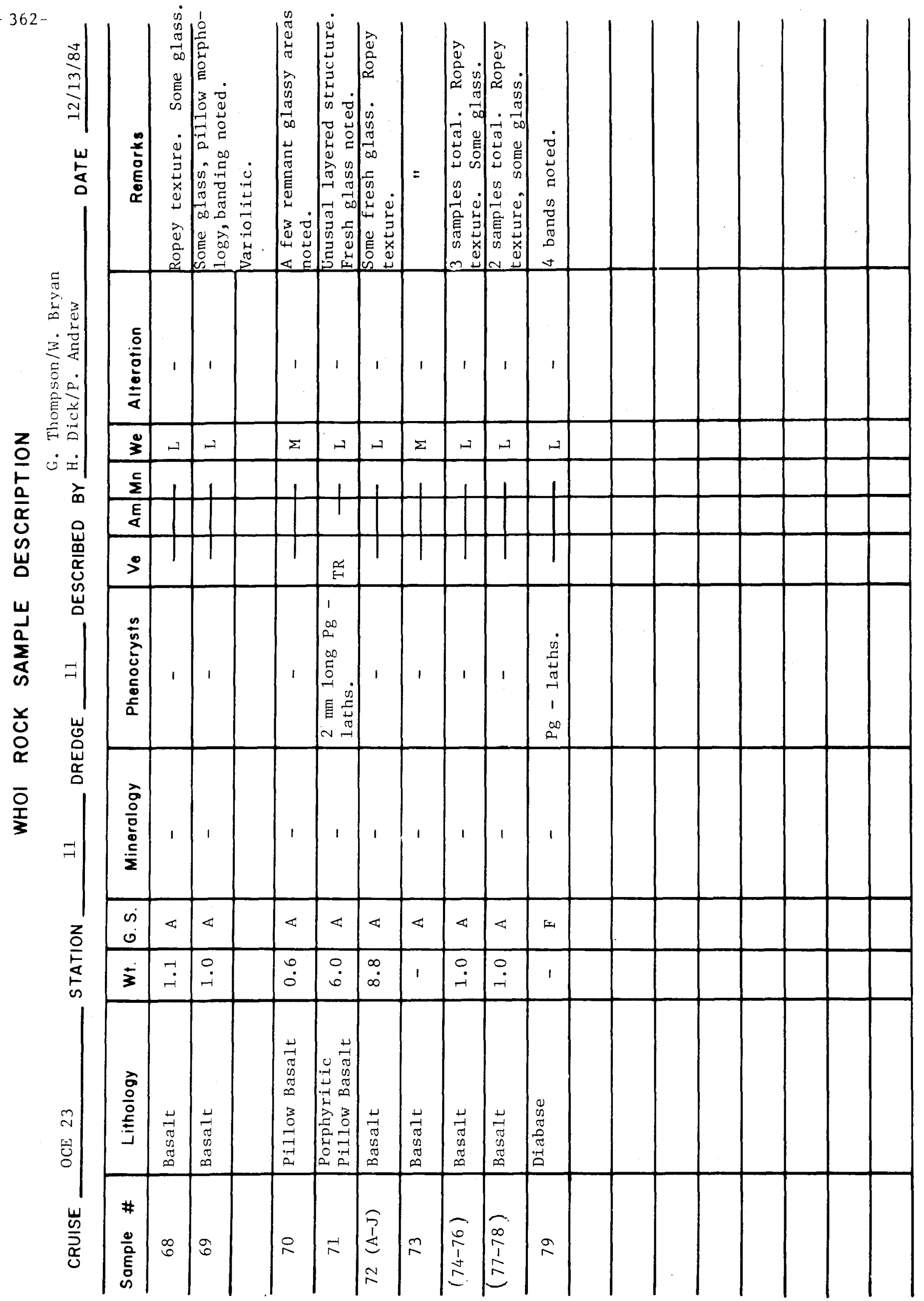




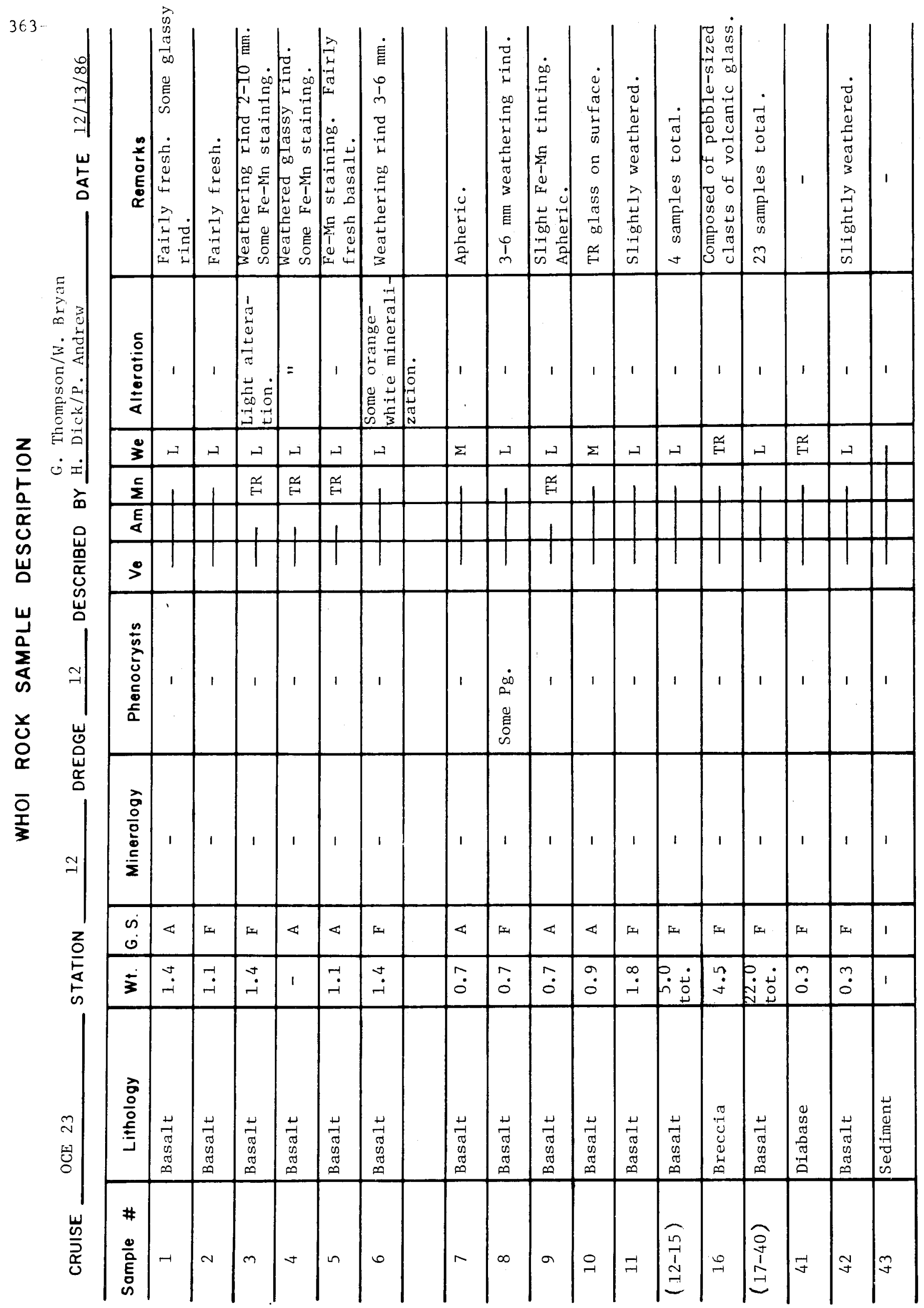




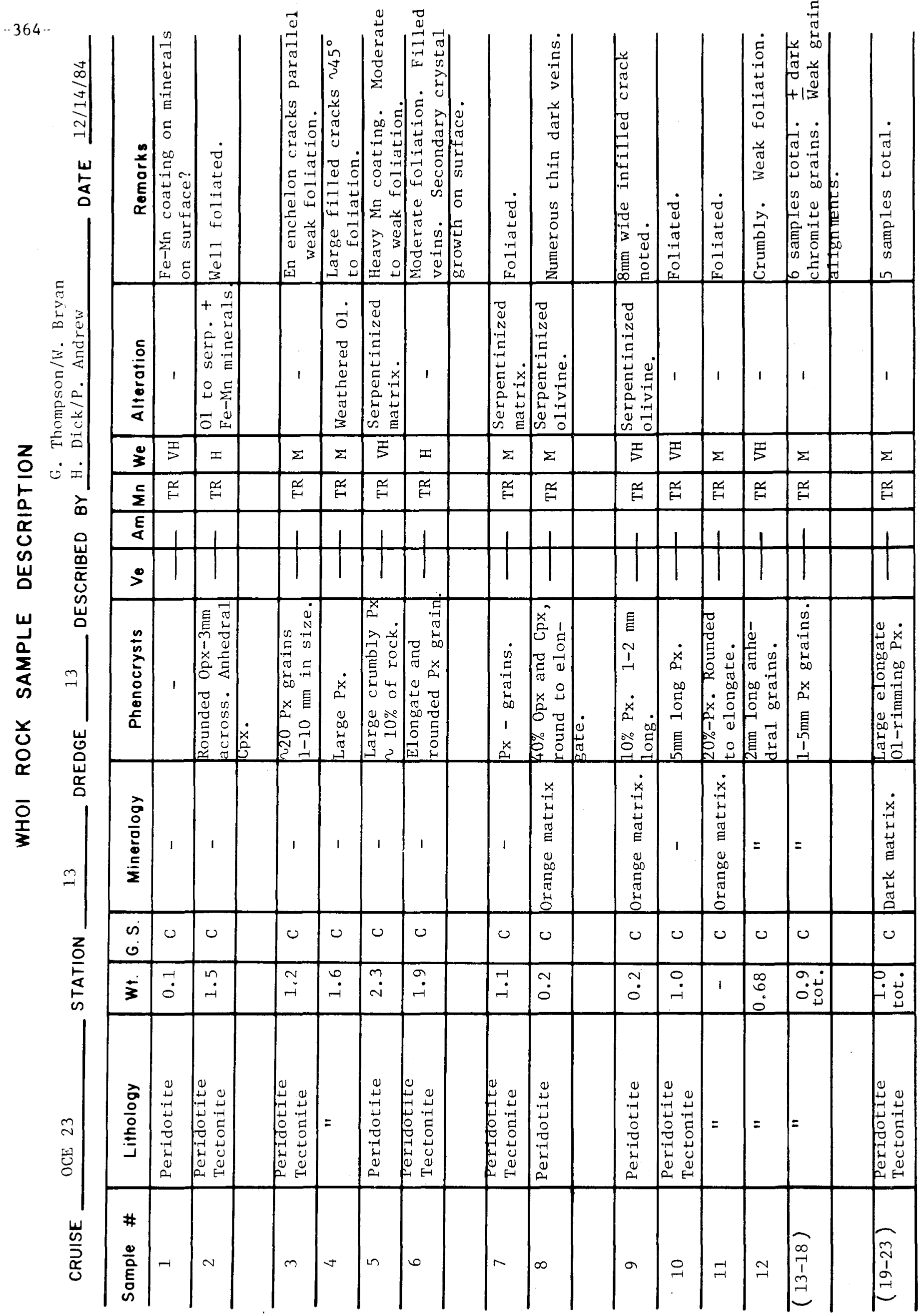




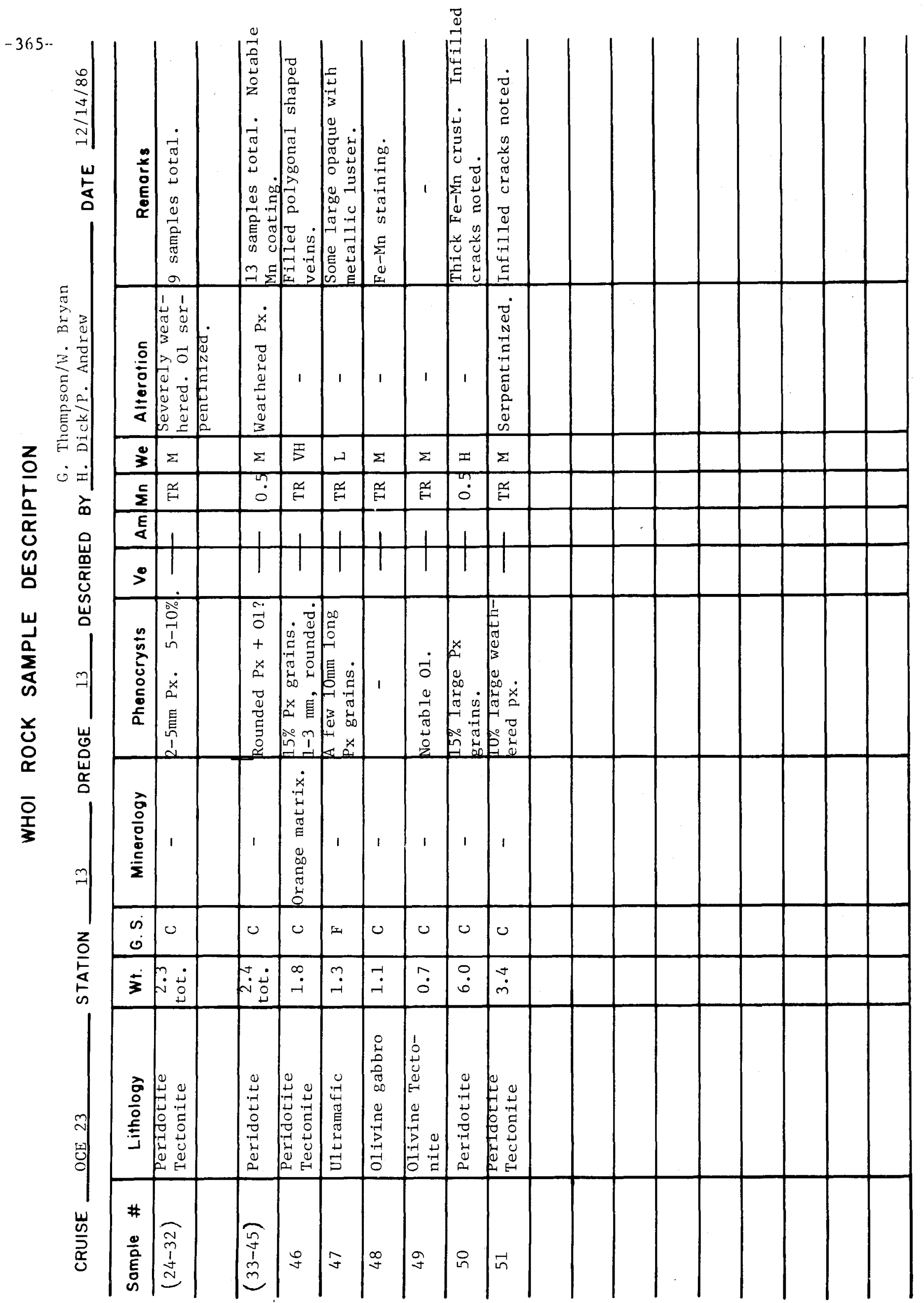




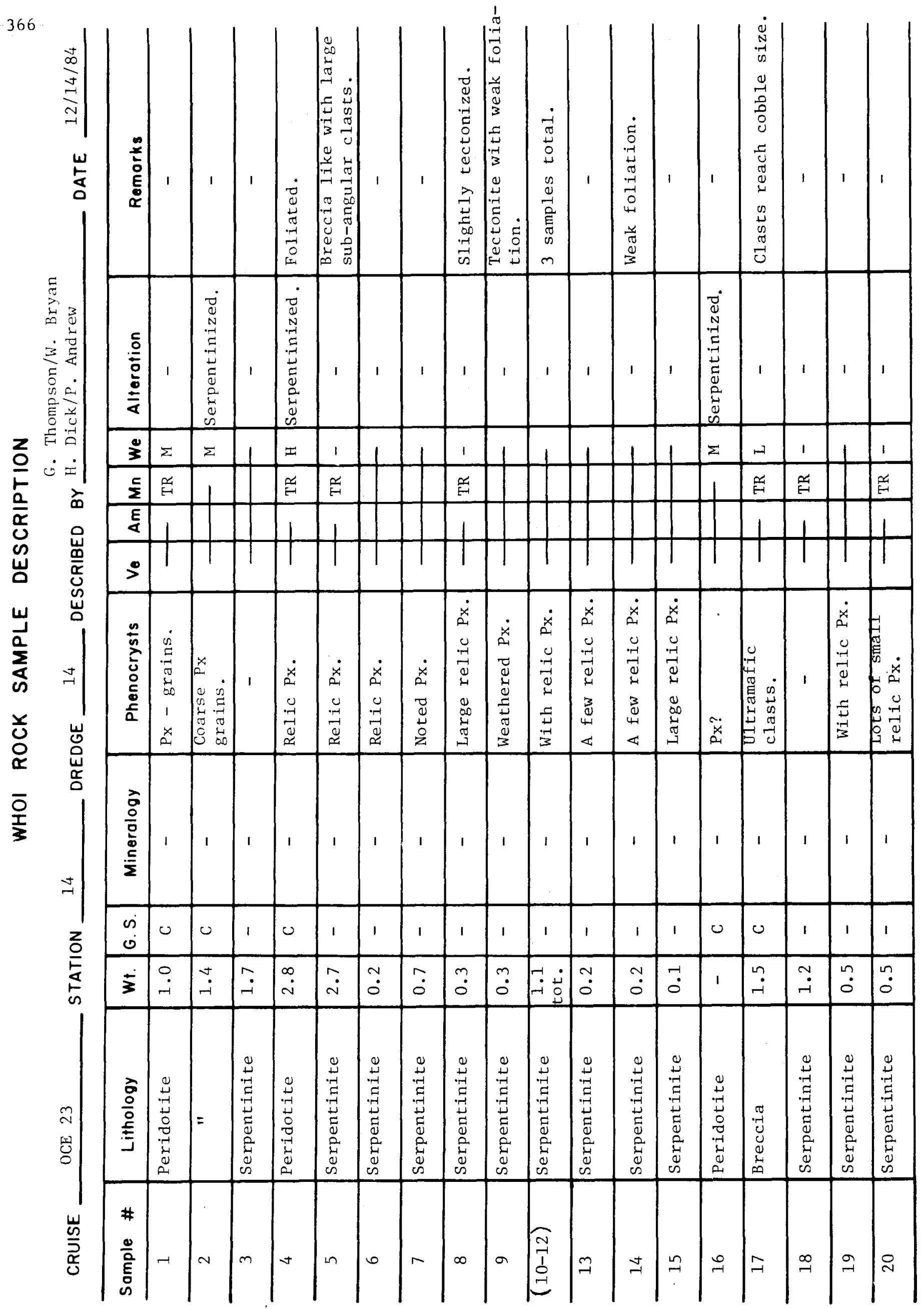




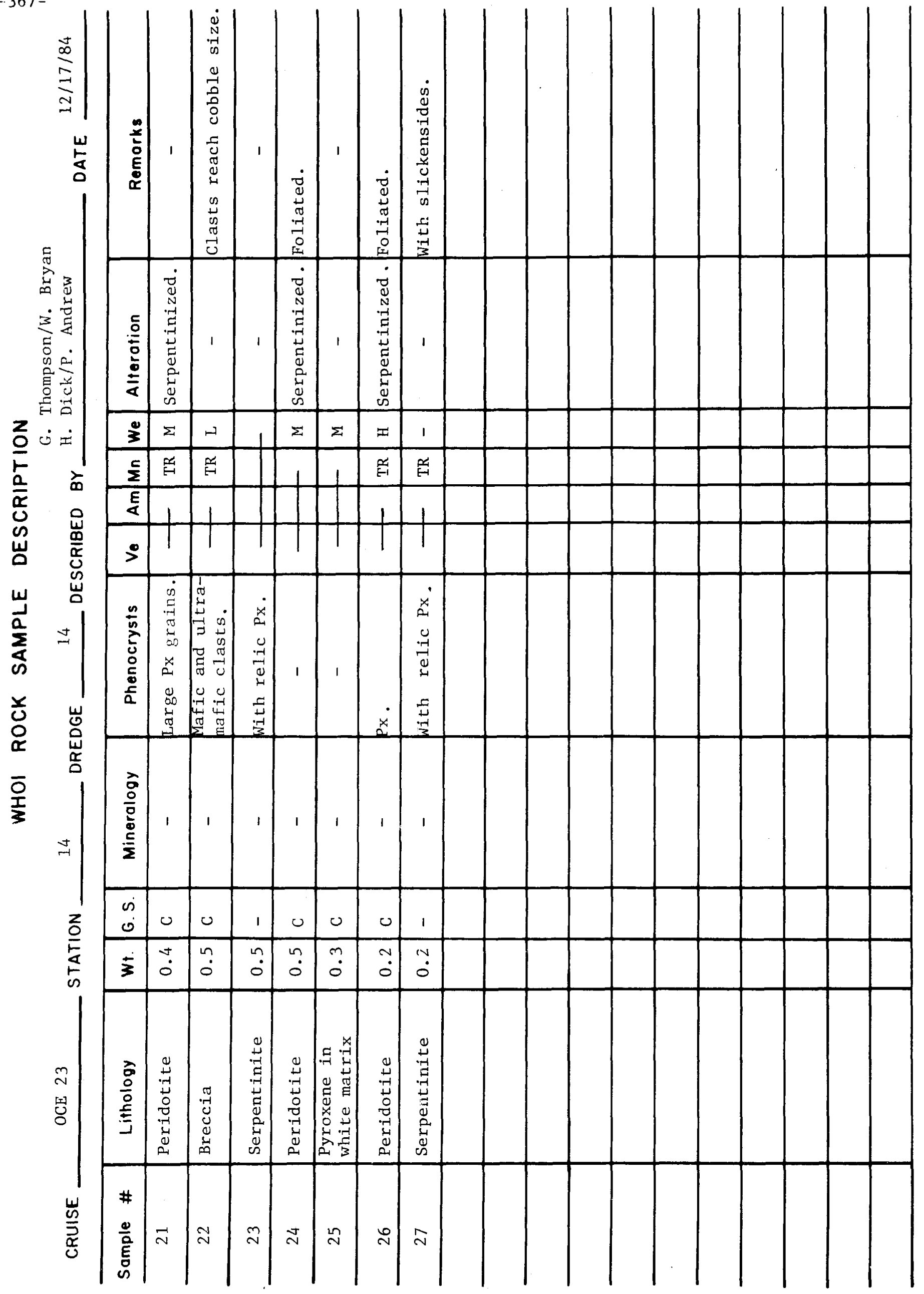




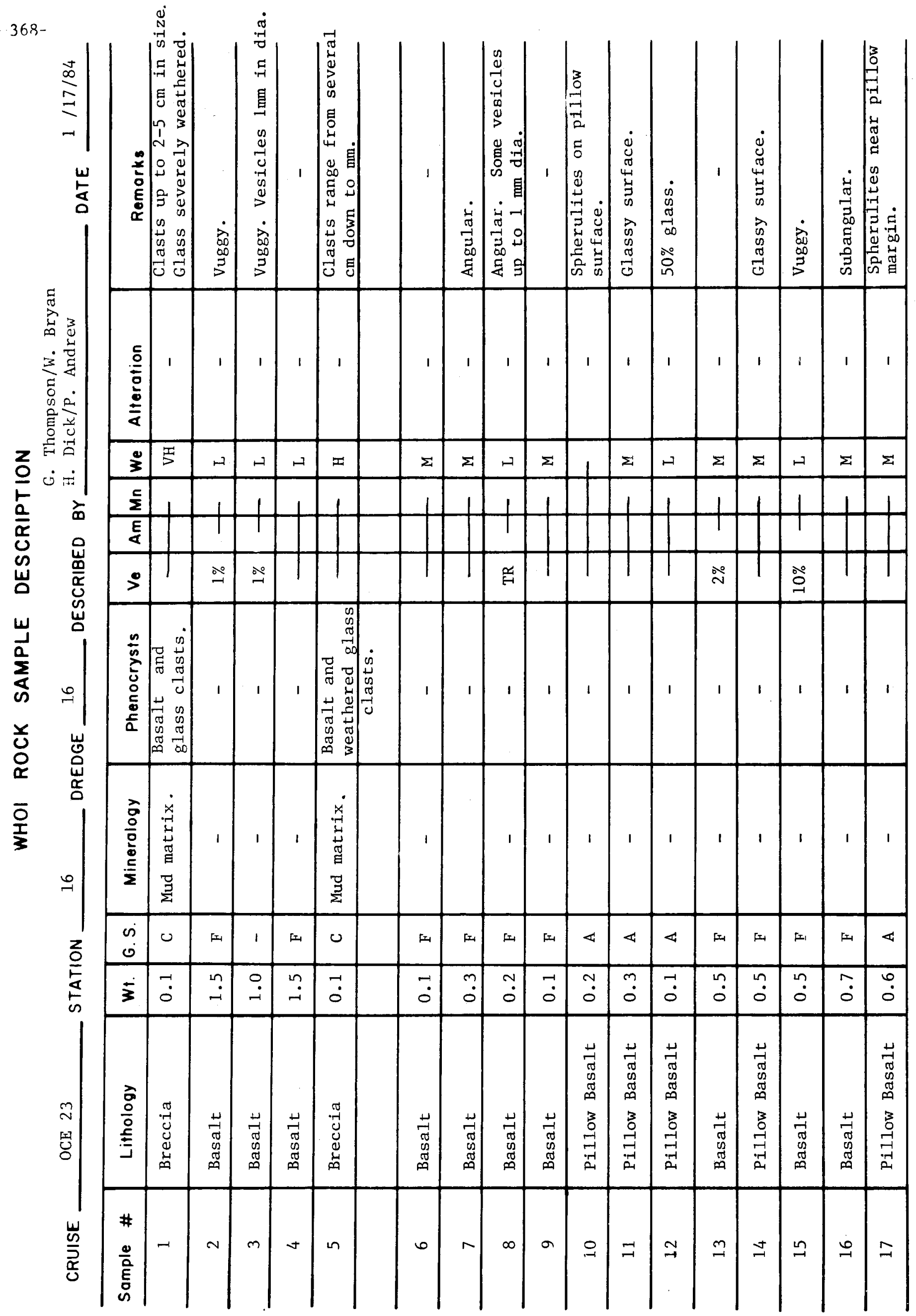




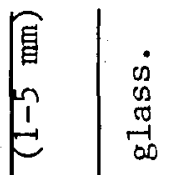

$\ddot{g} \dot{0}$

$\stackrel{-1}{\stackrel{9}{4}}$

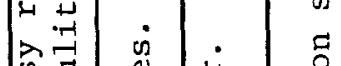

敢

3

उ

든

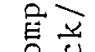

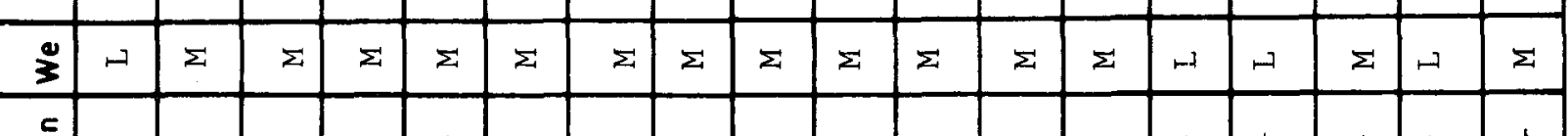

둥

a

哟 耑

岀

\begin{tabular}{|l|}
\hline$\Sigma$ \\
\hline$E$ \\
\hline
\end{tabular}

$>$ ㅇํํ

品

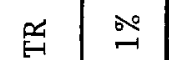

$\stackrel{\mathscr{N}}{\mathrm{H}}$

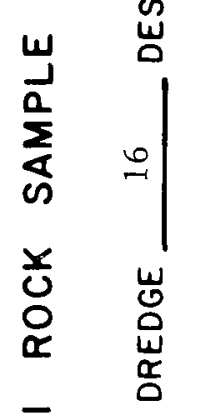

$\frac{\overline{0}}{3}$

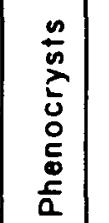

홍

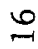

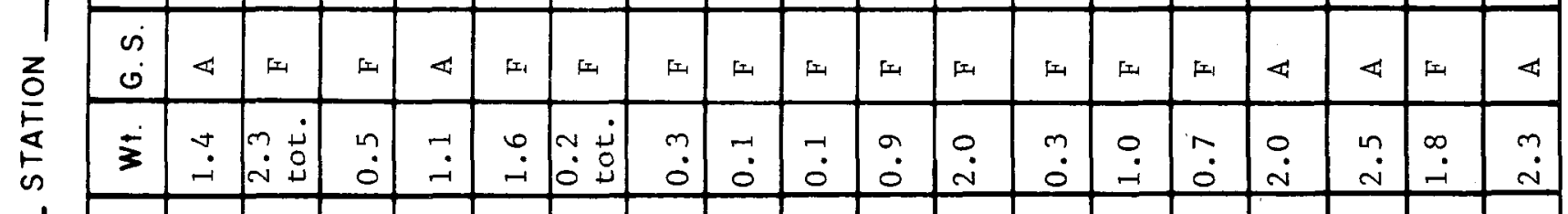

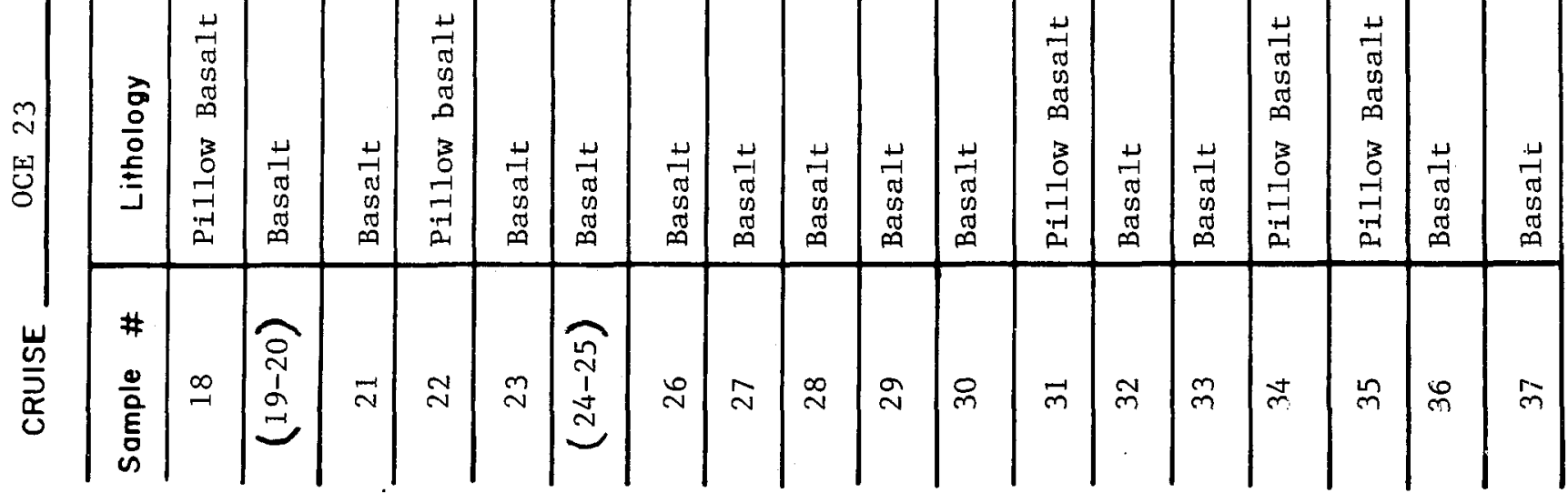


$-370-$
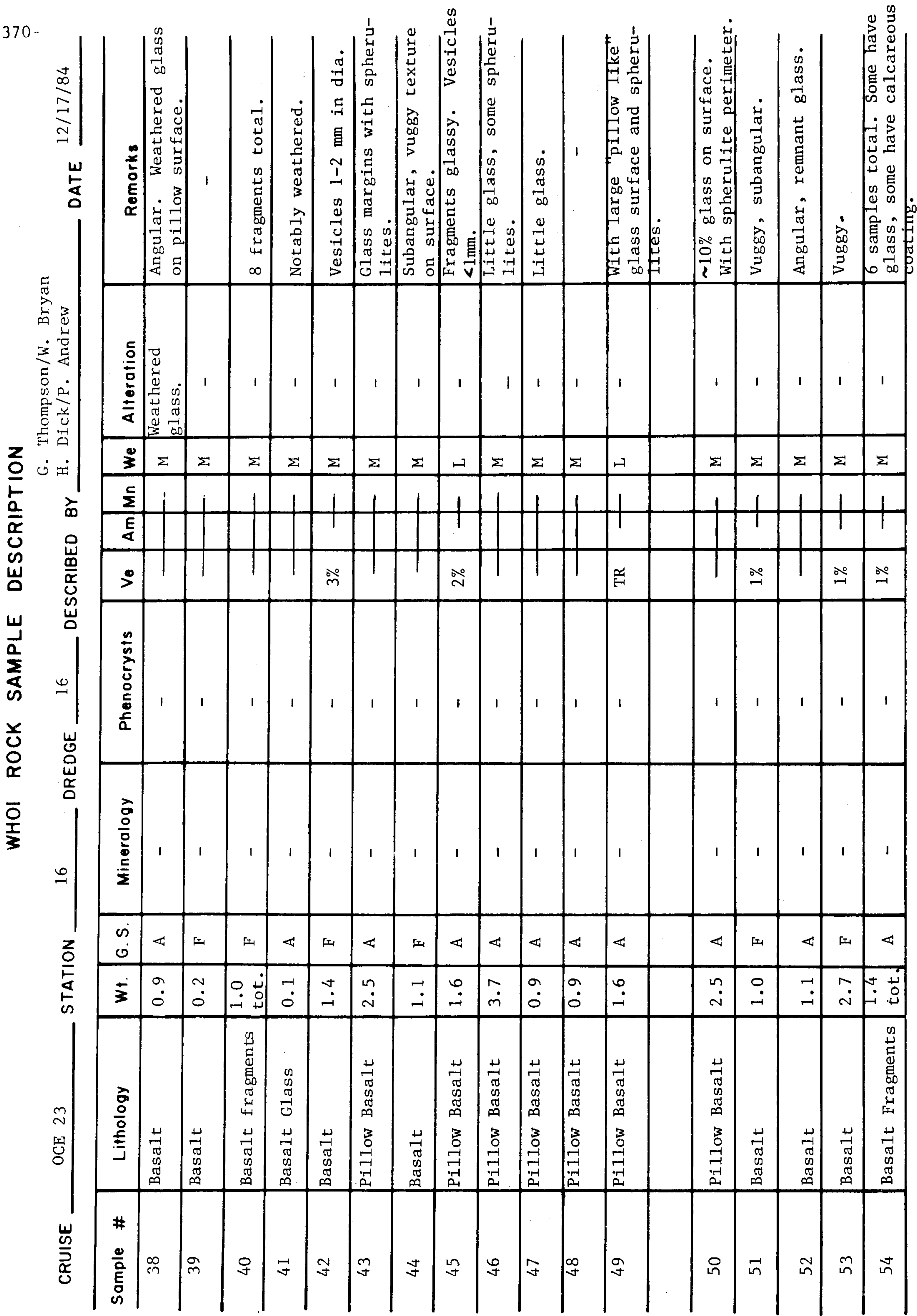


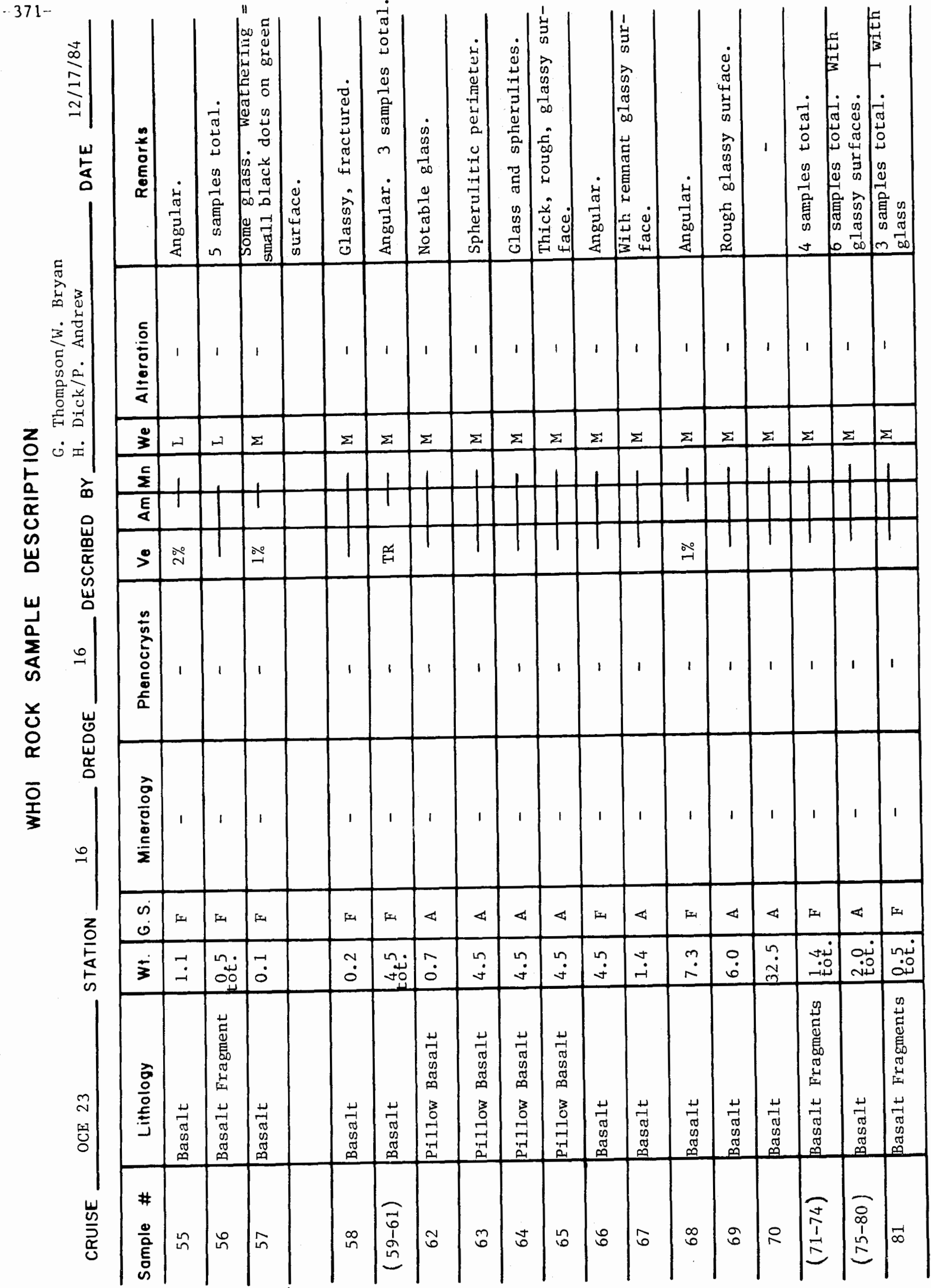




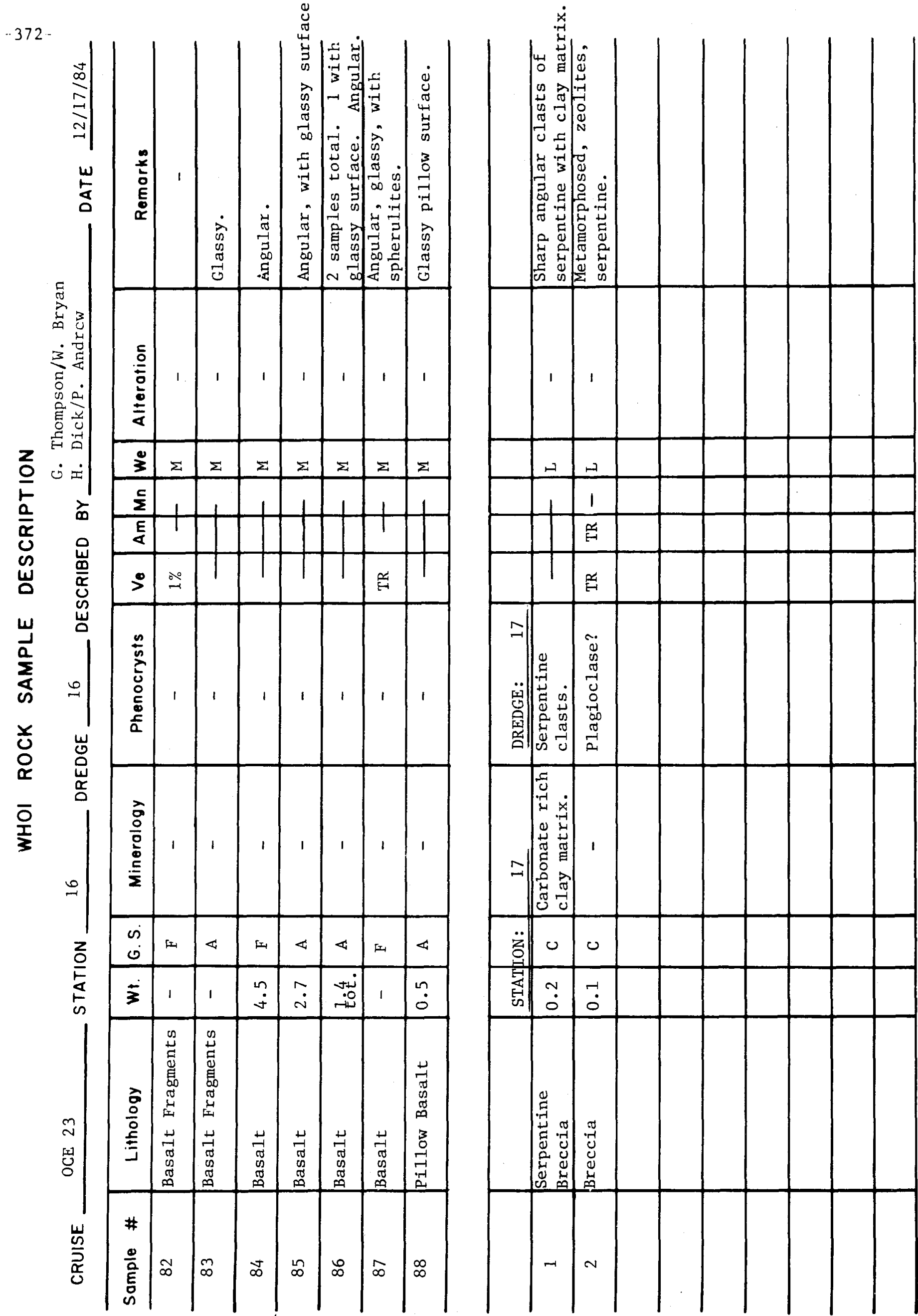



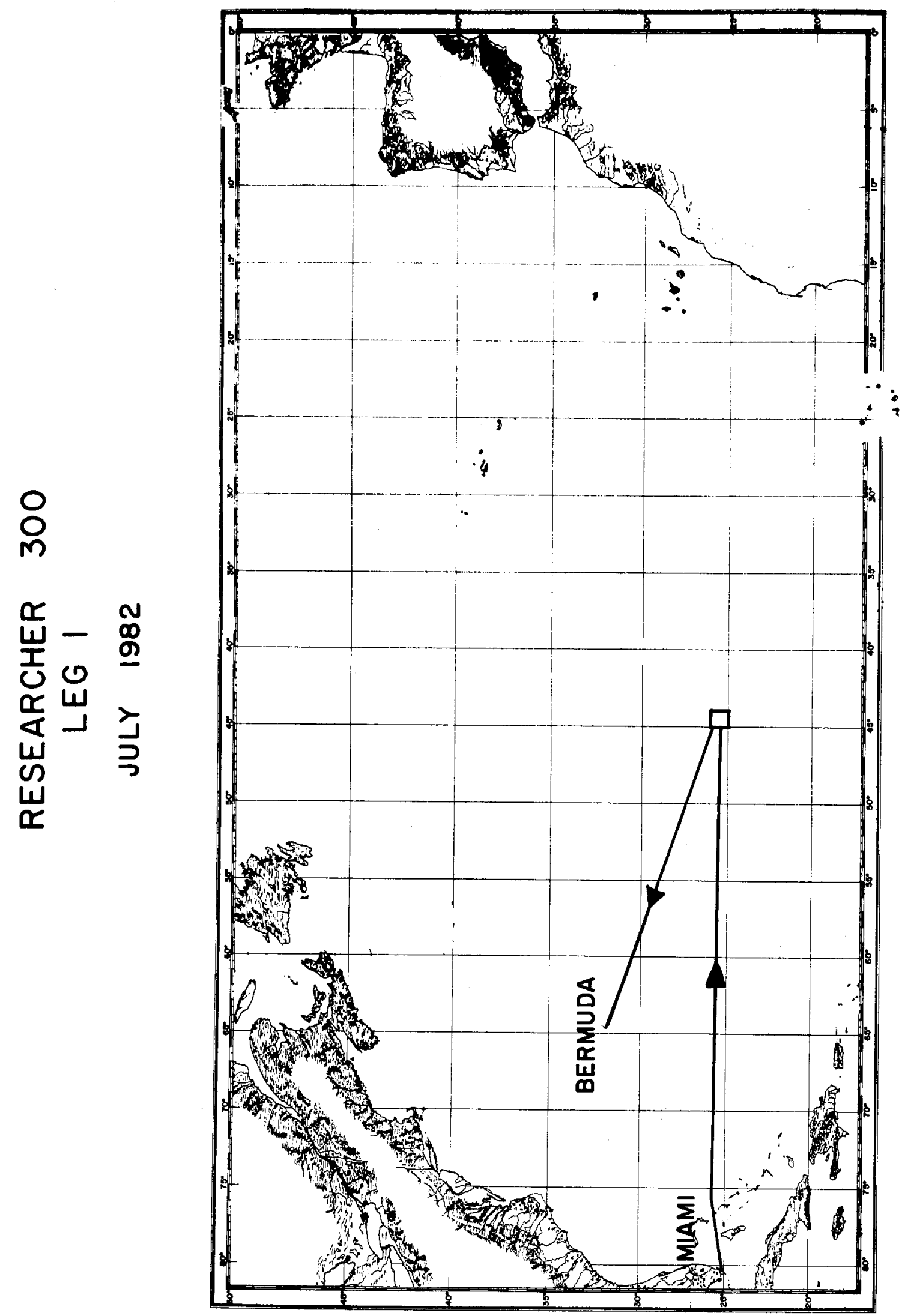


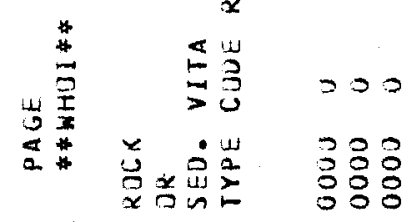

is

$\begin{array}{lll}2 & 0 & 0 \\ 0 & 0 & 0\end{array}$

$\lim _{\rightarrow \rightarrow 1}$

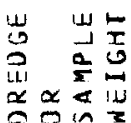

ำ

政

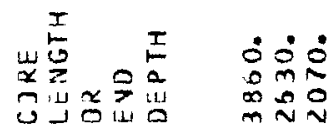

UN然

I $\quad \dot{0} \dot{0}$

号出 $\frac{\alpha}{4}$

以㟧

* 峟㟧

$1 \quad \frac{11}{2}$ :

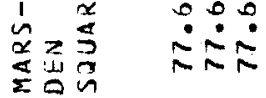

㟧市

4上 $\div 3$

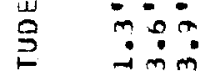

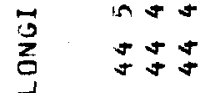

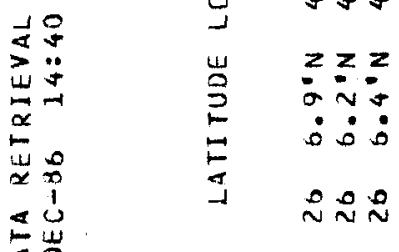

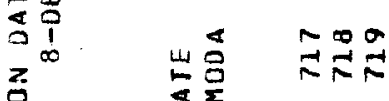

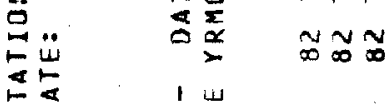

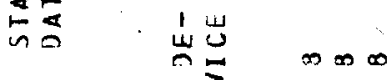

山出

岕岕

z

Е

出 $\leadsto \rightarrow$

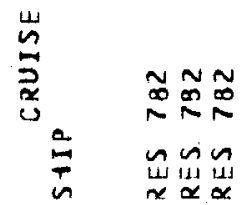




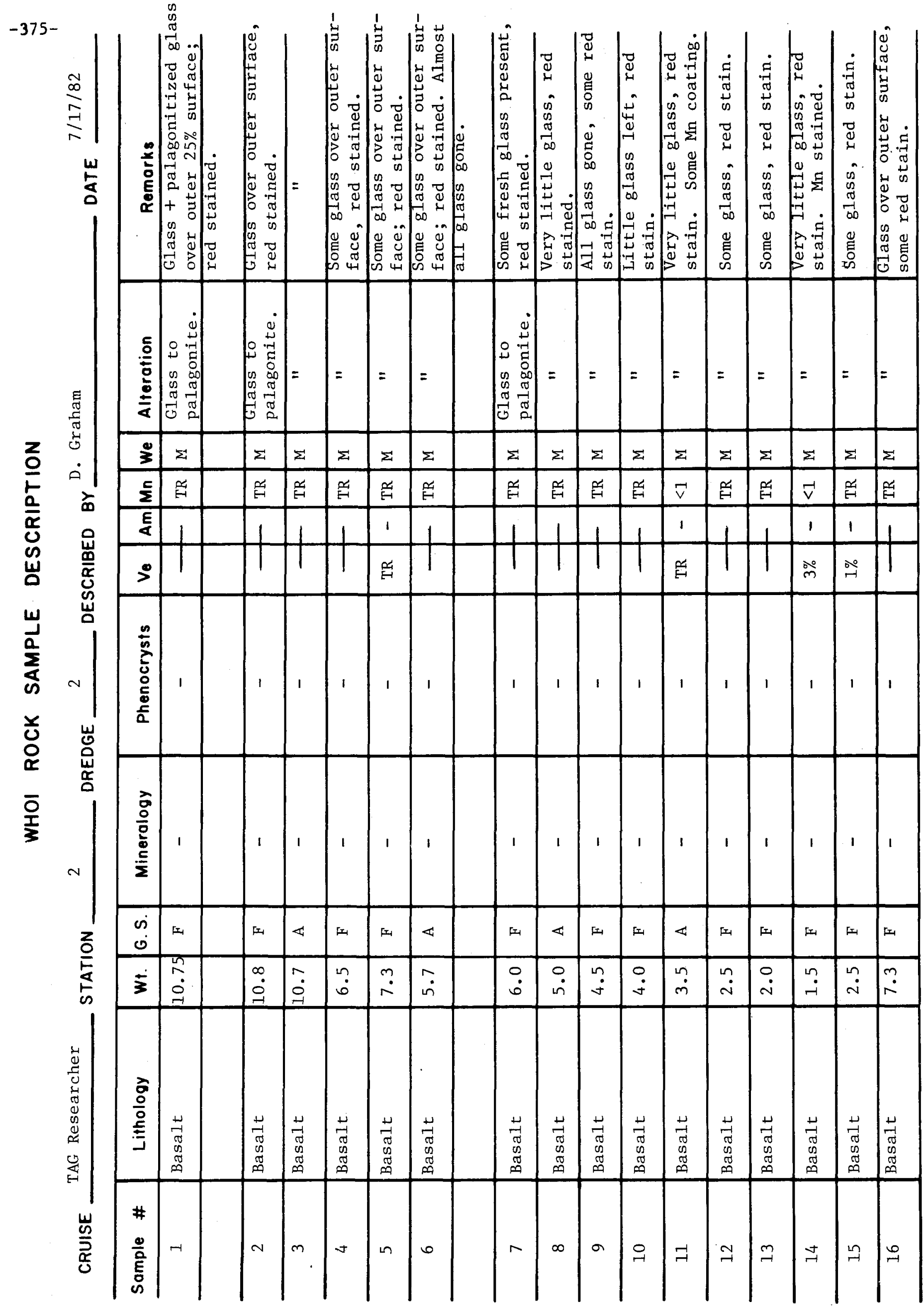



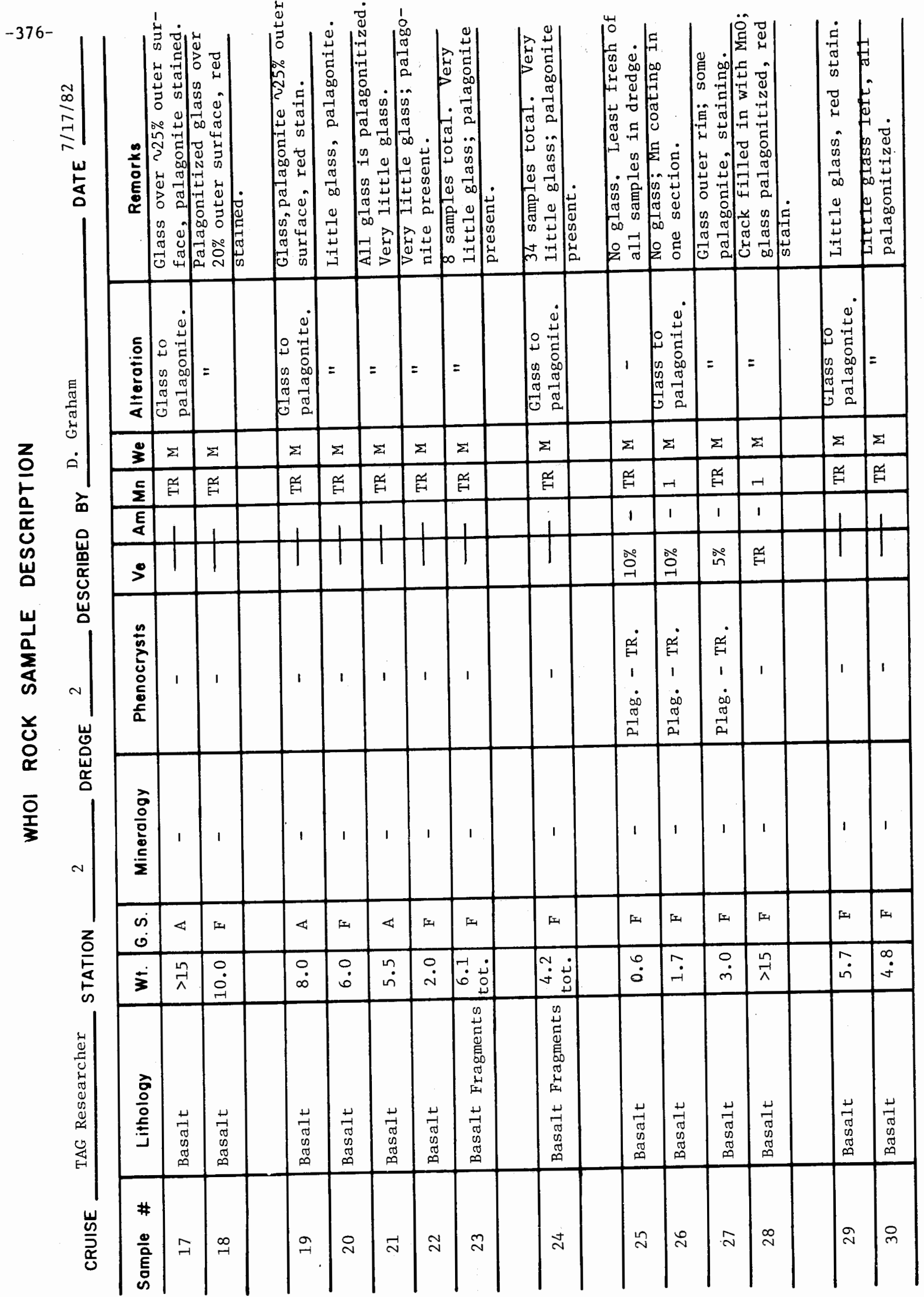


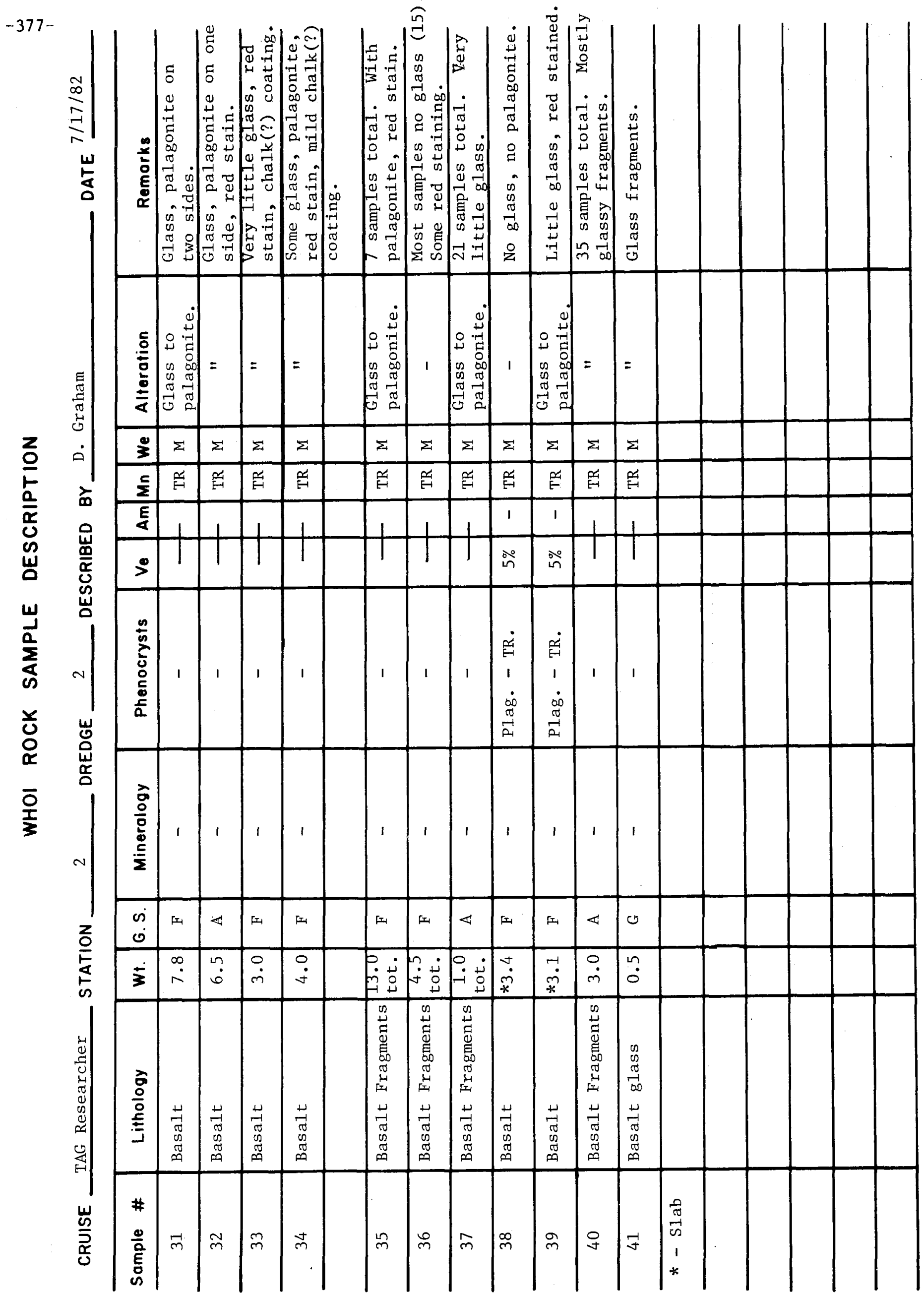




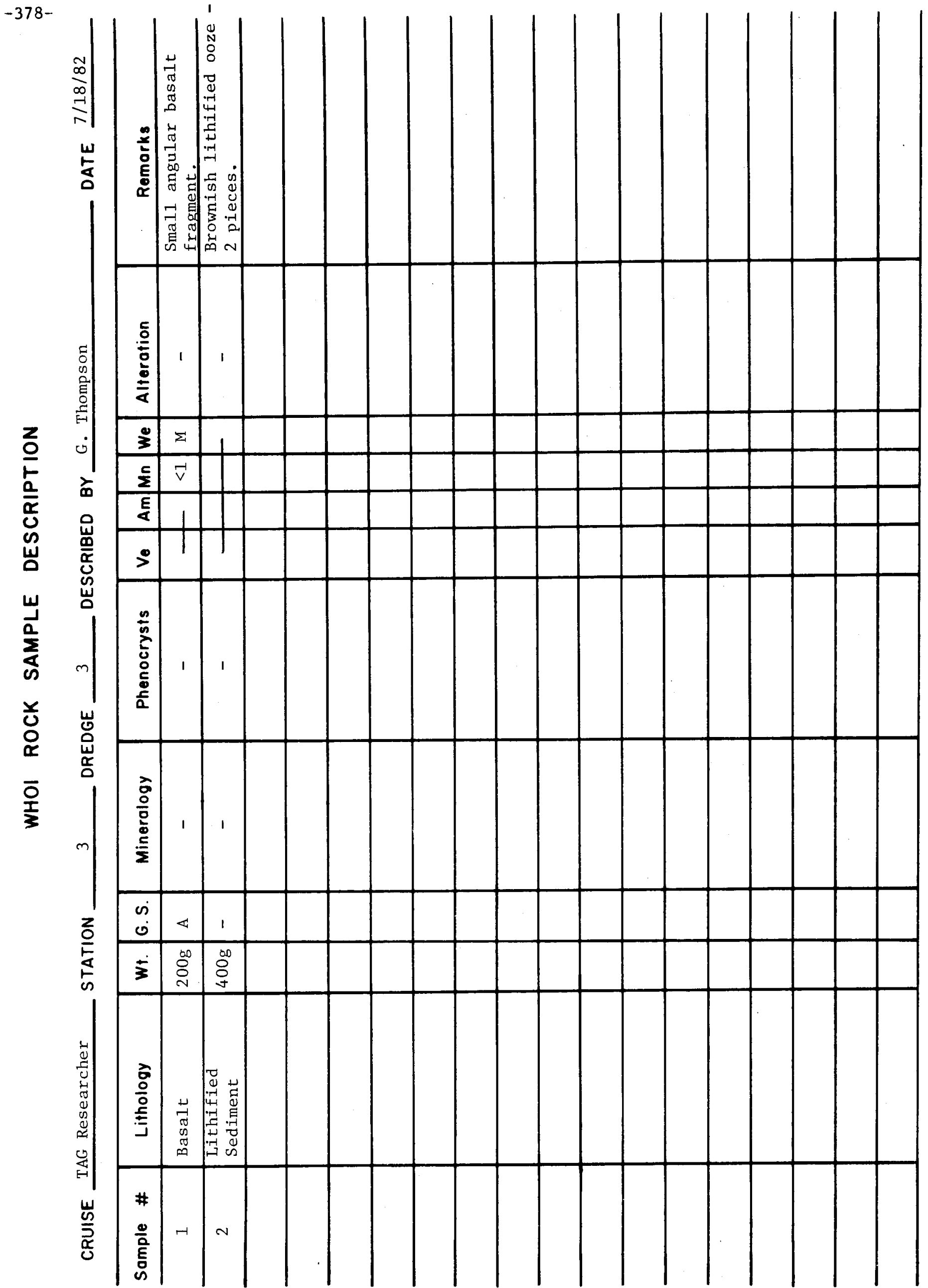




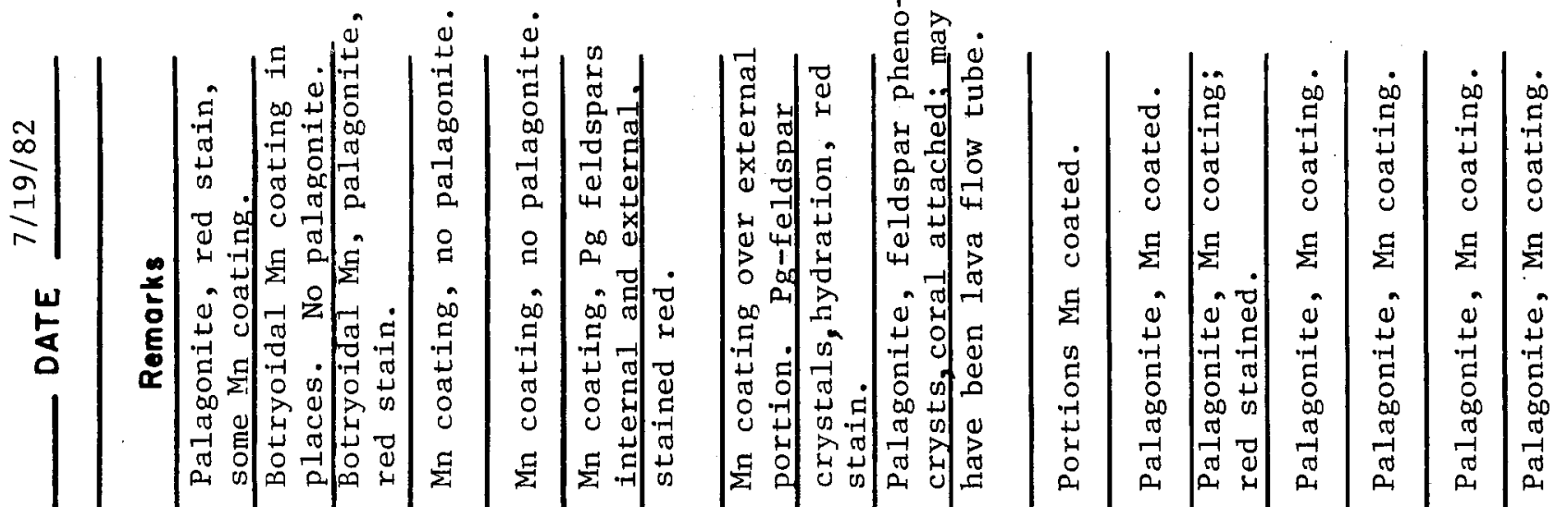

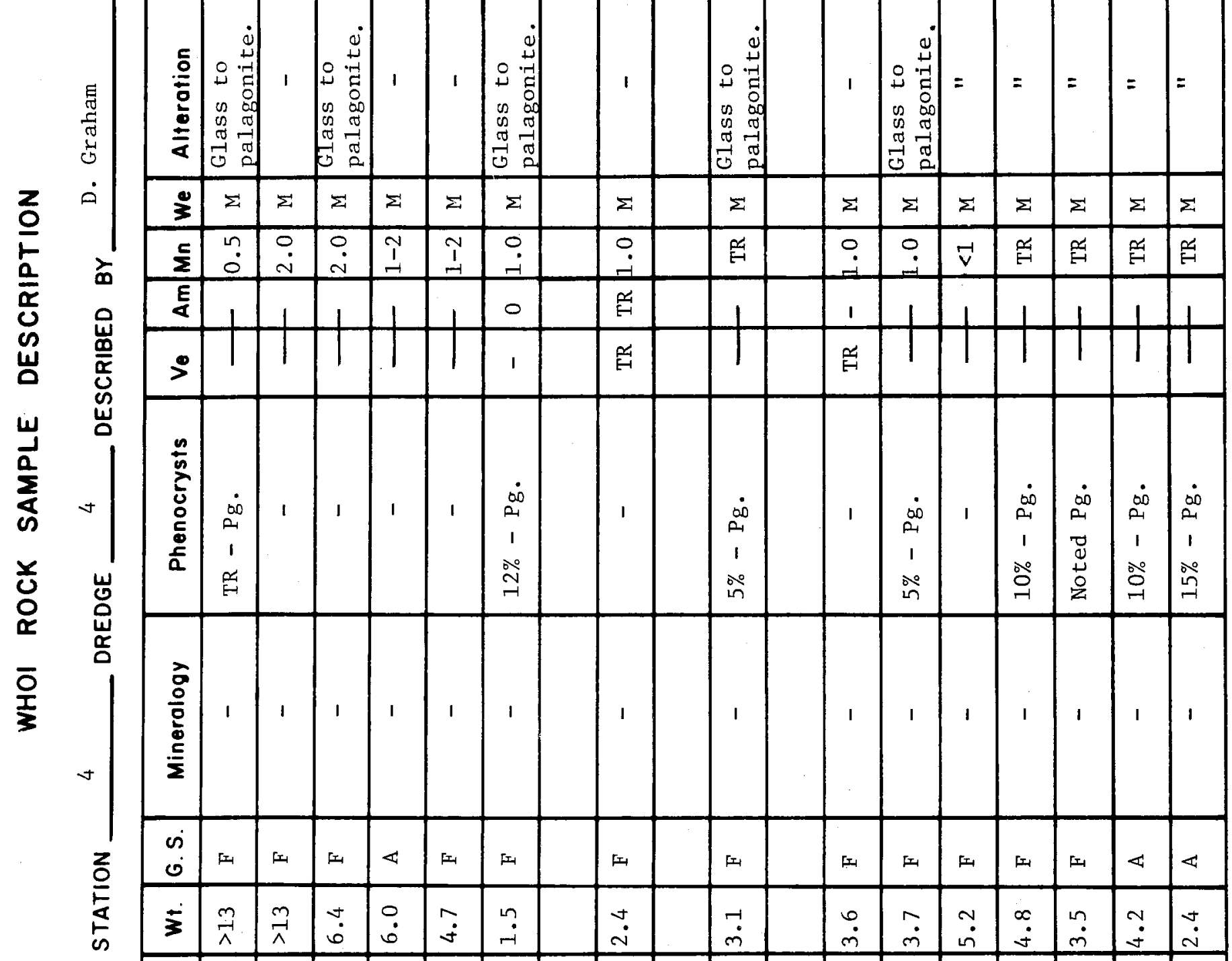

0
0
0
0
0
0
0
0
0
0
0

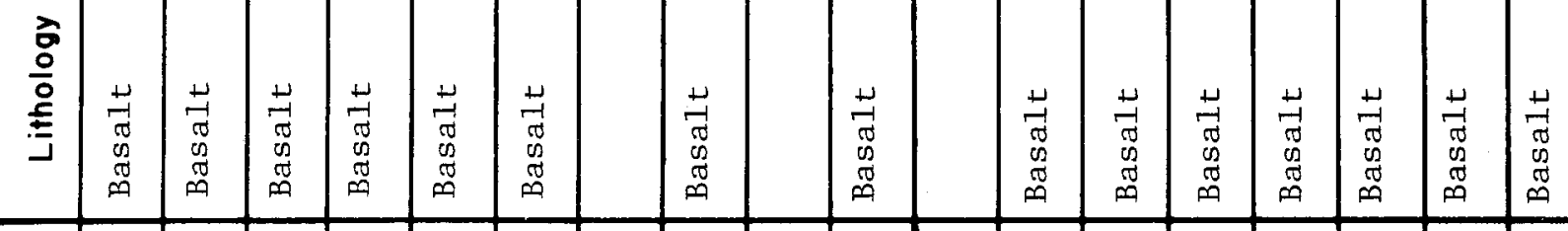

$\frac{w}{\frac{w}{5}}$

\#

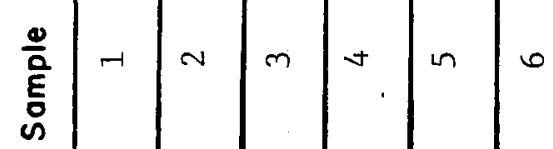

$\infty$

a 

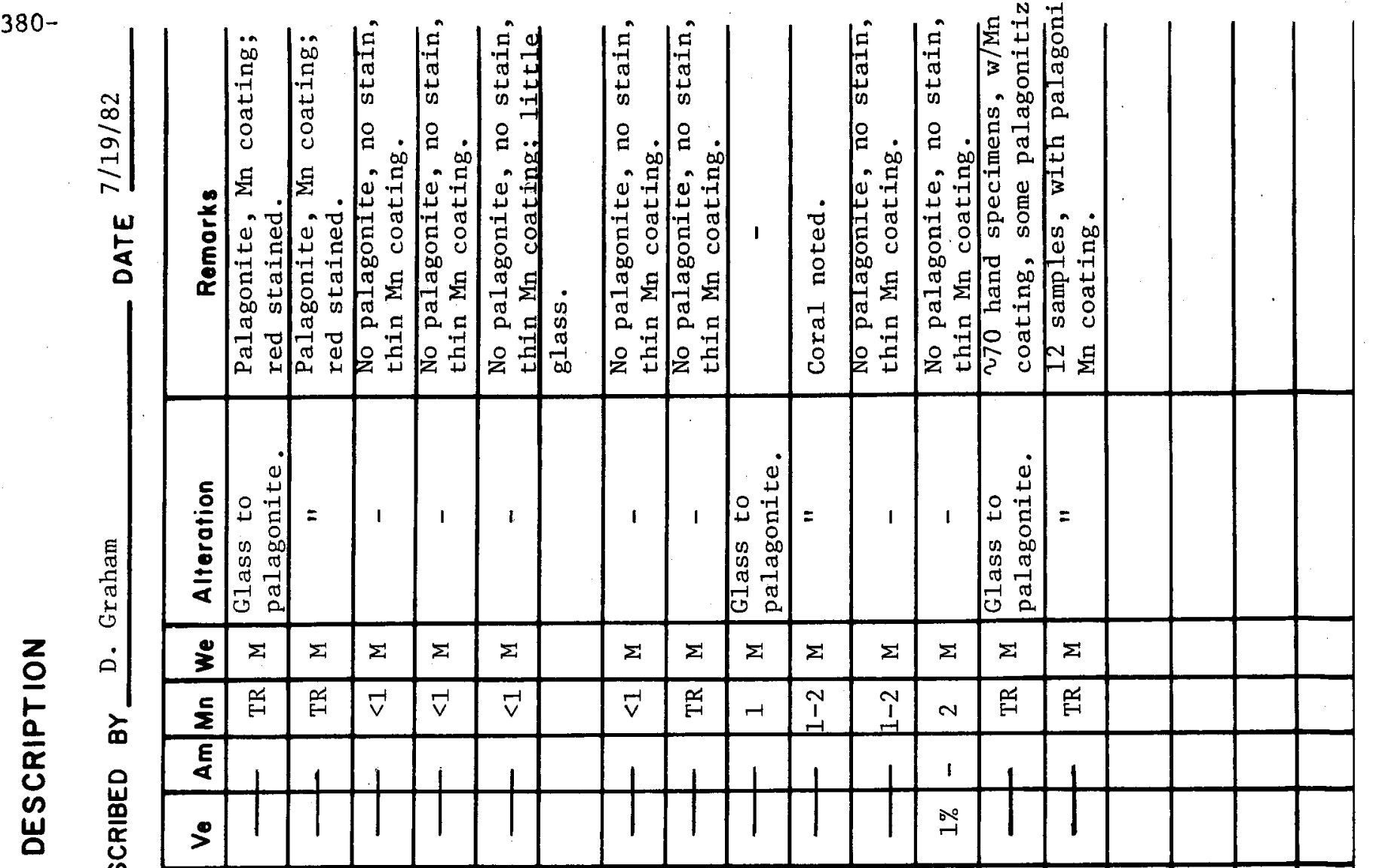

w 岁

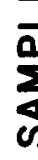

产

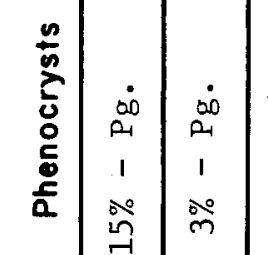

家

岁

운

$\frac{2}{\frac{0}{0}}$

is

is

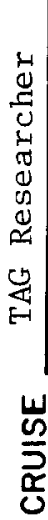

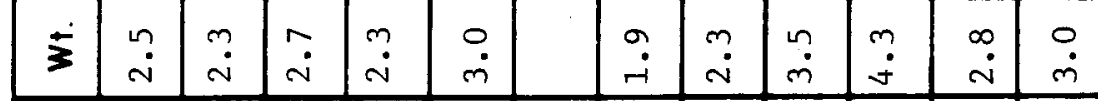

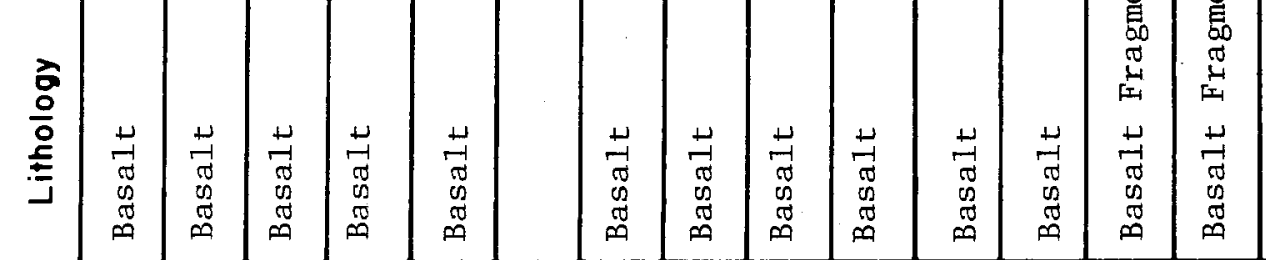

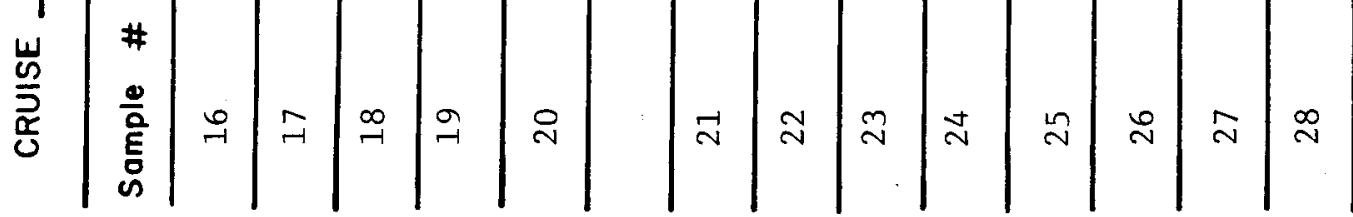




\section{DOCUMENT LIBRARY}

November 21, 1986

\section{Distribution List for Technical Report Exchange}

Institute of Marine Sciences Library

University of Alaska

O'Neill Building

905 Koyukuk Ave., North

Fairbanks, AK

Attn: Stella Sanchez-Wade

Documents Section

Scripps Institution of Oceanography

Library, Mail Code C-075C

La Jolla, CA 92093

Hancock Library of Biology \& Oceanography

Alan Hancock Laboratory

University of Southern California

University Park

Los Angeles, CA 90089-0371

Gifts \& Exchanges

Library

Bedford Institute of Oceanography

P.O. Box 1006

Dartmouth, NS, B2Y 4A2, CANADA

Office of the International

Ice Patrol

c/o Coast Guard R \& D Center

Avery Point

Groton, CT 06340

Library

Physical Oceanographic Laboratory

Nova University

8000 N. Ocean Drive

Dania, FL 33304

NOAA/EDIS Miami Library Center 4301 Rickenbacker Causeway

Miami, FL 33149

Library

Skidaway Institute of Oceanography

P.O. Box 13687

Savannah, GA 31416

Institute of Geophysics

University of Hawaii

Library Room 252

2525 Correa Road

Honolulu, HI 96822

Library

Chesapeake Bay Institute

4800 Atwell Road

Shady Side, MD 20876
MIT Libraries

Serial Journal Room 14E-210

Cambridge, MA 02139

Director, Ralph M. Parsons Laboratory

Room 48-311

MIT

Cambridge, MA 02139

Marine Resources Information Center

Bldg. E38-320

MIT

Cambridge, MA 02139

Library

Lamont-Doherty Geological Observatory

Colombia University

Palisades, NY 10964

Library

Serials Department

Oregon State University

Corvallis, OR 97331

Pell Marine Science Library

University of Rhode Island

Narragansett Bay Campus

Narragansett, RI 02882

Working Collection

Texas A\&M University

Dept. of Oceanography

College Station, TX 77843

Library

Virginia Institute of Marine Science

Gloucester Point, VA 23062

Fisheries-Oceanography Library 151 Oceanography Teaching Bldg.

University of Washington

Seattle, WA 98195

Library

R.S.M.A.S.

University of Miami

4600 Rickenbacker Causeway

Miami, FL 33149

Maury Oceanographic Library

Naval Oceanographic Office

Bay St. Louis

NSTL, MS 39522-5001

ATTN: Code 4601 


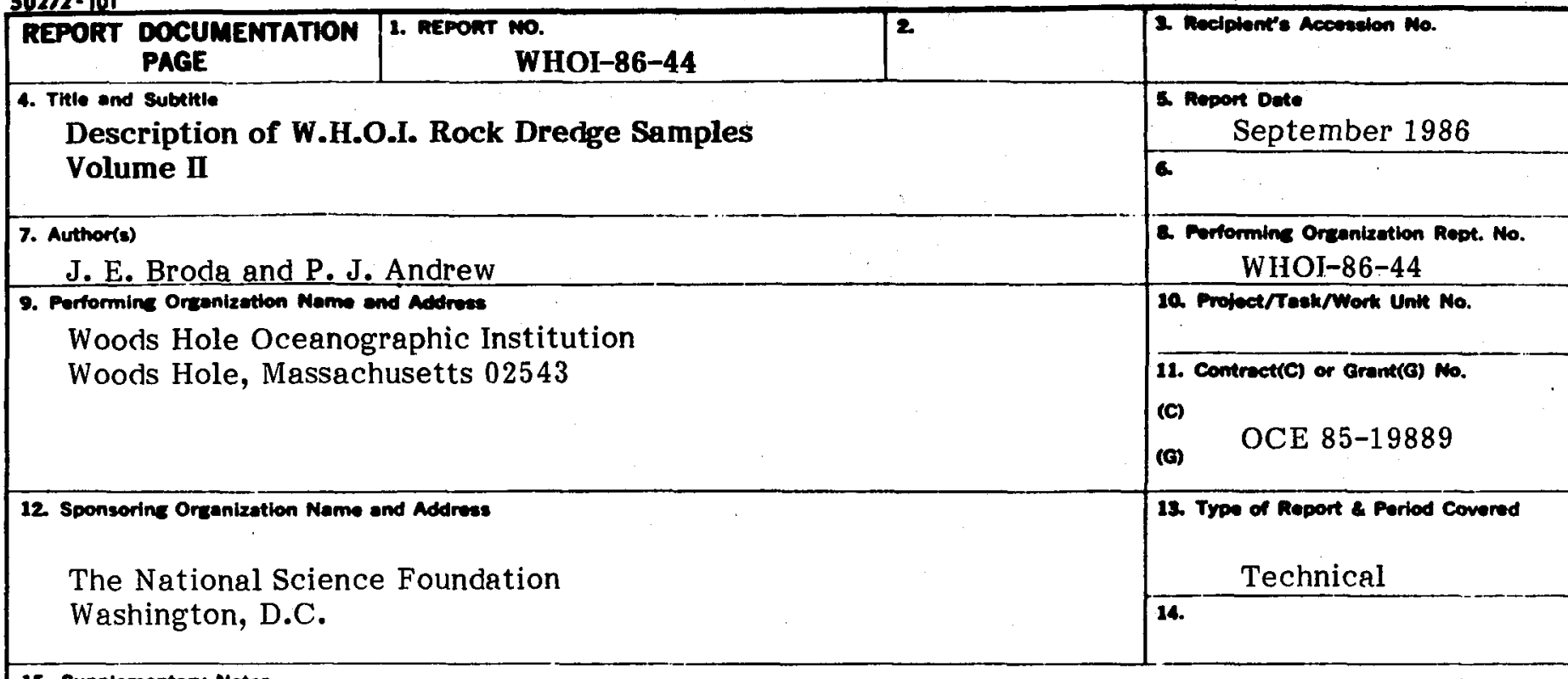

15. Supplementery Notes

This report should be cited as: Woods Hole Oceanog. Inst. Tech. Rept. WHOI-86-44.

16. Abstract (Limit: 200 words)

This report is Volume II in the series of reports entitled "Descriptions of WHOI Rock Dredge Samples". This volume represents the final step in the major effort to catalog and prepare initial descriptions for all rock dredge samples in the W.H.O.I. Sea Floor Samples Collection, and to distribute this information throughout the scientific community. The distribution of this report completes the initial description of the backlog of W.H.O.I. Dredge Samples. The data contained in this volume is an accumulation and transcription of initial descriptions made at sea, along with post-cruise descriptions performed at the lab by the curatorial staff.

Volume II contains individual stations executed during the period 1963 through 1986 . It also presents a digitized listing of all dredge station data for the entire W.H.O.I. Dredge Collection through 1986. The data are sorted by Marsden Squares and can serve as a regional index for all rock descriptions included in Volumes I-III.

17. Document Analysis a. Deserfoton

1. Dredges

2. Rocks

3. Deep Sea Rock Dredge Descriptions

b. Identifiers/Open-Ended Terms

c. COSATI Fleld/Group

18. Avallability statemen:

Approved for publication; distribution unlimited.

\begin{tabular}{|c|c|}
\hline $\begin{array}{c}\text { 19. Securtty Clase (This Report) } \\
\text { UNCLASSIFIED }\end{array}$ & $\begin{array}{c}\text { 21. No. of Pages } \\
380\end{array}$ \\
\hline 20. Socurity Clese (This Poge) & 22. Price \\
\hline
\end{tabular}

See Instructione on Reverse 